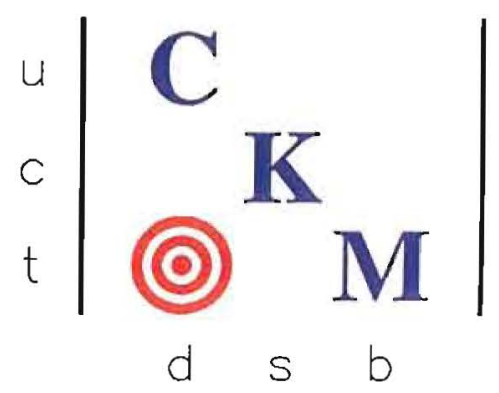

\title{
Charged Kaons at the Main Injector
}

\author{
April 2, 2001
}

\section{A Proposal for a Precision Measurement of the Decay $K^{+} \rightarrow \pi^{+} \nu \bar{\nu}$ and Other Rare $K^{+}$Processes at Fermilab Using the Main Injector}

\author{
J. Frank, S. Kettell, R. Strand \\ Brookhaven National Laboratory, Upton, NY, USA
}

L. Bellantoni, R. Coleman, P.S. Cooper*, T. R. Kobilarcik, A. Kushnirenko, C. Milstene, H. Nguyen, A. R. Pastsiak ${ }^{\dagger}$, E. Ramberg, R. S. Tschirhart, H. B. White, J. Y. Wu Fermi National Accelerator Laboratory, Batavia, IL, USA

G. Britvich, A. V. Inyakin, V. Kurshetsov, L. G. Landsberg,V. Molchanov,

V. Obraztsov, S. I. Petrenko, V. Polyakov, V. I. Rykalin, A. Soldatov, M. M. Shapkin, O. G. Tchikilev, D. Vavilov, O. Yushchenko Institute of High Energy Physics, Serpukhov, Russia

J. Engelfried, A. Morelos

Instituto de Fisica, Universidad Autonoma de San Luis Potosi, Mexico

M. Campbell, R. Gustafson, M. Longo, H. Park

University of Michigan, Ann. Arbor, Michigan 48109

K. Lang

University of Texas at Austin, Austin, Texas 78712

C. Dukes, R. Godang, L. Lu, K. Nelson

University of Virgina, Charlottesville, VA 22901

$\dagger$ Visitor from the Institute for Nuclear Research, Troitsk, Russia

* Spokesman: P.S. Cooper, pcooper@fnal.gov, (630) 840-2629

Web Address: www.fnal.gov/projects/ckm/Welcome.html 
edition history

1st edition submitted to Fermilab April 15, 1998

2st edition submitted to Fermilab April 2, 2001 


\section{SUMMARY}

We are proposing here an experiment to measure the branching ratio of the ultra-rare charged kaon decay $K^{+} \rightarrow \pi^{+} \nu \bar{\nu}$ by observing a large sample of $K^{+} \rightarrow \pi^{+} \nu \bar{\nu}$ decays with small background. Our pliysics goal is to measure the magnitude of the Cabibbo, Kobayashi, Maskawa matrix element $V_{t d}$ with a statistical precision of about $5 \%$ based upon a $\sim 100$ event sample with total backgrounds of less than 10 events. This decay mode is known to be theoretically clean. The only significant theoretical uncertainty in the calculation of this branching ratio is due to the charm contribution. A $10 \%$ measurement of the branching ratio will yield a $10 \%$ total uncertainty on the magnitude of $V_{t d}$.

The experimental technique we are proposing is decay in flight in a $\sim 22 \mathrm{GeV} / \mathrm{c} R \mathrm{RF}$ separated $K^{+}$beam with a kaon flux of $30 \mathrm{MHz}$ and a purity of higher than $70 \% K^{+}$generated with about $5 \times 10^{12} 120 \mathrm{GeV} / c$ protons per spill from the Main Injector. The detector will have redundant measurements for both the $K^{+}$and $\pi^{+}$with conventional high rate magnetic spectrometers and velocity spectrometers based on phototube ring imaging Cherenkov counters for each particle. A hermetic photon veto system capable of rejecting $\pi^{0}$ decays with an inefficiency less than $1.6 \times 10^{-7}$ and a muon range stack capable of rejecting $\mu^{+}$with an inefficiency less than $1 \times 10^{-5}$ are also required. The expected signal acceptance of this apparatus after all cuts will be $\sim 2 \%$. A data collection period of 2 years should yield a 100 event signal over a background of less than 10 events assuming a branching ratio of $1 \times 10^{-10}$.

This new experiment is known as "Charged Kaons at the Main injector", or "CKM".

This document is the second edition of this proposal. The first edition was submitted three years ago (April 15, 1998) and resulted in the approval of an R\&D project to assess the technical feasibility of the experiment. The results of these studies are reported here. Using prototypes, test beam studies and simulations qualified by data we have verified the assertions we liad made in the first edition. Initial prototypes of superconducting RF cavities have been procured and tested at superconducting temperatures. A first full 13 cell cavity is being fabricated now. The $\pi^{0}$ inefficiency of $1.6 \times 10^{-7}$ is achievable using $1 \mathrm{~mm}$ lead, $5 \mathrm{~mm}$ scintillator photon vetoes based upon a combination simulations at low photon energies and estimates based upon data in the high energy regime. A muon range stack prototype was tested in a muon test beam at IHEP Protvino. It achieved the required $\mu^{+}$inefficiency less than $1 \times 10^{-5}$. We have recently completed a full hit level simulation of both the separated beam line and the complete detector. The first and most straightforward reconstruction and analysis of these simulated data already achieve kinematic background rejection levels supportive of what was quoted in the first edition of this proposal

The only significant change to the experiment has been the adoptions of fine pitch, high rate, multi-wire proportional chambers as the detectors used to track the $50 \mathrm{MHz}$ beam. The advantages over the silicon strip detectors which we first proposed are significantly improved time resolution and ease of readout while maintaining a very low level of material in the beam. The University of Virginia group which successfully designed, built and operated such a chamber system in HyperC $C^{P}$ lias joined CKM and will lead this effort. In total the collaboration has grown from 19 physicists at 4 institutions three years ago to 40 physicists at 7 institutions today. This includes a group 
from our sister experiment E949 at Brookhaven. E949 and CKM have joined forces. Several members of CKM from Fermilab and IHEP have joined E949 with the goal of proceeding in a programmatic fashion from the one event signal observed by E787 at Brookhaven through a 5-10 signal events expected in E949 using an upgraded E787 apparatus to CKM's 100 events with a significant measurement of the branching ratio for this ultra-rare decay mode.

The rest of the world of kaon physics has vigorously evolved in the past three years. There are now two approved and funded experiments to observe and measure the branching ratio of $K_{L} \rightarrow \pi^{0} \nu \bar{\nu}$; KOPIO at Brookhaven and E391a At KEK, two approved extensions of the NA48 experiment at CERN to make high sensitivity studies of $K_{S}^{0}$ and $K^{+}$decays and planned programs of high sensitivity kaon studies at both IHEP Protvino and at the new Japanese Hadron Facility (JHF). This community of kaon physicists is now poised to perform a new generation of kaon experiments that can quantitatively challenge the Standard Model. The CKM experiment will be a key player in this world-wide program. 


\section{Contents}

1 Introduction $\quad 21$

2 CKM Physics Program 22

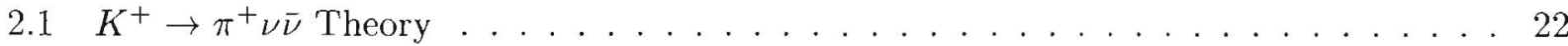

2.1 .1 General Considerations . . . . . . . . . . . . . . . . . 22

$2.1 .2 \quad K^{+} \rightarrow \pi^{+} \nu \bar{\nu}$ decay Probability . . . . . . . . . . . . . . 23

$2.1 .3\left|V_{t d}\right|$ Determination from $K^{+} \rightarrow \pi^{+} \nu \bar{\nu}$ Measurement . . . . . . . . . . 26

2.2 Related $K_{L}^{0}$ and B Measurement and Study of the Prediction of CP-violation Mech-

anisms in the SM and Beyond . . . . . . . . . . . . . . . . 29

2.2 .1 Future data of $K_{L}^{0}$ studies . . . . . . . . . . . . . . . . . . . . . . . . . . . . . . . .

$2.2 .2 \quad$ B-meson experiments and unitarity triangle . . . . . . . . . . . . 30

2.3 Over-constraining the Unitary Condition and Detecting Effects Beyond the SM . . . 31

2.4 Other $K^{+}$Physics . . . . . . . . . . . . . . . . . . . 40

2.4.1 Lepton Flavor Violating Processes in $K^{+}$Decays . . . . . . . . . . . . . 41

$2.4 .2 \mathrm{~T}_{\text {odd }}$ Correlations in $K^{+} \rightarrow \pi^{0} l^{+} \nu_{l} \gamma \ldots \ldots \ldots \ldots \ldots \ldots \ldots$

2.4.3 Search for Direct CP Violation in Hadronic $K^{ \pm}$decays . . . . . . . . . . 45

2.4.4 Low Energy Hadronic Physics . . . . . . . . . . . . . . . . . . . . . 46

2.4 .5 Conclusion . . . . . . . . . . . . . . . . . . 48

3 The Experimental Method $\quad 49$

3.1 Experimental Overview . . . . . . . . . . . . . . . . . . . . . . . . 49

3.2 Background Sources . . . . . . . . . . . . . . . . . . . . . . . 49

3.3 Beam Issues . . . . . . . . . . . . . . . . . . . . . . . . . . . . . . . 52

3.4 Detector Requirements . . . . . . . . . . . . . . . . . . . 52

3.4 .1 The Momentum Spectrometers . . . . . . . . . . . . . . . 52

3.4 .2 The Velocity Spectrometers . . . . . . . . . . . . . . . . . 53

3.4.3 Components of the $M_{\text {miss }}^{2}$ Resolution Function. . . . . . . . . . . . . . 55

3.4.4 The Kaon Entrance Angle Tracker System (KEAT) . . . . . . . . . . . . . 56

3.4 .5 The Beam Interaction Veto System (BIVS) . . . . . . . . . . . . 57

3.4 .6 The Photon Veto Systems . . . . . . . . . . . . . . . . . . . . 57

3.4 .7 The Muon Veto System . . . . . . . . . . . . . . . . . 6 63

3.5 Stopped versus In-flight Kaon Decays . . . . . . . . . . . . . . 65

4 The Separated Charged Kaon Beam $\quad 68$

4.1 Design Motivations for the CKM Beam . . . . . . . . . . . . . . . . 68

4.1 .1 CKM Beam Requirements . . . . . . . . . . . . . . . . . . 68

4.1.2 Design Motivations for a Debunched Beam . . . . . . . . . . . . . . 68

4.1.3 Selection of $K^{+}$beam and Negative Beam Options . . . . . . . . . . . 68 .

4.1.4 Comparison to other RF separated beams . . . . . . . . . . . . 68

4.2 Separated Beam Design . . . . . . . . . . . . . . . . . . . . . . . 69

4.2 .1 How RF Separation Works . . . . . . . . . . . . . . . . . . . 69

4.2 .2 Overview of the Separated Kaon Beam Design . . . . . . . . . . . . . 69

4.2.3 Stage-1: Collection and Preparation for RF Separation . . . . . . . . . . . . 70

4.2 .4 Stage-2: RF Separation . . . . . . . . . . . . . . . . . 72

4.2 .5 Stage-3: Transport to the Experiment . . . . . . . . . . . . . 73

4.2 .6 Beam Purity . . . . . . . . . . . . . . . . . . . . 77 
4.2 .7 Shielding . . . . . . . . . . . . . . . . . . . . . . 78

4.2 .8 Alignment . . . . . . . . . . . . . . . . . . . . . . . 78

4.2 .9 Tolerance to RF parameters . . . . . . . . . . . . . . . . . 79

4.3 The Superconducting RF Separator System . . . . . . . . . . . . . . . . . 79

4.3 .1 Cavity Design . . . . . . . . . . . . . . . . . . . . 79

4.3 .2 Review of R\&D . . . . . . . . . . . . . . . . . . . 82

4.4 GEANT Calculated Rates and Backgrounds . . . . . . . . . . . . . . 85

4.4 .1 Introduction . . . . . . . . . . . . . . . . 85

4.4 .2 Beam Rates and Purity . . . . . . . . . . . . . . . . 86

4.4.3 Effect of clean-up bends after beam stopper . . . . . . . . . . . . . 86

4.4.4 Muon shielding along the beamline. . . . . . . . . . . . . . . 88

4.5 Summary of Beam Section . . . . . . . . . . . . . . . . . 88

5 The Detector Systems $\quad 96$

5.1 The Upstream Magnetic Spectrometer . . . . . . . . . . . . . . . . . . . . . 96

5.1 .1 Description of the Wire Chambers . . . . . . . . . . . . . . . 97

5.1 .2 Performance of similar Hyper $C^{P}$ chambers . . . . . . . . . . . . . . . . 100

5.1 .3 Expected Performance of the UMS . . . . . . . . . . . . . . . . 101

5.1 .4 Wire Chamber Fabrication . . . . . . . . . . . . . . . . . 104

5.1 .5 Wire Chamber Electronics . . . . . . . . . . . . . . . . . . 105

5.2 The Beam Time Stamp Module . . . . . . . . . . . . . . . . . . . . . 108

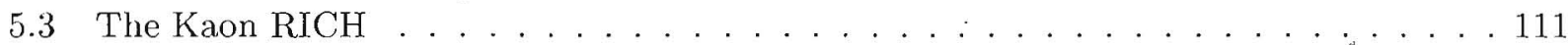

5.3 .1 Experience from the SELEX RICH . . . . . . . . . . . . . 111

5.3 .2 Further Studies with SELEX RICH data . . . . . . . . . . . . . . . 113

5.3 .3 The Kaon RICH . . . . . . . . . . . . . . . . . . . . . . . . . 114

5.3.4 Resolution of the Kaon RICH for different Gases . . . . . . . . . . . . . . . 114

5.3 .5 Kaon RICH readout system . . . . . . . . . . . . . . . . . . . 115

5.4 The Kaon Entrance Angle Tracker System . . . . . . . . . . . . . . . . . . . . 124

5.5 The Beam Interaction Veto System . . . . . . . . . . . . . . . . 126

5.5 .1 BIVS Design Considerations. . . . . . . . . . . . . . . 126

5.6 The Vacuum Veto System. . . . . . . . . . . . . . . . . . . 126

5.6 .1 The vacuum vessel. . . . . . . . . . . . . . . . . . . 126

5.6 .2 The vacuum veto system stations. . . . . . . . . . . . . . . . . 127

5.6 .3 The vacuum veto system station module. . . . . . . . . . . . . . . 127

5.6 .4 Pumping system requirements. . . . . . . . . . . . . . . . . . . 132

5.6 .5 Scintillator studies. . . . . . . . . . . . . . . . . . . . . . . 133

5.6 .6 Efficiency requirement for charged particles. . . . . . . . . . . . . 134

5.6 .7 Efficiency studies of low energy photons $(<20 \mathrm{MeV}) \ldots \ldots \ldots \ldots$

5.6.8 Effect of accidental activity on the detection efficiency of low energy photons. 135

5.6.9 Efficiency studies of medium energy photons $(20 \mathrm{MeV}-1000 \mathrm{MeV})$. . . 137

5.6 .10 Theoretical limits of high energy efficiency. . . . . . . . . . . . . 138

5.6 .11 Practical concerns of ligh energy efficiency. . . . . . . . . . . . . . . 142

5.6 .12 VVS Hermetic design strategy. . . . . . . . . . . . . . . . . 142

5.6 .13 Efficiency studies of high energy electrons. . . . . . . . . . . . . . . 143

5.6.14 Efficiency monitoring and measurement strategies. . . . . . . . . . . . . . 144

5.7 Downstream Magnetic Spectrometer . . . . . . . . . . . . . . . . 147

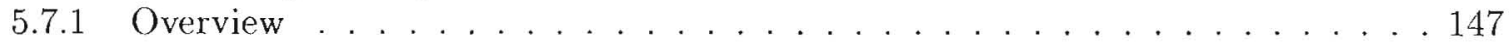

5.7 .2 Conceptual Design . . . . . . . . . . . . . . . . . . 148 
5.7 .3 Experience with Other Straw Systems . . . . . . . . . . . . . . . . . 149

5.7 .4 Straw Properties Under Vacuum . . . . . . . . . . . . . . . . . . 150

5.7 .5 DMS Simulation . . . . . . . . . . . . . . . . . . 152

5.7.6 The BNL-871 Momentum Reconstruction . . . . . . . . . . . . . . 157

5.8 The Pion RICH . . . . . . . . . . . . . . . . . . . . . 161

5.8 .1 Pion RICH readout system . . . . . . . . . . . . . . . . . 164

5.9 The Forward Veto Systems. . . . . . . . . . . . . . . . . . . . 166

5.9 .1 The PHENIX shashlik calorimeter. . . . . . . . . . . . . . 166

5.9 .2 The KTeV CsI calorimeter. . . . . . . . . . . . . . . . . . 167

5.9 .3 Studies of $\pi^{+}-\gamma$ separation performance. . . . . . . . . . . . . . 170

5.9.4 Impact of FVS energy and position resolution on measuring detector photon veto efficiencies with $K^{+} \rightarrow \pi^{+} \pi^{0}$ decays. . . . . . . . . . . . . 172

5.9 .5 Benefits and liabilities of longitudinal segmentation. . . . . . . . . . 177

5.9 .6 Other FVS technologies being considered. . . . . . . . . . . . . . 177

5.10 The Muor Veto System ． . . . . . . . . . . . . . . . . . . 177

5.10 .1 MVS prototype test beam results . . . . . . . . . . . . 177

5.10 .2 The Muon Veto System prototype . . . . . . . . . . . . . 178

5.10 .3 Analysis . . . . . . . . . . . . . . . . . . . . . . . . . . . . . . . . . . . . . . . . . . . . . . . . . .

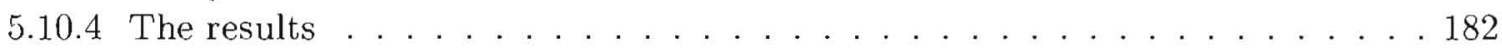

5.10 .5 Further tests . . . . . . . . . . . . . . . . . . . . 184

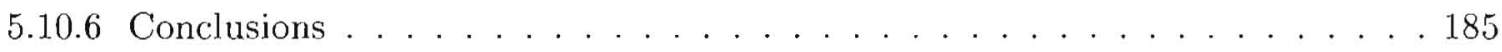

5.11 The Conversion Veto Plane . . . . . . . . . . . . . . . 185

5.12 The Beam Dump . . . . . . . . . . . . . . . . . . . . 186

6 The Trigger and Data Acquisition Systems $\quad 187$

6.1 Experiment Requirements on DAQ and Trigger Electronics . . . . . . . . . . 187

6.2 Architecture of CKM DAQ and Trigger System . . . . . . . . . . . . . . 188

6.2 .1 Front End . . . . . . . . . . . . . . . . . . . . . . . . . . 192

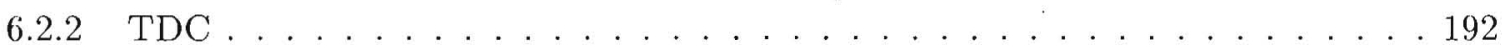

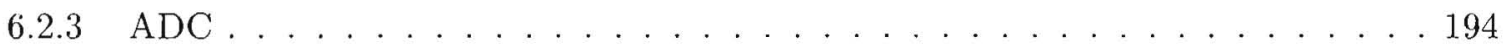

6.3 The Trigger System . . . . . . . . . . . . . . . . . . . . 194

6.3 .1 The Level 1 Trigger . . . . . . . . . . . . . . . . . . . . 195

6.3 .2 The Level 2 and the Level 2.5 Trigger . . . . . . . . . . . . . 196

6.3 .3 The Level 3 Trigger . . . . . . . . . . . . . . . . . . . . 197

6.4 Physical Structure of CKM DAQ and Trigger System . . . . . . . . . . 197

6.5 Detector Performance Monitoring . . . . . . . . . . . . . . . . . 199

$7 K^{+} \rightarrow \pi^{+} \nu \bar{\nu}$ Signal Acceptance and Background Analysis. $\quad 200$

7.1 Summary of Detector Changes Since the First Edition . . . . . . . . . . . . . . 201

7.2 Overview of the CKM_GEANT Detector Simulation. . . . . . . . . . . . . . 201

7.2 .1 Event Reconstruction in CKM_GEANT . . . . . . . . . . . . . 203

7.2.2 CKM_GEANT $K^{+} \rightarrow \pi^{+} \nu \bar{\nu}$ Signal Selection Cuts. . . . . . . . . . . . . . . . 204

7.2 .3 Developments in the $K^{+} \rightarrow \pi^{+} \nu \bar{\nu}$ Signal Acceptance. . . . . . . . . . . . 206

7.2.4 CKM_GEANT Studies of the $K^{+} \rightarrow \pi^{+} \pi^{0}$ Background Rejection. . . . . . . 207

7.2.5 Additional Kaon Decay Backgrounds Considered. . . . . . . . . . . . . . . . 210

7.3 Developments in Bearn Interaction Backgrounds. . . . . . . . . . . . . . . . 210

7.4 Summary of Global Efficiency and Required Running Time. . . . . . . . . . . . 217

7.4 .1 Estimated Yield of a Two-Year Run. . . . . . . . . . . . . 218 
7.5 Summary of Signal and Background Studies. . . . . . . . . . . . . . 218

8 The Defensive Play Book $\quad 219$

8.1 Measuring the Backgrounds . . . . . . . . . . . . . . . . . . 219

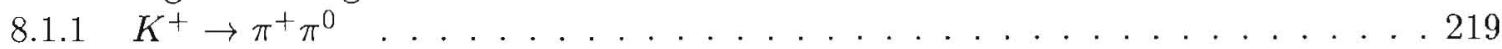

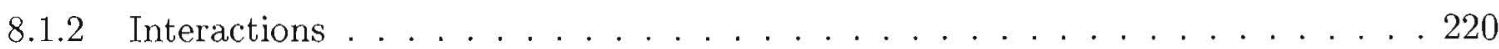

8.1 .3 Accidentals . . . . . . . . . . . . . . . . . . . . . . . . . . . . . . . . . . . . . . .

8.1 .4 Other Backgrounds . . . . . . . . . . . . . . . . . . . . . . . . . . . . . . . . . . . . . . . .

8.2 Measuring Detector Response Functions . . . . . . . . . . . . . . . . 222

8.3 Other Analysis Techniques . . . . . . . . . . . . . . . . . . . . 222

8.4 Safety Factors . . . . . . . . . . . . . . . . . . . . . . . 222

9 Siting and Conventional Construction 225

9.1 Siting Constraints . . . . . . . . . . . . . . . . . . 225

9.2 Geometry Constraints . . . . . . . . . . . . . . . . . . . . . 225

9.3 Superconducting RF Beamline . . . . . . . . . . . . . . . . . . 225

9.4 Meson Area Sites . . . . . . . . . . . . . . . . . . . . . . . . . . . 227

9.4 MP Beamline . . . . . . . . . . . . . . . . . . . . . 227

9.4 .2 ME Beamline . . . . . . . . . . . . . . . . . . . . . . 228

9.4.3 Experimental Apparatus . . . . . . . . . . . . . . . . . . 228

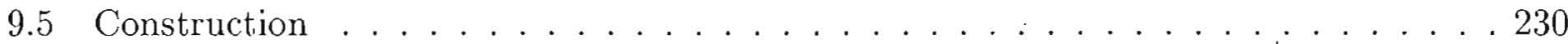

9.5.1 Target Enclosure . . . . . . . . . . . . . . . . . . 230

9.5.2 Experimental Hall . . . . . . . . . . . . . . . . . . . . . . 231

9.6 Environmental Assessment . . . . . . . . . . . . . . . . . . . . . . 232

10 Cost, Schedule and Staffing Estimates

10.1 Costs . . . . . . . . . . . . . . . . . . . . . . . . . . . . . . . . . . . . . . . .

10.1.1 Summary of Detector Cost Estimates . . . . . . . . . . . . . 233

10.1.2 Fermilab Facility Cost Estimates . . . . . . . . . . . . . . 233

10.2 Milestones . . . . . . . . . . . . . . . . . . . . . . . . . . . . . . . . . . . . . . .

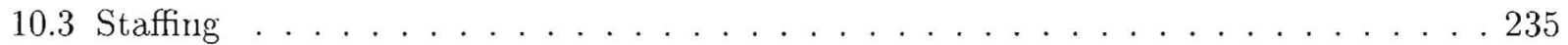

11 Conclusion $\quad 235$

12 Acknowledgements $\quad 236$

13 Appendix I - Simulation Results Chapter from 1st Edition 237

13.1 Monte Carlo Overview . . . . . . . . . . . . . . . . . . . . 237

$13.2 K^{+} \rightarrow \pi^{+} \nu \bar{\nu}$ Acceptance . . . . . . . . . . . . . . . . . . . 241

13.3 Kaon Decay Backgrounds . . . . . . . . . . . . . . . . . . . . 243

13.3.1 $K^{+} \rightarrow \pi^{+} \pi^{0}$ Background Study . . . . . . . . . . . . . . . 247

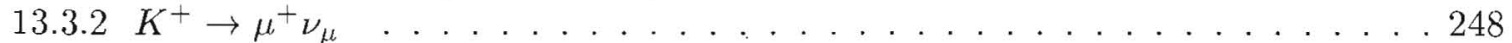

$13.3 .3 K^{+} \rightarrow \mu^{+} \nu_{\mu} \gamma$ Background Study . . . . . . . . . . . . 253

13.4 Photon loss mechanisms in detector material . . . . . . . . . . . . . 253

13.5 Beam-Detector Interaction Backgrounds . . . . . . . . . . . . . . 254

13.5.1 Charge-Exchange . . . . . . . . . . . . . . . . . 254

13.5.2 Other Interactions in Detectors . . . . . . . . . . . . . 257

13.5.3 Beam Gas Interactions . . . . . . . . . . . . . . . . . 257

13.6 Accidental Background Sources . . . . . . . . . . . . . . . . 258 


\section{List of Figures}

1 Diagrams for $\mathrm{FCNC} s \rightarrow d \nu \bar{\nu}$ decays ( $K \rightarrow \pi \nu \bar{\nu}$ decays): a) penguin diagrams, b) box diagram. . . . . . . . . . . . . . . . . . . . . 22

2 Diagrams containing SUSY contributions to the $Z d \bar{s}$ vertex. . . . . . . . . . . 22

3 Graphical representation in the $\bar{\rho}, i \bar{\eta}$ plane of the unitary condition $V_{u d} V_{u b}^{*}+V_{t d} V_{t b}^{*}=$

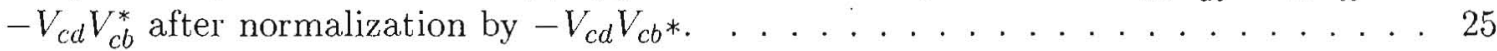

4 The ideal unitary triangle. For illustration, the value of $\bar{\eta}$ has been chosen to be higher than the fitted central value (reproduced from $[12,13]) \ldots \ldots \ldots 25$

5 A current snapshot of how parameters of the unitary triangle are constrained. $\varepsilon, \triangle m_{B_{d}}$, $\triangle m_{B_{s}},\left|V_{u b} / V_{c b}\right|, \sin 2 \beta\left(\right.$ from $\left.B^{0}\left(\bar{B}^{0}\right) \rightarrow J / \psi K_{S}^{0}\right)-$ see $[29] . \ldots \ldots \ldots$

6 A possible outcome for $\bar{\eta}, \bar{\rho}$ and $\beta$ obtained from future $\mathrm{K}$-meson and B-meson experiments: $K^{+} \rightarrow \pi^{+} \nu \bar{\nu}(\mathrm{CKM}) ; K_{L}^{0} \rightarrow \pi^{0} \nu \bar{\nu}(\mathrm{KAMI} / \mathrm{KOPI} 0)$ and $\triangle m_{B_{d}} / \triangle m_{B_{s}} ; B^{0}\left(\bar{B}^{0}\right) \rightarrow$ $J / \psi K_{S}^{0}$. The light region includes possible theoretical uncertainties in $B R\left(K^{+} \rightarrow\right.$ $\left.\pi^{+} \nu \bar{\nu}\right)$ connected with the influence of c-quarks. . . . . . . . . . . . 28

$7 \quad$ Correlation between $B R\left(K^{+} \rightarrow \pi^{+} \nu \bar{\nu}\right)$ and $R_{\triangle m_{B}}=\Delta m_{B_{d}} / \Delta m_{B_{s}}$ in the SM [41]. A scan over the presently allowed region $0<\bar{\rho}<0.27 ; 0.26<\bar{\eta}<0.37$ yields the dark region for central value $\triangle=P_{0} / A^{2} \cdot F\left(x_{t}\right)=0.42$ in (11) or light region for acceptable values $0.31<\triangle<0.54$.

8 A model with four generation of fundamental fermions with quark doublets [44]. a) Unitary quadrangle for this model. b) The correlation between $B R\left(K^{+} \rightarrow \pi^{+} \nu \bar{\nu}\right)$ and $B R\left(K_{L}^{0} \rightarrow \pi^{0} \nu \bar{\nu}\right)$ in this model; the dotted line rectangle corresponds for the $\mathrm{SM}$ predictions.

9 Diagrams using the horizontal gauge boson model. Dotted lines are the boson mediating an $S U(N)$ generational symmetry. (a) A generation conserving scalar or vector process; (b) A generation violating scalar or vector process; (c) A pseudoscalar or axial vector process. . . . . . . . . . . . . . . . . . . . . 43

$10 K \rightarrow \mu e$ and $K \rightarrow \pi \mu e$ in a SUSY model with $R$-parity nonconservation [68]. . . . 44

11 Decay angle versus momentum for $K^{+} \rightarrow \pi^{+} \nu \bar{\nu}$ events. Three distinct analysis regions are shown. The region defined by the signal cuts is also shown.

12 Ring radii for $K^{+}$and $\pi^{+}$versus their momentum for the kaon RICH system that measures the incident beam $K^{+}$momentum and decay angle. The black band indicates the momentum resolution of the kaon RICH system as described in section 5.3. Here we used $R=40 \mathrm{~m}$ and $n=1.0002765$.

13 Expected $M_{\text {miss }}^{2}$ resolution and contributions for the momentum(left) and velocity(right) spectrometers. The relevant mass scale is $m_{\pi^{0}}^{2}=18.2 \times 10^{-3} \mathrm{GeV}^{2} / \mathrm{c}^{4}$, where the dominant $K^{+} \rightarrow \pi^{+} \pi^{0}$ background resides. . . . . . . . . . . . 56

14 Energy distribution of the high energy photon in $K^{+} \rightarrow \pi^{+} \pi^{0}$ decay for the various veto detector subsystems.

Energy distribution of the low energy photon from the $K^{+} \rightarrow \pi^{+} \pi^{0}$ decay for the various veto detector subsystem.

Illustration of a $\mathrm{K}^{+} \rightarrow \pi^{+} \pi^{0}$ decaying within the vacuum decay volume surrounded by the VVS system. . . . . . . . . . . . . . . . . . . . . .

17 Energy distribution of the low energy photons in $K^{+} \rightarrow \pi^{+} \pi^{0}$ the VVS that intercept the gap and the active regions. . . . . . . . . . . . . . . 60 
19 The histogram plots the required VVS inefficiency. Overlaid are VVS GEANT studies at low and high energy, and the medium energy efficiency inferred from the BNL-E787 data set as described in section $5.6 .9 \ldots \ldots \ldots \ldots$. . . . . . . . . . . . .

20 Layout of the beam line. . . . . . . . . . . . . . . . . . 70

21 Layout of the beam line. . . . . . . . . . . . . . . . . . . 71

22 Vertical beam spot at the beam stopper. . . . . . . . . . . . . . . . 72

23 Kaon distribution at $0 \mathrm{~m}$ in detector coordinates(see figure 42 CKM Detector). Kaons are colored according to momentum bite (blue $<-0.01$, green -0.01 to 0.01 ,

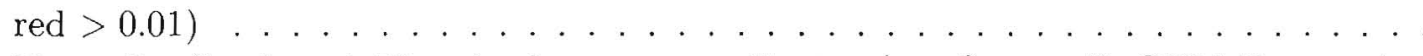

24 Kaon distribution at $50 \mathrm{~m}$ in detector coordinates (see figure 42 CKM Detector). Kaons are colored according to momentum bite (blue $<-0.01$, green -0.01 to 0.01 ,

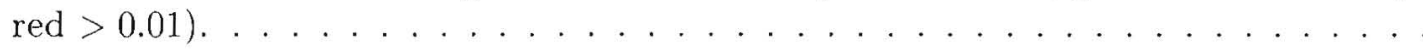

25 Kaon distribution at station $100 \mathrm{~m}$ in detector coordinates(see figure 42 CKM Detector). Kaons are colored according to momentum bite(blue $<-0.01$, green -0.01 to 0.01 , red $>0.01) \ldots \ldots \ldots \ldots \ldots \ldots$

26 Kaon rate $\left(\mathrm{MHz}\right.$ ) (at the exit of the last quadrupole for $5 \times 10^{12}$ protons per pulse) and purity (\%), as a function of beam stopper height $(\mathrm{mm})$. There are two regions where the purity is roughly independent of stopper height, and a transition region where the dependence is roughly linear. The kaon rate is roughly linear with stopper height. The vertical line indicates the $12 \mathrm{~mm}$ stopper width in the standard design. .

27 Beam envelopes. The apertures of the beam line elements are indicated. The curves represent the two standard deviation beam envelope. . . . . . . . . . . . . . 77

28 Sketch of two cavity cryostat, in the anticipated enclosing tunnel. . . . . . . . . 80

29 Beams-eye view of the cavities and their fields. At left, the magnetic field at the center of a cell; the two circles are the equator of the cavity and the beam pipe diameter. At right, the electric field at the iris between two cells. The cell length and cell-to-cell phase relationship are selected so that a particle timed to receive the maximum deflection sees these fields in all 13 cells and 12 irises. . . . . . . . . .

$30 Q$ vs $V_{\perp} / \mathrm{m}$ result for our single cell cavity. The horizontal axis is equivalent deflecting field, in $\mathrm{MV} / \mathrm{m}$; no correction for the difference between single and multiple cell structures has been applied. . . . . . . . . . . . . . . 83

31 Muon spatial distribution at the exit of the beam line. The source of the top (bottom) plot is beam pion (kaon) decays. . . . . . . . . . . . . . 87

32 Initial phase space in $\mathrm{x}, \mathrm{y}[\mathrm{cm}], \mathrm{x}$ angle, $\mathrm{y}$ angle $[\mathrm{rad}]$, and momentum $[\mathrm{GeV} / \mathrm{c}]$ for GEANT(solid line) and TURTLE(dashed line). . . . . . . . . . . . . . 89

33 Phase space after $\mathrm{Q} 32$ in $\mathrm{x}, \mathrm{y}[\mathrm{cm}], \mathrm{x}$ angle, $\mathrm{y}$ angle$[\mathrm{rad}]$, and momentum $[\mathrm{GeV} / \mathrm{c}]$ for GEANT(solid line) and TURTLE(dashed line). . . . . . . . . . . . . . 89

34 Phase space at $R F 2$ exit in $\mathrm{x}, \mathrm{y}[\mathrm{cm}], \mathrm{x}$ angle, $\mathrm{y}$ angle[ $[\mathrm{rad}]$, and momentum $[\mathrm{GeV} / \mathrm{c}]$ for GEANT(solid line) and TURTLE(dashed line). . . . . . . . . . . . . . . 89

35 Phase space at Stopper in $\mathrm{x}, \mathrm{y}[\mathrm{cm}], \mathrm{x}$ angle, $\mathrm{y}$ angle $[\mathrm{rad}]$, and momentum $[\mathrm{GeV} / \mathrm{c}]$ for GEANT(solid line) and TURTLE(dashed line). . . . . . . . . . . . . 89

36 Phase space at detector in $\mathrm{x}, \mathrm{y}[\mathrm{cm}], \mathrm{x}$ angle, $\mathrm{y}$ angle $[\mathrm{rad}]$, and momentum $[\mathrm{GeV} / \mathrm{c}]$ for GEANT(solid line) and TURTLE(dashed line). . . . . . . . . . . . . 90

37 Muon rate in $\mathrm{MHz}$ at last quadrupole exit within 1 meter transverse of the beam center vs the strength of the clean-up bends. . . . . . . . . . . . . 90

38 Decay location ( $\mathrm{cm}$ from target) for pion and kaon decays to muons that enter the

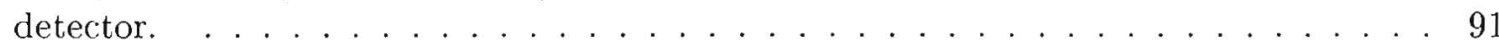


39 Momentum distribution ( $\mathrm{GeV}$ ) for muons from pion decay with and without the standard clean-up bend. . . . . . . . . . . . . . . . . . 92

40 Pi-decay muons reaching the end of the beamline. Shown is the RF stage of the beamline including the beam stopper. Pions are shown in red and muons are shown in green. This is the dominant source of beam muons. . . . . . . . . . . . 93

41 Muon shielding along the beam line. . . . . . . . . . . . . . 94

42 The CKM Detector. The lower section show the true proportions of the apparatus. . 95

43 Plan and elevation views of the upstream magnetic spectrometer. . . . . . . . . . 96

44 Momentum of the kaons at the entrance of the UMS. . . . . . . . . . . . . . . 97

45 The RF-separated beam at chamber C1. . . . . . . . . . . . . . . . 97

46 Front view of a UMS wire chamber. . . . . . . . . . . . . . . . . . 99

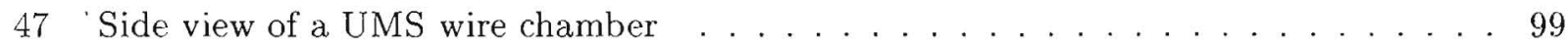

48 Magnified view of the center of a UMS wire chamber . . . . . . . . . . . . . . 99

49 Exploded view of a UMS wire chamber . . . . . . . . . . . . . . . . 100

$50 \quad$ Hyper $^{P}$ cliamber 1 efficiency vs intensity. . . . . . . . . . . . . . . 101

51 Comparison of $\mathrm{CF} 4$-isobutane and Argon-ethane inthe HyperCP wire chambers . . . 102

52 Gain vs time for the HyperCP wire chambers . . . . . . . . . . . . . . . . . 102

53 Momentum resolution without multiple scattering. . . . . . . . . . . 103

54 Momentum resolution with multiple scattering. . . . . . . . . . . . . 103

55 Momentum resolution with 1.53 tracks per event. . . . . . . . . . . 103

56 Angular resolution of the downstream tracks in the UMS. . . . . . . . . . . . 104

57 Track finding efficiency vs average single-plane efficiency . . . . . . . . . . . . . 104

58 UVa Hyper $C^{P}$ preamplifier test results . . . . . . . . . . . . . . . . . . . . . 106

59 The Beam Time Stamp Module . . . . . . . . . . . . . . . . . . . . . . . 108

60 Light output of Hyper $C^{P}$ hadronic calorimeter BCF-92 fibers . . . . . . . . . . . . . 11

61 Ring radius distribution in the SELEX RICH for multi track events for tracks with momentum between 95 and $105 \mathrm{GeV} / \mathrm{c}$. We fit a Gaussian to the pion peak of this

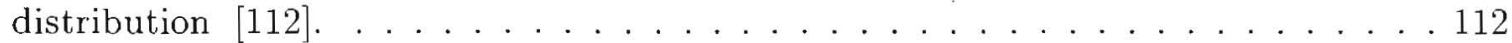

62 Fitted sigma of the proton ring radius distribution versus the mean radius. These fitted sigmas are larger than the $4.6 \mathrm{~mm}$ since they include finite track resolution and hits from overlapping rings. . . . . . . . . . . . . . . . . 113

63 The pull (upper) and ring radius (lower) distributions for $10.35 \mathrm{M} \beta=1$ beam tracks in the SELEX RICH. The shaded regions are a sub-sample with modest cuts on the predicted uncertainties of the ring fit parameters. . . . . . . . . . 116

64 left: Number of detectable photons. right: Distribution of detected photons in the kaon RICH focal plane for 1000 beam $K^{+}$and 1000 beam $\pi^{+}$events. . . . . . . . 117

65 Possible schematic of the kaon RICH optical path. The vessel drawn has a total length of $11 \mathrm{~m}$. The long arrow represents the beam; the thicker dots are the beam windows. The dashed line represents Cherenkov photons generated by a $K^{+}$, dotted line Cherenkov photons from a $\pi^{+}$. The flat mirror in the beam will only reflect part of the Cherenkov photons from the pions. . . . . . . . . . 117

66 Ring radius, ring radius resolution, and momentum resolution for the Kaon RICH, gas $C F_{4}$, Photomultiplier size $1 / 2$ ", R760 with quartz window. . . . . . . . . 118

67 Momentum (top) and angular (bottom) resolution for the Kaon RICH as a function of pressure and for different photomultiplier sizes. Left: $C F_{4}$, Right: $N_{2} \ldots \ldots$

68 Momentum (top) and angular (bottom) resolution for the Kaon RICH as a function of pressure, for 3 different wavelength cutoffs in $C F_{4} \ldots \ldots \ldots \ldots$ 
69 Momentum (top) and angular (bottom) resolution for the Kaon RICH as a function of pressure and for different photomultiplier sizes, for a $300 \mathrm{~nm}$ cutoff. Left: $C F_{4}$,

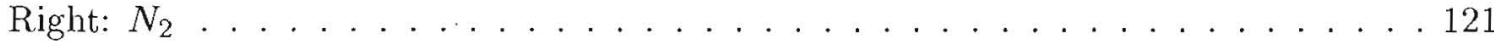

70 Difference in Ring Radius (in units of Gaussian $\sigma$ ) between Kaons and Pion in the Kaon RICH as a function of pressure. Gas: $C F_{4}$, PM Size 1/2 inch, cutoff $300 \mathrm{~nm} . \quad 122$

71 Distribution of rates in PMs for the Kaon RICH. 10000 tracks are scaled to our $30 \mathrm{MHz}$ nominal beam rate. Gas: $C F_{4}, \mathrm{PM}$ Size $1 / 2$ inch, cutoff $300 \mathrm{~nm} . \ldots . . .123$

72 A simulation of the expected angular resolution (projected) of the KEAT. Angles are in mrad. Multiple scattering, but not nuclear scattering, is included in the simulation.124

73 Layout of upstream chambers in HyperCP. Each chamber has four planes, two planes of vertical wires and two stereo views with the wires inclined angle at $\pm 26.6^{\circ}$ from the vertical. The wire spacing is $1.0 \mathrm{~mm}$ for $\mathrm{C} 1$ and $\mathrm{C} 2$, and $1.2 \mathrm{~mm}$ for $\mathrm{C} 3$ and $\mathrm{C} 4.125$

74 A simulation of the expected angular resolution (projected) for the HyperCP geometry. Angles are in mrad. Multiple scattering; but not nuclear scattering, is included

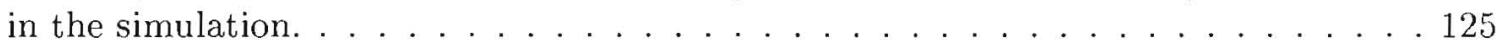

75 Measured angular resolution (projected) for the HyperCP geometry. The solid histogram is for 1-track events; the dashed is for events with 2-tracks. The RMS widths of both are in very good agreement with the simulation in Fig. $74 \ldots . . .125$

76 Elevation drawing of the upstream vacuum window, last tracking station of the KEAT, and the upstream section of the BIVS. . . . . . . . . . . 127

77 Elevation drawing of a section of vacuum decay volume showing the geometry of VVS stations. . . . . . . . . . . . . . . . . . . . . . . . . . . 128

78 Front Beams-eye view (elevation) drawing of one the 34 VVS stations. . . . . . . 129

79 Longitudinal slice of an annular. VVS station. Geometry of the lead-scintillator sampling and photomultiplier tubes is evident. . . . . . . . . . 130

80 Exploded view of the even-odd fiber routing scheme that maps fibers from alternate scintillator sectors to separate photomultiplier tubes. . . . . . . . . . . . 131

81 Illustration of the scintillator sector "clocking" scheme that eliminates first order cracks. the nominal fiber spacing is indicated. . . . . . . . . . . . 131

82 Photograph of a scintillator sector instrumented with the nominal fiber pattern bundled to a photomultiplier tube. This is one of many prototypes studied for light

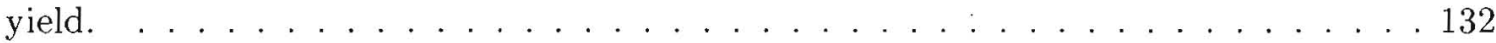

83 (a): Typical pulse (1 MeV deposited) from a MIP traversing a tile of BC404 readout with BCF-92 fibers. An average of 512 traces fit to extract a FWHM as shown. . . . 133

84 (a) Pulse height distribution of a MIP traversing a single tile. A Poisson convolved Landau fit is overlaid, from which the number of photoelectrons can be extracted. . 134

85 GEANT simulation of the low energy inefficiency $(0-20 \mathrm{MeV})$ for a threshold of 10 and 20 photoelectrons which corresponds to $3 \mathrm{MeV}$ and $6 \mathrm{MeV}$ incident photon energy respectively. . . . . . . . . . . . . . . . . . . . . . . . . . . . . . . . . .

86 Illustration of the pileup effect on a $1 \mathrm{MeV}$ deposit from an early muon that deposits $20 \mathrm{MeV}$ of energý $20 \mathrm{nsec}$ before the trigger in-time window. The pulse-shape parameters were taken from our BC404/BCF92 prototype measurements. . . . . . 136

87 Photon veto inefficiency of $\mathrm{Pb}$-scintillator sampling devices inferred from photonuclear cross section measurements of these devices at the INS test facility. The normal effects of electromagnetic shower fluctuations are not included. . . . . . . . . . 138

88 Angular distribution of high energy photons $(>1 \mathrm{GeV})$ incident of the VVS. . . . . 139

89 GEANT simulation of a $1 \mathrm{GeV}$ photon with grazing incidence from the left on the inner aperture of a VVS module. . . . . . . . . . . . . . . . . . 139 
90 Measured [166] cross section of $\gamma p \rightarrow \rho^{0} \rightarrow \pi^{+} \pi^{-}$photo-production .vs. energy. . . . 141

91 Measured [167] $t$ dependence of $\gamma p \rightarrow n \pi^{-}$. The diffractive peak at low $t$ is clearly evident. . . . . . . . . . . . . . . . . . . . . 141

92 One of the 56,000 tagged Ke3 events used to study the electron efficiency of the $\mathrm{Pb}$-scintillator sampling device behind the left CsI beam hole. . . . . . . . . . 145

93 Scatter plot of the measured energy in the Back-Anti .vs. the incident tagged electron momentum. The few events that straggle from the main population are indicated with "*". . . . . . . . . . . . . . . . . . . . . 146

94 Back-Anti energy distribution of incident $\pi^{+}$. The energy of the one electron tagged event with the least reported energy in the Back-Anti is indicated with a vertical bar, and is clearly greater than the MIP peak. . . . . . . . . . . . . 146

95 Layout of a BNL-871 Straw Chamber. . . . . . . . . . . . . . . . . . 150

96 Rate seen by the BNL-871 Straw Chambers. . . . . . . . . . . . . . . 150

97 End-detail of a BNL-871 Straw Chamber. . . . . . . . . . . . . . . . 151

98 DMS 'cassette' containing a primed-unprimed pair of straw planes. The cassette would inserted into the vacuum vessel. . . . . . . . . . . . . . . . . 153

99 DMS 'cassette' arranged into $\mathrm{X}, \mathrm{Y}, \mathrm{U}, \mathrm{V}$ views. The inner circle denotes the active area of the straws ( $80 \mathrm{~cm}$ diameter). The outer circle denotes the proposed vacuum vessel diameter. The straws would have to be significantly longer to sub-tend the vessel diameter. . . . . . . . . . . . . . . . . . . . . . . . . . . . . . . . . . . . . . . .

100 Momentum resolution $\left(P_{\text {recon }} / P_{\text {true }}\right)$ for pions in the momentum range $14-20 \mathrm{GeV} / \mathrm{c} .154$

101 The momentum dependence of the momentum resolution. . . . . . . . . . 154

102 Angular resolution $\left(\theta_{\text {recon }}-\theta_{\text {true }}\right)$ in the bend view for pions in the momentum range $14-20 \mathrm{GeV} / \mathrm{c} \ldots \ldots \ldots \ldots \ldots \ldots \ldots$. . . . . . . . . . . . . . . . . . . . . . . . . .

103 The momentum dependence of the angular resolution $\left(\theta_{\text {recon }}-\theta_{\text {true }}\right)$ in the bend view 154

104 Angular resolution $\left(\theta_{\text {recon }}-\theta_{\text {true }}\right)$ in the non-bend view for pions in the momentum

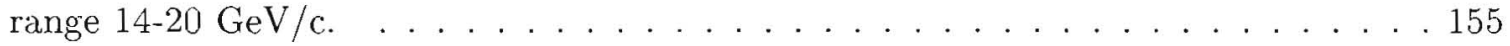

105 The momentum dependence of the angular resolution $\left(\theta_{\text {recon }}-\theta_{\text {true }}\right)$ in the non-bend

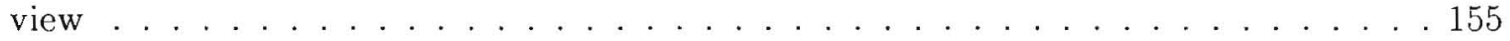

106 Momentum and angular resolution versus $\pi^{+}$predicted by an analytical calculation [114]. The slight shift from unity is due to a slight error (on our part) in converting from the desired magnet Pt kick into field strength (used in GEANT) . . . . . . . . 155

$107 M_{m i s s}^{2}$ of $K^{+} \rightarrow \pi^{+} \pi^{0}$ events reconstructed by the DMS assuming a perfect UMS and KEAT reconstruction. . . . . . . . . . . . . . . . . . . 155

$108 M_{m i s s}^{2}$ versus $p_{\pi}^{r e c o n} / p_{\pi}^{\text {true }}$ of $K^{+} \rightarrow \pi^{+} \pi^{0}$ events. A perfect UMS, KEAT, and DMS angular reconstruction is assumed. Hence, this focuses on the $M_{m i s s}^{2}$ tail that is due

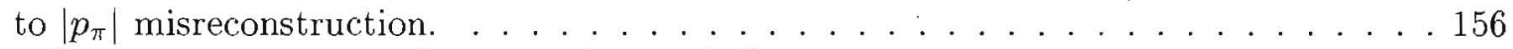

$109 M_{\text {miss }}^{2}$ versus $\theta_{\pi}^{\text {recon }}-\theta_{\pi}^{\text {true }}$ of $K^{+} \rightarrow \pi^{+} \pi^{0}$ events. A perfect UMS, KEAT, and DMS $\left|p_{\pi}\right|$ reconstruction is assumed. As shown, the non-Gaussian tails in the decay angle distribution do not have as big an impact on $M_{\text {miss }}^{2}$ as does the $\left|p_{\pi}\right|$ misreconstruction. . . . . . . . . . . . . . . . 156

110 Momentum reconstruction in the DMS $\left(p_{\pi D M S}\right)$ versus pion Rich $\left(p_{\pi R I C H}\right)$ coml-

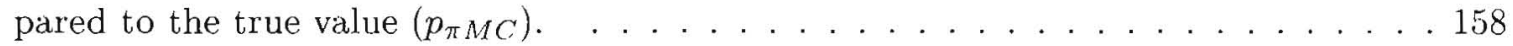

111 Decay angle reconstruction in the DMS $\left(\theta_{\pi D M S}\right)$ versus pion Rich $\left(\theta_{\pi R I C H}\right)$ compared to the true value $\left(\theta_{\pi M C}\right)$. A small correlation is seen. . . . . . . 158

112 Layout of the BNL-871 detector. . . . . . . . . . . . . . . . . . 159 
113 Relative difference between the momentum measurements of the upstream (straw) and downstream (conventional wire drift chamber) magnetic spectrometer in BNL871. Dots and histogram are data and simulation respectively. . . . . . . . . 159

114 Overlay of figure 113 showing BNL-871 data (dots) and figure 100 showing our DMS GEANT simulation (histogram). Our DMS GEANT simulation has smaller non Gaussian tails by a factor of 5-10.

115 Pion RICH momentum resolution for Pions as a function of momentum for different PM sizes . . . . . . . . . . . . . . . . . . . . . . 161

116 The functional relationship between the ring radius and the $\pi^{+}$track momentum. The shaded band is the accepted momentum range. The black stripe is a $1-\sigma$ band that illustrates the momentum-radius transfer function. . . . . . . . . . . . . 162

117 Pion (a) fractional RICH momentum resolution and (b) RICH angular resolution as a function of pion momentum from the pion $\mathrm{RICH}$ velocity spectrometer measurements. Contributions to the resolutions from optical dispersion and multiple Coulomb scattering in the Neon radiator gas and phototube size, are included. Downstream magnetic spectrometer (DMS) (c) fraction momentum resolution and (d) angular resolution as a function of pion momentum. Contributions to the resolutions from tracking resolution and multiple Coulomb scattering in the tracker are included. $16 \dot{3}$

118 Radius separation between $\mu^{-}$and $\pi^{-}$in the Pion RICH. PM size $1 / 2$ inch . . . . 164

119 Rate in PMs for the Pion RICH. 10000 tracks are scaled to our $30 \mathrm{MHz}$ nominal beam rate for Kaons and their decay products (top), and $2.95 \mathrm{MHz}$ nominal beam rate for pions and their decay products (bottom). Gas: $N e$, PM Size $1 / 2$ inch. . . . 165

120 Angular distribution of photons incident on the FVS . . . . . . . . . . 167

121 Conceptual drawing of one SHASHLIK $5 \mathrm{~cm} \times 5 \mathrm{~cm}$ tower. These scintillator towers are embedded in larger super-modules of continuous lead sheet. . . . . . . . 168

122 Conceptual drawing of the FVS with transverse segmentation and offset beam hole

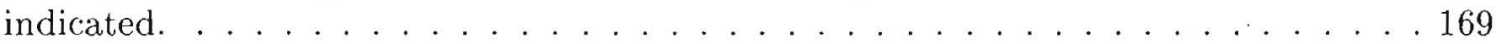

123 GEANT simulation of a $15 \mathrm{GeV} \pi^{+}$and $1.6 \mathrm{GeV}$ photon separated by $15 \mathrm{~cm}$ incident on a $16 X_{0}$ Pb-scintillator FVS. . . . . . . . . . . . . . . . . 172

$124 \mathrm{KTeV}$ event display of a $K_{L} \rightarrow \pi^{0} \pi^{+} \pi^{-}$decay. . . . . . . . . . . . . 173

125 Average transverse projection profile of $\mathrm{KTeV} \pi^{+}$(shaded) and photons (solid line) separated by $10 \mathrm{~cm}$ on the left and $15 \mathrm{~cm}$ on the right. . . . . . . . . . . 174

126 The average $\pi^{+}$shower projection profile in the KTeV data compared with a GEANT simulation of the $\mathrm{KTeV}$ CsI calorimeter. . . . . . . . . . . . . . . . . . 174

127 Measured from KTeV Data, Figure (a): Efficiency of accepting a $\pi^{+}$as a function of threshold energy of blocks outside of the central $3 \times 3$ cluster. Figure (b): The efficiency of detecting a $(1.0-2.0 \mathrm{GeV})$ photon near a $14-20 \mathrm{GeV} \pi^{+}$as a function of separation at a fixed $\pi^{+}$efficiency of $90 \%$.

128 Overlay of the KTeV shower library analysis and an identical analysis of a GEANT simulation of $27 X_{0}$ CsI. Figure (a) shows $\pi^{+}$efficiency as a function of outer block energy threshold. Figure (b): The efficiency of detecting a $(1.0-2.0 \mathrm{GeV})$ photon near a $14-20 \mathrm{GeV} \pi^{+}$as a function of separation at a fixed $\pi^{+}$efficiency of $90 \%$.

129 GEANT simulation of $18 X_{0}$.Pb-Scintillator and CsI. Figure (a) shows $\pi^{+}$efficiency as a function of outer block energy threshold. Arrows in figure (a) indicate the respective $90 \%$ efficiency points. Figure (b): The efficiency of detecting a $(1.0-$ $2.0 \mathrm{GeV}$ ) photon near a $14-20 \mathrm{GeV} \pi^{+}$as a function of separation at a fixed $\pi^{+}$efficiency of $90 \%$. 
130 Liner and $\log$ transverse shower profiles of CsI (solid) and shashlik Pb-scintillator (dashed) simulations in GEANT. . . . . . . . . . . . . . 176

131 The MVS prototype. . . . . . . . . . . . . . . . . . . . . . 179

132 The MVS prototype on the ISTRA-M beam. . . . . . . . . . . . 180

133 The total MVS signal for $25 \mathrm{GeV}$ pions(left), the muon beam momentum spectrum(right) . . . . . . . . . . . . . . . . . . . . 181

$134 \mathrm{x}$ (top) and $\mathrm{y}$ view of $25 \mathrm{GeV} \pi$ event (left) and muon event(right) in the MVS . . . 181

135 The $n 1$ distributions: (above) for the low intensity muon (left) and pion data(right)// (below) GEANT Monte Carlo:muons(left), $25 \mathrm{GeV}$ pions(center), $12 \mathrm{GeV}$ pions(right) 182

136 The $\mathrm{n} 3$ distributions: (above) for the low intensity muon (left) and pion data(right)// (below) GEANT Monte Carlo:muons(left), $25 \mathrm{GeV}$ pions(center), $12 \mathrm{GeV}$ pions(right) 183

$137 \mathrm{x}$ and $\mathrm{y}$ view of the e.m. shower caused by $\mu \rightarrow e \nu \bar{\nu}$ decay before the calorimeter(left) or inside.(low intensity muon run) . . . . . . . . . . . . . . 183

138 Block Diagram of CKM DAQ and Trigger System . . . . . . . . . . . . 189

139 CKM "Event" . . . . . . . . . . . . . . . . . . . . . . . . . . 190

140 Physical Structure of CKM DAQ and Trigger System . . . . . . . . . . . 198

141 The number of photomultiplier tube hits in the KRICH and PRICH for reconstructed $K^{+} \rightarrow \pi^{+} \nu \bar{\nu}$ events. . . . . . . . . . . . . . . . . . 202

142 Reconstructed vertex variables as obtained from the tracking system in good $K^{+} \rightarrow \pi^{+} \nu \bar{\nu}$ events: a) $\mathrm{Z}$ vertex resolution $(\mathrm{cm})$, b) distance of closest approach (DOCA), in $\mathrm{cm}, \mathrm{c}$ ) opening angle $\theta$ resolution $(\mathrm{mrad}) \ldots \ldots \ldots \ldots \ldots . \ldots \ldots \ldots$

143 Reconstructed spectrometer variables in good $K^{+} \rightarrow \pi^{+} \nu \bar{\nu}$ events: a) fractional difference between UMS and KRCH reconstruction for $K^{+}$momentum, b) fractional difference between DMS and PRCH reconstruction for $\pi^{+}$momentum c) difference between magnetic spectrometer and RICH spectrometer for $M_{\text {miss }}^{2}\left(\mathrm{GeV}^{2}\right)$. . . . . 206

144 Reconstructed missing mass variable of $K^{+} \rightarrow \pi^{+} \nu \bar{\nu}$ events that pass the spectrometer consistency cuts listed in section 7.2 .2 for: a) for the magnetic tracking spectrometer, b) for the RICH spectrometer, c) for the combined weighted average of both. All in $\mathrm{GeV}^{2} / c^{4}$. . . . . . . . . . . . . . . . . . . . 207

145 The combined measured missing mass squared $\left(M_{\nu \bar{\nu}}^{2}\right)$ of the two spectrometers for both the $K^{+} \rightarrow \pi^{+} \nu \bar{\nu}$ signal (light shade) and principal background $K^{+} \rightarrow \pi^{+} \pi^{0}$ are shown. The signal region is shown in dark shade. The $158 \mathrm{~K} K^{+} \rightarrow \pi^{+} \pi^{0}$ events are the residual background after all cuts expect for missing mass. . . . . . . . . . 208

146 The combined measured missing mass squared $\left(M_{\nu \bar{\nu}}^{2}\right)$ of the two spectrometers reconstructed in CKM_GEANT. The signal region is shaded. . . . . . . . . . . . . 209

147 The measured [191] $\pi^{+}$momentum line-shape of $K^{+} \rightarrow \pi^{+} \pi^{0}$ decays from BNL experiment E787. The different curves are the evolution from nio photon veto cuts, to online photon veto cuts, and finally full offline photon veto cuts. The stability of the

line-shape validates the factorization hypothesis for this pair of variables. . . . . . 211

$148 M_{\nu \bar{\nu}}^{V \text { elocity }}$.vs. $M_{\nu \bar{\nu}}^{\text {Nomentum }}$ in $\mathrm{GeV}^{2} / \mathrm{c}^{4}$ for one million $K^{+} \rightarrow \pi^{+} \pi^{0}$ decays in CKM_GEANT.212

149 The inclusive inelastic $\pi^{+}$production fraction as a function of momentum from an incident $22 \mathrm{GeV} K^{+}$on light nuclei. These fractions depend weakly on the atomic number of the struck nucleus. The SAS curve shown is from a straightforward fit in $\left(x_{F}, p_{t}\right)$. The curve is an extrapolation above $19 \mathrm{GeV} \ldots \ldots . \ldots 214$ 
150 The SAS data and simulation results for $\pi^{+}$production into the forward region $(\theta<15 \mathrm{mrad})$ from a $22 \mathrm{GeV} K^{+}$on light nuclei. These fractions depend weakly on the atomic number of the struck nucleus. The SAS data points are straightforward $\left(x_{F}, p_{t}\right)$ extrapolations of higher energy SAS data. The curve is an extrapolation above $19 \mathrm{GeV}$

151 Figure (a) shows the distribution of hit BIVS photomultipliers as a function of longitudinal position. Figure (b) shows the distribution of hit arrival time with respect to a common trigger time. . . . . . . . . . . . . . . . . 216

152 The dependence of BIVS residual inefficiency on the threshold of visible energy detected within a 5 nsec of $K^{+}$traversal. . . . . . . . . . . . . . 216

153 Fixed target experimental areas. The Meson Area is on the left. . . . . . . . . 226

154 The MP9 experimental hall configuration. . . . . . . . . . . . . . . . . 229

155 The CKM detector and the existing MP9 experimental hall. (meters) . . . . . . 230

156 Target enclosure. . . . . . . . . . . . . . . . . . . . . . . 231

157 A new experimental hall configuration. . . . . . . . . . . . . . . 232

158 The resolution in missing mass squared in $K^{+} \rightarrow \pi^{+} \pi^{0}$ decays for the three methods of determining it as described in the text. About 20,000 events are shown. . . . . . 240

159 Distance between measured vertex location and actual decay position in $K^{+} \rightarrow \pi^{+} \nu \bar{\nu}$ events. The fitted resolution sigma is $\sim 18 \mathrm{~cm}$, with non-Gaussian tails originating from hard scatters included in the tracking simulation. . . . . . . . . . . . . . 242

160 Measured distance of closest approach (DOCA ) for the $K^{+}$and $\pi^{+}$in $K^{+} \rightarrow \pi^{+} \nu \bar{\nu}$ events.244

161 Difference in measured missing mass squared from the momentum and velocity measurements $\left(M_{\text {magnetic }}^{2}-M_{\mathrm{RICH}}^{2}\right)$ for $K^{+} \rightarrow \pi^{+} \nu \bar{\nu}$ events. . . . . . . . . . 245

162 Measured missing mass squared $\left(M_{\text {combined }}^{2}\right)$ in $K^{+} \rightarrow \pi^{+} \nu \bar{\nu}$ events. The signal region is shaded. . . . . . . . . . . . . . . . . . . . . 246

163 Missing mass squared $\left(M_{\text {combined }}^{2}\right)$ for $K^{+} \rightarrow \pi^{+} \pi^{0}$ events passing all cuts superimposed on the signal (light shading). The sensitivity shown in this plot corresponds to the experimental sensitivity of $73 \mathrm{~K}^{+} \rightarrow \pi^{+} \nu \bar{\nu}$ events in our mass window (single event sensitivity of $1.4 \times 10^{-12}$ ) which are indicated with the dark shading. . . . . 249

164 Missing mass of $K^{ \pm} \rightarrow \mu^{ \pm} \nu_{\mu}$ decays where the muon is reconstructed under the pion mass hypothesis. . . . . . . . . . . . . . . . . . . . 250

165 A GEANT simulation of a common Dalitz decay topology where the electron and positron tracks are kicked out of the photon veto system coverage by the $100 \mathrm{MeV} / \mathrm{c}$ $p_{\perp}$-kick magnet. Fortunately the rejection of the remaining photon together with the low Dalitz decay branching fraction set the scale of this problem topology to be low at the outset. . . . . . . . . . . . . . . . . . . . . . 251

166 A typical GEANT event where one of the decay $K^{+} \rightarrow \pi^{+} \pi^{0}$ photons converts in the pion RICH gas. Photons are dot-trajectories and charged particles are dashedtrajectories. The photon entering the pion $\mathrm{RICH}$ volume is seen to convert in the neon gas, and the charged pion (lower track) produces a hard delta-ray in the neon gas as well. . . . . . . . . . . . . . . . . . . . . . 252

167 The location of 10,000 hadronic interactions in beamline material. In the z scale of this figure (not the same as figure 42) the beam RICH spans the range $-14 \mathrm{~m}<$ $z<-4 \mathrm{~m}$, with the mirror located at $z=-4 \mathrm{~m}$. The two beam vacuum trackers are located at $z=-2 \mathrm{~m}$ and $z=0 \ldots \ldots \ldots \ldots \ldots \ldots$ 


\section{List of Tables}

1 Some SM and CKM matrix parameters used for the evaluation of $\operatorname{BR}(K \rightarrow \pi \nu \nu)$ and other values. The value for $\lambda$ is taken from [116]. The rest comes from [31]. . . 27

2 Determination of $\sin 2 \beta$ and $\left|V_{t d}\right|$ from branching ratios $B R\left(K^{+} \rightarrow \pi^{+} \nu \bar{\nu}\right)$ and

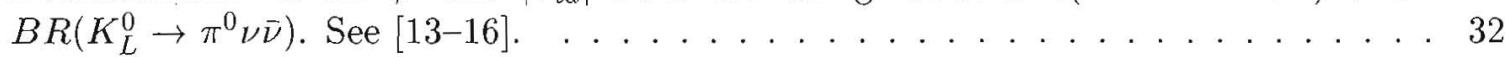

3 Branching ratios $B R\left(\pi^{+} \nu \bar{\nu}\right), B R\left(\pi^{0} \nu \bar{\nu}\right)$ and $B R\left(\pi^{0} e^{+} e^{-}\right)_{C P-d i r}$ for various new physics scenarios. . . . . . . . . . . . . . . . . . . . . . . . . . 35

4 The dependence of branching ratios for decays $K \rightarrow \pi \nu \bar{\nu}$ and $K_{L}^{0} \rightarrow \pi^{0} e^{+} e^{-} \mid C P-d i r$ in the SUSY model [48] on parameters $\lambda_{t}$ and $\Lambda_{t} \ldots \ldots \ldots \ldots$ The efficiency for the detection and sample size of several kaon decay modes in the CKM detector. . . . . . . . . . . . . . . . . . . . . . 40 Branching ratio limits (all limits are at the $90 \%$ C.L.) for lepton flavor violating kaon decays. . . . . . . . . . . . . . . . . . . . . 42 Classification by generation number change for some lepton flavor violating processes.

8 Sensitivity of performed and proposed experiments for CP-violation decay asymme-

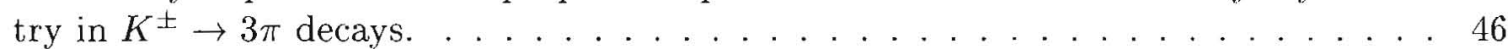

9 Theoretical predictions and experimental results for $\left|F_{v}+F_{a}\right|$ and $\left|F_{v}-F_{a}\right|$. We use the convention of where $F_{v}$ and $F_{a}$ are dimensionless and larger by a factor of $\sqrt{2}$ than in many papers, and there is a minus sign in the interference term. . . . . . . 48 Kaon decay modes which are potential backgrounds to $K^{+} \rightarrow \pi^{+} \nu \bar{\nu}$ in each of the three regions, defined in the text. The list is not exhaustive and includes only modes with relatively large branching ratios. . . . . . . . . . . . . . . . 50 Required inefficiency performance of the CKM photon veto systems. . . . . . . . 61 Typical alignment tolerances for beamline elements. . . . . . . . . . . . . . 78

13 Effect of quadrupole misalignments on bean centroid and phase ellipse. Refer to text for explanation. . . . . . . . . . . . . . . . . . . . . 79

14 Separator cavity parameters. Transverse shunt impedance is defined so that $P=$

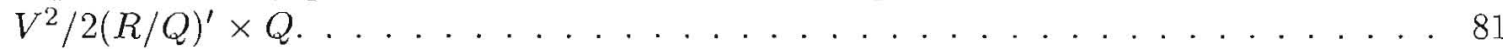

15 Beam rates at the exit of the last quadrupole required to produce $30 \mathrm{MHz}$ of kaons at the entrance to the kaon $\operatorname{rich}(\mathrm{z}=230 \mathrm{~m}) \ldots \ldots \ldots \ldots$ Dogleg and Spectrometer magnet parameters. . . . . . . . . . . . . . . . 97

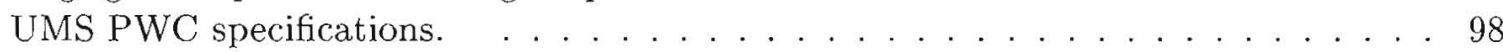
Wire chamber material. . . . . . . . . . . . . . . . . . . . . . . 98 BTSM and UMS spectrometer material. . . . . . . . . . . . . . . 103 Preamplifier bench-test comparisons. . . . . . . . . . . . . 106 HyperCP PWC electronic specifications. . . . . . . . . . . . . . 106 Bearm Time Stamp Module parameters. . . . . . . . . . . . . . . . . . . 108 Hamamatsu H7546 multi-anode photomultiplier characteristics. . . . . . . . . . 109 Bicron BCF-10 scintillating fiber parameters. . . . . . . . . . . . . . 110 Kuraray SCSF-3HF scintillating fiber parameters. . . . . . . . . . . . . . . 110

26 Rates .vs. visible energy threshold for CKM veto systems. Maximum phototube rates per channel (MAX) and global sub-system rates (OR) are listed. . . . . . 136 
31 Performance Summary of a CsI FVS solution. . . . . . . . . . . . . . . 171

32 The details of the data reduction . . . . . . . . . . . . . . . . . 184

33 The details of the Monte Carlo GEANT data reduction . . . . . . . . . . . . . . 184

34 CKM Detector Electronics Channel Summary: In this table, we have assumed that each TDC card or TDC+ADC card hosts 16 or 8 channels, respectively. . . . . . 188

35 Level 1 trigger rates. . . . . . . . . . . . . . . . . . . 196

36 Background estimates in terms of effective branching ratio. . . . . . . . . . . . 201

37 Losses to our signal acceptance due to deadtime and inefficiencies. . . . . . . . . . 217

38 April 1998 cost estimate of the CKM detector systems. . . . . . . . . . . . . . . . . 234

39 Parameters used in the Monte Carlo detector simulation. . . . . . . . . . . . . 238

40 Miscellaneous inefficiency table. . . . . . . . . . . . . . . . . . . . . . . . . . . . . . . . .

41 Background estimates in terms of effective branching ratio. . . . . . . . . . . 260 


\section{Introduction}

We are proposing here an experiment to measure the branching ratio of the ultra-rare charged kaon decay $K^{+} \rightarrow \pi^{+} \nu \bar{\nu}$ by observing a large sample of $K^{+} \rightarrow \pi^{+} \nu \bar{\nu}$ decays with small background. Our principal physics goal is to measure the magnitude of the Cabibbo, Kobayashi, Maskawa matrix element $V_{l d}$ with a statistical precision of about $5 \%$ based upon a 100 event sample. This measurement will play a critical role in testing the Standard Model hypothesis that the sole source of CP violation in nature resides in the imaginary parts of the $V_{t d}$ and $V_{u b}$ Cabibbo, Kobayashi, Maskawa matrix elements. Attacking this question in the kaon sector is both experimentally and theoretically independent of the ongoing programs to measure these same parameters in the B meson sector. Each sector provides an independent test of the Standard Model description of CP violation. Both must measure the same parameters for that description to be correct. Such a parallel approach is critical to confirm, with confidence, both the Standard Model description of $\mathrm{CP}$ violation and the veracity of the individual measurements.

The $K^{+} \rightarrow \pi^{+} \nu \bar{\nu}$ decay mode is the theoretically cleanest laboratory in which to measure the magnitude of $V_{t d}$ [1]. The calculation of this decay rate is relative to the $K^{+} \rightarrow \pi^{0} e^{+} \nu$ decay mode. This cancels uncertainties due to the hadronic structure of the $K^{+} \rightarrow \pi^{+} \mathrm{X}$ transition. Long distance contributions to this decay have been calculated [2] [3] [4] [5] to be negligible. The only significant uncertainty in the relationship between the branching ratio and $\left|V_{t d}\right|$ is a small contribution from the charmed quark which depends upon the poorly known charmed quark mass.

Evidence for this decay mode has recently been published [6] by experiment E787 at Brookhaven National Laboratory (BNL). They reported the observation of one event with an expected background of $0.08 \pm 0.03$ events and quote a branching ratio of $\left[1.5_{-1.2}^{+3.4}\right] \times 10^{-10}[6]$. This branching ratio is consistent with the current prediction of $[0.77 \pm 0.21] \times 10^{-10}$. The next important step is a measurement of this rate with sufficient precision to quantitatively challenge the Standard Model interpretation of the source of CP violation. That is the goal of this experiment. The design of our experiment has benefited enormously from the BNL E787 achievement, which has shown that all physics backgrounds to the $10^{-11}$ level have been identified and can be controlled.

The challenge of this measurement then is clearly experimental. A signal of 100 events if the branching ratio is at the predicted level requires that the apparatus control all backgrounds to less than the $10^{-11}$ level. In order to achieve an order of magnitude increase in sensitivity per year of data taking while maintaining excellent control of all backgrounds requires an apparatus with much higher rate capabilities than has been achieved in the BNL experiment. This leads us to propose a decay in flight experiment in contrast to the stopped kaon technique used at BNL. This new experiment is known as "Charged Kaons at the Main Injector", or "CKM".

In addition to the paramount goal of measuring the $K^{+} \rightarrow \pi^{+} \nu \bar{\nu}$ branching ratio we also plan a series of other measurements of rare charged kaon decay properties using the CKM apparatus. The high rate capabilities and redundant measurement capabilities of the CKM spectrometer will make it well suited to such a program of measurements. 


\section{CKM Physics Program}

\section{$2.1 K^{+} \rightarrow \pi^{+} \nu \bar{\nu}$ Theory}

\subsubsection{General Considerations}

The processes induced by Flavor Changing Neutral Currents (FCNC) play an important role in the study of weak decays and in verifying the Standard Model (SM) predictions for the mechanism of CP violation. Due to the unitarity of the Cabbibo-Kobayashi-Maskawa quark mixing matrix $V_{C K M}$, these processes cannot take place at tree level since the matrix elements of the corresponding nondiagonal currents vanish.

However, FCNC processes can take place via loop diagrams containing internal quarks and intermediate bosons, although with very low probability. The contributions from the various diagrams with different quark loops are unequal, as they usually depend on the quark mass. We shall consider the FCNC decays $K^{+} \rightarrow \pi^{+} \nu \bar{\nu}$ and $K_{L}^{0} \rightarrow \pi^{0} \nu \bar{\nu}$ (see the diagrams in figure 1 ). These decays are of particular interest and are called "gold-plated decays" since they can be predicted in the SM framework with very high theoretical accuracy.
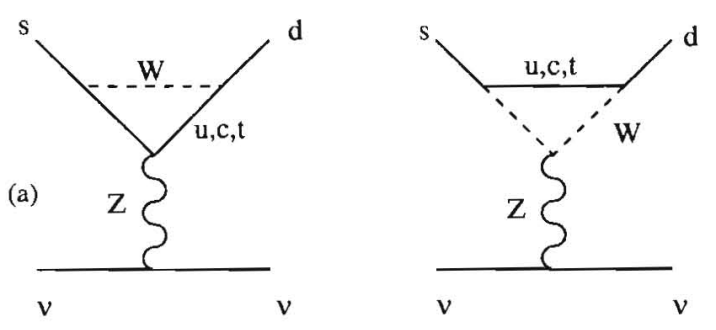

(b)

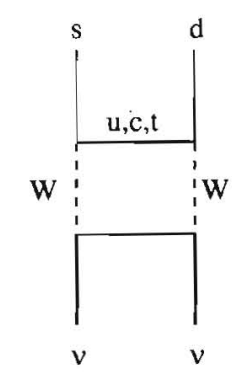

Figure 1: Diagrams for FCNC $s \rightarrow d \nu \bar{\nu}$ decays $(K \rightarrow \pi \nu \bar{\nu}$ decays): a) penguin diagrams, b) box diagram.
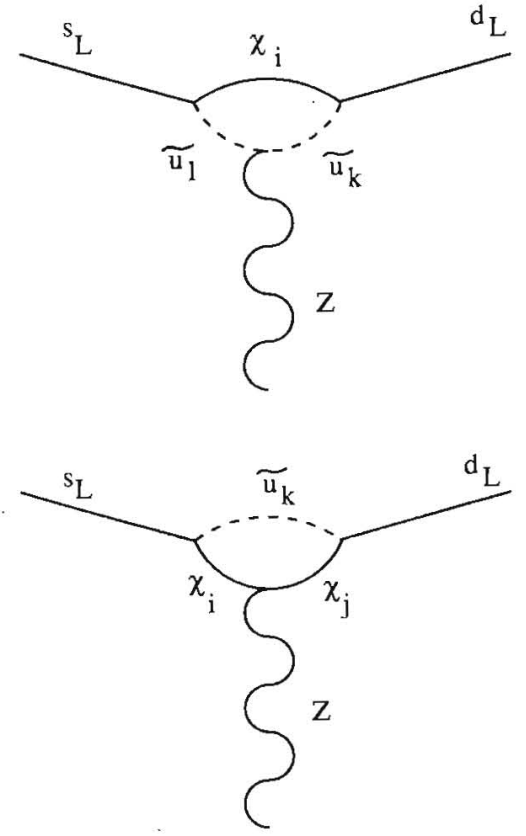

Figure 2: Diagrams containing SUSY contributions to the $Z d \bar{s}$ vertex.

The $K \rightarrow \pi \nu \bar{\nu}$ decays in the SM framework are treated in detail in a number of papers, reviews and monographs [1-24]. We enumerate here the interesting aspects of these decays.

a) The main contribution to the FCNC processes is made by the region of very small distances $r \sim 1 / m_{t}, 1 / m_{Z}$. Therefore in this case, a very accurate description for strong interactions is possible in the framework of perturbative QCD in the leading logarithmic order (LLO) with corrections in the next to leading order (NLO) approximation.

b) The calculation for the matrix element $\left\langle\pi\left|H_{w}\right| K\right\rangle_{\pi \nu \bar{\nu}}$ requires one to relate the quark-level processes to hadronic ones. This is difficult, as it involves long-distance physics. However, 
this can be avoided by using a novel renormalizing procedure developed by Inami and Lim [7]. Through isotopic-spin symmetry, the matrix element $\left\langle\pi\left|H_{w}\right| K\right\rangle_{\pi \nu \bar{\nu}}$ can be related to $\left\langle\pi\left|H_{w}\right| K\right\rangle_{\pi e \nu}$, the matrix element of the well-known decay mode $K^{+} \rightarrow \pi^{0} e^{+} \nu_{e}$.

The difficulties with long-distance physics is therefore minimized in the ratio $B R\left(K^{+}\left(K^{0}\right) \rightarrow\right.$ $\left.\pi^{+}\left(\pi^{0}\right) \nu \bar{\nu}\right) / B R\left(K^{+} \rightarrow \pi^{0} e^{+} \nu\right)$.

c) Since the effective vertex $Z d \bar{s}$ in the diagrams of figure 1 is determined by the region of small distances $R \sim 1 / m_{\ell}, 1 / m_{Z}$, these processes are also sensitive to the contributions from new heavy objects (e.g., supersymmetric particles - see figure 2). Consequently, the comparison of the experimental results with reliable theoretical estimates in the SM framework allows us to search for new physics in these rare decays.

\subsection{2 $K^{+} \rightarrow \pi^{+} \nu \bar{\nu}$ decay Probability}

In the Standard Model, the $K^{+} \rightarrow \pi^{+} \nu \bar{\nu}$ decay is described by penguin and box diagrams presented in figure 1. The partial width of the corresponding processes has the form:

$$
\Gamma\left(K^{+} \rightarrow \pi^{+} \nu \bar{\nu}\right)=\left(\frac{G_{F^{\prime}}}{\sqrt{2}}\right)^{2} \cdot\left|\left\langle\pi^{+} \nu \bar{\nu}\left|H_{w}\right| K^{+}\right\rangle\right|^{2} \cdot 3\left(\frac{\alpha}{2 \pi \sin ^{2} \vartheta_{w}}\right)^{2} \cdot\left|\lambda_{c} F\left(x_{c}\right)+\lambda_{l} F\left(x_{t}\right)\right|^{2}
$$

The factor 3 in the equation accounts for three types of neutrinos $\left(\nu_{e}, \nu_{\mu}, \nu_{\tau}\right)$ participating in the $K^{+} \rightarrow \pi^{+} \nu \bar{\nu}$ decays. The factors $\lambda_{c}$ and $\lambda_{t}$ are $V_{c s}^{*} V_{c d}$ and $V_{t s}^{*} V_{t d}$ respectively. The factors $F\left(x_{c}\right)$ and $F\left(x_{t}\right)$ are the Inami-Lim functions for the loop diagrams in figure 1. They depend on the variables $x_{c}=\left(m_{c} / m_{W}\right)^{2}$ for the c-quark and $x_{\ell}=\left(m_{t} / m_{W}\right)^{2}$ for the t-quark.

The QCD LLO and NLO corrections to the Inami-Lim functions have been evaluated $[15,16$, $21,22]$. After taking these into account, we get $F\left(x_{c}\right)=(9.5 \pm 1.4) \cdot 10^{-4}$ and $F\left(x_{t}\right)=1.53 \pm 0.05$ (the accuracy of QCD calculations improves with increasing quark mass).

The partial width for the well-known decay mode $K^{+} \rightarrow \pi^{0} e^{+} \nu_{e}$ is given by:

$$
\Gamma\left(K^{+} \rightarrow \pi^{0} e^{+} \nu_{e}\right)=\left(\frac{G_{F}}{\sqrt{2}}\right)^{2} \cdot\left|V_{u S}\right|^{2} \cdot\left|\left\langle\pi^{0} e^{+} \nu_{e}\left|H_{w}\right| K^{+}\right\rangle\right|^{2}
$$

As mentioned above, one can relate this to $\left\langle\pi^{+} \nu \bar{\nu}\left|H_{w}\right| K^{+}\right\rangle$with the help of isotopic-spin symmetry:

$$
\left|\frac{\left\langle\pi^{+} \nu \bar{\nu}\left|H_{w}\right| K^{+}\right\rangle}{\left\langle\pi^{0} e^{+} \nu_{e}\left|H_{w}\right| \bar{K}^{+}\right\rangle}\right|^{2}=\left|\frac{\left\langle\pi^{+}\left|H_{w}\right| K^{+}\right\rangle}{\left\langle\pi^{0}\left|H_{w}\right| K^{+}\right\rangle}\right|^{2}=2 r_{+}
$$

Here the factor $\sqrt{2}$ in the amplitude ratio accounts for the quark structure $\left|\pi^{0}\right\rangle=\frac{1}{\sqrt{2}}(u \bar{u}-d \bar{d})$ and $\left|\pi^{+}\right\rangle=|u \bar{d}\rangle$. The factor $r_{+}=0.901$ arises from the phase space correction and the breaking of isotopic symmetry [10].

Hence from $(1)-(3)$ the branching ratio for $K^{+} \rightarrow \pi^{+} \nu \bar{\nu}$ decay is:

$$
B R\left(K^{+} \rightarrow \pi^{+} \nu \bar{\nu}\right)=R_{+} \cdot \frac{\left|\lambda_{c} F\left(x_{c}\right)+\lambda_{t} F\left(x_{t}\right)\right|^{2}}{\left|V_{u s}\right|^{2}}
$$

where

$$
\begin{aligned}
R_{+} & =\left[B R\left(K^{+} \rightarrow \pi^{0} e^{+} \nu_{e}\right) \cdot \frac{3 \alpha^{2}}{2 \pi^{2} \sin ^{4} \vartheta_{w}} \cdot r_{+}\right] \\
& =7.50 \cdot 10^{-6}
\end{aligned}
$$


Here $\alpha=1 / 129, \sin ^{2} \vartheta_{w}=0.23$, and $B R\left(K^{+} \rightarrow \pi^{0} e^{+} \nu_{e}\right)=4.82 \cdot 10^{-2}$.

The values $V_{u s}, \lambda_{c}$, and $\lambda_{t}$ in (4) are determined by the elements of $V_{C K M}[25,26]$ :

$$
\left(\begin{array}{c}
d^{\prime} \\
s^{\prime} \\
b^{\prime}
\end{array}\right)=\left(\begin{array}{lll}
V_{u d} & V_{u s} & V_{u b} \\
V_{c d} & V_{c s} & V_{c b} \\
V_{t d} & V_{t s} & V_{t b}
\end{array}\right)\left(\begin{array}{c}
d \\
s \\
b
\end{array}\right)=V_{C K} M\left(\begin{array}{c}
d \\
s \\
b
\end{array}\right)
$$

The matrix $V_{C K M}$ in the Wolfenstein parameterization [27] is presented as an expansion in powers of $\lambda \equiv V_{u s}=0.2196 \pm 0.0023$. To order $\lambda^{4}$, the $V_{C K M}$ matrix has the form:

$$
V_{C K M}=\left(\begin{array}{ccc}
1-\frac{\lambda^{2}}{2} & \lambda & A \lambda^{3}(\varrho-i \eta) \\
-\lambda & 1-\frac{\lambda^{2}}{2} & A \lambda^{2} \\
A \lambda^{3}(1-\varrho-i \eta) & -A \lambda^{2} & 1
\end{array}\right)+O\left(\lambda^{4}\right) .
$$

In this representation the $V_{C K M}$ matrix is defined by four parameters $\lambda, A \equiv V_{c b} / \lambda^{2}, \rho, i \eta$, i.e. by three real values and one phase, which gives imaginary components to the matrix elements and is therefore responsible for describing all CP violating processes in the SM. Modified parameters

$$
\bar{\rho}=\rho\left(1-\frac{\lambda^{2}}{2}\right) \quad, \bar{\eta}=\eta\left(1-\frac{\lambda^{2}}{2}\right),
$$

are introduced to improve the $V_{C K M}$ decomposition to an accuracy not worse than $O\left(\lambda^{6}\right)$. The complex quantities in expression (4)

$$
\lambda_{c}=V_{c s}^{*} \cdot V_{c d} \quad, \lambda_{t}=V_{t s}^{*} \cdot V_{t d}
$$

have the following forms:

$$
\begin{aligned}
\operatorname{Re} \lambda_{c} & \simeq-\lambda\left(1-\frac{\lambda^{2}}{2}\right)+O\left(\lambda^{5}\right) \\
\operatorname{Im} \lambda_{c} & \simeq-\eta A^{2} \lambda^{5}+O\left(\lambda^{7}\right) \\
\operatorname{Re} \lambda_{t} & =-A^{2} \lambda^{5}\left(1-\frac{\lambda^{2}}{2}\right)(1-\bar{\rho})+O\left(\lambda^{7}\right) \\
\operatorname{Im} \lambda_{t} & =\eta A^{2} \lambda^{5}+O\left(\lambda^{9}\right)
\end{aligned}
$$

The contribution from the c-quark is evidently non-negligible. Although $F\left(x_{c}\right) / F\left(x_{t}\right) \sim 10^{-3}$, the c-quark contribution is enhanced by the fact that $\operatorname{Re} \lambda_{c}$ is much larger than $\operatorname{Re} \lambda_{t}$ and $\operatorname{Im} \lambda_{t}{ }^{1}$ Therefore:

$$
\begin{aligned}
\left|\lambda_{c} F\left(x_{c}\right)+\lambda_{t} F\left(x_{t}\right)\right|^{2} & =\left[\operatorname{Re} \lambda_{c} \cdot F\left(x_{c}\right)+\operatorname{Re} \lambda_{t} \cdot F\left(x_{t}\right)\right]^{2}+\left[\operatorname{Im} \lambda_{c} \cdot F\left(x_{c}\right)+\operatorname{Im} \lambda_{t} \cdot F\left(x_{t}\right)\right]^{2} \\
& \simeq\left[\operatorname{Re} \lambda_{c} \cdot F\left(x_{c}\right)+\operatorname{Re} \lambda_{t} \cdot F\left(x_{t}\right)\right]^{2}+\left[\operatorname{Im} \lambda_{t} \cdot F\left(x_{t}\right)\right]^{2} \\
& \simeq\left[-\lambda^{5}\left(1-\frac{\lambda^{2}}{2}\right) A^{2} F\left(x_{t}\right)\left(\rho_{0}-\bar{\rho}\right)\right]^{2}+\left[\lambda^{5}\left(1-\frac{\lambda^{2}}{2}\right) A^{2} F\left(x_{t}\right) \sigma \eta\right]^{2} \\
& \simeq \lambda^{10} \cdot A^{4} \cdot F\left(x_{t}\right)^{2} \cdot \frac{1}{\sigma}\left[\left(\rho_{0}-\bar{\rho}\right)^{2}+(\sigma \bar{\eta})^{2}\right]
\end{aligned}
$$

Here the following notations have been introduced:

$$
\begin{aligned}
\rho_{0} & =1+\Delta \\
\Delta & =F\left(x_{c}\right) /\left[A^{2} \lambda^{4} F\left(x_{t}\right)\right]=0.42 \pm 0.06 \\
\sigma & =1 /\left(1-\lambda^{2} / 2\right)^{2}=1.050
\end{aligned}
$$

\footnotetext{
${ }^{1} R e \lambda_{c} \sim \lambda$ while $R e \lambda_{t}, \operatorname{Im} \lambda_{t}$, and $\operatorname{Im} \lambda_{c}$ are less than $\lambda^{5}$.
} 
The quantity $\Delta=0.42 \pm 0.06$ is the c-quark contribution.

From (4), (10) and (11) one can obtain the SM prediction for the branching ratio of $K^{+} \rightarrow \pi^{+} \nu \bar{\nu}$.

$$
\begin{aligned}
B R\left(K^{+} \rightarrow \pi^{+} \nu \bar{\nu}\right) \mid S M & =R_{+} A^{4} \lambda^{8} F\left(x_{t}\right)^{2} \frac{1}{\sigma}\left[\left(\rho_{0}-\bar{\rho}\right)^{2}+(\sigma \bar{\eta})^{2}\right] \\
& =R_{+}\left|V_{c b}\right|^{4} F\left(x_{t}\right)^{2} \frac{1}{\sigma}\left[\left(\rho_{0}-\bar{\rho}\right)^{2}+(\sigma \bar{\eta})^{2}\right] \\
& =(0.77 \pm 0.21) \cdot 10^{-10}
\end{aligned}
$$

Here, we have used parameter values as shown in table 1 . It should be kept in mind that the accuracy of the theoretical prediction for $B R\left(K^{+} \rightarrow \pi^{+} \nu \bar{\nu}\right)$ is mainly limited by the c-quark contribution and is equal to $\simeq 5 \%$. It is hopeful that the uncertainty will improve as theoretical work on $\Delta$ continues [17].

However the uncertainty in the estimate for $B R\left(K^{+} \rightarrow \pi^{+} \nu \bar{\nu}\right)$ given in (12) is dominated by the current uncertainty in the $V_{C K M}$ parameters. The estimates for the matrix elements $V_{t d}, V_{t s}$ and $V_{t b}$ are obtained not from direct t-decays, but from other data and the $V_{C K M}$ unitarity conditions. One of the conditions is:

$$
V_{u d} \cdot V_{u b}^{*}+V_{t d} \cdot V_{t b}^{*}=-V_{c d} V_{c b}^{*}
$$

In the Wolfenstein parameterization, this is:

$$
A \lambda^{3}(\bar{\rho}+i \bar{\eta})+A \lambda^{3}(1-(\bar{\rho}+i \bar{\eta}))=A \lambda^{3}
$$

After normalizing by $A \lambda^{3}$ it may be represented as a triangle in the complex plane $(\bar{\rho}, i \bar{\eta})$ as shown in figure 3.

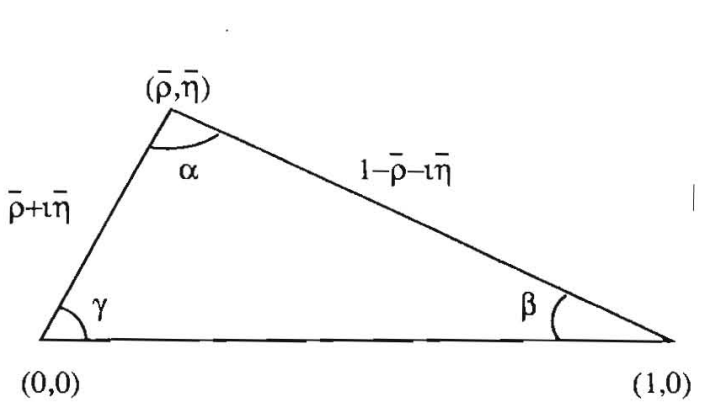

Figure 3: Graphical representation in the $\bar{\rho}, i \bar{\eta}$ plane of the unitary condition $V_{u d} V_{u b}^{*}+$ $V_{t d} V_{t b}^{*}=-V_{c d} V_{c b}^{*}$ after normalization by $-V_{c d} V_{c b} *$.

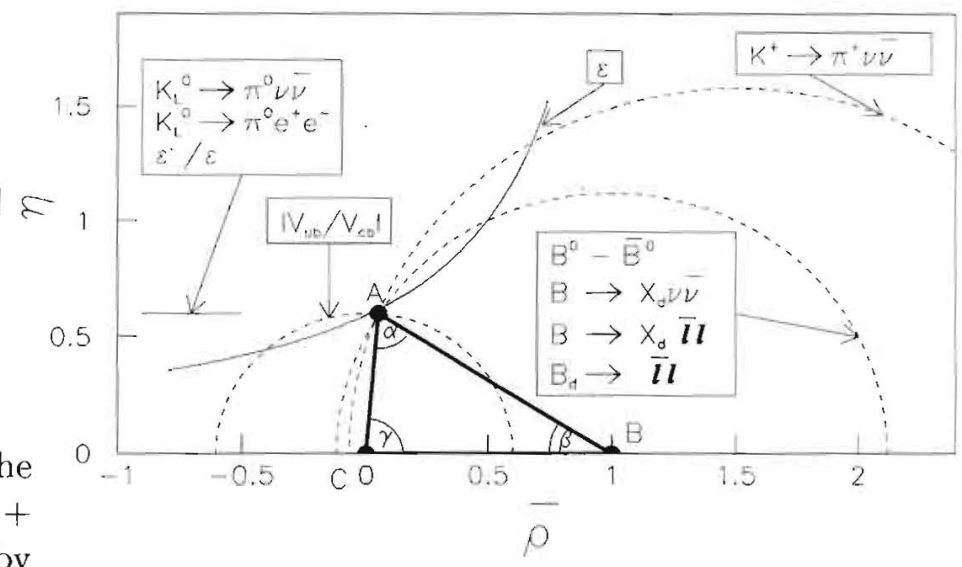

Figure 4: The ideal unitary triangle. For illustration, the value of $\bar{\eta}$ has been chosen to be higher than the fitted central value (reproduced from $[12,13]$ ).

Figure 4 is an idealized diagram showing the connection between the position of the apex of this triangle $(\bar{\rho}, \bar{\eta})$ and various physical processes. In reality, the current theoretical and experimental uncertainties constrain $\bar{\rho}, \bar{\eta}$ to lie in the, region as shown in figure 5 [29]. Detailed analyses of the 


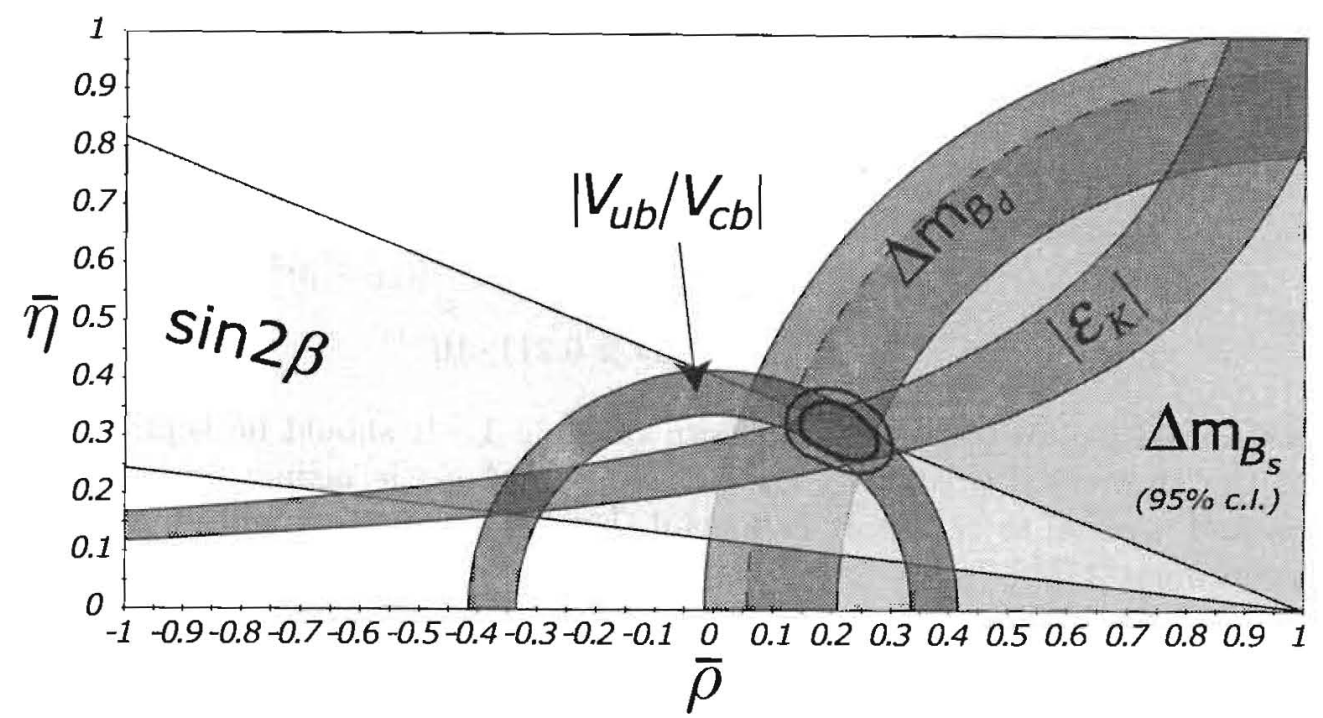

Figure 5: A current snapshot of how parameters of the unitary triangle are constrained. $\varepsilon, \Delta m_{B_{d}}$, $\triangle m_{B_{s}},\left|V_{u b} / V_{c b}\right|, \sin 2 \beta\left(\right.$ from $\left.B^{0}\left(\bar{B}^{0}\right) \rightarrow J / \psi K_{S}^{0}\right)$ - see [29].

$V_{C K M}$ matrix are given in references [28-32]. Table 1 presents the values of relevant parameters used to obtain numerical estimates for the $\left.B R\left(K^{+} \rightarrow \pi^{+} \nu \bar{\nu}\right)\right|_{S M}$ in (12) and some other processes to be considered later.

\subsection{3 $\left|V_{t d}\right|$ Determination from $K^{+} \rightarrow \pi^{+} \nu \bar{\nu}$ Measurement}

The matrix element $\left|V_{t d}\right|$ can be extracted from a measurement of $B R\left(K^{+} \rightarrow \pi^{+} \nu \bar{\nu}\right)$, which we propose to measure to $10 \%$ precision. The precision on the determination of $\left|V_{t d}\right|$, while it depends on the $B R\left(K^{+} \rightarrow \pi^{+} \nu \bar{\nu}\right)$ experimental uncertainty, also depends on other parameters appearing in equation (12), especially $V_{c b}$ and the mass of the charmed quark [16]:

$$
\frac{\sigma\left(\left|V_{t d}\right|\right)}{\left|V_{t d}\right|}= \pm 0.04_{\text {scale }} \pm \frac{\sigma\left(\left|V_{c b}\right|\right)}{\left|V_{c b}\right|} \pm 0.7 \frac{\sigma\left(\bar{m}_{c}\right)}{\bar{m}_{c}} \pm 0.65 \frac{\sigma\left[B R\left(K^{+} \rightarrow \pi^{+} \nu \bar{\nu}\right)\right]}{B R\left(K^{+} \rightarrow \pi^{+} \nu \bar{\nu}\right)}
$$

The \pm 0.04 represents the renormalization scale uncertainty stemming mainly from $\mu_{c}$. For $\sigma\left(\left|V_{c b}\right|\right)=$ $0.002, \sigma\left(m_{c}\right)=100 \mathrm{MeV}$, and a $10 \%$ branching ratio uncertainty, the parameter $\left|V_{t d}\right|$ is determined to $10 \%$ precision. This precision is reduced to $\pm 11 \%$ once the uncertainties due to $m_{t}, \alpha_{s}$, and $\left|V_{u b}\right| /\left|V_{c d}\right|$ are taken into account. This error has a somewhat larger contribution from the parameter uncertainties than from the branching ratio uncertainty.

A possible outcome for the $\bar{\rho}, \bar{\eta}$ plane from the CKM measurement of $K^{+} \rightarrow \pi^{+} \nu \bar{\nu}$ is shown figure 6. We can hope that future QCD calculations in the next approximation as well as a better determination of $\left|V_{c b}\right|$ will increase this precision. 


$\lambda=\left|V_{u s}\right|=0.2196 \pm 0.0023$
$\bar{\rho}=0.223 \pm 0.038$
$\bar{\eta}=0.316 \pm 0.039$
$\left|V_{c b}\right|=(41.0 \pm 1.6) \cdot 10^{-3}$
$\left|V_{u b}\right|=(35.5 \pm 3.6) \cdot 10^{-4}$
$A=V_{c b} / \lambda^{2}=0.819 \pm 0.049$
$\sin 2 \beta=0.70 \pm 0.07$
$m_{t}=\bar{m}_{t}=167 \pm 5 \mathrm{GeV}$
$F\left(x_{t}\right)=1.53 \pm 0.05$
$\rho_{0}=1+\frac{P_{0}}{A^{2} \cdot F\left(x_{t}\right)}=1+F\left(x_{c}\right) / A^{2} \lambda^{4} F\left(x_{t}\right)=1.42 \pm 0.06$
$\xi=f_{B_{s}} \sqrt{B_{s}} / f_{B_{d}} \sqrt{B_{d}}=1.14 \pm 0.06$
$m_{B_{d}^{0}}=5.2792 \mathrm{GeV}$
$m_{B_{s}^{0}}=5.3693 \mathrm{GeV}$
$m_{w}=80.41 \mathrm{GeV}$

Table 1: Some SM and CKM matrix parameters used for the evaluation of $\mathrm{BR}(K \rightarrow \pi \nu \nu)$ and other values. The value for $\lambda$ is taken from [116]. The rest comes from [31]. 


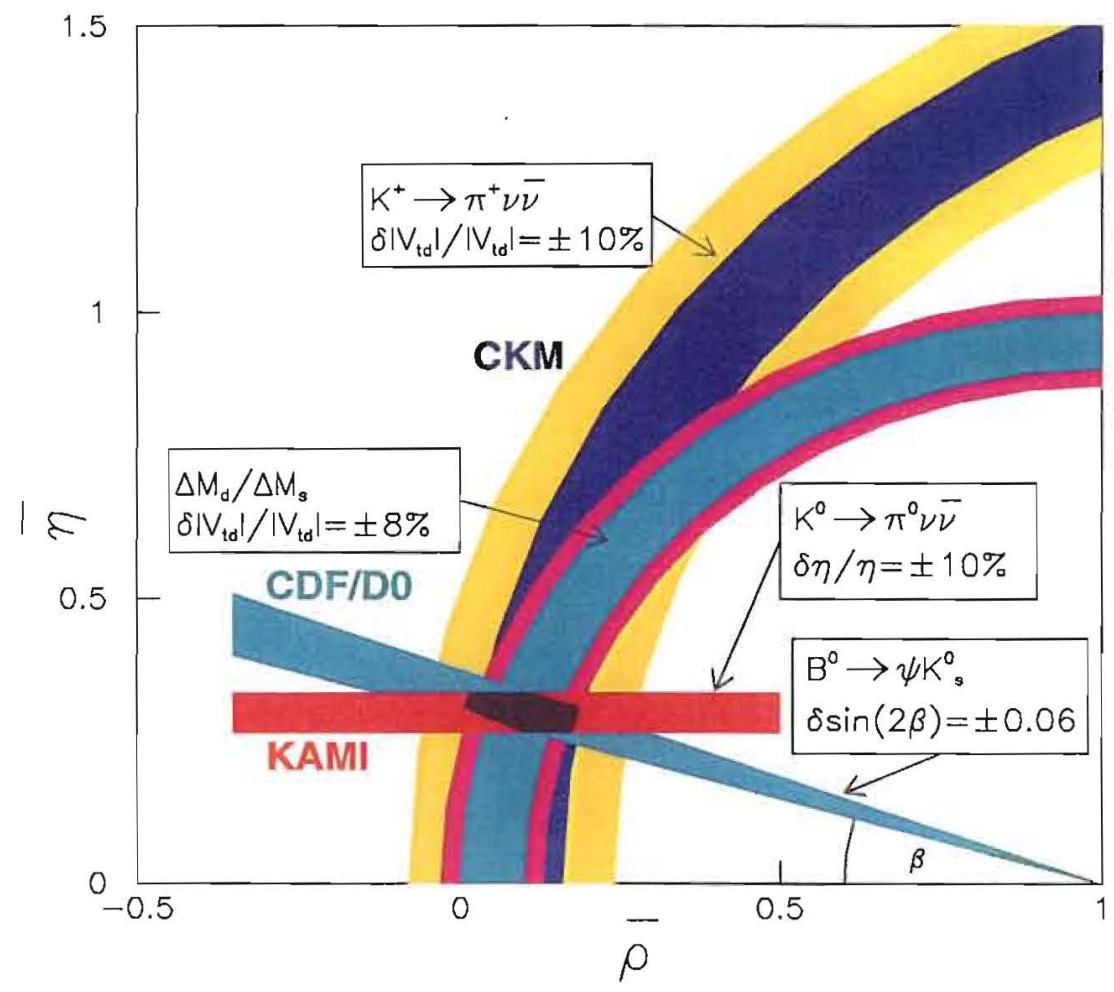

Figure 6: A possible outcome for $\bar{\eta}, \bar{\rho}$ and $\beta$ obtained from future $\mathrm{K}$-meson and B-meson experiments: $K^{+} \rightarrow \pi^{+} \nu \bar{\nu}(\mathrm{CKM}) ; K_{L}^{0} \rightarrow \pi^{0} \nu \bar{\nu}(\mathrm{KAMI} / \mathrm{K} 0 \mathrm{PI} 0)$ and $\triangle m_{B_{d}} / \triangle m_{B_{s}} ; B^{0}\left(\bar{B}^{0}\right) \rightarrow J / \psi K_{S}^{0}$. The light region includes possible theoretical uncertainties in $B R\left(K^{+} \rightarrow \pi^{+} \nu \bar{\nu}\right)$ connected with the influence of c-quarks. 


\subsection{Related $K_{L}^{0}$ and B Measurement and Study of the Prediction of CP-violation Mechanisms in the SM and Beyond}

\subsubsection{Future data of $K_{L}^{0}$ studies}

It was shown in $[22,33,34]$ that $K_{L}^{0} \rightarrow \pi^{0} \nu \bar{\nu}$ decay will be governed by direct CP-violation process ${ }^{2}$ with the branching ratio prediction in the SM of

$$
\begin{aligned}
\left.B R\left[K_{L}^{0} \rightarrow \pi^{0} \nu \vec{\nu}\right]\right|_{S M} & =R_{0} \cdot \lambda^{8} \cdot\left[\frac{\operatorname{Im} \lambda_{t}}{\lambda^{5}} F\left(x_{t}\right)\right]^{2} \\
& =R_{0} \cdot \lambda^{8} A^{4} \cdot F\left(x_{t}\right)^{2} \cdot \sigma|\bar{\eta}|^{2} \\
& =R_{0} \cdot\left|V_{c b}\right|^{4} \cdot F\left(x_{t}\right)^{2} \cdot \sigma|\bar{\eta}|^{2} \\
& =(2.6 \pm 1.8) \cdot 10^{-11}
\end{aligned}
$$

where

$$
\begin{aligned}
R_{0} & =B R\left(K^{+} \rightarrow \pi^{0} e^{+} \nu\right) \cdot \frac{3 \alpha^{2}}{2 \pi^{2} \sin ^{4} \vartheta_{w}} \cdot \frac{\tau\left(K_{L}^{0}\right)}{\tau\left(K^{+}\right)} r_{0} \\
& =R_{+} \frac{\tau\left(K_{L}^{0}\right)}{\tau\left(K^{+}\right)} \cdot \frac{r_{0}}{r_{+}} \\
& =3.28 \cdot 10^{-5}
\end{aligned}
$$

The factor $r_{0}=0.944$ corrects for phase space and the isotopic-spin symmetry breaking in the $K_{L}^{0} \rightarrow \pi^{0} \nu \bar{\nu}$ decay. The parameters $\tau\left(K_{L}^{0}\right)$ and $\tau\left(K^{+}\right)$are the lifetimes of $K_{L}^{0}$ and $K^{+}$respectively. The definitions are the same as in (12) and the parameter values were taken from table 1 . As shown in the above, the $\mathrm{SM}$ prediction for $\operatorname{BR}\left(K_{L}^{0} \rightarrow \pi^{0} \nu \bar{\nu}\right)$ is theoretically clean as it does not suffer from the c-quark correction uncertainty.

It is interesting and worthwhile to compare $\operatorname{BR}\left(K_{L}^{0} \rightarrow \pi^{0} \nu \bar{\nu}\right)$ to $\operatorname{BR}\left(K^{+} \rightarrow \pi^{+} \nu \bar{\nu}\right)$. From (12) and (14), we get:

$$
\frac{B R\left(K_{L}^{0} \rightarrow \pi^{0} \nu \bar{\nu}\right)}{B R\left(K^{+} \rightarrow \pi^{+} \nu \bar{\nu}\right)}=\frac{R_{0}}{R_{+}}\left[\frac{(\sigma \bar{\eta})^{2}}{\left(\rho_{0}-\bar{\rho}\right)^{2}+(\sigma \bar{\eta})^{2}}\right]=\frac{\tau\left(K_{L}^{0}\right)}{\tau\left(K^{+}\right)} \frac{r_{0}}{r_{+}}\left[\frac{(\sigma \bar{\eta})^{2}}{\left(\rho_{0}-\bar{\rho}\right)^{2}+(\sigma \bar{\eta})^{2}}\right]
$$

Hence in the ratio, one gets a theoretically clean constraint on the angle $\beta$ of the unitary triangle (see figure 3 ), as the large uncertainty from $V_{c b}^{4}$ is canceled. This is discussed later in table 2. However, the c-quark correction uncertainty remains as indicated by the presence of $\rho_{0}=1.42 \pm 0.06$.

Another technique is to combine the constraints from $\epsilon_{K}$ together with $K \rightarrow \pi \nu \nu$, when the latter two are fully established. In this manner, the uncertainty on $V_{c b}$ cancels, and the dominant theoretical uncertainties remain to be $B_{K}$ and $\Delta$ (the charm quark uncertainty). These three measurements would overconstrain one of the quark-mixing unitary relations, but only to the accuracy permitted by the current uncertainties on $B_{K}$ and $\Delta$.

Another very interesting decay is $K_{L}^{0} \rightarrow \pi^{0} e^{+} e^{-}$, as the branching ratio contains a direct-CP violating component $[13-16,35,36]$ :

$$
\begin{aligned}
\left.B R\left(K^{+} \rightarrow \pi^{0} e^{+} e^{-}\right)\right|_{C P-d i r .} & =2.45 \cdot 10^{-4} \cdot\left|V_{c b}\right|^{4} \lambda^{2} \sigma(\bar{\eta})^{2} \\
& =(4.1 \pm 1.3) \cdot 10^{-12}
\end{aligned}
$$

\footnotetext{
${ }^{2}$ More precisely, by the interference between the direct CP-violating amplitude $s \rightarrow d \nu \bar{\nu}$ and the amplitude for $K^{0} \rightleftarrows \bar{K}^{-0}$ mixing [34].
} 
Unfortunately the extraction of $\left.B R\left(K^{+} \rightarrow \pi^{0} e^{+} e^{-}\right)\right|_{C P-d i r}$ is complicated by the need to untangle the direct-CP violating amplitudes from the indirect-CP violating as well as $C P$ conserving amplitudes of this decay (see detailed discussion in $[15,16]$ ). There is also a very serious experimental difficulty to suppress the background from $K_{L}^{0} \rightarrow e^{+} e^{-} \gamma \gamma$ decay.

Up to now the existing upper limits for the branching ratios $K_{L}^{0} \rightarrow \pi^{0} \nu \bar{\nu}$ and $K_{L}^{0} \rightarrow \pi^{0} e^{+} e^{--}$ are much higher than their SM predictions. Thus the studies of these decays are the aims of very difficult and sophisticated experiments. The measurement of the $K_{L}^{0} \rightarrow \pi^{0} \nu \bar{\nu}$ decay have been proposed by KAMI [37] and KOPIO [38]. The KAMI proposal includes also the attempt to measure $K_{L}^{0} \rightarrow \pi^{0} l^{+} l^{-}$.

\subsubsection{B-meson experiments and unitarity triangle}

In the B-meson system this story is much longer and more complicated. The same authors have studied the theoretical limitations of the B sector measurements [12-16,22,24]. A summary of their conclusions is as follows:

1. The present uncertainty on $\left|V_{t d}\right|$ measured with the $B_{d}$ mixing parameter $x_{d}=\Delta m_{B_{d}} / \Gamma_{B_{d}}$ is already dominated by the uncertainties in the lattice calculations of $f_{B_{d}}$ and $B_{B_{d}}$. This is a theoretical limitation common to most of the hadronic B decays.

2. The $x_{s} / x_{d}$ ratio of these observable is significantly less sensitive to lattice calculation uncertainties. Currently, the $\mathrm{B}_{s}$ mixing parameter $x_{s}$ has not yet been firmly established. This would be measured in future hadronic $B$ production experiments, where good tagging is crucial for distinguishing $B_{s}$ from $B_{d}$ events.

3. Among the conventional measurements of the unitarity triangle angles $\alpha, \beta$ and $\gamma$ expected from the $B$ factories, only $\beta$ from the measurement of $B^{0} \rightarrow \psi K_{S}\left(K_{L}\right)$ is free from significant theoretical uncertainties.

4. $\alpha$ measured with $B^{0} \rightarrow \pi^{+} \pi^{-}$suffers from significant "penguin pollution" which must be overcome before this measurement can be useful for CKM parameter determinations. The isospin analysis required to control this problem requires measurements of modes which are probably too low in branching ratio to be well measured with the available luminosity.

5. The $B$ sector analogies of the ultra-rare kaon decays; $B^{0} \rightarrow X_{d} \nu \nu, B^{0} \rightarrow X_{s} \nu \bar{\nu}, B_{d}^{0} \rightarrow \mu^{+} \mu^{-}$ and $B_{s}^{0} \rightarrow \mu^{+} \mu^{-}$are likewise theoretically clean. The Standard Model branching ratios for these modes are believed to be beyond the sensitivity of all proposed experiments.

The $B$ sector experiments have more than ample experimental insurance. Eight experiments are in process of working or under construction: Babar, Belle, CDF, CLEO-III, Dzero, HERA-B, BTeV and LHCB.

The most theoretically unambiguous constraint on $V_{C K M}$ can be obtained in 2 types of measurements.

a) The intensity of $B_{d}^{0} \rightleftarrows \bar{B}_{d}^{0}$ and $B_{s}^{0} \rightleftarrows \bar{B}_{s}^{0}$ mixing depends on $\triangle m_{B_{d}}$ and $\triangle m_{B_{s}}$, the mass differences between the "heavy" and "light" eigenstates of $B^{0}$ mesons.

$$
\left.\begin{array}{l}
\Delta m_{B_{d}} \simeq \frac{G_{F}^{2}}{6 \pi^{2}} m_{W}^{2} m_{B_{d}} f_{B_{d}}^{2} B_{B_{d}} \eta_{B} S\left(x_{t}\right)\left|V_{t d} V_{t b}^{*}\right|^{2} \\
\Delta m_{B_{s}} \simeq \frac{G_{F}^{2}}{6 \pi^{2}} m_{W}^{2} m_{B_{s}} f_{B_{s}}^{2} B_{B_{s}} \eta_{B} S\left(x_{t}\right)\left|V_{t s} V_{t b}^{*}\right|^{2}
\end{array}\right\}
$$


Here $S\left(x_{l}\right)$ is the corresponding Inami-Lim function for the box diagrams responsible for $B^{0} \rightleftarrows \vec{B}^{0}$ mixing; $\eta_{B}=0.55 \pm 0.01$ is QCD correction factor; $f_{B_{d}}^{2}, B_{B_{d}}, f_{B_{s}}^{2}, B_{B_{s}}$ are coupling constants and decay parameters for $B_{d}^{0}$ and $B_{s}^{0}$ mesons and $m_{B_{d}}, m_{B_{s}}-$ their masses. The values of the coupling constants and decay parameters are obtained from the lattice QCD calculations and have large systematic uncertainties. Thus it is much more convenient to use the ratio:

$$
R_{\triangle m_{B}}=\frac{\triangle m_{B_{d}}}{\triangle m_{B_{s}}}=\frac{m_{B_{d}}}{m_{B_{s}}} \cdot \frac{f_{B_{d}}^{2}}{f_{B_{s}}^{2}} \cdot \frac{B_{B_{d}}}{B_{B_{s}}} \cdot \frac{\left|V_{t d}\right|^{2}}{\left|V_{t s}\right|^{2}}=\frac{m_{B_{d}}}{m_{B_{s}}} \cdot \frac{1}{\xi^{2}}\left|\frac{V_{t d}}{V_{t s}}\right|^{2}
$$

Here

$$
\begin{gathered}
\xi=f_{B_{s}} \cdot \sqrt{B_{s}} / f_{B_{d}} \cdot \sqrt{B_{d}}=1.14 \pm 0.06 \\
\Delta m_{B_{d}}=(0.473 \pm 0.016) p s^{-1} \\
\Delta m_{B_{s}}>15.0 p^{-1}(95 \% \text { C.L. })
\end{gathered}
$$

Thus

$$
R_{\triangle m_{B}}<3.0 \cdot 10^{-2} \text { and }\left|\frac{V_{t s}}{V_{t d}}\right|>5.0(95 \% \text { C.L. })
$$

b) Another important measurement is the search for the CP asymmetry in the decays $B_{d}\left(\bar{B}_{d}^{0}\right) \rightarrow$ $J / \psi K_{S}\left(K_{L}\right)$. For $B_{d}\left(\bar{B}_{d}^{0}\right) \rightarrow J / \psi K_{S}:$

$$
\begin{aligned}
A_{C P} & =\frac{\left[B(t)^{0} \rightarrow J / \psi K_{S}^{0}\right]-\left[\bar{B}(t)^{0} \rightarrow J / \psi K_{S}^{0}\right]}{\left[B(t)^{0} \rightarrow J / \psi K_{S}^{0}\right]+\left[\bar{B}(t)^{0} \rightarrow J / \psi K_{S}^{0}\right]} \\
& =-a_{\psi K_{S}^{0}} \cdot \sin \triangle M_{B_{d}} t=-\sin 2 \beta \cdot \sin \triangle M_{B_{d}} t
\end{aligned}
$$

The first results of measurements in the CDF (FNAL), electron B factories BaBar (SLAC), Belle (KEK) and LEP data in average gave [32]:

$$
\left\langle a_{\psi K^{0}}\right\rangle=\langle\sin 2 \beta\rangle=0.48 \pm 0.16
$$

The $\mathrm{SM}$ prediction is $\left.\sin 2 \beta\right|_{S M}=0.70 \pm 0.07$. It is expected that the precision for these very important asymmetry measurements will increase significantly in the next several years.

\subsection{Over-constraining the Unitary Condition and Detecting Effects Beyond the $\mathrm{SM}$}

In this section we discuss over-constraining the unitary triangle, with due emphasis on the measurements of $K \rightarrow \pi \nu \nu$ as they provide theoretically clean constraints. This gives the possibility to see effects beyond the SM, and we enumerate some of the possibilities here. This subject has been widely discussed in the recent literature, and we summarize the key ideas.

The apex of the unitary triangle can be reconstructed from the measurements of $B R\left(K^{+} \rightarrow\right.$ $\left.\pi^{+} \nu \bar{\nu}\right)$ and $B R\left(K_{L}^{0} \rightarrow \pi^{0} \nu \bar{\nu}\right)$ as the crossing of an ellipse (equation 12) and a horizontal line (equation 14) as shown in figure 6 . The combined $K \rightarrow \pi \nu \nu$ measurements can determine $\sin (2 \beta)$ without being affected by the uncertainty on $\left|V_{c b}\right|$. This is enumerated in table 2 and denoted as $\left.\sin (2 \beta)\right|_{K}$. The expected measurement accuracy is $\left.\sigma(\sin 2 \beta)\right|_{K}=0.07[15,16]$. This is comparable to the expected precision on $\sin (2 \beta)$ derived from the CP asymmetry in $B_{d}^{0}, \bar{B}_{d}^{0} \rightarrow J / \psi K_{S}^{0}$ (denoted as $\left.\left.\sin (2 \beta)\right|_{B}\right)$, which is $0.06-0.08$.

As a result one will be able to get independently the information on direct $\mathrm{CP}$-violating processes from $\mathrm{K}$ and $\mathrm{B}$ experiments. Obviously, if the $\mathrm{SM}$ is correct then $\left.\sin 2 \beta\right|_{B}=\left.\sin 2 \beta\right|_{K}$, as all CPviolating phenomena in the SM are described by a single phase. 
Branching ratios of $K \rightarrow \pi \nu \bar{\nu}$ decays in the SM (see (12) and (14))

$$
\begin{aligned}
B R\left(K^{+} \rightarrow \pi^{+} \nu \bar{\nu}\right) & =4.06 \cdot 10^{-11} A^{4} F\left(x_{t}\right)^{2} \cdot \frac{1}{\sigma}\left[\left(\rho_{0}-\bar{\rho}\right)^{2}+(\sigma \bar{\eta})^{2}\right]= \\
& =4.06 \cdot 10^{-11}\left[\left(\frac{\operatorname{Im} \lambda_{t}}{\lambda^{5}} F\left(x_{t}\right)\right)^{2}+\left(\frac{\operatorname{Re} \lambda_{c}}{\lambda} P_{0}+\frac{\operatorname{Re} \lambda_{t}}{\lambda^{5}} F\left(x_{t}\right)\right)^{2}\right] \\
B R\left(K_{L}^{0} \rightarrow \pi^{0} \nu \bar{\nu}\right) & =1.78 \cdot 10^{-10} A^{4} F\left(x_{t}\right)^{2} \sigma(\bar{\eta})^{2}=1.78 \cdot 10^{-10}\left(\frac{\operatorname{Im} \lambda_{t}}{\lambda^{5}} F\left(x_{t}\right)\right)^{2}
\end{aligned}
$$

Reduced branching ratios:

From (12) and (14):

$$
B_{1}=\frac{B R\left(K^{+} \rightarrow \pi^{+} \nu \bar{\nu}\right)}{4.06 \cdot 10^{-11}} ; B_{2}=\frac{B R\left(K_{L}^{0} \rightarrow \pi^{0} \nu \bar{\nu}\right)}{1.78 \cdot 10^{-10}}
$$

$$
\begin{aligned}
\operatorname{Im} \lambda_{t}=\lambda^{5} \frac{\sqrt{B_{2}}}{F\left(x_{t}\right)} \\
\operatorname{Re} \lambda_{t}=-\lambda^{5}\left[\frac{\frac{R e \lambda_{c}}{\lambda} P_{0}+\sqrt{B_{1}-B_{2}}}{F\left(x_{t}\right)}\right] \\
\operatorname{From}(-8):
\end{aligned} \quad\left\{\begin{aligned}
\bar{\rho}=1+\frac{P_{0}-\sqrt{\sigma\left(B_{1}-B_{2}\right)}}{A^{2} F\left(x_{t}\right)} \\
\bar{\eta}=\frac{\sqrt{B_{2}}}{\sqrt{\sigma} A^{2} F\left(x_{t}\right)} \\
V_{t d}=A \lambda^{3}(1-\bar{\rho}-i \bar{\eta}) \\
V_{t d} \mid=A \lambda^{3} \cdot \sqrt{(1-\bar{\rho})^{2}+\bar{\eta}^{2}}
\end{aligned}\right\} \begin{aligned}
& \text { These values } \\
& \text { can be } \\
& \text { determined } \\
& \text { from } \\
& \text { measurements } \\
& \text { of branching } \\
& \text { ratios for } \\
& K \rightarrow \pi \nu \bar{\nu} \text { decays } \\
& \text { i.e. } B_{1} \text { and } B_{2}
\end{aligned}
$$

We introduce

$$
\begin{aligned}
& r_{s}=\frac{1-\bar{\rho}}{\bar{\eta}}=\sqrt{\sigma} \frac{\left[\sqrt{\sigma\left(B_{1}-B_{2}\right)}-P_{0}\right]}{\sqrt{B_{2}}} \\
& \sin 2 \beta=\frac{2 \bar{\eta}(1-\bar{\rho})}{(1-\bar{\rho})^{2}+\bar{\eta}^{2}}=\frac{2 r_{s}}{1+r_{s}^{2}} \quad\left\{\begin{array}{l}
\text { for } \sin 2 \beta \text { from } K \rightarrow \pi \nu \bar{\nu} \text { decays is dependent } \\
\text { only on the precision of measurements of } B_{1} \\
\text { and } B_{2} \text { and from calculation of } P_{0}=F\left(x_{c}\right) / \lambda^{4}
\end{array}\right.
\end{aligned}
$$

$$
\text { For }\left.\mathrm{SM} \sin 2 \beta\right|_{K}=\frac{2 r_{S}}{1+r_{s}^{2}}=a_{\psi K_{S}^{0}}=\left.\sin 2 \beta\right|_{B}
$$

Table 2: Determination of $\sin 2 \beta$ and $\left|V_{t d}\right|$ from branching ratios $B R\left(K^{+} \rightarrow \pi^{+} \nu \bar{\nu}\right)$ and $B R\left(K_{L}^{0} \rightarrow\right.$ $\left.\pi^{0} \nu \bar{\nu}\right)$. See $[13-16]$. 
It is worth noting that $K \rightarrow \pi \nu \nu$ and $B_{d}^{0}\left(\bar{B}_{d}^{0}\right) \rightarrow J / \psi K_{S}$ are distinctly different processes, so that new physics might differentiate between these two. The FCNC decays $K \rightarrow \pi \nu \bar{\nu}$ are loop processes and therefore the $Z d \bar{s}$ vertex in these decays is especially sensitive to the contributions from new interactions. On the other hand, the decays $B_{d}^{0}\left(\bar{B}_{d}^{0}\right) \rightarrow J / \psi K_{S}$ can proceed at tree level via $b \rightarrow c \bar{c} s$ and $\bar{b} \rightarrow \bar{c} c \bar{s}$.

Regarding CP violation, the CP asymmetry $a_{\psi K_{S}}$ is caused by the relative phase between the $B_{d}^{0} \rightleftarrows \bar{B}_{d}^{0}$ mixing amplitude and that of the tree-level $b \rightarrow c \bar{c} s$ decay [39]. On the other hand, $\mathrm{CP}$ violation in the $K_{L} \rightarrow \pi^{0} \nu \bar{\nu}$ decay is due to the relative phase between the $K^{0} \rightleftarrows \bar{K}^{0}$ mixing amplitude and the FCNC amplitudes of $s \rightarrow d \nu \bar{\nu}$ shown in figure 1.

New physics such as supersymmetry, where there are additional Higgs doublets and other mechanisms of CP violation, may differentiate between $\mathrm{K}$ and $\mathrm{B}$ measurements [39]. In the $B_{d}^{0}\left(\bar{B}_{d}^{0}\right)^{\prime} \rightarrow J / \psi K_{S}$ decays these mechanisms are very likely to manifest themselves in the $B_{d}^{0} \rightleftarrows \bar{B}_{d}^{0}$ mixing amplitudes, which are loop diagrams at small distances and sensitive to the contribution from new heavy particles. The first order process $b \rightarrow c \bar{c} s$ would remain in this case essentially unchanged. The $a_{\psi K_{S}^{0}}$ asymmetry in the B-decays would now be determined not only by the CKM phase (which leads to the $\beta$ angle) but also by a new additional phase $\beta_{d}$ as well. Consequently, the CP asymmetry $a_{\psi K_{S}^{\circ}}$ will change and will have the form $a_{\psi K_{S}}=\sin \left(\beta+\beta_{d}\right)$.

As it was previously mentioned, new mechanisms for the $K \rightarrow \pi \nu \bar{\nu}$ decays may influence considerably the loop FCNC processes which are sensitive to the effects at small distances. In this case, the ratio of $\mathrm{BR}\left(K^{+} \rightarrow \pi^{+} \nu \bar{\nu}\right)$ and $\mathrm{BR}\left(K_{L}^{0} \rightarrow \pi^{0} \nu \bar{\nu}\right)$ is very likely to change, and then the value for $\left.\sin 2 \beta\right|_{K}$ will also change. Hence if there exists new mechanisms for $\mathrm{CP}$ violation, the values for $\left.\sin 2 \beta\right|_{B}$ and $\left.\sin 2 \beta\right|_{K}$ may become significantly different, and the proposed future $\mathrm{K}$ experiments may be able to detect this.

Some other comparisons between the experimental data for B-mesons and $K \rightarrow \pi \nu \bar{\nu}$ decays have been considered (see for example [41]). One of them is between $B R\left(K^{+} \rightarrow \pi^{+} \nu \bar{\nu}\right)$ and $R_{\triangle m_{B}}=\Delta m_{B_{d}} / \Delta m_{B_{s}}$ as shown in figure 7. Recall that these are independent measurements of $\left|V_{t d}\right|$. Violation of such correlations might indicate a possible manifestation of new physics. As it follows from the figure, a quite reliable upper limit for $B R\left(K^{+} \rightarrow \pi^{+} \nu \bar{\nu}\right)$ may be obtained for the $\mathrm{SM}$ if one uses the data on the B-meson mass difference (see also $[15,16]$ ):

$$
B R\left(K^{+} \rightarrow \pi^{+} \nu \bar{\nu}\right)_{\max }<(1.0-1.2) \cdot 10^{-10}
$$

The experimental limits for FCNC processes, presented in table 3, still leave a large region to search for new physics in $K \rightarrow \pi \nu \bar{\nu}$ decays. Such effects were dealt with in a number of papers $[16,19,39,42-58]$. The new physics considered encompasses new heavy particles, new interactions at small distances, and new mechanisms of $\mathrm{CP}$ violation, which is characterized by additional complex parameters (new phases). As shown in table 3, numerous predictions have been made as to the possible modification to the FCNC processes, in particular, for rare K-meson decays.

As shown in [42], in nearly all models with new physics but with only left-handed neutrinos, the effective Hamiltonian which describing $K \rightarrow \pi \nu \bar{\nu}$ decays has the form:

$$
H_{e f f}=\frac{2 G_{F}}{\sqrt{2}} \cdot \frac{\alpha}{\pi \sin ^{2} \vartheta_{w}} \lambda_{t} \cdot \bar{s} \gamma^{\mu}\left(X_{L} P_{L}+X_{R} P_{R}\right) d \cdot \bar{\nu}_{l} \gamma_{\mu} P_{L} \nu_{e}+\text { (herm.conjug.) }
$$

Here $P_{L}=\left(1-\gamma_{5}\right)$ and $P_{R}=\left(1+\gamma_{5}\right)$, which correspond to left and right currents (with $X_{L}$ and $X_{R}$ ).

This Hamiltonian (23) leads to the following branching ratios for $K \rightarrow \pi \nu \bar{\nu}$ decays:

$$
B R\left(K^{+} \rightarrow \pi^{+} \nu \bar{\nu}\right)=7.50 \cdot 10^{-6}\left|\frac{\lambda_{t}\left(X_{L}+X_{R}\right)}{\lambda}\right|^{2}
$$




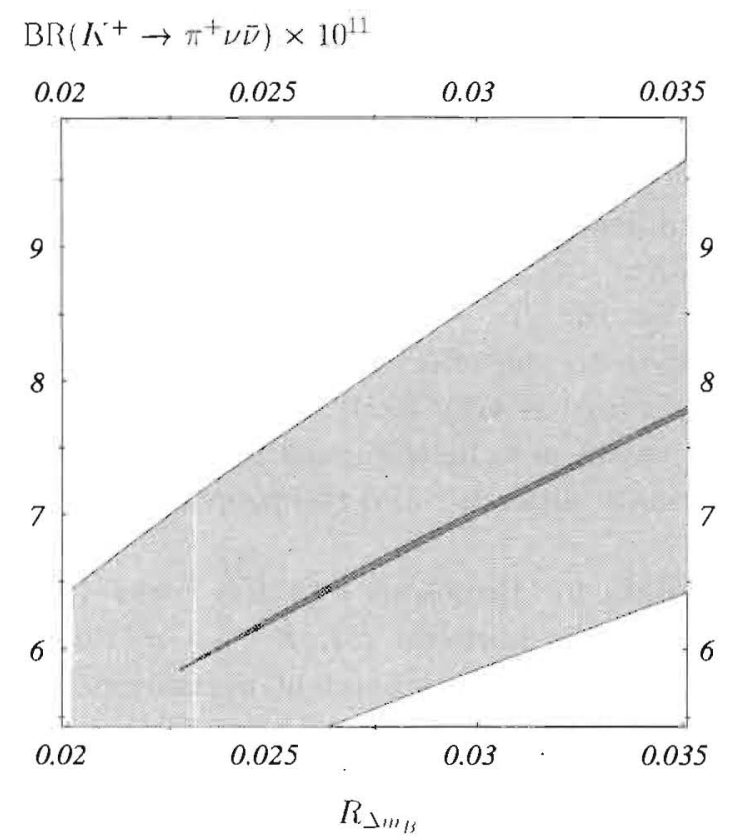

Figure 7: Correlation between $B R\left(K^{+} \rightarrow\right.$ $\left.\pi^{+} \nu \bar{\nu}\right)$ and $R_{\triangle m_{B}}=\Delta m_{B_{d}} / \Delta m_{B_{s}}$ in the SM [41]. A scan over the presently allowed region $0<\bar{\rho}<0.27 ; 0.26<\bar{\eta}<0.37$ yields the dark region for central value $\triangle=P_{0} / A^{2} \cdot F\left(x_{t}\right)=$ 0.42 in (11) or light region for acceptable values $0.31<\Delta<0.54$.
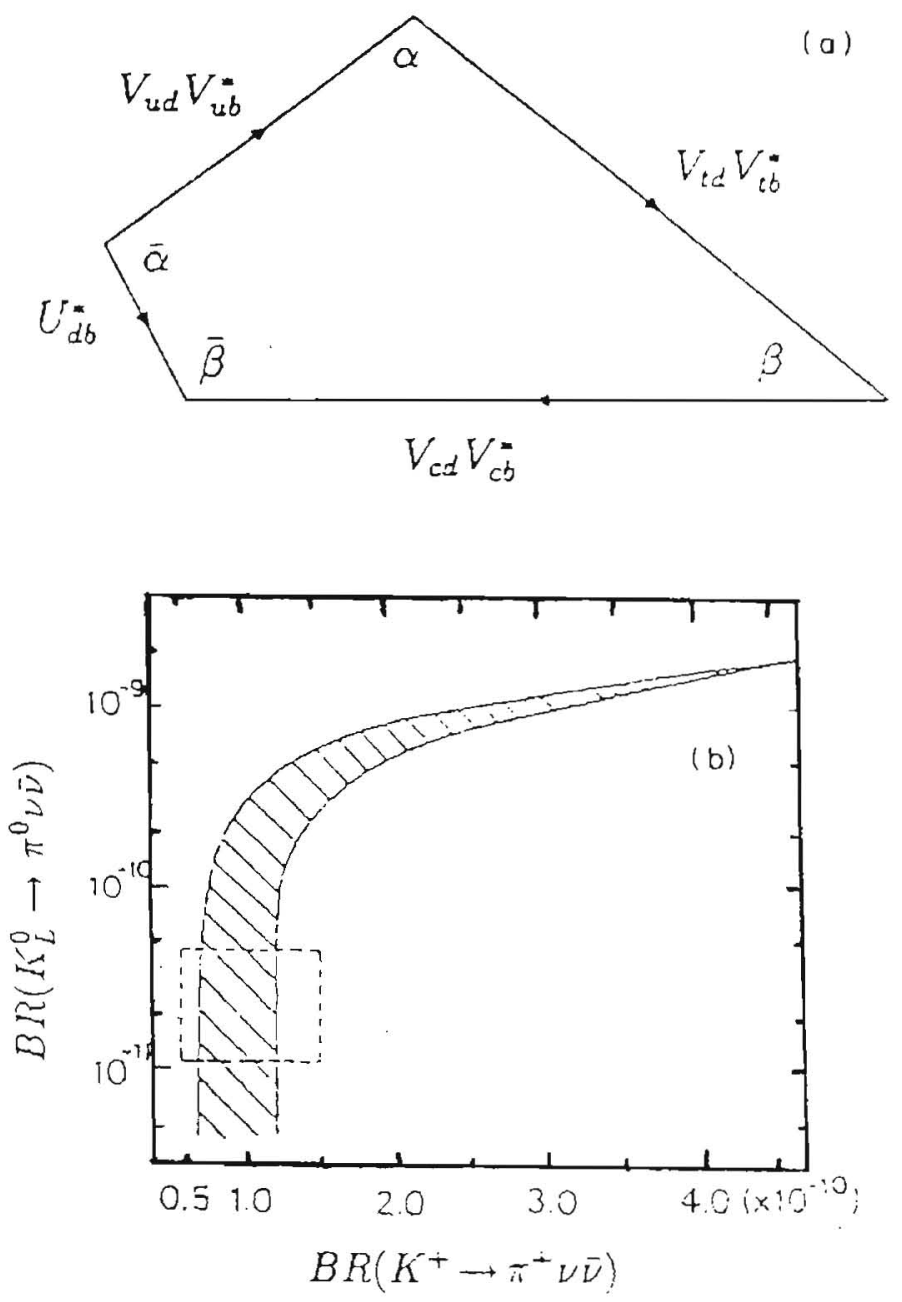

Figure 8: A model with four generation of fundamental fermions with quark doublets [44]. a) Unitary quadrangle for this model. b) The correlation between $B R\left(K^{+} \rightarrow \pi^{+} \nu \bar{\nu}\right)$ and $B R\left(K_{L}^{0} \rightarrow \pi^{0} \nu \bar{\nu}\right)$ in this model; the dotted line rectangle corresponds for the SM predictions. 


\begin{tabular}{l|l|l|l|l}
\hline & $B R\left(K^{+} \rightarrow \pi^{+} \nu \bar{\nu}\right)$ & $B R\left(K_{L}^{0} \rightarrow \pi^{0} \nu \bar{\nu}\right)$ & $B R\left(K_{L}^{0} \rightarrow \pi^{0} e^{+} e^{-}\right)_{C P-d i r}$ & Note \\
\hline $\begin{array}{l}\text { Standard } \\
\begin{array}{l}\text { Model pre- } \\
\text { diction }\end{array}\end{array}$ & $(7.7 \pm 2.1) \cdot 10^{-11}$ & $(2.3 \pm 0.7) \cdot 10^{-11}$ & $(3.6 \pm 1.1) \cdot 10^{-12}$ & \\
\hline $\begin{array}{l}\text { Experimental } \\
\text { data }\end{array}$ & $\left(15_{-12}^{+34}\right) \cdot 10^{-11}[6]$ & $\begin{array}{l}<5.9 \cdot 10^{-7}[59] \\
(90 \% \text { C.L. })\end{array}$ & $<5.1 \cdot 10^{-10}[60](90 \%$ C.L. $)$ & $\begin{array}{l}\text { From isospin symmetry there is a } \\
\text { model independent limit: } B R\left(K_{L}^{0} \rightarrow\right. \\
\left.\pi^{0} \nu \bar{\nu}\right)<4.4 B R\left(K^{+} \rightarrow \pi^{+} \nu \bar{\nu}\right) \leq 2.9 . \\
10^{-9}(90 \% \text { C.L. })[34]\end{array}$ \\
\hline
\end{tabular}

\begin{tabular}{|c|c|c|c|c|}
\hline $\begin{array}{l}\text { Models with } \\
\text { new pliysics }\end{array}$ & $\begin{array}{l}R_{(1)}=\operatorname{BR}\left(\pi^{+} \nu \bar{\nu}\right) / \\
\operatorname{BR}\left(\pi^{+} \nu \bar{\nu}\right)_{S M}\end{array}$ & $\begin{array}{l}R_{(2)}=\operatorname{BR}\left(\pi^{0} \nu \bar{\nu}\right) / \\
\operatorname{BR}\left(\pi^{0} \nu \bar{\nu}\right)_{S M}\end{array}$ & $\begin{array}{l}\dot{R}_{(3)}=\mathrm{BR}\left(\pi^{0} e^{+} e^{-}\right)_{C P-d i r} / \\
\mathrm{BR}\left(\pi^{0} e^{+} e^{-}\right)_{C P-\operatorname{dir} S M}\end{array}$ & \\
\hline $\begin{array}{l}\text { Super sym- } \\
\text { metry models } \\
\text { with general } \\
\text { flavor and } \\
\mathrm{CP} \text { violation } \\
\text { and with } \\
\text { modification } \\
\text { of } \\
Z d \bar{s} \text { structure } \\
{[47,48,50]}\end{array}$ & $<4$ & $<14-18$ & $<22-29$ & $\begin{array}{l}\text { The most probable limitations are: } \\
R_{1}<2.2 \\
R_{2}<4.3 \\
R_{3}<10\end{array}$ \\
\hline $\begin{array}{l}{[46]} \\
\text { (with some } \\
\text { additional } \\
\text { mechanisms) }\end{array}$ & $\begin{array}{l}\leq 3 \\
(\leq 6,5)\end{array}$ & $\begin{array}{l}\leq 8 \\
(<45)\end{array}$ & $\begin{array}{l}\leq 7 \\
(<45)\end{array}$ & \multirow[t]{2}{*}{$\begin{array}{l}\text { Values of } R_{(3)} \gtrsim 10 \text { can be due to in- } \\
\text { fluence of chromomagnetic operators. } \\
\text { Very large values of } R_{(1)}, R_{(2)} \text { and } R_{(3)} \\
\text { as a rule have been obtained without } \\
\text { taking into account all experimental } \\
\text { limitations ( }[45]) \text {, or by fine tuning } \\
\text { of some nodel parameters and are not } \\
\text { very probable. }\end{array}$} \\
\hline [45] & $<12$ & $<130$ & $\leq 120$ & \\
\hline
\end{tabular}

Table 3: Branching ratios $B R\left(\pi^{+} \nu \bar{\nu}\right), B R\left(\pi^{0} \nu \bar{\nu}\right)$ and $B R\left(\pi^{0} e^{+} e^{-}\right)_{C P-d i r}$ for various new physics scenarios. 


\begin{tabular}{|c|c|c|c|c|}
\hline & $R_{(1)}$ & $R_{(2)}$ & $R_{(3)}$ & \\
\hline $\begin{array}{l}\text { Minimal SUSY with Minimal Fla- } \\
\text { vor Violation (MFV). No new } \\
\text { mechanisms of CP violation }[49 \text {, } \\
50]\end{array}$ & $0.65-1.03$ & $0.41-1.03$ & $\begin{array}{l}0.48- \\
1.1\end{array}$ & $\begin{array}{l}\text { The decay probabilities are modified due to the super- } \\
\text { symmetry corrections in the loop diagrams. In this } \\
\text { model the decay probabilities as a rule are reduced in } \\
\text { comparison with SM predictions. }\end{array}$ \\
\hline $\begin{array}{l}\text { SUSY with real CKM matrix and } \\
\text { new mechanisms of CP viola- } \\
\text { tion (sd transition with gluino ex- } \\
\text { change) [53]. }\end{array}$ & $0.2-2$ & $\ll 1$ & & $\begin{array}{l}R_{(2)} \text { is very small since the CKM phase is } \simeq 0 \text { and the } \\
\text { gluino exchange mechanism makes very small contri- } \\
\text { butions to } K_{L}^{0} \rightarrow \pi^{0} \nu \bar{\nu} \text {. }\end{array}$ \\
\hline $\begin{array}{l}\text { Model with } 4 \text { fundainental gener- } \\
\text { ations of quarks and leptons [44] }\end{array}$ & $0.9-6$ & $0.2-36$ & & $\begin{array}{l}\text { Instead of a unitary triangle, there is instead a unitary } \\
\text { quadrangle }\end{array}$ \\
\hline Technicolor [54] & $<1-10$ & $<1-10$ & & $\begin{array}{l}K^{+} \rightarrow \pi^{+} \nu \bar{\nu} \text { data can be used to obtain the limits for } \\
\text { the parameters of technicolor models. }\end{array}$ \\
\hline $\begin{array}{l}\text { Model with lepton flavor violation } \\
\text { for the decay } K_{L}^{0} \rightarrow \pi^{0} \nu_{i} \bar{\nu}_{j}[34]\end{array}$ & & $\begin{array}{l}\text { CP conserv- } \\
\text { ing process } \\
\text { can be domi- } \\
\text { nant for this } \\
\text { decay }\end{array}$ & & $\begin{array}{l}\text { For SM CP conserving decay } K_{L}^{0} \rightarrow \pi^{0} \nu \bar{\nu} \text { is very small } \\
\left(\sim 10^{-4}\right)[52] .\end{array}$ \\
\hline $\begin{array}{l}\text { L-R model with } W_{L} \text { and } W_{R} \text { and } \\
X_{L} \text { and } X_{R} \text { amplitudes. In this } \\
\text { model there is an additional scalar } \\
\text { interaction which gives a CP con- } \\
\text { serving input to } K \rightarrow \pi \nu \bar{\nu}[55]\end{array}$ & 1 & 1.3 & & $\begin{array}{l}\text { In this model the scalar operator gives additional CP } \\
\text { conserving input even to } K_{L}^{0} \rightarrow \pi^{0} \nu \bar{\nu} \text { (in distinction } \\
\text { from V-A interaction of } \mathrm{SM} \text { ). Additional interaction } \\
\text { modifies the soft part of } \pi^{0} \text { momentum spectrum in } \\
K_{L}^{0} \rightarrow \pi^{0} \nu \bar{\nu} \text { and increases } R_{(2)} \text { by } \sim 30 \% \text {. }\end{array}$ \\
\hline $\begin{array}{l}\text { Anomalous interactions in the } \\
\text { triple boson } W W Z \text { vertex }[56]\end{array}$ & $0.1-2.8$ & & & $\begin{array}{l}\text { It was shown that if the anomalous coupling coustant } \\
\text { of WWZ interaction } \triangle g_{1}^{z} \text { vary from }-0.2 \text { to }+0.2 \text {, the } \\
\text { ratio } R_{(1)} \text { can vary from } 0.1 \text { up to } 2.8 \text {. This ratio is not } \\
\text { sensitive to } \triangle g_{5}^{z} \text {. }\end{array}$ \\
\hline $\begin{array}{l}\text { Several variants of MSSM, two } \\
\text { doublet Higgs models, several } \\
\text { other theories }[19,42,43,57,58]\end{array}$ & 1 & 1 & & $\begin{array}{l}\text { If the quark mixing and CP violation is governed by the } \\
\text { properties of CKM matrix (in the same way as in the } \\
\text { SM) the branching ratios of } K \rightarrow \pi \nu \bar{\nu} \text { decays would be } \\
\text { the same as in the SM. }\end{array}$ \\
\hline
\end{tabular}

Table 3: (continuation) 


$$
B R\left(K_{L}^{0} \rightarrow \pi^{0} \nu \bar{\nu}\right)=3.28 \cdot 10^{-5}\left|\operatorname{Im}\left[\frac{\lambda_{t}\left(X_{L}+X_{R}\right)}{\lambda}\right]\right|^{2}
$$

In the limit of the SM, as one can see from the comparison of (24) and (25) with (12) and (14), $X_{R}$ and $X_{L}$ reduces to $X_{R}=0$ and $X_{L}=F\left(x_{t}\right)+\lambda^{4} \lambda_{c} P_{0} / \lambda_{t}$. In different models with new physics, the expressions for $X_{L}$ and (or) $X_{R}$ are modified, as will be shown in what follows by several examples.

In models with an additional generation of fundamental fermions, the unitarity conditions for the $V_{C K M}$ matrix no longer hold. For example, instead of the unitarity relation $V_{u d} \cdot V_{u b}^{*}+V_{c d} \cdot V_{c b}^{*}+$ $V_{t d} \cdot V_{t b}^{*}=0$, the valid relation would be $V_{u d} \cdot V_{u b}^{*}+V_{c d} \cdot V_{c b}^{*}+V_{t d} \cdot V_{t b}^{*}=-V_{t^{\prime} d} V_{t^{\prime} b}^{*}$ which is represented by a quadrangle (see figure 8 ). In these models, the amplitude $X_{L}$ acquires an additional term: $X_{L}=\left(X_{L}\right)_{S M}+\frac{\lambda_{t^{\prime}}}{\lambda_{t}} F\left(x_{t^{\prime}}\right)$.

Depending on the mass $m_{t^{\prime}}$ the quantity $F\left(x_{t^{\prime}}\right)$ lies within the limits $1.5 \leq F\left(x_{t^{\prime}}\right) \leq 5$, and at sufficiently large values of $\frac{\lambda_{t^{\prime}}}{\lambda_{t}}$ the contribution from the fourth generation of quarks to the probabilities (24) and (25) may be quite significant and result in considerable deviations from the SM prediction in estimating the branching ratio for $K \rightarrow \pi \nu \nu$ decays. For instance, ref. [44] gives the following limitations for the branching ratios of FCNC decays of K-mesons in the model with 4 generations of fundamental fermions (having accounted for constraints arising from $\triangle m_{K}, \triangle m_{B_{d}}, \triangle m_{B_{s}}, \triangle m_{D}$ ): $B R\left(K^{+} \rightarrow \pi^{+} \nu \bar{\nu}\right) \simeq(0.7-4.4) 10^{-10}, B R\left(K_{L}^{0} \rightarrow \pi^{0} \nu \bar{\nu}\right) \simeq(0.05-10) 10^{-10}$ (see also figure 8).

The estimates of the branching ratios for $K \rightarrow \pi \nu \bar{\nu}$ and $K_{L}^{0} \rightarrow \pi^{0} l^{+} l^{-}$decays in different variants of supersymmetric models (SUSY) are of particular interest [45-51].

We consider here a more general variant of the SUSY model, where new mechanisms of CP violation and changes of quark flavors may take place. As was mentioned in Section 2.1, the contribution from supersymmetric particles may quite noticeably change the $Z d \bar{s}$ vertex, which will have a very significant impact on the branching ratios of kaon FCNC decays. In the general case such processes are described by 4 quark operators of the form $\frac{1}{M^{2}}(\bar{f} f)(\bar{f} f)$ where $M$ is some mass scale. Another SUSY contribution is related with the so-called chromomagnetic operators of the type $\frac{1}{M} \bar{q} \sigma^{\mu \nu} f_{\mu \nu} q$ which influences the photon or gluon radiation ( $f_{\mu \nu}$ field). These operators manifest themselves in $\left.B R\left(K_{L}^{0} \rightarrow \pi^{0} l^{+} l^{-}\right)\right|_{C P-d i r}$ and in $\varepsilon^{\prime} / \varepsilon[49,51]$.

Supersymmetric models lead to the following modification of the $X_{L}$ amplitude in (24), (25)

$$
X_{L}=\left(X_{L}\right)_{S M}+\frac{\tilde{\lambda}_{t}}{\lambda_{\ell}} H\left(x_{s}\right)=X
$$

Here $\tilde{\lambda}_{l}$ and $H\left(x_{s}\right)$ are defined by the masses and mixing of supersymmetric particles which make an additional contributions to the amplitudes of FCNC processes. The contributions depend on three effective constants: $\Lambda_{t}$ (accounts for the $Z d \bar{s}$ vertex modification) and $\Lambda_{g}^{ \pm}$(accounts for the contribution from chromomagetic operators). Here $\Lambda_{t}=\tilde{\lambda}_{l} H\left(x_{s}\right)$ and similar expressions are for $\Lambda_{g}^{ \pm}$with loop functions which describe chromomagnetic penguin diagrams.

In other words the supersymmetric contribution leads to the following modification of the expressions for probabilities of the FCNC processes:

$$
\lambda_{\ell} \cdot O\left(x_{t}\right) \rightarrow \lambda_{\ell} O\left(x_{t}\right)+\Lambda_{\ell}\left(\Lambda_{g}^{ \pm}\right)
$$

Here $\lambda_{t}$ is related to the CKM matrix, $\Lambda_{t}$ describes the contribution from new processes in $Z d \bar{s}$ vertex, and $\Lambda_{g}^{ \pm}$are for chromomagnetic operators.

Some upper estimates have beell obtained for these quantities:

$$
\left|\Lambda_{t}\right| \lesssim 3 \cdot 10^{-3}\left(\frac{500 \mathrm{GeV}}{m_{\tilde{q}}}\right) \text { and }\left|\Lambda_{g}^{ \pm}\right|<10^{-4}\left(\frac{500 \mathrm{GeV}}{m_{\tilde{q}}}\right) .
$$


The supersymmetric contribution to the $X_{L}$ amplitude has a rather complicated character and depends on the various supersymmetric models being considered, which are characterized by different masses and mixing mechanisms of new particles as well as by some other peculiarities. Various scenarios which lead to essentially different results, have been considered. Additional constraints on the model parameters, brought forward by the experimental data on $\varepsilon^{\prime} / \varepsilon$ and $B R\left(K_{L}^{0} \rightarrow \mu^{+} \mu^{-}\right)_{S D}$, have been taken into account.

Different supersymmetric contributions influence, each in its own way, the decay probabilities $B R\left(K^{+} \rightarrow \pi^{+} \nu \bar{\nu}\right), B R\left(K_{L}^{0} \rightarrow \pi^{0} \nu \bar{\nu}\right)$ and $B R\left(K_{L}^{0} \rightarrow \pi^{0} l^{+} l^{-}\right)_{C P-d i r}$. For instance, chromomagnetic penguin operators make a contribution only to $K_{L}^{0} \rightarrow \pi^{0} l^{+} l^{-}$and do not change predictions for the otlier $K \rightarrow \pi \nu \bar{\nu}$ decays. On the other hand, the $K_{L}^{0} \rightarrow \pi^{0} \nu \bar{\nu}$ decay is insensitive to the certain supersymmetric models where direct CP violation is no longer defined by the $V_{C K M}$ phase (which is quite small in such models), but by new mechanisms. This is the reason why it is so important to carry out experimental studies of all the three rare $\mathrm{K}$-meson processes and to compare the data to theoretical predictions in different variants of supersymmetric model.

We present here the results of the most consistent and conservative analysis made in [47, 48]. Various phenomenological scenarios of CP violation are treated in the analysis, where: a) $\operatorname{Im} \Lambda_{t}=0$ and $\operatorname{Im} \Lambda_{g}^{ \pm} \neq 0$, b) $\operatorname{Im} \Lambda_{t} \neq 0$ and $\operatorname{Im} \Lambda_{g}^{ \pm}=0$, c) $\operatorname{Im} \Lambda_{t} \neq 0$ and $\operatorname{Im} \Lambda_{g}^{ \pm} \neq 0$. At the same time various assumptions on $\lambda_{t}$ are made, which now can differ from the preferred SM values, but satisfy the unitarity condition of CKM matrix (e.g., the case has been considered where $\operatorname{Im} \lambda_{t}=0$ described above). To characterize qualitatively the influence of the parameters $\lambda_{t}$ and $\Lambda_{t}$ on the probability of rare kaon FCNC decays we present the relevant estimates in Table 4 [48].

\begin{tabular}{|c|c|c|c|}
\hline \multirow{2}{*}{$\Lambda_{t}$} & $\mathrm{BR}$ & $\lambda_{t}$ from SM & $\begin{array}{c}\lambda_{t} \text { is not from SM, but satisfies } \\
\text { the unitarity requirements } \\
\text { of the quark mixing matrix }\end{array}$ \\
\hline \multirow{3}{*}{$\Lambda_{t}>0$} & $B R\left(K_{L}^{0} \rightarrow \pi^{0} \nu \bar{\nu}\right)$ & $0.7 \cdot 10^{-10}$ & $0.9 \cdot 10^{-10}$ \\
\cline { 2 - 4 } & $B R\left(K_{L}^{0} \rightarrow \pi^{0} e^{+} e^{-}\right)_{C P-d i r}$ & $1.1 \cdot 10^{-11}$ & $1.3 \cdot 10^{-11}$ \\
\cline { 2 - 4 } & $B R\left(K^{+} \rightarrow \pi^{+} \nu \bar{\nu}\right)$ & $1.7 \cdot 10^{-10}$ & $2.0 \cdot 10^{-10}$ \\
\hline \hline \multirow{3}{*}{$\Lambda_{t}<0$} & $B R\left(K_{L}^{0} \rightarrow \pi^{0} \nu \bar{\nu}\right)$ & $0.8 \cdot 10^{-10}$ & $4.0 \cdot 10^{-10}$ \\
\cline { 2 - 4 } & $B R\left(K_{L}^{0} \rightarrow \pi^{0} e^{+} e^{-}\right) C P-d i r$ & $2.0 \cdot 10^{-11}$ & $5.9 \cdot 10^{-11}$ \\
\cline { 2 - 4 } & $B R\left(K^{+} \rightarrow \pi^{+} \nu \bar{\nu}\right)$ & $1.7 \cdot 10^{-10}$ & $2.7 \cdot 10^{-10}$ \\
\hline
\end{tabular}

Table 4: The dependence of branching ratios for decays $K \rightarrow \pi \nu \bar{\nu}$ and $\left.K_{L}^{0} \rightarrow \pi^{0} e^{+} e^{-}\right|_{C P-d i r}$ in the SUSY model [48] on parameters $\lambda_{t}$ and $\Lambda_{t}$.

Different scenarios of the model $[47,48]$ give the following limits for the decay probabilities:

$$
\left.\begin{array}{rl}
B R\left(K^{+} \rightarrow \pi^{+} \nu \bar{\nu}\right) & <2.7 \cdot 10^{-10}\left(<1.7 \cdot 10^{-10}\right) \\
B R\left(K_{L}^{0} \rightarrow \pi^{0} \nu \bar{\nu}\right) & <4 \cdot 10^{-10}\left(<1.2 \cdot 10^{-10}\right) \\
R\left(K_{L}^{0} \rightarrow \pi^{0} e^{+} e^{-}\right)_{C P-d i r} & <6 \cdot 10^{-11}\left(<2 \cdot 10^{-11}\right)
\end{array}\right\}
$$

Here in the brackets are the most probable limitations, which do not demand extreme assumptions. It should be noted that in this model for the variant with $\operatorname{Im} \lambda_{t}=0 B R\left(K_{L}^{0} \rightarrow \pi^{0} \nu \bar{\nu}\right)$ has a very small value $\left(<10^{-14}[52]\right)$.

Less stringent limitations quoted in $[45,46]$ seem to be artificial and hardly probable, though they cannot be completely neglected because of possible compensations between different supersymmetric contributions. 
Other variants of supersymmetric extensions of the Standard Model were also considered. For instance, papers in [49] analyze rare $\mathrm{K}$ and B-meson data and $\mathrm{CP}$ violation in the framework of 'least expansion' to the SM in which new processes with flavor and CP violation are absent (the so-called MSSM model with MFV (Minimal Flavor Violation) requirement). In this version, one complex phase in the CKM matrix remains as the sole source of CP violation. Quark flavor mixing is also defined by this matrix. The contributions from supersymmetric particles and new Higgs doublets manifest themselves through the corrections to the loop diagrams describing the processes of $K^{0} \rightleftarrows \bar{K}^{0}$ and $B^{0} \rightleftarrows \bar{B}^{0}$ mixing, FCNC decays, etc. The main contributions to these corrections are made by the charged Higgs bosons $H^{ \pm}$and supersymmetric charginos $\chi_{j}^{+}(j=1,2)$, which interact with bottom d-, s-, b-quarks and top superquarks $\tilde{u}, \tilde{c}, \tilde{t}$. The contribution from supersymmetric particles in this case tends to reduce the $K \rightarrow \pi \nu \nu$ and $K_{L}^{0} \rightarrow \pi^{0} l^{+} l^{-}$branching ratios [49]:

$$
\left.\begin{array}{l}
0.65 \leq \frac{B R\left(K^{+} \rightarrow \pi^{+} \nu \bar{\nu}\right)_{M S S M}}{B R\left(K^{+} \rightarrow \pi^{+} \nu \bar{\nu}\right)_{S M}} \leq 1.03 \\
0.41 \leq \frac{B R\left(K_{L}^{0} \rightarrow \pi^{0} \nu \bar{\nu}\right)_{M S S M}}{B R\left(K_{L}^{0} \rightarrow \pi^{0} \nu \bar{\nu}\right)_{S M}} \leq 1.03 \\
0.48<\frac{\left.B R\left(K_{L}^{0} \rightarrow \pi^{0} e^{+} e^{-}\right)_{C P-d i r .} \cdot \frac{M S M}{B R\left(K_{L}^{0} \rightarrow \pi^{0} e^{+}\right.} e^{-}\right)_{C P-\operatorname{dir} . S M}}{B}<1.1
\end{array}\right\}
$$

If the probabilities for these decays significantly exceed the SM predictions, it will imply, that more complicated mechanisms of CP violation, which are not described by one CKM phase, take place in nature.

In the supersymmetric model of [53] all $\mathrm{CP}$ violation in $\mathrm{K}$ decays are due to FCNC sd-transition with gluino exchange, and the CKM matrix does not contain complex elements. This mechanism makes no contribution to $\mathrm{CP}$ violating amplitude of $s \rightarrow d \nu \bar{\nu}$ decay. Therefore in the model under consideration the $K_{L}^{0} \rightarrow \pi^{0} \nu \bar{\nu}$ branching ratio will be very low. At the same time, the $K^{+} \rightarrow \pi^{+} \nu \bar{\nu}$ decay which is induced by CP-conserving as well as CP violating amplitudes, the expected branching ratio is $B R\left(K^{+} \rightarrow \pi^{+} \nu \bar{\nu}\right)=(8.8 \pm 7.2) \cdot 10^{-11}$. In other words, it can be comparable with the SM predictions.

In models with technicolor the probabilities for the $K \rightarrow \pi \nu \bar{\nu}$ decays may be one or two orders of magnitude larger than the SM predictions, which is due to the influence of new interactions on the $Z d \bar{s}$ vertex [54]. In fact, the existing data for the $K^{+} \rightarrow \pi^{+} \nu \bar{\nu}$ decay is used to constrain the technicolor model parameters. The resulting limits on the technicolor parameters still allow for a possible increase of 1-10 times the SM prediction of the $K^{+} \rightarrow \pi^{+} \nu \bar{\nu}$ and $K_{L}^{0} \rightarrow \pi^{0} \nu \bar{\nu}$ branching ratio.

The branching ratios for $K \rightarrow \pi \nu \bar{\nu}$ decays can greatly change in several exotic theories - in supersymmetric models with $\mathrm{R}$ parity violation, in models with leptoquarks as well as in some other models with significant modification of the structure of the FCNC $[42,43]$.

A few words must be said about the decays of $K^{+} \rightarrow \pi^{+}+X$ type, where $X$ is an invisible particle (or a pair of particles). Such processes could imitate the $K^{+} \rightarrow \pi^{+} \nu \bar{\nu}$ decays. For instance, in the models with extra dimensions the $K \rightarrow \pi+g$ decays with graviton emission into space with additional dimensions (i.e. with energy and momentum loss in our space), were considered. Such processes could imitate the $K \rightarrow \pi \nu \bar{\nu}$ decays. However the estimates of the $K \rightarrow \pi+g$ decay probabilities in the models with extra dimensions yield the value $<10^{-12}$ (or rather $\ll \cdot 10^{-12}[61]$ ). They are in fact unobservable. A very low probability is predicted for decays with an emission of a pair of very light supersymmetric particles (photino, goldstino - see [62]). Another hypothetical process of this type is the so-called familon decays $K \rightarrow \pi+f$, where $f$ is a neutral familon, which is characterized by weak interaction and does not manifest itself readily in experiments [42, 62]. 
Such decays can be singled out when one studies the $\pi$-meson spectrum (i.e., when extracting the monochromatic line). From the BNL E787 data, the following limits $B R\left(K^{+} \rightarrow \pi+X\right)<$ $1.1 \cdot 10^{-10}\left(90 \%\right.$ C.L.) and $F>5.6 \cdot 10^{11} \mathrm{GeV}$ have been obtained for the effective familion interaction described by the Lagrangian $L=\frac{1}{F} \gamma^{\mu} X\left(\bar{u} \gamma_{\mu} s\right)+$ (hermit.conjug.) [6].

In conclusion, table 3 presents the data and the possible manifestations of new physics in rare $K^{+} \rightarrow \pi^{+} \nu \bar{\nu}, K_{L}^{0} \rightarrow \pi^{0} \nu \bar{\nu}$ and $K_{L}^{0} \rightarrow \pi^{0} e^{+} e^{-}$decays, which were discussed in [42-58]. It shows that future experiments on the studies of rare kaon decays provide a unique opportunity to perform high precision tests of CP violation and flavor mixing within and beyond the SM. It was also stated, that in the kaon system large SUSY effects are naturally expected in any model with non-universal softbreaking terms. The proposed $K \rightarrow \pi \nu \bar{\nu}$ experiments have a real possibility of obtaining the first evidence for new physics including supersymmetry extensions of the Standard Model $[19,50,64]$.

\subsection{Other $K^{+}$Physics}

The CKM detector will have a spectrometer with an unparalleled rate capability and excellent spatial and time resolution, and RICHs that will provide particle ID through precise velocity measurements. It will provide the redundant measurement capabilities necessary for contemporary kaon rare decay studies. Table 5 shows the potential power of the CKM apparatus by listing our expected event rates for a number of interesting modes.

In this table and throughout this section, acceptances are from our Monte Carlo simulation, and we assume $1.4 \times 10^{5}$ spills/week. This corresponds to $5 \times 10^{11} K^{+}$decays per week, allowing for $30 \%$ DAQ dead time. We then assign an additional inefficiency of $70 \%$ for cuts against background, so we are working with $3.6 \times 10^{11} K^{+}$decays per week. In modes where photons must be measured, we assume that they must be measured in the FVS; if it is possible to measure photons well enough in the VVS for any specific mode with several photons in final state, the efficiency for detecting that mode is increased by a factor of 2 to 3 . Unless mentioned otherwise, our estimates are based on 2 years of 48 weeks each running time.

\begin{tabular}{|c|c|c|c|}
\hline Decay & $B R[23]$ & Efficiency & Events/week \\
\hline \hline$K^{+} \rightarrow \pi^{0} \mu^{+} \nu \gamma$ & $\sim 2 \times 10^{-5}$ & 0.0080 & $5.8 \times 10^{4}$ \\
$K^{+} \rightarrow \pi^{0} e^{+} \nu \gamma$ & $(2.62 \pm 0.20) \times 10^{-4}$ & 0.022 & $2.1 \times 10^{6}$ \\
\hline$K^{+} \rightarrow \pi^{+} \pi^{+} \pi^{-}$ & $(5.59 \pm 0.05) \times 10^{-2}$ & 0.20 & $4.0 \times 10^{9}$ \\
$K^{+} \rightarrow \pi^{+} \pi^{0} \pi^{0}$ & $(1.73 \pm 0.04) \times 10^{-2}$ & 0.0040 & $2.5 \times 10^{7}$ \\
$K^{+} \rightarrow \pi^{+} \pi^{\gamma}$ & $(2.75 \pm 0.15) \times 10^{-4}$ & 0.00112 & $1.1 \times 10^{6}$ \\
\hline$K^{+} \rightarrow \pi^{+} e^{+} e^{-}$ & $(2.88 \pm 0.13) \times 10^{-7}$ & 0.012 & $1.2 \times 10^{3}$ \\
$K^{+} \rightarrow \pi^{+} \mu^{+} \mu^{-}$ & $(7.6 \pm 2.1) \times 10^{-8}$ & 0.086 & $2.3 \times 10^{3}$ \\
$K^{+} \rightarrow \mu \nu \gamma$ & $(5.50 \pm 0.28) \times 10^{-3}$ & 0.050 & $1.0 \times 10^{8}$ \\
\hline$K^{+} \rightarrow \pi^{+} \pi^{-} e^{+} \nu$ & $(3.91 \pm 0.17) \times 10^{-5}$ & 0.066 & $9.4 \times 10^{5}$ \\
$K^{+} \rightarrow \pi^{0} \pi^{0} e^{+} \nu$ & $(2.1 \pm 0.4) \times 10^{-5}$ & 0.0093 & $7.2 \times 10^{4}$ \\
$K^{+} \rightarrow \pi^{+} \pi^{-} \mu^{+} \nu$ & $(1.4 \pm 0.9) \times 10^{-5}$ & 0.28 & $1.4 \times 10^{6}$ \\
$K^{+} \rightarrow \pi^{0} \pi^{0} e^{+} \nu$ & $\sim 0.7 \times 10^{-5}$ & 0.0024 & $6.1 \times 10^{3}$ \\
$K^{+} \rightarrow \pi^{+} \pi^{0} \gamma$ & $(2.75 \pm 0.15) \times 10^{-4}$ & 0.011 & $1.1 \times 10^{6}$ \\
\hline
\end{tabular}

Table 5: The efficiency for the detection and sample size of several kaon decay modes in the CKM detector.

While we will accept no compromises in either the sensitivity or background rejection required for $K^{+} \rightarrow \pi^{+} \nu \bar{\nu}$ in order to accommodate other physics goals, several other physics measurements 
should be possible in this apparatus. Broadening the physics scope of the experiment will increase the physics return, both on the capital and personnel investments in building this new apparatus. We intend, after we have completed a detailed technical design for a no-compromise $K^{+} \rightarrow \pi^{+} \nu \bar{\nu}$ apparatus, to fully investigate the possibilities for other pliysics goals. Modest incremental costs for apparatus additions and/or modifications and the possibility of dedicated running periods with different triggers will be seriously entertained by the collaboration at that time.

Within these boundary conditions we enumerate several possible physics measurements which will likely be within the reach of the CKM apparatus:

1. The search for lepton flavor violating processes.

2. The search for T-odd asymmetries in cases where it is a signal of new physics processes, it i.e., $K^{+} \rightarrow \pi^{0} \ell^{+} \nu \gamma$ decays.

3. The search for new directly CP violating processes in $K^{+}$decays through measurement of the charge asymmetry in $K^{ \pm} \rightarrow \pi^{ \pm} \pi \pi, \pi^{ \pm} \pi^{0} \gamma$.

4. The search for new S, P, T weak interactions in $K^{+} \rightarrow e^{+} \nu, e^{+} \pi^{0} \nu_{e}, \pi^{+} l^{+} l^{-}$and in other decays.

5. Low energy hadron physics investigations, in the context of chiral perturbation theory, QCD in lattice, etc.

\subsubsection{Lepton Flavor Violating Processes in $K^{+}$Decays}

It is well known that lepton flavor conservation is not predicted by the standard model. Rather, it is an essentially unexplained phenomena which we insert into our model with solely experimental justification. Indeed, if we allow for neutrino masses in the ranges suggested by recent atmospheric and solar neutrino experiments, lepton flavor is not conserved in the standard model, although the level of LFV in kaon decays is so low as to be non-observable [65-67].

In addition to being an open question from the experimental side, LFV frequently appears in extensions to the standard model; it appears in some SUSY [68-70] scenarios, left-right models with heavy neutrinos [71], technicolor, compositeness, horizontal boson [72] and leptoquark [73] models $[20,67]$.

Table 6 lists lepton flavor violating modes in kaon decay which have already elicited experimental interest. The branching ratio limits are from Brookhaven experiments 865, 871 and from $\mathrm{KTeV}$. The third column of the table gives our estimated sensitivity to these modes with the CKM apparatus. This numbers are based on statistical consideration and do not include possible background limitations. Based on these estimates, the CKM apparatus will be a powerful tool for the investigation of lepton flavor violation in the kaon sector. Certainly, we will need more careful investigations of possible backgrounds and their levels for these searches. Some of that work has already begun.

It is evident from table 6 that we have sensitivity to branching ratios on the order of $10^{-12}$, in comparison to limits on the order of $10^{-14}$ to $10^{-16}$ from new sophisticated projects for the search for $\mu \rightarrow e \gamma$ and $\mu-e$ conversion [74]. We stress however that searches for LFV in kaon decay are complimentary to searches in muonic processes. Kaon decays are the only processes which are sensitive to $s \rightarrow d \mu \bar{e}$ interactions with axial vector or pseudoscalar amplitudes for $K_{L}^{0} \rightarrow$ $\mu \bar{e}$ decays and vector, scalar or tensor structures for $K \rightarrow \pi \mu e$ decays. Furthermore, they can elucidate possible flavor structure in the quark sector through investigation of generation number conservation. 


\begin{tabular}{|l|l|c|}
\hline \multicolumn{1}{|c|}{ Mode } & $B R$ limit & Expected CKM $B R$ limits \\
\hline \hline$B R\left(K^{+} \rightarrow \pi^{+} \mu^{+} e^{-}\right)$ & $2.8 \times 10^{-11}[75]$ & $2.2 \times 10^{-12}$ \\
\hline$B R\left(K^{+} \rightarrow \pi^{+} \mu^{-} e^{+}\right)$ & $5.2 \times 10^{-10}[76]$ & $2.2 \times 10^{-12}$ \\
\hline$B R\left(K^{+} \rightarrow \pi^{-} \mu^{+} e^{+}\right)$ & $5.0 \times 10^{-10}[76]$ & $2.2 \times 10^{-12}$ \\
\hline$B R\left(K^{+} \rightarrow \pi^{--} \mu^{+} \mu^{+}\right)$ & $3.0 \times 10^{-9}[76]$ & $7.2 \times 10^{-13}$ \\
\hline$B R\left(K^{+} \rightarrow \pi^{-} e^{+} e^{+-}\right)$ & $6.4 \times 10^{-10}[76]$ & $5.6 \times 10^{-12}$ \\
\hline$B R\left(K_{L}^{0} \rightarrow \mu e\right)$ & $4.7 \times 10^{-12}[77]$ & \\
\hline$B R\left(K_{L}^{0} \rightarrow \pi^{0} \mu e\right)$ & $4.4 \times 10^{-10}[78]$ & \\
\hline
\end{tabular}

Table 6: Branching ratio limits (all limits are at the 90\% C.L.) for lepton flavor violating kaon decays.

The horizontal gauge boson model of Cahn and Harari [72] helps clarify the concept of generation number. Consider the three diagrams of figure 9 , which is drawn under the convention that the muon with charge -1 is a fermion of the same generation as the strange quark with charge $-1 / 3$. The concept of generation is not well defined, but the first diagram is fundamentally different from the second; one of them conserves generation number and one does not. If we define $G=1$ for $u, d$, $e^{-}$and $\nu_{e}$, and $G=2$ for $c, s, \mu^{-}$and $\nu_{\mu}$, then it is possible to classify different processes as shown in table 7 . The third diagram of figure 9 shows a two-body $K_{L}^{0}$ decay; because the $K_{L}^{0}$ is a mixture of $K^{0}$ and $\overline{K^{0}}$, both generation number conserving and generation number violating processes can contribute.

The strength of hypothetical $\Delta G$ selection rule is unclear now. Certainly, the new possibilities for the search for LFV processes in kaon decays to compare with muon processes will occur only if this strength is meaningful and every next order on $\Delta G$ will lead to significant suppression of corresponding process to compare with lower order.

\begin{tabular}{|l|l|c|}
\hline First order & $|\Delta G|=0$ & $K^{+} \rightarrow \pi^{+} \mu^{+} e^{-} ; K_{L}^{0} \rightarrow \mu^{\mp} e^{ \pm} ; K_{L}^{0} \rightarrow \pi^{0} \mu^{\mp} e^{ \pm}$ \\
\hline Second order & $|\Delta G|=1$ & $\mu \mu \rightarrow 3 e ; \mu \rightarrow e \gamma ; \mu^{-} N \rightarrow e^{-} N$ \\
\hline Third order & $|\Delta G|=2$ & $K^{0} \rightarrow K^{0}\left(\Delta M\left(K_{1}-K_{2}\right)\right) ; \mu^{-} e^{+} \rightarrow \mu^{+} e-; K^{+} \rightarrow \pi^{+} \mu^{-} e^{+}$ \\
\hline
\end{tabular}

Table 7: Classification by generation number change for some lepton flavor violating processes.

In the diagram in figure 9 the decay processes are governed by exchange of "horizontal" intermediate boson. But it is also possible to consider kaon decay LFV processes which are governed by exchange of leptoquarks $Y$.

There are specific examples of models in which the limits from muonic modes do not sharply constrain limits in kaon modes. Consider for example, SUSY with $R$-parity violation $[68,69]$. In the normal $R$-parity conserving case, say, the MSSM case [68], the universal GUT-scale parameters for sfermions and gauginos which are responsible for lepton flavor non-conservation are limited by data from $\mu^{-} \rightarrow e^{-} \gamma$ and $\mu \rightarrow 3 e$ so as to lead to branching ratio predictions for LFV kaon decays on the order of $10^{-16}$ to $10^{-18}$. But when $R$-parity is violated, there are additional Yukawa couplings in the superpotential of the form $\lambda L_{i} L_{j} \overline{E_{k}}, \lambda^{\prime} L_{i} W_{j} \bar{D}_{k}$, or $\lambda^{\prime \prime} \bar{U}_{i} \bar{D}_{j} \bar{D}_{k}$. Here $L(Q)$ are left handed lepton (quark) superfields and $\bar{E}_{k}\left(\bar{D}_{j}, \bar{U}_{j}\right)$ are the corresponding right handed superfields. Each of these terms give three Yukawa couplings; for example, the first term is

$$
\lambda_{i j k}\left(L_{i} L_{j} \tilde{E}_{k}+L_{i} \tilde{L}_{j} \bar{E}_{k}+\tilde{L}_{i} L_{j} \bar{E}_{k}\right),
$$



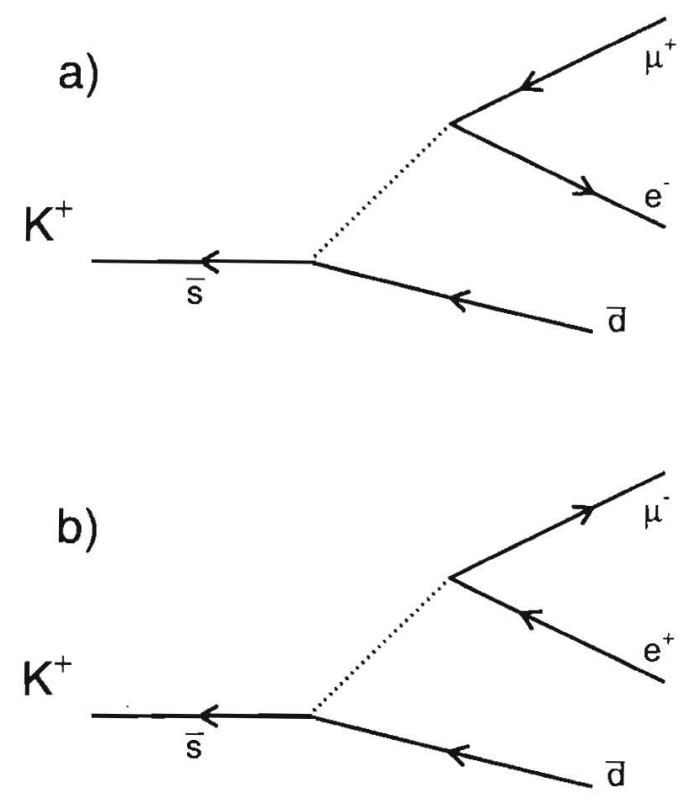

c)

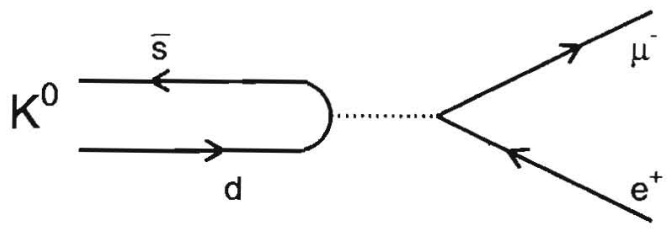

Figure 9: Diagrams using the horizontal gauge boson model. Dotted lines are the boson mediating an $S U(N)$ generational symmetry. (a) A generation conserving scalar or vector process; (b) A generation violating scalar or vector process; (c) A pseudoscalar or axial vector process. 
which contains a single $R$-parity breaking SUSY states. There are many experimental constraints on this these couplings, including those from lepton universality, from limits on proton decay, and others. In spite of these limits, the constraints are for several $R$-violating come only from existing LFV kaon decay experiments and can be tightened with improved branching ratio limits from kaon decay experiments. As an example, diagrams for $K^{0} \rightarrow l_{j}^{-} l_{k}^{+}$and $K \rightarrow \pi l_{j}^{-} l_{k}^{+}$decays in $R$-parity violating SUSY are shown in figure 10.
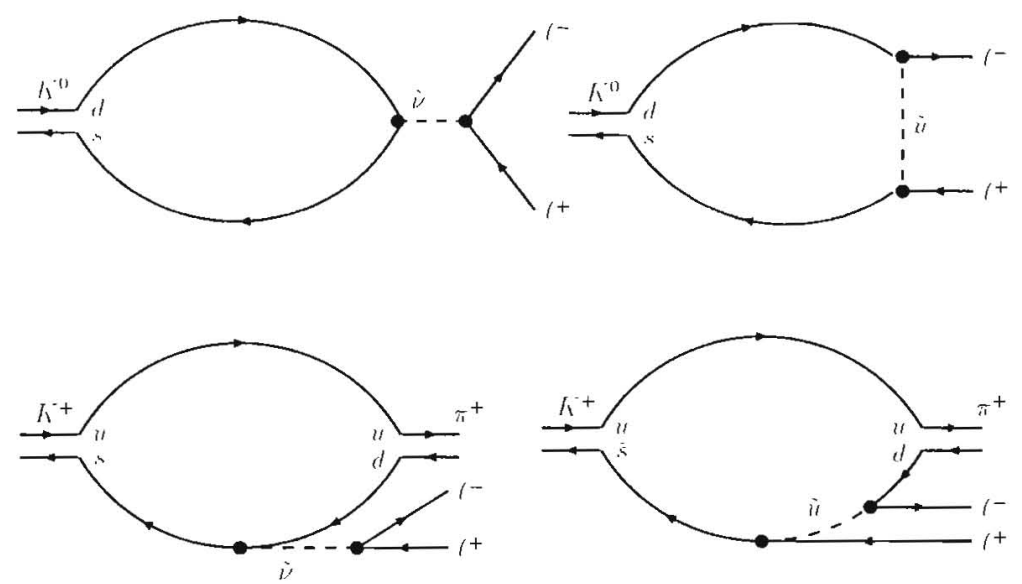

Figure 10: $K \rightarrow \mu e$ and $K \rightarrow \pi \mu e$ in a SUSY model with $R$-parity nonconservation [68].

Lepton flavor violation can also appear in $K^{+} \rightarrow \pi^{-} \mu^{+} \mu^{+}$, according to certain specific models $[69,97-99]$ with heavy Majorana neutrinos (i.e., $\sim 300 \mathrm{MeV}$ ) that have a small admixture of muon neutrino to them. The process proceeds via $K^{+} \rightarrow \nu_{h} \mu^{+} ; \nu_{h} \rightarrow \pi^{-} \mu^{+}$. It is also possible to use $K^{+} \rightarrow \pi^{-} e^{+} e^{+}$to search for the same type of neutrino, but with an electron neutrino admixture. Finally, we should note that there is a literature $[99,100]$ investigating other possible scenarios for LFV kaon decays.

These specific models thus provide examples of cases where further lepton flavor violation searches in kaon decays will be essential complements to similar searches in muonic processes.

\subsection{2 $\mathrm{T}_{\text {odd }}$ Correlations in $K^{+} \rightarrow \pi^{0} l^{+} \nu_{l} \gamma$}

$T$-invariance is a fundamental physical symnetry which has been experimentally examined [23] in just over a dozen physical systems to date. In electroweak processes, which we believe are described with quantum field theories that conserve $C P T, T$ violation occurs as a result of $C P$ violation.

Well known search methods include searches for electric dipole moments of elementary particles, and comparing the semileptonic decays of $K^{0}$ vs $\overline{K^{0}}$. Although not a completely direct method, looking for asymmetries in T-odd variables is also a valuable technique. One such variable is the angle, in $K_{L}^{0} \rightarrow \pi^{+} \pi^{-} e^{+} e^{-}$, between the plane containing the $\pi^{+} \pi^{-}$pair and the $e^{+} e^{-}$pair. Another [79-83] is $\sigma_{\perp}^{\mu}$, the polarization of the muon normal to the decay plane in $K^{+} \rightarrow \mu^{+} \nu \pi^{0}$. Our $K^{+}$beam energy is too high to permit a competitive measurement this parameter in the CKM detector, but we do want to measure or limit the T-odd parameter $T_{\pi \mu \gamma}$ in $K \rightarrow \pi^{0} \mu \nu \gamma$ :

$$
T_{\pi \mu \gamma}=\frac{\vec{p}_{\gamma} \cdot\left(\vec{p}_{\pi} \times \vec{p}_{\mu}\right)}{\left|\vec{p}_{\gamma}\right| \cdot\left|\vec{p}_{\pi} \times \vec{p}_{\mu}\right|}
$$

There is no firm prediction in the standard model for this parameter, which was first discussed by Gevais, Iliopoulous and Kaplan [84]. However theorists at IHEP are performing detailed calculations 
now of $T_{\pi \mu \gamma}$ in multi-Higgs doublet models [85] where there is a measurable $T$-violation effect, but no corresponding effect in the electronic mode. Constraints on these models can be extracted from experimental data on the neutron electrical dipole moment, the neutral kaon parameters $\epsilon$ and $\epsilon^{\prime}$, and $\sigma_{\perp}^{\mu}$. Even allowing for these constraints, the possibility of a large $T_{\pi \mu \gamma}$ does not appear to be excluded.

Final state interactions (FSI) that can fake $T_{\pi \mu \gamma}$ are problaby small: naively, they are similar to the same effects in $\sigma_{\perp}$ in $K \rightarrow \mu \nu \gamma$, which are $\sim 10^{-4}$. In principle, we can determine the FSI contribution to any possible signal by running with a $K^{-}$beam; final state interactions do not change sign when the charge of the initial beam does. We may also compare the triple vector product $T_{\pi \mu \gamma}$ with the same quantity for the electron mode, $T_{\pi e \gamma}$. To search for $T_{\pi e \gamma}$ we will no doubt need to develop kinematic cuts against $K \rightarrow \pi^{0} \mu \nu \gamma$ with internal bremsstrahlung. The events rejected by these selection criteria may also be used to assess the false asymmetry level.

The decay $K^{+} \rightarrow \pi^{0} \mu \nu \gamma$ has not yet been observed; the standard model prediction is $B R\left(K^{+} \rightarrow\right.$ $\left.\mu \nu \pi^{0} \gamma\right) \sim 2 \times 10^{-5}$. The OKA experiment plans to measure $T_{\pi \mu \gamma}$ with a sample of $\sim 10^{6} K^{+} \rightarrow$ $\pi^{0} \mu \nu \gamma$ decays $\left(\sim 10^{5}\right.$ events after cuts to suppress internal bremsstrahlung) [86]. At CKM, we expect a signal acceptance of 0.0080 , after requiring $E_{\gamma}>30 \mathrm{MeV}$ and $\theta_{\ell \gamma}>20^{\circ}$ to suppress internal radiation. While we may need even more stringent cuts for this purpose, with this level of analysis, we expect to collect $5.8 \times 10^{4}$ events per week of running, for a total sample of $5.6 \times 10^{6}$ events - an order of magnitude more data than OKA. Our statistical sensitivity limit in $T_{\pi \mu \gamma}$ should be $\pm 2.1 \times 10^{-4}$.

For the electron mode, the only experimental measurement of $T_{\pi e \gamma}$ is from the ISTRA set-up at IHEP's $70 \mathrm{GeV}$ accelerator [87]. From 192 reconstructed events, they found $T_{\pi e \gamma}=0.03 \pm 0.08$, corresponding to a branching ratio of $(2.7 \pm 0.2) \times 10^{-4} ;\left(E_{\gamma}^{c . m} .>10 \mathrm{MeV} ; 0.6<\cos \theta_{e, \gamma}<0.9\right)$. The OKA experiment [86] should obtain a sample of $\sim 10^{6} K^{+} \rightarrow \pi^{0} e \nu \gamma$ decays, before internal bremsstrahlung suppression. At CKM, 98 weeks of running should yield $1.7 \times 10^{7} \mathrm{~K}^{+} \rightarrow \pi^{0} e^{+} \nu_{e} \gamma^{\prime}$ events, with a statistical sensitivity for $T_{\pi e \gamma}$ of $\pm 3.5 \times 10^{-5}$.

The statistical sensitivity will not be reached if background processes and systematic uncertainties can not be controlled. We believe that the main background processes are $K^{+} \rightarrow \pi^{0} \pi^{0} \mu^{+} \nu$ with one lost photon, $K^{+} \rightarrow \pi^{+} \pi^{0} \gamma$ with $\pi^{+}$decay in flight, and $K^{+} \rightarrow \pi^{0} \mu^{+} \nu$ with accidentally coincident activity that appears as a photon. Monte Carlo simulation of $K^{+} \rightarrow \pi^{0} \pi^{0} \mu^{+} \nu$ says that this background will be about $6 \times 10^{3}$ events; other types of background and systematic uncertainties are now under study. The results of the OKA experiment will no doubt be of value in this work.

\subsubsection{Search for Direct CP Violation in Hadronic $K^{ \pm}$decays}

Studies of the nonleptonic charged kaon decays, $K^{ \pm} \rightarrow \pi^{ \pm} \pi^{+} \pi^{-}, K^{ \pm} \rightarrow \pi^{ \pm} \pi^{0} \pi^{0}, K^{ \pm} \rightarrow \pi^{ \pm} \pi^{0} \gamma$ and $K^{ \pm} \rightarrow \pi^{ \pm} \gamma \gamma$ are important for understanding $\Delta S=1$ hadronic weak interactions in the context of chiral perturbation theory (CHPT). But in addition to precision CHPT tests, studies of these processes with the large data samples available in the next generation of kaon experiments will permit the search for new and directly $\mathrm{CP}$ violating effects through the measurement of rate asymmetries in $\left(K^{+} \rightarrow \bar{f}\right) /\left(K^{-} \rightarrow f^{\prime}\right)$ or Dalitz plot asymmetries.

In principle it is straightforward to operate the charged kaon beam line at both polarities, allowing $K^{+}$and $K^{-}$data sets to be collected at different times. In practice, precision measurements could be vulnerable to the different beam and detector related backgrounds and rates in $K^{+}$vs. $K^{-}$running. Nevertheless, there is some value in addressing these potential measurements.

Direct CP violation could manifest itself (see for example [88]), in the decays $K^{ \pm} \rightarrow \pi^{ \pm} \pi^{ \pm} \pi^{\mp}$ $(\tau)$ and $K^{ \pm} \rightarrow \pi^{ \pm} \pi^{0} \pi^{0}\left(\tau^{\prime}\right)$ through the observation of a difference $\frac{\delta g_{ \pm}}{2 g}$ in the slope parameters $g^{ \pm}$ 
used in the conventional parameterization for the matrix element squared [23]:

$$
\left|M_{3 \pi}\right|^{2} \sim 1+g Y+h Y^{2}+k X^{2}+\ldots
$$

Here $Y=\frac{s_{3}-s_{0}}{m_{\pi}^{2}}, X=\frac{s_{2}-s_{1}}{m_{\pi}^{2}}, s_{i}=\left(p_{K}-p_{i}\right)^{2}, s_{0}=\frac{1}{3}\left(s_{1}+s_{2}+s_{3}\right)$ and $\pi_{3}$ is the odd pion. The world average values [23] are $g(\tau)=.2154 \pm 0.0035$ and $g\left(\tau^{\prime}\right)=0.652 \pm 0.031$. Theoretical predictions for $\frac{\delta g_{ \pm}}{2 g}$ in the standard model have converged to $\sim 10^{-5}[88,89]$. In models where $\mathrm{CP}$ violation is not constrained to the CKM matrix, larger values are possible. For example, Weinberg's three Higgs doublet model, where there is also CP in the Higgs sector, predicts [90] that $\frac{\delta g_{ \pm}}{2 g} \sim 2 \times 10^{-1}$.

Table 8 compares existing and proposed [86,91-94] searches for $C P$ violation in $K^{ \pm}$decays. The CKM entries are based on a scenario with 6.5 weeks $K^{+}$running and 9.5 weeks $K^{-}$running; this optimizes the statistical precision of the measurement, allowing that $K^{-}$running will provide half as many decays per week. The operation of $K^{-}$beam is considered in Section 4.1.3.

\begin{tabular}{|c|c|c|c|}
\hline $\begin{array}{l}\text { Experiment/Lab; } \\
\text { existing }\left(^{*}\right) \text { or } \\
\text { planned }\left(^{* *}\right)\end{array}$ & Decay & Statistics for $K^{ \pm} \rightarrow 3 \pi$ (Sec. 4.1.3) & $\begin{array}{l}\text { Statistical precision in } \\
\delta g_{ \pm} / 2 g \text { asymmetry }\end{array}$ \\
\hline $\begin{array}{l}\left(^{*}\right) \quad \text { Prince- } \\
\text { ton/BNL [92] }\end{array}$ & $K^{ \pm} \rightarrow \pi^{ \pm} \pi^{+} \pi^{-}$ & $3.2 \times 10^{6}\left(K^{+}+K^{-}\right)$ & $(-0.70 \pm 0.53) \times 10^{-2}$ \\
\hline $\begin{array}{l}\left(^{*}\right) \quad \text { Hy- } \\
\text { perCP/Fermilab } \\
{[93]}\end{array}$ & $K^{ \pm} \rightarrow \pi^{ \pm} \pi^{+} \pi^{-}$ & $2.04 \times 10^{8}\left(K^{+}\right)+0.75 \times 10^{8}\left(K^{-}\right)$ & $\sim 6 \times 10^{-4}(* *)$ \\
\hline $\begin{array}{ll}*) & \text { TNF } / \text { IHEP } \\
{[91]} & \end{array}$ & $K^{ \pm} \rightarrow \pi^{ \pm} \pi^{0} \pi^{0}$ & $10^{6}\left(K^{+}\right)+10^{6}\left(K^{-}\right)$ & $\sim 2 \times 10^{-3}(* *)$ \\
\hline $\begin{array}{l}(* *) \text { NA48/CERN } \\
{[94]}\end{array}$ & $\begin{array}{l}K^{ \pm} \rightarrow \pi^{ \pm} \pi^{+} \pi^{-} \\
K^{ \pm} \rightarrow \pi^{ \pm} \pi^{0} \pi^{0}\end{array}$ & $\begin{array}{l}1.3 \times 10^{9}\left(K^{+}\right)+0.75 \times 10^{9}\left(K^{-}\right) \\
0.82 \times 10^{8}\left(K^{+}\right)+0.47 \times 10^{8}\left(K^{-}\right)\end{array}$ & $\begin{array}{l}\sim 1.7 \times 10^{-4} \\
\sim 3 \times 10^{-4}\end{array}$ \\
\hline $\begin{array}{l}(* *) \\
{[86]}\end{array}$ & $\begin{array}{l}K^{ \pm} \rightarrow \pi^{ \pm} \pi^{+} \pi^{-} \\
K^{ \pm} \rightarrow \pi^{ \pm} \pi^{0} \pi^{0}\end{array}$ & $\begin{array}{l}3.8 \times 10^{9}\left(K^{+}\right)+2.1 \times 10^{9}\left(K^{-}\right) \\
3.3 \times 10^{8}\left(K^{+}\right)+1.9 \times 10^{8}\left(K^{-}\right)\end{array}$ & $\begin{array}{l}\sim 1.0 \times 10^{-4} \\
\sim 1.3 \times 10^{-4}\end{array}$ \\
\hline$(* *) \mathrm{CKM} / \mathrm{FNAL}$ & $\begin{array}{l}K^{ \pm} \rightarrow \pi^{ \pm} \pi^{+} \pi^{-} \\
K^{ \pm} \rightarrow \pi^{ \pm} \pi^{0} \pi^{0}\end{array}$ & $\begin{array}{l}2.6 \times 10^{10}\left(K^{+}\right)+1.9 \times 10^{10}\left(K^{-}\right) \\
1.6 \times 10^{8}\left(K^{+}\right)+1.2 \times 10^{8}\left(K^{-}\right)\end{array}$ & $\begin{array}{l}\sim 3.6 \times 10^{-5} \\
\sim 2.0 \times 10^{-4}\end{array}$ \\
\hline
\end{tabular}

Table 8: Sensitivity of performed and proposed experiments for CP-violation decay asymmetry in $K^{ \pm} \rightarrow 3 \pi$ decays.

Of course, table 8 does not reflect the difficulty of demonstrating that there are indeed no systematic uncertainties at the $<10^{-4}$ level. Possible sources of systematic uncertainty include different hadronic interactions with the detector for $\pi^{+}$and $\pi^{-}$, and different characteristics of $K^{+}$. and $K^{-}$beams. The first attempt to investigate possible systematic uncertainties was presented in [86], which found that these uncertainties are smaller than $10^{-3}$. However, it is clear that this is only a first estimate and much more serious investigation will be needed.

\subsubsection{Low Energy Hadronic Physics}

The channels $K^{+} \rightarrow \pi^{+} e^{+} e^{-}, K^{+} \rightarrow \pi^{+} \mu^{+} \mu^{-}$and $K^{+} \rightarrow \mu^{+} \nu \gamma$ are of interest in CHPT and also are relevant for certain new weak interaction $(\mathrm{S}, \mathrm{T})$ searches. Table 5 presents information on 
the acceptance for modes as determined from our Monte Carlo simulation, and the corresponding dataset sizes.

\section{$K^{+} \rightarrow \pi^{+} e^{+} e^{-}, K^{+} \rightarrow \pi^{+} \mu^{+} \mu^{-}$Measurements}

Measurement of $K^{+} \rightarrow \pi^{+} e^{+} e^{-}$and $K^{+} \rightarrow \pi^{+} \mu^{+} \mu^{-}$branching ratios and Dalitz plot analyses are excellent probes of CHPT predictions [95]. In the ratio of branching $\operatorname{ratios} \mathrm{R}(\mu \mu / e e)=$ $\mathrm{BR}\left[K^{+} \rightarrow \pi^{+} \mu^{+} \mu^{-}\right] / \mathrm{BR}\left[K^{+} \rightarrow \pi^{+} e^{+} e^{-}\right]$and in the differential rate ratios seen in the Dalitz plot $\rho(\mu \mu / e e)$, many theoretical uncertainties cancel. Predicted values for $\mathrm{R}(\mu \mu / e e)$ range fronı 0.2 to 0.3 ; measurements at the $\delta \mathrm{R}=2-3 \%$ level should give clean tests of the CHPT predictions. One early paper [96] suggested that the differential ratio $\rho(\mu \mu / e e)$ in the region of small $\pi^{+}$energies is sensitive to the contribution of second order weak diagrams.

The existing data for these two decay modes are dominated by results from the recent BNL $K^{+}$experiments, most notably E865 [101,102].

The large sample obtained for this decay mode(10300 events for electrons and 400 for muons) allow the following conclusions:"

1. The experimental data are consistent with a vector model for the decay, as expected in the standard model. New physics could generate either scalar or tensor interactions, but these have been limited at the $90 \%$ C.L. to less than a $2 \%$ contribution to $\Gamma\left(K^{+} \rightarrow \pi^{+} e^{+} e^{-}\right)$.

2. The form factor dependence [103] upon $q^{2}=m_{e e}^{2} / m_{K}^{2}$ can not be explained with $p^{4}$ CHPT. Terms out to $p^{6}$ are necessary [104], as are those from $\pi^{+}$loop graphs. These terms introduce a linear dependence upon $q^{2}$ not present in the $p^{4}$ expansion. It seems also that charge pion loop graphs are influenced the region of high $q^{2}$ [104].

It is important to note that the $K^{+} \rightarrow \pi^{+} \mu^{+} \mu^{-}$result from E787 [63], based on 196 events, differs from the E865 result by more than $3 \sigma$, for reasons which remain unclear.

In the CKM detector, data from the pion RICH could be used to suppress background for the muon mode from $K^{+} \rightarrow \pi^{+} \pi^{+} \pi^{-}$with two pion decays in flight. We expect to identify on the order of $10^{3}$ and $2 \times 10^{3}$ events per week for the electron and muon modes respectively. This large sample should permit precise measurements and further tests of $O\left(p^{6}\right)$ CHPT predictions.

\section{The Decay $K^{ \pm} \rightarrow \nu \mu \gamma$}

The radiative decay $K^{ \pm} \rightarrow \nu \mu \gamma$ can be used in order to test the standard model as expressed in CHPT. This processes proceeds both through bremsstrahlung from $K^{ \pm} \rightarrow \nu \mu$ (where the radiation can come from from either the $K^{+}$or the $\mu^{+}$) and through structure dependent (SD) amplitudes where the photon is emitted directly from the $K^{ \pm} \nu \mu$ vertex; there is also an interference term. The $\mathrm{SD}$ term is sensitive to the hadronic structure of the kaon as is the interference term. This field has been the subject of an extensive theoretical literature, beginning with Neville in 1961 and most recently framed in terms of CHPT [105].

The photon may be emitted with either left or right handed chirality; these are parameterized with form factors $F_{a}$ and $F_{v}$ respectively. The experimentally measurable quantities are the contributions to the Dalitz plot from the different amplitudes, and they are expressed in terms of $\left|F_{a}+F_{v}\right|^{2}$ and $\left|F_{a}-F_{v}\right|^{2}$. The polarizability of the $K^{+}$, an indicator of the rigidity of the meson in response to electromagnetic fields, proportional to the ratio $F_{a} / F_{v}$. 
This mode has been experimentally investigated at Brookhaven [106] in a two day special run with the E787 detector, yielding a final sample of 2693 events. There are also earlier results with smaller samples from KEK and ITEP [108]. Brookhaven measured $\left|F_{a}+F_{v}\right|$, and they improved the limit on $\left|F_{a}-F_{v}\right|$, as shown in table 9. The corresponding electronic mode was first investigated at CERN [107] with about 50 events.

\begin{tabular}{|c|c|c|}
\hline & $\left|F_{v}+F_{a}\right|$ & $\left|F_{v}-F_{a}\right|$ \\
\hline CHPT prediction [105] & 0.097 & 0.037 \\
\hline $\begin{array}{c}\text { CERN PS [107] } \\
(e \text { mode })\end{array}$ & $0.153 \pm 0.010$ & $-2.5<\left|F_{v}-F_{a}\right|<0.5$ \\
\hline $\begin{array}{c}\text { BNL 787 }[106] \\
(\mu \text { mode })\end{array}$ & $0.165 \pm 0.007 \pm 0.011$ & $-0.04<\left|F_{v}-F_{a}\right|<0.24$ \\
\hline
\end{tabular}

Table 9: Theoretical predictions and experimental results for $\left|F_{v}+F_{a}\right|$ and $\left|F_{v}-F_{a}\right|$. We use the convention of where $F_{v}$ and $F_{a}$ are dimensionless and larger by a factor of $\sqrt{2}$ than in many papers, and there is a minus sign in the interference term.

Our acceptance at CKM reaches $\simeq 5 \%$ and we expect [109] to accumulate about $10^{8}$ events in one week; after the tight final selection cuts this analysis needs, we hope to have on the order of $10^{5}$ events. We will very probably need to do a short special run because of trigger considerations.

\subsubsection{Conclusion}

There are numerous possibilities for the CKM experiment to search for exotic $K^{+}$rare decays and perform precision measurments that can challenge the Standard Model. The potential of this broad phyisics program is quite attractive, and directly results from the high decay rate and detector performance required to measure $K^{+} \rightarrow \pi^{+} \nu \bar{\nu}$. Although some of these auxiliary measurement require short special runs ( $K^{-}$running for example), none of the modes discussed require compromising the design of the $K^{+} \rightarrow \pi^{+} \nu \bar{\nu}$ beamline or detector. We are continuing to evaluate the backgrounds and systematic issues associated with this broad physics program. The focus the proposal now returns to the principal task: making the case for a precision measurement of $K^{+} \rightarrow \pi^{+} \nu \bar{\nu}$. 


\section{The Experimental Method}

\subsection{Experimental Overview}

The principal goal of CKM is to observe a sample (about 100 events) of $K^{+} \rightarrow \pi^{+} \nu \bar{\nu}$ decays with small background. A signal of 100 events if the branching ratio is $1 \times 10^{-10}$ would imply that CKM needs to reject all backgrounds to below the $10^{-11}$ level. Achieving this level of background rejection is the major challenge CKM faces. In addition, it is necessary to have methods for convincingly measuring the background(s), since an observation of $K^{+} \rightarrow \pi^{+} \nu \bar{\nu}$ will not be characterized by a clear signature, such as a mass peak. In this section we will discuss the backgrounds to $K^{+} \rightarrow \pi^{+} \nu \bar{\nu}$ and explore the requirements they impose on the detector.

An essential ingredient in any successful rare $K$ decay experiment is measurement redundancy. The experience of the most recent generation of rare $K$ decay experiments clearly teaches that. It would be unrealistic to seek to improve upon these experiments without building significant redundancy into the detector. Therefore, CKM will make completely redundant measurements of both beam kaons and charged decay products in the spectrometer. We will elaborate on these features of the detector after discussing the backgrounds and how they may be suppressed.

\subsection{Background Sources}

The most obvious backgrounds to $K^{+} \rightarrow \pi^{+} \nu \bar{\nu}$ are the decays $K^{+} \rightarrow \mu^{+} \nu$ and $K^{+} \rightarrow \pi^{+} \pi^{0}$, which have large branching ratios $(64 \%$ and $21 \%$, respectively). If the muon is misidentified as a pion in the former case, or if the photons from the $\pi^{0}$ decay are missed in the latter case, then these modes can resemble $K^{+} \rightarrow \pi^{+} \nu \bar{\nu}$ decays. Rejecting these decays depends on exploiting the kinematics of two-body decays and on highly effective vetoes of muons and photons. We will discuss the kinematic issues first.

In the two-body decays $K^{+} \rightarrow \mu^{+} \nu$ and $K^{+} \rightarrow \pi^{+} \pi^{0}$, the charged particle's center-of-mass momentum is fixed at $236 \mathrm{MeV}$ and $205 \mathrm{MeV}$, respectively. In stopped- $K$ experiments this results in narrow peaks in the observed momentum of the charged particles produced in these decays, and consequently these decays can be highly suppressed simply by excluding these peaks from the $K^{+} \rightarrow \pi^{+} \nu \bar{\nu}$ signal region. In the laboratory frame of a decay-in-flight experiment the kinematic rejection of two-body decays is similarly possible.

In the $K^{+} \rightarrow \pi^{+} \nu \bar{\nu}$ decay, for a specific value of the pion momentum in the kaon frame the mass $M_{\nu \bar{\nu}}$ of the lepton pair is fixed. The missing mass $M_{\text {miss }}$ in $K^{+} \rightarrow \pi^{+} \pi^{0}$ and $K^{+} \rightarrow \mu^{+} \nu$ decays is simply $m_{\pi^{0}}$ and 0 , respectively. Experimentally, $M_{\text {miss }}$ is readily obtained from measurements of the momenta and directions of the parent $K^{+}$and the daughter $\pi^{+}$. The relevant issue for background rejection is to what extent the kinematic resolution is adequate, and to what extent experimental mismeasurements lead to misclassification of events.

The measurable quantities in a decay-in-flight experiment are the momentum $p_{K}$ of the parent kaon, the momentum $p$ of the charged particle produced in the decay and the angle $\theta$ between the parent's trajectory and that of the charged daughter. In terms of these quantities, a sufficiently good approximation is that

$$
M_{\mathrm{miss}}^{2}=M_{K^{\prime}}^{2}\left(1-p / p_{K}\right)+m^{2}\left(1-p_{K} / p\right)-p p_{K} \theta^{2},
$$

where $M_{K}$ is the kaon mass and $m$ is the mass of the charged daughter. Consider the distribution of events from different decays in $p$ and $\theta$ for fixed $p_{K}$. For any two-body decay, $M_{\text {miss }}$ has a single value, so that allowed values of $p$ and $\theta$ form a curve. For a three-body decay, $p$ and $\theta$ can take on any pair of values which lie below a curve defined by the minimum value of $M_{\text {miss }}$. Figure 11 
illustrates this by showing the curve (solid line) corresponding to $K^{+} \rightarrow \pi^{+} \pi^{0}\left(M_{\text {miss }}=m_{\pi^{0}}\right.$ ) and the distribution of points for $K^{+} \rightarrow \pi^{+} \nu \bar{\nu}$ decays (minimum $M_{\text {miss }}=0$ ). A kaon energy of $22 \mathrm{GeV}$, which is the proposed CKM beam energy, is assumed. Figure 11 also shows a shaded area, which corresponds to the $(p, \theta)$ range of $K^{+} \rightarrow \pi^{+} \pi^{+} \pi^{-}$decays. These dark curves and shaded zone delineate regions where background rejection issues are somewhat different, as summarized in table 10. In the table the region above the solid line is referred to as "Region 1", between the solid and the shaded region as "Region 2", and in the shaded region as "Region 3." Traditionally $K^{+} \rightarrow \pi^{+} \nu \bar{\nu}$ searches have focussed on Region 1 (also referred to as "above the $\pi^{0}$ mass"). However, considerable acceptance can be gained by including Region 2 as well. Region 3 appears intractable, owing to the $K^{+} \rightarrow \pi^{+} \pi^{+} \pi^{-}$decay; it has a large branching ratio, lacks two-body kinematic rejection, lacks a particle identification veto on the observed charged particle, and has no photons to veto. In CKM our efforts thus far have been concentrated on understanding backgrounds in Region 1, but our intention is to study Region 2 as time permits and keep it as a goal.

\begin{tabular}{|c|l|c|c|l|}
\hline \multirow{2}{*}{ Region } & K-decay & $\begin{array}{c}\text { Branching } \\
\text { Ratio }\end{array}$ & $\begin{array}{c}\text { Minimum } \\
M_{\text {miss }}(\mathrm{MeV})\end{array}$ & Rejection Methods \\
\hline 1 & $K^{+} \rightarrow \mu^{+} \nu$ & 0.635 & 0 & 2-body kinematics, $\mu$-veto \\
& $K^{+} \rightarrow \pi^{+} \pi^{0}$ & 0.212 & 135 & 2-body kinematics, $\gamma$-veto \\
& $K^{+} \rightarrow \mu^{+} \nu \gamma$ & $5.5 \times 10^{-3}$ & 0 & $\mu$-veto, $\gamma$-veto \\
& $K^{+} \rightarrow \pi^{+} \gamma \gamma$ & $6.0 \times 10^{-7}$ & 0 & $\gamma$-veto \\
& $K^{+} \rightarrow \pi^{+} e^{+} e^{-}$ & $2.9 \times 10^{-7}$ & 1 & $e$-veto \\
\hline 2 & $K^{+} \rightarrow \pi^{+} \pi^{0}$ & 0.212 & 135 & 2-body kinematics, $\gamma$-veto \\
& $K^{+} \rightarrow \pi^{0} e^{+} \nu$ & 0.048 & 135 & $e$-veto, $\gamma$-veto \\
& $K^{+} \rightarrow \pi^{0} \mu^{+} \nu$ & 0.032 & 135 & $\mu$-veto, $\gamma$-veto \\
& $K^{+} \rightarrow \pi^{+} \pi^{0} \pi^{0}$ & 0.017 & 270 & $\gamma$-veto \\
& $K^{+} \rightarrow \mu^{+} \nu \gamma$ & $5.5 \times 10^{-3}$ & 0 & $\mu$-veto, $\gamma$-veto \\
& $K^{+} \rightarrow \pi^{+} \pi^{0} \gamma$ & $2.8 \times 10^{-4}$ & 135 & $\gamma$-veto \\
& $K^{+} \rightarrow \pi^{+} \pi^{-} e^{+} \nu$ & $3.9 \times 10^{-5}$ & 139 & $\pi^{ \pm}$-veto, $e^{\mp}$-veto \\
\hline 3 & Same as region 2, plus & & & \\
& $K^{+} \rightarrow \pi^{+} \pi^{+} \pi^{-}$ & 0.056 & 279 & charged particle veto \\
\hline
\end{tabular}

Table 10: Kaon decay modes which are potential backgrounds to $K^{+} \rightarrow \pi^{+} \nu \bar{\nu}$ in each of the three regions, defined in the text. The list is not exhaustive and includes only modes with relatively large branching ratios.

Rejecting the two-body decay backgrounds depends upon accurately determining $M_{\text {miss }}$. From equation 33, we can estimate the effect on $M_{\text {miss }}$ of mismeasurements of the underlying quantities. We need to measure all three quantities to about $1 \%$ or better of themselves in order to achieve sufficient resolution on $M_{\text {miss }}$ to make a $K^{+} \rightarrow \pi^{+} \nu \bar{\nu}$ search viable. Ultimately however, it is not measurement resolution which limits background rejection. Physical processes, such as multiple or single Coulomb scattering and inelastic hadronic scattering, which occur in the materials which make up the detector introduce unavoidable mismeasurements. These effects can only be reduced by minimizing the amount of material the charged particles traverse before the measurements are made. Minimizing material drives much of the CKM design.

For the $K^{+} \rightarrow \mu^{+} \nu$ and some other kaon decays which may introduce backgrounds, considerable rejection can be obtained from particle identification. As a consequence, exceptionally good particle identification is necessary for CKM. 


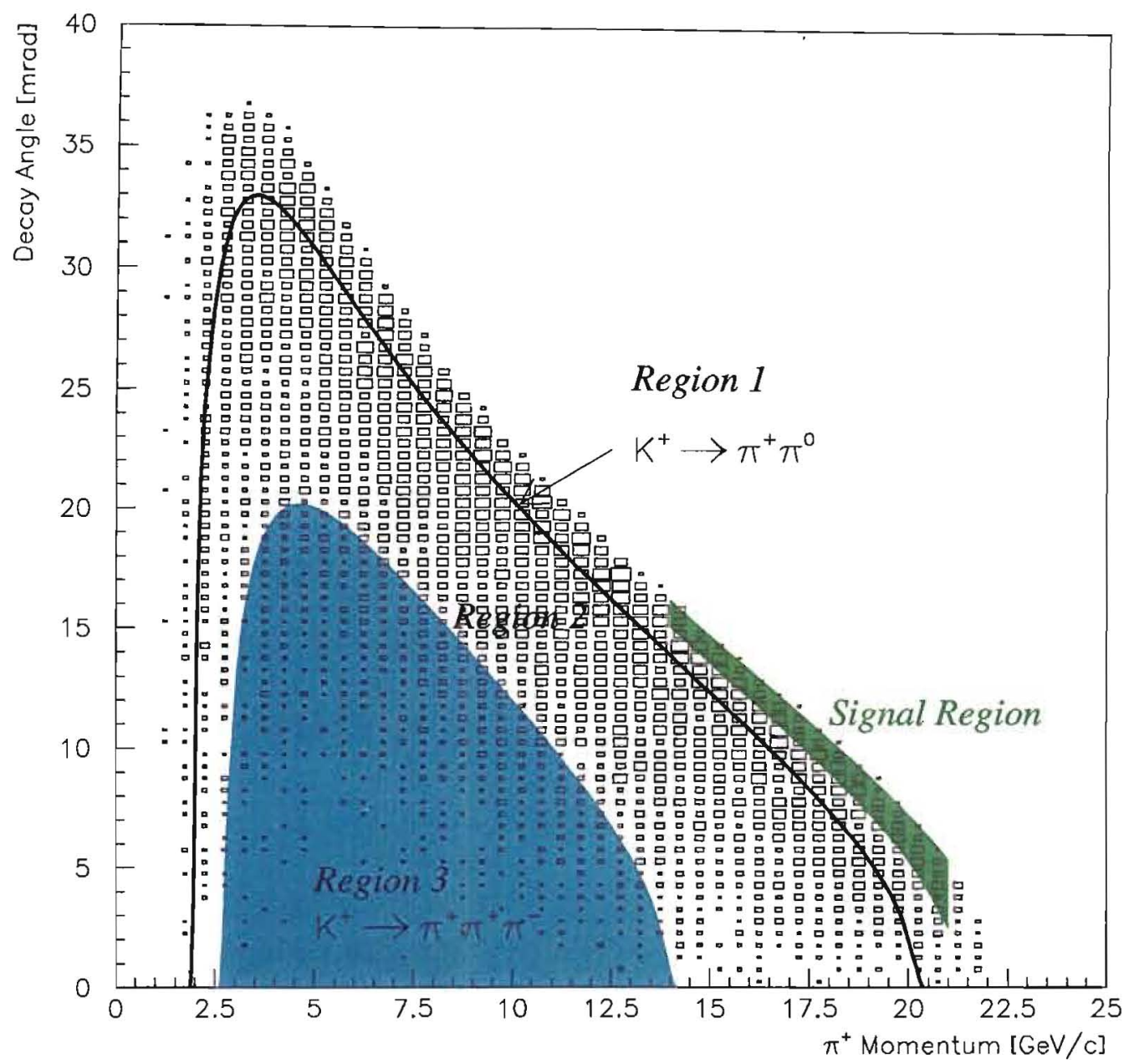

Figure 11: Decay angle versus momentum for $K^{+} \rightarrow \pi^{+} \nu \bar{\nu}$ events. Three distinct analysis regions are shown. The region defined by the signal cuts is also shown. 
For the $K^{+} \rightarrow \pi^{+} \pi^{0}$ and some other kaon decays which may introduce backgrounds, considerable rejection can be obtained by observing photons. As a consequence, a virtually hermetic photon veto system is required for CKM.

In addition to kaon decays, other sources of backgrounds are possible. Any process in which a $K^{+}$enters the detector and a $\pi^{+}$exits is a candidate background. An interesting example is a charge exchange interaction where the beam $K^{+}$emerges as a $K^{0}$ which subsequently decays via $K_{L}^{0} \rightarrow \pi^{+} e^{-} \nu$. The electron can have arbitrarily low momentum and go unobserved, leaving an event which satisfies the $K^{+} \rightarrow \pi^{+} \nu \bar{\nu}$ topology. Other more mundane hadronic interaction scenarios of the beam $K^{+}$can produce a single observed $\pi^{+}$. Also, since the beam will inevitably contain some pions, it is possible for an incoming $\pi^{+}$which scatters to be mistaken for a $K^{+}$and again simulate the $\mathrm{K}^{+} \rightarrow \pi^{+}$topology. Of course, handles are available to suppress these hadronic interaction backgrounds, such as requiring that the putative $K^{+}$and $\pi^{+}$make a good vertex which is well separated from any material, as well as vetoing events in which extra particles from the interactions are observed. These capabilities are important for CKM.

Finally, various accidental scenarios - for example, two kaon decays close together in time can introduce backgrounds. Many of the same tools which remove other backgrounds are effective here, but in addition the CKM detector must be capable of precision timing.

\subsection{Beam Issues}

CKM must sample about $5 \times 10^{6} K^{+}$decays per spill in order to reach a single event sensitivity of $1 \times 10^{-12}$ in two years of running at the Main Injector. This implies the beam must provide a high flux of debunched $K^{+}$'s, in the neighborhood of $3 \times 10^{7}$ per spill. For background rejection, CKM must measure the identity, momentum, and trajectory of each beam particle. That is, particle identification and tracking detectors must operate in this beam. A phototube-based ring-imaging Cherenkov counter can operate in this environment. Various high-rate tracking technologies are viable provided they have sufficient segmentation to reduce the rate through any single cell to an acceptable level. However, it is clear that a $\sim 50 \mathrm{MHz}$ beam rate (of which $30 \mathrm{MHz}$ are $K^{+}$) poses significant technical challenges for tracking detectors. Therefore, it is important for CKM that the beam consist primarily of kaons.

\subsection{Detector Requirements}

\subsubsection{The Momentum Spectrometers}

The momentum spectrometers must measure the vector momentum of each beam particle and decay daughter(s) to the resolution required for the suppression of background processes previously discussed. In addition, beam interactions with material must be minimal and exquisite time resolution is required to control accidental activity. The $50 \mathrm{MHz}$ rate of beam particles poses a significant challenge for the design of these detectors. Previous rare kaon and hyperon decay experiments have approached these rates, such as Brookhaven experiment E871 $\left(K_{L} \rightarrow \mu^{ \pm} e^{\mp}\right.$ search $)$ which ran at $\sim 20 \mathrm{MHz} / \mathrm{m}^{2}$, and the Fermilab HyperCPexperiment which ran at $\sim 30 \mathrm{MHz} / \mathrm{m}^{2}$.

Two momentum spectrometers compose the complete system: "Upstream", to measure the momentum of the $\sim 22 \mathrm{GeV} / c$ beam kaons with the accuracy of $0.3 \%$ and "Downstream", to measure the momentum of charged kaon decay daughters with an accuracy of $0.9 \%$. The ultimate resolution requirement is a $M_{\text {miss }}^{2}$ resolution $\sim M_{\pi^{0}}^{2} / 10$ for $K^{+} \rightarrow \pi^{+} \pi^{0}$ decays measured with the momentum vectors from both spectrometers.

The momentum spectrometers also provide the tracking measurements necessary to reconstruct the position and quality of the kaon decay vertex. The distance of closest approach (DOCA) of the 
kaon and daughter trajectories that form this vertex is an important cut in rejecting interactions and decay in flight of kaon daughters.

The high beam rate requires that the detectors must have excellent time resolution. This will help to suppress accidentals, pile-up and tracking errors. A time resolution of \pm 1 nsec is desirable for these spectrometers. Since neither the straw tube or MWPC tracking technologies have the required speed to achieve this level of time resolution, a fiber tracking plane is included in each spectrometer; the "Beam Time Stamp" system in the upstream momentum spectrometer and a fiber plane at the end of the downstream momentum spectrometer that will tag candidate spectrometer tracks with precise times.

Both of these spectrometers have $\sim 50 \mathrm{MHz}$ of beam running through them. Minimizing interactions in the material of these spectrometers is critical to control backgrounds. The greatest sensitivity to material in the beam is in the planes closest to the fiducial decay volume. The upstream momentum spectrometer must momentum analyze each beam particle. Based on the proven high-rate performance of fine pitch high-speed MWPC chambers developed by the HyperCP experiment, we have adopted this low mass technology for our baseline upstream tracking design. Copies of these MWPCs are placed immediately upstream of a low mass vacuum window to measure the incident angle of the $K^{+}$entering the evacuated decay volume.

The downstream momentum spectrometer must momentum analyze all daughters and track undecayed beam particles out of the decay volume. Minimum material is particularly important here, and the instrumented area required is $\sim 0.6 \mathrm{~m}^{2}$. The tracking technology of choice is straw tubes in vacuum with either deadened wires in the beam region. The magnet requirements are a $1 \times 1 \mathrm{~m}^{2}$ aperture with a $p_{\perp}$ kick of $100 \mathrm{MeV} / c$. This requires a $0.3 \mathrm{~T}$ field for a $1 \mathrm{~m}$ effective length. The beam hole costs some acceptance but the beam rate is too high for straw technology to handle with high efficiency. We have considered and rejected a fiber tracker since multiple Coulomb scattering alone from even thin $(500 \mu \mathrm{m})$ fibers is larger than the resolution required. There is a final plane of fiber tracker to track the beam and provide a high resolution time measurement on each track. The straw tube occupancy and rates are high but tolerable, while the material is as low as practical. This straw tracker must be located in the vacuum of the decay volume; multiple windows would be prohibitive in terms of material in the beam. This is a significant engineering challenge.

The channel count for the momentum spectrometers is driven by the rate capability of the detectors, as well as by dead time, naximum occupancy and resolution considerations. Low occupancy is important to avoid ambiguity in track reconstruction. The upstream and downstream trackers have about 15,500 wires and 5000 straws, respectively. Each fiber plane has $\sim 800$ channels.

The momentum spectrometers have demanding performance parameters but are fundamentally conventional tracking magnetic spectrometers. The tracking technologies are much slower in resolving time and time resolution than the velocity spectrometers. This requires a debunched separated beam to keep the rates seen by these detectors within acceptable limits. Without the redundant measurements they provide the required level of background rejection is unachievable.

\subsubsection{The Velocity Spectrometers}

The "Velocity Spectrometer" is the most novel component of the CKM apparatus, which measures the velocity vectors of the incoming $K^{+}$and the exiting $\pi^{+}$. This high speed, essentially isochronous detector system, measures $K^{+}$and $\pi^{+}$kinematics completely independent of the Momentum Spectrometer. The mis-reconstruction mechanisms of the Momentum and Velocity spectrometers are essentially orthogonal, which is a critical requirement of genuinely redundant detector systems. The independence of the momentum and velocity spectrometers is demonstrated with a 
detailed GEANT simulation of the apparatus presented in chapter 7.

The velocity spectrometer is composed of two phototube based "Ring Imaging Cherenkov" (RICH) systems, the "kaon RICH" and the "pion RICH", shown in figure 42. Conventional RICH systems operate well above the turn-on momentum threshold and primarily serve to separate particle species by mass. The CKM RICH systems in contrast operate in a momentum band centered on the RICH turn-on thresholds where the ring radii depend sensitively on the velocities. The $\pi^{+}$and $K^{+}$threshold curves of the kaon RICH are shown in figure 12, and in figure 116 for the pion RICH. The principles and sensitivity of the Velocity Spectrometer are determined by the simple formulae below.

Another significant difference between the CKM RICH systems and most of the previous large $\mathrm{RICH}$ detector systems is the Cherenkov photon detection technology. The significant challenge associated with realizing large RICH detectors based on gaseous detection of the Cherenkov, photons is well known in the field. The speed requirements of the CKM RICH requires the use of photomultiplier tube based photon detection. Although this is an apparent cost liability, phototube readout has the enormous advantage that the photon detection system is built from one of the most reliable and well understood detector components available. Indeed, the high performance [110] [112] of the large phototube based SELEX RICH system, the HERA-B RICH system, and earlier phototube based RICH systems (SPHINX) [113] bears this out.

A spectrometer with time resolution sufficiently good to cleanly resolve individual particles in a $50 \mathrm{MHz}$ beam environment is of critical importance in controlling accidental background sources. A PMT array provides 1 nsec leading edge time resolution and $10 \mathrm{nsec}$ wide pulses with ultra-low crosstalk for each individual PMT signal. Data recently shown from the HERA-B RICH system have demonstrated a single PMT time resolution of $\sim 1.5 \mathrm{nsec}$ [111]:

In a Ring Imaging Cherenkov detector, the basic formulas apply:

$$
\cos \Theta_{c}=\frac{1}{\beta \cdot n}
$$

where $\Theta_{c}$ denotes the Cherenkov angle, $\beta$ is the velocity of the particle relative to the velocity of light in vacuum, and $n$ is the refractive index of the medium.

$$
N_{\text {det }}=N_{0} \cdot L \cdot \sin ^{2} \Theta_{c}
$$

where $N_{\text {det }}$ is the number of detected photons, which is given by a proportional constant $N_{0}$, and the length of the radiator $L$. The spectrum of the Cherenkov photons is given by:

$$
\frac{d N}{d \lambda} \propto \frac{1}{\lambda^{2}} .
$$

In a RICH detector the photons are focused onto a ring of radius $r$, which is given by:

$$
r=\frac{R}{2} \cdot \tan \Theta_{c}
$$

where $R$ is the radius of the spherical mirror.

For relativistic kinematics, we obtain the radius of the ring $r$ as a function of the momentum $p$ of a particle with mass $m$ :

$$
r=\frac{R}{2} \sqrt{2-\frac{2}{n} \sqrt{1+\frac{m^{2} c^{2}}{p^{2}}}}
$$




$$
\frac{d r}{d p}=\frac{R^{2}}{4} \frac{m^{2} c^{3}}{r n p^{3} \sqrt{1+\frac{m^{2} c^{2}}{p^{2}}}}
$$

In figure 12 we illustrate equation 37 and 39 for a RICH detector with $R=40 \mathrm{~m}$, and $n=$ 1.000287 .

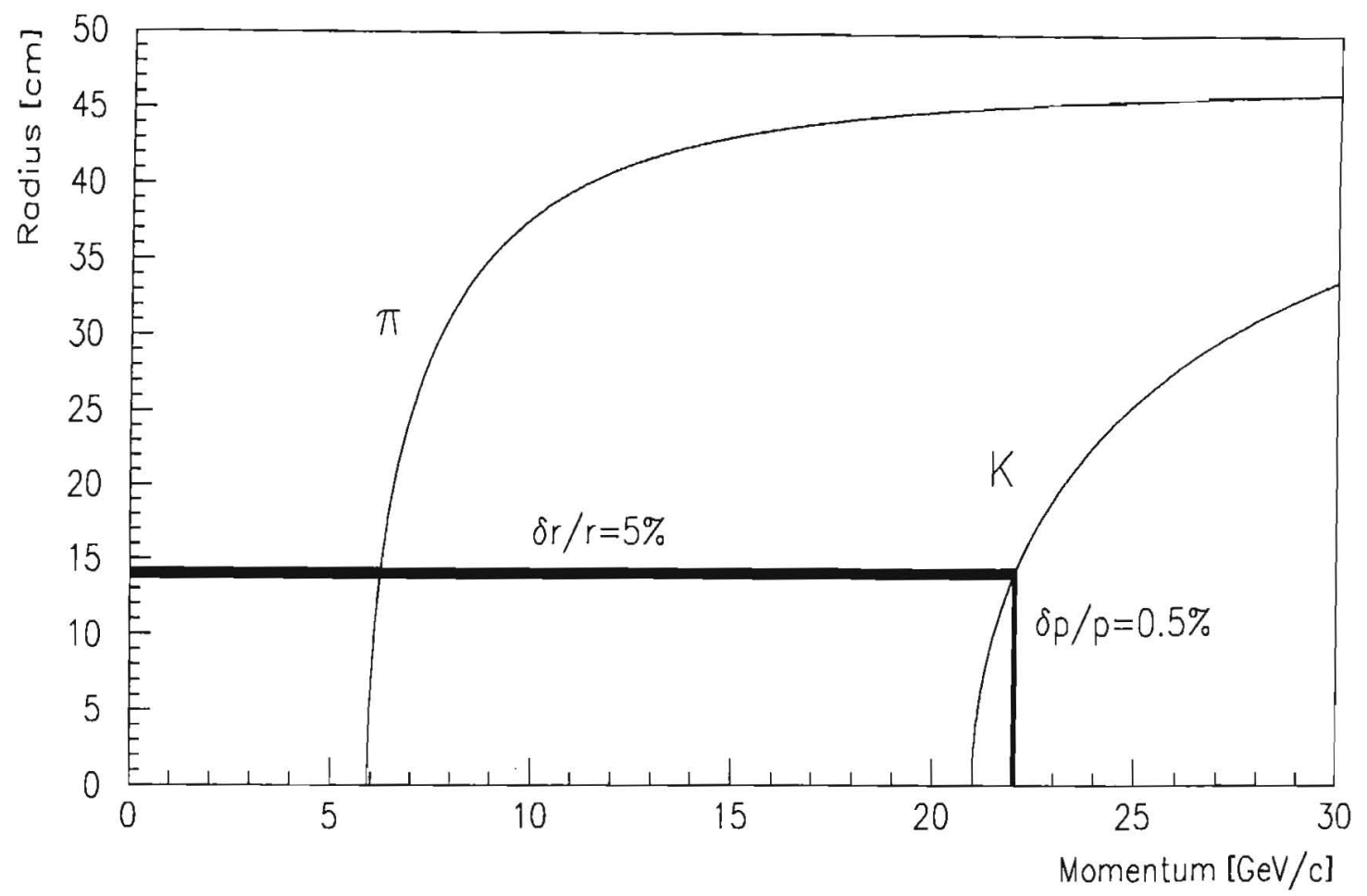

Figure 12: Ring radii for $K^{+}$and $\pi^{+}$versus their momentum for the kaon RICH system that measures the incident beam $\mathrm{K}^{+}$momentum and decay angle. The black band indicates the momentum resolution of the kaon RICH system as described in section 5.3 . Here we used $R=40 \mathrm{~m}$ and $n=1.0002765$.

The ring radius resolution is inversely proportional to $\sqrt{N_{\operatorname{det}}-3}$, which alone suggests that the largest ring radii produce the best momentum resolution. From figure 12 it is clear however that $d r / d p$ decreases with increasing ring radius, which degrades the momentum resolution. Other contributions including multiple scattering in the RICH gas and chromatic dispersion (the dependence of the refractive index on the wavelength $n=n(\lambda)$ ) must also be considered in optimizing the overall resolution. Chromatic dispersion is the dominant effect limiting the resolution of the Kaon RICH.

\subsubsection{Components of the $M_{\text {miss }}^{2}$ Resolution Function.}

The missing mass squared $\left(M_{\text {miss }}^{2}\right)$ resolution from the momentum and velocity spectrometers for $K^{+} \rightarrow \pi^{+} \pi^{0}$ or $K^{+} \rightarrow \pi^{+} \nu \bar{\nu}$ and the contributions to those resolutions from the individual momentum and opening angle measurements are shown in figure 13. These resolutions are calculated analytically with SPEC_RES [114]; which includes multiple Coulomb scattering in the Gaussian 
limit for the magnetic spectrometers and with the full CKM GEANT simulation for the velocity spectrometers. As discussed previously the relevant mass scale is $m_{\pi^{0}}^{2}=18.2 \times 10^{-3} \mathrm{GeV}^{2} / \mathrm{c}^{4}$, where the dominant $K^{+} \rightarrow \pi^{+} \pi^{0}$ background resides.

In both cases the mass resolution is dominated by the measurement of the $\pi^{+}$momentum. In the region of our acceptance $(14-21 \mathrm{GeV} / \mathrm{c})$ these resolutions are comparable. We are not using Cherenkov counters for particle identification is this application. It would be just as reasonable to say that we were measuring trajectories (vector velocities) with the RICHs and identifying particle species with the magnetic spectrometers.

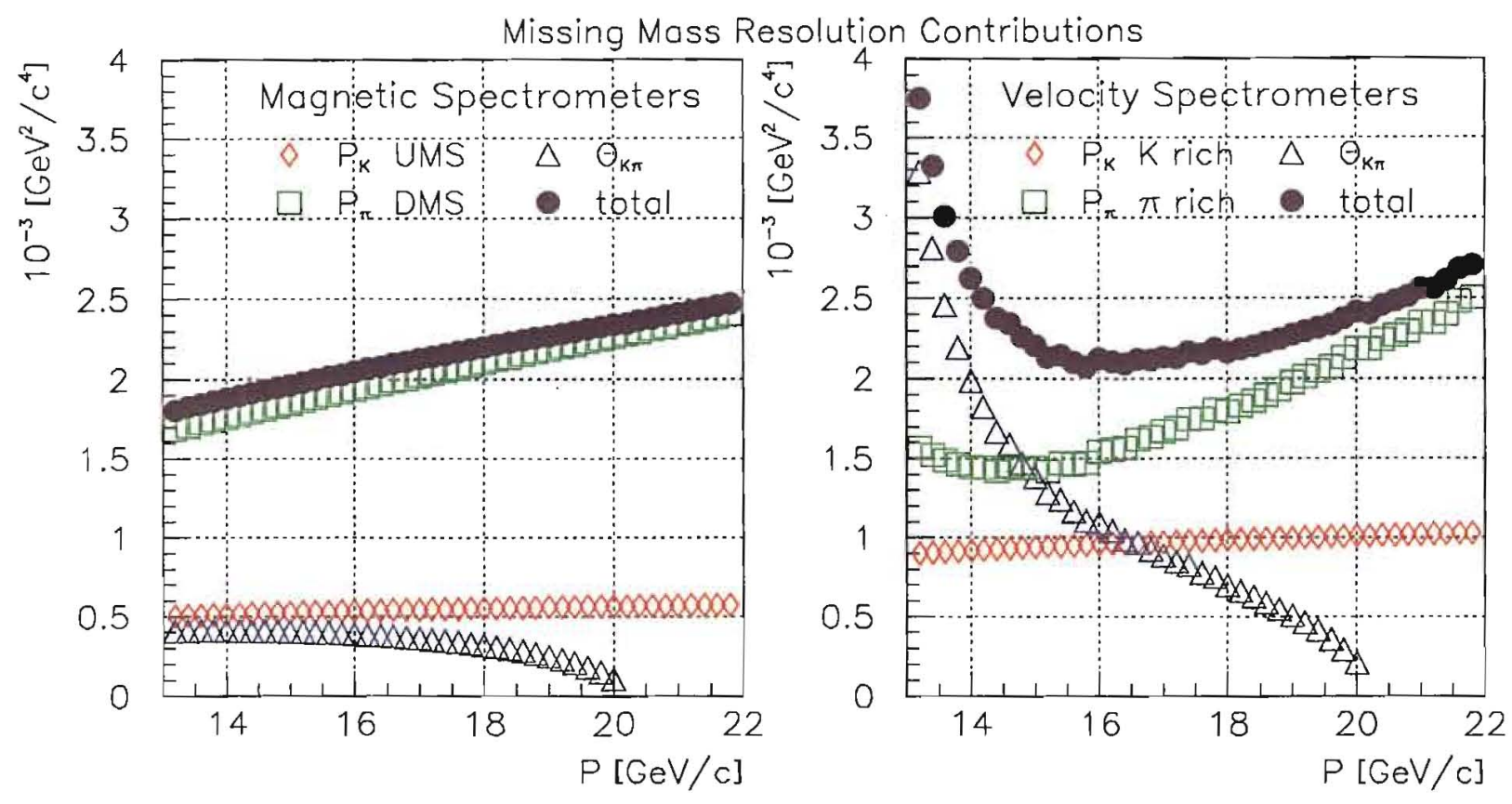

Figure 13: Expected $M_{\text {miss }}^{2}$ resolution and contributions for the momentum(left) and velocity(right) spectrometers. The relevant mass scale is $m_{\pi^{0}}^{2}=18.2 \times 10^{-3} \mathrm{GeV}^{2} / \mathrm{c}^{4}$, where the dominant $K^{+} \rightarrow \pi^{+} \pi^{0}$ background resides.

\subsubsection{The Kaon Entrance Angle Tracker System (KEAT)}

The material upstream of the vacuum decay volume illustrated in figure 42 corresponds to $\sim 8 \%$ of a radiation length which significantly alters the $K^{+}$entrance angle on the scale of interest. A pair of fine pitched high-speed MWPC tracking chambers is employed immediately upstream of the evacuated decay volume to precisely measure the entrance angle of the beam particle measured and tagged as a $\mathrm{K}^{+}$by the kaon RICH. This system must have minimal mass and have rate capability adequate to precisely track beam particles. 


\subsubsection{The Beam Interaction Veto System (BIVS)}

Incident $K^{+}$beam particles can scatter inelastically in the material upstream of the decay volume, including the KEAT system. These inelastic scatters can produce a fast forward $\pi^{+}$into the detector acceptance that can mimic the $K^{+} \rightarrow \pi^{+} \nu \bar{\nu}$ signature. Beam interactions at the most downstream plane of KEAT material are particularly troublesome, since it is much more likely that interactions there will be incorrectly reconstructed into the fiducial decay volume. The Beam Interaction Veto System (BIVS) shown in figure 42 and figure 76 is a cylindrical fine sarnpling leadscintillator veto system with a narrow beam hole that instruments essentially all of the forward scattering hemisphere downstream of the KEAT. The BIVS must efficiently veto the charged and neutral hadrons associated with the fast forward $\pi^{+}$, while maintaining low detector deadtime in the presence of high ambient bearn interaction rate. The $50 \mathrm{MHz}$ of beam particles traversing the $\sim 4 \% \lambda_{I}\left(K^{+}\right)$generates an ambient rate of about $2 \mathrm{MHz}$ of inelastic beam interactions that intercept the BIVS. The BIVS design strategy is described in section 5.5.1.

\subsubsection{The Photon Veto Systems}

One of the most challenging detector issues facing CKM is the efficient detection of all photons produced by background events along the $30 \mathrm{~m}$ long evacuated decay volume shown in figure 42 . A very high level of hermeticity for high energy $(>1 \mathrm{GeV}$ ) photons and efficient photon detection down to energies as low as $10-20 \mathrm{MeV}$ is required. The veto systems must also efficiently tag minimum ionizing particles and charged particles with $\beta,\left(\frac{v}{c}\right)$, as low as 0.97 that are produced in beam interactions with detector material. The veto systems in addition must provide reasonably precise measurement of photon energies and positions so that well measured control samples of $K^{+} \rightarrow \pi^{+} \pi^{0}$ decays can be used to monitor and study the global system efficiency.

The required level of photon veto inefficiency for CKM was determined by iterative studies of all backgrounds described in sections 7 and 13. These studies conclude that the performance of the photon veto systems is primarily driven by the $K^{+} \rightarrow \pi^{+} \pi^{0}$ background, where the associated $\pi^{0}$ must be vetoed with an inefficiency of less than $1.6 \times 10^{-7}$.

The signal analysis cuts described in chapter 7 include cuts to ensure a well measured decay vertex within the fiducial volume. One of these cuts is that the decay angle $\theta\left(K^{+}, \pi^{+}\right)$be greater than $2.5 \mathrm{mrad}$. As can be seen from the solid curve in figure 11 , given a $22 \mathrm{GeV} / \mathrm{c} K^{+}$beam this minimum decay angle cut implies a minimum combined photon energy of $2 \mathrm{GeV}$ from $K^{+} \rightarrow \pi^{+} \pi^{0}$ decay is present within the detector. The two-body kinematics of $K^{+} \rightarrow \pi^{+} \pi^{0}$ provides further key constraints in determining the required inefficiency. The following figures illustrate the effect of the detector geometry and $K^{+} \rightarrow \pi^{+} X$ kinematics on the photon energy spectra that the veto systems must contend with.

Figure 14 shows the distribution of the higher energy photon in $K^{+} \rightarrow \pi^{+} \pi^{0}$ decays in the various veto sub-systems. The sub-systems are referred to as the "Vacuum Veto System" (VVS) which surrounds the decay volume, the "Forward Veto System" (FVS) which instruments the forward region that the decay $\pi^{+}$traverses, and the "Hole Veto System" (HVS) which instruments the beam-hole behind the FVS. From this figure it's clear that all systems, including the "barrel" VVS must contend with photon with energies up to $8 \mathrm{GeV}$. Figure 15 shows the distribution of the lower energy photon in $K^{+} \rightarrow \pi^{+} \pi^{0}$ decays in the the different sub-systems. The lowest energy photon that the FVS and HVS systems must detect from $K^{+} \rightarrow \pi^{+} \pi^{0}$ decays is about $900 \mathrm{MeV} / \mathrm{c}$, relaxing the low energy performance requirements of the forward veto systems accordingly. In contrast the barrel VVS must detect photons with energies as low as 10-20 MeV, a considerable challenge in a high rate and high energy experiment. Fortunately there is a strong anti-correlation 
between the energy of the two photons from $K^{+} \rightarrow \pi^{+} \pi^{0}$ decays: When one photon has low energy the other photon necessarily is high energy. This correlation places a large premium on the efficiency to detect high energy photons.
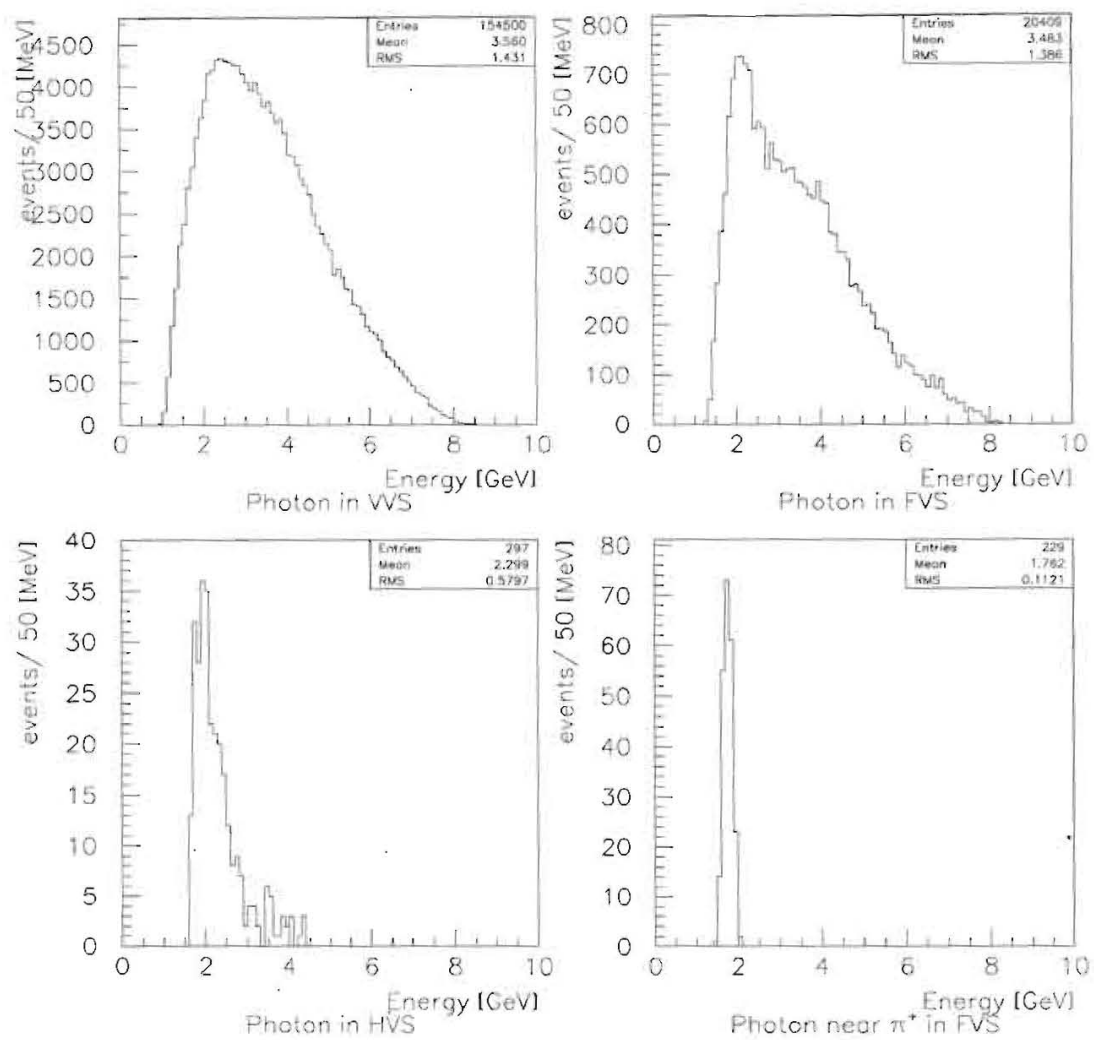

Figure 14: Energy distribution of the high energy photon in $K^{+} \rightarrow \pi^{+} \pi^{0}$ decay for the various veto detector subsystems.

Another important effect in $K^{+} \rightarrow \pi^{+} \pi^{0}$ background decays is the correlation between angle and energy: As the polar-angle in the laboratory of the decay photon increases upwards toward the VVS acceptance, the corresponding energy is reduced. This correlation is particularly strong for large angle photons. Consideration of this effect together with a desire to simplify the VVS system led us toward a baseline conceptual design with only half of the inner surface of the evacuated decay volume instrumented, as shown in figure 16. This concept dramatically eases the engineering challenge, and allows us to base the design on the successful $\mathrm{KTeV}$ vacuum veto system. The $\mathrm{KTeV}$ system employed annular modules in vacuum whose design can be augmented in a straightforward manner to serve as the base element of a modular system for CKM. A consequence of this scheme is the loss in geometric acceptance for about half of the $K^{+} \rightarrow \pi^{+} \pi^{0}$ decay photons with angles greater than $45^{\circ}$. The corresponding energy spectra of photons lost and not lost in these gaps is shown in figure 17. From figure 17 it is clear that the geometrically lost photons in this design are typically less than $10 \mathrm{MeV}$ where our detection efficiency is already relatively poor even if they were geometrically accepted. Losing this class of low energy photons has a small effect of the final background as will be discussed later. 

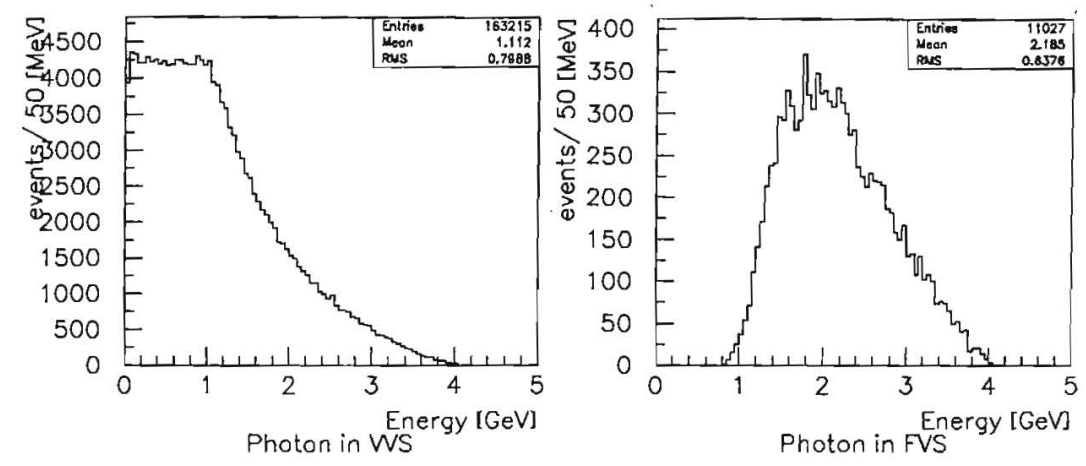

Figure 15: Energy distribution of the low energy photon from the $K^{+} \rightarrow \pi^{+} \pi^{0}$ decay for the various veto detector subsystem.

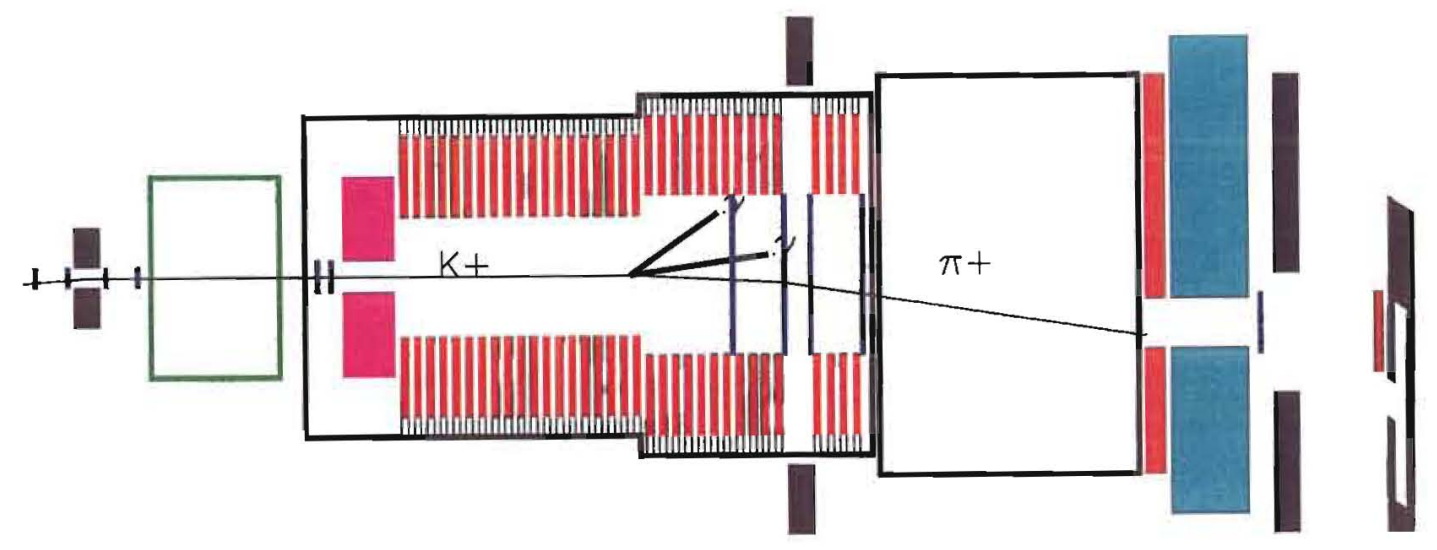

Figure 16: Illustration of a $K^{+} \rightarrow \pi^{+} \pi^{0}$ decaying within the vacuum decay volume surrounded by the VVS system. 

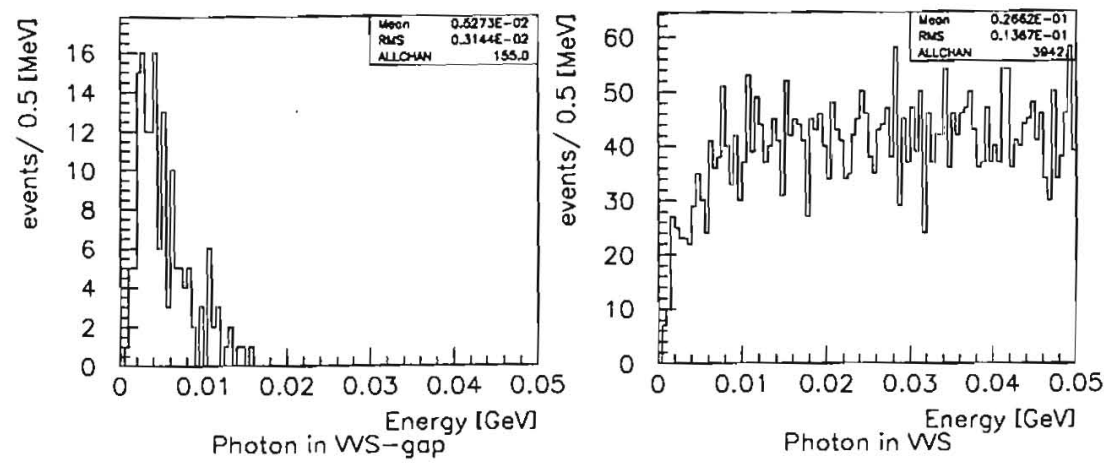

Figure 17: Energy distribution of the low energy photons in $K^{+} \rightarrow \pi^{+} \pi^{0}$ the VVS that intercept the gap and the active regions.

Although the forward veto systems don't share the challenge of detecting low energy photons, the FVS suffers from a "blind spot" near the charged pion that traverses it. In order to meet the stringent detection efficiency requirement for high energy photons, the FVS must be at least $18 X_{0}$ radiation lengths which corresponds to about one interaction length for the traversing pion. Hence most pions from $K^{+} \rightarrow \pi^{+} \pi^{0}$ (and $K^{+} \rightarrow \pi^{+} \nu \bar{\nu}$ ) decays begin showering in the FVS, thereby reducing the ability to distinguish photon clusters near the hadron shower. In order for a photon from $K^{+} \rightarrow \pi^{+} \pi^{0}$ decay to be near the associated pion it must have been emitted in the same direction as the pion in both the laboratory and center-of-mass frames. This constraint kinematically restricts the photon energy to be an almost monochromatic $1.6 \mathrm{GeV}$ when it lands near the pion as shown in figure 18 . This constraint is mildly smeared by the presence of the spectrometer magnetic field that bends the pion away from its original trajectory. This effect is included in figure 18.

We have studied the problem of maintaining both good acceptance for the charged pion and good rejection of nearby 1-2 GeV photons with GEANT shower simulations and KTeV data. These studies are presented in section 5.9.3, and allow us to conclude that we can identify 1-2 GeV photons with sufficient efficiency outside of a $10 \mathrm{~cm}$ radius from the charged pion while maintaining high signal acceptance. In this class of events, the other $K^{+} \rightarrow \pi^{+} \pi^{0}$ photon is always in the VVS with an energy greater than $1 \mathrm{GeV}$.

These considerations of the $K^{+} \rightarrow \pi^{+} \pi^{0}$ background as well as other backgrounds have led us to a photon veto system design based on lead-scintillator sampling detectors. There is substantial experience with the fine sampling ratio of $1 \mathrm{~mm}-\mathrm{Pb} / 5 \mathrm{~mm}$-Scintillator that is used in BNL experiments E787 and E949. In addition, this sampling ratio has been studied in detail with a variety of prototypes that will discussed in section 5.6.5. The VVS efficiency profile required to keep the $K^{+} \rightarrow \pi^{+} \pi^{0}$ background to a negligible level is shown in figure 19. The required efficiencies for all veto systems are listed in table 11. Superimposed on the VVS requirements in figure 19 are the results of full GEANT detector simulation of the low-energy and high-energy efficiencies, and the BNL-E787 measured inefficiency of this sampling ratio for intermediate energies of $20-200 \mathrm{MeV} / \mathrm{c}$. The details of these inefficiency studies are discussed in sections 5.6.

Folding the inefficiency profile of table 11 with the $K^{+} \rightarrow \pi^{+} \pi^{0}$ photon energy spectrum and $\pi^{0}$ kinematics leads to a global $\pi^{0}$ inefficiency of $1.6 \times 10^{-7}$. The classes of photon combinations that compose this residual inefficiency are: 

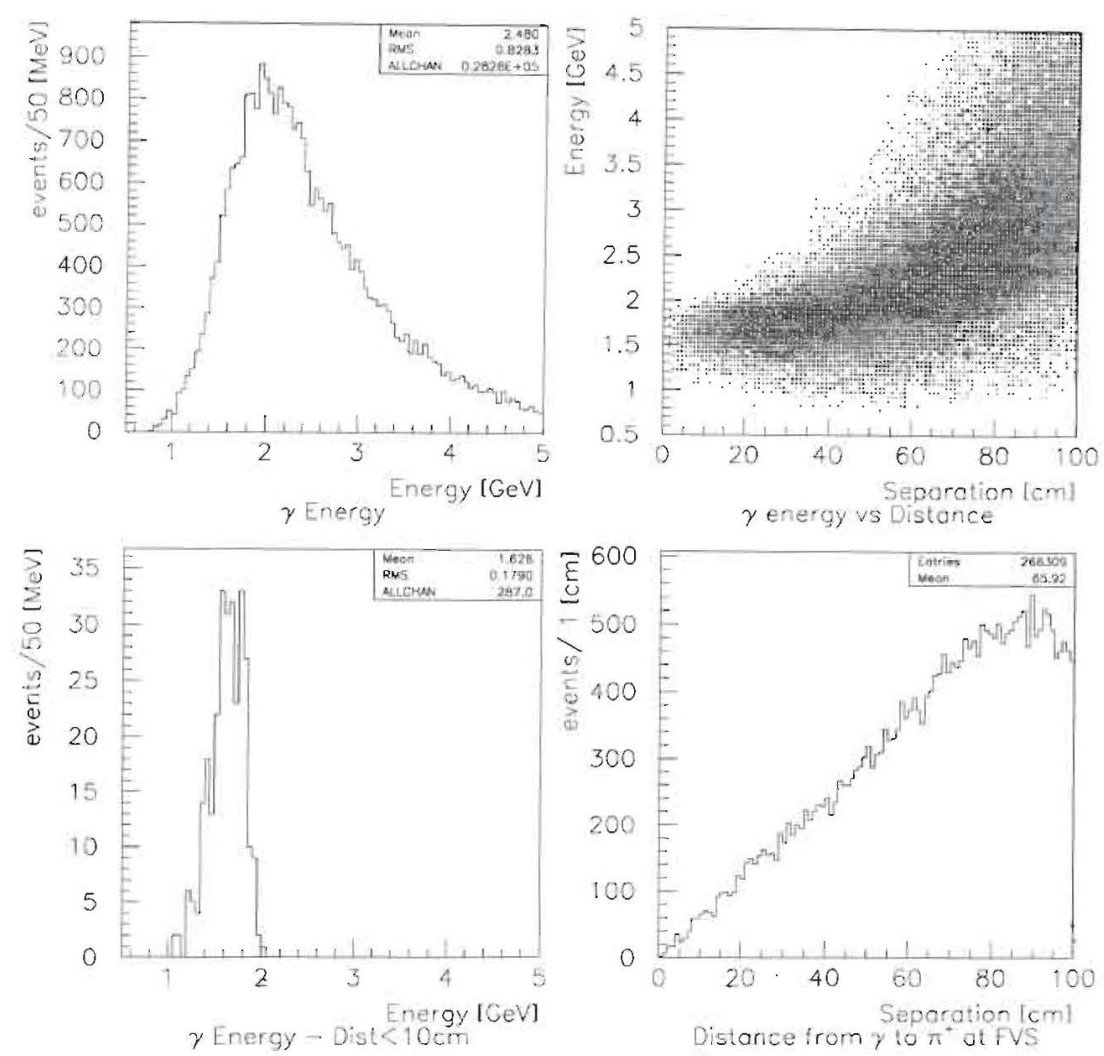

Figure 18: FVS distributions of the $E_{\gamma},\left(E_{\gamma} \cdot v s . r_{\pi \gamma}\right),\left(E_{\gamma}\right.$ with $\left.r_{\pi \gamma}<10 \mathrm{~cm}\right)$, and $r_{\pi \gamma}$.

\begin{tabular}{|r|r|r|r|}
\hline $\begin{array}{r}\text { Energy } \\
\text { Low-High (GeV) }\end{array}$ & $\begin{array}{r}(\mathrm{VVS}) \\
\text { Inefficiency }\end{array}$ & $\begin{array}{r}(\mathrm{FVS}) \\
\text { Inefficiency }\end{array}$ & $\begin{array}{r}(\mathrm{HVS}) \\
\text { Inefficiency }\end{array}$ \\
\hline \hline $0.0-0.02$ & $4 \times 10^{-1}$ & 1 & 1 \\
$0.02-0.04$ & $3 \times 10^{-2}$ & $5 \times 10^{-1}$ & 1 \\
$0.04-0.06$ & $3 \times 10^{-3}$ & $5 \times 10^{-2}$ & 1 \\
$0.06-0.10$ & $7 \times 10^{-4}$ & $5 \times 10^{-3}$ & 1 \\
$0.10-0.20$ & $4 \times 10^{-4}$ & $2 \times 10^{-3}$ & $2 \times 10^{-2}$ \\
$0.20-0.40$ & $1 \times 10^{-4}$ & $5 \times 10^{-4}$ & $5 \times 10^{-3}$ \\
$0.40-1.00$ & $1 \times 10^{-4}$ & $2 \times 10^{-4}$ & $2 \times 10^{-3}$ \\
$1.00-10.00$ & $3 \times 10^{-5}$ & $1 \times 10^{-4}$ & $2 \times 10^{-3}$ \\
\hline
\end{tabular}

Table 11: Required inefficiency performance of the CKM photon veto systems. 


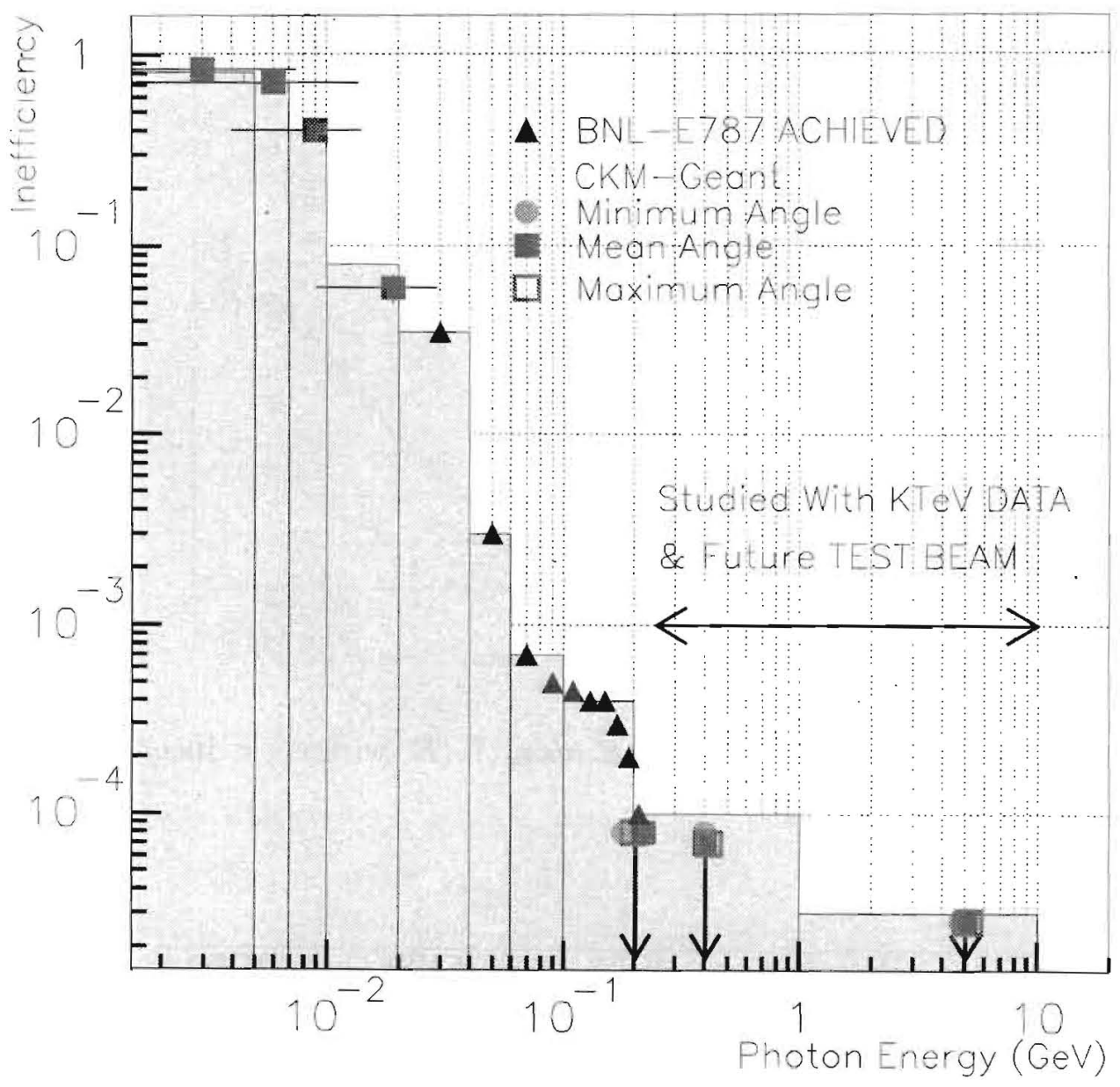

Figure 19: The histogram plots the required VVS inefficiency. Overlaid are VVS GEANT studies at low and high energy, and the medium energy efficiency inferred from the BNL-E787 data set as described in section 5.6.9. 
- $K^{+} \rightarrow \pi^{+} \pi^{0}$ Inefficiency Mechanisms:

- $5 \%$ (In VVS, $E>1 \mathrm{GeV}) \times$ (In FVS, nominal efficiency).

- 19\% (In VVS, $E>1 \mathrm{GeV}) \times($ In VVS-gap)

- 10\% (In VVS, $E>1 \mathrm{GeV}$ ) $\times$ (In VVS, nominal efficiency, $E>20 \mathrm{MeV}$ ).

- $36 \%$ (In VVS, $E>1 \mathrm{GeV}$ ) $\times$ (In VVS, nominal efficiency, $E<20 \mathrm{MeV}$ ).

- $30 \%$ (In VVS, $E>1 \mathrm{GeV}) \times\left(\mathrm{FVS} \pi^{+} / \gamma\right.$ fusion $)$.

This background classification allows the following conclusions regarding the $K^{+} \rightarrow \pi^{+} \pi^{0}$ background:

- This background is directly proportional to the VVS high energy inefficiency.

- The VVS filling fraction (50\%) is validated. Only $19 \%$ of this background comes from photons intercepting a VVS gap. The other photon by necessity is high energy, and most often in the VVS.

- The background combination from a photon in the VVS and a photon in the FVS more than $10 \mathrm{~cm}\left(\pi^{+} / \gamma\right.$ fusion radius) away from the $\pi^{+}$is small.

- This background is not very sensitive to the class of events where a photon lands near the charged pion in the FVS.

- The total background depends weakly on the VVS performance between $(20 \mathrm{MeV}-1000 \mathrm{MeV})$.

- Although the low energy VVS performance is important, the total background is not dominated by the low-energy $(<20 \mathrm{MeV})$ inefficiency. Only $36 \%$ of the background is proportional to the very low energy performance of the VVS system.

Most notable among these observations is that the $K^{+} \rightarrow \pi^{+} \pi^{0}$ background depends critically on the veto performance of high energy $(>1.0 \mathrm{GeV})$ photons. As will be discussed in section 5.6, the required inefficiency performance of $3 \times 10^{-5}$ in the VVS is well above loss mechanisms due to sampling fluctuations or photo-nuclear effects. The challenge of meeting this high energy performance will be driven by controlling inefficiencies due to undetected cracks, failures in the readout chain, and rate effects. This crucial issue of system hermeticity will be addressed in section 5.6.

\subsubsection{The Muon Veto System}

Muon rejection in CKM is critical to background rejection. Studies of potential backgrounds from $K^{+} \rightarrow \mu^{+} \nu$ and $K^{+} \rightarrow \mu^{+} \nu \gamma$ have shown that muon rejection of $1 \times 10^{-5}$ or better is needed for muons with momentum above $14 \mathrm{GeV}$. Our design goal for a muon veto system will exceed $1 \times 10^{-5}$ by a conservative safety factor.

The most common version of the problem of $\mu: \pi$ separation is that of trying to positively identify directly produced muons in an environment where pion decays contribute a background. This is the problem, for example, in a $K_{L}^{0} \rightarrow \mu^{ \pm} e^{\mp}$ search where decays of $K_{\epsilon 3}$ pions are a source of background events, or in a collider experiment attempting to use prompt muons to tag heavy quark decays. In these cases misidentification probabilities of a few percent are typical. In CKM the problem is the reverse. The requirement is to avoid misclassifying a muon as a pion. Fortunately, if a pion decays to a muon, that does not introduce background in CKM; rather it introduces a small inefficiency. 
It is possible to reject muons well below the $1 \times 10^{-5}$ level. The technique relies on the penetrating nature of muons versus pions. For example, a one meter thick iron absorber followed by a hodoscope can accomplish this task since most muons will penetrate and make signals in the hodoscope, while only a few pions will create such signals (via the punch-through of cascade particles from the pion's hadronic interaction). Those pions producing such false muon signals introduce an inefficiency, not background. The limitation on this technique is that not all muons penetrate the iron absorber. Some decay inside the absorber and others catastrophically loose a large fraction of their energy - most often but not exclusively via bremsstrahlung - and then range out in the absorber. These occurrence will fake the pion signature.

By longitudinally segmenting the absorber and instrumenting the layers, it is possible to improve the muon rejection considerably. Muon decays produce electrons which will typically induce observable electromagnetic showers. Most catastrophic energy losses will induce observable electromagnetic showers. Only a small fraction of muon interactions induce hadronic showers similar to those associated with pion interactions. That is, by standard techniques for discriminating between hadronic and electromagnetic showers, it is possible to enhance the muon rejection by a large factor. The ultimate limitation on this technique comes from the cross section for inelastic muon scattering on nuclei which creates hadronic showers.

The probability for a $14 \mathrm{GeV}$ muon to decay in 1 meter is $1 \times 10^{-5}$. The probability of an interaction can be estimated from the bremsstrahlung cross section, since bremsstrahlung is the dominant interaction process. It is useful to define the "inelasticity" $y$ of the muon interaction, $y=E_{\mu(\text { initial })}-E_{\mu(\text { final })} / E_{\mu \text { (initial) })}$. If the deptli of the absorber corresponds to $1 \mathrm{GeV}$ of energy loss (roughly one meter of iron), then a $14 \mathrm{GeV}$ muon must interact with $y>13.5 / 14=0.96$ (assuming it interacts half-way through the absorber) in order to be lost. The bremsstrahlung cross section as a function of $E_{\mu(\text { initial })}$ and $y$ is given by [115]:

$$
\frac{d \sigma}{d y}=\alpha Z^{2} r_{e}^{2}\left(\frac{m_{e}}{m_{\mu}}\right)^{2} \frac{4}{y}\left[1+(1-y)^{2}-\frac{2}{3}(1-y)\right]\left[\ln \left(\frac{2 E_{\mu(\text { initial })}}{m_{\mu}} \frac{\hbar}{m_{\mu} r_{n}} \frac{1-y}{y}\right)-\frac{1}{2}\right],
$$

where $r_{e}$ is the classical radius of the electron and $r_{n}=0.49 r_{e} A^{1 / 3}$. From this we can estimate the probability of a muon bremsstrahlung with $y>0.96$ in $1 \mathrm{~m}$ of iron to be about $7 \times 10^{-6}$.

We conclude therefore that the muon rejection of a simple $1 \mathrm{~m}$ iron absorber followed by a hodoscope is about $2 \times 10^{-5}$. This is not as good as CKM needs, but can be improved upon by having an active absorber with multiple hodoscope layers (every 2-4 $X_{0}$ ) rather than a passive one, so that the characteristic shape of electromagnetic showers created by these muons can be observed. The irreducible misidentification comes from muon interactions which produce hadrons and consequently hadronic showers. This process is more than an order of magnitude smaller than bremsstrahlung, so that muon rejection better than $1 \times 10^{-6}$ should be possible.

In view of this discussion, the CKM muon veto system should consist of a relatively short range stack instrumented longitudinally to provide shower shape information. Transverse segmentation will be driven by rate considerations. A traditional arrangement of iron and scintillator would be suitable, for example, provided it is free of cracks and dead material that could permit energy deposits to go unobserved. An additional complication is provided by the forward photon veto system which is immediately upstream of the muon veto system. The photon veto is where the pions (or muons) will first see significant material and begin to interact. The gap between these detectors must be small (to minimize shower blooming and muon decays) and the gap should not contain passive material which could absorb particles. These constraints must be met in the design of both systems. 


\subsection{Stopped versus In-flight Kaon Decays}

All prior searches for $K^{+} \rightarrow \pi^{+} \nu \bar{\nu}$ have utilized stopped-K's [116]. We are aware of a few instances of in-flight experiments being considered in the past, but before the 1989 Main Injector Workshop report [117] nothing seems to have been written down. A comparison of the two methods is not simple, since some subjective factors (such as how much weight to put on the accumulated experience using the stopped- $K$ method) enter in. Nonetheless, it is an interesting question which has been discussed within the experimental kaon community for many years. Two developinents make the question particularly timely today: (1) the Main Injector will be able to provide intense $K$ beams during Collider and MINOS operation and (2) ring-imaging Cherenkov counters (RICHs) have reached a maturity that makes them viable as a core component of an experiment. Fortuitously, typical energies of secondary kaons from Main Injector beams are suitable for RICH detectors.

Ultimately the comparison between a stopped- $K$ and in-flight $K^{+} \rightarrow \pi^{+} \nu \bar{\nu}$ experiment depends on achievable sensitivity and background rejection. We believe an in-flight experiment at the Main Injector has advantages on both scores, but suffers from the possible criticism that, lacking prior experience, there may be unanticipated problems and/or a significant learning curve to surmount. While such criticism can be leveled against any new approach, it should be kept in mind that most kaon experiments have been conducted in-flight, so there is a huge body of experience with the ill-flight technique (including rare decay experiments down to near $1 \times 10^{-12}$ for some modes).

The branching ratio sensitivity of a rare $K$ decay experiment is simply the reciprocal of the number of $K$ decays which occur during the experiment multiplied by the acceptance for the decay of interest. As described elsewhere in this proposal, CKM will utilize about $5 \times 10^{12}$ protons per spill (about $15 \%$ of the Main Injector) to produce a $K^{+}$beam of $3 \times 10^{7} K^{+}$per spill, of which about $17 \%$ will decay in our decay volume. For a $1.6 \%$ acceptance, the branching ratio sensitivity is $1.2 \times 10^{-5}$ per spill. It is reasonable to expect $1.4 \times 10^{5}$ Main Injector spills per week (120 hours with $3 \mathrm{sec}$ cycle time). Thus, CKM should achieve a sensitivity of about $8.5 \times 10^{-11}$ per week. In 2 years of running, at 39 weeks per year, CKM would acquire 95 signal events corresponding to a sensitivity of $1 \times 10^{-12}$. While 2 years is a long run for a fixed-target experiment,this running can take place in parallel with the MINOS and Collider program at low incremental cost.

To put this proposal in current context, the BNL E787 experiment has reported discovery of $K^{+} \rightarrow \pi^{+} \nu \bar{\nu}$ through the observation of one event with a corresponding branching fraction of $\left(15_{-12}^{+34} \times 10^{-11}\right)[6]$. Ongoing analysis of the remaining E787 data set will increase the sensitivity of this measurement by a factor of $\times(2-3)$. Since submission of the first edition of the CKM proposal an extension of the $K^{+} \rightarrow \pi^{+} \nu \bar{\nu}$ program at BNL has been approved based on an upgraded E787 apparatus. This new experiment is E949, which is currently taking commissioning bearn with first production running scheduled for the summer of 2001. The approved running schedule of BNL E949 with the attendant detector upgrades will increase the final sensitivity of E787 by a factor of $\times(5-10)$ to a final sensitivity of $1 \times 10^{-11}$.

Another important development since the original submission of the CKM proposal is that ten physicists from BNL, Fermilab, and IHEP/Protvino now collaborate on both E949 and CKM. Access to the integrated experience of BNL-E787 and the direct experience of E949 will be invaluable to the final design of CKM that aims at a sensitivity of $1 \times 10^{-12}$.

Ultimately though, sensitivity will be made irrelevant if backgrounds prevail. The most important message for CKM from the landmark E787 measurement is that backgrounds can convincingly be controlled to the $1 \times 10^{-11}$ at least somewhere. There are several aspects to a comparison of background rejection in the stopped- $K$ versus in-flight approaches. We will discuss in turn redundancy, kinematic resolution, particle identification, photon vetoes, and strategies for measuring backgrounds with data. 
An aspect of BNL E787/E949 which is appropriately emphasized is redundancy. In particular, the $\pi^{+}$is measured three times: momentum, energy, and range. The momentum is measured by trang in a magnetic field. The energy is measured by pulse height in an active (scintillator) range ste $k$. which also provides a range. Comparison of the three measurements improves background rejection and relaxing cuts on one can help to understand the effect of cuts on the others. The analogous three quantities in CKM are momentum, velocity, and shower energy. Momentum of both beam particles and charged decay products will be measured by tracking through magnets. Velocities will be measured by RICH's. The shower energy for the charged decay products will be measured in the muon veto system, which is really a calorimeter. We formulate this as a parallel with E787 in these terms simply to make the point that CKM has the same high degree of redundancy in similar quantities.

More important is the fact that CKM will make redundant independent measurements of both the incoming beam particle and outgoing putative $\pi^{+}$. The "momentum spectrometer" and the "velocity spectrometer" use completely independent hardware to measure vector quantities (magnitude and direction) for both the incoming and outgoing particles. For a given mass hypothesis the momentum and velocity vectors can be compared for consistency. Both measurements will have similar experimental resolutions and each spectrometer separately provides all the information needed to calculate the missing mass in the $K^{+}$decay.

Kinematic resolution is important in a $K^{+} \rightarrow \pi^{+} \nu \bar{\nu}$ experiment since one wants to reject the two-body backgrounds kinematically. For stopped- $K$ 's this is conceptually simple since the observed charged particles have fixed momentum. Of course, there is no fundamental advantage in the fact that the center-of-mass frame and the laboratory frame are the same. As a practical matter, the stopped- $K$ technique has one clear advantage - the kaon's momentum is known to effectively perfect precision. The value of this information depends on whether other measurements are of sufficient precision to fully exploit it. BNL E787/E949 has about a $2.5 \mathrm{MeV}$ momentum resolution of the $\pi^{+}$from $K^{+} \rightarrow \pi^{+} \pi^{0}$ (out of $205 \mathrm{MeV}$ for the momentum). The energy and range measurements are less precise in relative terms. In CKM we will reconstruct the missing mass $M_{\text {miss }}$ as discussed earlier. For a given value of $M_{\text {miss }}$ in any $K^{+} \rightarrow \pi^{+} X$ decay, the center-of-mass momentum $p^{*}$ of the $\pi^{+}$is fixed. Thus, we can compare CKM to E787 by simulating CKM in a Monte Carlo and determining how our expected $p^{*}$ resolution compares to that achieved in E787. This includes the uncertainty in our determination of the center-of-mass frame from our $K^{+}$resolution. Using the RICHs alone, we expect a $2.9 \mathrm{MeV}$ resolution. Using the tracking (momentum) spectrometers, we likewise expect $2.6 \mathrm{MeV}$ resolution. The combined resolution using both sets of measurements is $1.9 \mathrm{MeV}$. The Gaussian resolution, however, is not the whole story, since non-Gaussian tails are the more likely source of background. The tails generally result from scattering, but to the extent that they are due to detector mismeasurement, CKM has an advantage of comparing two measurements with similar intrinsic errors.

Particle identification, in particular avoiding misidentification of a muon as a pion, is very good in both the stopped- $K$ and in-flight situations. In the former the technique is to follow the entire decay chain $\pi \rightarrow \mu \rightarrow e$. In CKM both the RICH and the muon veto system independently provide powerful muon rejection. In the $\mathrm{RICH}$, for example, the ring radii for $20 \mathrm{GeV}$ (the highest momentum of interest) $\pi^{+}$'s and $\mu^{+}$'s differ by $11.6 \sigma$.

Following the full $\pi \rightarrow \mu \rightarrow e$ decay chain, of course, takes time - a few microseconds to be efficient - while the RICH and muon veto time scales are at most a few nanloseconds.

Key to a $K^{+} \rightarrow \pi^{+} \nu \bar{\nu}$ experiment is powerful photon vetoes, since $K^{+} \rightarrow \pi^{+} \pi^{0}$ and $K^{+} \rightarrow \mu^{+} \nu_{\mu} \gamma$ must be heavily suppressed based on the photon(s) in the decays. Higher photon energies favor better rejection. However, there is one caveat. A $K^{+} \rightarrow \pi^{+} \pi^{0}$ decay at rest never results in a photon below $20 \mathrm{MeV}$. In contrast, $K^{+} \rightarrow \pi^{+} \pi^{0^{*}}$ decays in-flight (for $22 \mathrm{GeV} K^{+}$'s) result in pho- 
tons below $20 \mathrm{MeV}$ (and all the way down to zero) in about $0.1 \%$ of decays. Fortunately, since CKM will impose a minimum $\left(\theta\left(K^{+}, \pi^{+}\right)>2.5 \mathrm{mRad}\right)$ decay angle cut to ensure a well measured vertex within the decay volume, the other photon in such asymmetric $K^{+} \rightarrow \pi^{+} \pi^{0}$ decays must have energy greater than $2 \mathrm{GeV}$. Such high energy photons are relatively easy to veto, at least in principle. While the subject of photon vetoes is a very involved one, higher energies seem to be a net benefit and we believe $\pi^{0}$ rejection better $2 \times 10^{-7}$ is achievable. This case is presented in detail within this proposal. BNL E787 has achieved $\sim 6 \times 10^{-7}$ in practice in the stopped- $K$ case, which is limited principally by photonuclear reactions which are significant at low energies.

Another important issue is how backgrounds can be measured in the two approaches. To the extent that scattering or interactions in matter are a source of background, the in-flight method has a fundarnental advantage. In the case of stopped- $K$, it is necessary for the incoming $K^{+}$to pass through enough material to stop it, an amount that depends on the $K^{+}$beam energy. In the in-flight case, initially one minimizes the amount of matter traversed by the particles. Of course there is some practical lower limit which sets the level at which the background processes will occur. The advantage of the in-flight technique is that material can be added to the paths of the particles in a controlled way so that the backgrounds can be measured directly as a function of the amount of material. Since one cannot change the amount of material that stops a given $K^{+}$, we believe this is a major advantage of the in-flight technique.

The alternative, of course, as convincingly demonstrated by E787 to the $1 \times 10^{-11}$ level is to study backgrounds by varying cuts. These techniques have the advantage of minimal reliance on Monte Carlo simulation of backgrounds. The redundant set of measurement tools available to both E787/E949 and CKM enable such a "data-based" analysis strategy. To the extent that different cuts reject the same background, one can estimate the background level by relaxing cuts in an astute way, determining the rejection of each cut separately, and then combining the separate rejections into a final background estimate. Key to this strategy is the demonstration that an orthogonal set' of cuts can be established. A striking example of such orthogonality within the E787 analysis is evident in figure 147, which demonstrates measurement of $p_{\pi^{+}}$in stopped $K^{+} \rightarrow \pi^{+} \pi^{0}$ decays is independent of vetoing the associated $\pi^{0}$ to the $6 \times 10^{-7}$ level. These data based analysis strategies will be employed in CKM as well.

In summary, considerable experience exists with the stopped- $K$ approach, and BNL-E949 is now ramping up to fully exploit the apparatus to reach the $1 \times 10^{-11}$ level of sensitivity. Our estimate is that CKM using the in-flight technique at the Main Injector can achieve a sensitivity of $1 \times 10^{-12}$ with a reasonable amount of running time. Backgrounds are the real issue. Clearly, CKM must have background rejection commensurate with this sensitivity. We discuss background estimates in detail elsewhere in this proposal. Our conclusion is that the in-flight technique has several potential advantages. These include powerful muon rejection at high rates, better photon rejection, and the improved ability to measure interaction backgrounds. 


\section{The Separated Charged Kaon Beam}

\subsection{Design Motivations for the CKM Beam}

\subsubsection{CKM Beam Requirements}

CKM requires a $K^{+}$beam of $30 \mathrm{MHz}$ to reach the desired sensitivity. For background rejection each beam particle must have its identity, momentum, and trajectory measured. Therefore to minimize the required rate in detectors operating in the beam, it is vital that the beam consist of primarily $K^{+}$. CKM has set a goal of a total charged particle rate of $50 \mathrm{MHz}$ in order to limit beam related accidental background sources. The design incorporates a safety factor of two or a maximum rate of $100 \mathrm{MHz}$. The beam energy is dictated by the use of ring-imaging Cherenkov counters and the gases available which operate near atmospheric pressures. Further discussions of small variations about this design energy are contained in the CKM internal memo [119]. At $22 \mathrm{GeV} / \mathrm{c}$ the required purity can only be achieved via RF separation. We have a design that can provide $30 \mathrm{MHz}$ of $\sim 22 \mathrm{GeV}$ $K^{+}$with an estimated contamination roughly $30 \%$ with $4 \times 10^{12}$ protons per spill incident on the production target. The beam divergence envelope is approximately $\pm 500 \mu \mathrm{rad}$ and the momentum bite is about $\pm 2 \%$ to match the size and properties of the spectrometer.

\subsubsection{Design Motivations for a Debunched Beam}

In this high rate environment it is advantageous to minimize the instantaneous rate by debunching the $53 \mathrm{MHz}$ beam structure. Some of the time variation of the beam must remain in order for the RF pickups to measure beam positions. The debunching of the beam was discussed with the Main Injector accelerator experts at a workshop on fixed target physics at the Main Injector [120]. Two different schemes to minimize the RF structure were discussed [121]. These RF manipulations may add $10 \%$ to the cycle time and care must be taken to avoid beam loading effects in the cavities which maintain the RF structure.

\subsubsection{Selection of $K^{+}$beam and Negative Beam Options}

The motivation for running $K^{+}$beam rather than $K^{-}$is proton economics. Estimates from several sources indicate that the $K^{+}$yield for $120 \mathrm{GeV} / c$ incident protons is about twice the $K^{-}$ yield $[122-124]$.

Still, the possibility of reducing the non-K component by simply running with a negative beam is attractive. At production using the Malensek [124] parameterization the $p^{-} / K^{-}$ratio is $\sim 0.3$, while the $p^{+} / K^{+}$ratio is $\sim 3.0$ (forward production, $7 \mathrm{mrad}$ angular cone, momentum $22 \mathrm{GeV}$ $\pm 2 \%$ ). For the present design the hadronic purity (ratio of $K^{+}$to all hadrons) of $\sim 80 \%$ with positives is improved to $\sim 90 \%$ with negative polarity. Given the relatively small additional expense of reversing switches on the beam line magnets and designing the beam dump to work with either case, this remains a possible option. Additionally, running with a $K^{-}$beam could permit some additional physics results, as described in section 2.4.3.

\subsubsection{Comparison to other RF separated beams}

The use of RF to separate particles is not new. An excellent review of the early activity in the field is given by P. Bernard [125]. Some of the original work we found are proposals by Panofsky [126], Montague [127], and Schnell [128] for two-cavity schemes. In 1965 two-cavity RF separated beams were in use at the AGS [129] and CERN [130]. At CERN in 1967 a 3-cavity separator system was successfully in operation [131]. This $19 \mathrm{GeV} K^{+}$beam line (U3) achieved a particle enhancement 
(desired species/background) of $\times 700$. The observed ratio of desired particles to all particles was $80 \%$. The background particles were $15-20 \%$ muon and $3-5 \%$ hadrons. The inter-cavity distance was $50 \mathrm{~m}$, and the cavities operated at $2.9 \mathrm{GHz}$ (S-band). However the pulse length of the RF separator did not exceed 8 microseconds, restricting the separation to very low duty-factor beams. The very high rate nature of the CKM experiment requires the relatively large duty factor of the Main Injector $(30 \%)$. The 1-second long Main Injector spill requires to the use of superconducting $\mathrm{RF}$ separators from power consumption considerations.

At present there are no high energy RF separated beams in operation. In addition to warm RF separator beams that operated in the past, a superconducting separator beam was developed and commissioned at the CERN laboratory during the late 1970's [132]. There a two-cavity scheme was operated at S-band with an $83 \mathrm{~m}$ inter-cavity distance. The design goal for the these cavities was $2 \mathrm{MV} / \mathrm{m}$, they achieved values of $1.2-1.4 \mathrm{MV} / \mathrm{m}$. The enhancement factor observed for $\mathrm{K}^{-} / \pi^{-}$ was $\times 31$ at $16 \mathrm{GeV} / c$.

The CERN cavities for that beam were preserved, and presently are components of the U-70 RF separated beam project under construction at IHEP, Protvino, Russian Federation. The IHEP collaboration is building a high energy (12-18) GeV, high intensity $\left(\sim 5 \times 10^{6} /\right.$ spill $)$ separated beam of $\mathrm{K}$ particles per $\sim 1 \times 10^{13}$ protons/spill. We mention this work for completeness in this document, and anticipate similarities to the CKM work. The original proponents of a superconducting RF separated beam at Fermilab have exchanged information and calculations with the builders of the U-70 beam at Protvino. These are the only two superconducting RF separated beams projects currently under development. CKM will benefit from the operational experience of the U-70 RF separated beam at Protvino, where first experiments are scheduled for the end of 2002.

\subsection{Separated Beam Design}

\subsubsection{How RF Separation Works}

Extensive literature exists regarding the design and operation of RF separated beamlines $[125,132$, 133]. This section will present a basic explanation of a two-cavity system.

Conceptually, the technique is quite simple: different species of particles, having the same momentum, will have different speeds; the time-of-flight between two deflecting cavities will be different for different species; thus, the distance between cavities may be chosen so that the unwanted particle receives no net deflection (and is absorbed by a beam stopper) while the wanted particle receives a time-varying deflection (and a portion of them miss the beam-stopper). This is illustrated in Figure 20.

Given the RF cavities are operating at some frequency, $f$ the distance between the two cavities is $L$, and the speed of the particle is $v$, the time-of-flight $L / v$, and the number of RF cycles elapsed is $f L / v$. For two particles traveling at different speeds, the difference in $\mathrm{RF}$ cycles is:

$$
\frac{L f}{v_{2}}-\frac{L f}{v_{1}}=L f \frac{v_{1}-v_{2}}{v_{2} v_{1}}
$$

or, equally, the difference in phase is:

$$
\Delta \phi=2 \pi L f \frac{\Delta v}{v_{2} v_{1}}
$$

\subsubsection{Overview of the Separated Kaon Beam Design}

The design of this beam was evolved from a design proposed by Jaap Dornbos [133]. Conceptually, the beamline consists of three stages: 


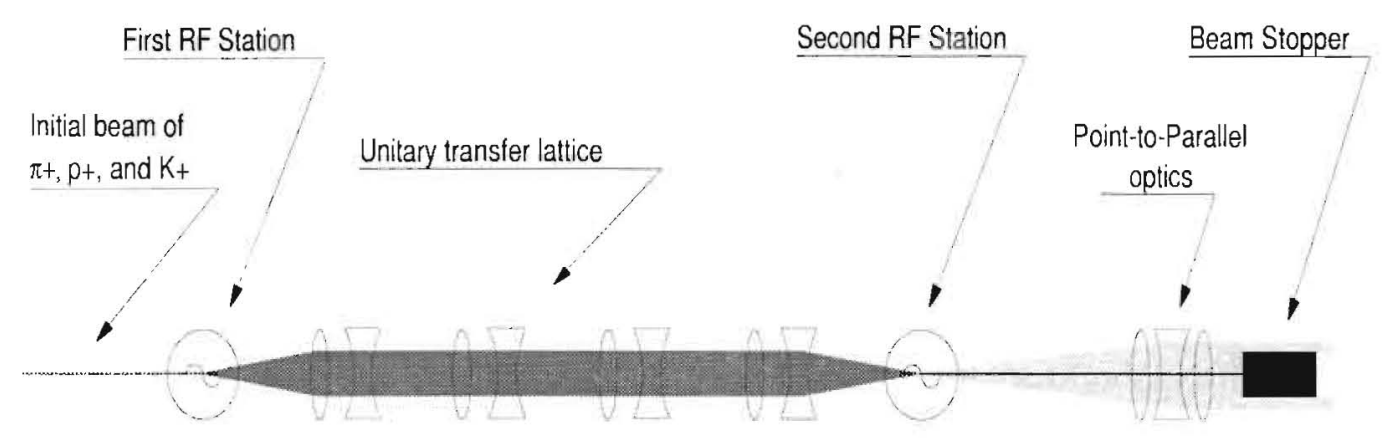

Due to their differing velocities,

$\pi+, p+$ and $K_{+}$"slip" in phase

by different amount.

\begin{abstract}
$\pi+$ and $p+$ arrive 180 degrees out of phase with respect to first RF station and receive no net deflection.

K+ arrive $\sim 93$ degrees out of phase with respect to first RF station and receive time-varying deflection.
\end{abstract}

Figure 20: Layout of the beam line.

1. Collection and preparation for RF separation.

2. RF separation.

3. Preparation for the experiment.

Figure 21 is a schematic of the beamline. Stage one extends from the target to Q32. Stage two extends from RF1 to the beam stopper. Staige three extends from B5 to BQ7.

\title{
4.2.3 Stage-1: Collection and Preparation for RF Separation
}

The first stage (see figure 21) consists of a large aperture, asymmetric triplet (Q11-13); a chicane (B1-B4), in which the particles are dispersed, momentum selected, and recombined; and final shaping (Q31-32) for the first RF station.

The triplet is composed of "5Q36" quadrupoles. Each quadrupole is 36 inches long and has a 5 inch aperture. At $22 \mathrm{GeV} / \mathrm{c}$, and $0.75 \mathrm{~mm}$ spot, the quadrupoles will accept 9.6 mrad horizontally and $6.6 \mathrm{mrad}$ vertically.

The chicane is composed of four bend points, each bend point consisting of two "BM105" magnets. The BM105 is 94 inches long, and has a 6 inch gap and 18 inch width. Each magnet bends the beam $35.6 \mathrm{mrad}$. The bending scheme results in maximum dispersion between $\mathrm{B} 2$ and $\mathrm{B} 3$, and momentum recombination after B4. There is sufficient space between B1 and B2 for a primary beam dump. The optics are such that there is a horizontal waist between B2 and B3; because this is also the point of maximum dispersion, momentum selection takes place here.

The beam is oriented for RF1 by Q31 and Q32. 


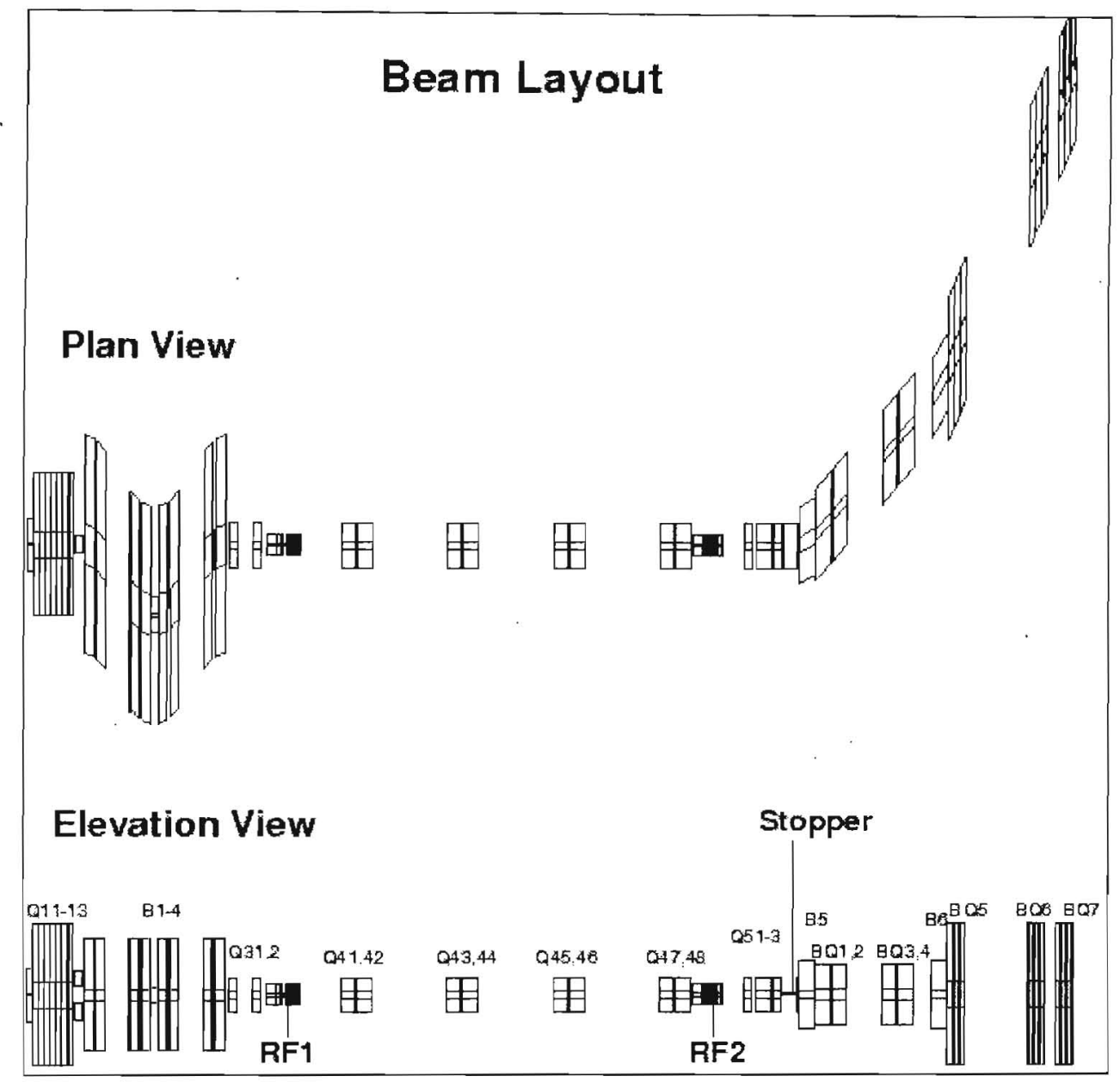

Figure 21: Layout of the beam line. 


\subsubsection{Stage-2: RF Separation}

The separation mechanism relies on the time-of-flight difference between pions, protons, and kaons. The RF stations run at $3.9 \mathrm{GHz}$ and are separated by $86 \mathrm{~m}$. The time-of-flight difference between a $22 \mathrm{GeV} / c$ pion and proton, traveling this distance, is $257 \mathrm{psec}$, or one RF period. However, the time-of-flight difference between a pion and kaon is $67 \mathrm{psec}$, or 93.8 degrees. The F0D0 channel formed by the Q41-48 quadrupole string provide a unitary transformation between the two RF stations. Therefore, if RF2 is run 180 degrees out of phase with respect to RF1, the kick received at RF2 will cancel the kick received at RF1 for pions and protons, but will double the kick received at RF1 by the kaons; thus, upon exiting RF2, the kaons have a net deflection, but the pions and protons do not.

The quadrupole triplet Q51-Q53 transforms this angle into a position at the beam stopper. Pions and protons are dumped in the bearn stopper and a large fraction of the kaons pass around the stopper - refer to figure 22 (and note the log scale).

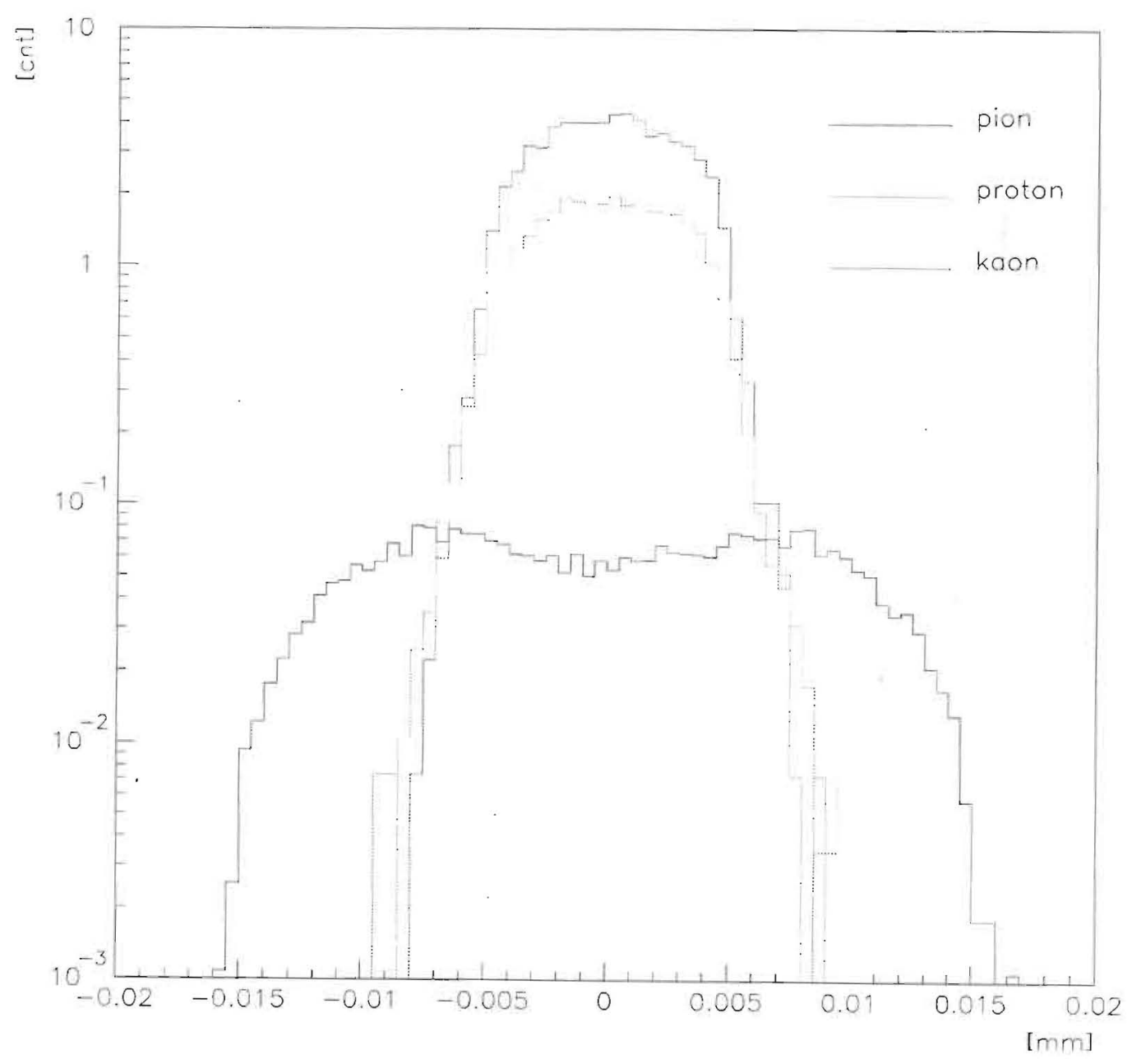

Figure 22: Vertical beam spot at the beam stopper. 
It is interesting to note that the resulting kaon beam has an associated time structure at the $3.9 \mathrm{GHz} \mathrm{RF}$ frequency, which is beneath the time resolution of the CKM detector.

\subsubsection{Stage-3: Transport to the Experiment}

Bending magnets B5 and B6 serve as a clean-up bend for the stopper. The $110 \mathrm{mrad}$ (total) bend provides enough momentum selection and displacement to reduce the number of muons reaching the experiment significantly (see section 4.4.3). Quadrupole string BQ1-4 gives a -1 transformation between the two bends, allowing the beam to remain achromatic.

The final triplet, BQ5-7, orients the beam correctly for the experiment. Plots of the size, phase space, and dispersion, of the kaon beam are shown in Figures 23-25.
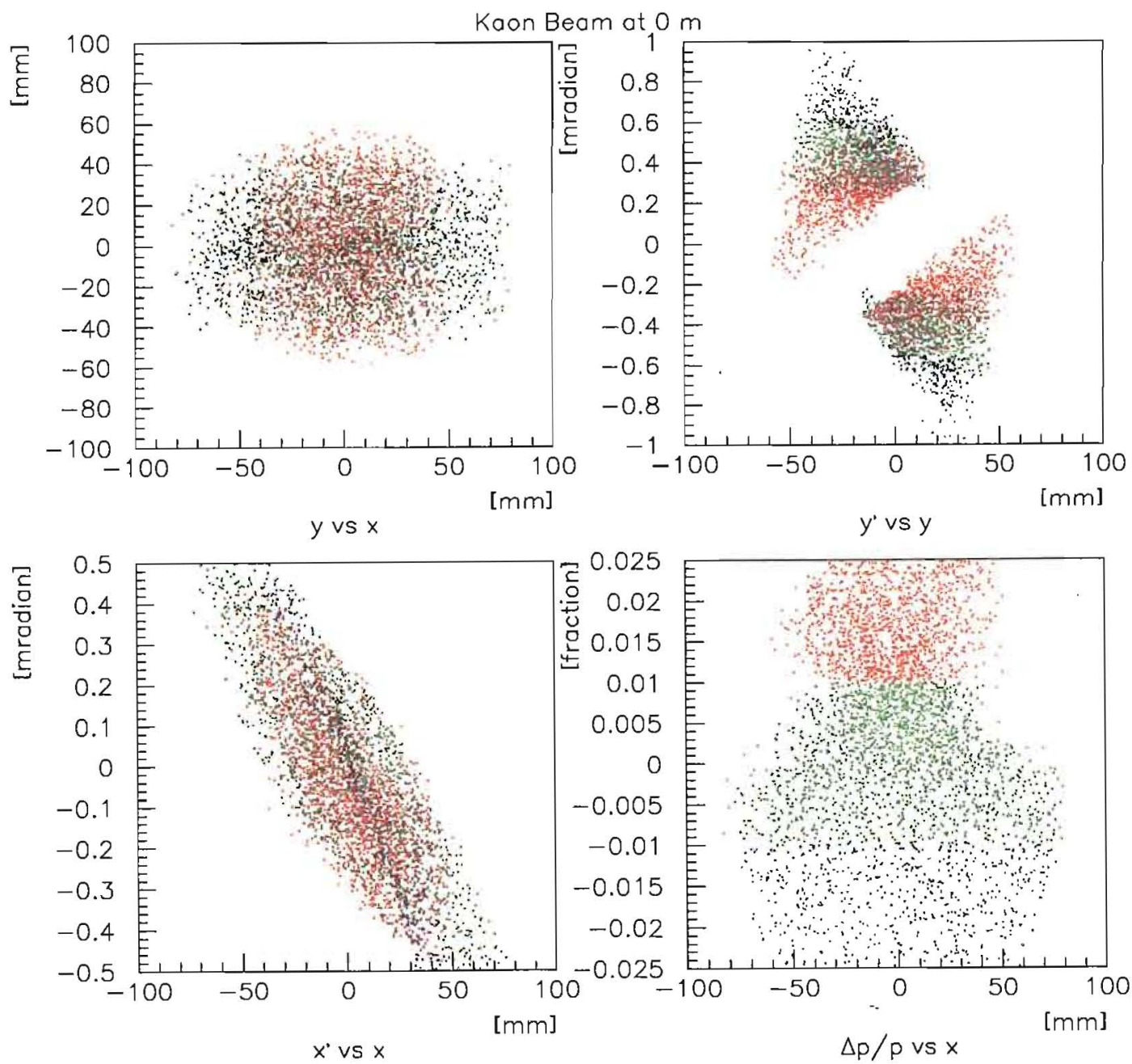

Figure 23: Kaon distribution at $0 \mathrm{~m}$ in detector coordinates(see figure $42 \mathrm{CKM}$ Detector). Kaons are colored according to momentum bite (blue $<-0.01$, green -0.01 to 0.01 , red $>0.01$ ) 

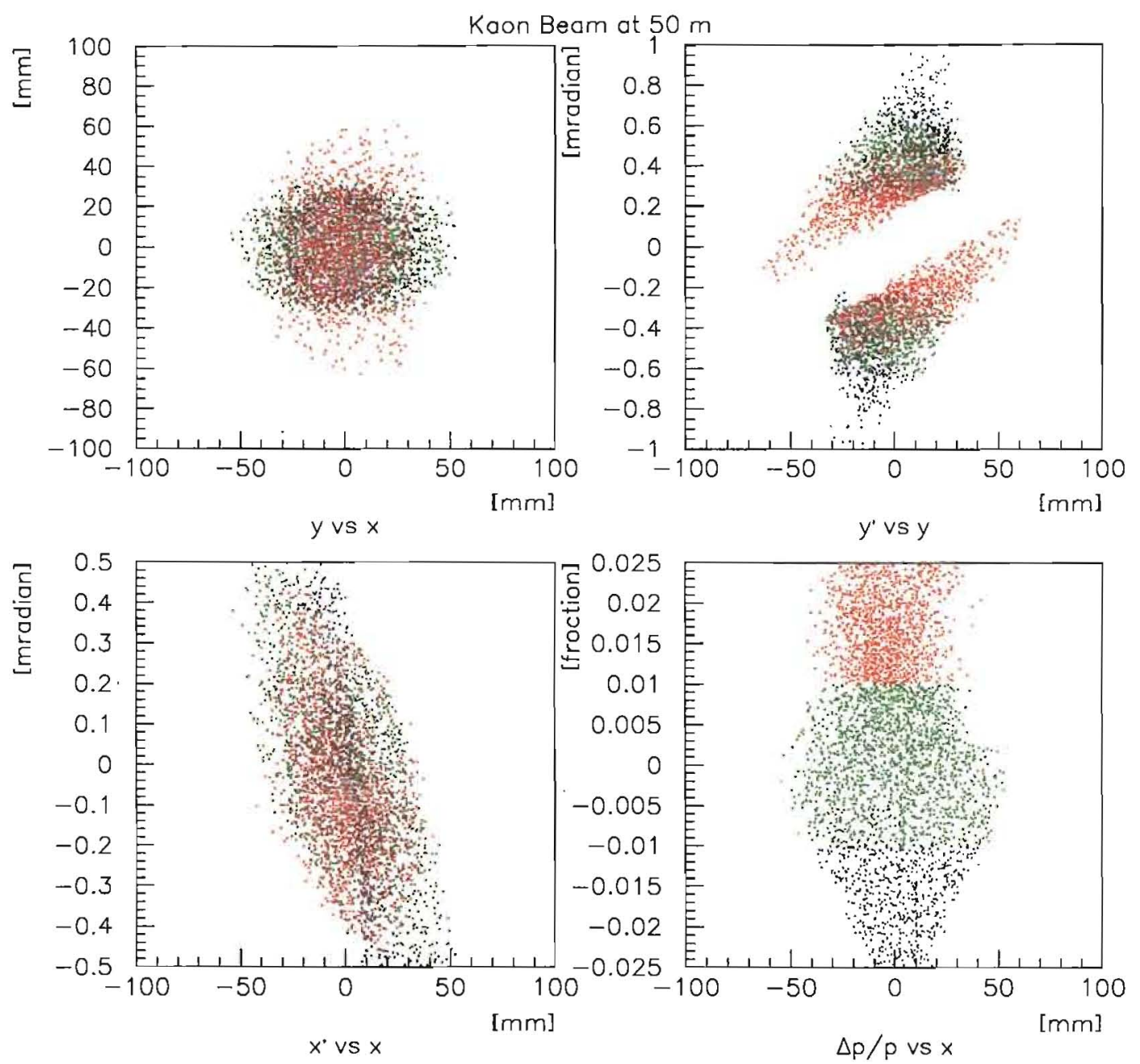

Figure 24: Kaon distribution at $50 \mathrm{~m}$ in detector coordinates (see figure $42 \mathrm{CKM}$ Detector). Kaons are colored according to momentum bite (blue $<-0.01$, green -0.01 to 0.01 , red $>0.01$ ). 

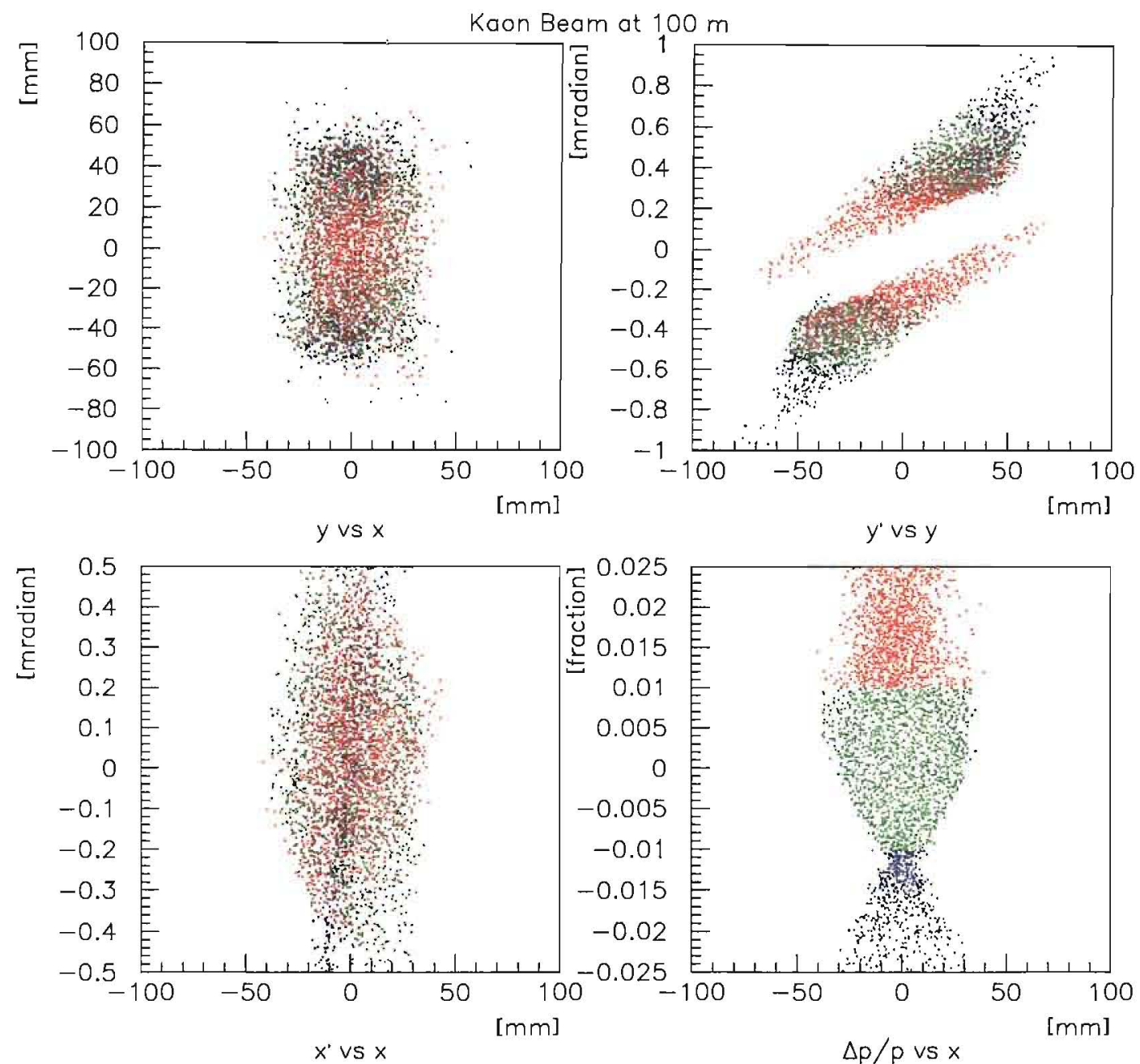

Figure 25: Kaon distribution at station $100 \mathrm{~m}$ in detector coordinates(see figure $42 \mathrm{CKM}$ Detector). Kaons are colored according to momentum bite(blue $<-0.01$, green $=0.01$ to 0.01 , red $>0.01$ ). 


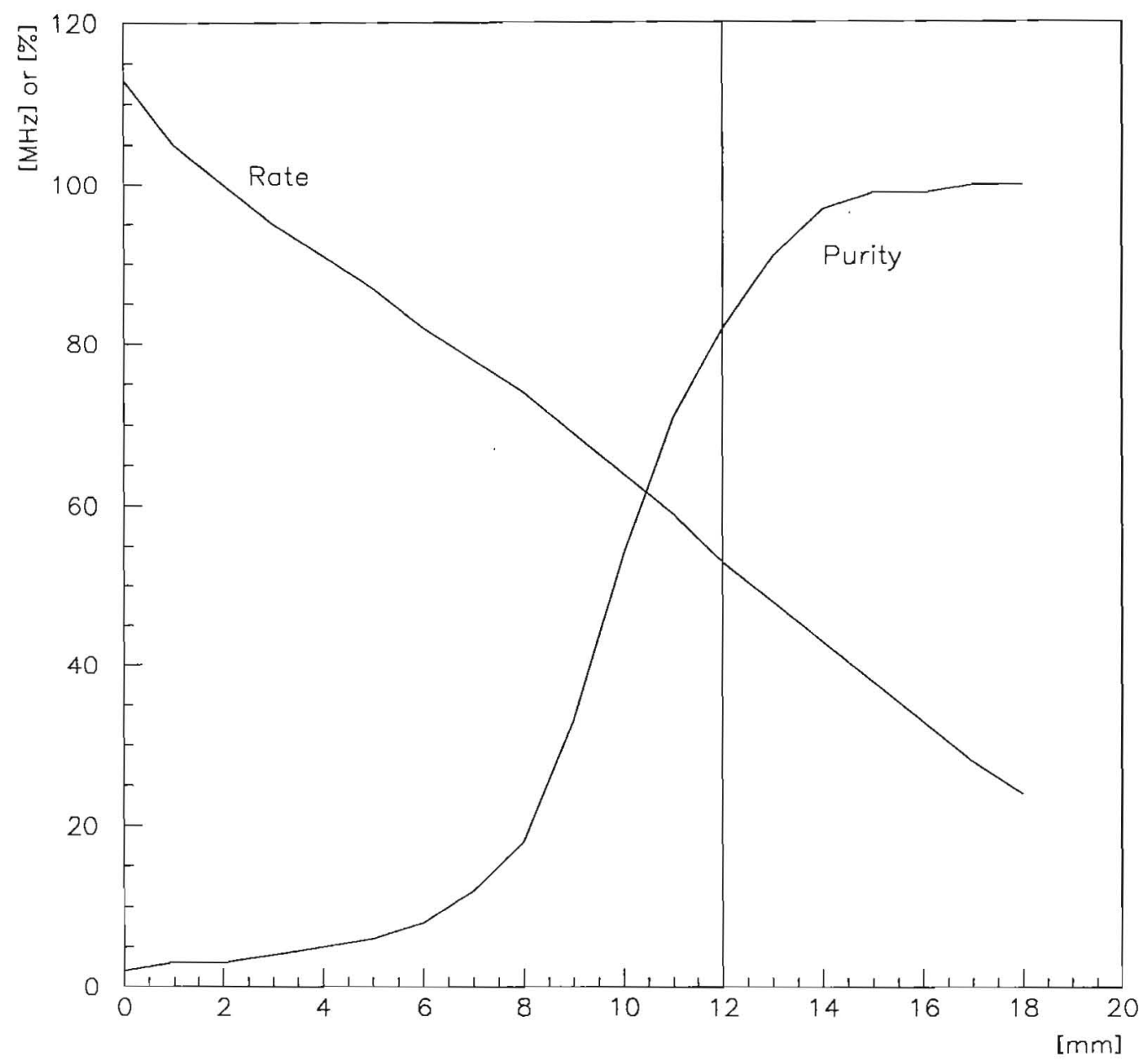

Figure 26: Kaon rate $(\mathrm{MHz})$ (at the exit of the last quadrupole for $5 \times 10^{12}$ protons per pulse) and purity $(\%)$, as a function of beam stopper height $(\mathrm{mm})$. There are two regions where the purity is roughly independent of stopper height, and a transition region where the dependence is roughly linear. The kaon rate is roughly linear with stopper height. The vertical line indicates the $12 \mathrm{~mm}$ stopper width in the standard design. 


\subsubsection{Beam Purity}

Of critical importance is the size of the beam stopper. Figure 26 shows the kaon rate and the beam purity as a function of the vertical size of the beam stop. The rate falls roughly linearly with the size of the beam stop, while the purity shows a shoulder. This can be understood by looking at Figure 22, which shows the kaons more uniformly distributed than the pions and protons. A 12 $\mathrm{mm}$ width beam stopper results in $53 \mathrm{MHz} K^{+}$rate $5 \times 10^{12}$ protons per pulse and $82 \%$ hadronic purity. This exceeds the design specifications.

The envelope and aperture plot of the beamline are shown in figure 27). The curves represent the two standard deviation beam envelope.
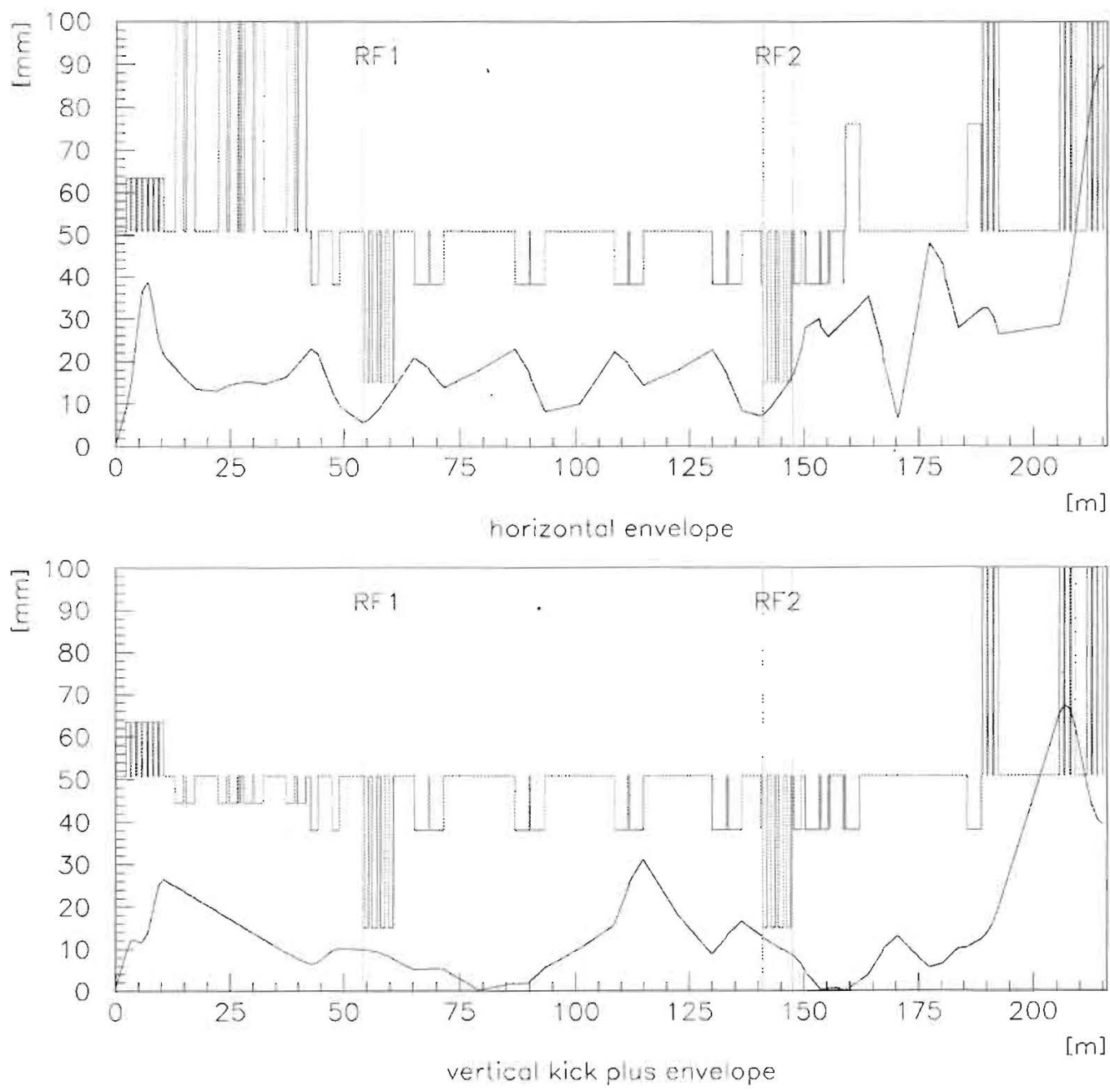

Figure 27: Beam envelopes. The apertures of the beam line elements are indicated. The curves represent the two standard deviation beam envelope. 


\subsubsection{Shielding}

A preliminary analysis of shielding for the CKM target pile [134], and beamline [135] has been completed. The results indicate that a total of $80 \mathrm{~cm}$ of iron, surrounding the interaction region along the beamline, is sufficient to reduce the residual rates on the surface of the target pile to acceptable levels. Combining the above shielding with a one foot concrete floor reduces any possible ground water or surface water contamination to several orders of magnitude below mandated limits. Furthermore, an additional 14 feet of earth-equivalent shielding will reduce the prompt rate outside of the target berm to acceptable levels.

Along the beamline, eight feet of earth-equivalent will shield against accidental beam-loss for one hour. Accidental beam loss is modeled as losing the full secondary intensity at one point. This amount of shielding is also consistent with that needed to shield from muons produced in the decay of the secondary beam (see section 4.4.4).

\subsubsection{Alignment}

The success of any transport line depends on the alignment of the elements. The effect of misaligning the quadrupoles in the FOD0 section was studied. The program TRANSPORT [136] was used for the study, and the misalignment of a single quadrupole, using the TRANSPORT formalism, was calculated by hand as a check.

Typical alignment tolerances in a beamline are 10 mils transversely, and tape measure accuracy longitudinally $(1 \mathrm{~cm})$. The roll $\left(\theta_{z}\right)$ of a magnet can be set to approximately 3 mils divided by the lever arm (on a 3Q120, this is taken as 15 inches). The tolerances of $\theta_{x}$ and $\theta_{y}$ are calculated by adding the longitudinal errors in quadrature and dividing by the lever arm (in this case, 5 feet). The tolerances quoted are the $96 \%$ tolerances [137]. Table 12 summarizes the information.

$$
\begin{array}{cc}
\delta_{x} & 10 \mathrm{mils} \\
\theta_{x} & 333 \mathrm{mrad} \\
\delta_{y} & 10 \mathrm{mils} \\
\theta_{y} & 333 \mathrm{mrad} \\
\delta_{z} & 250 \mathrm{mils} \\
\theta_{z} & 200 \mathrm{mrad}
\end{array}
$$

Table 12: Typical alignment tolerances for beamline elements.

Table 13 lists the effects of the quadrupole misalignments at the beam stopper. Specifically, the effects listed are those if each quadrupole is randomly and independently misaligned within the given tolerances. The results listed for "Beam Sigma" are the broadening of the beam in phase space, that is, the percent by which the nominal beam is increased in $x(y)$ and $x^{\prime}\left(y^{\prime}\right)$. The results listed for "Beam Centroid" are the axis on which the centroid is has a 95\% probability of being located-because misalignment errors cause steering of the centroid, the centroid will be located at one position, but because the exact sign and magnitude of the errors are unknown, that position cannot be determined a priori.

Because the centroid will be shifted by a definite amount, the error may be eliminated by the judicious placement of correction dipoles. However, this will not, in general, eliminate the broadening of the beam.

In the vertical plane - the plane in which separation takes place - the beam may be broadened by nearly $10 \%$. Applied to an approximately $10 \mathrm{~mm}$ beam, this is an increase of $1 \mathrm{~mm}$. Consequently, 


\begin{tabular}{cccc}
\multicolumn{4}{c}{ Beam Sigma } \\
$\mathrm{x}$ & (per cent $)$ increase \\
3.2 & $\mathrm{x}^{\prime}$ & $\mathrm{y}$ & $\mathrm{y}^{\prime}$ \\
& 0.9 & 9.9 & 3.3 \\
\hline \multicolumn{4}{c}{ Centroid Uncertainty } \\
$\mathrm{x}[\mathrm{mm}]$ & $\mathrm{x}^{\prime}[\mathrm{mrad}]$ & $\mathrm{y}[\mathrm{mm}]$ & $\mathrm{y}^{\prime}[\mathrm{mrad}]$ \\
3.3 & 0.1 & 1.8 & 0.2
\end{tabular}

Table 13: Effect of quadrupole misalignments on beam centroid and phase ellipse. Refer to text for explanation.

the unwanted particle spot size grows, increasing the pion and proton transmission. As the kaon beam is also smeared, more of the kaons strike the stopper, decreasing the kaon transmission somewhat.

An obvious solution is to increase the stopper size from $12 \mathrm{~mm}$ to $13 \mathrm{~mm}$. This will reduce the $K^{+}$rate somewhat, but should maintain purity. To compensate we would then need to raise the primary proton intensity from $3.1 \times 10^{12}$ to $3.9 \times 10^{13}$. Another approach is better alignment, in particular, we should be able to do better than we assumed on elements in close proximity.

\subsubsection{Tolerance to RF parameters}

We expect to control both the relative amplitude and the phase between RF station 1 and $\mathrm{RF}$ station 2 via a RF feedback loop. The error on the relative amplitude is about $1 \%$ and the phase error is a few picoseconds. The absolute frequency is given by the loaded $\mathrm{Q}$ and is known very well. None of the small variations have a significant effect on beam purity. The momentum spread in the bean by far dominates these effects.

\subsection{The Superconducting RF Separator System}

We have been working with the Fermilab Beams Division to define and carry out the R\&D required to build a $3.9 \mathrm{GHz}$ superconducting separator system. An extensive discussion of the RF properties, cryogenic requirements, fabrication considerations and required cavity processing technologies is available in [138], [139] and [140]. Here we present the existing design and review the relevant $\mathrm{R} \& \mathrm{D}$.

\subsubsection{Cavity Design}

Figure 28 shows the layout of two 13 cell cavities in a horizontal cryostat. The cavities will operate in the $\mathrm{TM}_{110}$ mode, applying fields to the beam shown in figure 29 . With a $p_{\perp}$ kick of $5 \mathrm{MeV} / \mathrm{m}$, each RF station will need $3 \mathrm{~m}$ long to give the required deflection of $15 \mathrm{MeV}$ per station. Each station will consist of three cryostats holding two 13 cell cavities each.

Table 14 lists the baseline design parameters of the cavities. Note that a $p_{\perp}$ kick of $5 \mathrm{MeV} / \mathrm{m}$ corresponds to a peak magnetic field on the surface of the superconductor $\left(\mathrm{B}_{\mathrm{M} A X}\right)$ of $77 \mathrm{mT}$. The TESLA collaboration at DESY, planning to build a $T e V$-scale superconducting linac, has investigated and advanced SCRF technology considerably. As of this writing, the TESLA collaboration reports [141] that it can routinely achieve $25 \mathrm{MeV} / \mathrm{m}$ accelerating gradients, corresponding to $\mathrm{B}_{\mathrm{MAX}}=107 \mathrm{mT}$. 


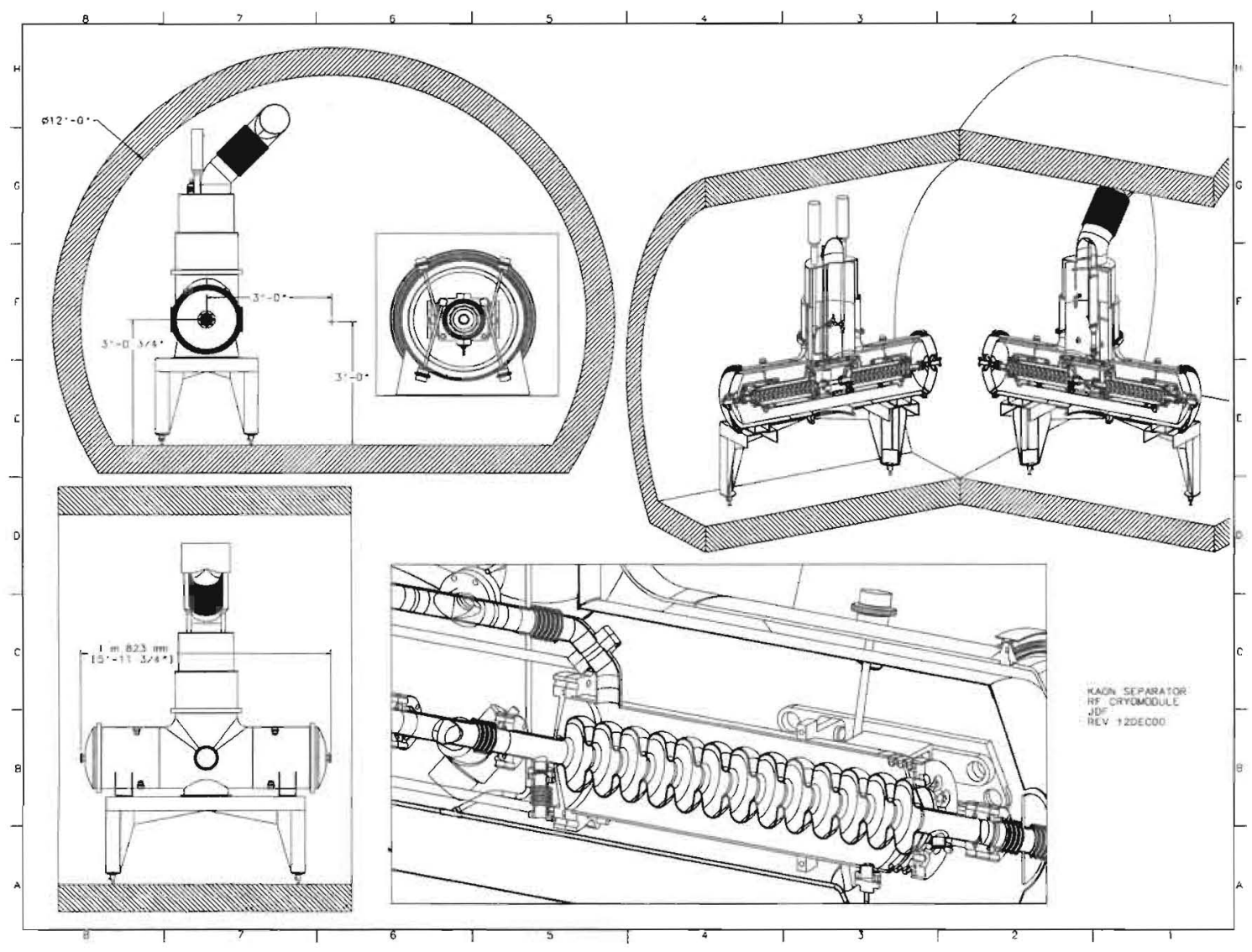

Figure 28: Sketch of two cavity cryostat, in the anticipated enclosing tunnel.
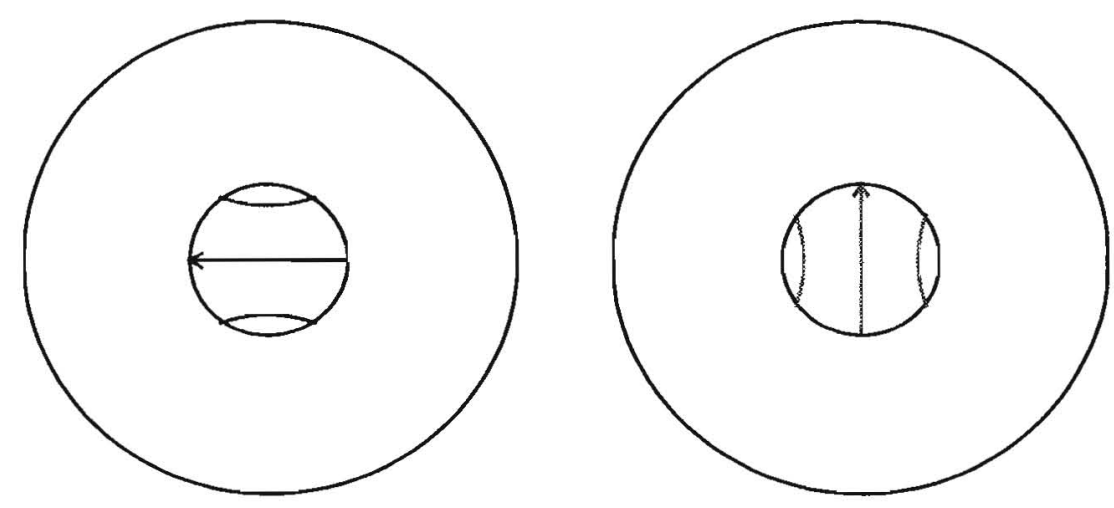

Figure 29: Beams-eye view of the cavities and their fields. At left, the magnetic field at the center of a cell; the two circles are the equator of the cavity and the beam pipe diameter. At right, the electric field at the iris between two cells. The cell length and cell-to-cell phase relationship are selected so that a particle timed to receive the maximum deflection sees these fields in all 13 cells and 12 irises. 


\begin{tabular}{|l|c|}
\hline Frequency & $3.9 \mathrm{GHz}$ \\
Mode & $\mathrm{TM}_{110}, \pi$ intercell phase advance \\
\hline Equator radius & $47.18 \mathrm{~mm}$ \\
Iris radius & $15.00 \mathrm{~mm}$ \\
Iris curvature & $5.50 \mathrm{~mm}$ \\
Cell length & $38.40 \mathrm{~mm}$ \\
Cells / cavity & 13 \\
Total cavity length & $660.0 \mathrm{~mm}$ \\
\hline$(\mathrm{R} / Q)^{\prime}$ & $351 \Omega / \mathrm{m}=701 \Omega /$ cell \\
$V_{\perp} / \mathrm{m}$ & $5 \mathrm{MeV} / \mathrm{m}$ \\
$E_{\mathrm{M} A X}$ & $18.5 \mathrm{MeV} / \mathrm{m}$ \\
$\mathrm{B}_{\mathrm{MAX}}$ & $77 \mathrm{mT}$ \\
\hline Intercell coupling & $4.3 \%$ \\
$\left(f_{\pi / 13}-f_{\pi}\right) / f_{7 \pi / 13}$ & $1.0 \mathrm{MHz}$ \\
$f_{12 \pi / 13}-f_{\pi}$ & $10 \sim 40 \mathrm{MHz}$ \\
Polarization split & $\pm 1 \mathrm{MHz}$ \\
Tuning range & $14 \times 10^{3}$ \\
$Q_{C u}$, room temp & $Q_{C u} / 2.89$ \\
$Q_{N b}$, room temp & $228 \Omega$ \\
\hline$G=Q \times R_{\text {surf }}$ & $110 n \Omega$ \\
$R_{\text {surf }}, 2 \mathrm{~K}$ & $0.73 \mathrm{~J} / \mathrm{m}$ \\
Stored Energy & $8.5 \mathrm{~W} / \mathrm{m}$ \\
Dissipated Power & $6 \times 10^{7}$ \\
$Q_{\text {external }}$ & $\mathrm{TE} \mathrm{F}_{11}$ \\
\hline Beam tube mode & $10.9 \mathrm{~mm}$ \\
Evanescent length & $5.86 \mathrm{GHz}$ \\
Beam tube cutoff freq. & \\
\hline
\end{tabular}

Table 14: Separator cavity parameters. Transverse shunt impedance is defined so that $P=$ $V^{2} / 2(R / Q)^{\prime} \times Q$. 
The separator project was externally reviewed in October 1998 by a panel headed by Maury Tigner of Cornell University and including experts from Cornell, JLab, Los Alamos and FNAL. The review panel concluded that the proposed system is technically feasible, and that the development program laid out in [138] would be required to provide a beam line in the 2003 time frame.

\subsubsection{Review of R\&D}

The goal of the R\&D program is to test a complete cryomodule in 2002 , and to test it in a beam during 2003. To accomplisl this, we need to do three things. We need (1) a robust design for the RF fields in the cavity, (2) to be able to treat the cavity surfaces so that they can hold the required field levels without breakdown, and (3) we need to fully specify, manufacture and test a cryostat of the final design.

(1) We must be satisfied that we have a robust and optimal design for the shape of the RF fields inside the cavity. This includes addressing issues such as multipacting and cell polarization, as well as issues involving the interaction of the fields with the mechanical properties of the cavities.

Towards this end, we have constructed demountable aluminum models and measured their RF properties in all modes below the beam pipe cutoff. The baseline design for the cavities has been modeled in great detail with the finite-element RF field package MAFIA, and we have developed an accurate lumped element equivalent circuit model. We also have polarized, bead-pull tested and warm tuned (i.e. correct for cell-to-cell manufacturing variations) a five cell cavity constructed of low grade niobium. The warm tuning of the five cell cavity revealed that we need to control the polarization of the individual cells during the tuning process. The design and manufacture of a cell tuning machine to do this is underway now. A numerical analysis of sensitivity to multipacting is underway, and multipacting has not appeared in any of our tests to date. We also plan to numerically quantify effects from higher order modes and the accelerating mode.

There are also several ways in which the mechanical properties of the cavity can interact with the RF fields. The best known of these is the Lorentz pressure, where the fields inside the cavity exert forces on the surface charges and currents on the inner wall of the cavity. We have begun to model this process numerically. The second potential hazard is microphonic pickup; a mechanical vibration of relative size comparable to $1 / Q \sim 17 \times 10^{-9}$ could move the cavity off resonance. With the cavity tests to date, we have introduced a feedback loop into the RF circuitry to compensate. For a system such as shown in figure 28, we will need to solve the problem again, using a cold mechanical tuning device to keep the frequency fixed.

(2) We need to assimilate the quality control practices of other SCRF producing laboratories so that we have the cleanliness and surface processing methodology necessary to build and test cavities capable of achieving the required field levels.

High quality niobium can be obtained commercially, but it is necessary to examine the surface of the material for defects. This is done with eddy current scanning, for which we are using the DESY facilities and manpower. The major steps in the standard surface treatment are an acid bath and a high pressure rinse with ultra-pure water. We have been relying on Cornell and Jefferson Lab facilities for the acid bath, and are in the process of commissioning a high power rinse system. While rinsing systems do exist at other labs, their design does reflect the specific cavity geometry of the application.

To measure the achieved field levels, we have built a test stand which holds the cavities so that their axis is vertical. Upgrades and modifications for different measurements of course continue as needed for the testing process. In this vertical dewar we have cold-tested high quality one and two cell cavities lent to us by Cornell University, which operate in the accelerating mode at just under $3 \mathrm{GHz}$. We have also cold tested the five cell cavity and a single cell cavity made here from high 
quality niobium. A second single cell cavity is being manufactured as this is being written, and the first test of our first 13 cell cavity will also be in this Dewar.

The primary indicator of cavity quality from these tests is a plot of the resonator's $Q$ as a function of the deflecting (or accelerating, or peak) field strength. $Q$ values on the order of $10^{9}$ are determined in $\mathrm{GHz}$ cavities by measuring the time for the oscillator to dampen down after power has been turned off. The value of the field where the cavity's $Q$ begins to fall off is an indicator of what is happening in the cavity [142]. So for example, a sharp fall in $Q$ at fields corresponding to a peak electric field on the surface $\left(\mathrm{E}_{\mathrm{M} A \lambda}\right)$ of roughly $5 \mathrm{MV} / \mathrm{m}$ indicates multipacting. Similarly. for failure at higher levels, when $\mathrm{E}_{\mathrm{M} A X}$ is anywhere in the range from 25 to $\sim 50 \mathrm{MV} / \mathrm{m}$, field emission is a cause of $Q$ dropping. This effect is created by particles on the order of $10 \mu \mathrm{m}$ in size, from which electrons tunnel out into the central part of the cavity under extremely high localized fields. The electrons are then accelerated in the cavity, absorbing energy from the fields and smash into cavity walls at high energies, creating hot spots. At the highest field levels, when $\mathrm{B}_{\mathrm{MAX}} \sim 120 \mathrm{mT}$ or more, the limit is global thermal breakdown. It must be emphasized that knowing the field level where $Q$ drops off, while useful, is not a perfectly reliable indicator of what is happening in the cavity.

The high quality cavities lent to us by Cornell maintained a high $Q$ up to field levels where the typical failure mode is field emission. This phenomena can be better diagnosed with thermometry and $\mathrm{X}$ ray detection to identify the impact of the electrons on other walls of the cavity. We have been upgrading our thermometry; our $\mathrm{X}$ ray detection still needs improvement. The most effective technique to prevent field emission is to rinse the interior of the cavity with ultra-pure water $(\sim 18 \mathrm{M} \Omega)$ at very high pressures $(\sim 100$ atmospheres). The only cavity which we have tested which has received this treatment is our single cell cavity, and that cavity has performed quite well. Figure 30 shows the $Q$ vs $V_{\perp} / \mathrm{m}$ result for this cavity. The highest field achieved before thermal breakdown corresponds to a deflecting gradient of $9.5 \mathrm{MV} / \mathrm{m}$, a $\mathrm{B}_{\mathrm{MAX}}$ of $104 \mathrm{~m}$ Tand an $\mathrm{E}_{\mathrm{MAX}}$ of $32 \mathrm{MV} / \mathrm{m}$. Remember that our multi-cell design calls for a deflecting gradient of $5.0 \mathrm{MV} / \mathrm{m}$ at a $\mathrm{B}_{\mathrm{MA \lambda}}$ of $77 \mathrm{mT}$.

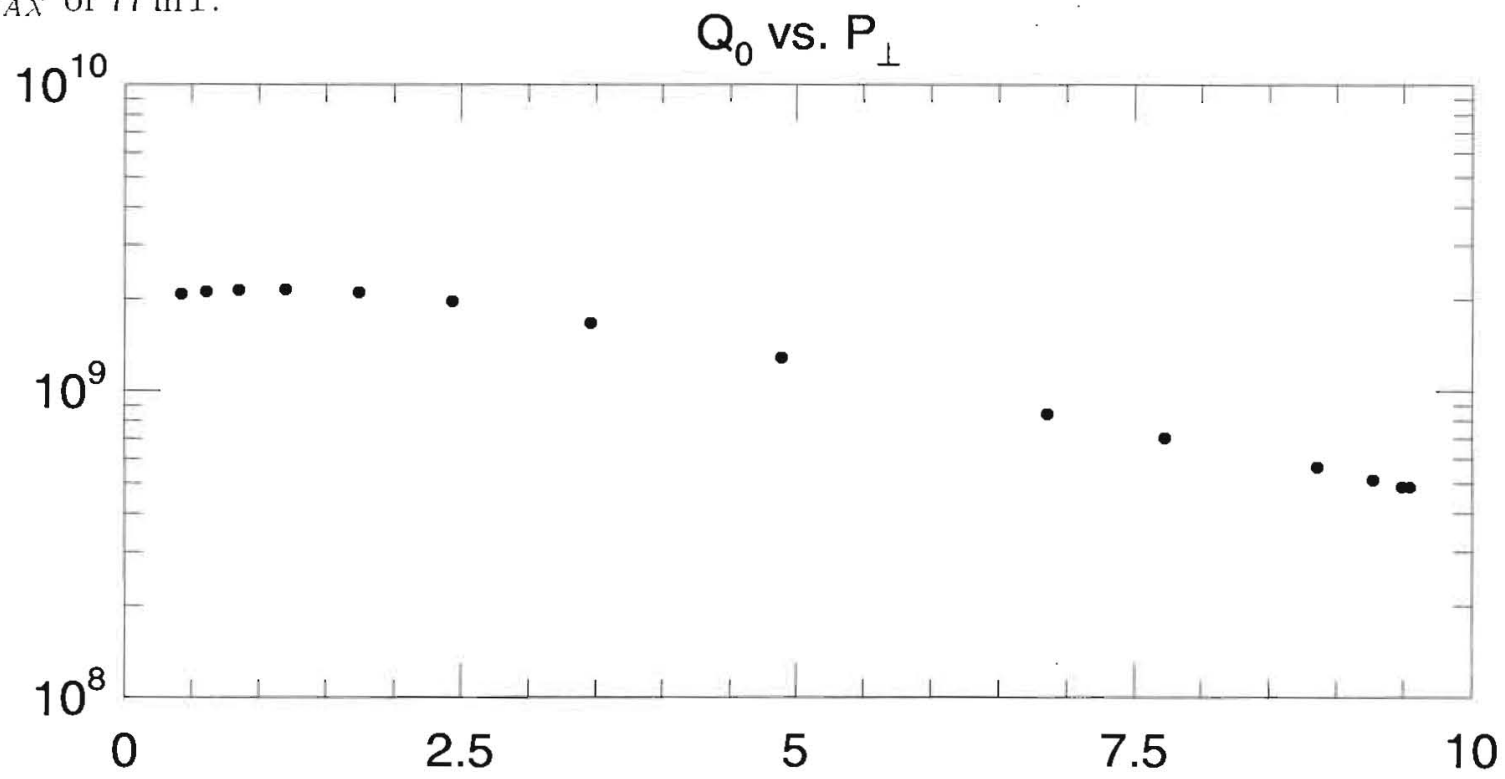

Figure 30: $Q$ vs $V_{\perp} / \mathrm{m}$ result for our single cell cavity. The horizontal axis is equivalent deflecting field, in $\mathrm{MV} / \mathrm{m}$; no correction for the difference between single and multiple cell structures has been applied. 
The five cell cavity was constructed of low quality niobium, and was originally made in order to determine good machining and $e$-beam welding parameters. It shows a low $Q$ and has been able to sustain deflecting fields of only $0.9 \mathrm{MeV} / \mathrm{m}$; we suspect that a small bead with poor thermal contact to the rest of the cavity is experiencing thermal runaway, quenching the fields in some fraginent of the cavity. This cavity will be re-etched and re-measured.

(3) Third, we need to finish the engineering for the device shown in figure 28. This will also prepare us for the mass production and commissioning of a full set of 12 cavities plus spares.

We have the basic cavity-making technique in hand, although we have not yet built 13 cell structures and wish to develop RF testing of smaller structures from which the 13 cell cavity will be built. The design and manufacture of the horizontal cryostat itself is underway. The design of a cold tuner has just started. The design of the side ports to the beam tubes, which are use to drive the cavity and monitor the field level inside the system is underway.

The testing of the first horizontal cryomodule obviously requires supplies of cryogenic helium, high power RF, RF diagnostic facilities, a beamline into which the cryomodule can be inserted, and the ability to monitor the beam position, direction, size and shape both entering and leaving the cavities. The cryogenic facilities now in $\mathrm{A} 0$ are adequate to run a two cavity cryostat, but the 200W RF amplifier now being used for the tests in the vertical Dewar will not be sufficient; we will need the final klystron power unit. The A0 beamline has flexible beam measurement facilities. It is too early to specify the sequence for these tests in excruciating detail, but it will probably look something like this:

1. Verify basic functionality of cryogenics, thermometry, RF power windows.

2. Check that microphonics and vibrations are acceptably low.

3. Exercise and verify the performance of the cold tuner, and possibly a piezoelectric tuner, at low RF power.

4. Verify that the low level RF control works as needed.

5. Investigate and select the optimal coupling, coupler effective $Q$, and $\mathrm{RF}$ power level for operation.

6. Put high power RF into the cavities, and verify frequency shift due to Lorentz pressure, RF window functionality. Here, we will probably use a transmitted power probe to measure the stored energy.

7. Measure static and dynamic heat loss.

8. Look for $\mathrm{X}$ rays from the cavities.

9. Put beam through the cavity without RF, and see if HOM excitation matches predictions for the given beam structure.

10. Deflect the beam with some known power level and measure transverse shunt impedance. To startwith, this need not be a full power deflection.

11. Run the system for long periods and monitor stability of oscillation phase and deflection amplitude. Verify that the polarization axis does not wander. 


\subsection{GEANT Calculated Rates and Backgrounds}

\subsubsection{Introduction}

The issues explored here are what beam purity of $K^{+}$can be accomplished and what are the background rates at the experiment. The simulations are based on the optics design [143] made using the program TURTLE [144]. The GEANT program was modified to read the same magnet positions and fields as used for the optics code. The Malensek production parameterization is used for the absolute rates [124] in both TURTLE and GEANT.

A detailed description of the model used in GEANT Version 321 [145] follows:

1. Secondary products are generated from the target into a cone $\pm 8(8)$ mrad in $x(y)$ within $\pm 10 \%$ of central momentum of $22 \mathrm{GeV} / c$. This is larger that the acceptance of about $\pm 8(4) \mathrm{mrad}$ in $\mathrm{x}(\mathrm{y})$ with $\pm 2 \%$ momentum spread. The source size was taken as $3 \mathrm{~mm} \times 3 \mathrm{~mm}$ and 10 cm long.

2. All optics is first order, i.e. quadrupole fields are taken as proportional to the displacement off axis with the appropriate gradient.

3. Magnets are modeled as boxes of iron with proper dimensions. The fields in the magnet steel are taken from POISSON. Then these are read into GEANT.

4. The transverse deflection of $5 \mathrm{MeV} / m$ in the $\mathrm{RF}$ cavities is simulated using the magnetic field code available in GEANT. The GEANT time of flight is used to get the proper time phasing of the transverse kicks for each particle type. The cavity frequency was $3.9 \mathrm{GHz}$. The RF cavity materials were not modeled in detail. As a check, first the cavity was modeled as a tube of iron with inner aperture of $3.0 \mathrm{~cm}$ and onter aperture of $20 \mathrm{~cm}$, then the iron was replaced by vacuum. Since we are placing a $2 \mathrm{~m}$ iron shadow collimator with the same transverse dimensions as the cavity itself upstream of each RF station, the material in the cavity does not affect the simulation results significantly. Each RF station has 4 mil Mylar windows on each end. Each of the two RF stations provides $15 \mathrm{MeV}$ of kick in $3 \mathrm{~m}$ of active length.

5. Vacuum is assumed everywhere except where elements are inserted, i.e. beam pipes are not yet simulated explicitly.

6. In addition to the RF shadow collimators we have several other collimators. The first is a iron fixed hole collimator just after the target and before the first collection quadrupoles. The fixed hole collimator limits the initial particle angles and shields the collection quadrupoles. Three other collimators are placed in the beamline to restrict the momentum, $\mathrm{x}$ angle and $\mathrm{y}$ angle. These collimators are modeled after existing Fermilab variable gap "jaw" collimators.

7. To facilitate reasonable running times a $1 \mathrm{GeV}$ threshold was taken. Studies with a $10 \mathrm{MeV}$ thresholds showed only the photon content of the beam was underestimated using a $1 \mathrm{GeV}$ threshold ( by about 2-3 times).

8. The tungsten beam stopper which absorbs unwanted particles was $3 \mathrm{~m}$ long and had a vertical widtlı of $12 \mathrm{~mm}$.

Since TURTLE was used for the optics design and GEANT for the background calculations it was important that both programs give very similar results. The comparison also provided a excellent check on the code. 
For the comparison of GEANT and TURTLE, both individual rays and phase space distributions were compared. GEANT uses a stepping procedure while TURTLE propagates with matrix multiplication and since the beamline is quite long $(215 \mathrm{~m})$, so stepping errors can accumulate. For this reason, we converted GEANT to double precision [146] to get good agreement. The results of the comparison between GEANT and TURTLE are:

1. The transmission for kaons is in excellent agreement, TURTLE gives 0.42 while GEANT gives 0.40 .

2. A comparison of the major beam loss vs element is also in good agreement [147].

3. The distribution of kaon positions, angles, and momenta at different $z$ locations along the beamline are in agreement, Figures 32, 33, 34, 35, 36 show these results.

4. The beam purity predicted from TURTLE is 0.82 while GEANT predicts 0.81 .

\subsubsection{Beam Rates and Purity}

GEANT was used to generate positively charged particles at the target proportionally to their respective production taken from Malensek [124](10M pions, $1 \mathrm{M}$ kaons, and 3.5M protons). Table 15 gives the normalized results at the exit to the last quadrupole ( $215 \mathrm{~m}$ from the target). The beam size at the last quadrupole is constrained by the $25.4 \mathrm{~cm}$ diameter of the quadrupole. The $\mathrm{K}^{+}$ beam rate is normalized such that the rate $15 \mathrm{~m}$ downstream of the last quadrupole, after the UMS and just before the kaon RICH is $30 \mathrm{MHz}$. The rates are for a $1 \mathrm{~m} \times 1 \mathrm{~m}$ box cut around the beam as it exits the last quadrupole. $95 \%$ of the hadrons are contained in a box cut around the beam of $8 \mathrm{~cm} \times 8 \mathrm{~cm}$, while $80 \%$ of the muons are contained in the same cut.

\begin{tabular}{|l|l|l|l|l|l|}
\hline & $K^{+}$ & pion+proton & muons & total charged & photons \\
\hline Rate $(\mathrm{MHz})$ & 33 & 7.5 & 7.4 & 48 & 1.0 \\
\hline \%hadronic & 81 & 19 & $N A$ & 100 & NA \\
\hline \%total & 69 & 16 & 15 & 100 & NA \\
\hline
\end{tabular}

Table 15: Beam rates at the exit of the last quadrupole required to produce $30 \mathrm{MHz}$ of kaons at the entrance to the kaon $\operatorname{rich}(z=230 \mathrm{~m})$.

The hadronic purity meets the CKM requirement of $70 \%$. The required kaon rate for CKM is $30 \mathrm{MHz}$ this is achievable with $4 \times 10^{12}$ protons per pulse.

The muon spatial distribution at exit of last quad is concentrated mainly in the beam region(see figure 31).

\subsubsection{Effect of clean-up bends after beam stopper}

To investigate the necessity for a clean-up bend after the beam stopper, we repeated the results of the previous section varying the fields in B5 and B6 in figure 21. This straight bearnline configuration was also more compatible with some of the early site selection options such as Meson MP beamline and experimental hall.

The only significant change to the rates without the bends is the increase in muons both in detector and in the beam. To investigate muon rate vs clean-up bend, several runs were taken varying the value of the bends. (Two bends and quadrupoles between the bends are needed to make the beam achromatic.) The results of several runs with different values for the sum of the 


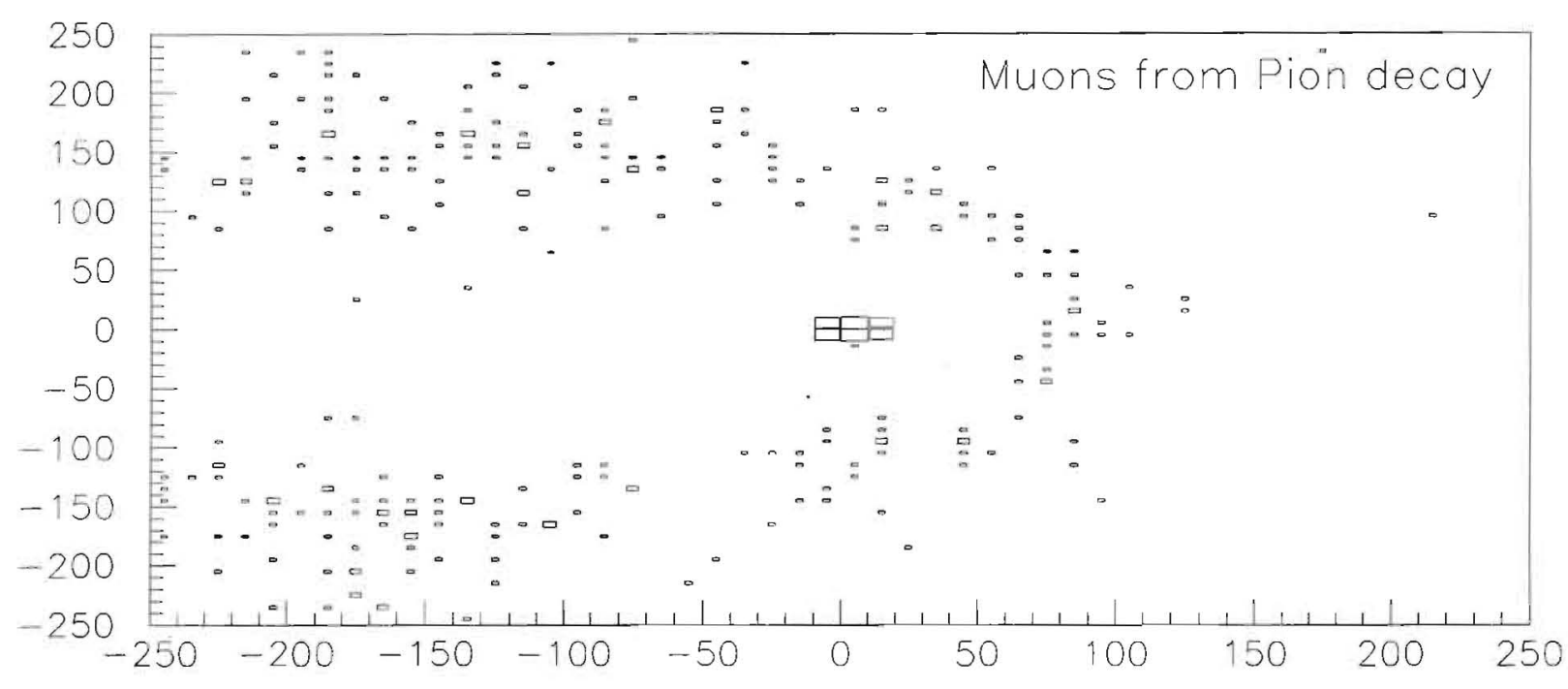

xy at. Detector-Muons

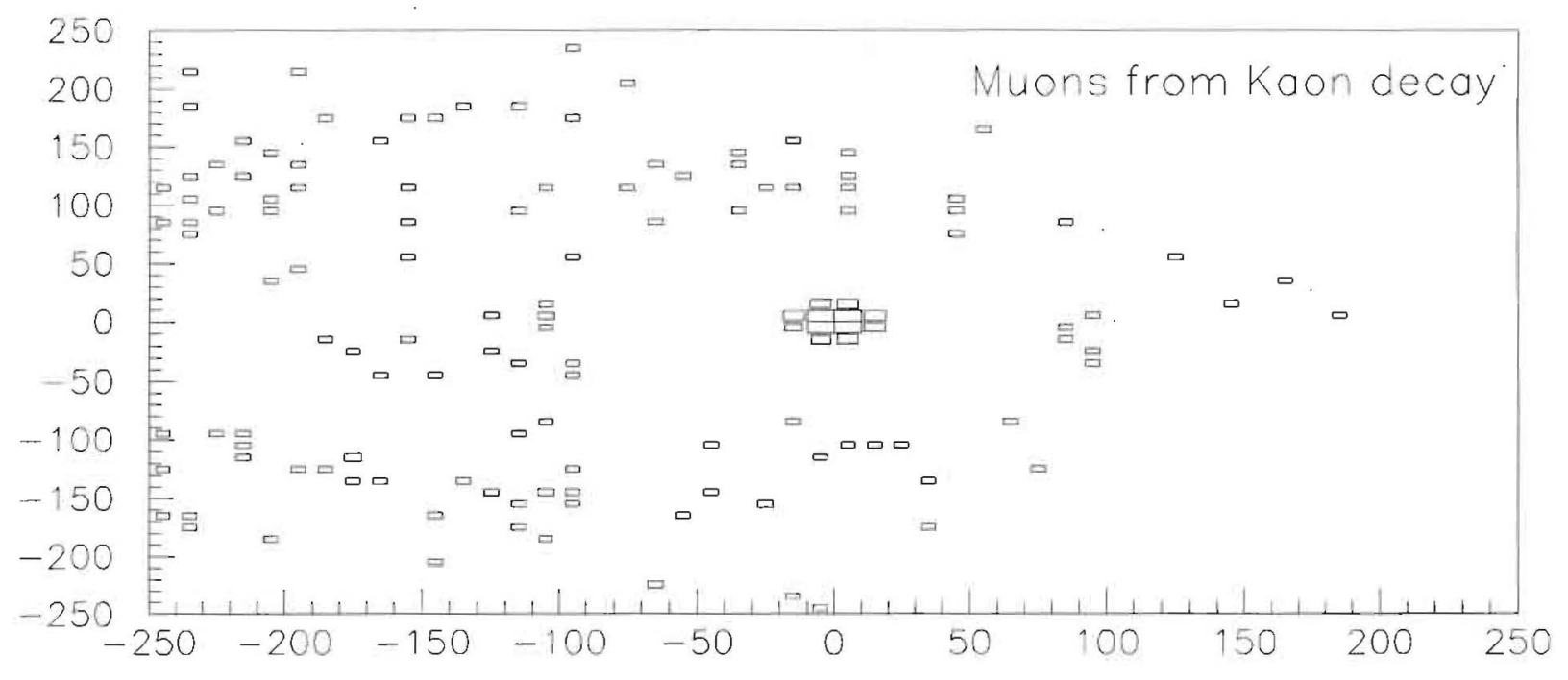

- xy at Detector-Muons

Figure 31: Muon spatial distribution at the exit of the beam line. The source of the top (bottom) plot is beam pion (kaon) decays. 
two clean-up bends is shown in figure 37. The number of muons striking the upstream detector plane increases by a factor of 4 in the comparison of no bend and the standard 6.3 degree bend.

Figure 38 compares the $z$ origin of the beam muons reaching the detector with the standard bends after the stopper (B5/6). The pion decays dominate and originate upstream of the beam stopper, so they are sensitive to the final bend. The kaon decays pass the beam apertures only if they originate downstream (because of the larger opening angle), so they are insensitive to the choice of final bends.

Figure 40 shows the interactive GEANT display of 44 events which have a muon from pion decay which enters the detector. In Figure 40 pions are shown as solid lines while muons are dashed lines. These muons are sufficiently close to the beam momentum that their trajectories resemble the nominal beam particles closely (see Figure 39). The absence of downstream bends opens the momentum acceptance up, and give a broader peak in the case with no clean bend.

Neutrals: The dominant source of neutrals in the beam/detector come from kaon decays near the end of the beam, similar to the muons from kaon decay shown in figure 38 . The photon rate is $1.2 \mathrm{MHz}(1.5 \mathrm{MHz})$ with (without) the clean-up bends. The $K_{L}^{0}$ /neutron rate is two orders of magnitude smaller.

\subsubsection{Muon shielding along the beamline.}

To investigate the muon shielding along the beamline, a tube of concrete $3 \mathrm{~m}$ thick with an inner radius of $1.5 \mathrm{~m}$ was placed along the beamline. An additional tube of concrete was placed around the stopper. The stopper shielding was $0.5 \mathrm{~m}$ thick with an inner radius of $0.3 \mathrm{~m}$ and had a length of $2.88 \mathrm{~m}$ centered on the stopper. Figure 41 shows the layout along with a sample of events generated by initial pions. With $3 \mathrm{~m}$ of concrete the peak radiation levels are $2 \mathrm{mrem} / \mathrm{hr}$ just outside the shield. Decreasing the concrete tube thickness from $3 \mathrm{~m}$ to $2 \mathrm{~m}$; would increase the peak dose from $2 \mathrm{mrem} / \mathrm{hr}$ to $5 \mathrm{mrem} / \mathrm{hr}$. A more complete discussion of radiation shielding in given in section 4.2 .7 .

\subsection{Summary of Beam Section}

A conceptual beam design which meets the CKM requirements has been completed. Beam simulation is fully incorporated into the detector simulations. The critical component of the beam is the superconducting RF cavities. An R\&D program continues on this effort. The required $5 \mathrm{MV} / \mathrm{m}$ deflecting field strength has been exceeded in a single cell structure. The outstanding remaining task is obtaining this result in the final 13-cell cryostat. 


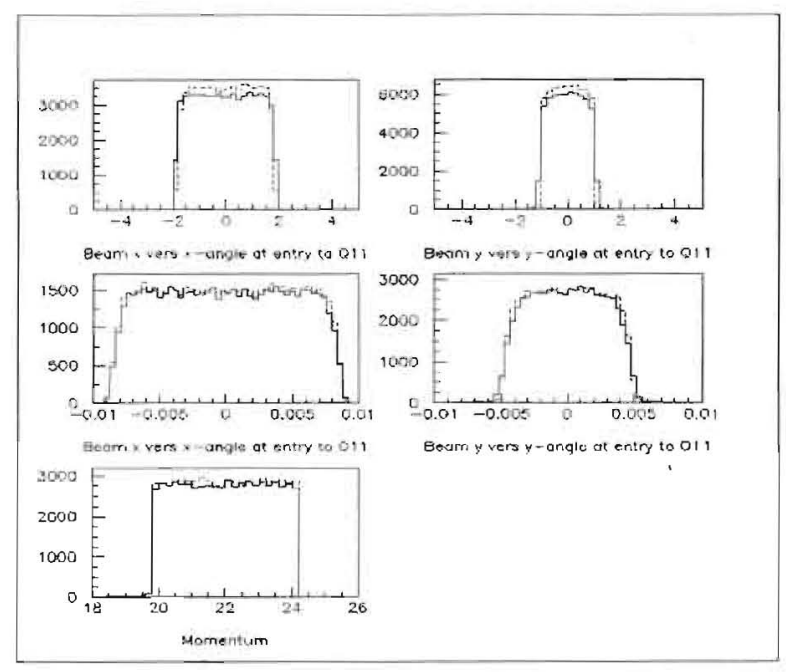

Figure 32: Initial phase space in $\mathrm{x}, \mathrm{y}[\mathrm{cm}], \mathrm{x}$ angle, $y$ angle $[\mathrm{rad}]$, and momentum[ $\mathrm{GeV} / c]$ for GEANT(solid line) and TURTLE(dashed line).

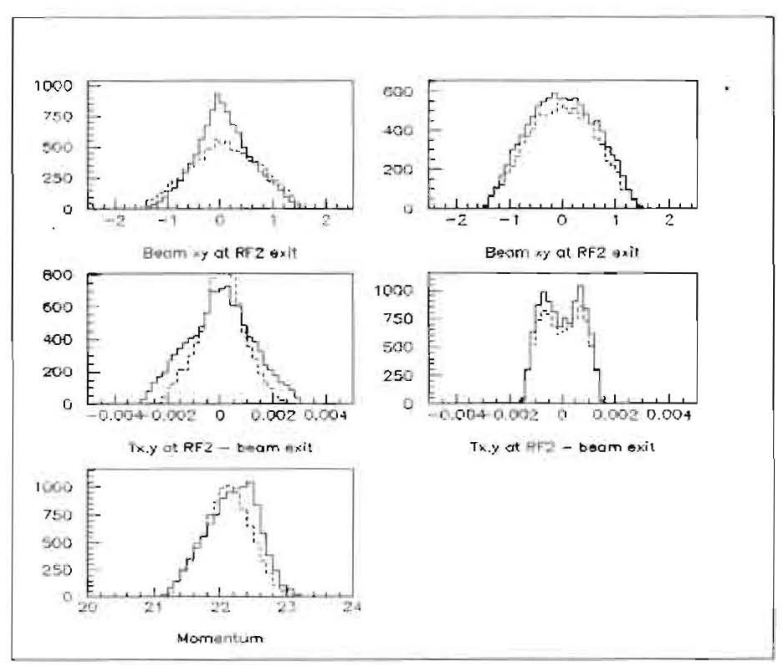

Figure 34: Phase space at RF2 exit in $\mathrm{x}, \mathrm{y}[\mathrm{cm}], \mathrm{x}$ angle, $\mathrm{y}$ angle $[\mathrm{rad}]$, and momentum $[\mathrm{GeV} / \mathrm{c}]$ for GEANT(solid line) and TURTLE(dashed line).

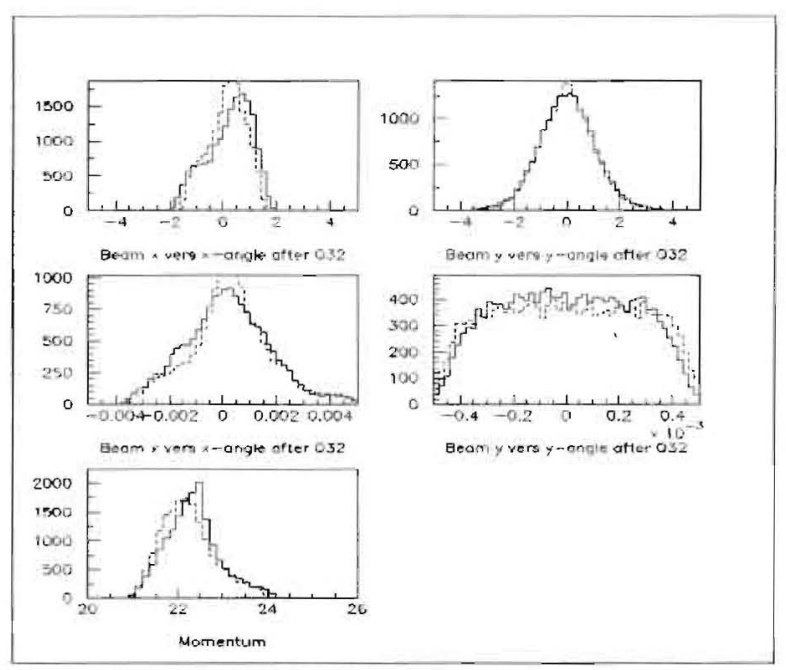

Figure 33: Phase space after $\mathrm{Q} 32$ in $\mathrm{x}, \mathrm{y}[\mathrm{cm}], \mathrm{x}$ angle, y angle[rad], and momentum[GeV/c] for GEANT(solid line) and TURTLE(dashed line).

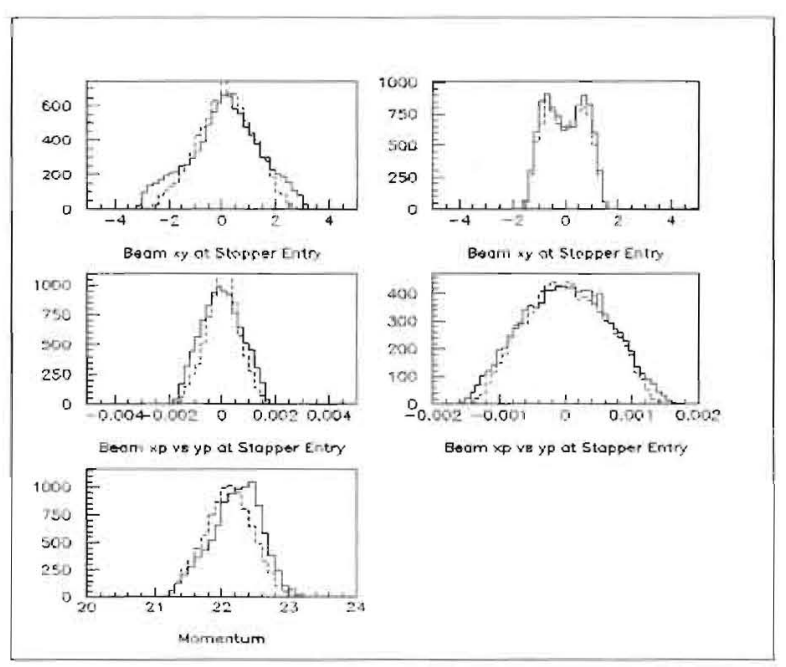

Figure 35: Phase space at Stopper in $\mathrm{x}, \mathrm{y}[\mathrm{cm}], \mathrm{x}$ angle, $\mathrm{y}$ angle[ $\mathrm{rad}]$, and momentum $[\mathrm{GeV} / \mathrm{c}]$ for GEANT(solid line) and TURTLE(dashed line). 


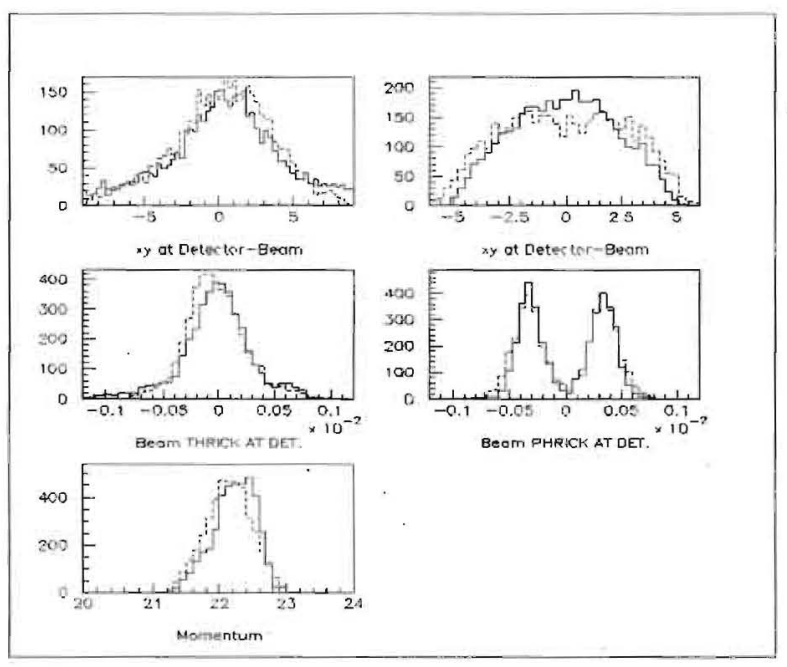

Figure 36: Phase space at detector in $\mathrm{x}, \mathrm{y}[\mathrm{cm}], \mathrm{x}$ angle, $\mathrm{y}$ angle[rad], and momen$\operatorname{tum}[\mathrm{GeV} / \mathrm{c}]$ for GEANT(solid line) and TURTLE(dashed line).

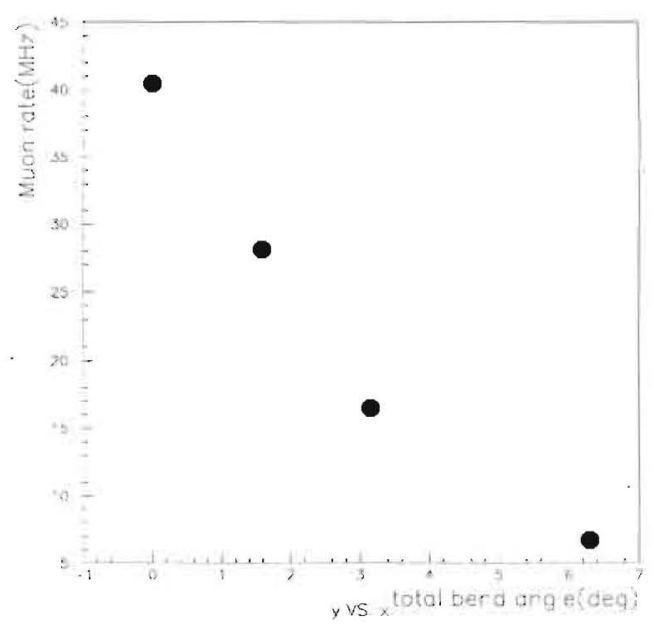

Figure 37: Muon rate in $\mathrm{MHz}$ at last quadrupole exit within 1 meter transverse of the beam center vs the strength of the cleanup bends. 

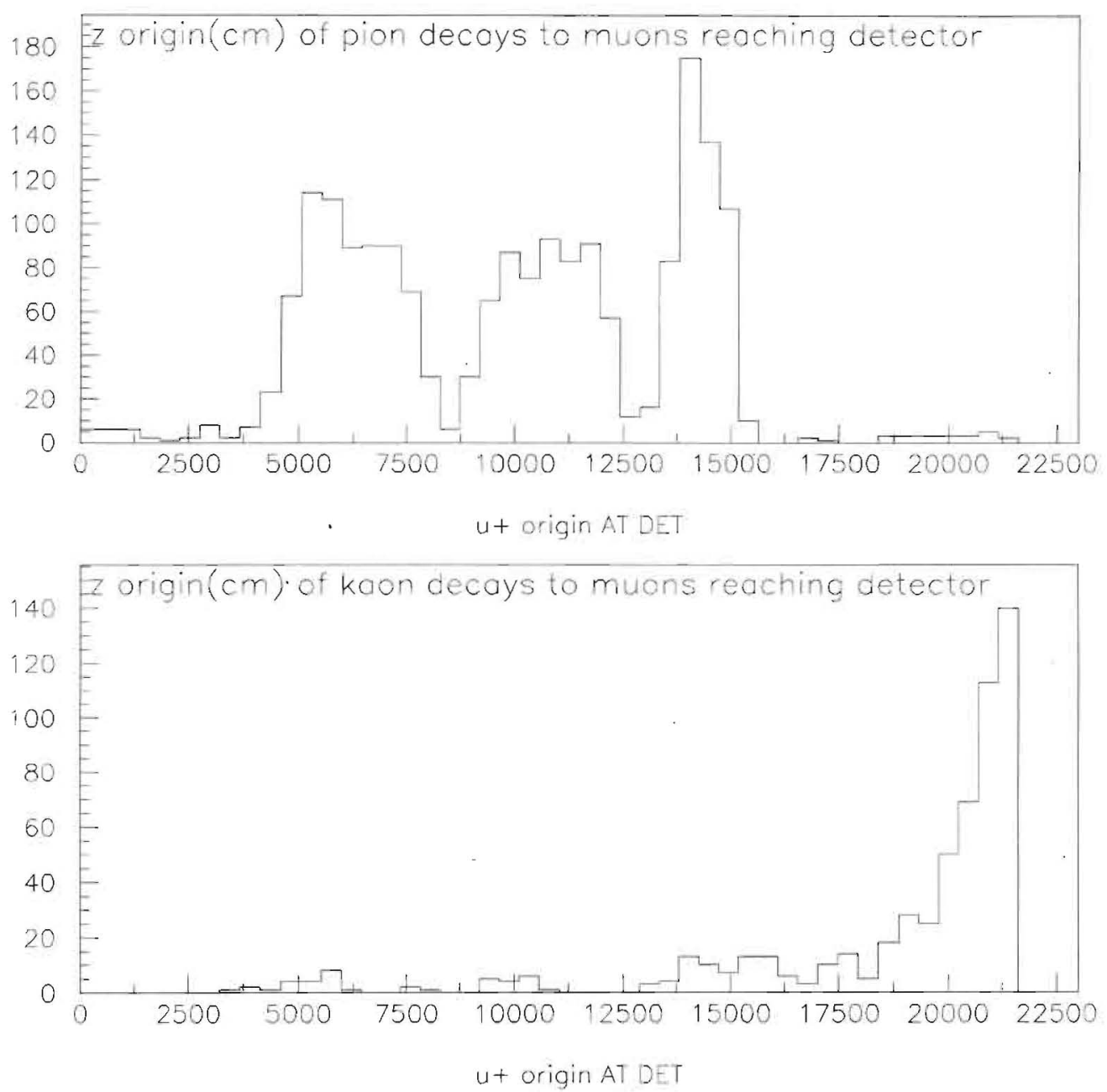

Figure 38: Decay location ( $\mathrm{cm}$ from target) for pion and kaon decays to muons that enter the detector. 


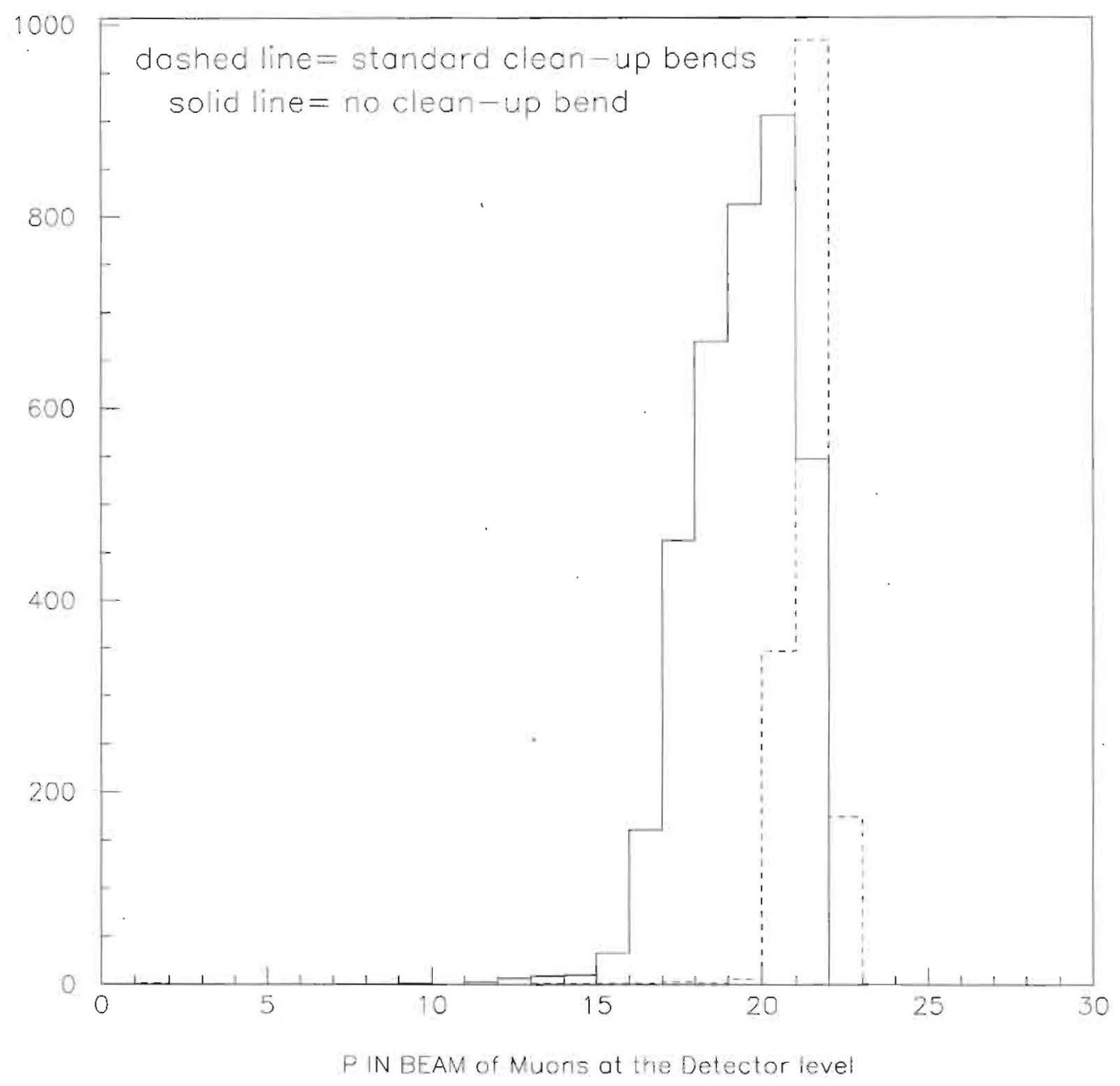

Figure 39: Momentum distribution $(\mathrm{GeV})$ for muons from pion decay with and without the standard clean-up bend. 


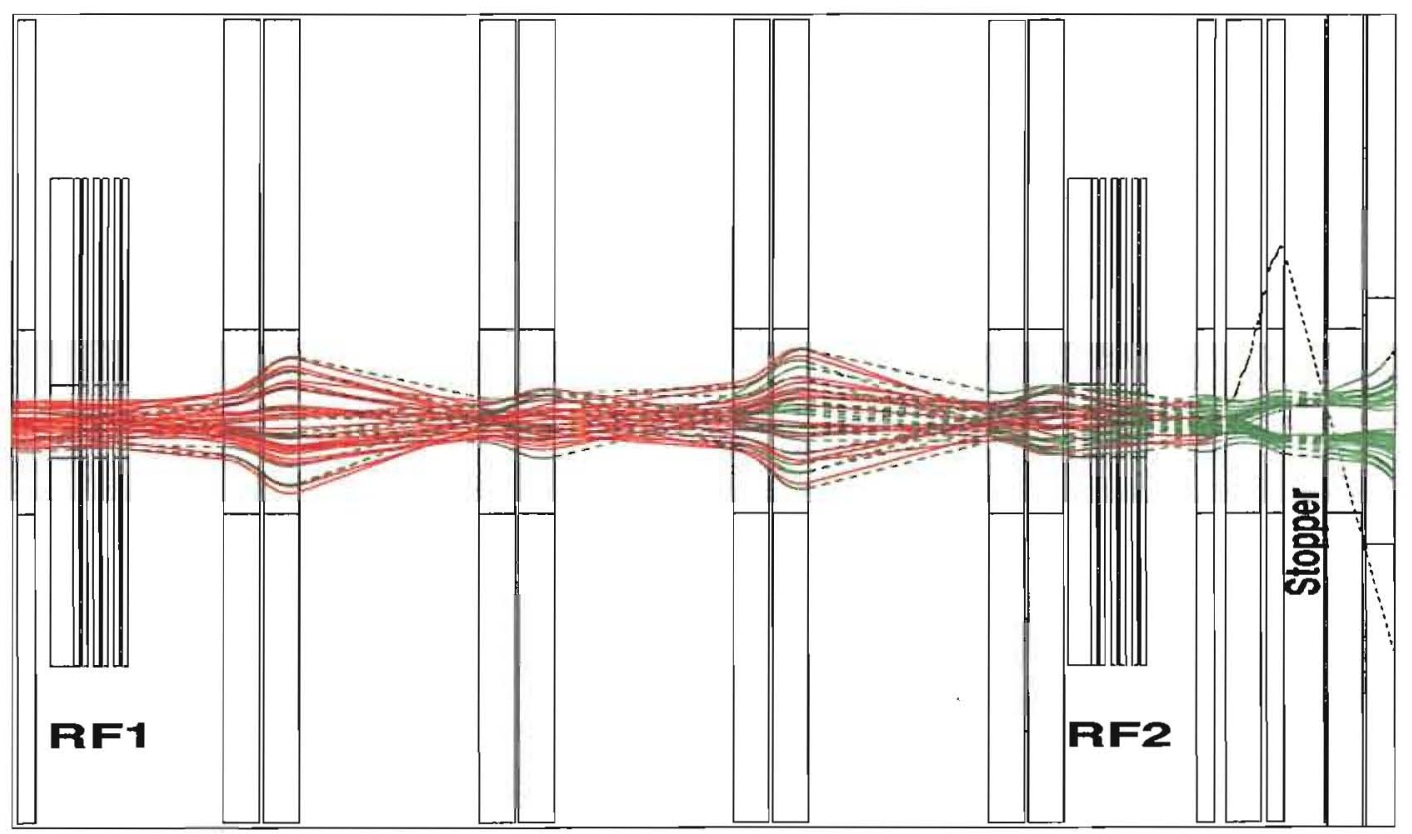

Figure 40: Pi-decay muons reaching the end of the beamline. Shown is the RF stage of the beamline including the beam stopper. Pions are shown in red and muons are shown in green. This is the dominant source of beam muons. 


\section{Pi-decay muons and beam shielding}

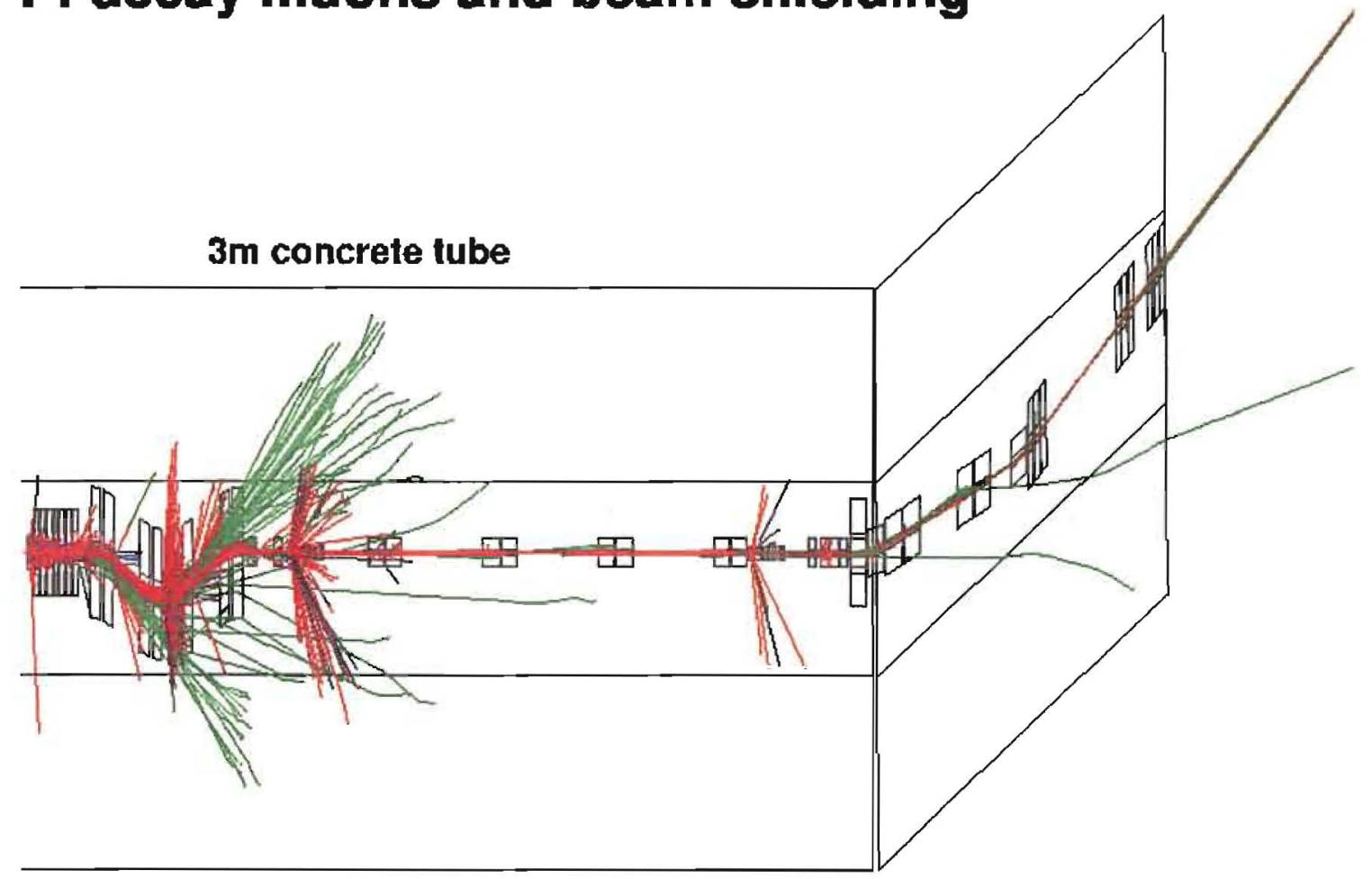

Figure 41: Muon shielding along the beam line. 


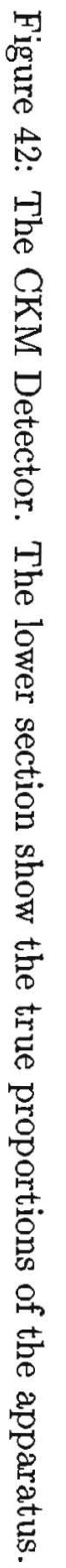

CKM Apparatus
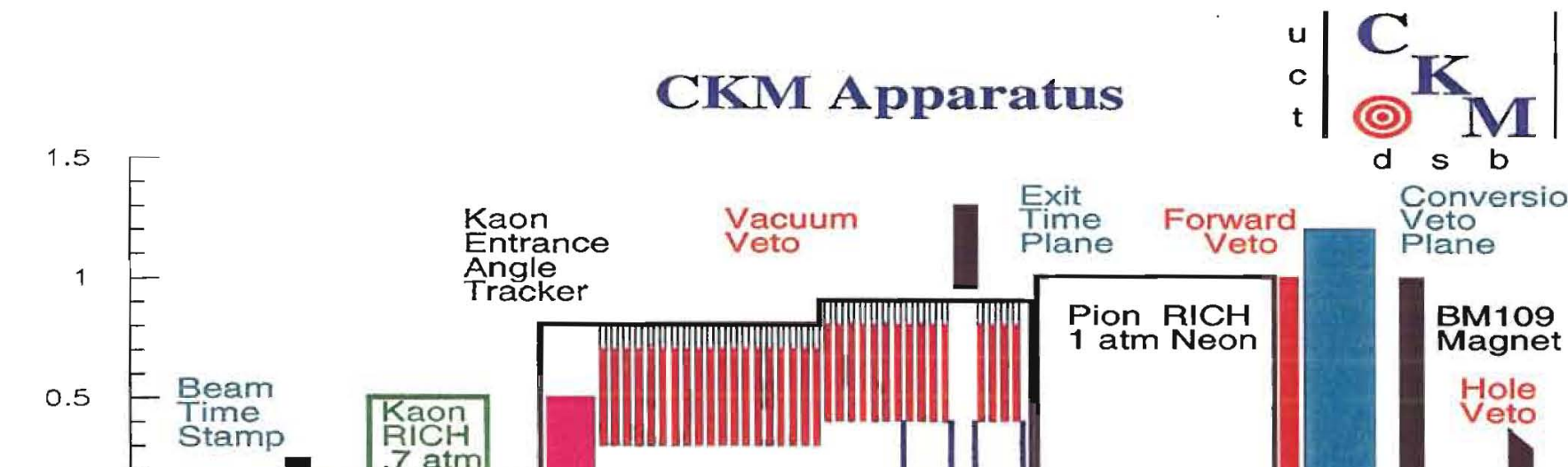

$\begin{array}{ll}\text { Kaon } & \text { Vacuum } \\ \text { Entrance } & \text { Veto }\end{array}$

Entrance

Angle

Exit

Conversion Conver

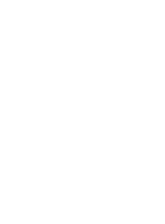

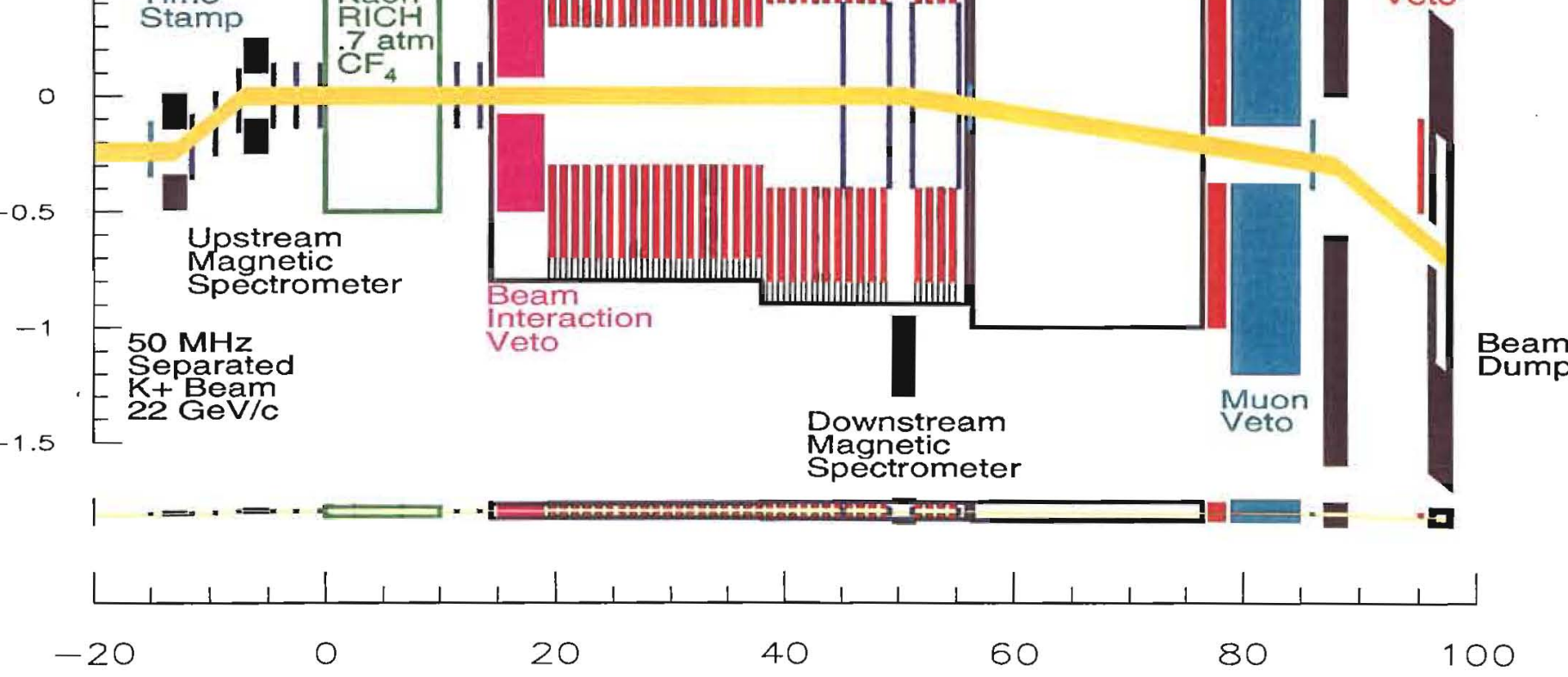




\section{$5 \quad$ The Detector Systems}

\subsection{The Upstream Magnetic Spectrometer}

The first element of the CKM spectrometer is the Upstream Magnetic Spectrometer (UMS). Its purpose is to measure the momentum of the particles leaving the RF-separated beam line and to measure the direction of the particles incident on the Kaon RICH. The UMS must have good acceptance, the ability to track reliably in a high-rate environment and to survive extended use in a high-rate environment, the ability to separate tracks that are close both in time and in space, and it must have better than $0.5 \%$ momentum resolution. Furthermore, it must present a minimal amount of material to the RF-separated beam to keep non-Gaussian tails under control.
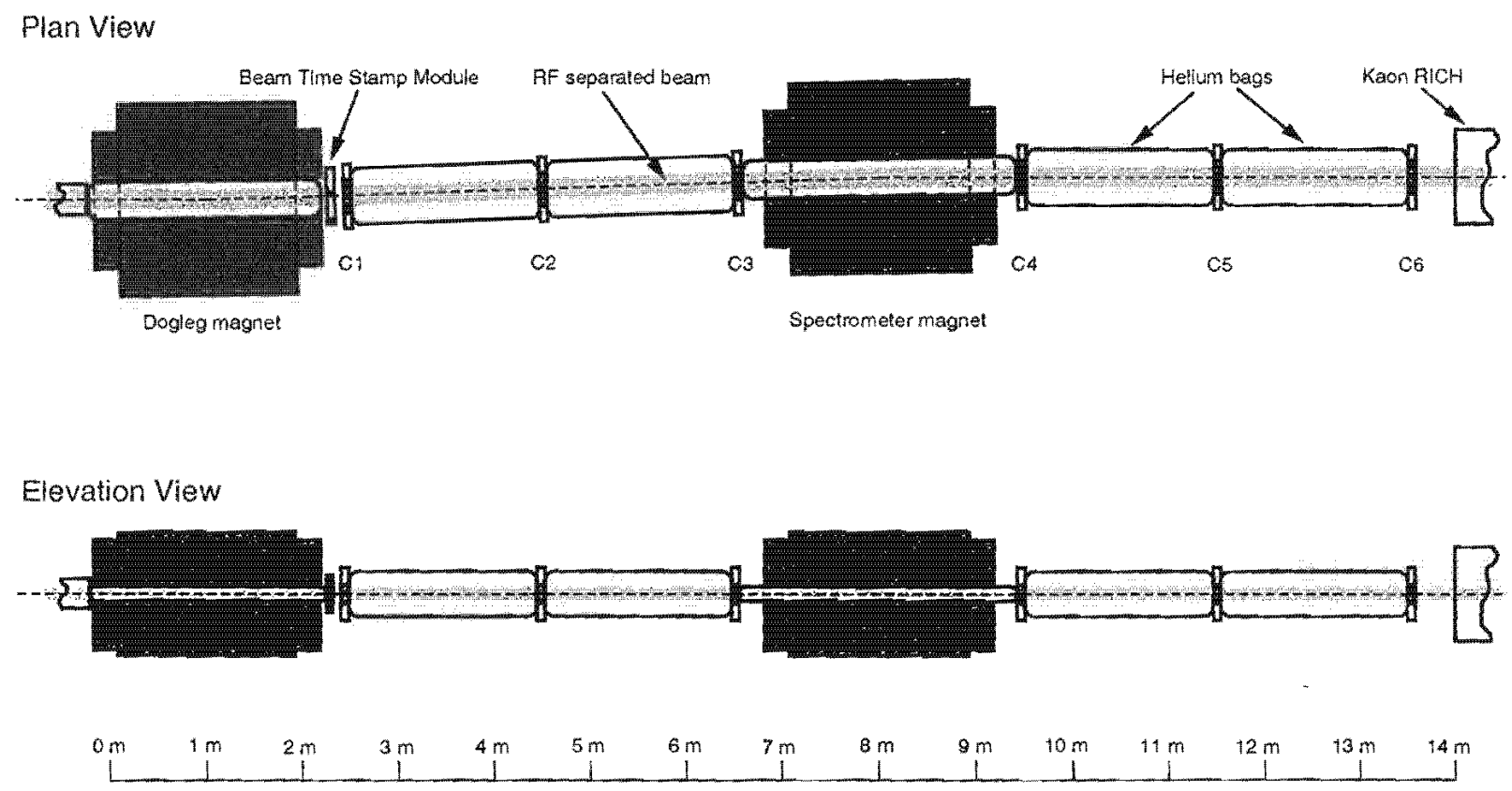

Figure 43: Plan and elevation views of the upstream magnetic spectrometer.

The RF-separated beam has $51 \mathrm{MHz}$ intensity (of which $30 \mathrm{MHz}$ are $K^{+}$). It's mean momentum is $22 \mathrm{GeV} / \mathrm{c}$ with a dispersion of about $\pm 4 \%$ (see Fig. 44). The beam inhabits an area of approximately $20 \times 10=200 \mathrm{~cm}^{2}$, with very little divergence either horizontally or vertically (Fig. 45). The maximum flux is $0.9 \mathrm{MHz} / \mathrm{cm}^{2}$.

The layout of the UMS is shown in Fig. 43. The spectrometer is approximately $14 \mathrm{~m}$ long. Two BM105 dipole magnets separated by $7 \mathrm{~m}$ are run with equal, but opposite magnetic fields, giving $22.0 \mathrm{GeV} / \mathrm{c}$ particles a temporary bend of $2^{\circ}$ and then restoring them to their original direction after a horizontal shift of $24.44 \mathrm{~cm}$. There are 6 wire chamber stations, three in front and three behind the downstream (Spectrometer) magnet. Helium bags between the chambers minimize material in the beam. Just upstream of the UMS is a bank of scintillating fibers - the Beam Time Stamp Module (BTSM) - which provide the timing information for the experiment. The transition from vacuum to atmospheric pressure is done before the upstream (Dogleg) dipole since beam-material interactions are less damaging when they occur upstream. Immediately following the UMS is the Kaon RICH. About $5.9 \%$ of the kaons decay in the UMS. 


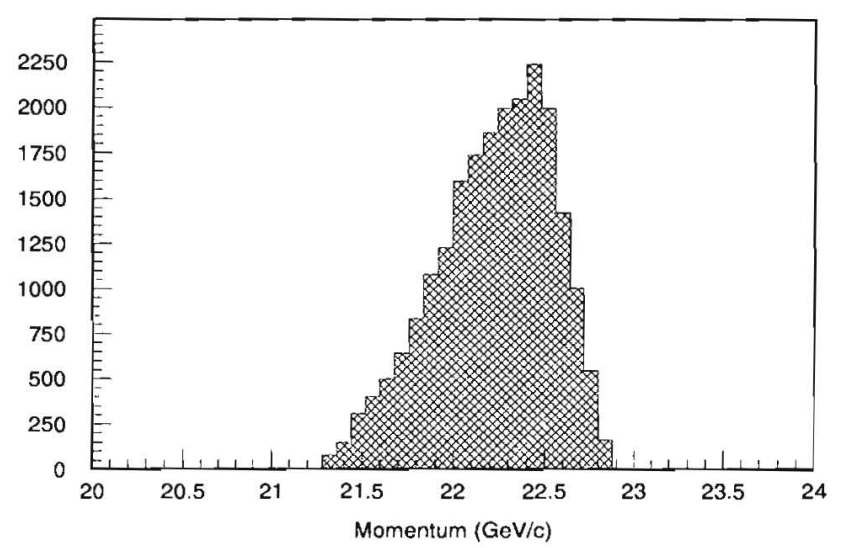

Figure 44: Momentum of the kaons at the entrance of the UMS.

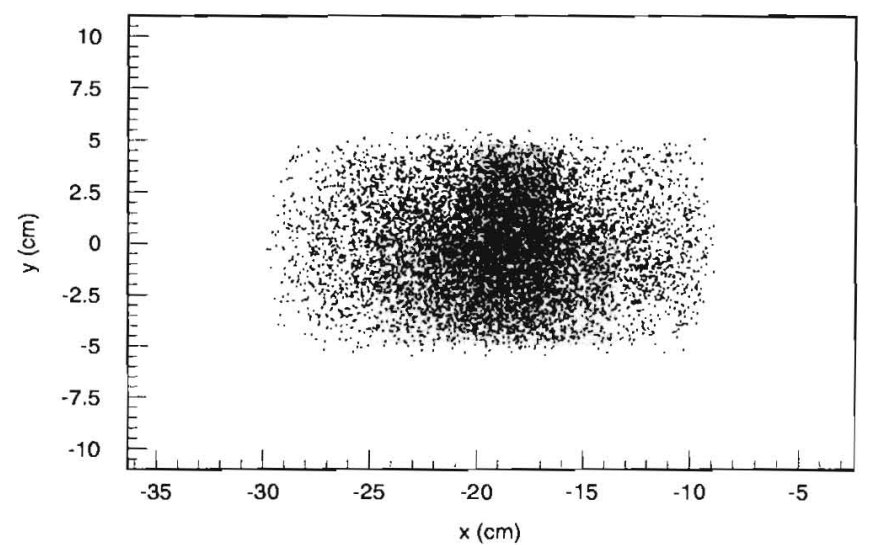

Figure 45: The RF-separated beam at chamber $\mathrm{C} 1$.

\begin{aligned} & \hline \hline Type: $\overline{\text { BM105 }} \\ &$ Horizontal gap: $45.72 \mathrm{~cm} \\ &$ Vertical gap: $15.24 \mathrm{~cm} \\ &$ Length: $182.88 \mathrm{~cm} \\ &$ Field integral: $2.5610 \mathrm{~T} \cdot \mathrm{m} \\ &$\hline\end{aligned}

Table 16: Dogleg and Spectrometer magnet parameters.

\subsubsection{Description of the Wire Chambers}

The UMS wire chambers must survive a maximum rate of $0.9 \mathrm{MHz} / \mathrm{cm}^{2}$ (in the busiest region), reliably, for a two-year run. This is a very high intensity, but not above that encountered in other high-rate experiments. The HyperCP wire chambers built by the University of Virginia (UVa) group ran at a maximum rate of $0.75 \mathrm{MHz} / \mathrm{cm}^{2}$ with no untoward effects, and wire chambers operating at rates of several times $10 \mathrm{MHz} / \mathrm{cm}^{2}$ have successfully been built [148]. Nonetheless, the intensity and size of the RF-separated beam, mandates state-of-the-art wire chambers which push the technology to the limit.

We favor wire chambers as a tracking device for several reasons: they are able to handle the needed rates (more on this below); they have the required spatial and timing resolution; and they present a minimal amount of material to the charged beam. The latter reason is the most compelling. Alternative designs employing silicon, or gas microstrip chambers, present significant amounts of material to the RF-separated beam, degrading the momentum resolution and introducing significant non-Gaussian tails.

A disadvantage of wire chambers is the projective read out, which leads to high channel rates and track finding ambiguities. Space point tracking devices would be preferable and we have investigated the use of silicon pixel devices. Unfortunately, besides the material issue, they suffer from the added disadvantage of lack of a suitable read out. Read out chips that are either presently available or are currently under development are too slow and hence would result in multiple tracks. (Of course the Beam Time Stamp Module would eliminate many of the out-of-time tracks.) Developing a custom read out chip suited for the CKM specifications would be a daunting and expensive task. Wire chambers with pad read out can provide space points - although they too suffer from the problem of additional unwanted material. We are considering fabricating such a 


\begin{aligned} & \hline \hline Number of cathode planes: 6 \\ & Wire orientation: $x:$ vertical \\ &$u:+60^{\circ}$ from vertical \\ &$v:-60^{\circ}$ from vertical \\ &$x, x^{\prime}: 390 \\ & u, u^{\prime}: 342 \\ & v, v^{\prime}: 342 \\ &$ Total: 2148 \\ &$x, x^{\prime}: 18.0 \mathrm{~cm} \\ & u, u^{\prime}: 28.0 \mathrm{~cm} \\ & v, v^{\prime}: 28.0 \mathrm{~cm} \\ &$ Maximum wire lengther of wires $x, x^{\prime}: 9.5 \mathrm{~cm} \\ & u, u^{\prime}: 8.5 \mathrm{~cm} \\ & v, v^{\prime}: 8.5 \mathrm{~cm} \\ &$ Minimum wire length: $0.8 \mathrm{~mm} \\ &$ Wire pitch: Au-coated W \\ & Wire type: $2.5 \mathrm{~mm} \\ &$ Wire diameter: $12 \mu \mathrm{m} \\ &$ Cathode material: $1000 \AA$ Au coated $0.001^{\prime \prime}$ kapton \\ & Gas: $50: 50 \mathrm{CF} 4$-isobutane \\ & \hline \hline\end{aligned}

Table 17: UMS PWC specifications.

chamber that would be placed at the entrance of the UMS, where interactions produce the least harm.

The wire chambers have been designed to survive high rates while providing a robust space point and maximizing the two-track separation resolution. Front and side views of a wire chamber are shown in Fig. 46 and Fig. 47 and an exploded view showing the anode planes is shown in Fig. 49. Table 17 gives the chamber specifications. Each of the six wire cliambers is identical: 6 anode planes of $0.8 \mathrm{~mm}$ pitch: two $x$ planes, two $u$ planes, and two $v$ planes, the $u$ and $v$ planes being rotated by $\pm 60^{\circ}$ from vertical (see Fig. 48). The stereo angles have been chosen to maximize the two-track scparation resolution, which in the worst case is only $0.92 \mathrm{~mm}$. The primed planes are shifted by half a pitcl to improve the chamber resolution and allow drift-time ambiguities to be resolved. Since any $x, u, v$ combination produces a space point the chambers will provide such space points in the presence of inefficiencies.

There are seven cathode planes composed of gold-coated Kapton. Outside of the outer cathode planes are two identical planes used to provide electrostatic balancing for the outer cathode foils. And outside of the outer cathode foils are two more sheets of Al-coated Mylar used as an RF shield. The total thickness of a wire chamber is $0.067 \%$ of an interaction length and $0.21 \%$ of a radiation length (st Table 18).

\begin{tabular}{|l|l|r|r|c|}
\hline \multicolumn{1}{|c|}{ Item } & \multicolumn{1}{c|}{ Material } & Length $(\mathrm{cm})$ & $z / \lambda_{I}$ & $z / X_{0}$ \\
\hline \hline Outer & Al & $2 \cdot\left(2.54 \times 10^{-3}\right)=5.08 \times 10^{-3}$ & $1.29 \times 10^{-4}$ & $5.71 \times 10^{-4}$ \\
windows & Mylar & $2 \cdot\left(2.54 \times 10^{-3}\right)=5.08 \times 10^{-3}$ & $0.82 \times 10^{-4}$ & $1.27 \times 10^{-4}$ \\
\hline Cathode & Au & $7 \cdot\left(1000 \times 10^{-8}\right)=7.00 \times 10^{-5}$ & $0.07 \times 10^{-4}$ & $2.05 \times 10^{-1}$ \\
foils & Kapton & $7 \cdot\left(2.54 \times 10^{-3}\right)=1.78 \times 10^{-2}$ & $2.94 \times 10^{-4}$ & $6.22 \times 10^{-4}$ \\
\hline Gas & $50 \%$ CF4, 50\%Iso. & 4.0 & $1.52 \times 10^{-4}$ & $3.47 \times 10^{-4}$ \\
\hline Wires & W & $6 \cdot \pi\left(12 \times 10^{-4} / 2\right)^{2}(12.5)=8.46 \times 10^{-5}$ & $0.09 \times 10^{-4}$ & $2.42 \times 10^{-4}$ \\
\hline \hline \multicolumn{2}{l|}{ Total: } & & $6.7 \times 10^{-4}$ & $2.1 \times 10^{-3}$ \\
\hline
\end{tabular}

Table 18: Wire chamber material. 


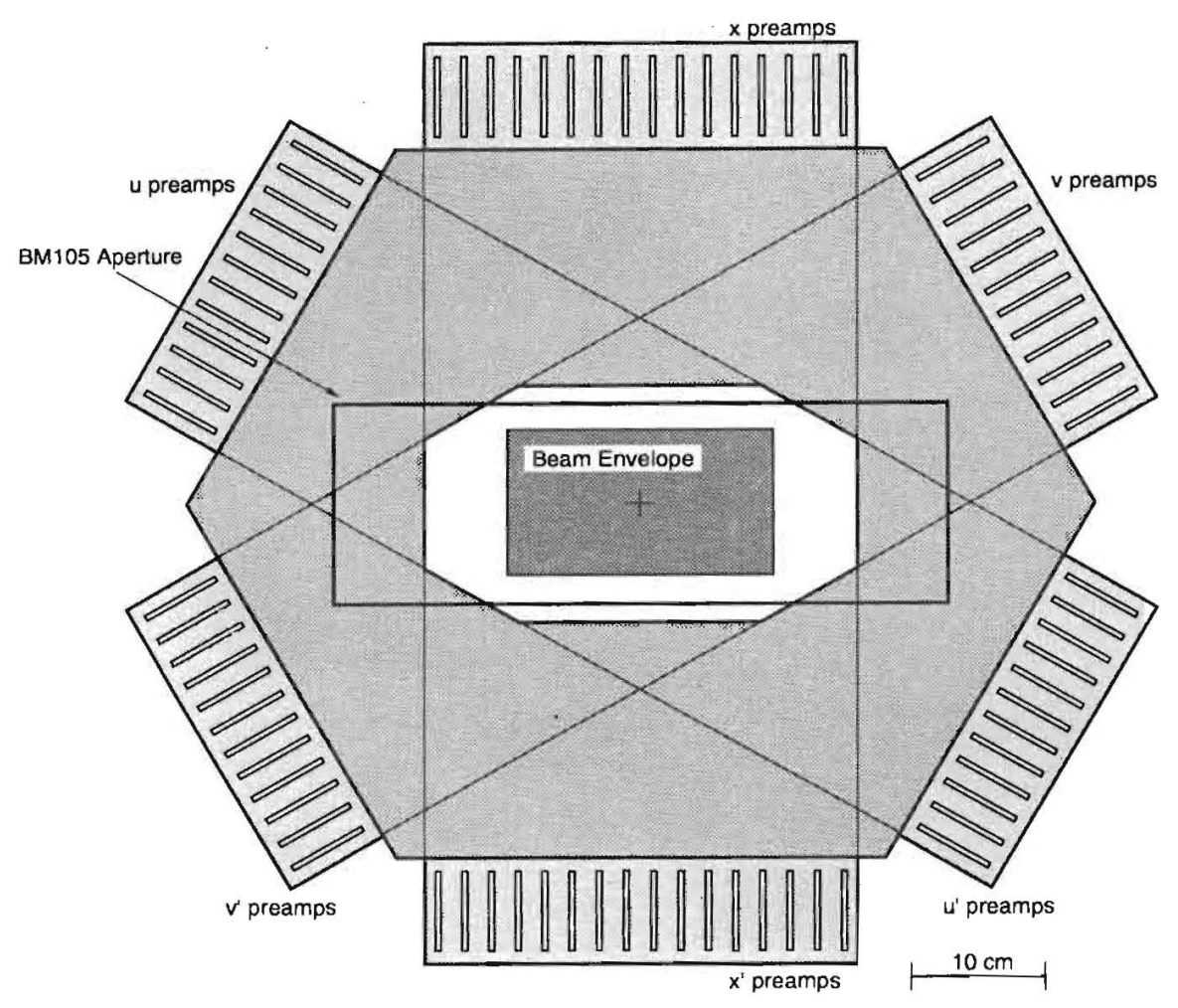

Figure 46: Front view of a UMS wire chamber.

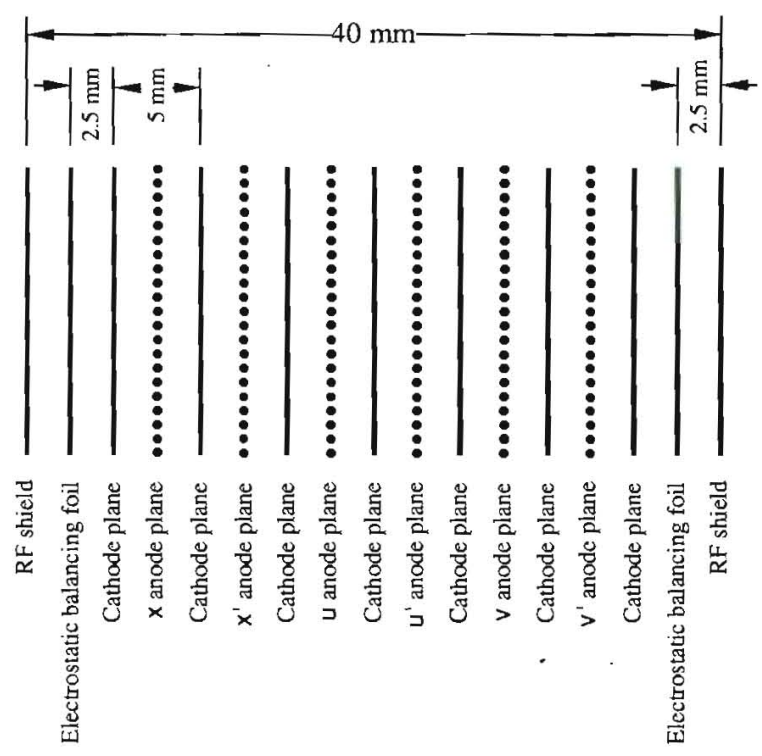

Figure 47: Side view of a UMS wire chamber showing the cathode and anode plane arrangement.
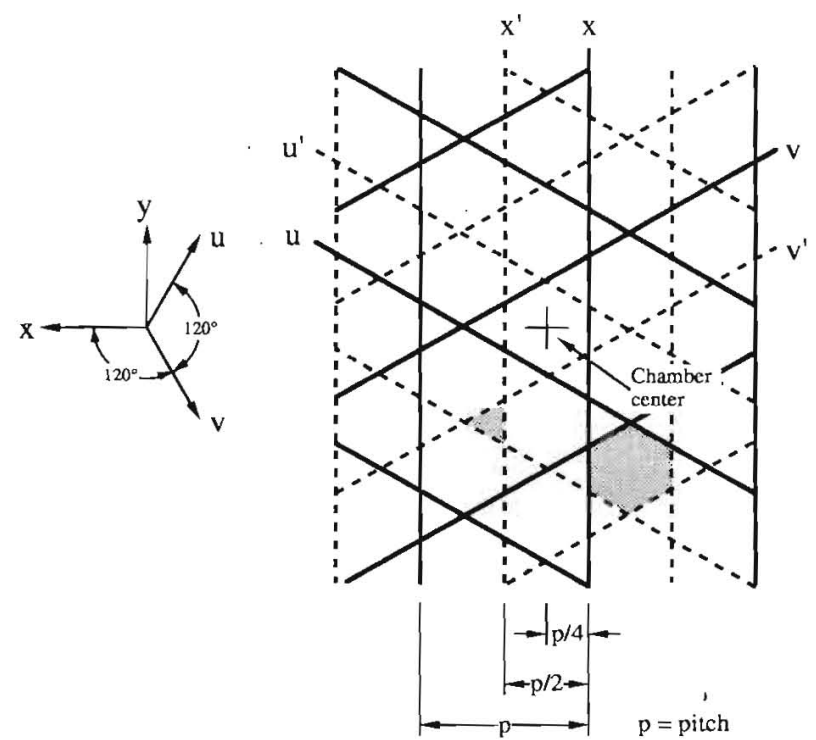

Figure 48: Magnified view of the center of a UMS wire chamber showing the wire geometry. The primed planes are shown as dashed lines. 


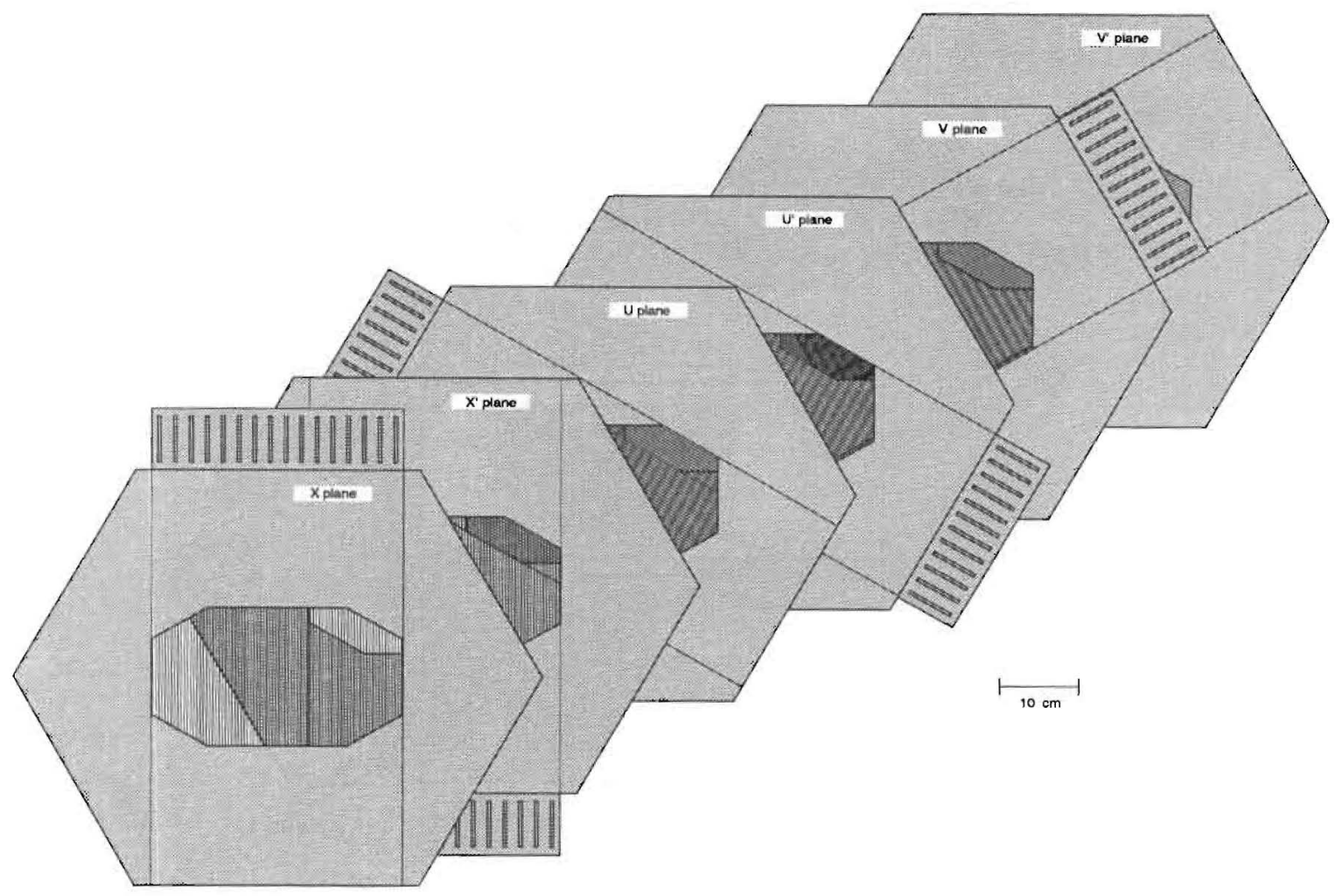

Figure 49: Exploded view of a UMS wire chamber showing the anode planes.

With a $0.8 \mathrm{~mm}$ pitch the maximum rate per wire is expected to be $0.61 \mathrm{MHz}$. To reduce space charge and aging effects the wire chambers will have a small anode-to-cathode gap $(2.5 \mathrm{~mm})$ and will be operated at a very low total avalanche gain of about $\sim 2 \times 10^{4}$. At such a gain the maximum amount of charge accumulated on a wire - the maximum rate estimated to be $0.9 \mathrm{MHz} / \mathrm{cm}^{2}$ - will be $0.005 \mathrm{C} / \mathrm{cm}$ in a run of two years $\left(2 \times 10^{7} \mathrm{~s}\right)$. This is well within the accepted limit of $\sim 1 \mathrm{C} / \mathrm{cm}$ for $25 \mu \mathrm{m}$ wires in traditional chamber gases. A so-called "fast-gas" mixture of 50:50 CF4-isobutane will be used. Besides its speed this mixture has been found to have very good ageing properties.

To facilitate precision alignment the wire chambers will be mounted on two tables, one in front and one behind the Spectrometer magnet. To reduce tracking errors due to ghost tracks, chambers $\mathrm{C} 2$ and $\mathrm{C} 5$ will be tilted at $+15^{\circ}$ and chambers $\mathrm{C} 3$ and $\mathrm{C} 6$ at $-15^{\circ}$.

\subsubsection{Performance of similar $\mathrm{HyperC}^{P}$ chambers}

The UVa group built for the HyperCP (E871) experiment high-rate wire chambers very similar to these proposed for CKM. They performed exceedingly well, with only one broken wire in about 12 months of higll-intensity operation, and no sign of any drop in gain over their course of operation. They had a $1 \mathrm{~mm}$ pitch, $3.0 \mathrm{~mm}$ anode-cathode gap, and four anode planes: $x, x^{\prime}, u$, and $v$. The channeled beam in HyperCP had a routine intensity of $13 \mathrm{MHz}$ over an area of about $66 \mathrm{~cm}^{2}$, with a rate of $0.27 \mathrm{MHz} / \mathrm{cm}^{2}$ in the busiest region - one-third the maximum rate anticipated for the UMS. Higher intensity data were taken, up to rates of $0.75 \mathrm{MHz} / \mathrm{cm}^{2}$ and $0.64 \mathrm{MHz} /$ wire, and these data show a small drop in efficiency consistent with that expected from the electronic dead-time (see Fig. 50). The limitation on the channeled beam intensity in HyperCP was not the wire chambers, but the trigger and DAQ. 


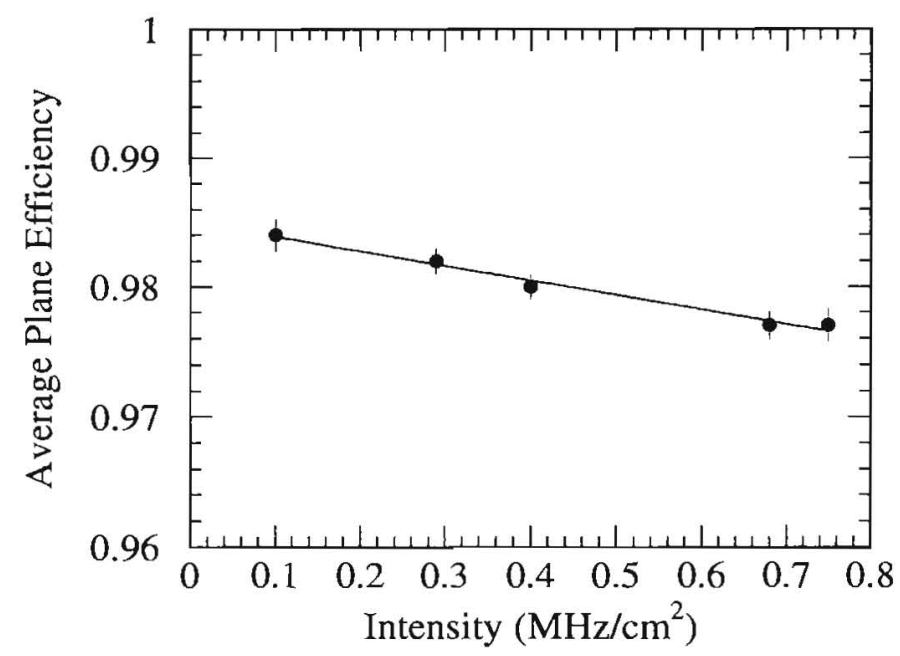

Figure 50: Hyper $\dot{C}$ P chamber 1 efficiency vs intensity.

In the 1997 HyperCP run an 80:20 argon-ethane gas mixture was originally employed, which worked well with no untoward effects. Nevertheless, to improve the chamber speed, after about a month of rumning we switched to a mixture of 50:50 CF4-isobutane because of its superior timing characteristics as well as its reputed favorable ageing properties. (See Fig. 51 for a comparison of the two gas mixtures.) Despite the need to increase the chamber voltage by about $200 \mathrm{~V}$, the chambers performed exceedingly well with this gas mixture. Periodic gain tests with an ${ }^{55} \mathrm{Fe}$ source showed no sign of gain drop off during either the 1997 or the 1999 runs (see Fig. 52).

\subsubsection{Expected Performance of the UMS}

The UMS performance has been modeled using a detailed GEANT-based Monte Carlo simulation coupled with an existing tracking program. modified to the UMS specifications. The simulation includes multiple scattering, interactions, chamber inefficiencies, and multiple hits. The tracking program is projection-based ${ }^{3}$ and heuce is not optimal for multi-track events. A space-point based tracking program is being written which should improve the ability to resolve events with multiple tracks.

The momentum resolution of the spectrometer is shown in Figures 53, 54, and 55 which give the reconstructed momentum of 50,000 Monte Carlo tracks generated with exactly $22.0 \mathrm{GeV} / \mathrm{c}$ momentum, with respectively: no multiple scattering, multiple scattering, and multiple scattering and event pile-up. The spectrometer resolution with no multiple scattering is $\sigma / p=0.15 \%$, which increases to $\sigma / p=0.18 \%$ with the addition of multiple scattering.

It is anticipated that the mean number of tracks per event will be 1.53 , assuming a $51 \mathrm{MHz}$ rate and a (conservative) $30 \mathrm{~ns}$ time resolution. Track finding errors for multi-track events will degrade the momentum resolution. We have estimated the effect of track pile-up on the momentum resolution by generating events with a Poisson track nultiplicity with a mean of 1.53 , and find it to be $\sigma / p=0.20 \%$, over a factor of two better than the benchmark value of $0.50 \%$. In addition to this slight increase in the width of the distribution with event pile-up is an increase in the tails as well.

\footnotetext{
${ }^{3}$ A projection based tracking program has been used because: 1) one existed that could be easily modified, and 2) projection tracking is more general, and can be used for other chamber geometries that we have modeled that do not provide space points.
} 


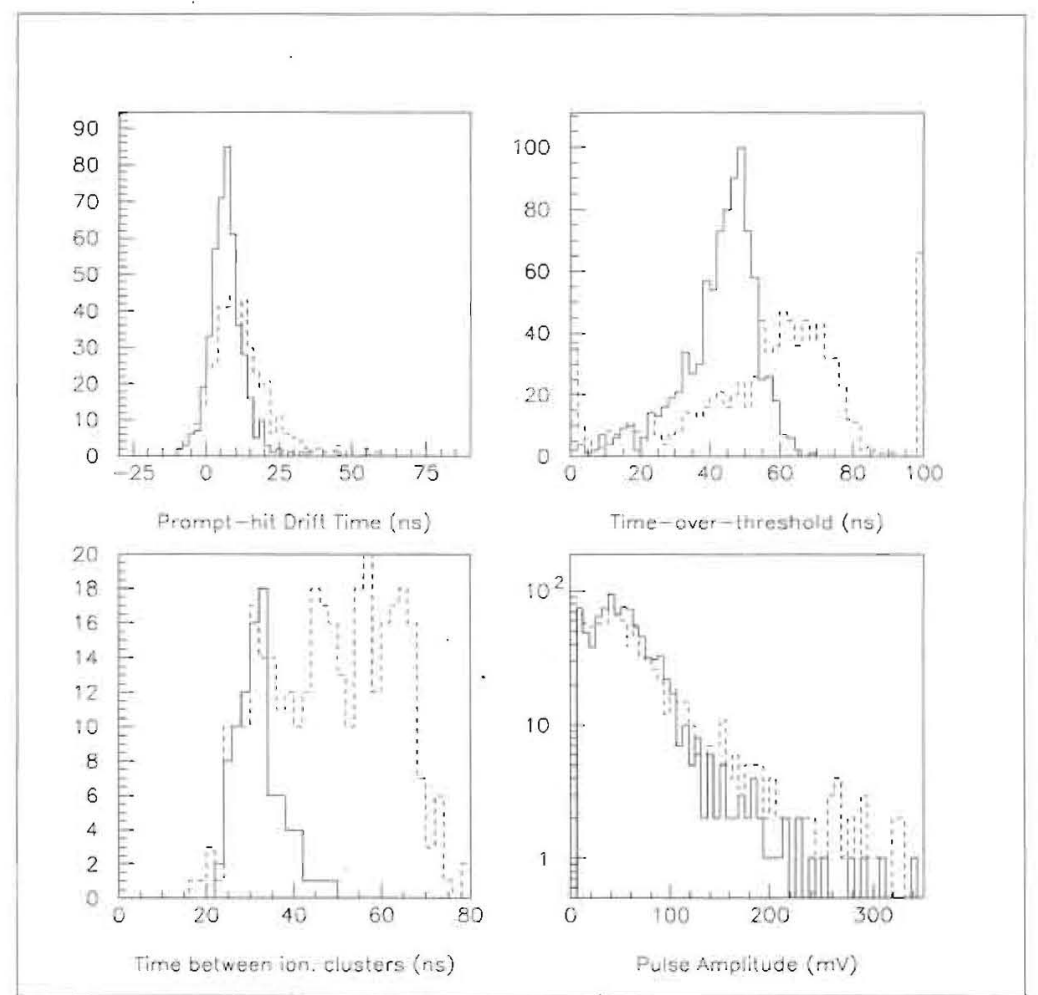

Figure 51: Comparison of CF4-isobutane (solid) with Argon-ethane (dashed) for the HyperCP wire chambers.

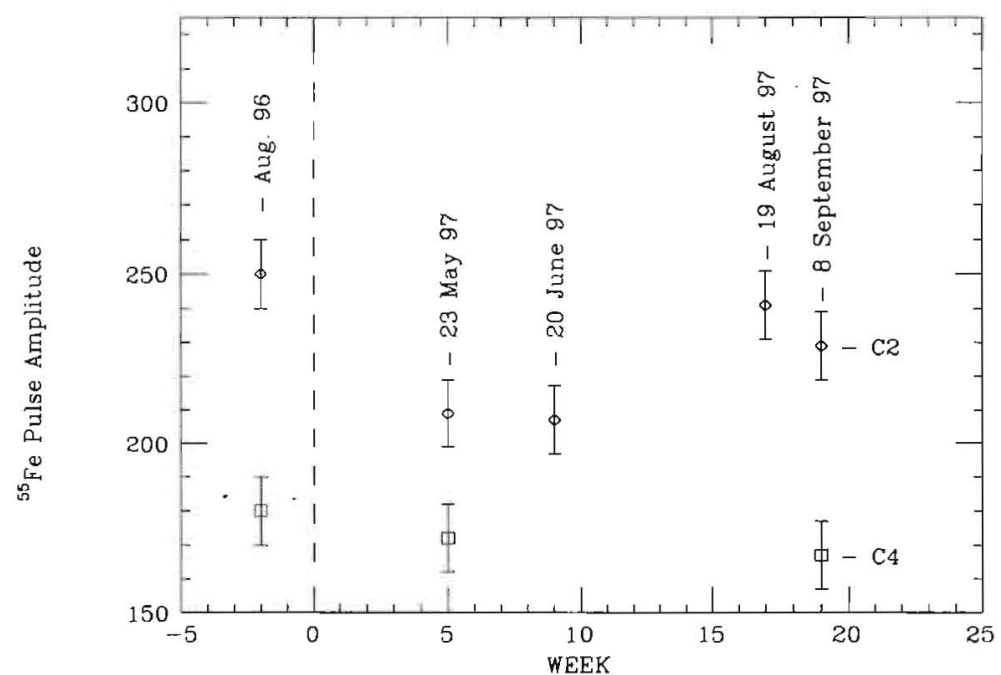

Figure 52: Gain vs time for HyperCP chambers $\mathrm{C} 2$ and $\mathrm{C} 4$, measured with an ${ }^{55} \mathrm{Fe}$ source. 


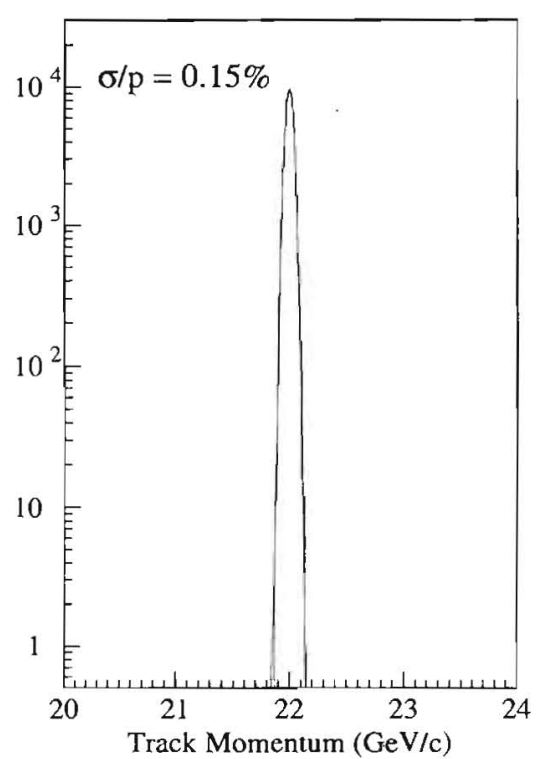

Figure 53: Momentum resolution without multiple scattering.

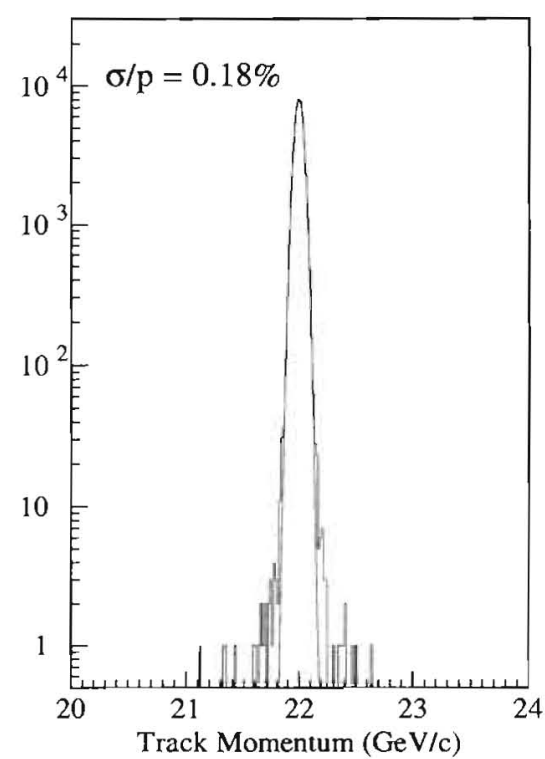

Figure 54: Momentum resolution with multiple scattering.

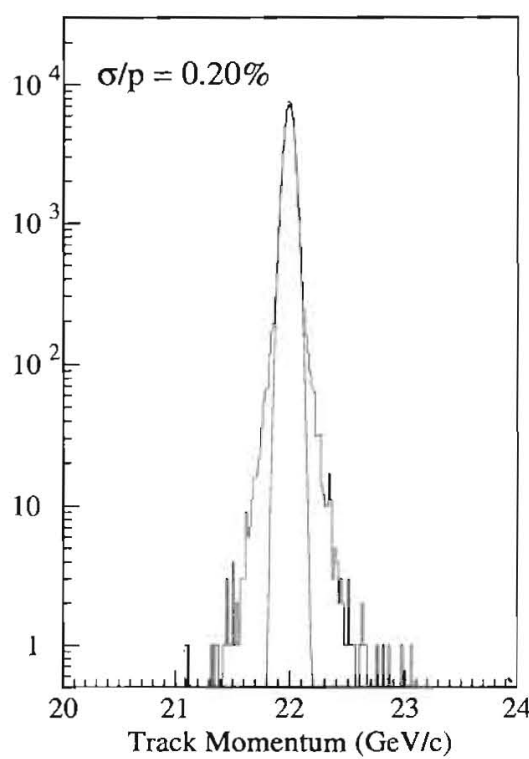

Figure 55: Momentum resolution with 1.53 tracks per event.

\begin{tabular}{|l|l|r|r|r|}
\hline & \multicolumn{1}{|c|}{ Matem } & \multicolumn{1}{c|}{ Length $(\mathrm{cm})$} & $z / \lambda_{I}$ & \multicolumn{1}{c|}{$z / \mathrm{X}_{0}$} \\
\hline \hline Vacuum pipe exit window & Titanium & $2.54 \times 10^{-2}$ & $7.13 \times 10^{-3}$ & $0.92 \times 10^{-3}$ \\
Beam Time Stamp Module (BTSM) & Polystyrene & $1.57 \times 10^{-1}$ & $1.98 \times 10^{-3}$ & $3.70 \times 10^{-3}$ \\
Dogleg magnet He bag & Mylar/He & $0.002^{\prime \prime} / 285.0 \mathrm{~cm}$ & $8.36 \times 10^{-4}$ & $7.16 \times 10^{-4}$ \\
\hline C1 & - & - & $6.73 \times 10^{-4}$ & $2.11 \times 10^{-3}$ \\
C1-C2 He bag & Mylar/He & $0.002^{\prime \prime} / 200.0 \mathrm{~cm}$ & $6.30 \times 10^{-4}$ & $5.55 \times 10^{-4}$ \\
C2 & - & - & $6.73 \times 10^{-4}$ & $2.11 \times 10^{-3}$ \\
C2-C3 He bag & Mylar/He & $0.002^{\prime \prime} / 200.0 \mathrm{~cm}$ & $6.30 \times 10^{-4}$ & $5.55 \times 10^{-4}$ \\
C3 & - & - & $6.73 \times 10^{-4}$ & $2.11 \times 10^{-3}$ \\
Spectrometer magnet He bag & Mylar/He & $0.002^{\prime \prime} / 285.0 \mathrm{~cm}$ & $8.36 \times 10^{-4}$ & $7.16 \times 10^{-4}$ \\
C4 & - & - & $6.73 \times 10^{-4}$ & $2.11 \times 10^{-3}$ \\
C4-C5 He bag & Mylar/He & $0.002^{\prime \prime} / 200.0 \mathrm{~cm}$ & $6.30 \times 10^{-4}$ & $5.55 \times 10^{-4}$ \\
C5 & - & - & $6.73 \times 10^{-4}$ & $2.11 \times 10^{-3}$ \\
C5-C6 He bag & Mylar/He & $0.002^{\prime \prime} / 200.0 \mathrm{~cm}$ & $6.30 \times 10^{-4}$ & $5.55 \times 10^{-4}$ \\
C6 & - & - & $6.73 \times 10^{-4}$ & $2.11 \times 10^{-3}$ \\
\hline Subtotal (spectrometer only): & - & $0.7 \times 10^{-2}$ & $1.6 \times 10^{-2}$ \\
\hline Grand Total: & - & $1.7 \times 10^{-2}$ & $2.1 \times 10^{-2}$ \\
\hline
\end{tabular}

Table 19: BTSM and UMS spectrometer material. 
We expect the tails to be significantly reduced by use of a space-point tracker and the inclusion of the TDC information.

The angular resolution of the spectrometer for downstream tracks is shown in Fig. 56 which gives the angle between the Monte Carlo and reconstructed tracks exiting the UMS. With no multiple scattering the mean value is $47 \mu \mathrm{rad}$ - multiple scattering increases the mean error to $54 \mu \mathrm{rad}$.

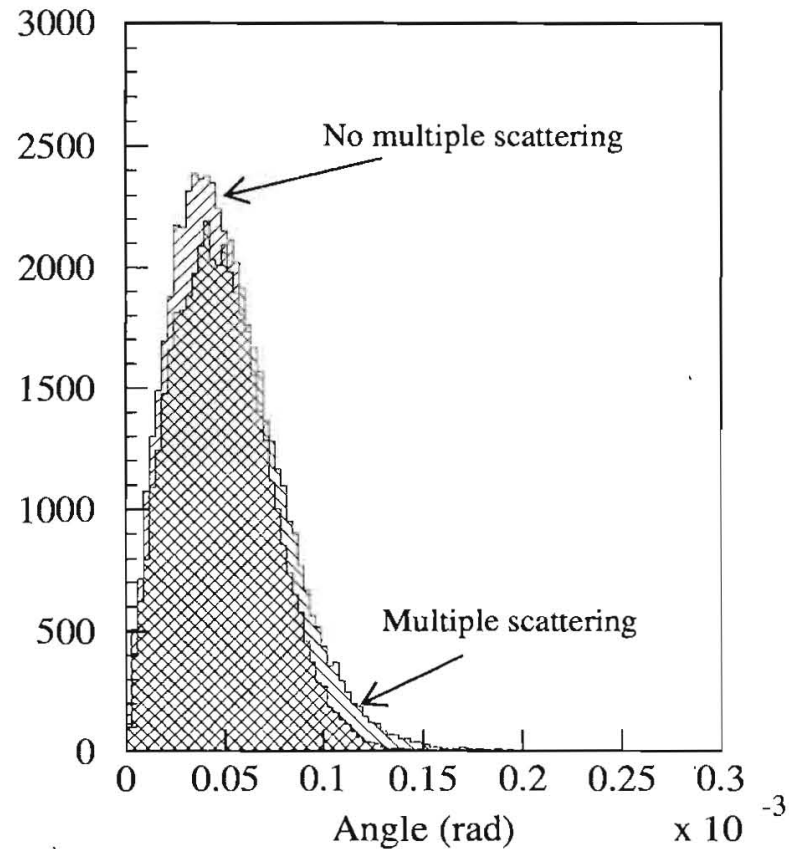

Figure 56: Angular resolution of the downstream tracks in the UMS.

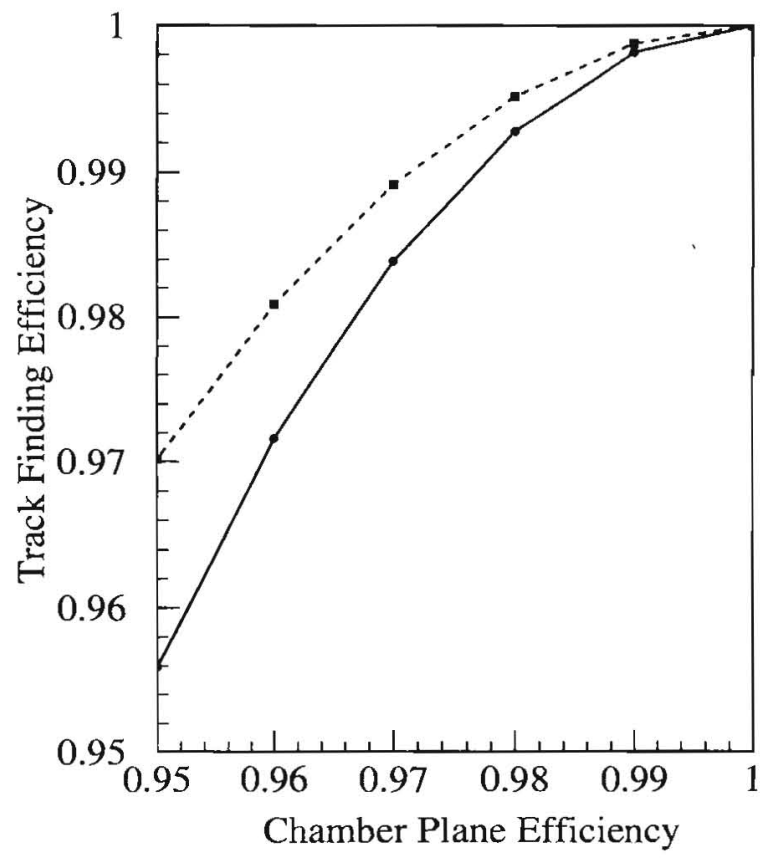

Figure 57: Track finding efficiency vs average single-plane efficiency for the two algorithms described in the text.

Assuming an electronic dead-time of about $25 \mathrm{~ns}$, the expected chamber plane efficiency will be about $97 \%$ in the busiest region, and higher elsewhere. The corresponding track finding efficiency depends on the algorithm used. If space points (with one of each of $x, u, v$ planes) are required in all chambers then the solid curve in Fig. 57 gives the corresponding track finding efficiency. If $x, u, v$ space points are required only for the seed planes, and any three planes for the confirming chamber's (C2 and $\mathrm{C} 5$ ), then the dashed curved in Fig. 57 gives the track finding efficiency. In either case the tracking efficiency is better than $98 \%$ for a chamber plane efficiency of $97 \%$. We expect the average chamber plane efficiency to be about $98 \%$ with a corresponding track finding efficiency of greater than $99 \%$.

Note that our simulation does not include timing information, which we estimate will improve the individual plane resolution by about a factor of two to around $100 \mu \mathrm{m}$. Although this will improve both the momentum and spatial resolution of the spectrometer, the timing information will be of greatest help in resolving track finding ambiguities in multi-track events. The BTSM, which has not been used in the simulation, will also help in this respect.

\subsubsection{Wire Chamber Fabrication}

The detailed design of the wire chambers is being done with Auto-CAD at UVa. The wire chambers will be fabricated at Fermilab and UVa, in a similar manner to the HyperCP wire cliambers. 
Exceptional care must be taken in the fabrication if the chambers are to perform at the desired rates with good efficiencies.

In chambers having a narrow anode-cathode gap one can only achieve a uniform gain across the anode plane by keeping the gap spacing as uniform as possible. Our past experience is that vendors have difficulty in achieving the required tolerance of a $\pm 25 \mu \mathrm{m}$ modulation in the plane thickness. Hence we will order thicker planes than needed and have them ground down by a commercial vendor. The anode and cathode planes will be machined on the Thermwood machines at Fermilab, a process we expect to take 8 weeks. The Al chamber frame and preamp card cages will be machined at UVa while the front and rear chamber tables will be fabricated at Fermilab.

The anode planes will be strung at Fermilab using the small winding machine in Lab 6, after which they will be shipped out to UVa. We expect the winding to take 12 weeks. We do not anticipate the need for support wires: the longest wires are $28 \mathrm{~cm}$, just under the $32 \mathrm{~cm}$ theoretical maximum length for our anode-cathode gap and wire diameter. Cathode foil stretching will be done at UVa using a jig (built by Carl Lindenmeyer) borrowed from Lab 8, as was done for the Hyper $C P$ wire chambers. Finally chamber assembly and testing will be done at UVa.

A prototype wire chamber is being designed at UVa using Auto-CAD in order to achieve compatibility with the NC machines at Fermilab, which will be used to machine the anode and cathode frames. Fabrication is expected to begin this summer and the chamber will be tested in the summer of 2002 in a Fermilab test beam. With this beam test we wish to: 1) test the ability of the chamber to operate reliably at the anticipated CKM rates, 2) find the ultimate rate limit of the wire chamber, and 3) measure the resolution of the chamber.

It is vital that a fully instrumented wire chamber be tested as only then can collective effects be tested. For example, the HyperCP wire chambers were practically impervious to oscillations when only partially instrumented, but needed careful grounding to avoid oscillations when fully instrumented. Unless we decide to use the existing Hyper $C P$ preamplifiers, we will not have enough electronics by the summer of 2002 , and would expect the full chamber test to be performed in the summer of 2003.

\subsubsection{Wire Chamber Electronics}

The UMS front-end electronics consists of an amplifier followed by a discriminator mounted on the chamber. In view of the inverse relationship between noise and bandwidth there are two somewhat opposing requirements for the amplifier. The system noise when the amplifier is connected to the detector must be minimal since, in either the wire chamber (or Si $\mu$-strip options) the expected signal size is of order $10^{4} \mathrm{e}$. Furthermore the sliaping time should be limited to the range of $\sim 10 \mathrm{~ns}$ due to the high rates $\left(\sim 1 \mathrm{MHz} / \mathrm{cm}^{2}\right)$ in the detector. Currently these requirements are best met by bipolar technology rather than MOS.

To illustrate what is achievable Table 20 lists bench-test measurements made by the UVa group of several bipolar preamplifiers built from commercial ICs and two ASICs; MB43458 (Fujitsu) and MQS104 (LeCroy). All circuits, with the exception of the MB43458, had shaping to cancel the ion-tail occurring in wire chamber current waveforms.

The PWCs of the HyperCP experiment employed the MQS104 because of its relatively low cost, good performance characteristics, and relatively high channel density. The uniformity of these chips is shown in Fig. 58. The output of the amplifier was conveyed through about $30^{\prime}$ of ribbon cable to discriminators located nearby in the experimental hall. Final performance characteristics measured on the detector are given in Table 21.

The HyperCP amplifiers and discriminators could be used for the CKM UMS. However there are some undesirable features of these parts. The time-over-threshold, which is mainly a property 


\begin{tabular}{|l|l|r|r|r|r|r|}
\hline \multicolumn{1}{|c|}{ Amplifier } & Configuration & \multicolumn{1}{c|}{$T_{R}$} & \multicolumn{1}{c|}{ FWHM } & Gain & ENC 5pf & ENC 27pf \\
\hline \hline E690/Nevis & CE & $4 \mathrm{~ns}$ & $8 \mathrm{~ns}$ & $1 \mathrm{mV} / \mathrm{fC}$ & $3,000 e$ & $5,000 e$ \\
\hline E711/MB43458 & $\mathrm{CB}$ & $6 \mathrm{~ns}$ & $20 \mathrm{~ns}$ & $0.35 \mathrm{mV} / \mathrm{fC}$ & $3,500 e$ & $5,500 e$ \\
\hline "Home-built" & $\mathrm{CB}$ & $6 \mathrm{~ns}$ & $11 \mathrm{~ns}$ & $19 \mathrm{mV} / \mathrm{fC}$ & $1,500 e$ & $2,700 e$ \\
\hline LRS MQS104TB & CE cascade & $11 \mathrm{~ns}$ & $20 \mathrm{~ns}$ & $18 \mathrm{mV} / \mathrm{fC}$ & $1,800 e$ & $3,000 e$ \\
\hline LM359 & CE cascade & $10 \mathrm{~ns}$ & $20 \mathrm{~ns}$ & $67 \mathrm{mV} / \mathrm{fC}$ & $2,000 e$ & $2,900 e$ \\
\hline
\end{tabular}

Table 20: Preamplifier bench-test comparisons.

\begin{aligned} & \hline Avalanche Gain $1.6 \times 10^{4} \\ &$ M.I.P. signal $7 \times 10^{4} e$ average \\ & Threshold $1.5 \times 10^{4} e \\ &$ Noise rate $<100 \mathrm{~Hz} \\ &$ Tirne-over-threshold $\leq 50 \mathrm{~ns} \\ &$\hline \hline\end{aligned}

Table 21: HyperCP PWC electronic specifications.
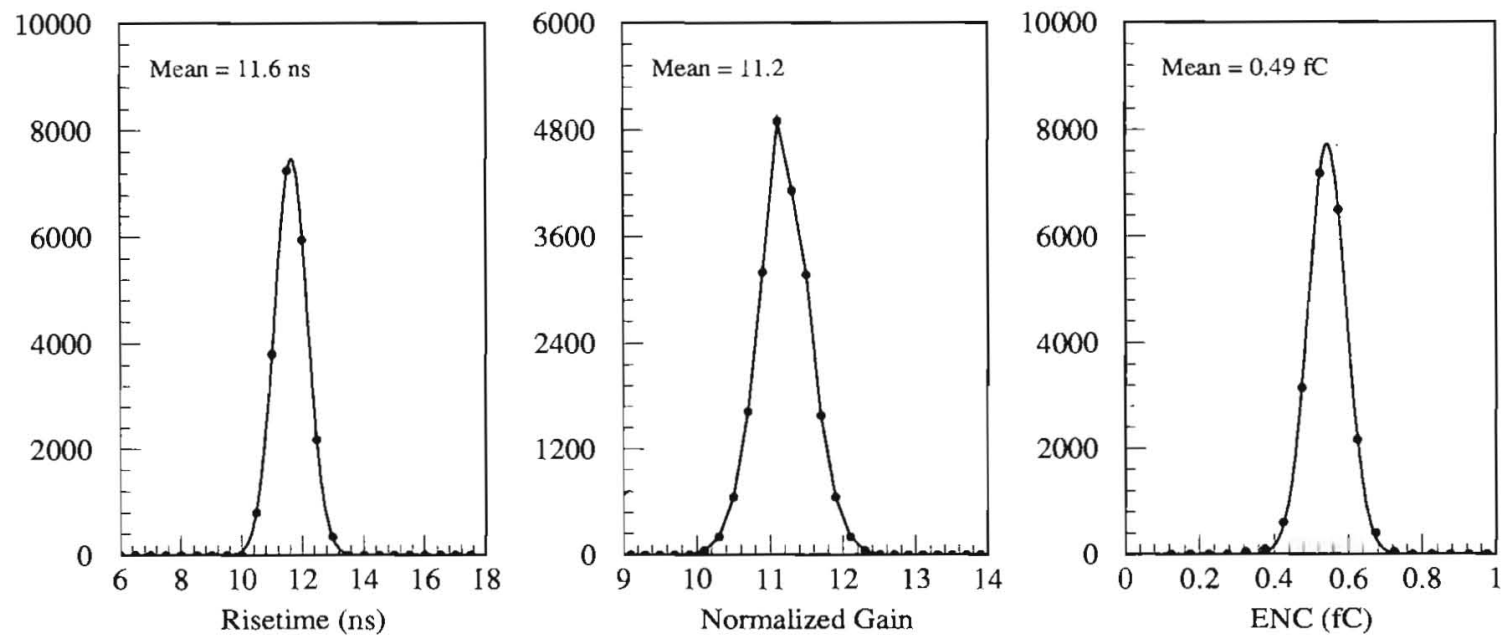

Figure 58: UVa Hyper $C P$ preamplifier test results. Shown is the risetime, gain and equivalent noise charge for 20,800 chanuels. 
of the amplifier's bandwidth, is somewhat longer than desirable. When the amplifier is followed by a simple leading edge discriminator, as in $H_{y p e r} C$, one can anticipate a pile-up induced inefficiency of about $5 \%$ at the beam flux envisaged in CKM. The amplifier and discriminator were separated by cable which, although good for system stability, creates more interconnections and reliability problems than a fully integrated solution. The power dissipation is somewhat high at about $180 \mathrm{~mW}$ and $250 \mathrm{~mW}$ per channel respectively for the amplifier and discriminator. Some forced air cooling would be necessary at the channel density of the UMS chambers. An improvement over the HyperCP amplifier/discriminator would be the ASDQ ASIC chip developed for the CDF Central Outer Tracker (COT) of Run II. The reported noise performance of this chip is comparable to the MQS104 but the pulse shaping is about a factor of three more narrow than achieved with preamplifier plus cable in Hyper $C P$. As in many large systems employing many channels of sensitive and high bandwidth electronics packed in close proximity, we would need to instrument an entire chamber with the ASDQ to verify its stability and sensitivity in our situation. 


\subsection{The Beam Time Stamp Module}

The purpose of the Beam Time Stamp Module (BTSM) is to tag the time of each beam track with a precision of about $1 \mathrm{~ns}$. (Recall that the RF-separated beam is not bunched and that the average time between tracks will be about 20 ns.) Parameters of the BTSM are given in Table 22 below.

\begin{aligned} & \hline Active area: $25.5 \times 16.0 \mathrm{~cm}^{2} \\ &$ Effective thickness: $0.16 \mathrm{~cm} \\ &$ Number of fibers: $512 \mathrm{in}$ two layers \\ & Fiber diameter: $1.0 \mathrm{~mm} \\ &$ Fiber type: Kuraray SCSF-3HF or Bicron BCF-10 \\ & Read Out: $8 \mathrm{Hamamatsu} \mathrm{H} 754664-\mathrm{channel} \mathrm{PMTs} \\ &$ Expected photo-electron yield: $8(\mathrm{SCSF}-3 \mathrm{HF})$ or $13(\mathrm{BCF}-10) \\ &$ Time resolution: $\sim 1 \mathrm{~ns} \\ &$\hline\end{aligned}

Table 22: Beam Time Stamp Module parameters.

The BTSM is placed just after the Dogleg magnet and in front of the first UMS wire chamber (see Fig. 43). The layout of the BTSM is shown in Fig. 59. It consists of two layers of 256 scintillating fibers, each fiber oriented vertically. The fibers are $1.0 \mathrm{~mm}$ in diameter, giving a module width of $25.5 \mathrm{~cm}$. The entire vertical gap of the Dogleg magnet, $15.24 \mathrm{~cm}$, is covered by the fibers. The fibers are read out on the top end; their bottom end has an $\mathrm{Al}$ reflective coating.

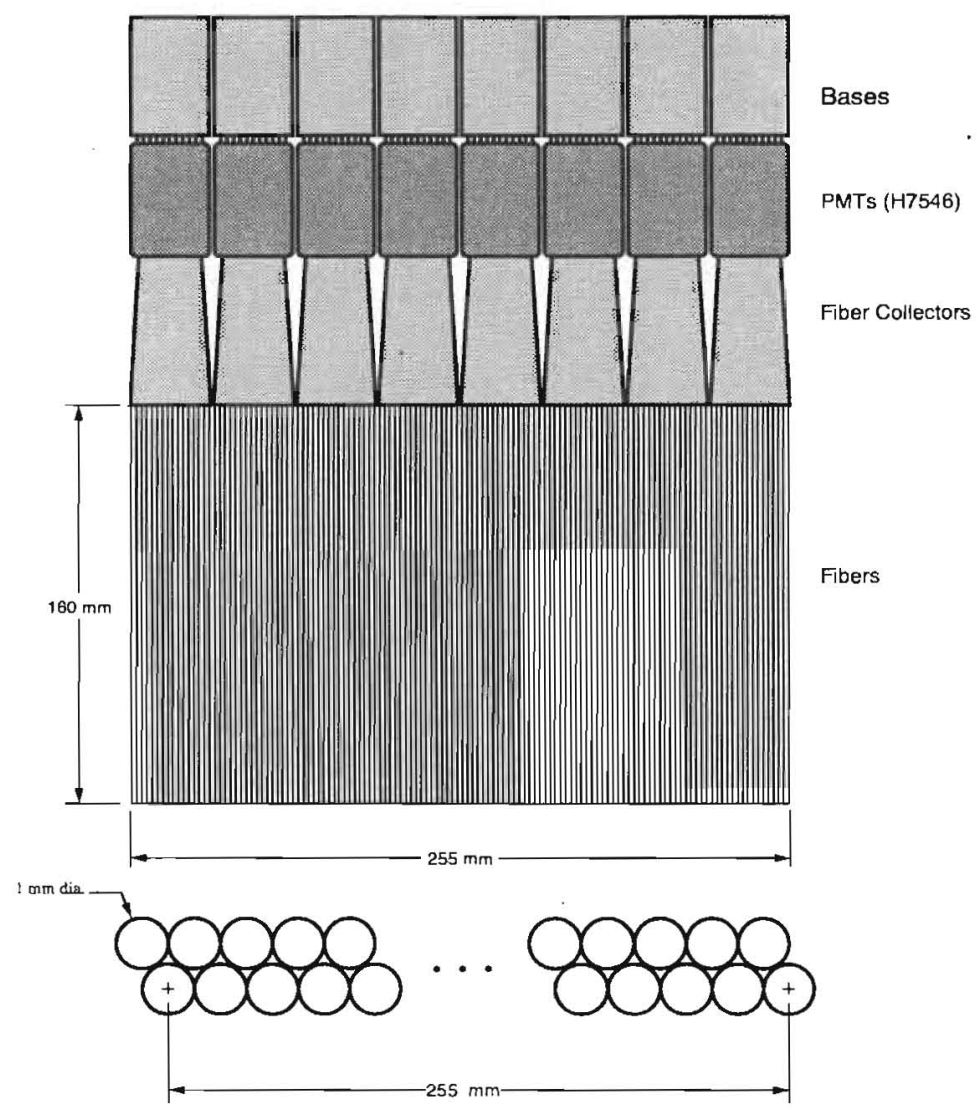

Figure 59: The Beam Time Stamp Module read out with a Hamamatsu multi-anode PMT. 
The scintillating fibers will not be mated to longer attenuation length clear fibers to bring the light to the photo-detectors, as if often done, because the fibers are sufficiently short and since the region where the fibers are 'scrambled' to place them with the proper PMT pixel is shielded by the magnet steel. Mating schemes typically produce a loss of 10 to $20 \%$ in photo-electron yield.

The photodetector for the BTSM must have good quantum efficiency, high gain, high rate capability, and excellent time resolution. Because of the large fiber density it should also be compact and reasonably inexpensive. Both multianode photomultipliers (PMT) and visible light photon counters (VLPC) satisfy these criteria.

The VLPC has the advantage of a very high quantum efficiency which extends out into the red: at $\sim 60 \%$ about three times that of a typical PMT. They have excellent gain (around $10^{6}$ ) and are currently produced in chips with $2 \times 4$ pads for a convenient conmection to the fibers. Their main disadvantage - unfortunately a non-trivial one - is their low operating temperature of $6-7 \mathrm{~K}$, which requires liquid helium cooling.

Because of the very short fiber length in the BTSM, attenuation is not a serious problem, and multianode PMTs, despite their low quantum efficiency relative to VLPCs, present a viable read out solution. The Hamamatsu H7546, 64-channel multianode photomultiplier [150], is particularly attractive. This new multianode PMT has a metal channel dynode, an $8 \times 8$ anode array, good gain, high speed response, and low cross-talk. It has a quantum efficiency of only $20 \%$ at $432 \mathrm{~nm}$ and $9 \%$ at $530 \mathrm{~nm}$ (with a standard non-green-extended photocathode). See Table 23 for its characteristics. It also has compact cross section of $3.0 \times 3.0 \mathrm{~cm}^{2}$, allowing the photomultipliers to be placed near the fibers, as shown in Fig. 59. Rate effects on the photomultiplier gain do not appear to be a concern: we estimate that the mean anode current of the busiest PMT will be only $\sim 12 \mu \mathrm{A}$. This is well below the limit of conventional photomultipliers - in HyperCP we saw no untoward effects on the gain with anode currents up to $300 \mu \mathrm{A}$ with a Hamamatsu R329 photomultiplier using a high-rate transitorized base. Tests will be done to see how much anode current can be tolerated before the gain starts falling. If it is a problem simple solutions are to balance the rate over all the PMTs or to use more photomultipliers.

We currently favor the multianode PMT read out over the VLPC and will use it if tests give the desired photoelectron yield.

\begin{aligned} & \hline Anodes: $8 \times 8=64 \\ &$ Quantum efficiency: $20 \%$ (at $432 \mathrm{~nm}$ ), 9\% (at $530 \mathrm{~ns}$ ) \\ & Gain: $3.0 \times 10^{5} \\ &$ Anode pulse rise time: $1.5 \mathrm{~ns} \\ &$ Transit time spread (FWHW): $0.3 \mathrm{~ns} \\ &$ Cross-talk (with 1 mm optical fiber): $2 \% \\ &$ Pulse linearity per channel ( $\pm 5 \%$ deviation): $0.6 \mathrm{~mA} \\ &$ Uniformity among all anodes: $1: 3 \\ &$\hline\end{aligned}

Table 23: Hamamatsu H7546 multi-anode photomultiplier characteristics.

The scintillating fibers must survive a high continuous fluence of charged particles. In one year of running $\left(1 \times 10^{7} \mathrm{~s}\right)$ the detector will receive $2.8 \mathrm{kGy}(0.28 \mathrm{MRad})$ of radiation. This is within the limits of radiation hard green-shifted scintillating fibers, such as those based on the 3HF wave shifter.

Nevertheless we are considering using a non-green-shifted fiber, such as Bicron's BCF-10 [149] (see Table 24) for several reasons. It is emits light where photomultipliers have good quantum efficiency and is faster than $3 \mathrm{HF}$-based fibers. The disadvantage of such a fiber is its propensity toward radiation damage. However, because the BTSM fiber length is quite short even a relatively 
large decrease in the attenuation length will not produce a significant loss in photo-electron yit $\mathrm{A}$. Also, given the modest amount of fiber needed for the BTSM, it is entirely feasible to periodically replace with fibers when damaged. We have not yet been able to find any suitable studies of radiation damage to $\mathrm{BCF}-10$ fibers.

Should the radiation damage prove to be a show-stopper then we will use the Kuraray SCSF$3 \mathrm{HF}$ scintillating fiber (see Table 25) [153] which will certainly be able to survive the anticipated radiation dose.

\begin{aligned} & \hline \hline Type: multi-clad \\ & Emission peak: $432 \mathrm{~nm} \\ &$ Decay time: $2.7 \mathrm{~ns} \\ &$ Attenuation length: $1.9 \mathrm{~m} \\ &$ Number of photons per MeV: $\sim 8000 \\ &$\hline \hline\end{aligned}

Table 24: Bicron BCF-10 scintiliating fiber parameters.

\begin{aligned} & \hline \hline Type: multiclad \\ & Emission peak: $530 \mathrm{~nm} \\ &$ Decay time: $8.2 \mathrm{~ns} \\ &$ Attenuation length: $5.5 \mathrm{~m} \\ &$ Number of photons per MeV: $\sim 10000 \\ &$\hline \hline\end{aligned}

Table 25: Kuraray SCSF-3HF scintillating fiber parameters.

The photo-electron yield has been estimated by scaling measurements of the D0 fiber tracker [151] to our BTSM parameters. D0 measures a photo-electron yield of 9.6 using Kuraray SCSF$3 \mathrm{HF}$ fibers of 5-6 m length and with a $5.5 \mathrm{~m}$ attenuation length, mated to clear fibers of $10-12 \mathrm{~m}$ length and $10.4 \mathrm{~m}$ attenuation length [152]. The D0 fibers are read out by VLPCs. With this yield D0 has achieved a timing resolution of $0.75 \mathrm{~ns}$ [154]. Scaling these to our parameters gives a photo-electron yield of 13 with the Bicron BCF-10 fibers read out by a Hamamatsu H7546 PMT, or a photo-electron yield of 8 with the Kuraray SCSF-3HF fibers read out by the same PMT. Both of these yields are adequate for the desired timing resolution.

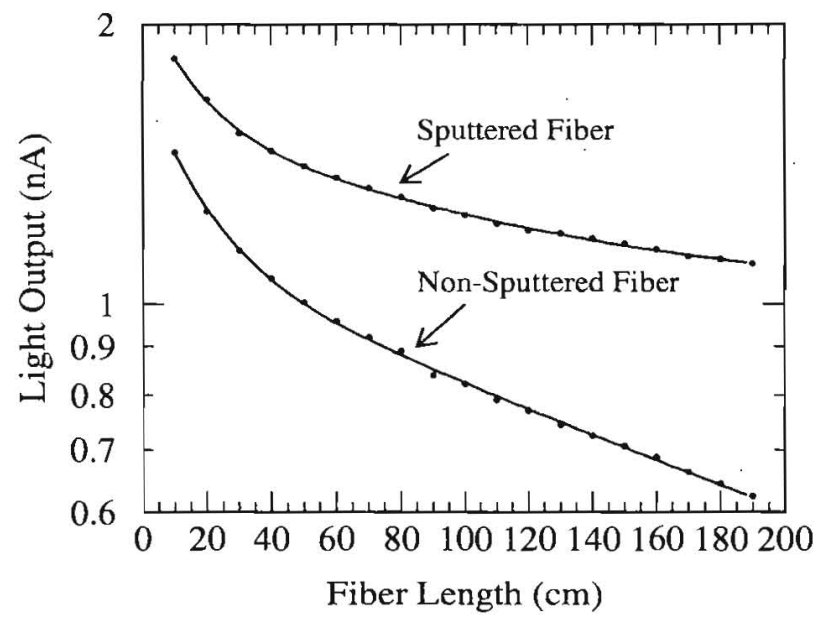

Figure 60: Light output of HyperCP hadronic calorimeter BCF-92 fibers. Note the large increase in light output at short distances.

Note that these photo-electron yields do not include: 1) the used of a green-extended photocathode (which we will use if we can convince Hamamatsu to produce the H7546 with it); 2) the gain in not mating clear fibers; and 3) the expected increase in fiber yield from the short wavelength component. The light yield as a function of length of all scintillating fibers is well fit by a double exponential (see Fig. 60). The short-attenuation-length component - typical values being on the order of $20 \mathrm{~cm}$ or so - is attenuated out rather rapidly and hence is ignored in most fiber detectors, 
and only the long attenuation length is quoted. The BTSM fibers are sufficiently short that these photons could possibly make a rather significant contribution to the total photo-electron yield. We have not found any measurements of the short attenuation length for BCF-10 fibers and hence have not added their contribution to our estimated yield.

\subsection{The Kaon RICH}

The kaon RICH provides a precision measurement of the vector velocity for kaons in the $\sim 22 \mathrm{GeV}$ beam. $\beta=1$ particles $\left(\pi^{+}, \mu^{+}, e^{+}\right)$and sub-threshold particles $\left(p^{+}\right)$are completely separated from kaons (> 40 standard deviations) in ring radius. Any mis-identification will be dominated by accidental effects. The momentum of $K^{+}$particles must be measured to an accuracy better than $\pm 0.5 \%$ and the $K^{+}$angles to $\pm 0.3 \mathrm{mrad}$. The time resolution for of this phototube based spectrometer is $\sim 1 \mathrm{nsec}$

\subsubsection{Experience from the SELEX RICH}

Fermilab experiment 781 (SELEX) built and successfully operated a phototube based RICH detector $[110,112,155,156]$. This detector used neon gas at atmospheric pressure as the radiator, a $R=20 \mathrm{~m}$ mirror. The photon detection was performed with an array of $28481 / 2$ " photomultipliers. Performance parameters [112] of special interest are described here. For Hamamatsu R760 photomultipliers, the figure of merit achieved was $N_{0}=162 \mathrm{~cm}^{-1}$. The single hit resolution excluding the contribution of tracking errors is $4.6 \mathrm{~mm}$, with a contribution of $4.0 \mathrm{~mm}$ from the size of the phototubes, and $1.3 \mathrm{~mm}$ from optical dispersion in the neon $n(\lambda)$ for a $\beta=1$ particle. The typical $\pi^{+} / K^{+}$separation for a narrow momentum band is shown in figure 61 . The single hit resolution is essentially independent of the ring radius, as shown in fig. 62 . This result was obtained by fitting a Gaussian to the measured ring radii of proton tracks in narrow $(1 \mathrm{GeV} / c)$ momentum bins.

In order to achieve the required kinematic rejection $\left(\sim 3 \times 10^{4}\right)$ of the $K^{+} \rightarrow \pi^{+} \pi^{0}$ background from the $K^{+} \rightarrow \pi^{+} \nu \bar{\nu}$ signal when the $\pi^{0}$ is not detected, it is crucial that the ring radius and center resolution functions be well understood with limited non-Gaussian tails over many decades.

To demonstrate the resolution functions for this type of spectrometer we used a 10.35 Million event sample of $\sim 600 \mathrm{GeV} / c$ single tracks from SELEX. The outcome of this study is that with modest and efficient cuts applied to the fit rings the fraction of events beyond $5 \sigma$ is held to the $\sim 8 \times 10^{-5}$ level. We chose this definition for the tails since it takes about a $5 \sigma$ upward fluctuation of the ring radius in either the kaon of pion RICH to move a $K^{+} \rightarrow \pi^{+} \pi^{0}$ event down into the $K^{+} \rightarrow \pi^{+} \nu \bar{\nu}$ signal region.

The pattern recognition scheme for selecting the PMT's on the ring was taken from SELEX [112]. Using information from a magnetic spectrometer the ring center and radius can be predicted. We selected PMT's that are $\pm 1.27 \mathrm{~cm}$ from the predicted circumference. With those selected PMT's we perform a three parameter fit to get the ring radius and center. For scale, the tube spacing in the SELEX RICH is $1.6 \mathrm{~cm}$

The single track events were also selected as only pions with the aid of the SELEX beam TRD, and $\beta=1$ particles with a measured momentum in the range $550 \mathrm{GeV} / c<p<750 \mathrm{GeV} / c$. The basic sample defining cuts were a well reconstructed single beam track SELEX event with more than 4 PMT's on the ring, less than 30 hits in all the entire PMT matrix, and a reduced- $\chi^{2}<4$ for the ring fit.

A total of $1.035 \times 10^{7}$ events pass these cuts, of them 5080 events or $4.9 \times 10^{-4}$ are in the tails beyond $5 \sigma$. The standard deviation of the ring fit is calculated event by event assuming a fixed resolution for each PMT taking into account both the number and distribution of PMT's around 


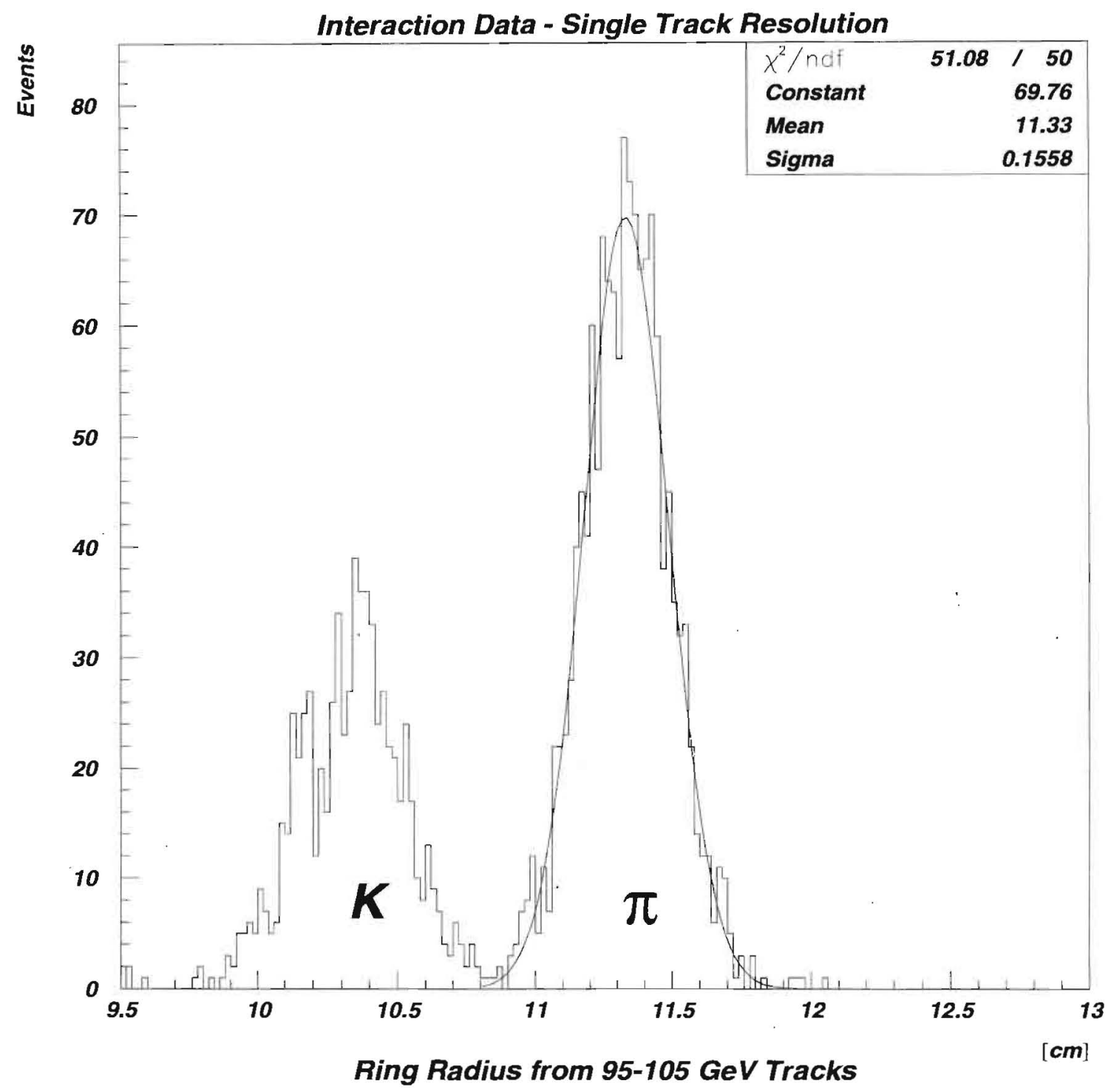

Figure 61: Ring radius distribution in the SELEX RICH for multi track events for tracks with momentum between 95 and $105 \mathrm{GeV} / c$. We fit a Gaussian to the pion peak of this distribution [112]. 


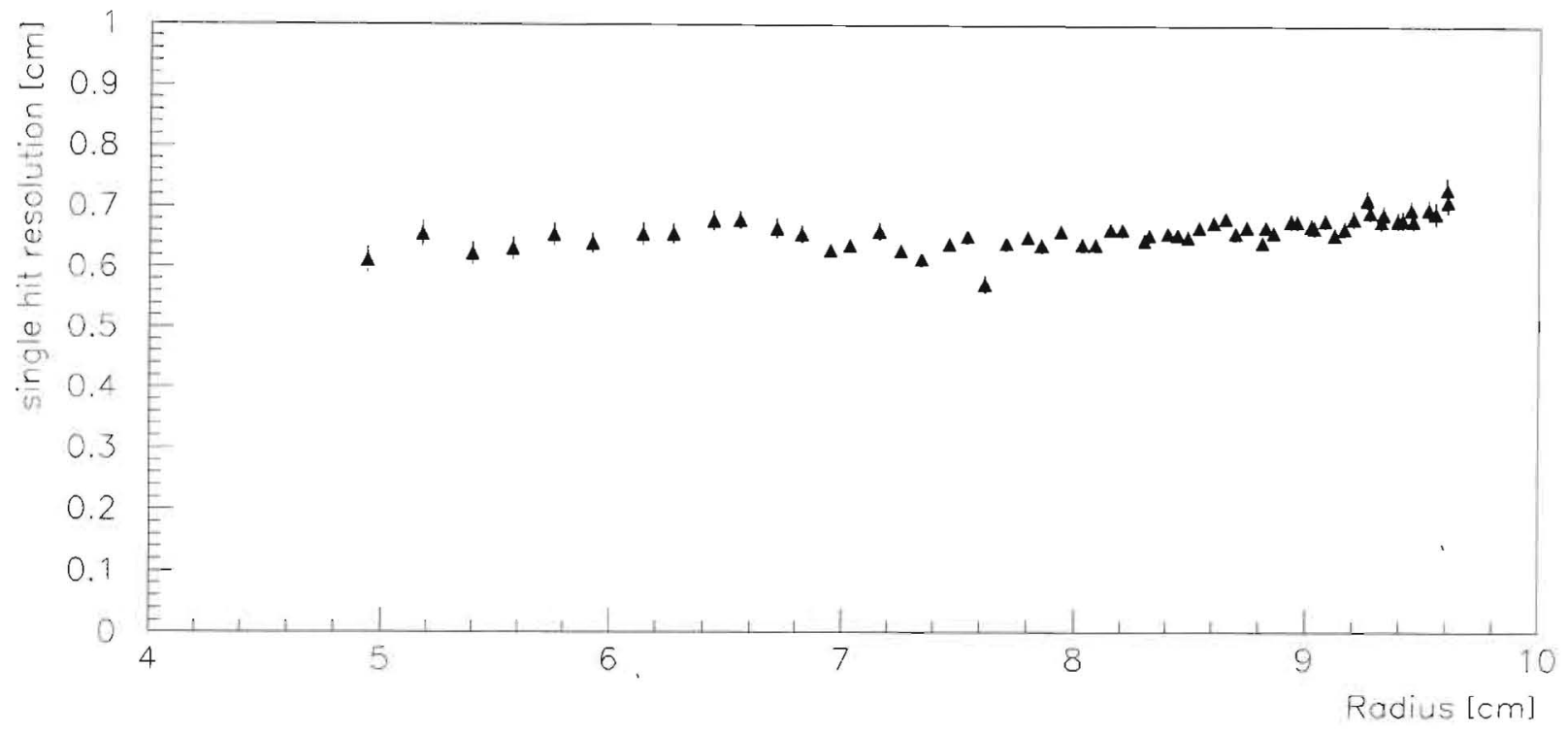

Figure 62: Fitted sigma of the proton ring radius distribution versus the mean radius. These fitted sigmas are larger than the $4.6 \mathrm{~mm}$ since they include finite track resolution and hits from overlapping rings.

the ring. These data are shown in the open histograms of figure 63. A simple set of additional cuts on the predicted uncertainties of the ring parameters and the distribution of hits around the ring reduces the fraction of tail events to $\left(7.9 \times 10^{-5}\right)$ while rejecting $11 \%$ of the events. We require that these tails be held to the $\left(3 \times 10^{-5}\right)$ level when combined with the measurements from the magnetic spectrometer (UMS or DMS). At this cut level the RICH can achieve most of the required suppression of these tails alone.

These resolution functions are show as the solid curves in figure 63. This level of cut efficiency applies to the kaon RICH where the average number of PMT's expected of a ring (10.8) is close to that observed in the SELEX data. For the pion RICH, where $\sim 20$ PMT's per ring are expected these cuts are much more efficient.

These results from the SELEX RICH were used to fine-tune a GEANT simulation for both RICHs. All optical properties (reflectivities of mirror, efficiencies of photomultiplier) of all materials were used from SELEX measurements, the chromatic dispersion of gases were taken from [157-159]. The number of photons detected was compared with SELEX, to make sure that all effect are considered correctly.

\subsubsection{Further Studies with SELEX RICH data}

All these results use a ring pattern recognition algorithm based on a found track segment in one to the SELEX magnetic spectrometers. Our goal is to develop a stand alone pattern recognition algorithm so that the magnetic and velocity spectrometer measurements are kept as independent of each other as possible. This work is in progress now. The SELEX RICH was read out in 180 nsec time bins and had a relatively large amount of material in the beam upstream of the RICH. The SELEX RICH data are considerably noisier than we expect in an equivalent 3 nsec time gate for 
the CKM RICHs. This make the pattern recognition algorithm more difficult with SELEX data.

\subsubsection{The Kaon RICH}

The kaon RICH physically is a $10 \mathrm{~m}$ long radiator gas vessel with an internal optical system that images the Cherenkov photons to an array of about $6001 / 2$ " photomultiplier tubes that measure the the ring image.

To reduce the occupancy of a single photomultiplier tube, a focal length $F=20 \mathrm{~m}$ will be used that magnifies the size of the ring image by $\times 2$ at the photomultiplier focal plane array. The optical path will be folded with one or two flat mirrors so to fit into a vessel of $10 \mathrm{~m}$ length. The mirror that first reflects the Cherenkov photons must sit in the beam and will be a minimal mass small flat mirror. Figure 65 illustrates a candidate optical path inside the kaon RICH. The details of the optical path and focal plane array are currently under active study. The system defined here provides a $K^{+}$momentum resolution of $0.5 \%$ as shown in figure 12 , which illustrates the Cherenkov threshold curve for this design.

A : radiator gases we are considering $C F_{4}$ or $N_{2}$. Exterssive GEANT Studies have been made for these two gases. The resolution in momentum depends on the threshold, or on the pressure. Multiple Scattering will increase with pressure. The angular resolution depends only on the ring radius resolution, which is limited by chromatic dispersion.

Also plotted in figure 64 is the distribution of detected photoelectrons for 1000 bean kaons and 1000 beam pions. A beam momentum of $22 \mathrm{GeV} / c( \pm 1 \%)$, a beam divergence of $\pm 100 \mu \mathrm{rad}, L=$ $10 \mathrm{~m}, F=20 \mathrm{~m}, N_{0}=150 \mathrm{~cm}^{-1}$ and $n=1.000287$ are assumed. The two rings in fig 64 correspond to the $K^{+}$(inner) and $\pi^{+}$(outer) respectively. To reduce the number of photomultipliers, we will only instrument part of the $\pi^{+}$ring region. The arrangement shown corresponds to an average of 10 detected photoelectrons for beam pions. In this proposed arrangement the average rate in the phototubes on the kaon ring (for a $30 \mathrm{MHz}$ kaon beam) will be $63 \mathrm{kHz}$ and the maximum rate will be $1.4 \mathrm{MHz} 71$. Although these rates are high, the tubes are counting single photo-electrons so this should be tolerable with fast high quality tubes such as the Hamamatsu R647 tube.

\subsubsection{Resolution of the Kaon RICH for different Gases}

To answer the question of what gas to use we made use of CKM_GEANT and simulated different gases, pressures, and photomultiplier sizes.

To obtain the resolutions, we made a pressure scan with 10000 events for each point. All Kaons were started at the center location, but with an small angle smear. For every event, we fitted a ring with to the obtained points. For every setting, we obtained the mean ring radius and its resolution vit a Gaussian fit to the distribution. In fig. 66 we show the procedure

As a first attempt we tried to use photomultipliers of the same quality as were used in SELEX, e.g. Hamamatsu R760 with $\frac{1}{2}$ inch outer diameter, with a quartz window (transparent down to $160 \mathrm{~nm})$. Other size photomultipliers $\left(\frac{3}{8} \mathrm{inch}, \frac{3}{4} \mathrm{inch}\right)$, assuming the same wavelength range and efficiencies, were simulated as well. A summary of the results is shown in fig. 67 .

The momentum and angular resolution required for this experiment cannot be achieved with these design criteria. The resolution is independent on the photomultiplier size. The limited factor for the ring radius (and the angular) resolutions is the chromatic dispersion $(n=n(\lambda))$ of the gases, with $\mathrm{N}_{2}$ showing the worse resolution, as expected.

Dispersion (dependence of the refractive index on the wavelength of the Cherenkov photon) is a small fraction of the resolution function for the SELEX RICH since the particles considered are high on the threshold curve where the Cherenkov angle is changing slowly. In contrast, CKM 
operates on the steep region of the threshold curve where the Cherenkov angle is changing rapidly. Dispersion effects are more significant near threshold, since the fractional contribution to the resolution function is inversely proportional to the Cherenkov angle. To minimize this effect we have been considering [157-159] only low dispersion radiator gases such as Freon-14 $\left(C F_{4}\right)$, and nitrogen.

Since dispersion is more pronounced at shorter wavelength, it is in principle possible to reduce its influence by using an appropriate window which is nontransparent below a certain wavelength. To study this, we simulated with CKM_GEANT the response to different wavelength ranges. In figure 68 a study for different cutoff is shown.

A cutoff of $300 \mathrm{~nm}$ was chosen to match the Hamamatsu R647 photomultiplier tube. The tube is identical to the R760 tube (used in SELEX) but with a glass rather than quartz window. It has an optical cutoff at $300 \mathrm{~nm}$.

In figure 69 we show the resolutions, with a $300 \mathrm{~nm}$ cutoff, for $N_{2}$ and $C F_{4}$.

As a conclusion, we will use $C F_{4}$ at a pressure around $0.7 \mathrm{~atm}$ as radiator gas for our RICH. As a backup solution we will keep $N_{2}$ at a pressure of around $1.2 \mathrm{~atm}$. We will use $1 / 2$ inch photomultipliers, R647 from Hamamatsu which have glass windows with an optical cutoff at $300 \mathrm{~nm}$. About 600 tube will be required

We will also detect the $\pi^{+}$in the beam. The mirrors in the Kaon RICH will be big enough only to reflect part of the Cherenkov-photons from pions. For a particular mirror size, we show in figure 70 the difference radius as a function of pressure, for $C F_{4}$.

To evaluate the rate in the photomultipliers, we ran we our now standard setup ( $1 / 2$ inch PM, $C F_{4}$ at $\left.0.7 \mathrm{~atm}, 300 \mathrm{~nm}\right) 10000$ Kaons from out beam file. The corresponding distribution of the number of hits is shown in figure 71 .

\subsubsection{Kaon $\mathrm{RICH}$ readout system}

The photomultiplier tubes (PMT's) that instrument the kaon RICH focal plane must have excellent timing and noise characteristics. PMT tubes under consideration now (Hamamatsu 1/2" R647) have timing resolutions better than $\pm 1 \mathrm{nsec}$, and deadtimes less than $25 \mathrm{nsec}$. PMT's are intrinsically very low noise devices, and extrapolating from the SELEX experience the CKM RICH systems should have noises rates of less than $500 \mathrm{~Hz} /$ tube. The PMT's are readout with the pipeline TDC system described in section 6.2 .2 . 

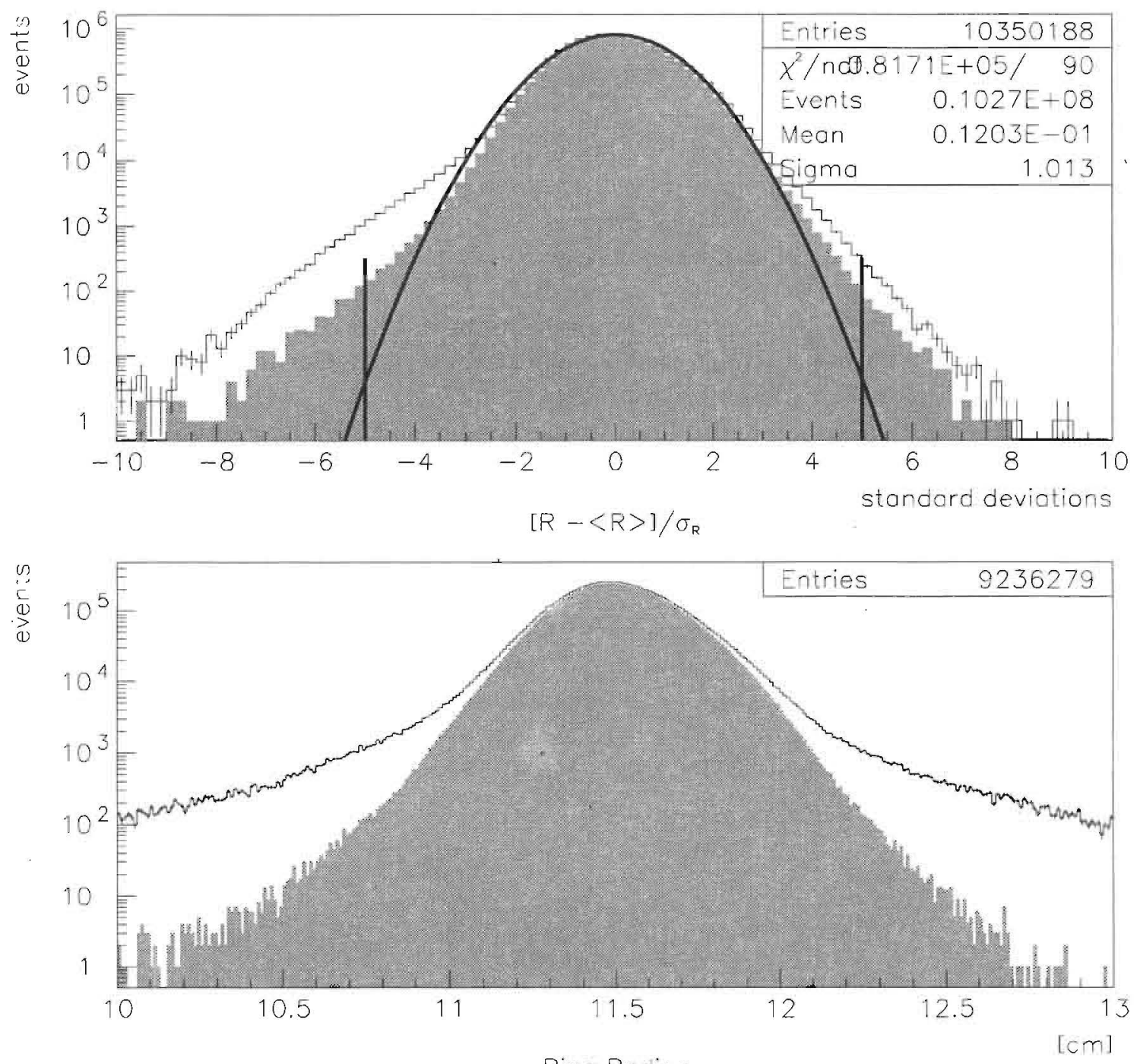

Ring Radius

Figure 63: The pull (upper) and ring radius (lower) distributions for $10.35 \mathrm{M} \beta=1$ beam tracks in the SELEX RICH. The shaded regions are a sub-sample with modest cuts on the predicted uncertainties of the ring fit parameters. 

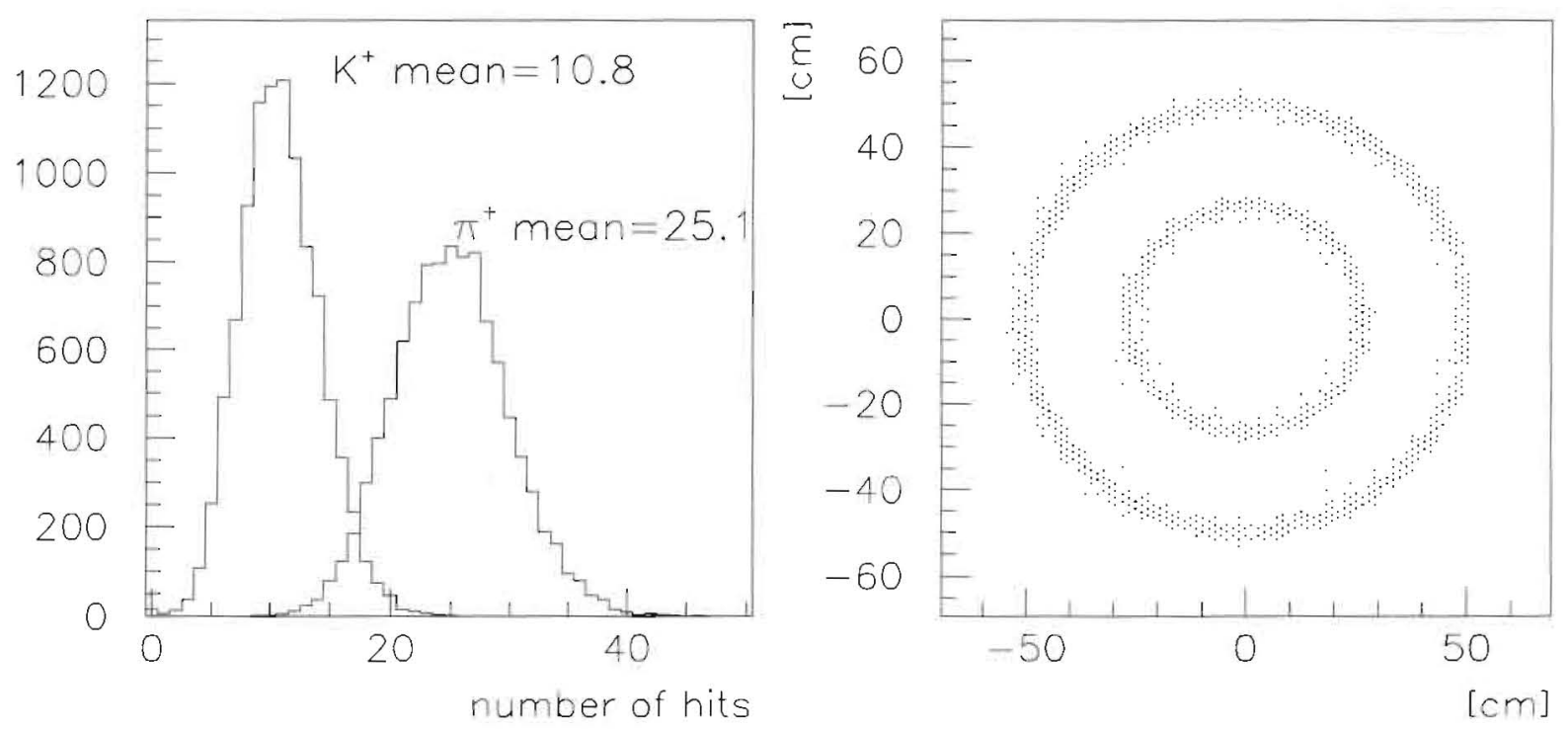

Figure 64: left: Number of detectable photons, right: Distribution of detected photons in the kaon RICH focal plane for 1000 beam $K^{+}$and 1000 beam $\pi^{+}$events.

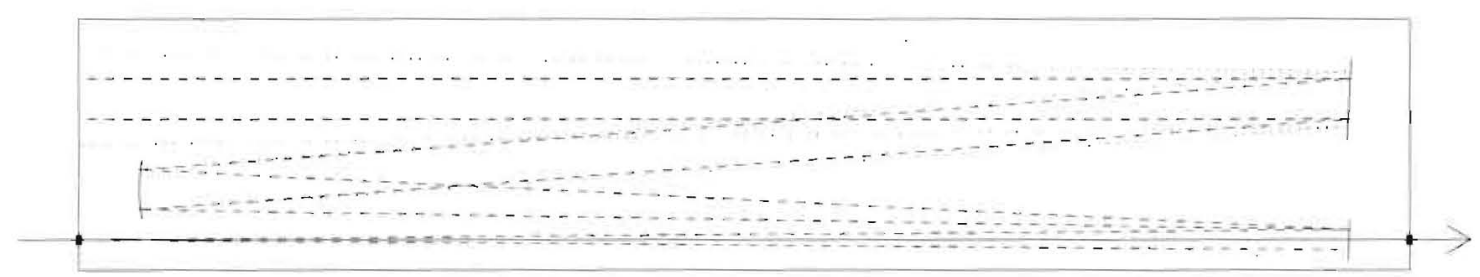

Figure 65: Possible schematic of the kaon RICH optical path. The vessel drawn has a total length of $11 \mathrm{~m}$. The long arrow represents the beam; the thicker dots are the beam windows. The dashed line represents Cherenkov photons generated by a $K^{+}$, dotted line Cherenkov photons from a $\pi^{+}$. The flat mirror in the beam will only reflect part of the Cherenkov photons from the pions. 

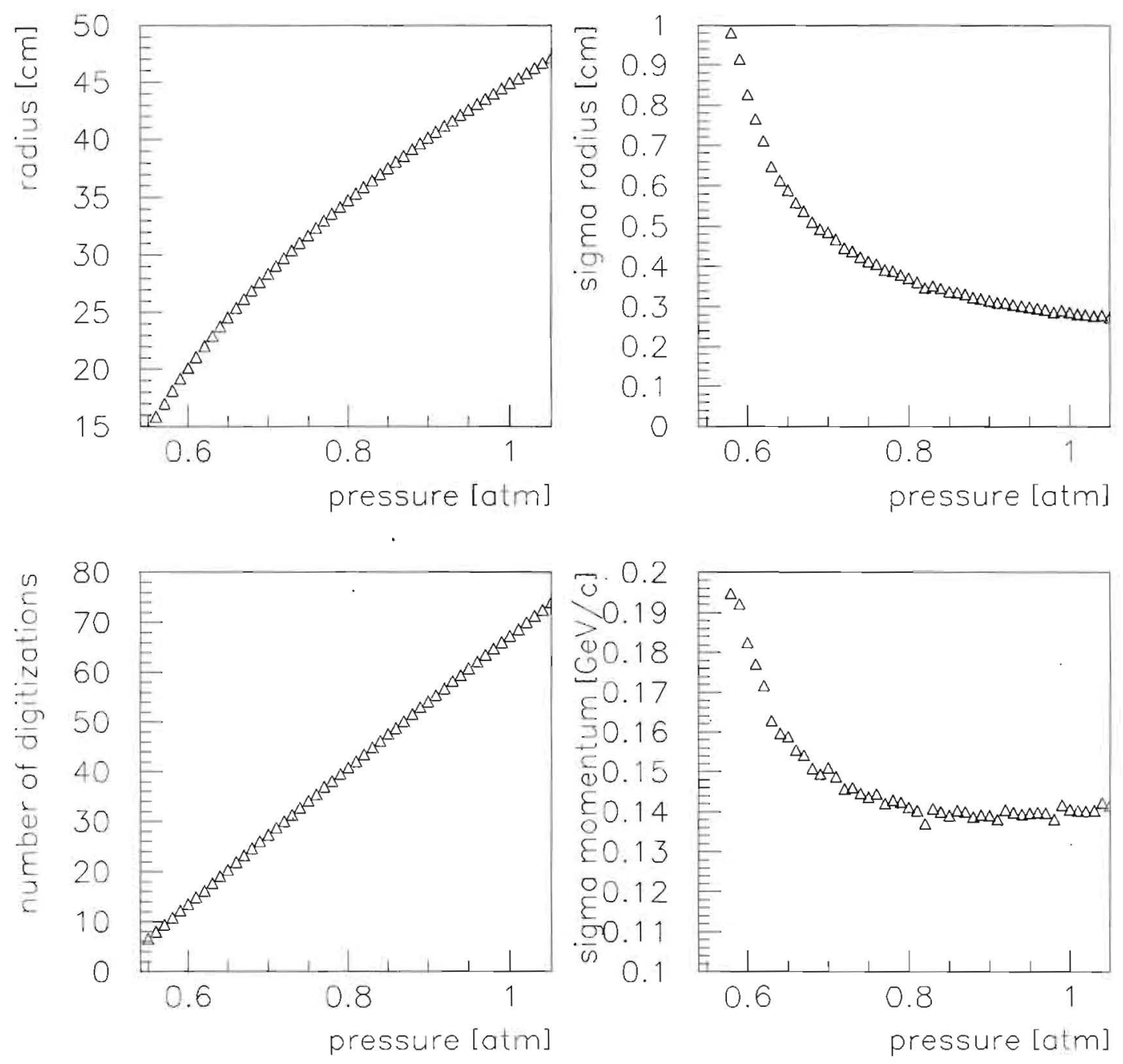

Figure 66: Ring radius, ring radius resolution, and momentum resolution for the Kaon RICH, gas $C F_{4}$, Photomultiplier size $1 / 2 "$, R760 with quartz window. 

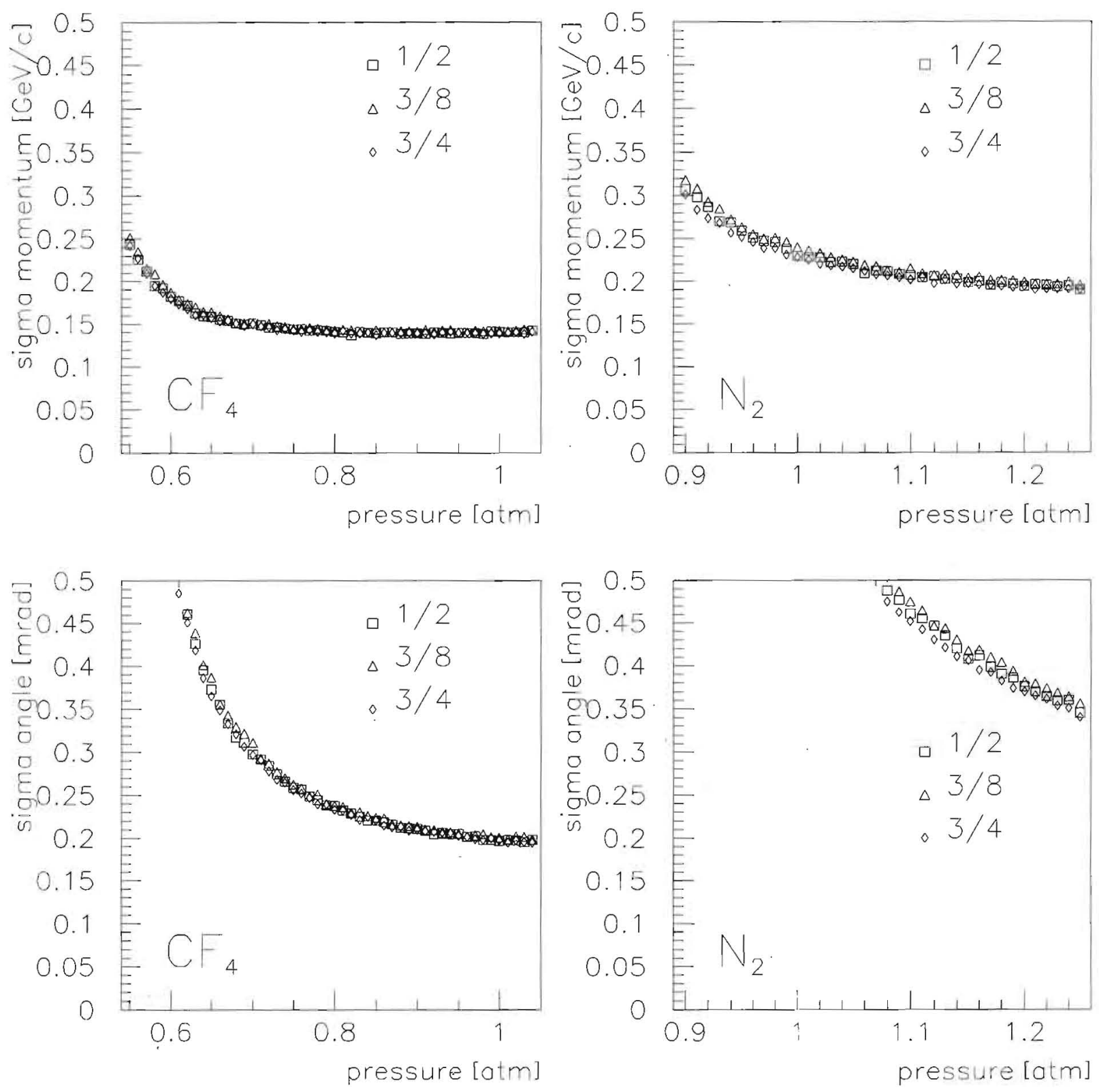

Figure 67: Momentum (top) and angular (bottom) resolution for the Kaon RICH as a function of pressure and for different photomultiplier sizes. Left: $C F_{4}$, Right: $N_{2}$ 

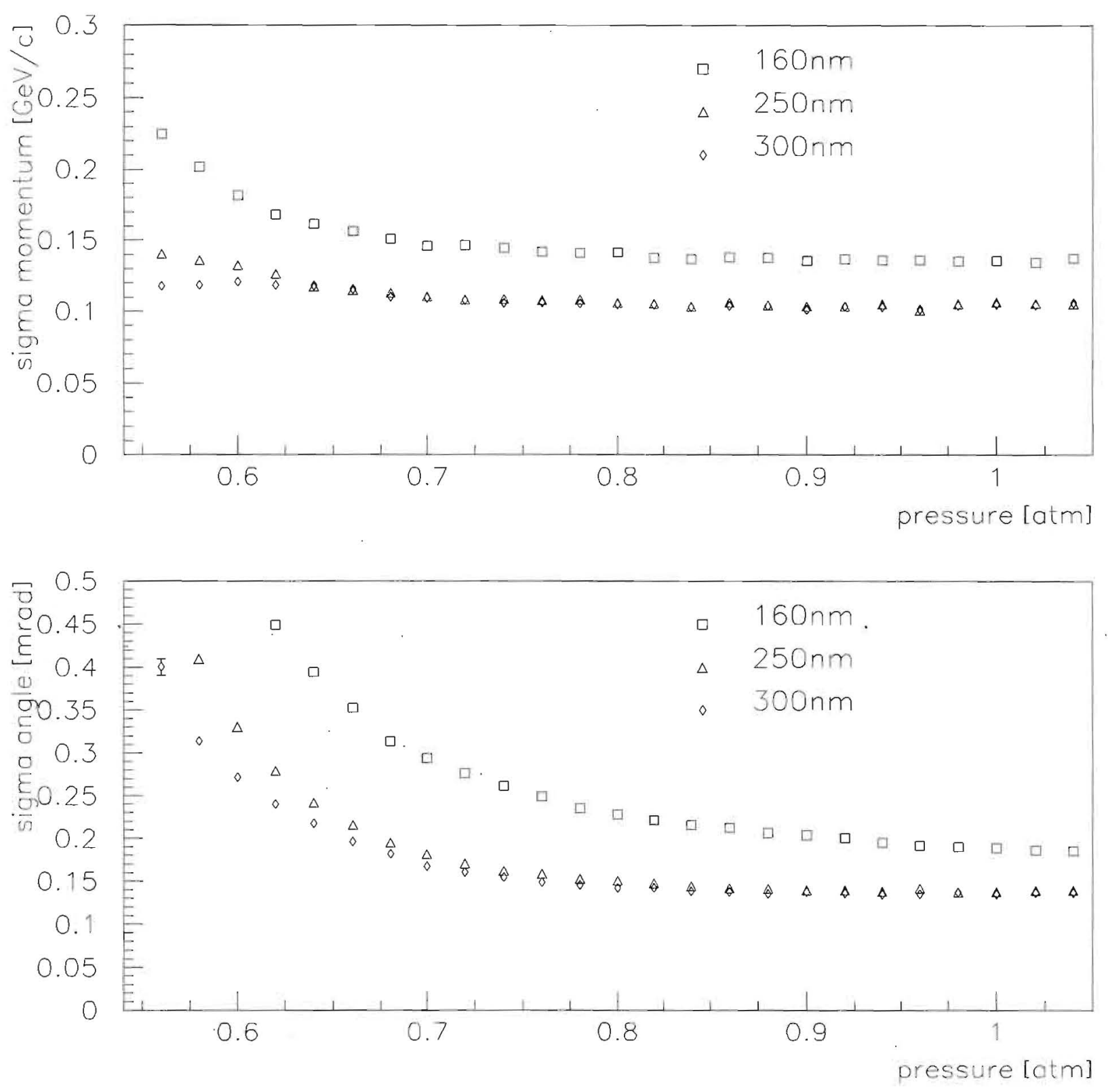

Figure 68: Momentum (top) and angular (bottom) resolution for the Kaon RICH as a function of pressure, for 3 different wavelength cutoffs in $C F_{4}$. 

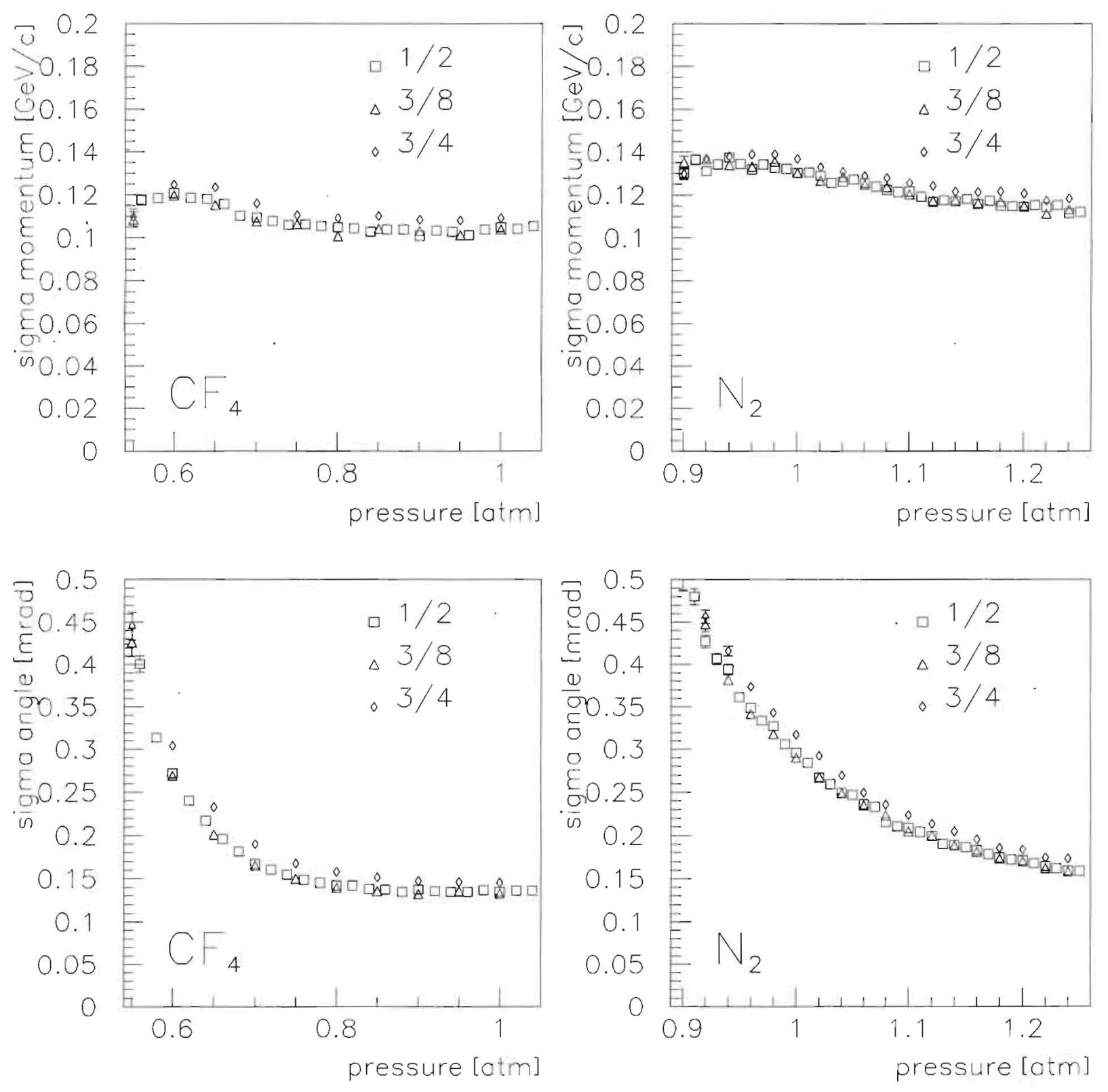

Figure 69: Momentum (top) and angular (bottom) resolution for the Kaon RICH as a function of pressure and for different photomultiplier sizes, for a $300 \mathrm{~nm}$ cutoff. Left: $C F_{4}$, Right: $N_{2}$ 


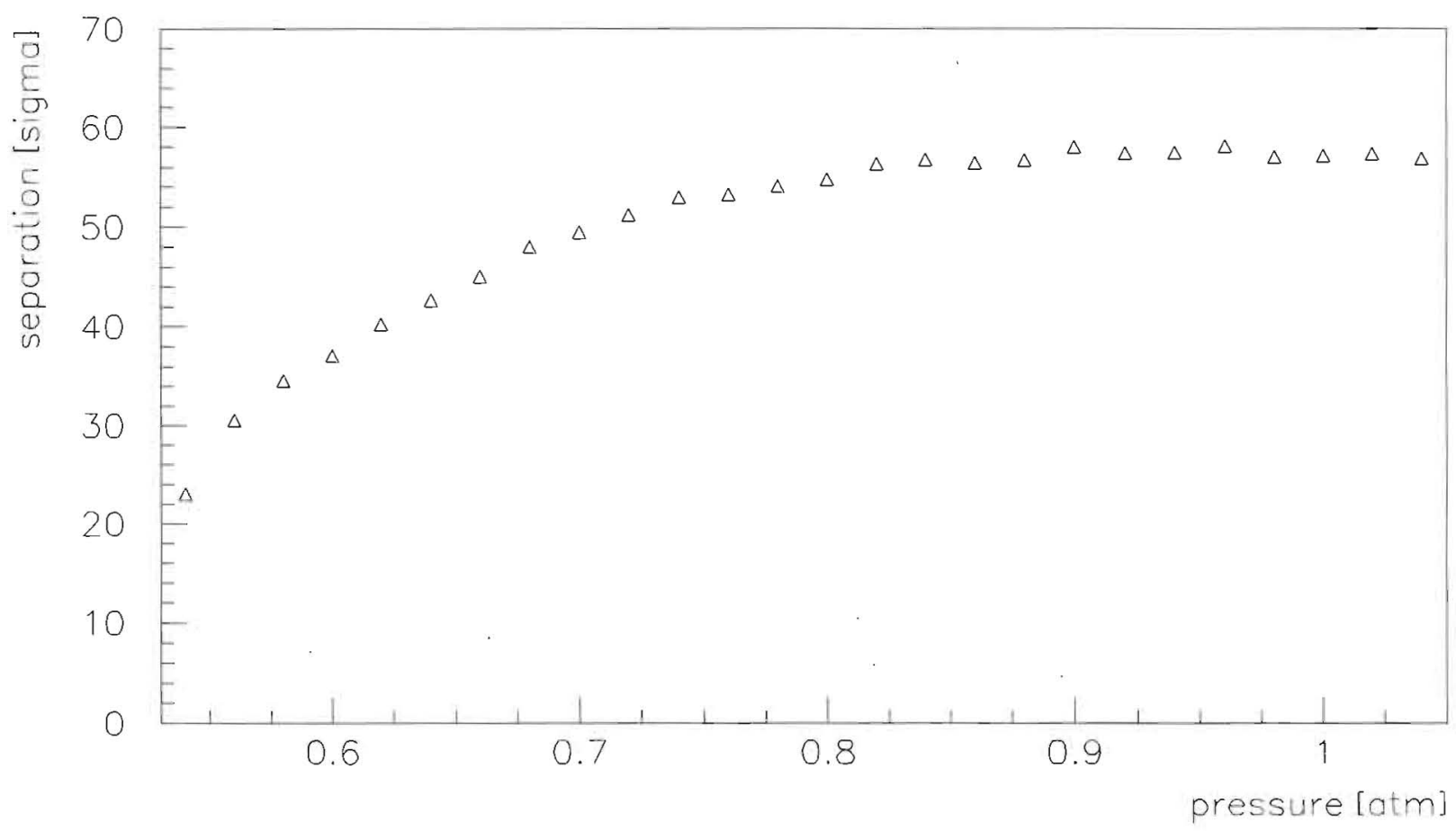

Figure 70: Difference in Ring Radius (in units of Gaussian $\sigma$ ) between Kaons and Pion in the Kaon $\mathrm{RICH}$ as a function of pressure. Gas: $C F_{4}, \mathrm{PM}$ Size $1 / 2$ inch, cutoff $300 \mathrm{~nm}$. 


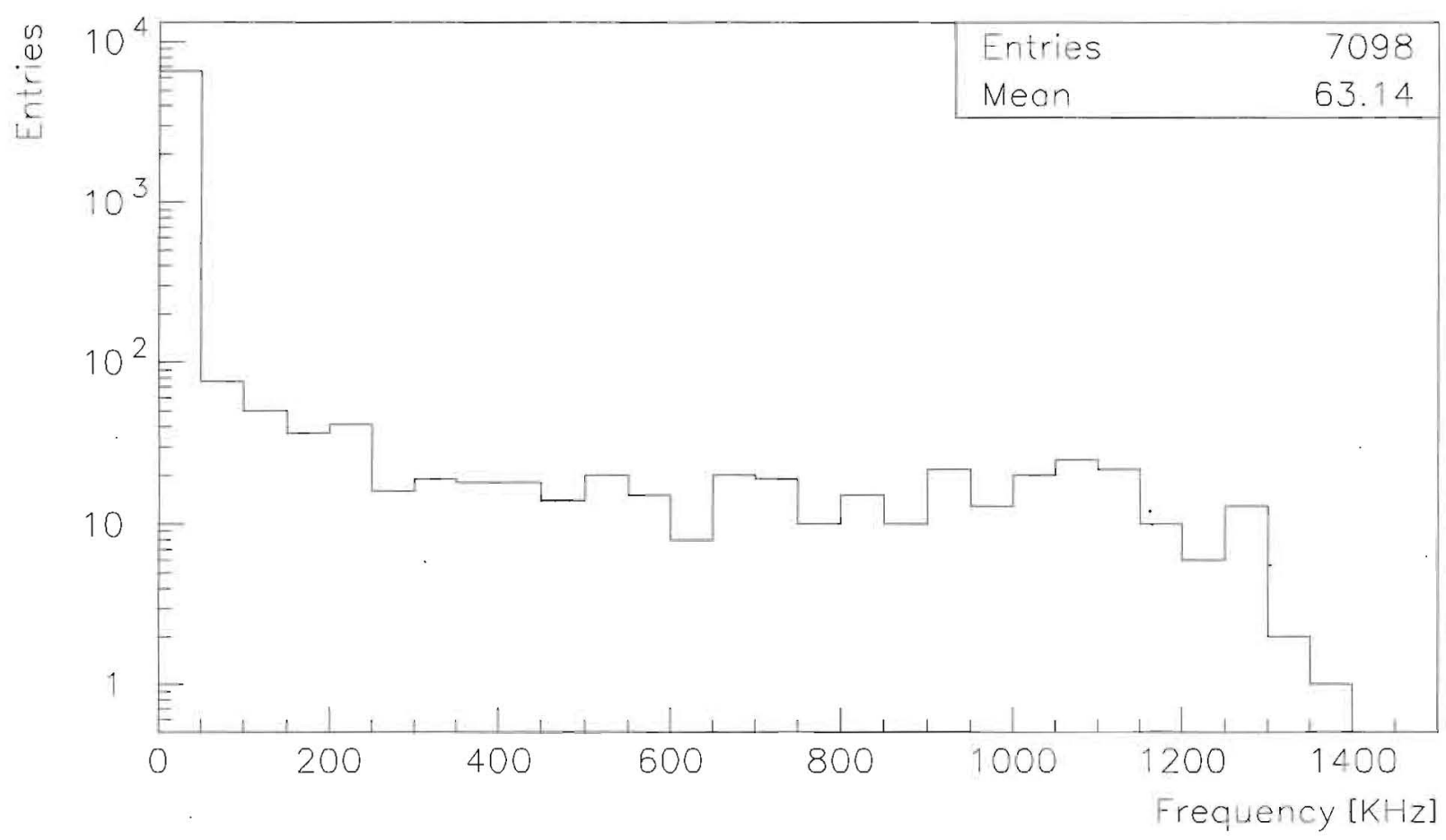

Figure 71: Distribution of rates in PMs for the Kaon RICH. 10000 tracks are scaled to our $30 \mathrm{MHz}$ nominal beam rate. Gas: $C F_{4}, P M$ Size $1 / 2$ inch, cutoff $300 \mathrm{~nm}$. 


\subsection{The Kaon Entrance Angle Tracker System}

The Kaon Entrance Angle Tracker (KEAT) is a critical element in CKM; it must have excellent angular resolution to accurately tie the incoming kaon track to the pion track from the $\mathrm{K}^{+} \rightarrow$ $\pi^{+}+\nu+\bar{\nu}$ decays and a minimum amount of material. These are essential to reduce background from interactions and multiple scattering, particularly in the Kaon RICH, which may contain $\sim 0.1$ radiation lengths depending on the choice of radiator gas.

The required resolution, rates, size, and other characteristics of the KEAT are very similar to those for the Upstream Magnetic Spectrometer described in Sect. 5.2. It is therefore reasonable to use the same technology for the KEAT. As presently planned, the KEAT will consist of two stations of high-resolution, high-rate PWCs with a total of 12 planes with the same readout system as the UMS.

The angular resolution of the KEAT should be as good or better than that of the UMS (Sect. 5.2.3) and the DMS (Sect. 5.8.5). This can be achieved with chambers of the same design as the UMS with two stations separated by approximately 2.6 meters placed just ahead of the window of the vacuum decay region. Each station will have 6 anode planes and a material inventory similar to that of the UMS chambers (Table 18 ), so that $z / \lambda_{I} \approx 6.7 \times 10^{-4}$ and $z / X_{O} \approx 2.1 \times 10^{-3}$.

A Monte Carlo simulation of the KEAT angular resolution(projected) is shown in Fig. 72. The simulation includes multiple coulomb scattering but not nuclear scattering. The width (RMS) of the distribution is $76 \mu \mathrm{rad}$ for a 2.6 meter spacing of the KEAT stations.

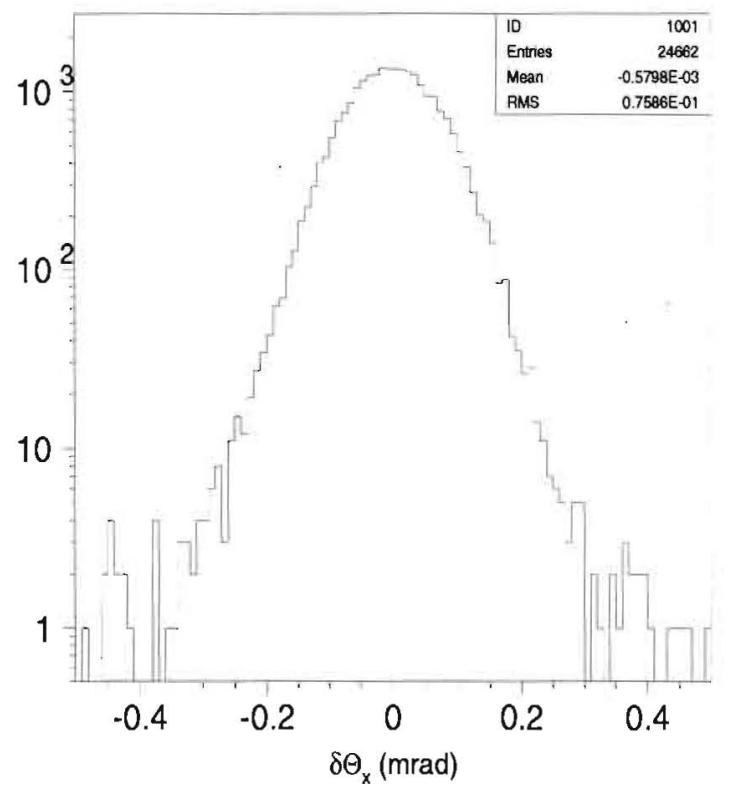

Figure 72: A simulation of the expected angular resolution (projected) of the KEAT. Angles are in mrad. Multiple scattering, but not nuclear scattering, is included in the simulation.

In order to verify that the simulation in Fig. 72 is realistic, we have analyzed data from HyperCP (E871) in which similar chambers (built by the U. Va. Group) were used. The layout of upstream chambers in HyperCP is shown in Fig. 73. The geometry for the HyperCP chambers upstream of the spectrometer magnet is similar to that for the KEAT with 4 chamber stations, C1, C2, C3, C4. The planes in C1 and C2 had a pitch of $1.0 \mathrm{~mm}$ and those in C3 and C4 had a pitch of $1.2 \mathrm{~mm}$. Each chamber had only 4 planes: 2 with vertical wires shifted by half a wire spacing and 2 with 
wires inclined at $\pm 26.6^{\circ}$ with respect to the vertical. C1 and C2 were separated by $2.0 \mathrm{~m}$ as were C3 and C4. Thus it is possible to "measure" the angular resolution in a geometry very similar to the KEAT geometry by comparing the angles of tracks measured with C1-C2 with those from C3-C4. The same Monte Carlo simulation used for the KEAT in Fig. 72 gives for the E871 geometry the angular resolution shown in Fig. 74 with a width (RMS) of $158 \mu$ rad and no significant tail. In Fig. 75 we show the actual distributions for the E871 geometry as measured with high-momentum muon tracks. The standard HyperCP tracking codes were used for fitting the tracks. The solid listogram is for one-track events; the dashed curve is for events with two tracks in the spectrometer. The RMS width of both is $160 \mu \mathrm{rad}$, in excellent agreement with the Monte Carlo expectation, and the tails on the distributions are very small, about $3 \times 10^{-5}$ for the one-track and $5 \times 10^{-4}$ for the two-track events. This shows that tracking ambiguities due to accidental tracks in CKM will not cause appreciable tails on the angular resolution.

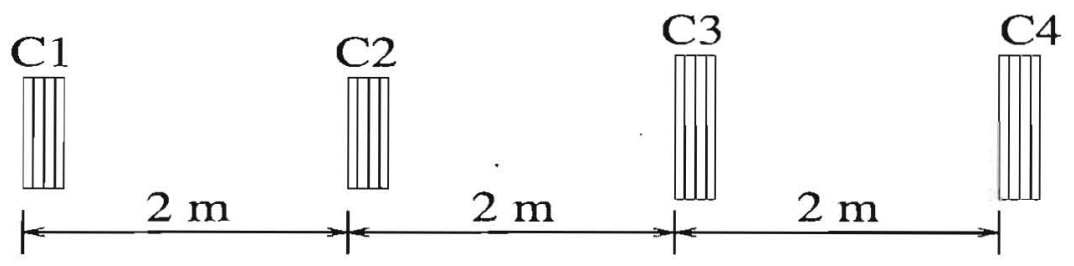

Figure 73: Layout of upstream chambers in HyperCP. Each chamber has four planes, two planes of vertical wires and two stereo views with the wires inclined angle at $\pm 26.6^{\circ}$ from the vertical. The wire spacing is $1.0 \mathrm{~mm}$ for $\mathrm{C} 1$ and $\mathrm{C} 2$, and $1.2 \mathrm{~mm}$ for $\mathrm{C} 3$ and $\mathrm{C} 4$.

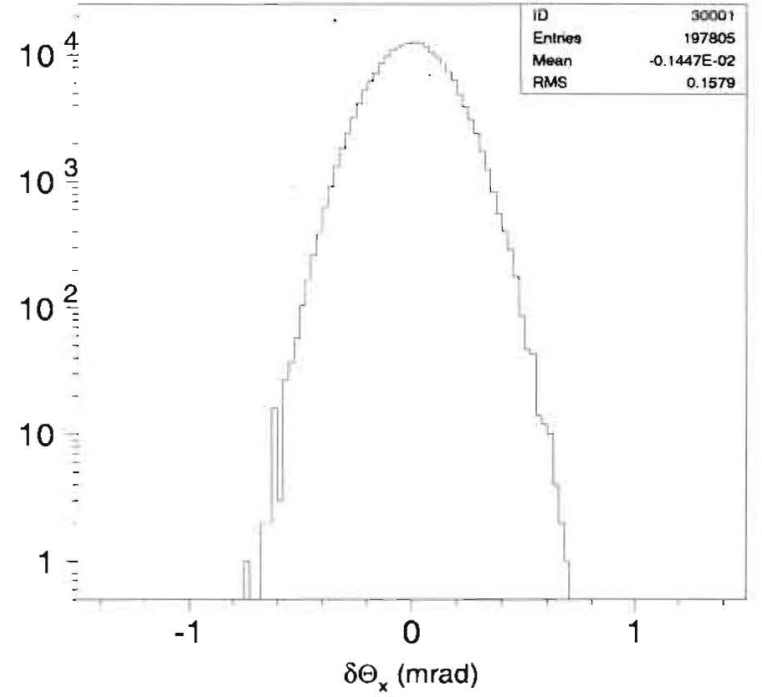

Figure 74: A simulation of the expected angular resolution (projected) for the $\mathrm{Hy}$ perCP geometry. Angles are in mrad. Multiple scattering, but not nuclear scattering, is included in the simulation.

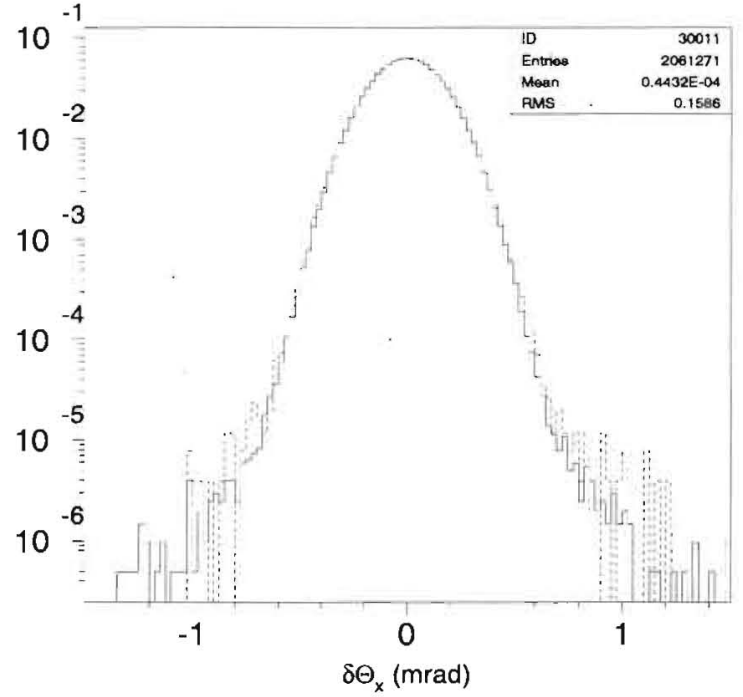

Figure 75: Measured angular resolution (projected) for the HyperCP geometry. The solid histogram is for 1-track events; the dashed is for events with 2-tracks. The RMS widths of both are in very good agreement with the simulation in Fig. 74

Note that the HyperCP tracking code was not optimized for the CKM situation, so that it should be possible to reduce the tails considerably below those in Fig. 75 witlı optimized code. The 
simulations do not contain nuclear scattering. The Veto systems described in the following sections are designed to greatly reduce background from this source

\subsection{The Beam Interaction Veto System}

Despite the minimal material of the KEAT system, elastic and inelastic interactions in the KEAT mass can be a source of background particles as well as corrupt the incident kaon momentum. The role of the Beam Interaction Veto System (BIVS) situated in figure 42 is to veto particles associated with inelastic scattering of beam particles in material close to the upstream vacuum window. The inner layer of the BIVS must be very efficient for electrons, muons, pions, and protons that arrive within a geometry corrected 5 nsec timing gate. The bulk sampling material of the BIVS must be thick enough to efficiently detect neutral hadrons and photons associated with the upstream interaction.

\subsubsection{BIVS Design Considerations.}

The Beam Interaction Veto System (BIVS) geometry illustrated in figure 76 is essentially a narrow bore (20 cm diameter) extended version of a VVS station that is 4 meters long and 1 meter in diameter. The downstream end of the BIVS marks the beginning of the fiducial decay volume. Analogous to a VVS station, the BIVS is segmented both azimuthally and longitudinally and instrumented with high speed photomultiplier tubes. Given the beam proximity and the immediate upstream interaction rate of $\sim 2 M H z$, the BIVS photomultipliers see the highest rate of large pulses within the experiment. The maximum BIVS PMT rate is $\sim 2 \mathrm{MHz}$ for a $10 \mathrm{MeV}$ threshold, which is $\times 10$ the hottest VVS channel running with a $1 \mathrm{MeV}$ threshold. Fortunately, as shown in section 7 , the required BIVS visible threshold to efficiently veto interactions is not less than $10 \mathrm{MeV}$. In addition to the critical role of vetoing beam interactions, the large BIVS mass plays an important role in shielding the downstream VVS stations from upstream beam interactions.

\subsection{The Vacuum Veto System.}

\subsubsection{The vacuum vessel.}

The vacuum decay vessel and embedded veto stations shown in figure 42 extends from the downstream KEAT station to the beginning of the pion RICH. The vessel is $38 \mathrm{~m}$ long, increasing from an upstream outer diameter of $135 \mathrm{~cm}$ to a downstream outer diameter of $192 \mathrm{~cm}$ and is composed of $12 \mathrm{~mm}$ thick steel between the annular veto modules. The upstream vacuum window is immediately downstream of the last KEAT tracking plane. As motivated by the discussion of beam interaction induced backgrounds in section 7 , it is critical to minimize the combined interaction length of the last KEAT plane and the upstream vacuum window. To minimize hadronic scattering the upstream vacuum window is a $200 \mu \mathrm{m}$ thick aluminum foil that is $20 \mathrm{~cm}$ diameter. This window contributes $2.4 \times 10^{-4} K^{+}$interaction lengths to a total of $\sim 6 \times 10^{-4} K^{+}$interaction lengths of the KEAT/window station. A drawing of the downstream KEAT station, vacuum window, and beginning of the Beam Interaction Veto System (BIVS) in shown in figure 76. Design considerations of the BIVS were discussed previously in section 5.5 The downstream end of the vacuum decay volume is terminated with a $96 \mathrm{~cm}$ diameter vacuum window composed of mylar-kevlar sandwich similar to the $\mathrm{KTeV}$ vacuum windows. Since this window is downstream of the tracking stations the thickness is not as critical as the upstream window. 


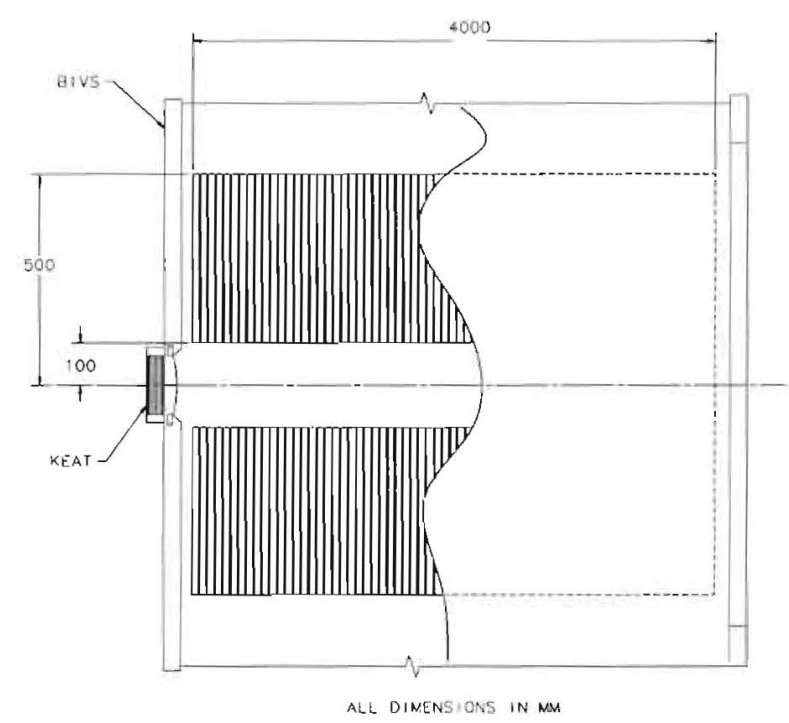

Figure 76: Elevation drawing of the upstream vacuum window, last tracking station of the KEAT, and the upstream section of the BIVS.

\subsubsection{The vacuum veto system stations.}

The vacuum veto system shown in figure 77 is composed of 34 annular lead-scintillator veto stations integrated into the vacuum vessel. The spatial filling fraction of $50 \%$ allows for straightforward modular design based largely on the successful design of the $\mathrm{KTeV}$ vacuum veto system. The impact of the residual lost spatial coverage is discussed in section 3.4.6, which concludes that the final background increase is small. Each station is longitudinally composed of 81 layers of 1-mm lead/5$\mathrm{mm}$ scintillators corresponding to a longitudinal depth of of $50 \mathrm{~cm}$ and $\sim 15$ radiation lengths. The effective radiation length of this sampling material is $\sim 3.6 \mathrm{~cm}$. Each annular station is segmented into 16 azimuthal sectors, with four photomultiplier tubes instrumenting the longitudinal depth of each sector. The irner surfaces the VVS modules are designed with a minimum of inactive material. The mechanical load is supported entirely at the outer radius of each station. Detailed mechanical drawings of a veto station can be found in figures $78,79,80$, and 81 .

\subsubsection{The vacuum veto system station module.}

The VVS sector module scintillator stack shown in figure 80 is readout with a plant of wavelength shifting (WLS) blue $\rightarrow$ green scintillating fibers that are alternately bundled, tile by tile, to different photomultiplier tubes. This is the base element of a system composed of 25 tons of fast bright scintillator readout with $450 \mathrm{~km}$ of WLS fiber. The fiber of choice is a fast $1 \mathrm{~mm}$ diameter doubleclad fiber, Bicron BCF-92/99, which is an affordable high performance fiber. We have studied a variety of combinations of this fiber with different scintillators and coupling schemes. The results of these studies are described in section 5.6.5. A photograph of our baseline prototype tile is shown in figure 82. The end of the fiber at the inner radius of the station module is mirrored with an aluminum vacuum evaporation process to increase the light yield. We will use a "green-extended" photonıultiplier tube (PMT) such as the "Electron Tubes (formerly EMI)" type 9954, to optimize 


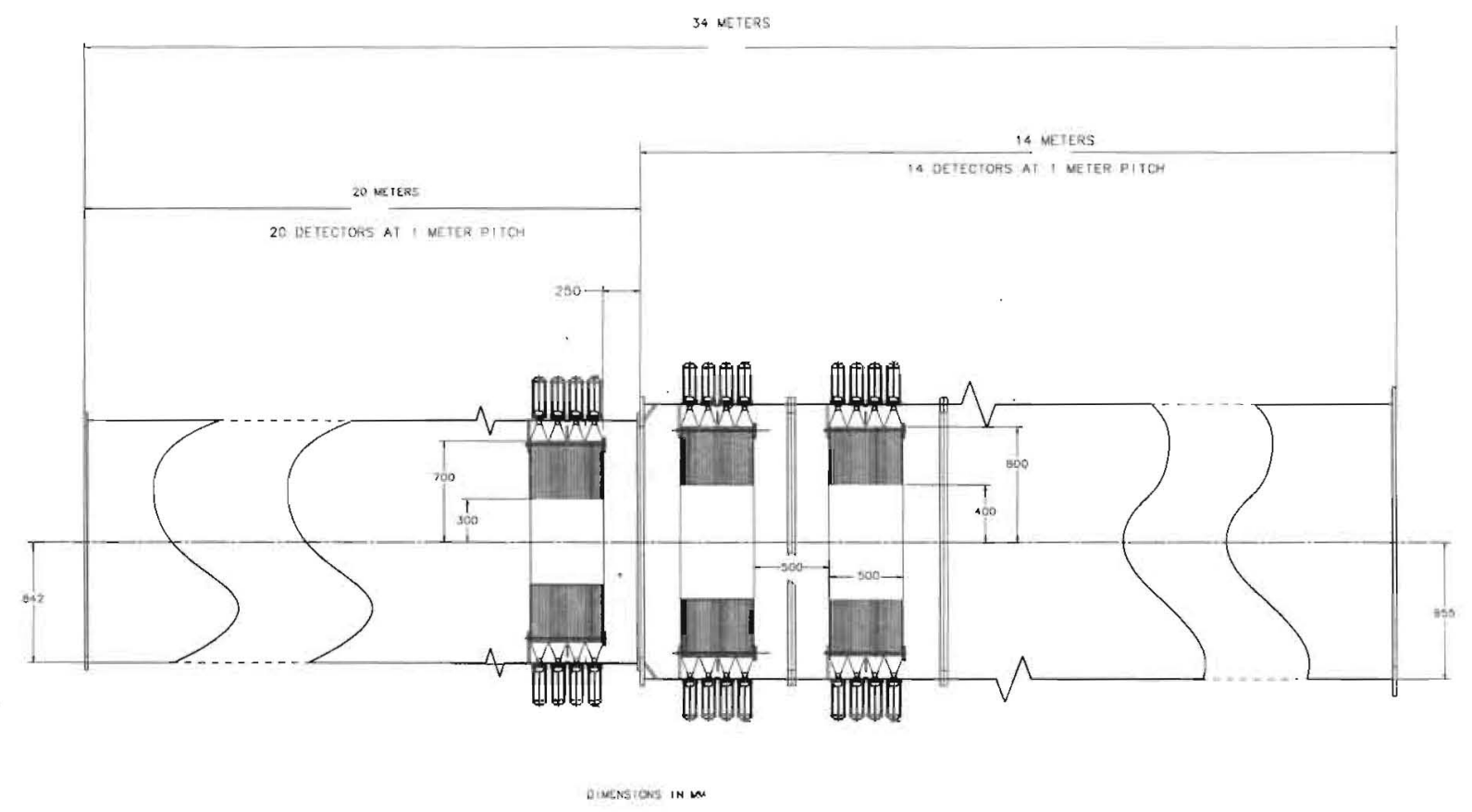

Figure 77: Elevation drawing of a section of vacuum decay volume showing the geometry of VVS stations. 


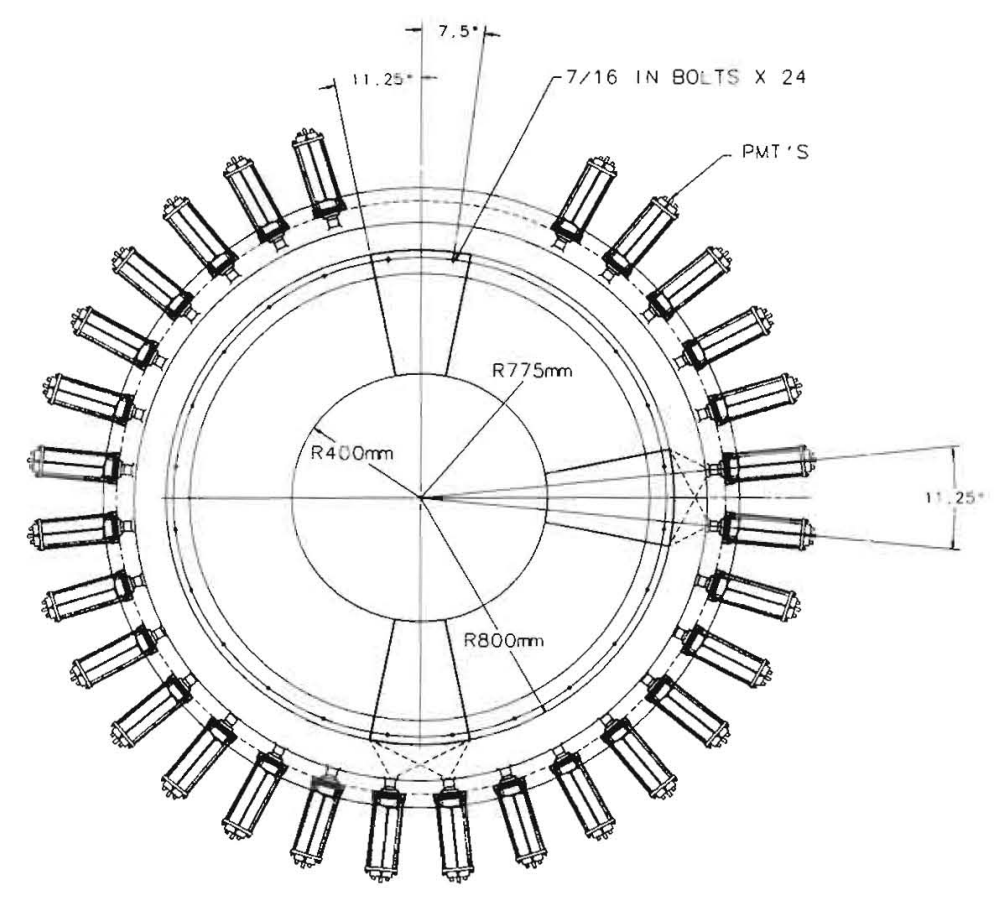

ALL DIMENSIONS IN MM EXCEPT WHERE NOTED

Figure 78: Front Beams-eye view (elevation) drawing of one the 34 VVS stations.

the spectral match to the BCF-92/99 fiber. The PMT will be grease-fitted at the outside of the vacuum vessel onto a window that will terminate the fiber bundle on the inside of the vacuum.

As a baseline design, we have extensively studied the light yield of Bicron-404 scintillator read out with mirrored BCF-92/99 fibers. This scintillator/fiber combination delivers about 22 photoelectrons/MeV for an optimal fiber-scintillator coupling. Various degradations of the optimal coupling can simplify the construction problem at the cost of light yield. Likewise, lower performance scintillator can significantly reduce the material costs at the expense of light yield. These tradeoffs are discussed in section 5.6.5. Our minimum light yield specification is 10 photo-electrons/MeV in order to remain efficient for low energy photons and remain well above electronic readout noise. With this Pb-scintillator sampling fraction a photon deposits about $1 / 3$ of its energy in the scintillator. As evident in figure 85, we don't require or expect any efficiency for photons below 5 $\mathrm{MeV}$, which would correspond to 16 photo-electrons at our minimum specification. In order to maintain high rate performance of the readout PMT we will operate at the relatively low gain of $1 \times 10^{5}$ corresponding to $250 \mathrm{fC}$ of signal charge for a $5 \mathrm{MeV}$ photon, about $12 \mathrm{ADC}$ counts for the electronic readout system described in section 6 .

Our baseline design has each piece of scintillator wrapped with white Tyvek in order to increase the light yield and protect the scintillator optical surfaces from the lead pieces. This wrapping technique was employed successfully in the $\mathrm{KTeV}$ vacuum veto system. We have studied the mechanical properties of $1 \mathrm{~mm}$ thick lead pieces from Vulcan Lead and find the mechanical tolerances to be sufficient. The Young's modulus of lead is high enough that are no creep concerns with $1 \mathrm{~mm}$ thick sheets supported only at the outer edges of the VVS sector modules. The lead pieces from Vulcan are calcified for additional rigidity and clad with a thin polymer layer (Vulclad process) to ease handling concerns during construction. We have a fabrication quote for all of the lead pieces, 


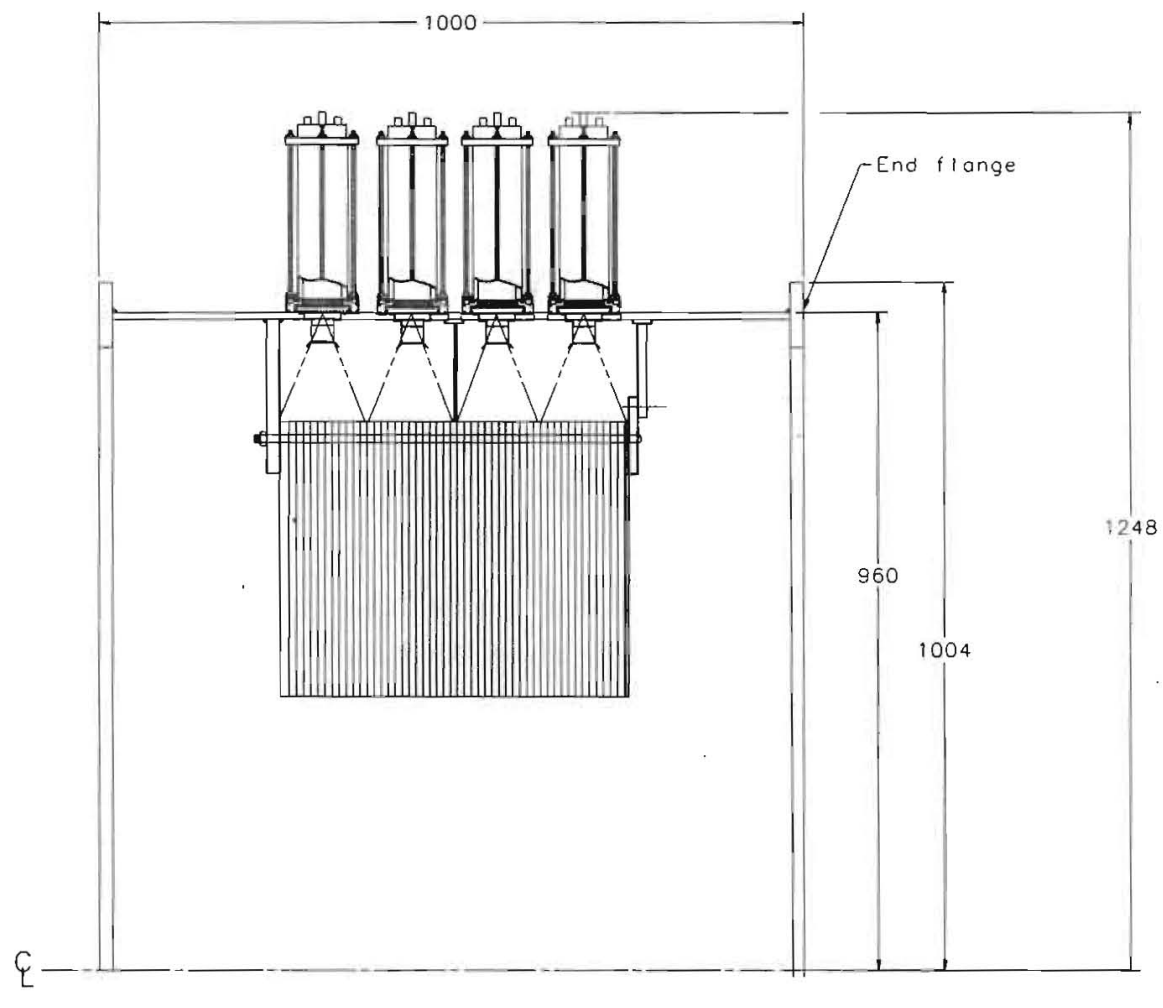

ALL DIMENSIONS IN MM

Figure 79: Longitudinal slice of an annular VVS station. Geometry of the lead-scintillator sampling and photomultiplier tubes is evident. 


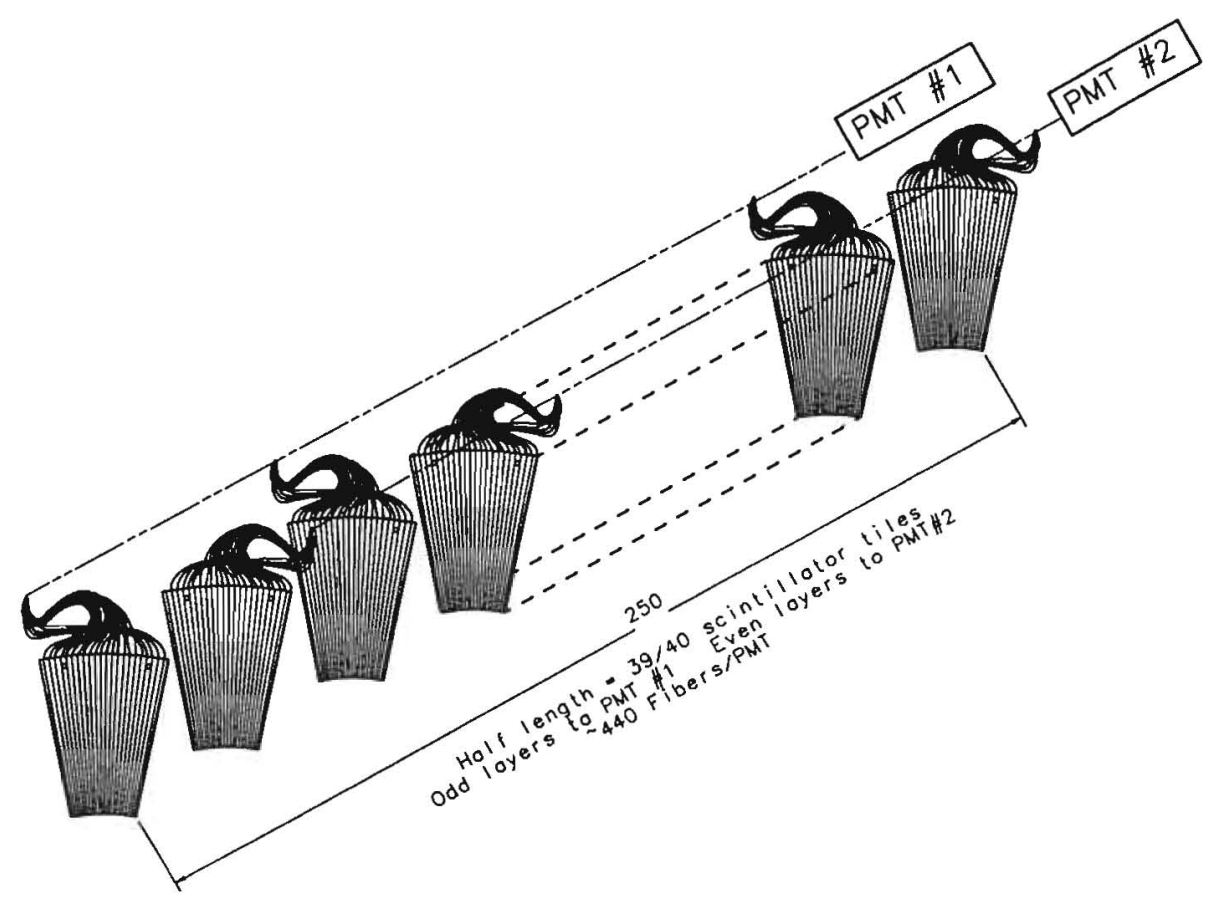

Figure 80: Exploded view of the even-odd fiber routing scheme that maps fibers from alternate scintillator sectors to separate photomultiplier tubes.

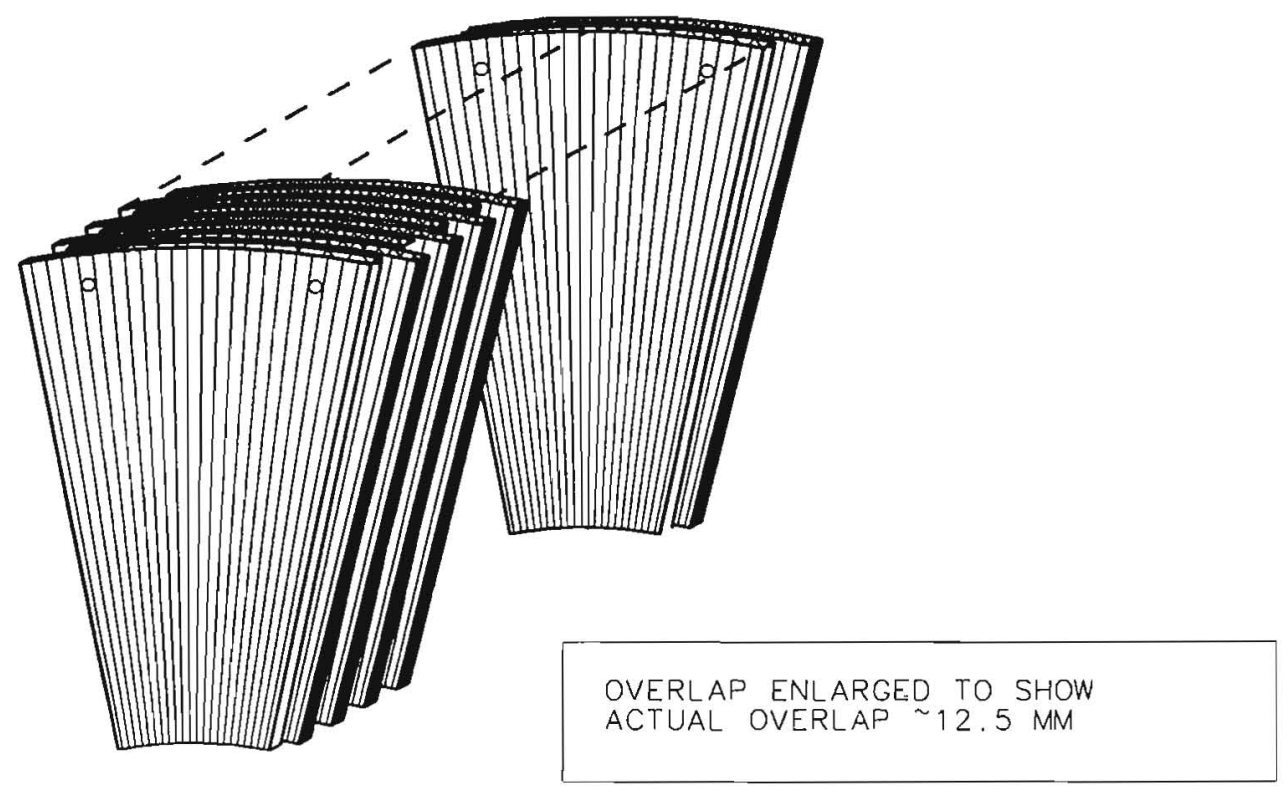

Figure 81: Illustration of the scintillator sector "clocking" scheme that eliminates first order cracks. the nominal fiber spacing is indicated. 


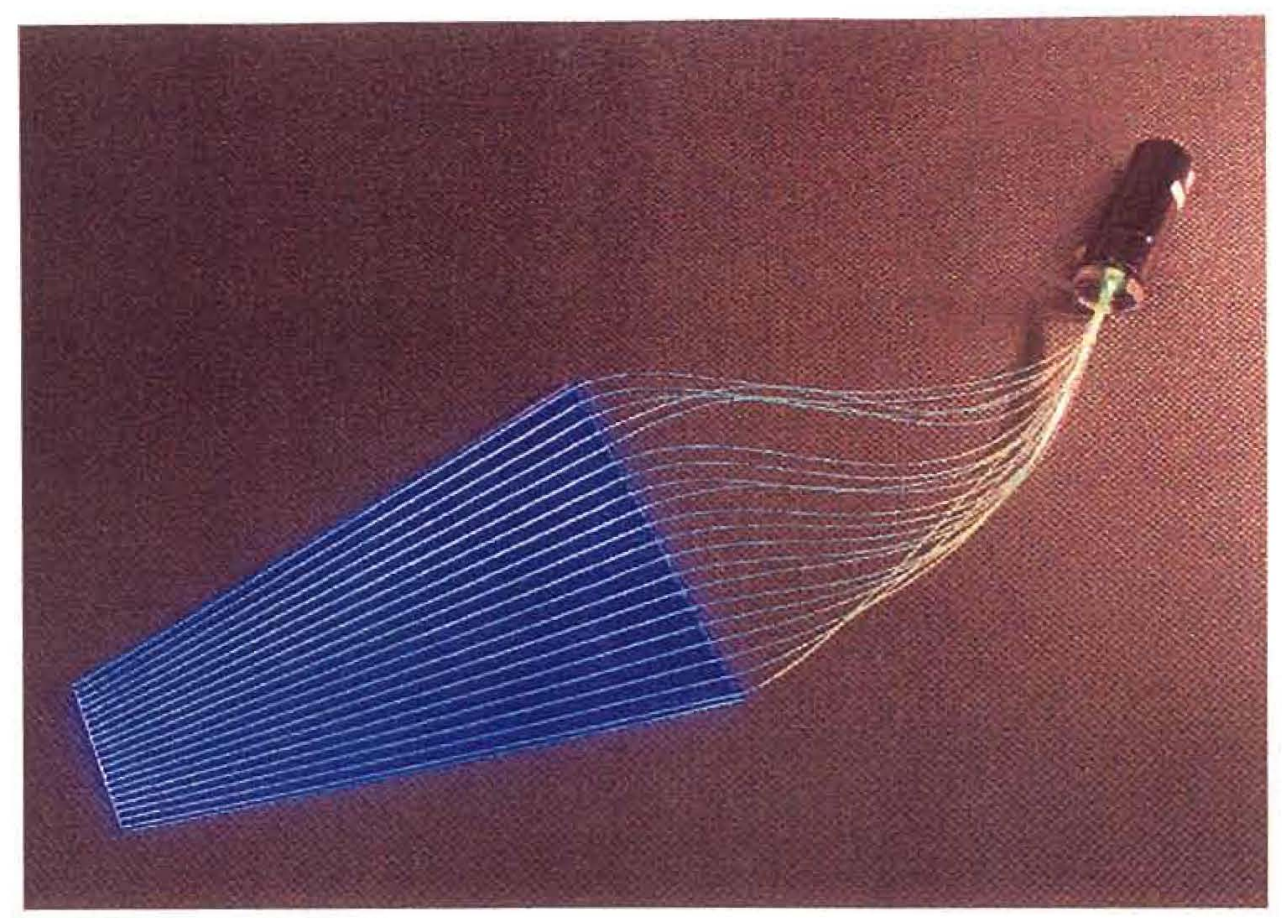

Figure 82: Photograph of a scintillator sector instrumented with the nominal fiber pattern bundled to a photomultiplier tube. This is one of many prototypes studied for light yield.

which is a small fraction of the expected scintillator cost.

\subsubsection{Pumping system requirements.}

In order to keep background from beam interactions with the residual gas at a negligible level the vacuum system must maintain a pressure of less than $1 \times 10^{-6}$ torr. Maintaining this pressure is significantly complicated by the out-gassing load of the $\sim 8000$ straws that compose the DMS, and the $\sim 27$ tons of plastic scintillator that compose the VVS and BIVS detectors. The out-gassing rate of the total straw system has been extrapolated from prototype measurements (refer to DMS section5.7) to a negligible load of 105 liters/second at $1 \times 10^{-6}$ torr.

In contrast the outgassing load from the $\sim 50,000$ plastic scintillator tiles is quite large. Based on outgassing measurements of plastic scintillator tiles and lead [162] the raw gas load corresponds to $600 \mathrm{~K}$ liters/second at $1 \times 10^{-6}$ torr after 24 hours of pumping. The VVS and BIVS designs will employ a "virtual vacuum" thin membrane encapsulating each VVS module that is pumped separately but is connected to the main decay volume with a small leak. This scheme shunts local out-gassing away from the decay volume vacuum into a lesser quality auxiliary vacuum. This shunting strategy was used successfully in the $\mathrm{KTeV}$ vacuum veto system. Based on similar estimates of the $\mathrm{KTeV}$ outgassing load and the actual pressure achieved in the field, this virtual vacuum scheme corresponds to to a reduction factor of about $\times 8$ [163] in outgassing into the main decay volume. The residual load on the decay volume of $75 \mathrm{~K}$ liters/second at $1 \times 10^{-6}$ torr can be handled with a $\mathrm{KTeV}$ sized system, that delivered a pumping capacity of $100 \mathrm{~K}$ liters/second at $1 \times 10^{-6}$ torr. We regard this outgassing load as an upper limit since it is based on measurements after only 24 hours of pumping. 


\subsubsection{Scintillator studies.}

We have made extensive measurements of a variety of (BC-404 tile/BCF-92 fiber) geometries and coupling. A cosmic ray telescope with an $80 \mathrm{~cm}$ steel muon filter slab was employed as the primary apparatus to measure scintillator response to traversing minimum ionizing particles (MIPs). A MIP deposits $1 \mathrm{MeV}$ on average as it traverses the tile, and the corresponding pulse shape is shown in figure 83. A typical pulse height distribution for traversing MIPs is shown in figure 84. A fit to this pulse height distribution based on a convolution of the expected Landau shape with a Poisson distribution of photoelectrons is also shown in figure 84. From this fit the number of photoelectrons per $\mathrm{MeV}$ can be extracted.

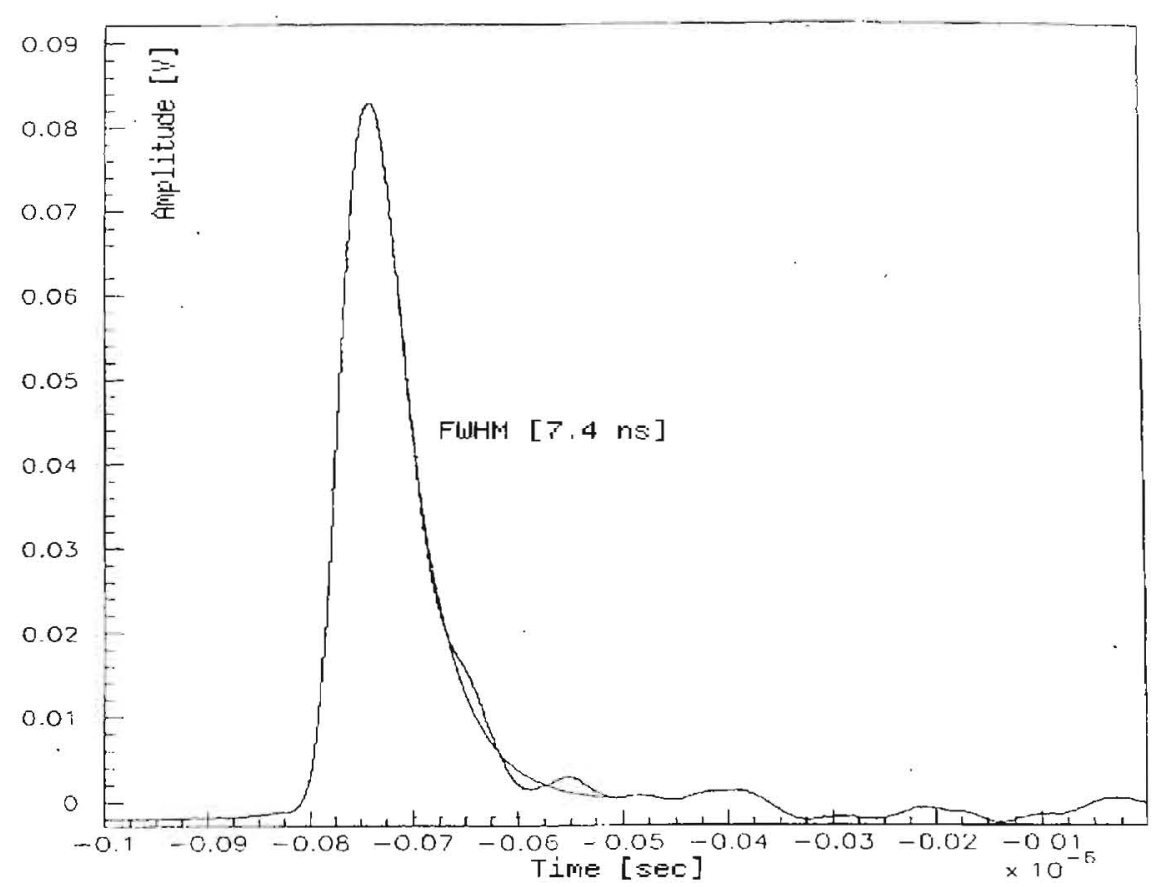

Figure 83: (a): Typical pulse (1 MeV deposited) from a MIP traversing a tile of BC404 readout with BCF-92 fibers. An average of 512 traces fit to extract a FWHM as shown.

The photoelectrons per $\mathrm{MeV}$ deposited has been independently determined by measuring the gain of the photomultiplier with single photo-electron measurements. These direct measurements of the photo-electron yield agree well with yield determined from the Landau-Poisson convolved fits.

Our baseline tile geometry and fiber coupling has fibers embedded in $1.1 \mathrm{~mm}$ diameter grooves $1.7 \mathrm{~mm}$ beneath the scintillator surface, with an average transverse spacing of $1 \mathrm{~cm}$. The fiber ends in the scintillator are mirrored with vacuum evaporation of aluminum, and the tiles are wrapped in white Tyvek. Initially we paid only modest attention to the surface quality of the cut groove, and typically achieved 11-15 photo-electrons/MeV. We have found that cutting and polishing the grooves with attention paid to the final surface finish significantly raises the light yield upwards to about 20 photo-electrons/MeV. We have also found that gluing the fibers into nominally cut grooves with attention paid to the quality of the glue joint (no air bubbles, etc) can likewise raise the light yield to to about 20 photo-electrons/MeV. Finally the use of green extended photomultiplier tubes (EMI-9954) has further increased the output of a polished groove tile up to about 25 photoelectrons/MeV. 


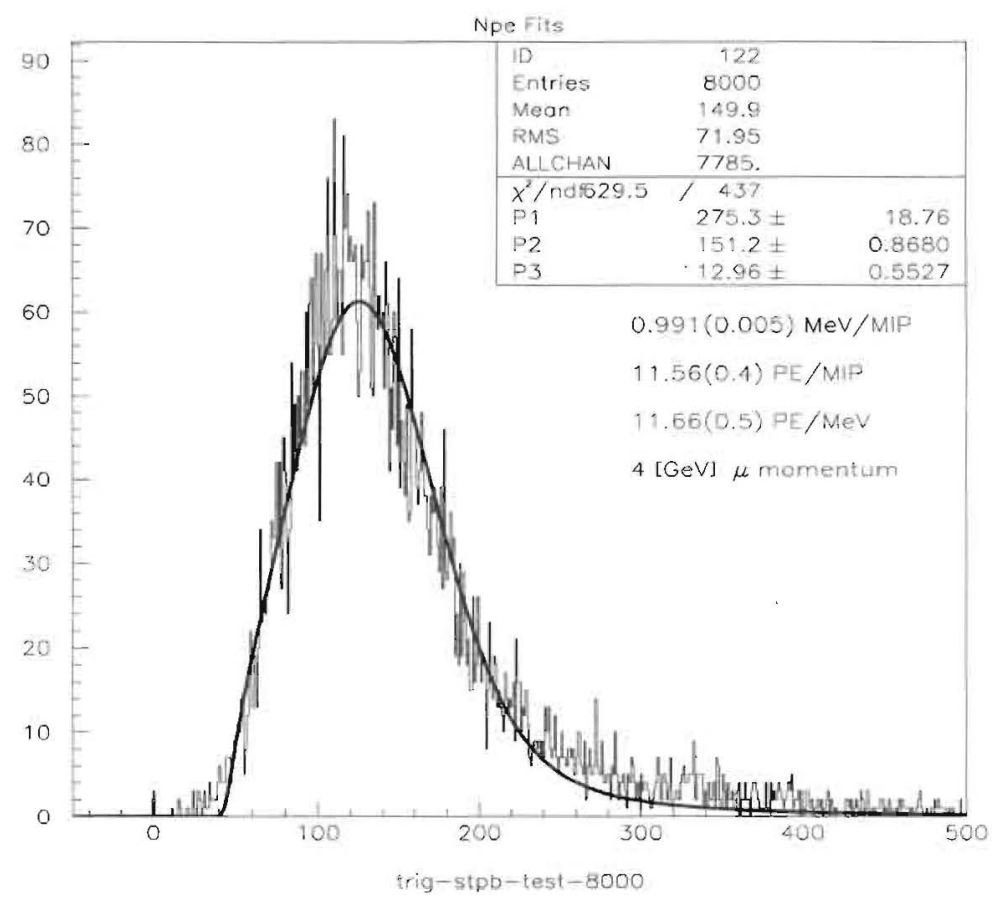

Figure 84: (a) Pulse height distribution of a MIP traversing a single tile. A Poisson convolved Landau fit is overlaid, from which the number of photoelectrons can be extracted.

In addition to these tile studies based on the BC-404/BCF-92 solution, our IHEP/Protvino colleagues have studied the relative light yield of a wide variety of scintillator-fiber combinations. Several combinations of fast scintillator fabricated by Russian vendors readout with BCF-92 fiber yield light outputs of $(60 \%-70 \%)$ of the BC- 404 reference. It is possible that the Russian scintillator vendors can provide scintillator at considerably lower cost than Bicron. Given the headroom of the $\mathrm{BC}-404$ light output over the CKM specification of 10 p.e. / MeV deposited, these alternative sources of scintillator will be investigated further.

We have yet to embark on the complex optimization between scintillator type, fabrication difficulty and cost. Although we clearly must carefully consider this optimization, there is reason to be optimistic from our prototype studies that sufficient flexibility exists for a robust and affordable solution to be found.

\subsubsection{Efficiency requirement for charged particles.}

The VVS must efficiently detect charged particles from kaon decay backgrounds and the inelastic interaction of beam particles with residual gas in the vacuum decay volume. As an example, The background process of $\left(\mathrm{K}^{+} \rightarrow \pi^{+} e^{+} e^{-}\right)$requires that the associated electrons are vetoed with high efficiency. These decay and interaction background mechanisms are discussed in section 7 . To facilitate rejection of charged particles, the upstream face of the VVS sampling structure will start with scintillator.

\subsubsection{Efficiency studies of low energy photons $(<20 \mathrm{MeV})$.}

The detection efficiency of low energy photons in a sampling device is limited by the fraction of deposited energy in the active medium fluctuating below the detection threshold. This effect 
drives the VVS design toward the fine sampling ratio of $1 \mathrm{~mm}-\mathrm{Pb} / 5 \mathrm{~mm}$-scintillator, where the relatively high average fraction of $30 \%$ is deposited in the active medium. The detection threshold is determined by the combined effect of accidental energy deposits and the photo-electron (p.e.) statistics of 10 p.e/(deposited $\mathrm{MeV})$. Based on these considerations we have assumed a threshold of $1 \mathrm{MeV}$ (10 p.e.) on the detected energy, corresponding to an average. incident ploton energy of about $3 \mathrm{MeV}$.

In the photon energy range of $6-20 \mathrm{MeV}$ the inefficiency decreases from $50 \%$ to about $7 \%$ due to the combined effect of the half-filled VVS geometry and the smaller probability of the deposited energy fluctuating below the detection threshold (1-2) MeV. At the $1 \%$ level of uncertainty GEANT electromagnetic shower simulations can be used to accurately model these dominant sources of inefficiency. Figure 85 plots the inefficiency of low energy $K^{+} \rightarrow \pi^{+} \pi^{0}$ photons illuminating a full GEANT simulation of the VVS. Below $6 \mathrm{MeV}$ the inefficiency increases rapidly due to the combined effect of the half-filled geometry and the $1 \mathrm{MeV}$ visible energy threshold. The effect of raising the visible detection threshold from $1 \mathrm{MeV}$ to $2 \mathrm{MeV}$ raises the average inefficiency in the $0-20 \mathrm{MeV}$ bin from $40 \%$ to $54 \%$.

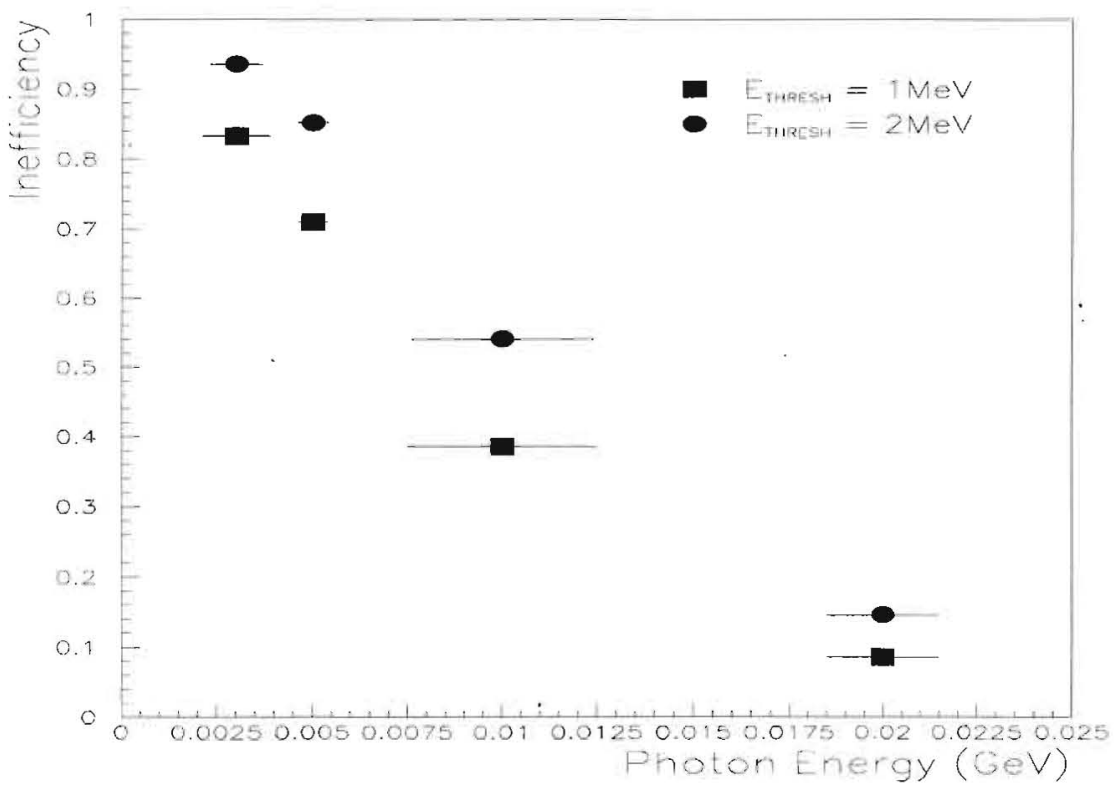

Figure 85: GEANT simulation of the low energy inefficiency $(0-20 \mathrm{MeV})$ for a threshold of 10 and 20 photoelectrons which corresponds to $3 \mathrm{MeV}$ and $6 \mathrm{MeV}$ incident photon energy respectively.

\subsubsection{Effect of accidental activity on the detection efficiency of low energy photons.}

Accidental energy deposits that result from high rate can corrupt the detection of low energy photons in two ways: First, an accidental energy deposit in a VVS module unassociated with a $K^{+} \rightarrow \pi^{+} \pi^{0}$ (or $K^{+} \rightarrow \pi^{+} \nu \bar{\nu}$ ) decay but within the experiment time resolution window can veto the event. This doesn't increase the $K^{+} \rightarrow \pi^{+} \pi^{0}$ background but does directly compromise the $K^{+} \rightarrow \pi^{+} \nu \bar{\nu}$ acceptance. Second, an accidental energy deposit that arrives before the the triggertime but outside of the experimental time resolution can mask detection of a small energy deposit within the in-time trigger window. This pileup effect on a $1 \mathrm{MeV}$ in-time energy deposit is illustrated 
in figure 86 , which plots the time development of a muon that arrives 20 nsec before the trigger time. Clearly both these rate effects are reduced with faster veto response, which motivates the choice of high speed scintillator shown in figure 83. The magnitude of these two rate effects will be addressed in turn.

From figure 83 it is reasonable to assume that the VVS can deliver a leading edge time resolution of $\sigma=1 \mathrm{nsec}$, even for small pulses. Given a leading edge time resolution of $\sigma=1 \mathrm{nsec}$ we can expect that an offline leading edge separation of $\pm 3 \sigma= \pm 3 \mathrm{nsec}$ between hit channels is feasible. We have estimated the rate as function of visible energy threshold from a GEANT simulation of the beam and detector described in section 4 and 7 . In these estimates we have accounted for the $K^{+}, \pi^{+}$, and proton content of the beam. The rates for the various veto systems are listed below in table 26 .

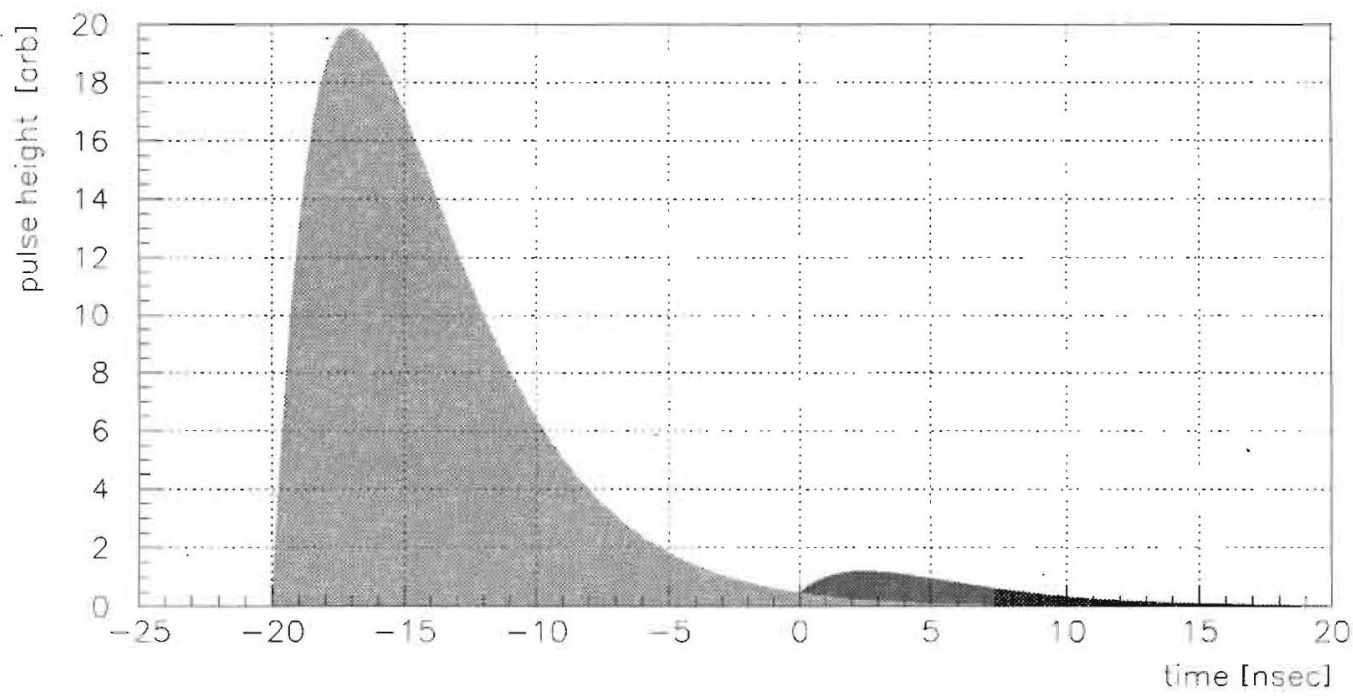

Figure 86: Illustration of the pileup effect on a $1 \mathrm{MeV}$ deposit from an early muon that deposits 20 $\mathrm{MeV}$ of energy $20 \mathrm{nsec}$ before the trigger in-time window. The pulse-shape parameters were taken from our BC404/BCF92 prototype measurements.

\begin{tabular}{|l|l|l|l|l|l|l|}
\hline $\begin{array}{l}\text { Visible Energy } \\
(\mathrm{MeV})\end{array}$ & $\begin{array}{l}\text { BIVS-OR } \\
(\mathrm{MHz})\end{array}$ & $\begin{array}{l}\text { BIVS-MAX } \\
(\mathrm{MHz})\end{array}$ & $\begin{array}{l}\text { VVS-OR } \\
(\mathrm{MHz})\end{array}$ & $\begin{array}{l}\text { VVS-MAX } \\
(\mathrm{MHz})\end{array}$ & $\begin{array}{l}\text { FVS-OR } \\
(\mathrm{MHz})\end{array}$ & $\begin{array}{l}\text { FVS-MAX } \\
(\mathrm{MHz})\end{array}$ \\
\hline \hline 1 & 7.1 & 2.6 & 6.5 & 0.4 & 13 & 1.5 \\
3 & 6.8 & 2.4 & 6.0 & 0.2 & 13 & 1.3 \\
30 & 3.8 & 1.5 & 1.5 & 0.01 & 12 & 0.69 \\
300 & 2.5 & 0.3 & $\sim 0$ & $\sim 0$ & 4.0 & 0.15 \\
\hline
\end{tabular}

Table 26: Rates .vs. visible energy threshold for CKM veto systems. Maximum phototube rates per channel (MAX) and global sub-system rates (OR) are listed.

A few general comments regarding table 26 should be made. Regarding the VVS, a maximum 
tube rate of $400 \mathrm{kHz}$ for a $1 \mathrm{MeV}$ is tolerable, as will be discussed below. The global VVS sub-system "OR" rate of $6.5 \mathrm{MHz}$ is dominated by kaon decays within the decay volume. As expected, rates in the BIVS are high, but as will be shown in section 7 the threshold per tube doesn't need to be less than $20 \mathrm{MeV}$. Rates in the FVS are likewise high, but offline veto thresholds can be correspondingly higher and cluster-based given the much higher energy spectrum. The VVS show a significant drop in rate between $3 \mathrm{MeV}$ and $30 \mathrm{MeV}$-which corresponds to crossing the traversing muon energy deposit threshold $(\sim 25 \mathrm{MeV})$. A similiar drop in rate is seen in the FVS between $30 \mathrm{MeV}$ and $300 \mathrm{MeV}$ which crosses the higher $100 \mathrm{MeV}$ MIP threshold in the FVS.

The 6.5 MHz global VVS rate within a $6 \mathrm{nsec}$ ( $\pm 3 \mathrm{nsec}$ ) window straddling the trigger time corresponds to a $4 \%$ increase in the experimental deadtime. From table 26 one can see that the hottest VVS channel runs at $400 \mathrm{kHz}$ for a $1 \mathrm{MeV}$ threshold. This rate can mask detection of a $1 \mathrm{MeV}$ deposit as shown in figure 86. Assuming a channel recovery time of $20 \mathrm{nsec}$ to a $1 \mathrm{MeV}$ veto threshold, this pileup mechanism contributes an additional 1\% to the deadtime. This estimate is based on figure 86 and assumes a MIP early energy deposit. Table 26 shows that VVS rate in fact is dominated by MIP deposits, but to be conservative we assume a recovery time of $40 \mathrm{nsec}$ to account for higher average energy deposits, which raises this component of the dead time to $2 \%$. Our readout system will allow full access to the time profile in 1 nsec bins for several hundred nsec prior to the event. This available history will allow us to directly monitor these pileup effects.

\subsubsection{Efficiency studies of medium energy photons $(20 \mathrm{MeV}-1000 \mathrm{MeV})$.}

The dissection of the $K^{+} \rightarrow \pi^{+} \pi^{0}$ background in section 3.4.6 demonstrates our relative insensitivity to the medium energy $(20 \mathrm{MeV}-400 \mathrm{MeV})$ inefficiency in the VVS. Increasing this inefficiency by $\times 4$ only raises the total background by $16 \%$. Despite this insensitivity, it is worthwhile to understand what we can conclude from relevant measurements that have been made in this energy region. Foremost among these are measurements of the photon veto inefficiencies of BNL experiment E787. Integrated over the photon energy spectrum of $\pi^{0}$ 's from stopped $K^{+} \rightarrow \pi^{+} \pi^{0}$ decays $(20-205 \mathrm{MeV})$, the BNL experiment has demonstrated a $\pi^{0}$ inefficiency of $\sim 1.7 \times 10^{-6}$ for a 14 $\mathrm{L}_{\text {rad }} 1 \mathrm{~mm}$-Pb/5 mm-scintillator sampling veto detector [164]. An internal E787 study [165] has examined the unfolding of the $\pi^{0}$ inefficiency into the inefficiencies of the two constituent photons. There are some systematic issues in such an analysis associated with the requirement of positively identifying one of the $\pi^{0}$ photons. Nevertheless, the corresponding inefficiency [165] plotted in figure 19 serves as a useful benchmark in this energy region for CKM.

In addition to these in-situ measurements, the limits of what can be achieved in this medium energy region have been probed in a series of tagged photon test beam experiments at the Institute for Nuclear Science (INS) in Japan. The technique and results will be briefly discussed.

The photon veto efficiency of both sampling and fully active detectors is ultimately limited by photo-nuclear and and photo-nucleon processes where a large fraction of the incident energy can be converted into minimum or non-ionizing particles. The dominant loss mechanism for medium energy photons $(20 \mathrm{MeV}-400 \mathrm{MeV}$ ) on heavy nuclei is the production of multi-neutron final states where the incident photon interacts coherently with the entire nucleus. The INS technique measures this cross section by illuminating a test device with energy tagged bremsstrahlung photons $(20 \mathrm{MeV}$ $1100 \mathrm{MeV}$ ) produced from an intense electron beam incident on a thin foil. Unfortunately the effects of multiple bremsstrahlung and scattering in the foil result in a photon false-tag probability of $\sim 1 \%$. This residual false-tag rate precludes a direct photon inefficiency measurement at the relevant scale. To circumvent this tagging problem the incident "photon" trigger requires coincident activity in a neutron detector (liquid scintillator) surrounding the test device. Hence the INS inefficiency measurements are essentially direct measurement of photo-nuclear processes that produce slow 
neutrons.

This test beam program has measured [161] the photo-nuclear cross sections of several $\mathrm{Pb}$ scintillator sampling and fully active CsI prototype veto modules in the $(20 \mathrm{MeV}-1100 \mathrm{MeV})$ medium energy range.

The measured photo-nuclear cross section can then be converted into a detection inefficiency shown in figure 87 . Inefficiency due to electromagnetic shower fluctuations in $1 \mathrm{~mm}-\mathrm{Pb} / 5 \mathrm{~mm}$ scintillator are not included in figure 87. It is evident from figure 87 that the photo-nuclear contribution to the inefficiency of a $1 \mathrm{~mm}-\mathrm{Pb} / 5 \mathrm{~mm}$-scintillator sampling design is safely below both our medium energy and high energy requirements.

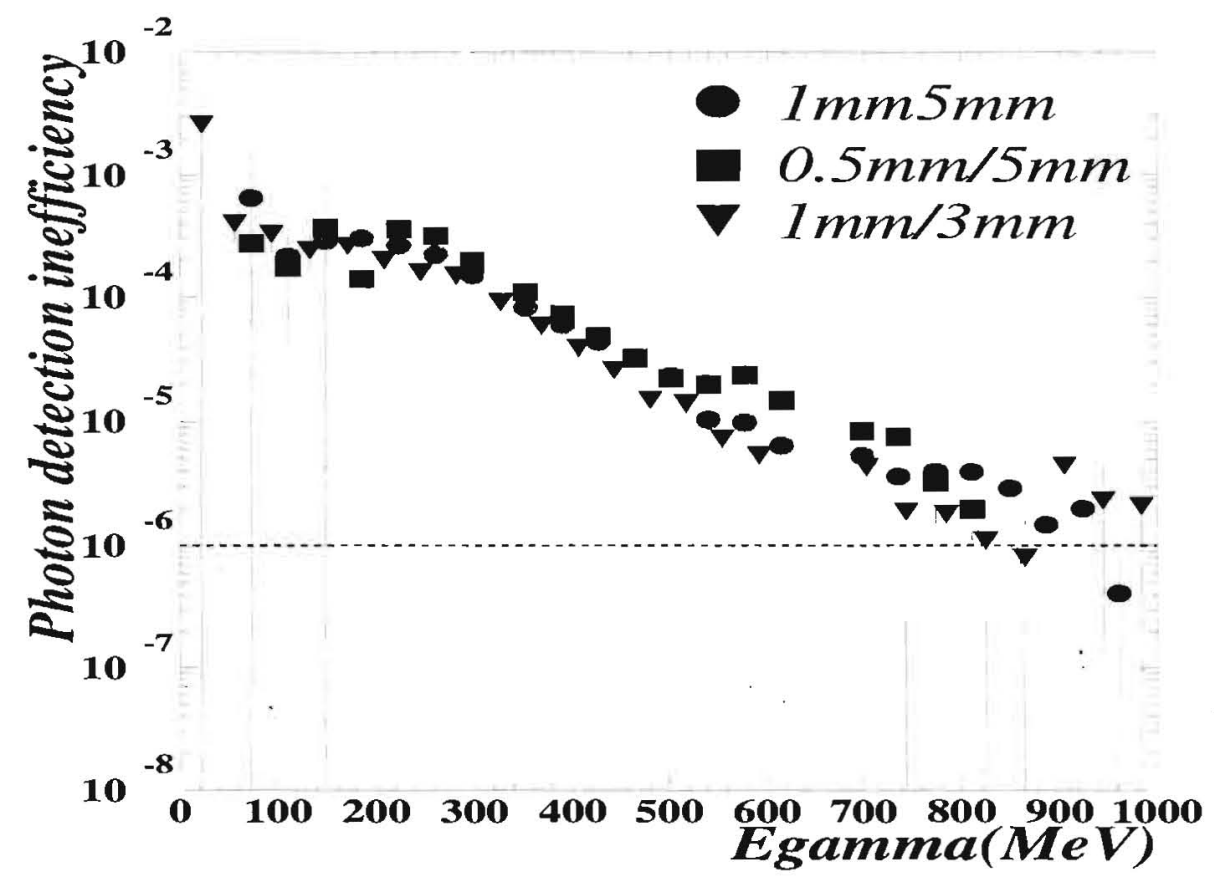

Figure 87: Photon veto inefficiency of Pb-scintillator sampling devices inferred from photonuclear cross section measurements of these devices at the INS test facility. The normal effects of electromagnetic shower fluctuations are not included.

\subsubsection{Theoretical limits of high energy efficiency.}

The detection efficiency of high energy photons is ultimately limited by considerations of conversion length, shower fluctuations in a sampling device, and photo-nuclear effects. These mechanisms will be discussed in turn. Regarding conversion probability, the conversion length in the VVS is effectively infinite. Each VVS module is $15 X_{0}$ deep and the angle of incidence of high energy photons is in the $10-100$ mrad range as shown in figure 88. Any leakage out the back of one VVS module will transport directly to the next module downstream. A more significant concern is that of shower fluctuations in the active material. For a $1 \mathrm{GeV}$ photon normally incident on a bulk $1 \mathrm{~mm}-\mathrm{Pb} / 5 \mathrm{~mm}$-scintillator, the probability of a mean energy deposit of $\sim 300 \mathrm{MeV}$ fluctuating below a $1 \mathrm{MeV}$ deposition threshold is negligible. This confortable geometry of course is not the real scenario for a grazing high energy photon incident on the inner aperture of a VVS module as shown in figure 89 . 


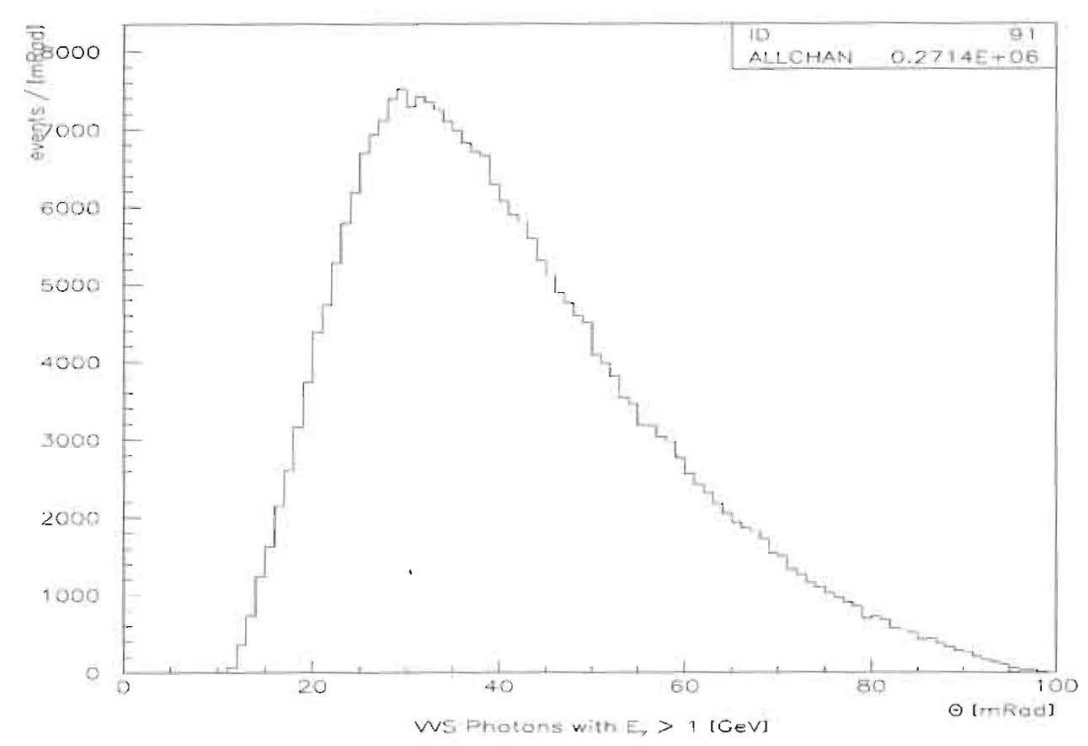

Figure 88: Angular distribution of high energy photons $(>1 \mathrm{GeV})$ incident of the VVS.

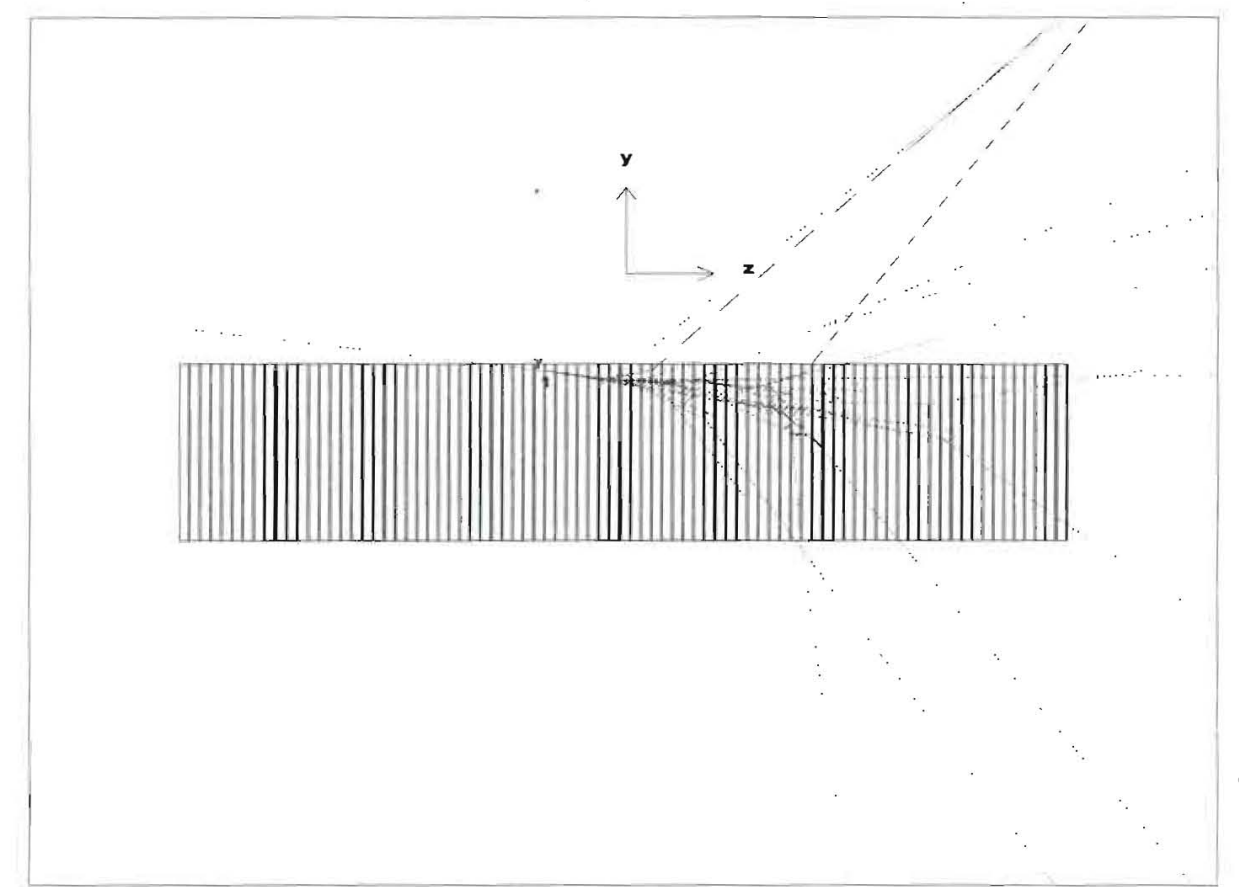

Figure 89: GEANT simulation of a $1 \mathrm{GeV}$ photon with grazing incidence from the left on the inner aperture of a VVS module. 
From figure 88 it is clear that most high energy photons incident on the VVS strike a module close to the inner aperture with a minimum angle of $10 \mathrm{mrad}$. Further, about $\sim 1 / 2$ of the photons have entry points distributed uniformly along the inner annular surface of a VVS module. In such geometry, a significant fraction of the incident energy leaks out of the inner aperture back into the decay volume. As an extreme case, a photon could convert early in the lead and both the conversion $e^{+}$and $e^{--}$could scatter out back into the decay volume. If this renegade conversion pair encounters the spectrometer magnetic field before encountering another VVS module it could be lost. These loss mechanisms are strongly suppressed by the minimum incident angle of $10 \mathrm{mrad}$ but still remain a concern given the required inefficiency. We have addressed these concerns with a GEANT shower simulation of incident $K^{+} \rightarrow \pi^{+} \pi^{0}$ high energy photons illuminating a VVS module defined in detail. As indicated in figure 19, no inefficient events were found at a sensitivity of $3 \times 10^{-5}$. In this GEANT simulation we assumed any energy that leaked back into the decay volume was lost. Most of any leaked energy is destined for an away-side VVS module, but in order to preserve the best possible timing to combat rate effects we assumed this energy is lost. Traversing the decay volume accumulates a $\sim 3 n s e c$ time of flight. Hence we conclude that inefficiency mechanisms associated with high energy photon conversion and electromagnetic shower development are below our requirement of $3 \times 10^{-5}$.

Regarding photo-nuclear effects, at photon energies exceeding $(800-1000 \mathrm{MeV})$ loss mechanisms are dominated by the photo-production of hadrons off nucleons. At these high energies the $\sim 100 \mu B$ Barn nucleon cross section corresponds to an effective hadronic "branching fraction" (normalized to one radiation length) of photons in lead of about $1-2 \times 10^{-4}$. Given a high energy VVS inefficiency requirement of $3 \times 10^{-5}$ for $>1 \mathrm{GeV}$ photons, it is necessary to understand the nature of these processes. Fortunately the relevant photo-production processes have been well measured off both. nucleons [166] and heavy nuclei at SLAC and DESY. Processes of greatest concern are those where the target nucleon and nucleus remains intact, and where the final state is composed of a small number of minimum or non-ionizing particles. This class of interactions is dominated by diffractive $\gamma N \rightarrow \rho^{0}, \omega^{0}, \phi^{0}$ and $\gamma p^{+} \rightarrow n^{0} \pi^{+}$production, which together correspond to about $17 \%$ of the total cross section in the $1-3 \mathrm{GeV}$ region. The cross section of the process $\gamma N \rightarrow \rho^{0} \rightarrow \pi^{+} \pi^{-}$is shown in figure 90 , with the resonant and diffractive components fitted. The measured $t$ dependence of the $\gamma p^{+} \rightarrow n^{0} \pi^{+}$reaction is shown in figure 91 where the steep forward diffractive peak is evident at low $t$.

These data are well described by a sum of known resonances $\left(N^{*}, \Delta\right.$, etc) and non-resonant diffraction $\left(e^{-8|t|}\right)$, where the target nucleon recoils slowly. Even if the struck nucleon leaves the nucleus, this slow particle will be quickly absorbed in the lead. Hence these processes are in principle quite problematic since a high energy photon can transfer essentially all of it's incident energy to a small number of forward hadrons. The cross sections off lead (assuming $A^{1.0}$ dependence for $V^{0}$ photo-production and $Z^{1.0}$ dependence for $\gamma p^{+} \rightarrow n \pi^{+}$production) and the corresponding effective branching ratio are listed in table 27.

The sum of problematic exclusive processes in table 27 would correspond to an inefficiency of about $4 \times 10^{-5}$ if the hadronic final states were to pass undetected. The VVS will however have good efficiency for detecting these final states. Each charged hadron will deposit a minimum of $1 \mathrm{MeV}$ for each plane of scintillator traversed, summing to at least $80 \mathrm{MeV}$ for each VVS module travérsed. In addition, the VVS presents a large integrated hadronic interaction length which will initiate a hadronic shower ensuring detection. It is clear however that from the level of these processes it is important to maintain sub-MIP thresholds to veto high energy photons to the required level.

A more difficult task than the task of vetoing a cleanly traversing photo-produced $\pi^{+} \pi^{-}$pair or single produced $\pi^{+}$is the case where the pion(s) is produced and then scatters off a lead nucleus away from the VVS acceptance. We have not simulated this or other mechanisms where photo- 


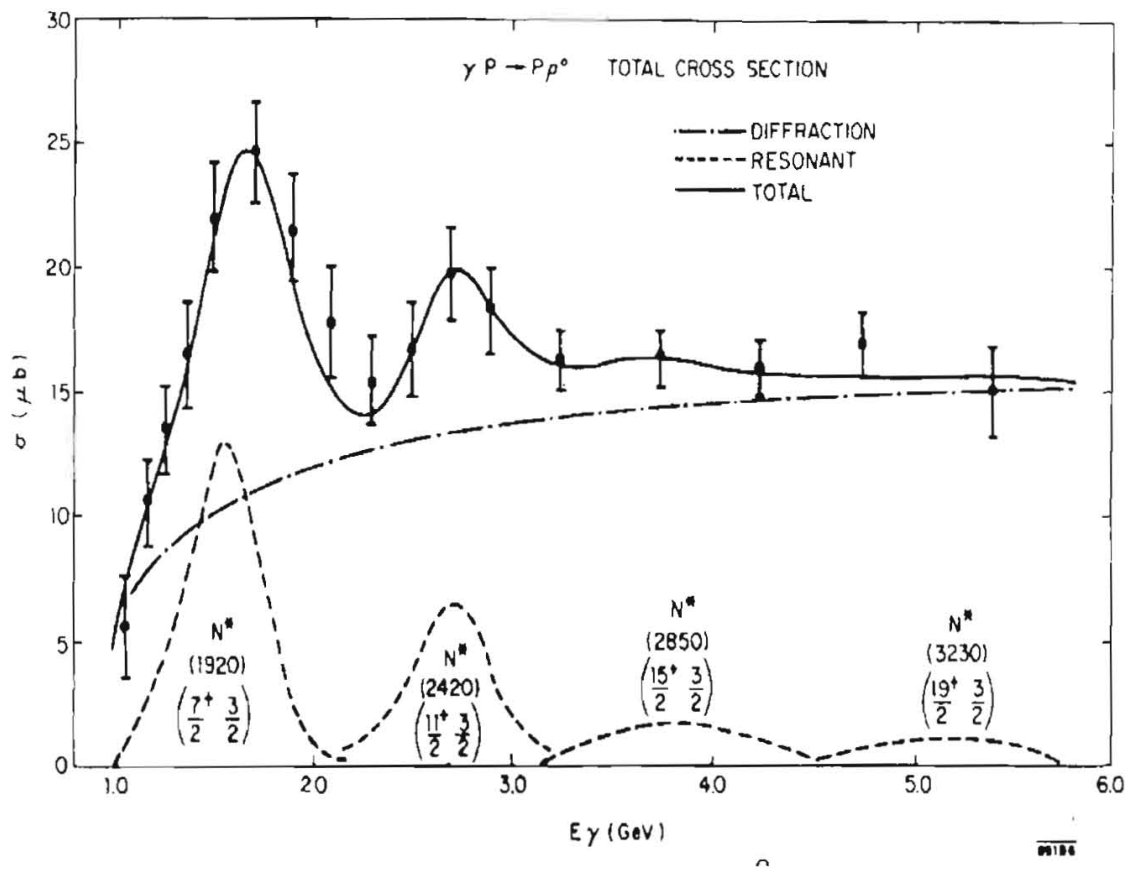

Figure 90: Measured [166] cross section of $\gamma p \rightarrow \rho^{0} \rightarrow \pi^{+} \pi^{-}$photo-production .vs. energy.

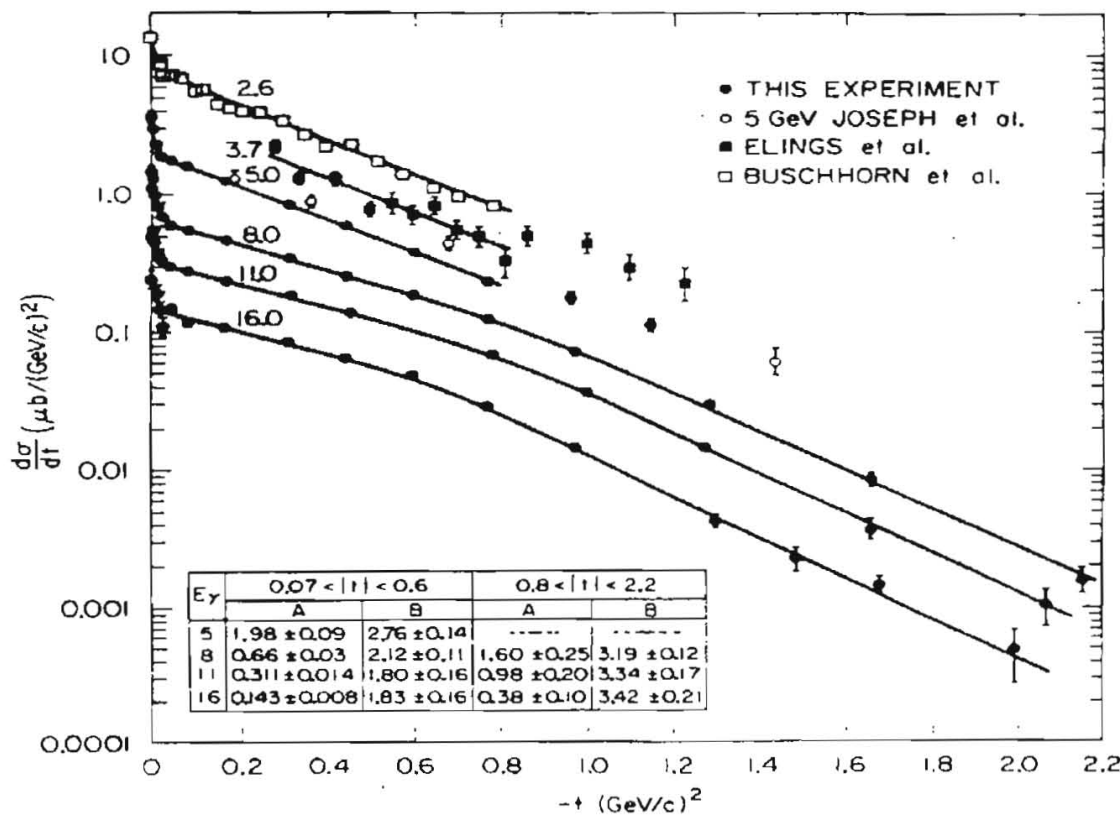

Figure 91: Measured [167] $t$ dependence of $\gamma p \rightarrow n \pi^{-}$. The diffractive peak at low $t$ is clearly evident. 


\begin{tabular}{|l|r|l|}
\hline Photo-process & $\begin{array}{r}\text { Mean cross-section on Pb } \\
(E: 1-3 \mathrm{GeV})\end{array}$ & Effective branching fraction \\
\hline \hline$\gamma-P b \rightarrow A l l$ & $\sim 20,000 \mu B$ & $1-2 \times 10^{-4}$ \\
\hline$\gamma-P b \rightarrow \rho^{0} \rightarrow \pi^{+} \pi^{-}$ & $3300 \mu B$ & $3 \times 10^{-5}$ \\
$\gamma-P b \rightarrow n \pi^{+}$ & $410 \mu B$ & $4 \times 10^{-6}$ \\
$\gamma-P b \rightarrow \Lambda K^{0}$ & $480 \mu B$ & $4 \times 10^{-6}$ \\
$\gamma-P b \rightarrow \phi \rightarrow K^{+} K^{-}$ & $3 \mu B$ & $3 \times 10^{-7}$ \\
$\gamma-P b \rightarrow \phi \rightarrow K_{L} K_{S}$ & $2 \mu B$ & $2 \times 10^{-7}$ \\
\hline
\end{tabular}

Table 27: Cross section of relevant photo-production processes.

produced hadrons can get lost, but it is reasonable to expect an efficiency of greater than $90 \%$ for these processes which suppresses the corresponding inefficiency to less than $4 \times 10^{-6}$. It is possible that the ultimate level of inefficiency is even considerably less than $4 \times 10^{-6}$, but making that case would require careful simulation of these loss mechanisms.

\subsubsection{Practical concerns of high energy efficiency.}

In the previous section we argued that the sum of expected physical processes that can cause high energy inefficiency is less than the requirement of $3 \times 10^{-5}$ for $E_{\gamma}>1 \mathrm{GeV}$. A parallel, and more difficult, case must be made that instrumental losses can be controlled to less than $3 \times 10^{-5}$ as well. The first order concern of hermetic spatial coverage and sufficient conversion depth is well addressed by the VVS design previously described. The angular distribution of high energy $1 \mathrm{GeV}$ $K^{+} \rightarrow \pi^{+} \pi^{0}$ photons in figure 88 is completely covered by the VVS and forward systems.

Of greater concern is hermeticity in time: Effects from readout chain reliability and high counting rates can induce unmonitored deadtime - the most likely mechanism for losing a $1 G e V$ photon is that you weren't looking and you didn't know it. This concern is common to all CKM detector systems to varying degrees, but the stringent VVS inefficiency requirement and corresponding deadtime specification is particularly challenging.

To set the scale, an unmonitored coherent VVS failure during just three spills $(3 \mathrm{sec})$ per live year of running violates the VVS inefficiency requirement. This dramatic example is in fact straightforward, although not trivial, to detect. A far more troublesome and likely example of breaking the inefficiency budget is if three of the 1,088 VVS sector modules have a $\sim 1 \%$ inefficiency that goes undetected. Clearly these examples underscore the importance of readout redundancy and monitoring.

\subsubsection{VVS Hermetic design strategy.}

As previously discussed, the high energy inefficiency requirement of $3 \times 10^{-5}$ implies controlling VVS module readout and rate-induced deadtime to a comparable level. Regarding the readout chain, each VVS module has two levels of redundancy. First, every other layer of the scintillator stack composing a module is readout by a separate photomultiplier tube: The 41 odd layers are mapped to one tube, the 40 even layers are mapped to a second tube. This cross-tube monitoring scheme enables the constraint that both tubes viewing the same sector must agree for large signals. The second level of redundancy is that each channel will be digitized with both a pipeline ADC (QIE) and a two-level (high/low) TDC system. Veto signals from the ADC and TDC systems serve as cross monitors and will be constrained to concur with each other. 
In addition to cross-monitoring energy deposits, each VVS photomultiplier will be monitored with a LED or laser flash running at $1 \mathrm{~Hz}$ to monitor the tube gain and provide a diagnostic tool. The sector gain and efficiency will be further monitored by the in-situ sample of muons that will be traversing VVS stations (depositing $80 \mathrm{MeV}$ ) which can provide course tracking. As discussed in the trigger and data acquisition section, the readout time aperture per event will be about $1 \mu \mathrm{sec}$, during which one or more muons will be "tracked" in the VVS. These muons will be a very useful sample to continuously monitor the VVS efficiency. An extended time record is also important to monitor deadtime induced by accidental activity within the sarne channel. The effect of high rate on deadtime is primarily a concern at low energy as illustrated in figure 86 , where the the traversing $\sim 7 M H z$ muon flux deposits $20 \mathrm{MeV}$ per channel compared to an offline veto threshold of $1 \mathrm{MeV}$. At the analysis stage the low energy veto threshold will be a function of previous recorded activity. Given the high speed of candidate veto scintillator evident in figure 83, a deadtime of $20 \mathrm{nsec}$ following a $80 \mathrm{MeV}$ muon deposit to return to a $1-2 \mathrm{MeV}$ threshold is reasonable. As discussed previously, this corresponds to a 1-2\% accountable deadtime for maximum rate of $\sim 400 k H z$ per VVS channel.

\subsubsection{Efficiency studies of high energy electrons.}

In principle the best way to probe the limits of high energy photon inefficiency is to illuminate test structures with a tagged photon beam which has a false-tag rate commensurate with $\sim 1 \times 10^{-5}$. Unfortunately this is essentially impossible to achieve: After several rounds of careful design the INS tagged photon test beam was able to achieve a false tag rate of about $1 \%$. This level of tagging is useful for studying low energy photon detection efficiency, but falls far short of what is required at high energy.

As argued previously, the list of physical processes that lead to photon detection inefficiency at high energy are less than the requirement of $3 \times 10^{-5}$. A more likely inefficiency scenario is that of inadequate hermeticity in space and time. To address these concerns we have studied two different $\mathrm{KTeV}$ data sets: one where very well measured electrons illuminate a Pb-Scintillator sampling calorimeter, and another where electrons illuminate the CsI calorimeter in a high rate environment. These two studies will be presented in turn.

The $\mathrm{Pb}$-scintillator data set was acquired during test-beam running at the end of $1999 \mathrm{KTeV}$ run. The sampling device studied was the $\mathrm{KTeV}$ "Back-Anti" [168] veto system which sits in the neutral kaon beam downstream of the CsI calorimeter to veto photons that escape through the beam holes in the calorimeter. The device tested consisted of two contiguous longitudinal $10 X_{0}$ modules composed of relatively course $3 \mathrm{~mm}-\mathrm{Pb} / 10 \mathrm{~mm}$-scintillator sampling. The scintillator was fast radiation hard $3 \mathrm{HF}$ material. The upstream $10 X_{0}$ module had $12 \mathrm{x}$-view and $12 \mathrm{y}$-view (transverse) $2.5 \mathrm{~cm}$-wide slats readout, and the downstream $10 X_{0}$ module had only $12 \mathrm{x}$-view slats readout. The energy detected by the device for the purposes of this test beam was the sum of the 36 slats that compose the composite $20 X_{0}$ deep structure. The beamline and detector were configured in the following way for this test run:

- One of the two neutral parallel beams was plugged, and a high purity relatively low intensity $K_{L}$ beam was established in the other beam. The Back-Anti being studied was behind the CsI hole that had no beam.

- A special trigger was operated that selected $K_{L} \rightarrow \pi^{ \pm} e^{\mp} \nu_{e}$ (Ke3) decays where the electron passed through the "empty" CsI beam hole and illuminated the Back-Anti. An example of one of these events is shown in figure 92. 
- The 8-module KTeV Transition Radiation Detector (TRD) system was configured to analyze tracks that illuminated the Back-Anti detector under test.

Stringent cuts on the TRD system provided a $\pi / e$ rejection of $\times 700$, which together with Ke3 kinematic cuts, cuts on the muon veto system and CsI calorimeter cuts, define a sample of 56,000 electrons with a background of less then two events. A scatter plot of the measured .vs. electron track momentum is slown in figure 93 . From this plot it's clear that the vast majority of the high momentum electrons as determined by the spectrometer are cleanly detected by the Pb-scintillator sampling device. The few events that straggle from the main population are indicated with an asterisk "*", and one straggler in particular has an anomalously low energy measured in the BackAnti. The response of the Back-Anti to minimum ionizing particles (MIPs) is shown in figure 94 together with the energy of the one low energy electron candidate. This track is likely a pion from a background event, and would in any case be vetoed with an energy cut of greater than the peak MIP energy deposit. Assuming all candidate electrons are counted as detected, this sample corresponds to a high energy electron inefficiency of less than about $4 \times 10^{-5}$ at the $90 \%$ C.L. for a $\mathrm{Pb}$-scintillator sampling device. To our knowledge this is the first measurement of sampling calorimetric efficiency to this level.

Although this measurement of sampling calorimetric efficiency supports the case that a system inefficiency of less than $3 \times 10^{-5}$ is achievable, it suffers from two caveats. First, the momentum spectrum of the test sample is about $\times 5-10$ higher than the corresponding "high energy" photon energy spectrum illuminating the VVS. Second, the data were collected over a short period (18 hours) in a relatively low rate beam environment which doesn't invite real experiment "battle conditions" that can lead to a loss of hermeticity.

To address these caveats we have begun studying the detection efficiency of Ke3 electrons that illuminate the $\mathrm{KTeV} \mathrm{CsI}$ calorimeter. These $\mathrm{Ke} 3$ electrons were collected through heavily prescaled minimum bias data streams acquired during a year of high intensity neutral beam running. These data sets are large enough to study the relevant level of hermeticity as a function of electron energy under "battlefield" conditions. We have initiated these studies with analysis of a small subset of $\mathrm{Ke} 3$ decays acquired during high rate running rare decay running (E799). The E799 data set is particularly useful since the TRD system provides a very clean electron tag independent of the calorimeter. On examining a $80 \mathrm{~K}$ tagged $\mathrm{Ke} 3$ electrons we have found the small remaining class of electron tracks that do not have an associated calorimeter cluster are due to upstream scatters in the tracking spectrometer. Based on this preliminary analysis we can limit inefficiency mechanims to less than about $3 \times 10^{-5}$ at a $90 \%$ C.L. integrated over the Ke3 electron energy spectrum. We are continuing to studies with a goal of a high statistics energy dependent analysis in the near future.

\subsubsection{Efficiency monitoring and measurement strategies.}

It is clear from the previous discussion that in - situ monitoring of photon veto inefficiency is critical to the success of the experiment. Our strategy is to monitor the efficiency with a threetiered approach. At the lowest level each photomultiplier will be monitored with an LED that will be flashed at $1 \mathrm{~Hz}$. Physical energy deposits within a veto module will cross-monitored with the redundant fiber-phototube mapping scheme described previously.

The next level of monitoring will exploit the high fluence $(\sim 7 \mathrm{MHz})$ of muons from upstream $K^{+} \rightarrow \mu^{+} \nu_{\mu}$ decays that traverse the VVS stations and the FVS. Muons that traverse the VVS can be coarsely tracked by upstream and downstream VVS stations and can be used to measure the efficiency of $\sim 20 \mathrm{MeV}$ energy deposits from traversing muons. Muons from $\mathrm{K}^{+} \rightarrow \mu^{+} \nu_{\mu}$ decays within the decay volume can be tracked with the downstream spectrometers into the FVS and 

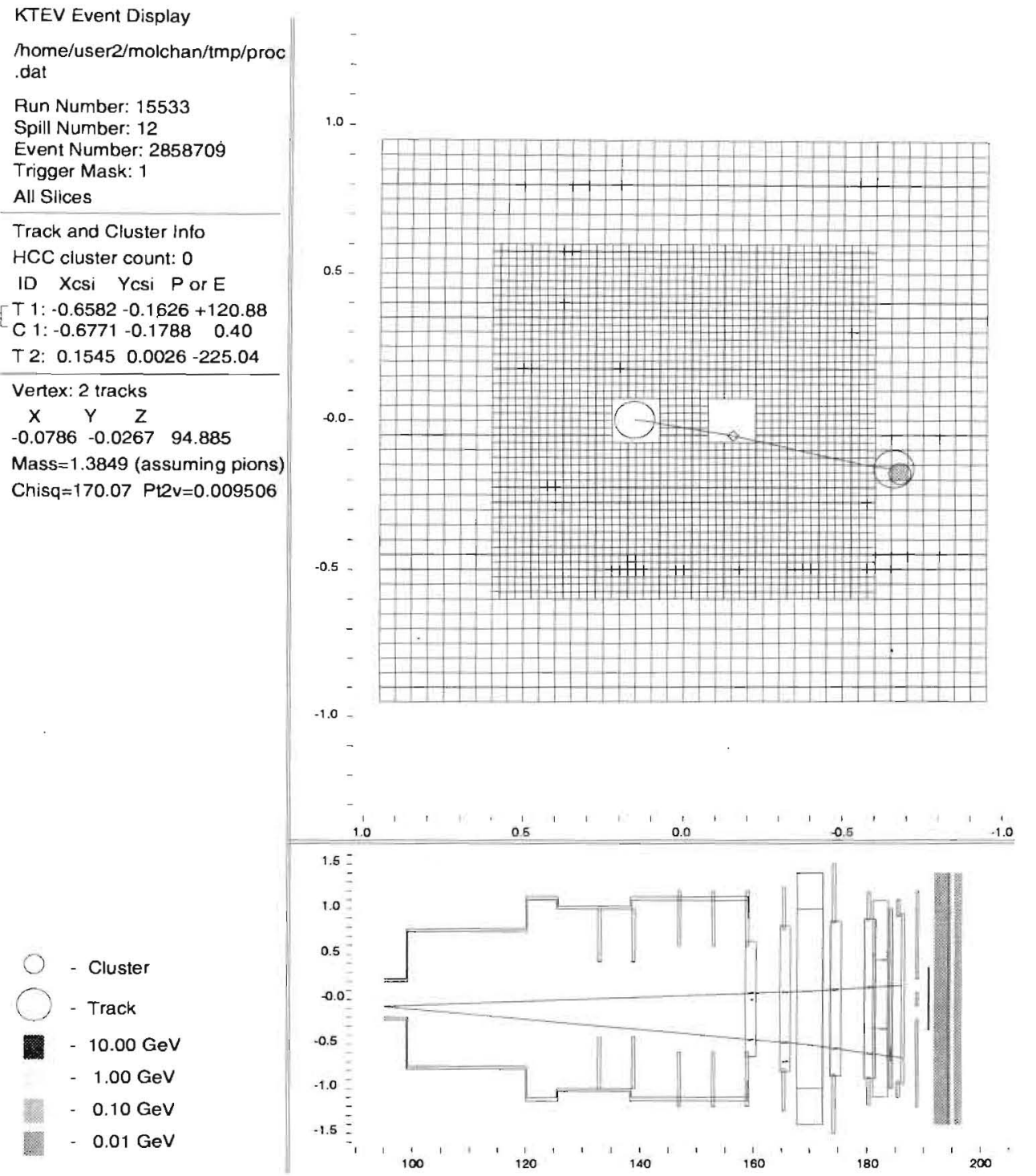

Figure 92: One of the 56,000 tagged Ke3 events used to study the electron efficiency of the Pbscintillator sampling device behind the left CsI beam hole. 


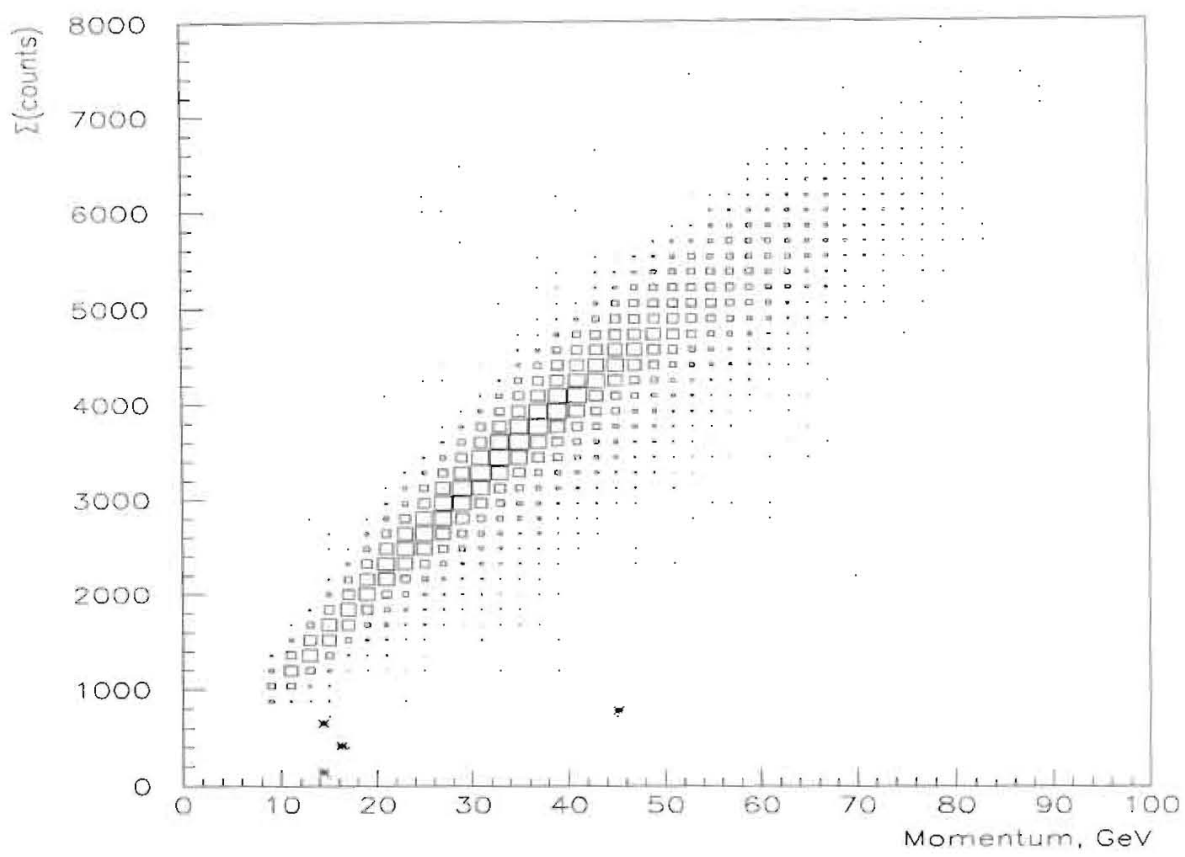

Figure 93: Scatter plot of the measured energy in the Back-Anti .vs. the incident tagged electron momentum. The few events that straggle from the main population are indicated with "*".

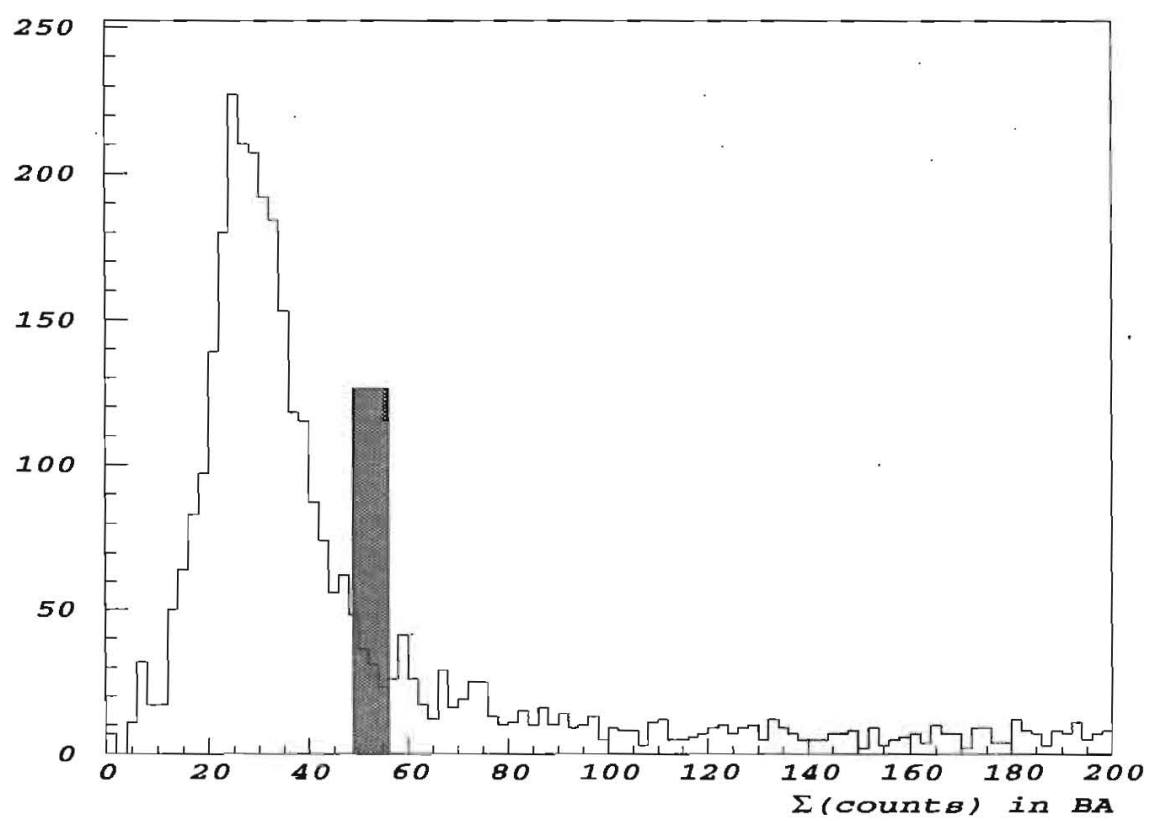

Figure 94: Back-Anti energy distribution of incident $\pi^{+}$. The energy of the one electron tagged event with the least reported energy in the Back-Anti is indicated with a vertical bar, and is clearly greater than the MIP peak. 
verified by hits in the Muon Veto System. These FVS muons deposit $\sim 90 \mathrm{MeV}$ of visible energy, and can be used to measure the efficiency of the FVS to the required level.

In principle $\mathrm{K}^{+} \rightarrow \pi^{+} \pi^{0}$ decays offer an ideal laboratory to kinematically tag probe photons that can be used to measure the real desired photon inefficiency. These kinematic tools were exploited successfully in the BNL E787 data set to provide the only real measurement of photon veto inefficiency in the field today. Unfortunately it is difficult to carry these tecliniques to a high energy in-flight experiment. This difficulty arises from the fact that measurements of the longitudinal $K^{+}$and $\pi^{+}$momenta are made at the $\sim(0.5-1.0) \%$ level of precision, which corresponds to a longitudinal energy uncertainty at the $100-200 \mathrm{MeV}$ level. This level of energy uncertainty completely washes out the pointing accuracy of photons of less than $200 \mathrm{MeV}$, and significantly degrades the pointing accuracy of higher energy photons as well.

Despite this absence of pointing accuracy however, the geometry of the CKM detector together with a good measurement of the $K^{+} \rightarrow \pi^{+} \pi^{0}$ decay vertex can be exploited to measure the global photon veto efficiency of the Vacuum Veto System. This technique employs a sample of $K^{+} \rightarrow \pi^{+} \pi^{0}$ decays with a well measured decay vertex and a well measured isolated photon in the FVS. The detector geometry constrains the other probe photon from the $K^{+} \rightarrow \pi^{+} \pi^{0}$ decay to intercept the Vacuum Veto System. Although the expected location of the probe photon within the VVS is not measured well, it's containment within the VVS acceptance is assured. Hence the global photon veto efficiency as a function of energy can be measured in the range of $200 \mathrm{MeV}$ to $5 \mathrm{GeV}$, and with some pointing accuracy for $1-5 \mathrm{GeV}$ photons. With the loss of probe photon energy resolution below $200 \mathrm{MeV}$, only the integrated VVS global inefficiency from 3-200 MeV can be measured. Given the rapidly falling inefficiency with energy, this is equivalent to measuring the inefficiency of the $3-20 \mathrm{MeV}$ range which matters most.

Although these photon efficiency measurements are limited by the in-flight environment, the suite of measurements match our requirements well. As previously discussed, control of the $K^{+} \rightarrow \pi^{+} \pi^{0}$ background hinges on the ability of the VVS to veto high energy photons, where this technique will work well. These high energy inefficiency measurements combined with integrated low energy inefficiency measurements will adequately characterize the VVS system performance with real in - situ data.

\subsection{Downstream Magnetic Spectrometer}

\subsubsection{Overview}

The $K^{+} \rightarrow \pi^{+} \pi^{0}$ background can be identified by the presence of photons in the veto systems or the charged kinematics. Regarding the kinematics, recall that the missing mass recoiling against the $\pi^{+}$is given by:

$$
M_{m i s s}^{2}=M_{K}^{2}\left(1-\frac{p_{\pi}}{p_{K}}\right)+m_{\pi}^{2}\left(1-\frac{p_{K}}{p_{\pi}}\right)-p_{\pi} p_{K} \theta^{2}
$$

Here, $\theta$ is the $\pi^{+}$decay angle in the lab frame.

The downstream magnetic spectrometer (DMS) will be used to measure the momentum of the $K^{+}$charged decay products, while the combination of the upstream magnetic spectrometer (UMS) and the kaon entrance angle tracker (KEAT) will measure the $K^{+}$momentum. Together, the charged tracking measures the kinematics as well as determine the position of the decay vertex.

An important technique in CKM is to have multiple measurements of the decay kinematics. While the devices are designed to have a sufficient resolution, it is crucial to have multiple measurements in order to remove non-Gaussian tails. For this, the pion and kaon riches will provide an independent measurement of the kinematics. 


\subsubsection{Conceptual Design}

The two major concerns for the DMS are the following. (1) The chambers must be as thin as possible and have sufficient momentum and angular resolution. The measurement errors have to be sufficiently Gaussian. (2) The DMS must be sufficiently live and survive the $50 \mathrm{MHz}$ beam of kaons through the center of the decay volume.

The conceptual design we have chosen are 4 planar straw drift chambers, two on either side of a $100 \mathrm{MeV}$ dipole magnet. Chamber 1 has 5 layers to measure the $\mathrm{X}, \mathrm{X}, \mathrm{Y}, \mathrm{Y}^{\prime}, \mathrm{U}$ views. Chambers 2 through 4 each have 8 layers to measure $X, X^{\prime}, Y^{\prime}, Y^{\prime}, U^{\prime}, U^{\prime}, V, V^{\prime}$ views. The primed and unprimed layers are offset by $1 / 2$ cell-size in order to resolve the left-right ambiguity. The straws are $5 \mathrm{~mm}$ diameter and are arranged in the "close-packed" geometry. That is, there are no gaps between neighboring straws and between primed and unprimed planes. Here, the $\mathrm{X}(\mathrm{Y})$ view denotes the bend(non-bend) view. The $\mathrm{U}$ and $\mathrm{V}$ views are rotated $\pm 45^{\circ}$ with respect to the $\mathrm{X}$ view. Table 28 shows the relevant parameters for the DMS.

Table 28: Parameters for the Downstream Magnetic Spectrometer.

\begin{tabular}{ll}
\hline Number of chambers & 4 (in vacuum decay volume) \\
Analysis magnet ptkick & $100 \mathrm{MeV} / \mathrm{c}$ \\
Approximate spacing between chambers & 2.5 meters \\
Number of straw layers in 1st chamber & $5\left(\mathrm{X}, \mathrm{X}^{\prime}, \mathrm{Y}, \mathrm{Y}^{\prime}, \mathrm{U}\right)$ \\
Number of straw layers in 2nd-4th chambers & $8\left(\mathrm{X}, \mathrm{X}^{\prime}, \mathrm{Y}, \mathrm{Y}^{\prime}, \mathrm{U}, \mathrm{U}^{\prime}, \mathrm{V}, \mathrm{V}^{\prime}\right)$ \\
$\mathrm{X}, \mathrm{X}^{\prime}$ straw orientation & $0^{\circ}$ (bend view) \\
Y,Y' straw orientation & $90^{\circ}$ (non-bend view) \\
U,U' straw orientation & $45^{\circ}$ \\
V,V' straw orientation & $-45^{\circ}$ \\
Active straw length & $80 \mathrm{~cm}$ (gain reduced in beam region) \\
Straw diameter & $0.5 \mathrm{~cm}$ \\
Straw construction & 2 layers of 0.5 mil Kapton, $100 \mathrm{~nm} \mathrm{Cu}$ deposit \\
Anode wires & $20 \mu \mathrm{m} \mathrm{Au-W}$ wires \\
Gas & $\mathrm{CF} 4$-isobutane or CF4-ethane \\
Drift time & $\approx 25 \mathrm{nsec}$ \\
Maximum rate per straw & $120 \mathrm{kHz}$ \\
\hline
\end{tabular}

In order to minimize the measurement tails due to scattering, we have chosen to place the chambers and field inside the vacuum decay volume. Straws are the thinnest devices that would meet this requirement. Aside from the requirement of the operation in vacuum, straw drift chambers have several advantages over wire drift chambers. The drift cells are isolated from one another thereby minimizing cross talk. If the anode wire breaks or is loose, the channel could be easily disabled without affecting neighboring channels. Since there are no low E-field region, the maximum drift time is precisely defined, and so one minimizes the effect of late-hits which plague wire drift chambers.

As mentioned above, we have chosen to instrument the $U$ and $V$ views, which are independent measurements of $\mathrm{X}$ and $\mathrm{Y}$. So our design is not the absolute thinnest, but the extra measurements give useful redundancy and increased precision. Table 29 compares the amount of material for this and other configurations. Having redundant measurements give protection against tails due to pattern recognition errors. Wrong hits can be assigned to tracks by several mechanisms. Good 
Table 29: Comparison of material for this and other configurations.

\begin{tabular}{ll} 
Layout & Radiation Length $\left(10^{-3} X_{0}\right)$ \\
\hline 5 straw layers & 2.1 \\
8 straw layers & 3.4 \\
KTeV Drift Chamber (X,X',Y,Y') & 1.1 \\
KTeV Drift Chamber and vacuum window & 3.2 \\
4-layer 500 $\mu \mathrm{m}$ scintillating fibers & 5.7 \\
4-layer 835 $\mu \mathrm{m}$ scintillating fibers & 9.5 \\
$300 \mu m$ double-sided silicon strips & 3.2 \\
BTeV silicon pixels & 8.9 \\
\hline
\end{tabular}

hits can be lost if the signal sizes are below the chamber electronics threshold. This will happen at the boundaries between two straw cells, where the track path length in the gas can be quite small. Good hits can also be lost due to finite chamber deadtime if there were early hits on the same wire. The early hits can come from accidental tracks or delta rays from the track in question.

Once good hits are lost, the left-right ambiguity is not as easily resolved and incorrect hits can be assigned in place of the correct ones. In section 5.7.5, we present GEANT simulations of the measurement tails. In section 7, we present a GEANT simulation of how well the combination of kaon/pion riches and UMS/DMS work to remove the $K^{+} \rightarrow \pi^{+} \pi^{0}$ background.

Concerning the deadtime and related effects due to undecayed beam kaons, we plan to reduce the gain of the center of the chambers. This is possible as we do not need to momentum-analyze these particles. There are well-established techniques to achieve this without introducing significant additional material. For instance, in the $\mathrm{KTeV}$ TRD chambers, the beam region of the wires were electro-plated so as to increase the diameter and therefore lower the gain [171]. After making the chambers insensitive to beam kaons, we expect a maximum rate of $120 \mathrm{kHz}$ per straw due to kaon decays and residual beam particles.

Other schemes to achieve the effect of beam-region deadening have been proposed, for instance by $\mathrm{BTeV}$ and ALICE. They involve terminating the straws at the central (beam) region, which is understandable, as the designs have to accommodate a beam pipe through the straw chambers. For us, terminating the straws at the beam region would introduce material needed for the gas seal and wire centering, as well as increase the complexity of the design.

Reducing the chamber gain in the beam region in the manner of wire-deadening keeps our design flexible. For instance, if we find that the chambers can sustain a higher rate than we thought, we can envision recovering the efficiency in the beam region by replacing the wires. This efficiency increase is also quite useful for other physics topics that involve 3 -body decays, where the acceptance in the forward region is quite high.

A remaining issue is the straw drift time. Fast gases such as CF4-isobutane and CF4-ethane have been used in high-rate experiments such as BNL-871 and FNAL-HyperCP with good reliability. These drift velocities are of the order $100 \mu \mathrm{m}$ per nsec. Since we plan to use $5 \mathrm{~mm}$ diameter straws and a fast gas, we can achieve a maximum drift time of around $25 \mathrm{nsec}$.

\subsubsection{Experience with Other Straw Systems}

Many straw chambers have been built for use in HEP, and it is acknowledged that their construction involve a unique set of problems that are not all trivial to solve. The straw-end termination, gas 
feed-through, and electrical power/signal routing can be quite complex. The straws have to be kept sufficiently rigid and straight, otherwise the wire offsets could prevent the straws from holding the required high voltage. One must also take care to prevent the conductive plating from etching due to ionization and possible gas impurities [172].

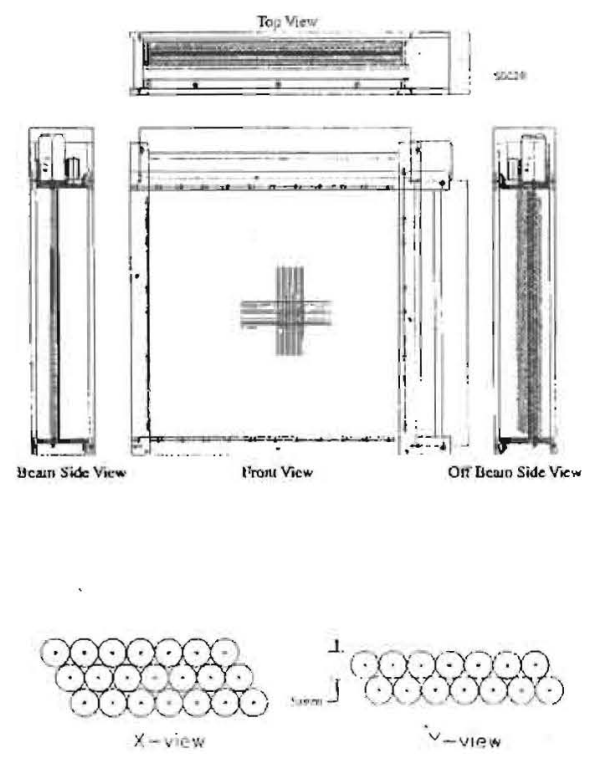

Figure 95: Layout of a BNL-871 Straw Chamber.

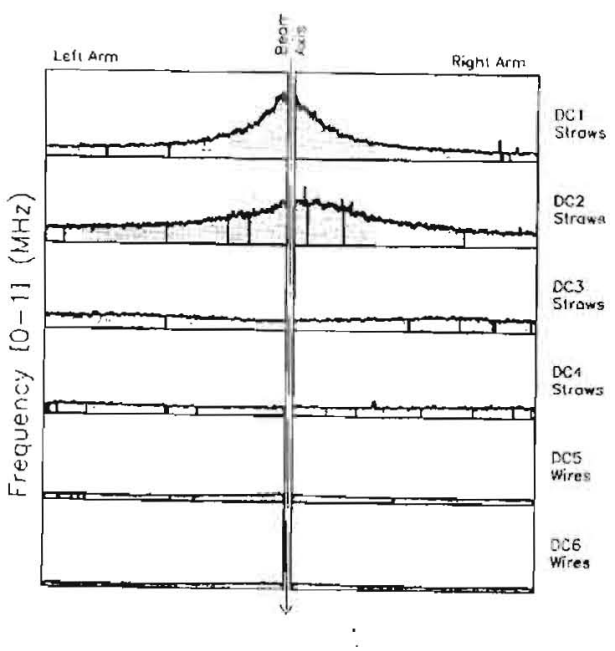

Figure 96: Rate seen by the BNL-871 Straw Chambers.

One of us (K.L.) led the design and construction of the BNL-871 straw drift chambers [173]. It is natural then to borrow heavily from this experience. In fact, we are proposing to use the BNL871 "ultem" for the DMS. The ultem is a clever wire-centering device that also provides chamber gas routing to the straws and plays a key role in the electrical connections (see 97).

The BNL-871 straw chambers achieved a $160 \mu \mathrm{m}$ resolution in the face of more than $100 \mathrm{kHz}$ hit rate. Figures 95 and 97 shows the construction and end-detail of the BNL-871 chambers. Figures 96 shows the rate seen by the BNL- 871 chambers. These figures were taken from the thesis work of D. Ambrose [174].

Following the decommissioning of BNL-871, one of the chambers was sent to Fermilab including the amplifier/discriminator electronics. As all of the straws of this chamber were damaged, we installed a small set of new straws and wires. This chamber is now operational, and we have performed studies of the hit resolution and signal properties. It will continue to be useful for many other studies, especially for tuning the simulation of the chamber signals.

\subsubsection{Straw Properties Under Vacuum}

While there have been high-pressure straw chambers used in HEP such as the CLEO and AMY vertex detectors, which operated at 2-3 atmosphere overpressure, CKM and MECO are the first to propose usage of straws in a vacuum. We have studied the straw mechanical strength and behavior 


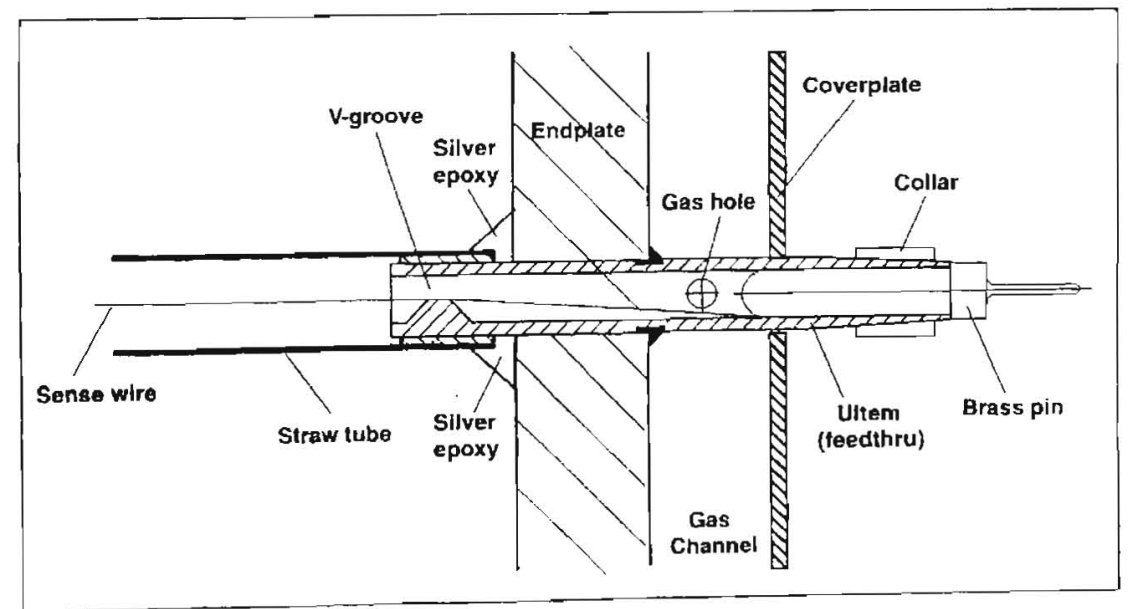

The layout of the straw tube ends into the endplate.

The tensioned wire rests in a $V$ shaped groove in the ultem. a plastic insulating feedihrough located at eicher end of the siraw tube. The "Ultem" serves as a support for keeping the wire loce the gasply and accepts the connection to the long the symmelry axis of the lube, enass pin). The wire is held tensioned due to friction high voltage supply and electronics (brass pin). The wire is held tensioned due to friction collar.

Figure 97: End-detail of a BNL-871 Straw Chamber.

under vacuum. These include a measurement of the straw leak rate under vacuum, its strength and size change under 1 atmosphere overpressure. There is also a wealth of recent data on straws as they were heavily studied for the LHC [175-177].

The bulk kapton material appears to be sufficiently strong. Under a 1 atmosphere overpressure, the hoop stress experienced by a $5 \mathrm{~mm}$ straw of 1 mil wall thickness is $1500 \mathrm{lb} / \mathrm{in}^{2}$. The yield point of kapton is $10^{4} \mathrm{lbs} / \mathrm{in}^{2}$, hence the straw is at $1 / 6$ of its yield point. We have pressured the straw to 100 psi without failure.

Similarly, we may wish to apply a linear tension to the straw to keep it straight. The straw cross sectional area is $6.3 \cdot 10^{-4} \mathrm{in}^{2}$. Hence, the yield point is equivalent to applying a $6 \mathrm{lbs}$ linear tension. We have loaded a straw with $6 \mathrm{lbs}$ for $1 / 2$ year without failure. In practice, we need stretching of order 1 mil out of 40 inches. Given a modulus of elasticity of kapton is $55 \mathrm{lbs} / \mathrm{in}^{2}$, we need only to apply $1 / 8 \mathrm{oz}$. tension to get a 1 mil stretch.

We find an interesting effect that a 1 meter length straw stretches by about 1 mil under 1 atmosphere overpressure. This must be compensated for in the assembly, since an unloaded 1 meter length straw will bow by almost $0.5 \mathrm{~cm}$ when pressurized. Other effects to consider are the dependences of expansion on temperature and humidity. They cause length changes of similar magnitude [175-177]. For these reasons, we may wish to assemble the straws under load in order to absorb all these variations, which are unavoidable in practice.

A mitigating effect is that straws, when glued together into planes appear to be quite robust under environmental variations normal to an experiment [178]. In fact, no stress was applied to the BNL-871 straws as they were assemble into their frames. The straws were, however, glued to their neighbors to increase the stiffness. With this procedure, the BNL- 871 straw system was not affected by their temperature variations. Regarding the humidity variation, this effect was mitigated by an outer helium volume that enveloped the straws. • 
A concern is whether the diffusion of chamber gas through the straw walls would spoil the vacuum: We expect this not to be a problem as there is a $100 \mathrm{~nm}$ coating of $\mathrm{Cu}$. Diffusion through membranes have been studied for other applications, with the result that a thin metal deposition greatly reduces this effect [179].

We have placed a 1 meter length straw under vacuum. With the straw back-filled with helium, we measured a leak rate of approximately $15 \mathrm{liter} / \mathrm{sec}$ at a $1.4 \cdot 10^{-8}$ Torr. Assuming conservatively that this is entirely due to the straw and not the vacuum vessel, this represents a leak of 0.2 liters $/ \mathrm{sec} / \mathrm{straw}$ at $10^{-6}$ Torr. This value of the pressure was selected based on the requirement of minimizing the scattering from the residual gas. For a 5000 straw system, the total leak rate would be 105 liters/sec which is well within the reach of commercially-available pumps. We have not measured the leak through the straw ends. This depends on the details of the end-termination, whose design is still evolving.

Since a major straw leak would be quite undesirable for the experiment, we will want the ability to 'easily' swap out a primed-unprimed straw plane pair, or the ability to disable the flow of gas to the leaky straw. A potential headache is that it maybe difficult to identify the source of the leak. Our conceptual design is a 'cassette' of a prime-unprimed straw plane pair (see figure 98), which is inserted into the vacuum vessel. The amplifier/discriminators are currently envisioned to remain outside the vacuum vessel. We will maintain the rotational symmetry such that a cassette can be used for any view (see figure 99). We have specified that the active straw length is $80 \mathrm{~cm}$. The true straw length could be significantly longer $(\approx 160 \mathrm{~cm})$ in order to subtend the vacuum vessel diameter, which is necessarily larger.

\subsubsection{DMS Simulation}

In this section, we examine the DMS resolution in the kinematic variables. A reasonably detailed GEANT simulation was used to generate straw hits from ionizing tracks. We relied on GEANT to simulate adequately the relevant processes such as multiple Coulomb and hadronic scattering, and delta-ray production.

For each GEANT hit, we calculated a single drift time. Here, we did not simulate the signal shapes or added any after-pulsing effects. The simulation also did not include hits from out-oftime particles. Regarding the description of delta-rays, while we used GEANT to sinulate the momentum transfer to the primary particle, we did not digitize ${ }^{4}$ the hits from the delta-ray itself unless it was more energetic than $2 \mathrm{MeV}$. While we recognize this limitation, we did it in order to reduce the simulation CPU time. Noise hits from delta-ray and accidentals will be included as we improve the simulation. Our goal was to see if there were any limitations from the scattering process itself.

Using the drift times, tracks were reconstructed using a 5-constraint fit [180]. Figures 100 and 101 show the momentum resolution and the momentum dependence for charged pions. Figures 102, 103, 104, and 105 show the angular resolutions and their momentum dependence. In summary, the DMS has rouglhly a $1.1 \%$ resolution in momentum, a $39 \mu \mathrm{rad}$ resolution in the decay angle, and a mild momentum dependence for these quantities.

We have developed an analytical calculation that assumes Gaussian processes for the scattering and hit resolution [114]. The analytical calculations are in reasonable agreement with our GEANT simulation. Figure 106 shows this analytical calculation of the DMS momentum and angular resolution.

The previous figures show significant non-Gaussian tails. We can now examine the impact the tails have on the kinematic reconstruction for $K^{+} \rightarrow \pi^{+} \pi^{0}$. To do this, we look at $M_{\text {miss }}^{2}$ (equation

\footnotetext{
iI.e. we did not allow GEANT to propagate the electron.
} 


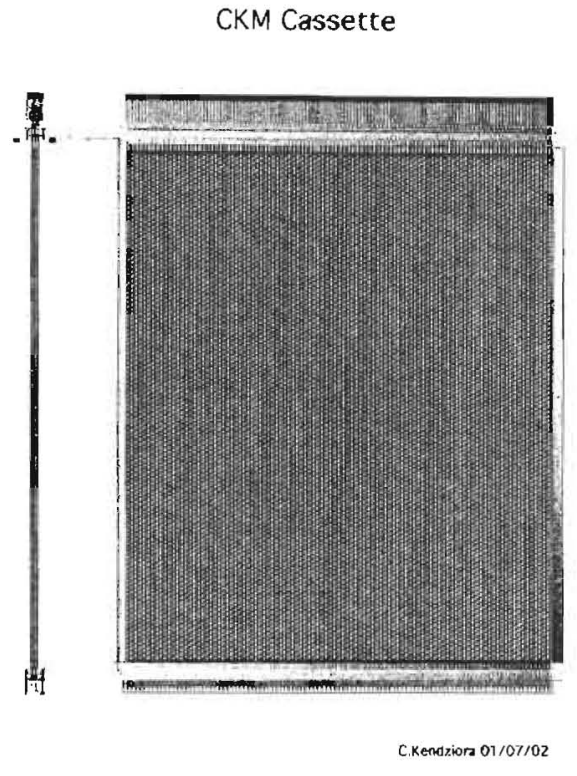

Figure 98: DMS 'cassette' containing a primed-unprimed pair of straw planes. The cassette would inserted into the vacuum vessel.

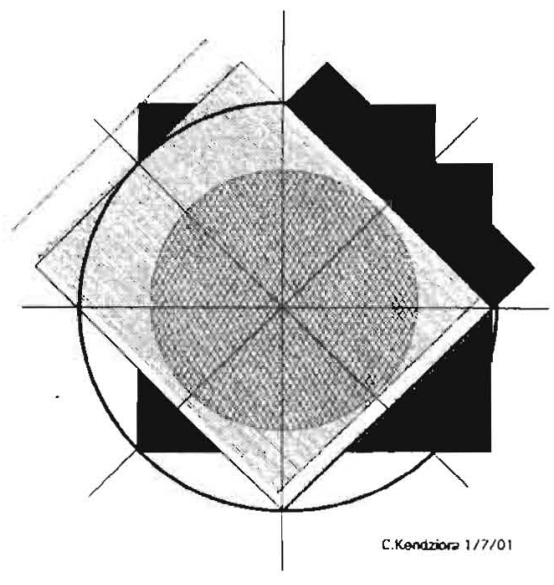

Figure 99: DMS 'cassette' arranged into $\mathrm{X}, \mathrm{Y}, \mathrm{U}, \mathrm{V}$ views. The inner circle denotes the active area of the straws $(80 \mathrm{~cm}$ diameter). The outer circle denotes the proposed vacuum vessel diameter. The straws would have to be significantly longer to sub-tend the vessel diameter. 


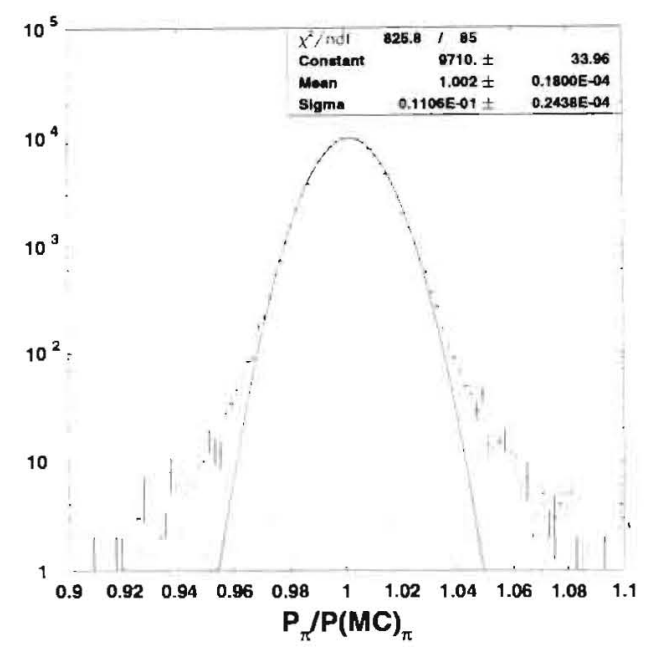

Figure 100: Momentum resolution $\left(P_{\text {recon }} / P_{\text {truc }}\right)$ for pions in the momentum range $14-20 \mathrm{GeV} / \mathrm{c}$.

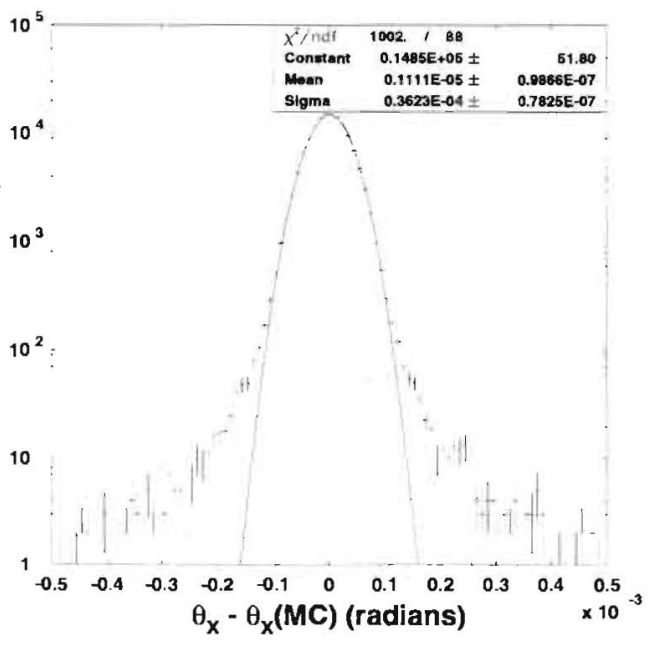

Figure 102: Angular resolution $\left(\theta_{\text {recon }}-\theta_{\text {true }}\right)$ in the bend view for pions in the momentum range $14-20 \mathrm{GeV} / \mathrm{c}$.

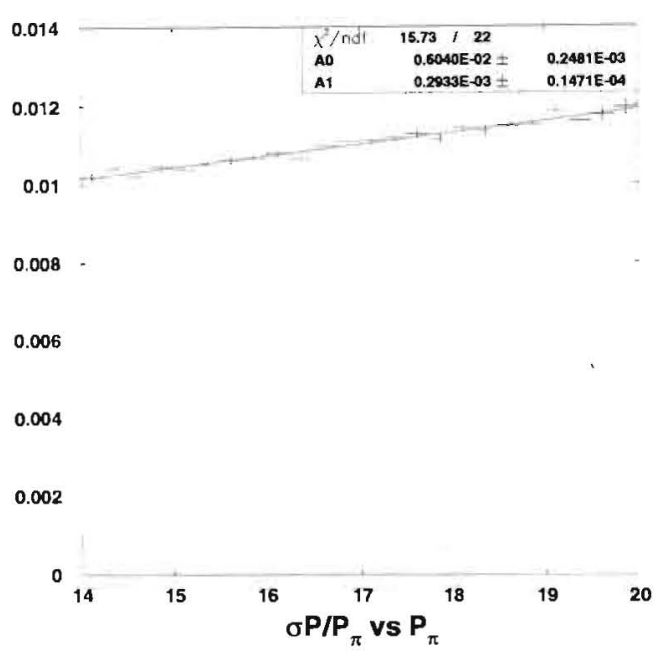

Figure 101: The momentum dependence of the momentum resolution.

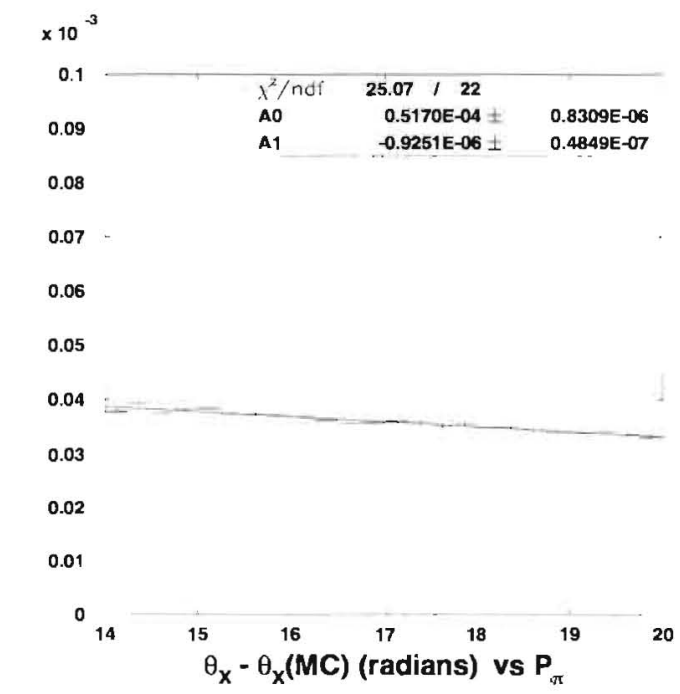

Figure 103: The momentum dependence of the angular resolution $\left(\theta_{\text {recon }}-\theta_{\text {true }}\right)$ in the bend view 


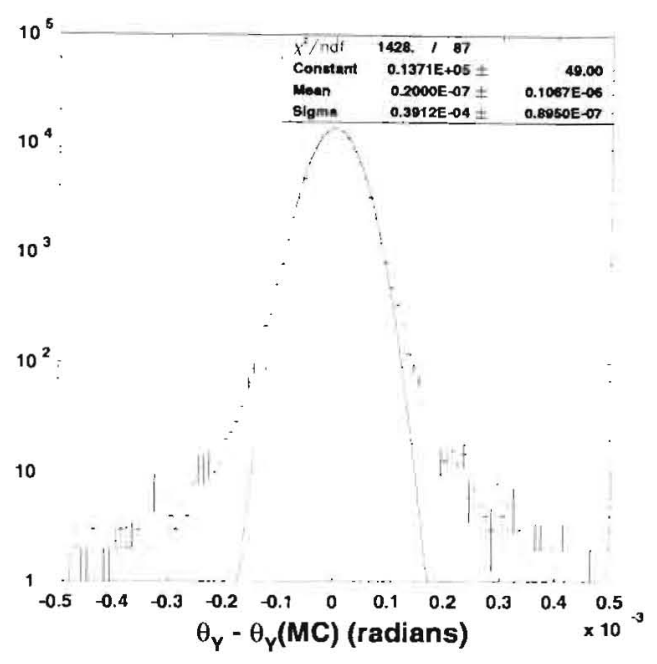

Figure 104: Angular resolution $\left(\theta_{\text {recon }}-\theta_{\text {true }}\right)$ in the non-bend view for pions in the momentum range $14-20 \mathrm{GeV} / \mathrm{c}$.

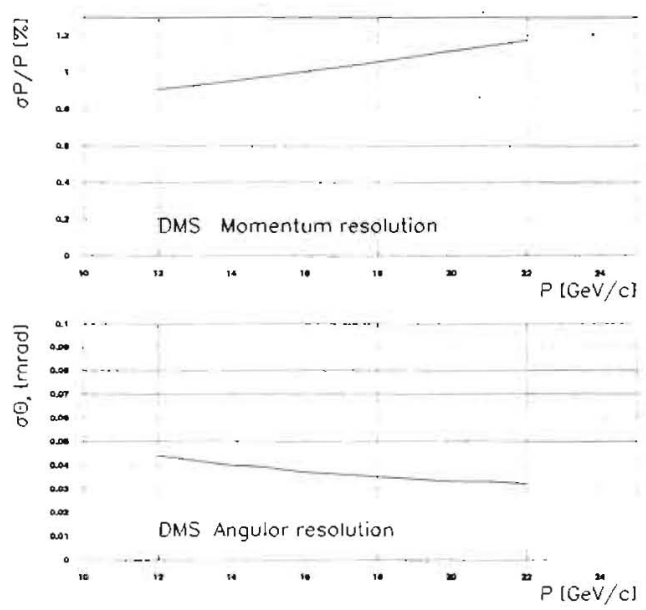

Figure 106: Momentum and angular resolution versus $\pi^{+}$predicted by an analytical calculation [114]. The slight shift from unity is due to a slight error (on our part) in converting from the desired magnet $\mathrm{Pt}$ kick into field strength (used in GEANT).

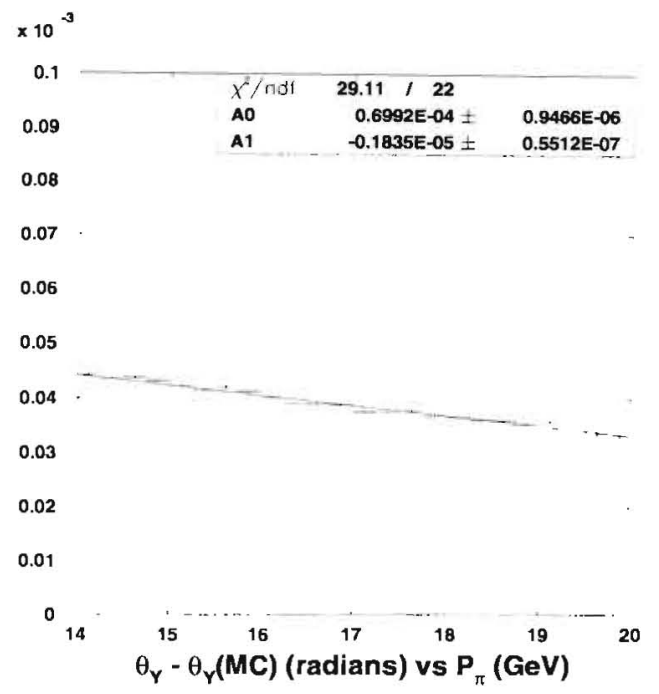

Figure 105: The momentum dependence of the angular resolution $\left(\theta_{\text {recon }}-\theta_{\text {true }}\right)$ in the non-bend view

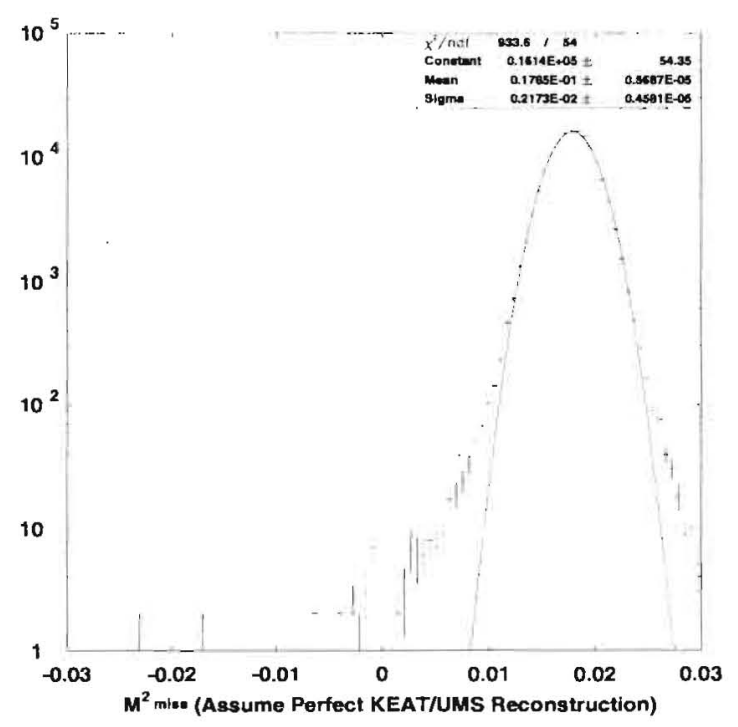

Figure 107: $M_{\text {miss }}^{2}$ of $K^{+} \rightarrow \pi^{+} \pi^{0}$ events reconstructed by the DMS assuming a perfect UMS and KEAT reconstruction. 


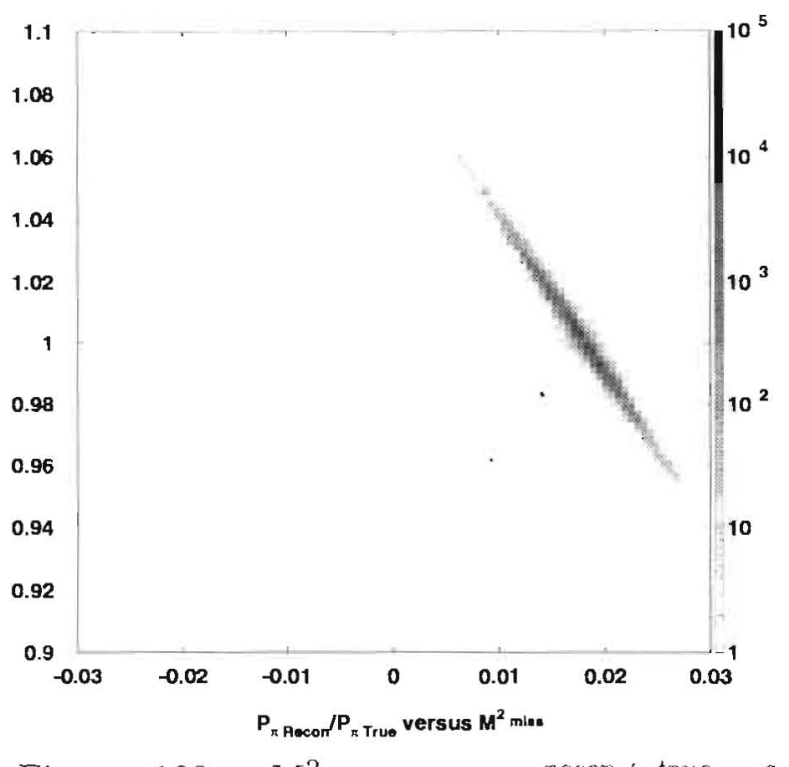

Figure 108: $M_{\text {miss }}^{2}$ versus $p_{\pi}^{\text {recon }} / p_{\pi}^{\text {true }}$ of $K^{+} \rightarrow \pi^{+} \pi^{0}$ events. A perfect UMS, KEAT, and DMS angular reconstruction is assumed. Hence, this focuses on the $M_{\text {mis.s }}^{2}$ tail that is due to $\left|p_{\pi}\right|$ misreconstruction.

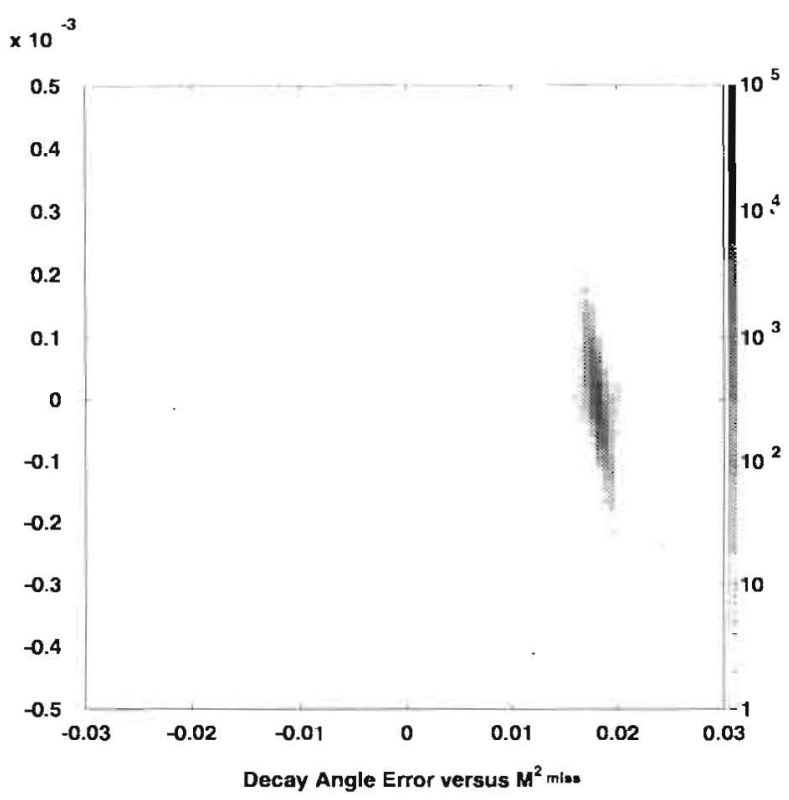

Figure 109: $M_{m i s s}^{2}$ versus $\theta_{\pi}^{\text {recon }}-\theta_{\pi}^{\text {true }}$ of $K^{+} \rightarrow \pi^{+} \pi^{0}$ events. A perfect. UMS, KEAT, and DMS $\left|p_{\pi}\right|$ reconstruction is assumed. As shown, the non-Gaussian tails in the decay angle distribution do not have as big an impact on $M_{\text {miss }}^{2}$ as does the $\left|p_{\pi}\right|$ mis-reconstruction. 
43) for the case assuming a perfect reconstruction of the $K^{+}$kinematics by the UMS and KEAT. This is shown in figure 107, which shows a significant tail in the signal region, which is the region below $M_{m i s s}^{2}=0.008 \mathrm{GeV}^{2}$. As a reminder, we will utilize the information from the Riches in order to remove these tails, as it is clear that the magnetic spectrometer information alone does not have enough rejection power.

To see the dependence on the tails, figure 108 and 109 show the dependence of $M_{\text {miss }}^{2}$ on the $\pi^{+}$momentum and angle resolution. As shown, the tails in $M_{m i s s}^{2}$ are due primarily to momentum mis-reconstructions. The tails due to the decay angle mis-reconstruction are negligible.

Upon closer inspection, the $p_{\pi}$ momentum mis-reconstructions are entirely due to scatters in the 2nd or 3rd DMS chamber. There were no mis-reconstructions due to the pattern recognition using incorrect hits. However, it is not yet sophisticated enough to detect scatters by identifying poor fits. We believe that this will be eventually possible with a more sophisticated algorithm.

In order for mis-reconstructions to leak into the $K^{+} \rightarrow \pi^{+} \nu \nu$ region, the $\left|p_{\pi}\right|$ has to be reconstructed too high by $5 \%$ of itself. Since the analysis magnet is designed for a strength of 100 $\mathrm{MeV} / \mathrm{c}$, the problematic scatters are ones with more than $5 \mathrm{MeV} / \mathrm{c}$ transverse momentum. Since it is difficult to reduce the material any further without sacrificing redundancy, we will be exploring increasing the field strength. This reduces the momentum errors as well as help the reconstruction better identify poor track fits.

As mentioned at the outset, the DMS and the pion Rich (assuming the pion mass) both measure $\left|p_{\pi}\right|$ to comparable precision, with the pion Rich being slighter better. With data, we will use the pion Rich reconstruction to study and improve the non-Gaussian tails in the DMS. This is valid insofar as the scattering processes do not introduce correlations in the DMS and pion Rich. We can use our GEANT simulation to examine a whether there are correlations in the $\left|p_{\pi}\right|$ measurement. As shown in figure 110, we do not see any correlations as yet.

Regarding the reconstruction of the $\pi^{+}$decay angle, we do not expect to gain useful information in the comparison between the DMS and pion Rich for a number of reasons. Firstly, the angular resolutions are not well matched between these two devices. Secondly, a scattering in the first straw chamber (DMS1) would introduce a correlation in the pion Rich. Our GEANT simulation shows this effect (see figure 111), though it is rather small in size. Lastly, while the DMS decay angle measurement is insensitive to scattering in material between DMS2 and the downstream vacuum window, the decay angle measurement pion rich is affected by material in this region.

\subsubsection{The BNL-871 Momentum Reconstruction}

Since we are quite concerned about non-Gaussian tails in the DMS $\left|p_{\pi}\right|$ reconstruction, it is illustrative to look at this behavior in the BNL-871 magnetic spectrometer. We will see that it is difficult to draw hard conclusions about our own design. Short of simulating the BNL871 spectrometer for ourselves, we can make only some qualitative statements.

BNL-871 had two magnetic spectrometers (see figure 112) that measured independently the momenta of the decay particles, whose mean is between 3-4 GeV. The upstream spectrometer chambers consisted of 4 planar straw chambers, two on either side of a $400 \mathrm{MeV} / \mathrm{c}$ analysis magnet. Chambers 1 through 3 each had 5 straw layers. Chamber 4 had only 3 straw layers. The straw construction is identical to our proposed design. The straw chamber spacing ranged from 1.5 meters to 0.5 meters. It is interesting to note that this straw magnetic spectrometer is thinner than what we propose for the DMS.

The downstream magnetic spectrometer consisted of straw chambers 3 and 4 for its upstream portion, a $200 \mathrm{MeV} / \mathrm{c}$ analysis magnet, and two conventional wire drift chambers for its downstream portion. Helium bags were used in between all the chambers. We emphasize here that these two 


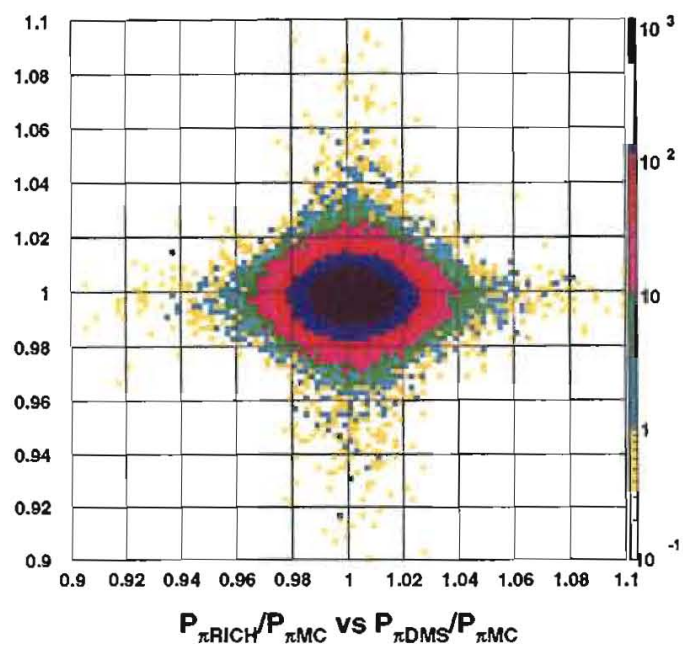

Figure 110: Momentum reconstruction in the DMS $\left(p_{\pi D M S}\right)$ versus pion Rich $\left(p_{\pi R I C H}\right)$ compared to the true value $\left(p_{\pi M C}\right)$.

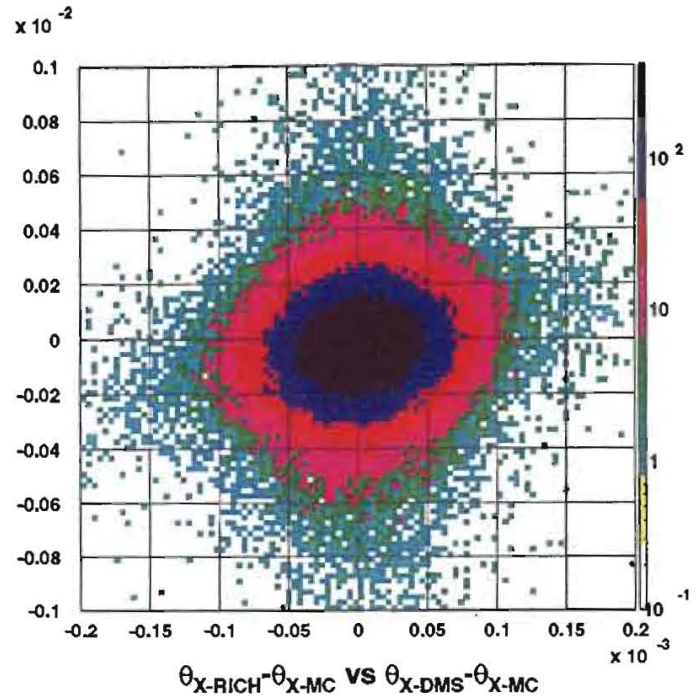

Figure 111: Decay angle reconstruction in the DMS $\left(\theta_{\pi D M S}\right)$ versus pion Rich $\left(\theta_{\pi R I C H}\right)$ compared to the true value $\left(\theta_{\pi M C}\right)$. A small correlation is seen.

BNL magnetic spectrometers give independent measurements of $|p|$.

Figure 113, taken from the thesis work of Roy Lee [181], shows the difference in momentum measurements between the upstream and downstream spectrometer. The tracks used are primarily from $K_{L} \rightarrow \pi^{+} \pi^{-}$events, with some background from $\mathrm{Ke} 3$ and $\mathrm{Kmu} 3$. Also shown is a simulation of this difference, which fails to describe the tail beyond $5 \%$. The reason for this mismatch is not entirely clear, but it could be due to the absence of large angle (hadronic) scatters in the simulation, short-comings in the simulation of noise and accidental hits, or errors in the field map [182].

We compare now figure 113 with figure 100. The overlay is shown in figure 114. The overlay shows comparable resolution in the bulk. But our DMS GEANT simulation result has 5-10 times fewer events outside of $5 \%$. Recall that $5 \%$ is the scale for which mis-reconstructions begin to leak into the signal region of $M_{\text {miss }}^{2}$. However, it is difficult to say if this means that our DMS GEANT simulation is underestimating the non Gaussian tail by a factor of 5-10 in the size of the tail.

A number of qualitative statements can be made to support our case that the DMS design would have smaller non Gaussian tails. (1) The non Gaussian tail contribution from the straw (upstream) portion of BNL-871 must be smaller than what is shown in figure 113, as part of the tail in the plot can be attributed to the downstream part. (2) Our proposal uses more tracking planes than the BNL-871 layout, which would help the pattern recognition. In addition, our proposed intensity is about 6 times lower than the BNL-871 peak intensity in their first straw chamber. (3) While the $p_{t}$ transfer due to multiple coulomb scattering is nearly independent of the momentum, the large angle hadronic scattering should decrease with increasing momentum. For us, we will be measuring pions in the range $14-20 \mathrm{GeV}$, rather than the $3-4 \mathrm{GeV}$ of BNL-871. (4) Lastly, in the comparison shown in figure 114, the BNL data points were the result of an analysis without using the particle-ID and so there would be $\pi$ decay in-flight. On the other hand, the analysis of our DMS simulation uses the $\mu$ rejection power expected from the FVS and MVS. 


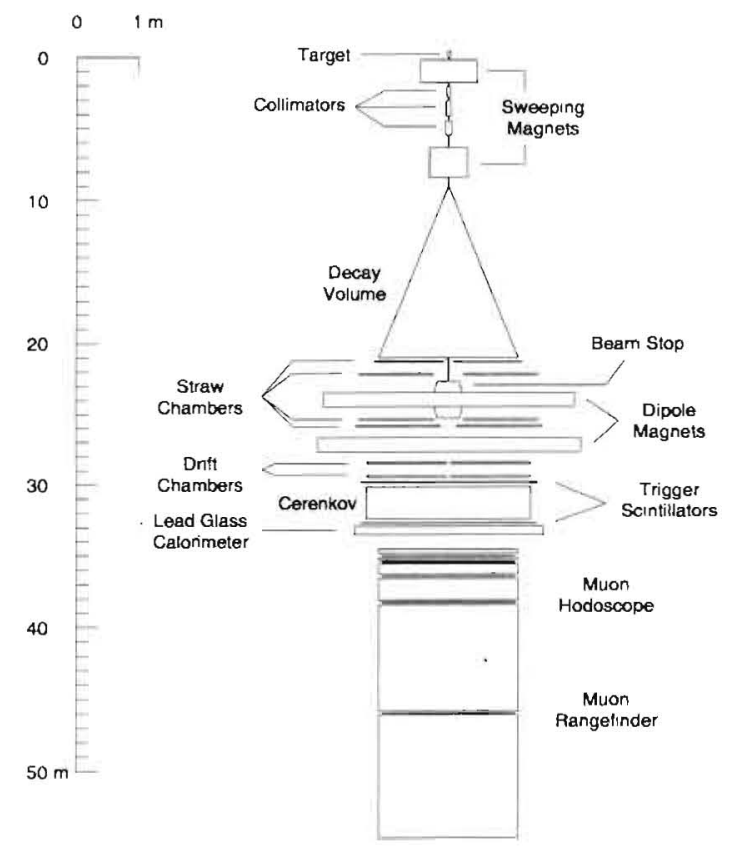

Figure 3.2: The E871 spectrometer, plan view.

32

Figure 112: Layout of the BNL-871 detector.

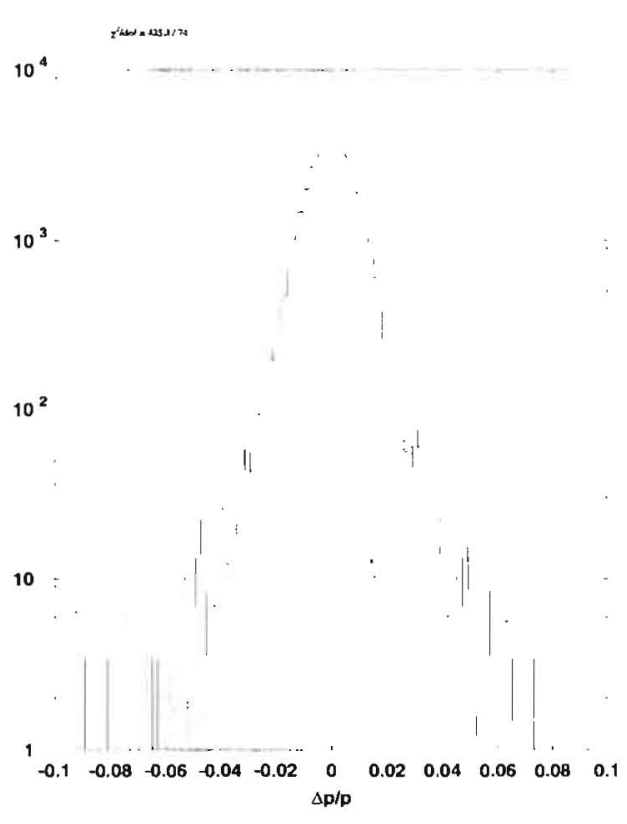

Figure 113: Relative difference between the momentum measurements of the upstream (straw) and downstream (conventional wire drift chamber) magnetic spectrometer in BNL-871. Dots and histogram are data and simulation respectively. 


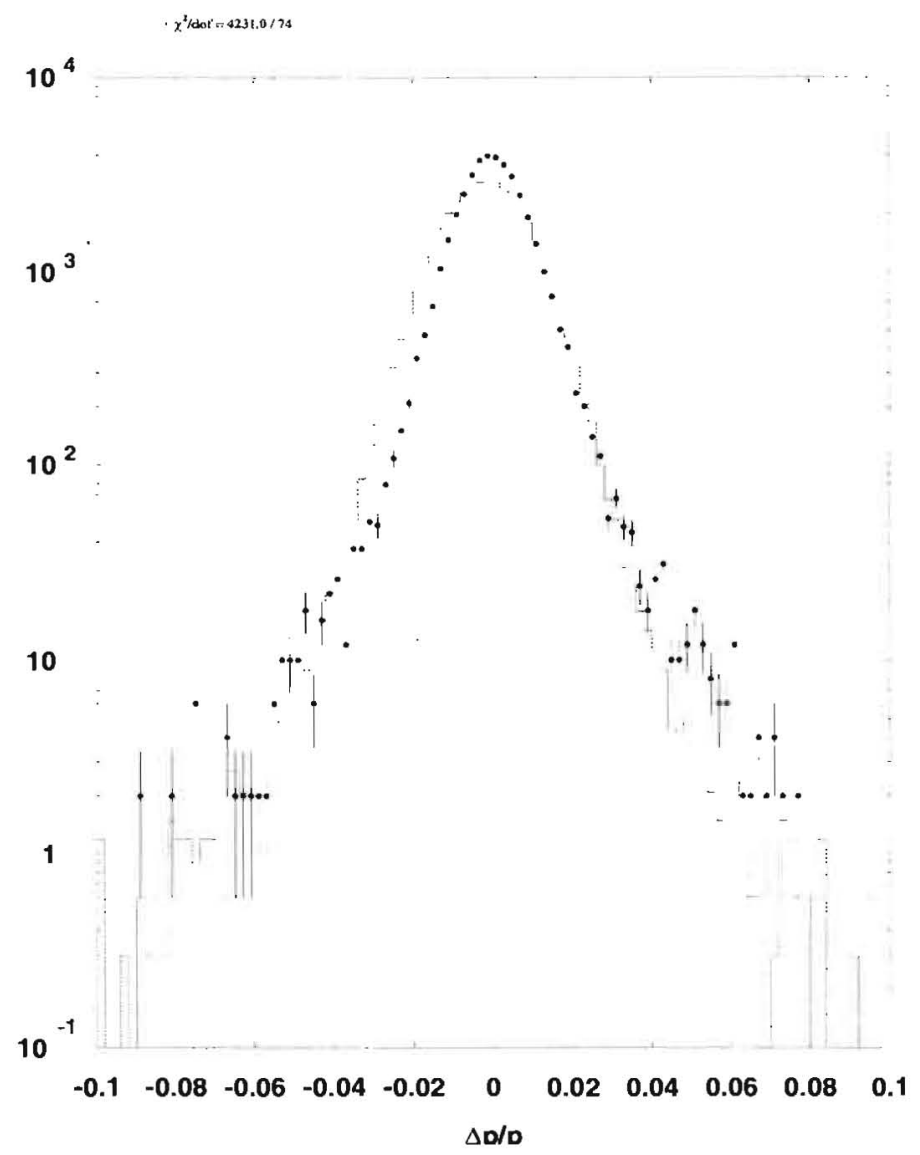

Figure 114: Overlay of figure 113 showing BNL-871 data (dots) and figure 100 showing our DMS GEANT simulation (histogram). Our DMS GEANT simulation has smaller non Gaussian tails by a factor of 5-10. 


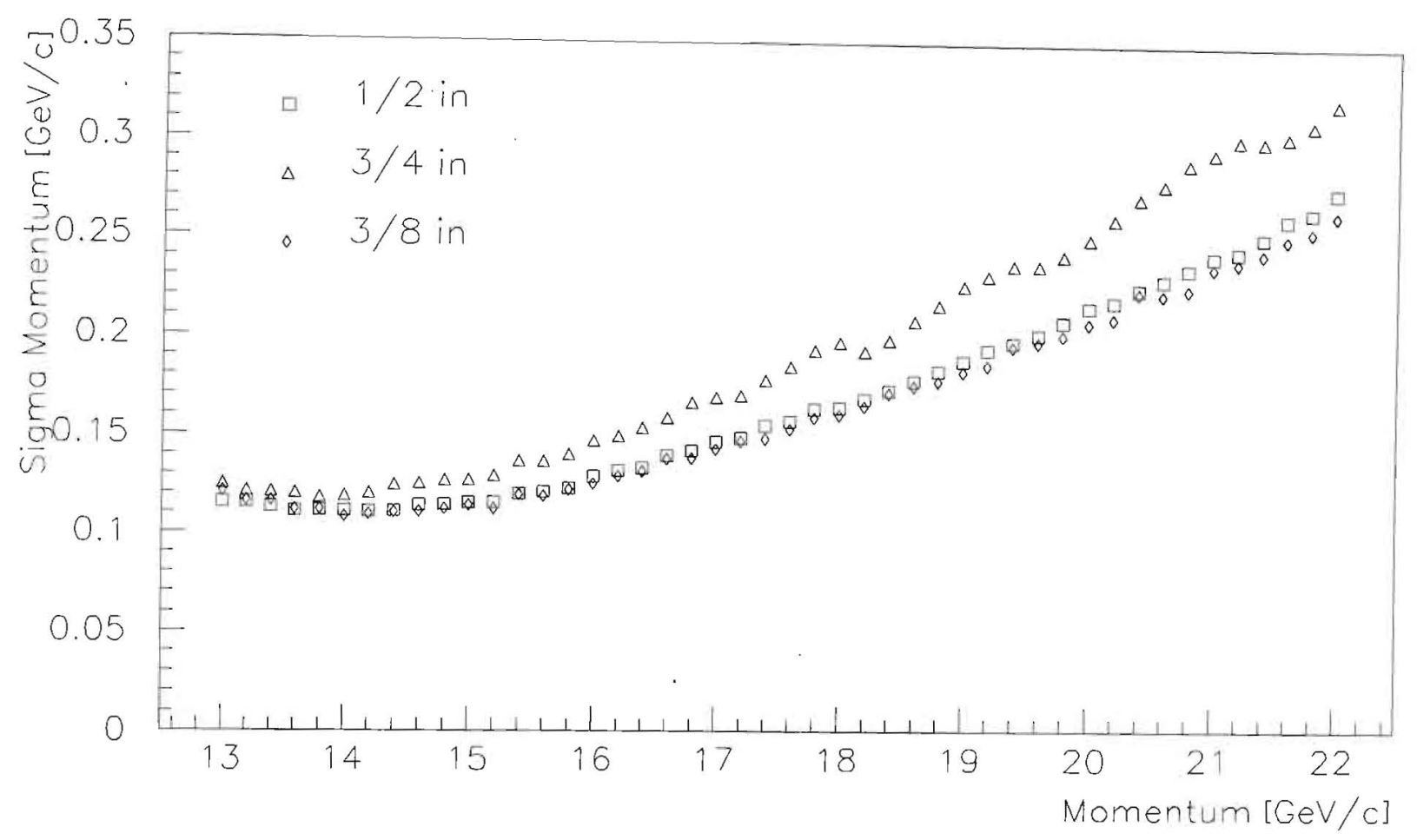

Figure 115: Pion RICH momentum resolution for Pions as a function of momentum for different PM sizes

\subsection{The Pion RICH}

The pion RICH provides a precision measurement of the vector velocity for pions from kaon decays. $\beta=1$ particles $\left(\pi^{+}, \mu^{+}, e^{+}\right)$and sub-threshold particles $\left(K^{+}\right.$and $\left.p^{+}\right)$are completely separated from kaons $\left(\pi^{+} \mu^{+}\right.$separation is $>10$ standard deviations in ring radius). The momentum of $\pi^{+}$ particles must be measured to an accuracy of $\pm 1.0 \%$ and the $\pi^{+}$angles to $\pm 0.2 \mathrm{mrad}$. The time resolution for of this phototube based spectrometer is $\sim 1 \mathrm{nsec}$

The relationship between ring radius and $\pi^{+}$track momentum is shown in figure 116, with the accepted momentum band indicated as a shaded band. The momentum and track angle resolution as determined from the fitted ring radius and ring center is shown figure 115 for different phototube sizes.

and summarized in figure 117. The momentum resolution is better than $1.2 \%$ over the full accepted $\pi^{+}$momentum band. For comparison the momentum and track angle resolution of the Downstream Magnetic Spectrometer is shown as well in figure 117.

The pion RICH physically is a relatively large diameter $(2 \mathrm{~m}) 20 \mathrm{~m}$ long vessel of neon operating at atmospheric pressure. Maintaining acceptance for decay particles requires that the pion RICH apertures be sealed with relatively large thin windows which practically limits the operating pressure to the ambient atmospheric level. As discussed previously neon is a low dispersion gas, and is additionally motivated by the high performance achieved with the large SELEX RICH. The 20 meters of neon radiator gas present about $6 \%$ and $2 \%$ of radiation and interaction lengths to the beam and traversing decay particles.

In contrast to the kaon RICH, the relatively large mirrors in the pion RICH optical system preclude the use of the flat thin mirror scheme outlined in the proceeding kaon RICH section. The 


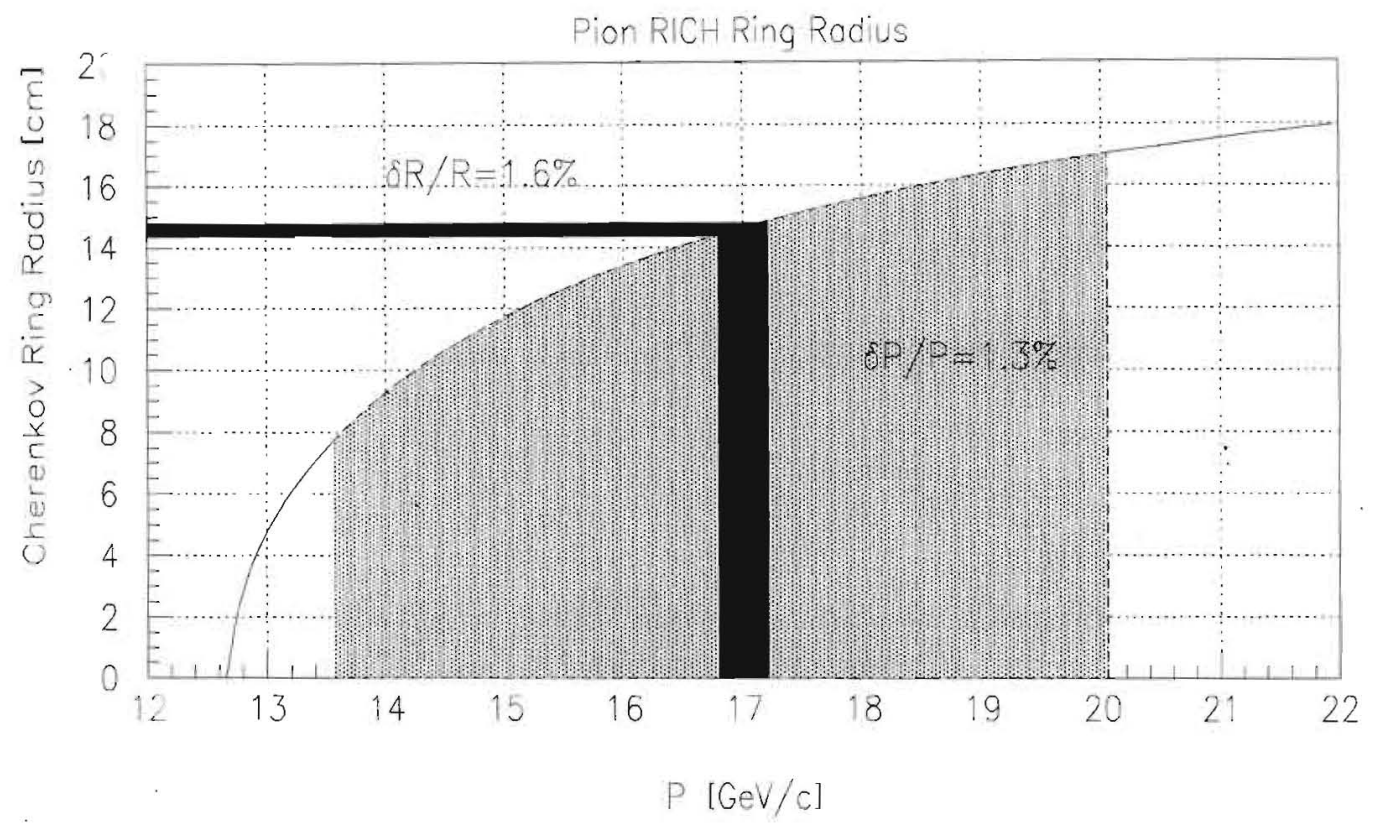

Figure 116: The functional relationship between the ring radius and the $\pi^{+}$track mornentum. The shaded band is the accepted momentum range. The black stripe is a $1-\sigma$ band that illustrates the momentum-radius transfer function. 

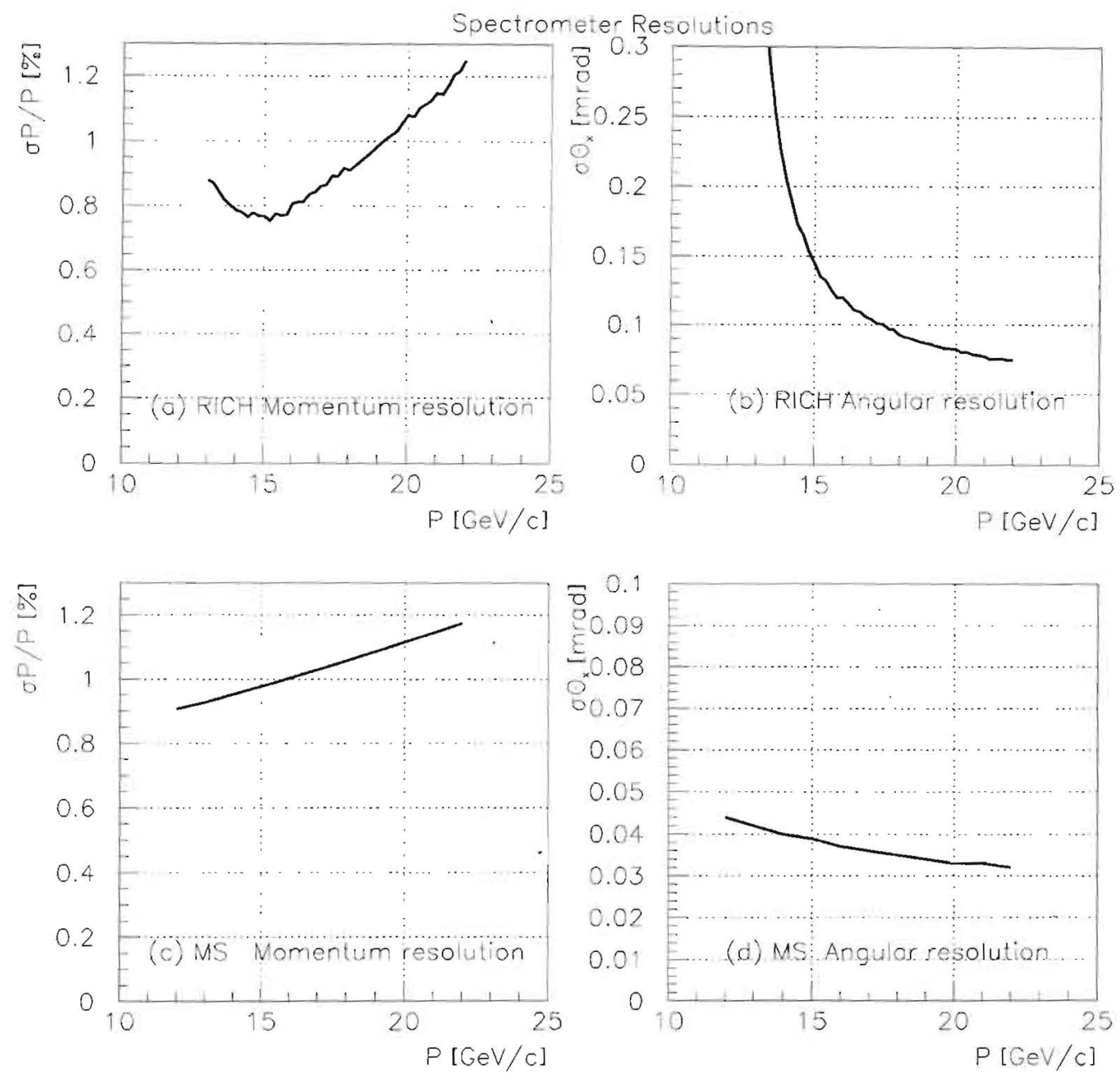

Figure 117: Pion (a) fractional RICH momentum resolution and (b) RICH angular resolution as a function of pion momentum from the pion RICH velocity spectrometer measurements. Contributions to the resolutions from optical dispersion and multiple Coulomb scattering in the Neon radiator gas and phototube size, are included. Downstream magnetic spectrometer (DMS) (c) fraction momentum resolution and (d) angular resolution as a function of pion momentum. Contributions to the resolutions from tracking resolution and multiple Coulomb scattering in the tracker are included. 


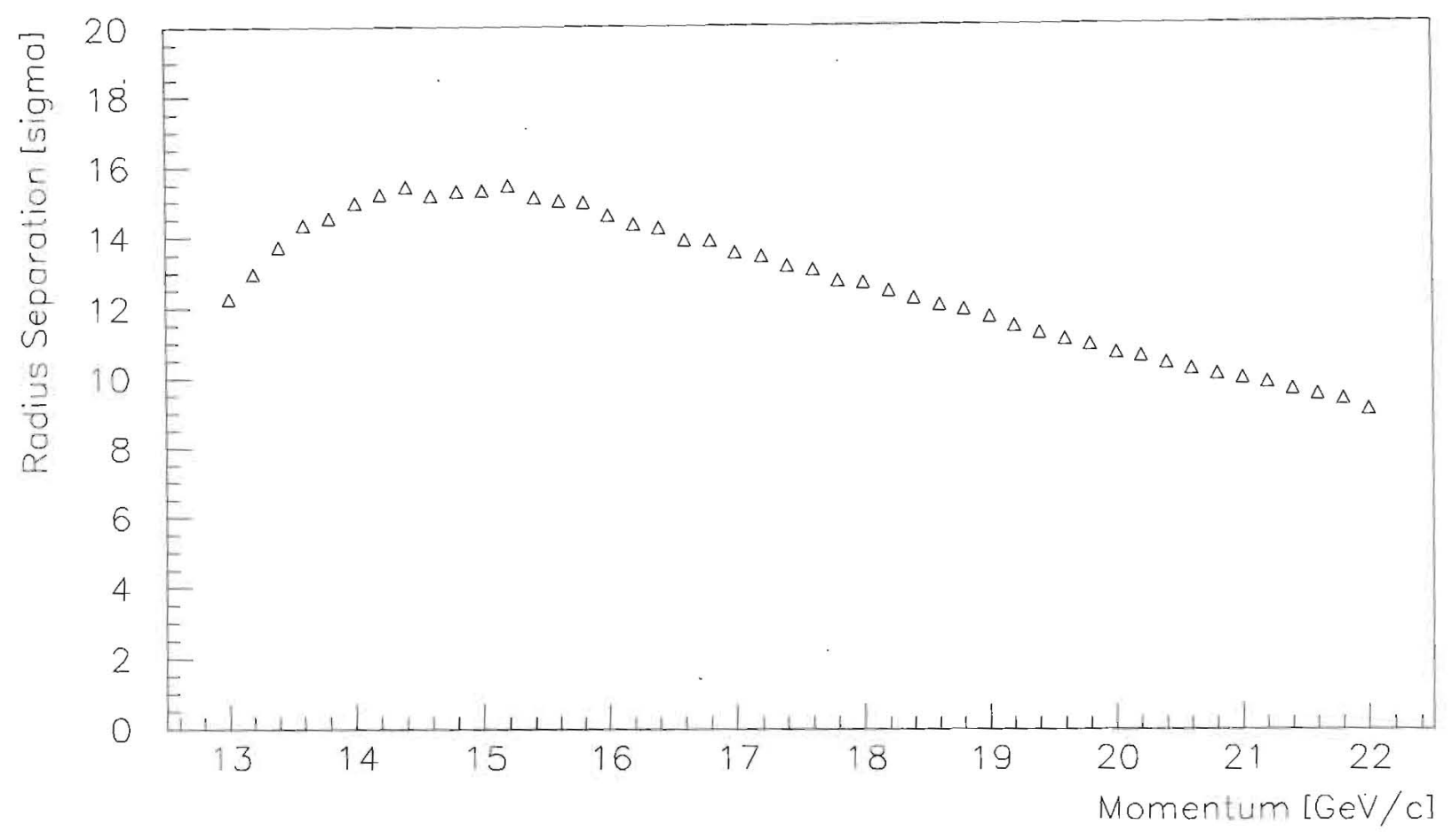

Figure 118: Radius separation between $\mu^{-}$and $\pi^{-}$in the Pion RICH. PM size 1/2 inch

downstream end of the pion RICH is fitted with a matrix of spherical mirrors mounted on a low mass rigid carbon-fiber lattice structure. The mirrors and mounting system present an additional $7.0 \%$ and $2.0 \%$ radiation and interaction lengths to the beam and traversing decay particles. The spherical mirrors image the ultraviolet Cherenkov photons to a close-packed array of $\sim 30001 / 2$ " photomultiplier tubes that measure the the ring image. As shown in figure 119 photomultiplier tubes in the "beam" ring would run at an maximum rate of about $400 \mathrm{kHz}$, with a nominal $\pi^{+}$rate of $2.95 \mathrm{MHz}$ in the beam, which should be no problem for reliable operation. Candidate rings for analysis are required to have at least eight photoelectrons. The rate/tube outside the "beam" ring from all kaon decays is at most $90 \mathrm{kHz}$ (see fig. 119).

The separation for pions and muons from Kaon decays is shown in figure 118 as a function of momentum.

\subsubsection{Pion $\mathrm{RICH}$ readout system}

The photomultiplier tubes (PMTs) that instrument the pion RICH focal plane have excellent timing and noise characteristics. PMT tubes under consideration now (Hamamatsu 1/2" R760) timing resolutions better than $\pm 1 \mathrm{nsec}$, and dead-times less than $25 \mathrm{nsec}$. PMTs are intrinsically very low noise devices, and extrapolating from the SELEX experience the CKM RICH systems should have noises rates of less than $500 \mathrm{~Hz} /$ tube. The 3000 PMTs are readout with the pipeline TDC system described in section 6.2.2. 

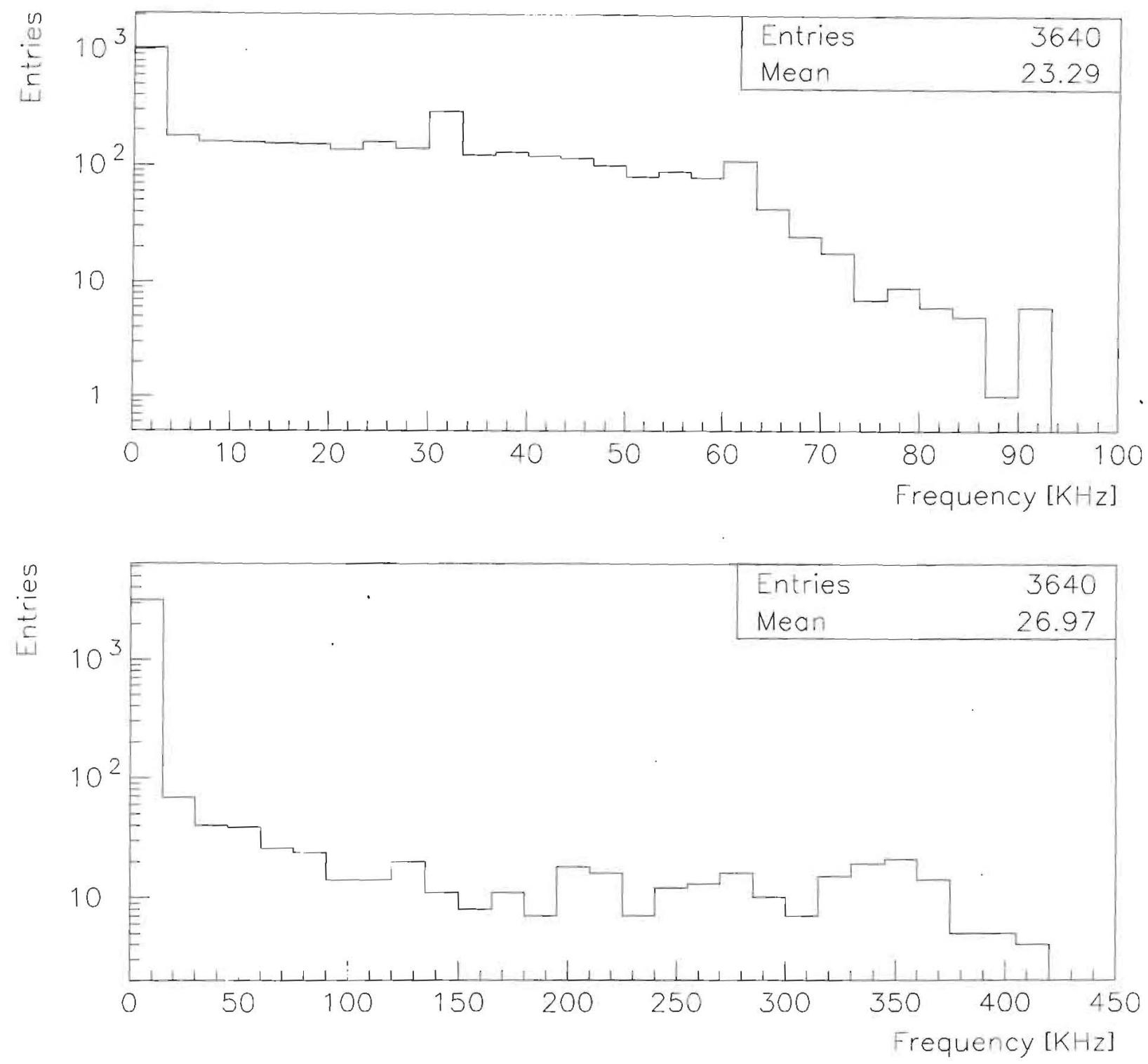

Figure 119: Rate in PMs for the Pion RICH. 10000 tracks are scaled to our $30 \mathrm{MHz}$ nominal beam rate for Kaons and their decay products (top), and $2.95 \mathrm{MHz}$ nominal beam rate for pions and their decay products (bottom). Gas: Ne, PM Size 1/2 inch. 


\subsection{The Forward Veto Systems.}

The principal role of the Forward Veto System (FVS) and the Hole Veto System (HVS) is to work together with the Vacuum Veto System (VVS) to veto photons from $K^{+} \rightarrow \pi^{+} \pi^{0}$ and $K^{+} \rightarrow \mu^{+} \nu_{\mu} \gamma$ decays. For $\mathrm{K}^{+} \rightarrow \pi^{+} \pi^{0}$ decays the photons intercepting the FVS are greater than $1 \mathrm{GeV}$ as shown in figure 14. About $5 \%$ of these incident photons convert to $e^{+} e^{-}$pairs in the pion RICH material material between the vacuum decay volume and the FVS. We have studied inefficiency mechanisms associated with these pair conversions and find them to be negligible compared to the required high energy inefficiency requirement of $1 \times 10^{-4}$ The result of a GEANT simulation of these conversion processes as well as Dalitz $\pi^{0} \rightarrow e^{+} e^{-} \gamma$ conversions are discussed in section 13 .

In addition to this principal veto function, the FVS must provide good measurements of photon energies and positions in a large control sample of $K^{+} \rightarrow \pi^{+} \pi^{0}$ decays. As discussed in section 5.6.14, these well measured photons contribute kinematic anchors to the task of measuring the total veto system performance. Central to the principal veto role are the concerns of high energy $(>1 \mathrm{GeV})$ hermeticity and $\pi^{+}-\gamma$ fusion discrimination as discussed in section 3.4.6. These two concerns are fundamentally in conflict, and the FVS design must strike a balance between them. A hermetic design calls for a thick device to ensure conversion and detection of high energy photons, and relatively course transverse segmentation to minimize cracks. A design capable of distinguishing nearby photons from charged pions calls for a relatively thin device to minimize hadronic interactions and fine transverse segmentation so that photon clusters can be distinguished from pion clusters. Maintaining good photon position and energy resolution requires relatively deep ( $>16 X_{0}$ ), uniform, and fine longitudinal sampling as well as fine transverse segmentation. Minimizing rate effects also pushes in the direction of transverse segmentation.

In order to maintain hermeticity in a design with fine transverse segmentation it is critical that cracks in any tower geometry not project back into the $K^{+} \rightarrow \pi^{+} \pi^{0}$ decay angle space. The angular distribution of $K^{+} \rightarrow \pi^{+} \pi^{0}$ photons intercepting the FVS is shown in figure 120. It is clear from figure 120 that canting the FVS by $50 \mathrm{mrad}$ in $\theta_{x}$ or $\theta_{y}$ is sufficient to ensure that no $K^{+} \rightarrow \pi^{+} \pi^{0}$ photons intercept cracks. Canting the FVS by $50 \mathrm{mrad}$ in $\theta_{x}$ is the natural choice since the $K^{+}$beam is already bent by $5 \mathrm{mrad}$ by the spectrometer magnet.

Balancing these conflicting demands and considering the higher rate environment of the FVS, the inefficiency requirement listed in table 11 is substantially relaxed with respect to the VVS. The inefficiency requirement of the HVS is even further relaxed yet. Although the HVS does not directly intercept the beam, it is downstream of the pion RICH beam volume and the FVS where hadronic showers often initiate. Only $\sim 10 \%$ of $\mathrm{K}^{+} \rightarrow \pi^{+} \pi^{0}$ photons intercept the FVS and HVS, and these relaxed efficiencies are included in the final expected background described in section 3.4.6.

As possible solutions for the FVS and HVS we have studied the performance of shashlik sampling designs and fully active crystal readout designs. Both designs have the capacity of providing the necessary hermeticity $\left(<1 \times 10^{-4}\right)$, and $\pi^{+}-\gamma$ fusion splitting $(>10 \mathrm{~cm})$. As baseline solutions to examine, we will compare and contrast the capabilities of two proven devices in the field: the PHENIX [183] shashlik calorimeter and the KTeV pure CsI calorimeter. The properties of the two calorimeter technologies are listed in table 30) and table 31. Our baseline design is shashlik given the expected lower cost.

\subsubsection{The PHENIX shashlik calorimeter.}

The shashlik readout scheme extracts the scintillation light from behind a Pb-scintillator stack with

an array of wavelength shifting fibers that skewer the stack with a typical density of $\sim 1$ fiber $/ \mathrm{cm}^{2}$. This architecture has the potential for being extremely hermetic, and is similar in principle to the 


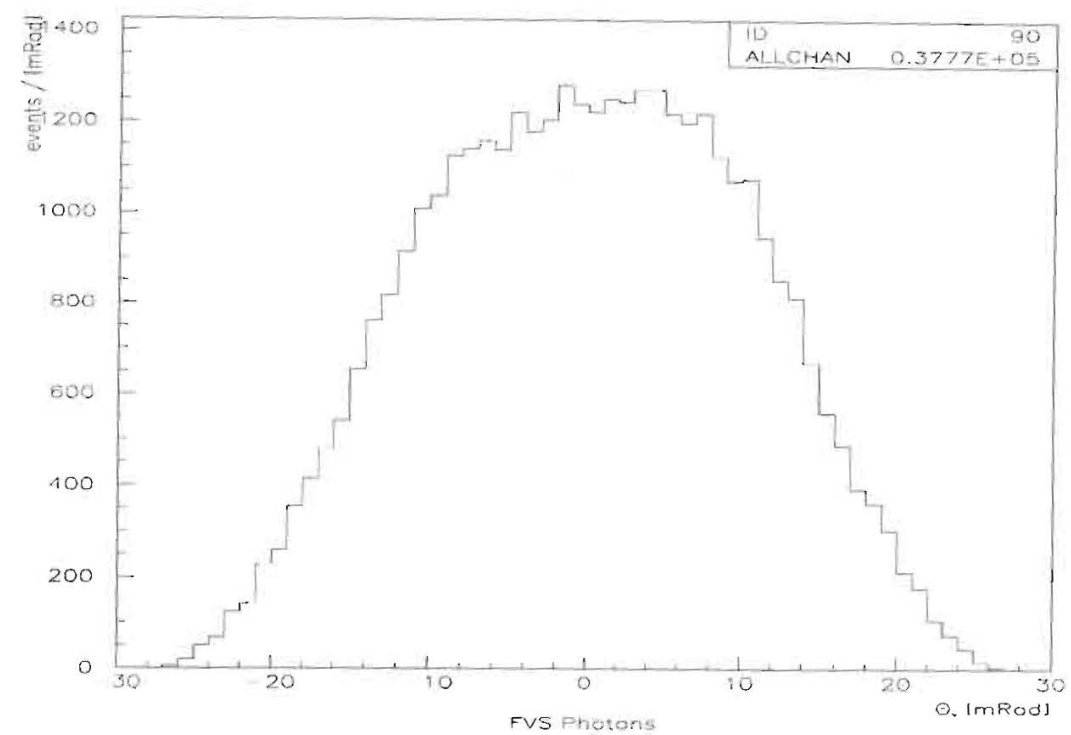

Figure 120: Angular distribution of photons incident on the FVS.

architecture of the Vacuum Veto System where the scintillator slabs are readout with embedded wavelength shifting fibers. To ensure hermeticity, one can reasonably consider large super-modules built from continuous sheets of lead with the scintillator planes segmented by narrow grooves cut in the scintillator sheet. A similar segmentation technique is being employed in the CMS HCAL design. For CKM we are studying a design of four overlapping super-modules in the Forward Veto System, with a transverse segmentation of $5 \times 5 \mathrm{~cm}^{2}$ cut into the scintillator planes. Drawings of the conceptual design indicating the transverse segmentation are shown in figure 121, and figure 122.

The lower shashlik fiber density as compared to other lead-scintillator fine sampling calorimeters reduces the amount of collected light, and consequently the energy resolution suffers somewhat. Despite this lower light yield, a shashlik prototype calorimeter tested at CERN [184] produced 12 photons/ $\mathrm{MeV}$, corresponding to approximately $\sim 50$ photo-electrons for a ( $20 \mathrm{MeV})$ photon. The lower shashlik fiber does have the advantage of reducing losses and distortions due to channeling effects. Operating at a non-projective angle of $50 \mathrm{mrad}$ this prototype nonuniformity envelope was $\pm 3 \%$ as a function of position and angle. The performance of the shashlik calorimetry employed in the PHENIX detector is listed below in table 30.

\subsubsection{The KTeV CsI calorimeter.}

The $\mathrm{KTeV}$ pure CsI calorimeter was designed primarily for the precise reconstruction of $K_{L} \rightarrow$ $2 \pi^{0}$ decays. The front face of $1.9 m \times 1.9 m$ device can be seen in the KTeV event display of a $K_{L} \rightarrow \pi^{+} \pi^{-} \pi^{0}$ decay shown in figure 124. Precise reconstruction of $K_{L} \rightarrow 2 \pi^{0}$ lead to a design that emphasizes high resolution and excellent linearity over a large range of photon energies in a moderate rate environment, that is radiation hard to the $20 \mathrm{kRad}$ level. These requirements are met well with the $\mathrm{KTeV}$ design of a deep $\left(27 X_{0}\right)$ pure CsI tower geometry with fine transverse segmentation.

An FVS made from pure CsI crystals with parameters shown in table 31 could meet CKM's FVS 


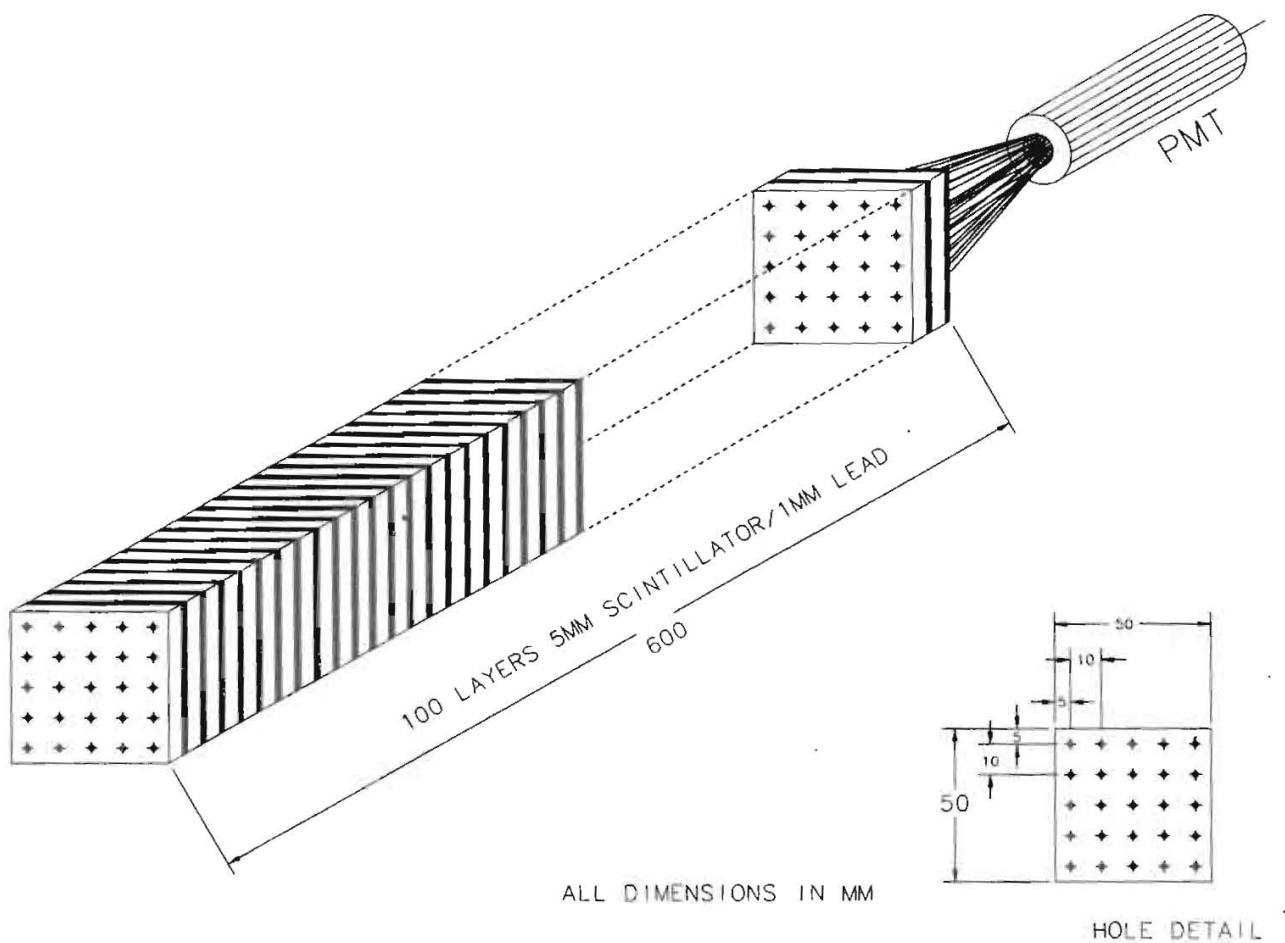

Figure 121: Conceptual drawing of one SHASHLIK $5 \mathrm{~cm} \times 5 \mathrm{~cm}$ tower. These scintillator towers are embedded in larger super-modules of continuous lead sheet. 

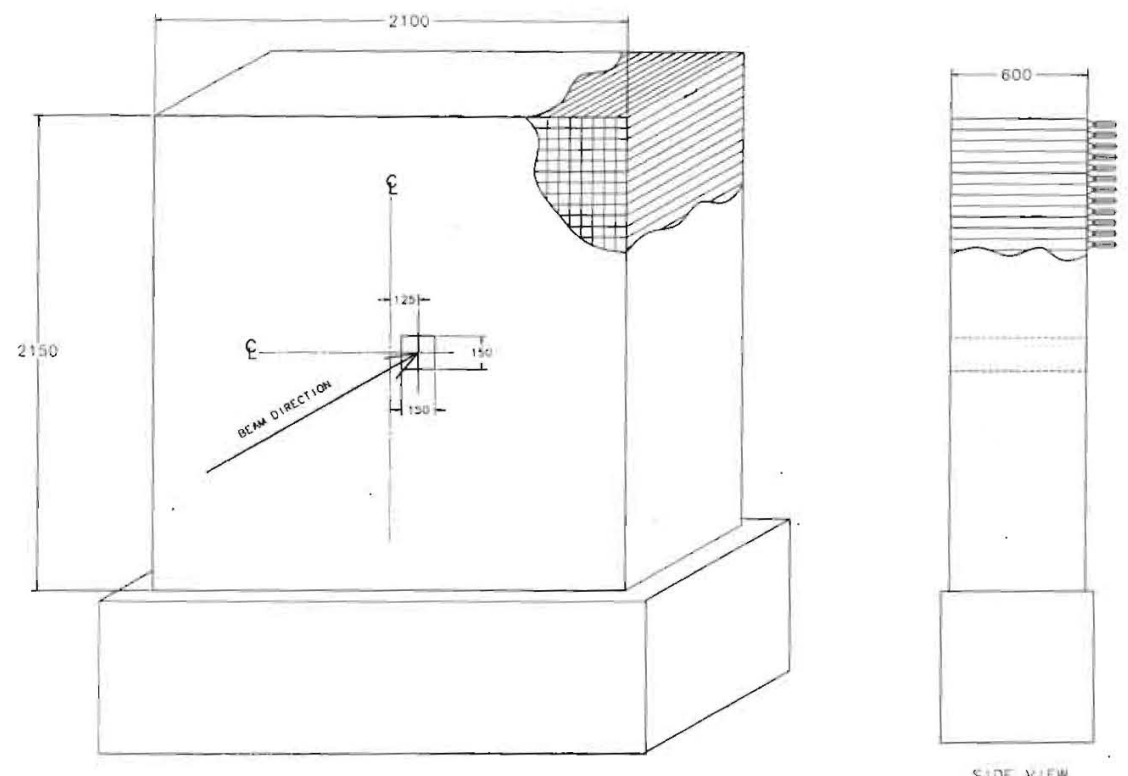

SIDE VIEW

Figure 122: Conceptual drawing of the FVS with transverse segmentation and offset beam hole indicated. 


\begin{tabular}{|l|l|l|}
\hline & PHENIX/shashlik & CKM/shashlik-FVS \\
\hline Outer Diameter. & Large barrel, $48 \mathrm{~m}^{2}$ & $210 \mathrm{~cm} \times 215 \mathrm{~cm}$ \\
Inner Dianeter (Beam Hole). & - & $15 \mathrm{~cm} \times 15 \mathrm{~cm}$ \\
Transverse Cell Size. & $5.25 \times 5.25 \mathrm{~cm}^{2}$ & $5.0 \times 5.0 \mathrm{~cm}^{2}$ \\
Number of Cells. & 15552 & 1797 \\
Longitudinal Depth. & $37.5 \mathrm{~cm}$ & $50.0 \mathrm{~cm}$ \\
Downstream Dead Gap. & $\sim 20 \mathrm{~cm}$ & $30 \mathrm{~cm}$ \\
Radiation Length Depth. & $19 X_{0}$ & $18 X_{0}$ \\
Interaction Length Depth. & $1.4 \lambda_{I}$ & $1.2 \lambda_{I}$ \\
Time Resolution. & $0.1 \mathrm{nsec}$ & $1 \mathrm{nsec}$ \\
Channel Deadtime. & $25 \mathrm{nsec}$ & $25 \mathrm{rsec}$ \\
Energy Resolution. & $8 \% / \sqrt{E}+1.5 \%$ & $5 \% / \sqrt{E}+1.0 \%$ \\
Position Resolution. & $6 \mathrm{~mm} / \sqrt{E}+1.5 \mathrm{~mm}$ & $5.0 \mathrm{~mm} / \sqrt{E}+1.0 \mathrm{~mm}$ \\
Transverse Non-uniformity. & $3 \% \mathrm{max}$. & $3 \% \mathrm{max}$. \\
Fiber Diameter. & $1.0 \mathrm{~mm}$ & $1.0 \mathrm{~mm}$ \\
Lead/Scintillator thickness. & $1.5 \mathrm{~mm} / 4 \mathrm{~mm}$ & $1 \mathrm{~mm} / 5 \mathrm{~mm}$ \\
Fibers/cell & 36 & 25 \\
Moliere Radius. & $3.40 \mathrm{~cm}$ & $3.40 \mathrm{~cm}$ \\
\hline
\end{tabular}

Table 30: Performance Summary of a shashlik FVS solution.

requirements. The principal difference with the $\mathrm{KTeV}$ design is courser transverse segmentation $(5 \mathrm{~cm} \times 5 \mathrm{~cm})$, and much shorter depth $\left(18 X_{0}\right)$. Such a design has the advantage of being fully active, and better energy and position resolution than $\mathrm{Pb}$-scintillator Shashlik. A design based on CsI crystals does have the disadvantage of finite cracks between blocks, and definitely will require canting the FVS by $\sim 50 \mathrm{mrad}$ in order to be non-projective.

\subsubsection{Studies of $\pi^{+}-\gamma$ separation performance.}

The dissection of $\mathrm{K}^{+} \rightarrow \pi^{+} \pi^{0}$ background in section 3.4.6 demonstrates the importance of separating nearby high energy photons $(\sim 1.6 \mathrm{GeV})$ from $(14-21 \mathrm{GeV})$ pions that traverse the FVS. The problem is evident in figure 123 which shows the result of a GEANT simulation where a $15 \mathrm{GeV}$ $\pi^{+}$and $1.6 \mathrm{GeV}$ photon separated by $15 \mathrm{~cm}$ are incident on a $16 X_{0}$ Pb-scintillator shashlik FVS array. Analysis of the $K^{+} \rightarrow \pi^{+} \pi^{0}$ background demonstrates that if these photons can be cleanly identified outside of a radius of $10 \mathrm{~cm}$ from the pion at the FVS, then this component of the $K^{+} \rightarrow \pi^{+} \pi^{0}$ background can be held in check. The viability of this separation radius has been tested with studies of $\pi^{+} / \gamma$ cluster data in the KTeV CsI array, and GEANT shower simulations. These studies will be described in turn.

Given the breadth of hadronic shower generators available and the uncertain accuracy of the FLUKA hadronic generator in the GEANT3 package, it is useful to establish a $\pi^{+} / \gamma$ separation benchmark based on data. We have studied a minimum bias low intensity $\mathrm{KTeV}$ data set containing a large sample of $K_{L} \rightarrow \pi^{+} \pi^{-} \pi^{0}$ decays. An example of one of these decays is shown in figure 124 . The sample studied had $146 \mathrm{~K} 14-19 \mathrm{GeV} \pi^{+}$tracks and 55K $1-2 \mathrm{GeV}$ photons cleanly separated from one another in the $\mathrm{KTeV}$ CsI calorimeter. The region of the CsI array studied was the outer region of $5 \times 5 \mathrm{~cm}^{2}$ crystals, where each crystal was $27 X_{0}$ and $0.7 \lambda_{I}^{\pi^{+}}$. The depth of the $\mathrm{KTeV}$ array was optimized for photon energy resolution and linearity, and hence is considerably longer than the nominal CKM length of $18 X_{0}$. Nevertheless, this data set serves as a useful benchmark 


\begin{tabular}{|l|l|l|}
\hline & KTeV $/$ CsI calorimeter & CKM/CsI-FVS \\
\hline \hline Outer Diameter. & $200 \mathrm{~cm}$ & $210 \mathrm{~cm} \times 215 \mathrm{~cm}$ \\
Inner Diameter (Beam Hole). & $2-15 \mathrm{~cm} \times 15 \mathrm{~cm}$ & $15 \mathrm{~cm} \times 15 \mathrm{~cm}$ \\
Transverse Cell Size. & $2.5 \times 2.5 \mathrm{~cm}^{2}$ & $5.0 \times 5.0 \mathrm{~cm}^{2}$ \\
Number of Cells. & 3100 & 1797 \\
Longitudinal Depth. & $50.0 \mathrm{~cm}$ & $33 \mathrm{~cm}$ \\
Downstream Dead Gap. & $\sim 50 \mathrm{~cm}$ & $30 \mathrm{~cm}$ \\
Radiation Length Depth. & $27 X_{0}$ & $18 X_{0}$ \\
Interaction Length Depth. & $0.7 \lambda_{I}$ & $0.5 \lambda_{I}$ \\
Time Resolution. & $<0.5 \mathrm{nsec}$ & $<1 \mathrm{nsec}$ \\
Channel Deadtime. & $25 \mathrm{nsec}$ & $25 \mathrm{nsec}$ \\
Energy Resolution. & $2 \% / \sqrt{E}+0.4 \%$ & $4 \% / \sqrt{E}+1.0 \%$ \\
Position Resolution. & $\sim 1 \mathrm{~mm}$ & $\sim 2 \mathrm{~mm}$ \\
Transverse Non-uniformity. & $2 \%, \mathrm{max}$. & $2 \%, \mathrm{max}$. \\
Fiber Diameter. & - & - \\
Lead/Scintillator thickness. & - & - \\
Fibers/cell & - & $3.8 \mathrm{~cm}$ \\
Moliere Radius. & $3.8 \mathrm{~cm}$ & - \\
\hline
\end{tabular}

Table 31: Performance Summary of a CsI FVS solution.

for the hadronic shower simulations.

The $\pi^{+} / \gamma$ separation in the $\mathrm{KTeV}$ data was studied by first preparing a, shower library of individual $\pi^{+}$and photon clusters from $K_{L} \rightarrow \pi^{0} \pi^{+} \pi^{-}$decays. The excellent linearity of the CsI calorimeter allows study of $\pi^{+} / \gamma$ separation by successively merging shower shapes in software as a function of separation. Figure 125 shows the average transverse projection of $(14-19) \mathrm{GeV}$ $\pi^{+}$showers overlaid with an average transverse projection of $(1.0-2.0) \mathrm{GeV}$ photon showers offset by $10 \mathrm{~cm}$ and $15 \mathrm{~cm}$. In figure 126 a comparison of the mean transverse profile of $\mathrm{KTeV} / \mathrm{CsI}$ $18-19 \mathrm{GeV} \pi^{+}$clusters with a GEANT3(FLUKA) simulation of the KTeV CsI array is slown. The level of agreement supports the use of GEANT3(FLUKA) to estimate $\pi^{+} / \gamma$ separation in these energy ranges.

A straightforward algorithm has been developed to study the separation of photon clusters from nearby traversing charged pions in the $\mathrm{KTeV}$ data. This algorithm first associates the center of a $3 \times 3$ cluster of blocks with the $\pi^{+}$track. Next the algorithm searches the $5 \times 5$ and $7 \times 7$ ring surrounding the $3 \times 3 \pi^{+}$cluster for any outer block that exceeds a fixed threshold. The efficiency for accepting the $\pi^{+}$(no self veto) as a function of this outer-block threshold is shown in figure 127. The outer-block threshold that corresponds to a $90 \% \pi^{+}$acceptance is $400 \mathrm{MeV}$, and is indicated with an arrow in figure 127. For a fixed $90 \%$ acceptance of $14-20 \mathrm{GeV}$ pions then, the efficiency for vetoing $1.0-2.0 \mathrm{GeV}$ photons as a function of distance can be measured by successively merging the $\pi^{+}$and photon clusters from the shower library. The photon veto efficiency as a function of separation distance is shown in figure 127. In order to maintain very high veto efficiency for $1.0-2.0 \mathrm{GeV}$ photons, the separation algorithm was modified outside of $20 \mathrm{~cm}$ to cluster photons to one tower. This was done to avoid spurious photon inefficiency from photon shower development where a $>1 \mathrm{GeV}$ photon had no individual blocks greater than $400 \mathrm{MeV}$ (incident near a corner for example).

From figure 127 it is clear that photons can be identified with high efficiency outside of a 10 


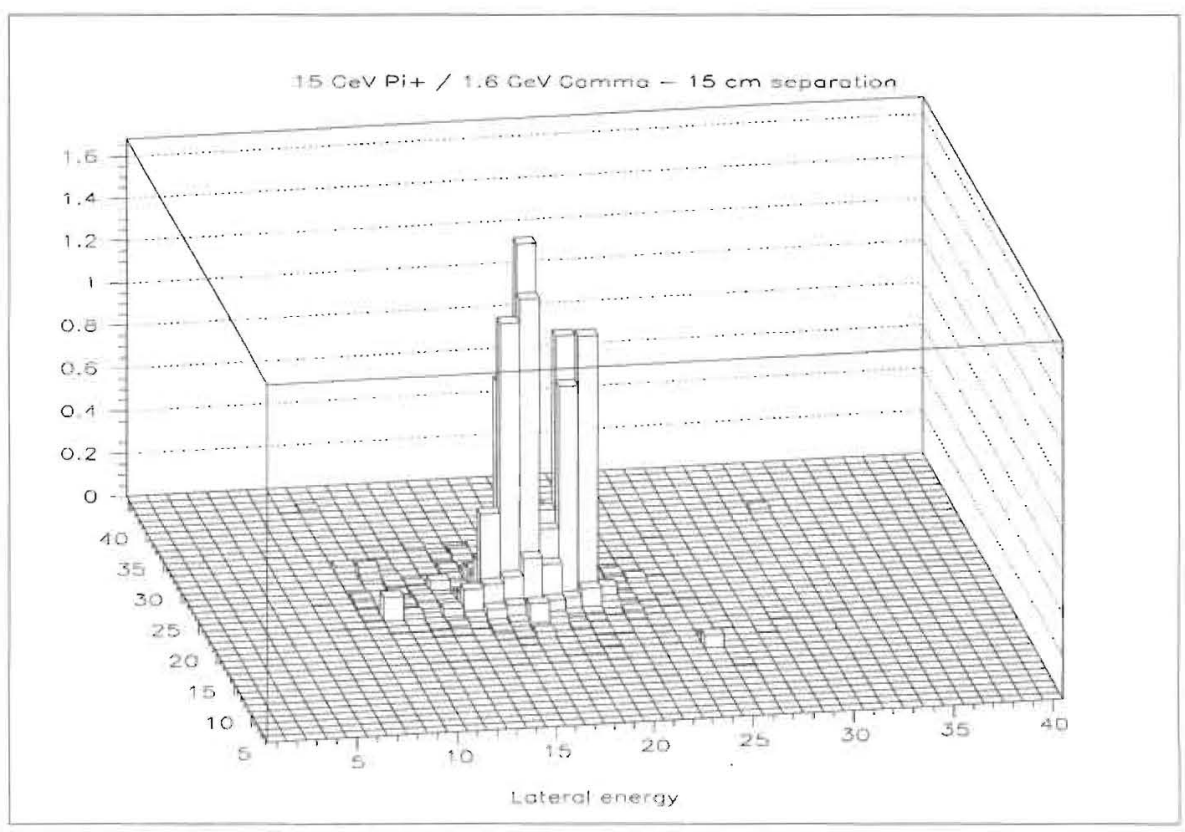

Figure 123: GEANT simulation of a $15 \mathrm{GeV} \pi^{+}$and $1.6 \mathrm{GeV}$ photon separated by $15 \mathrm{~cm}$ incident on a $16 X_{0} \mathrm{~Pb}$-scintillator FVS.

$\mathrm{cm}$ radius, and with some finite efficiency within this radius as well. A GEANT simulation of $\pi / \gamma$ separation in $27 X_{0}$ of CsI is shown overlaid on the data in figure 128 , and reasonably models the data. A similar analysis of GEANT clusters in a $18 X_{0}$ CsI and $1 \mathrm{~mm}-\mathrm{Pb} / 5 \mathrm{~mm}$-Scintillator FVS is shown in figure 129. From these simulations and analysis, it is evident that with $5 \mathrm{~cm} \times 5 \mathrm{~cm}$ transverse segmentation, the $\pi^{+}$efficiency and $\pi^{+} / \gamma$ separation depends, weakly on the calorimeter material and depth, and is dominated by the tower cell geometry. Figure 130 illustrates the difference in transverse shower profile of CsI and $\mathrm{Pb}$-scintillator as calculated in GEANT. More sophisticated clustering and/or finer transverse segmentation in the FVS could in principle provide further enhanced $\pi^{+} / \gamma$ separation. For the purposes of this proposal however, we will assume a $90 \% \pi^{+}$efficiency and use the corresponding $\epsilon\left\{\pi^{+} / \gamma\right\}(r)$ photon efficiency curve determined from $\mathrm{KTeV}$ data shown in figure 127.

\subsubsection{Impact of FVS energy and position resolution on measuring detector photon veto efficiencies with $K^{+} \rightarrow \pi^{+} \pi^{0}$ decays.}

As shown in section 3.4.6, CKM's principal background from $K^{+} \rightarrow \pi^{+} \pi^{0}$ decays has a significant component from $\pi^{+} / \gamma$ separation inefficiency in the FVS, and is directly proportional to the VVS high energy inefficiency. The corresponding very large sample of $K^{+} \rightarrow \pi^{+} \pi^{0}$ decays that can be collected with the CKM apparatus can be exploited to measure these two key system efficiencies. Employing similar analysis techniques to those described in the previous discussion of $\pi^{+} / \gamma$ separation, it is clear that FVS clusters in the CKM data set can be employed to accurately measure the FVS $\pi^{+} / \gamma$ separation efficiency. The $K^{+} \rightarrow \pi^{+} \pi^{0}$ data set which includes one well measured photon cluster in the FVS and a well measured $\pi^{+}$in the spectrometers can be used to measure the global VVS photon veto efficiency as described in section 5.6.14.

Both of these efficiency measurements require good calorimetric performance from the FVS: on 


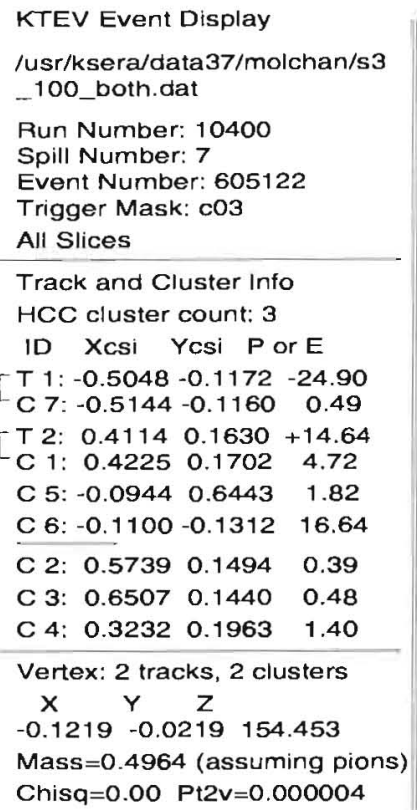

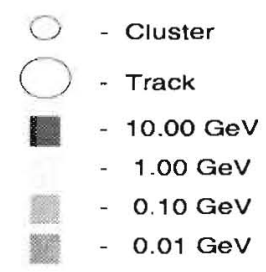

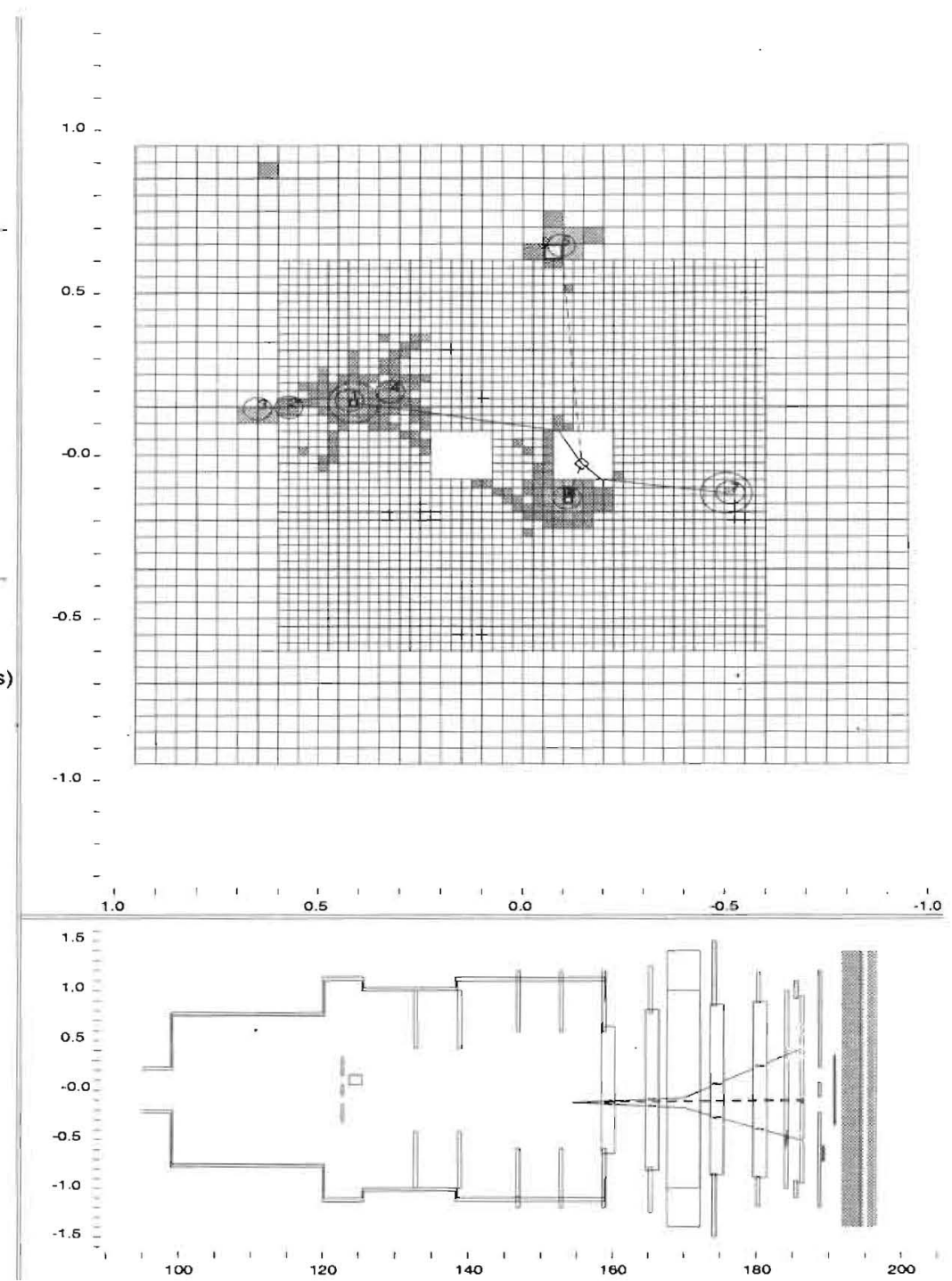

Figure 124: $\mathrm{KTeV}$ event display of ' a $K_{L} \rightarrow \pi^{0} \pi^{+} \pi^{-}$decay. 


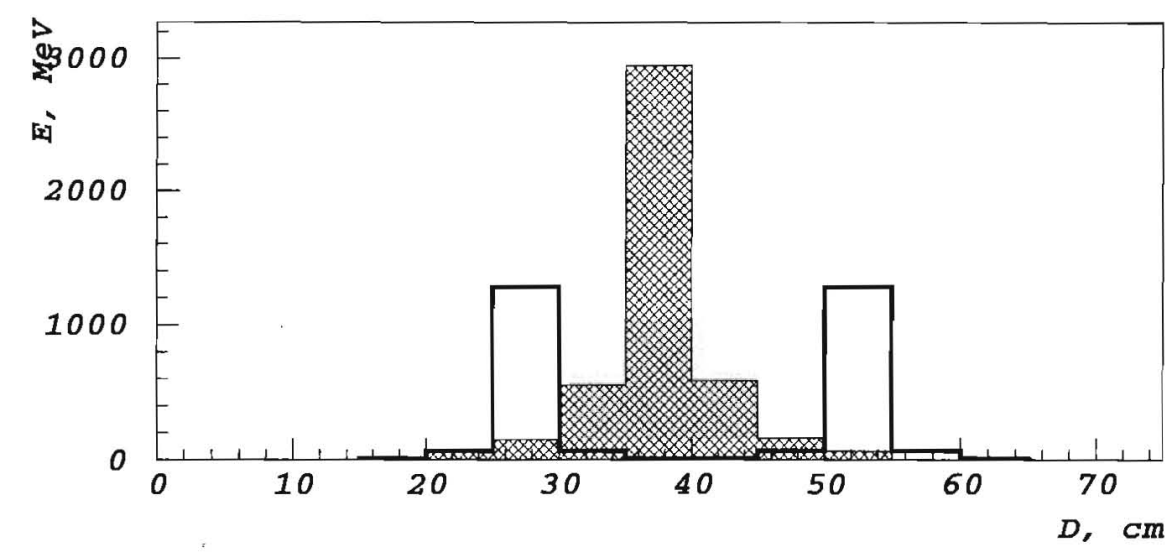

Figure 125: Average transverse projection profile of $\mathrm{KTeV} \pi^{+}$(shaded) and photons (solid line) separated by $10 \mathrm{~cm}$ on the left and $15 \mathrm{~cm}$ on the right.

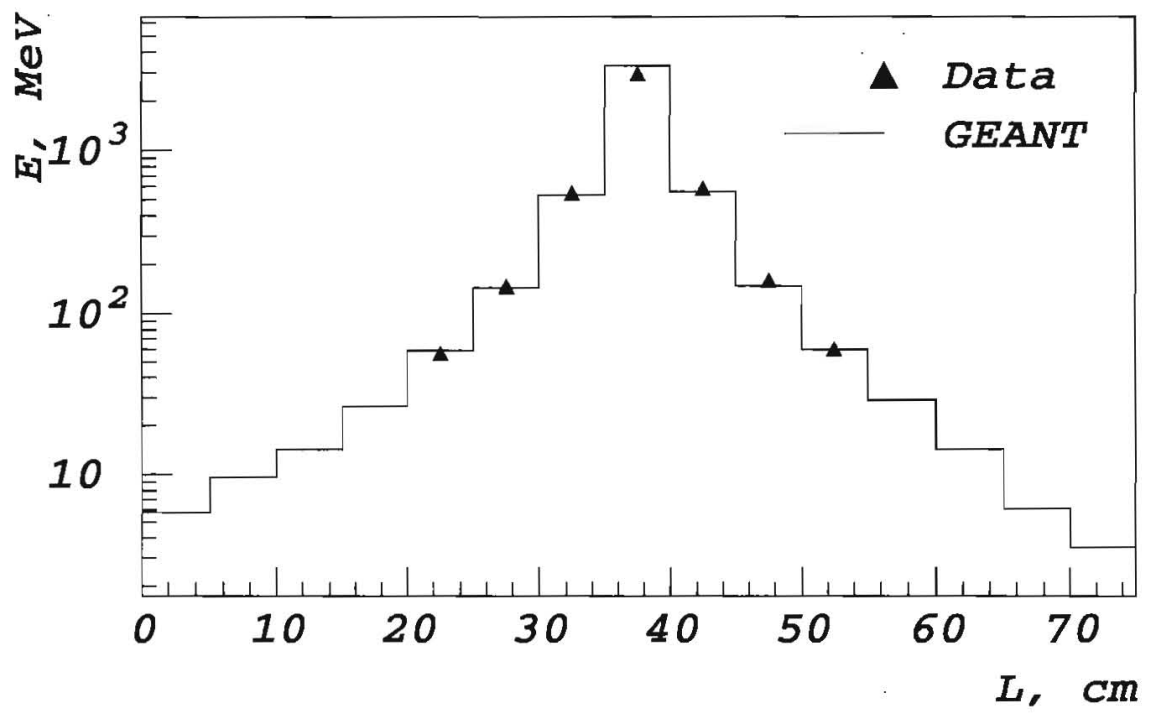

Figure 126: The average $\pi^{+}$shower projection profile in the $\mathrm{KTeV}$ data compared with a GEANT simulation of the $\mathrm{KTeV}$ CsI calorimeter. 

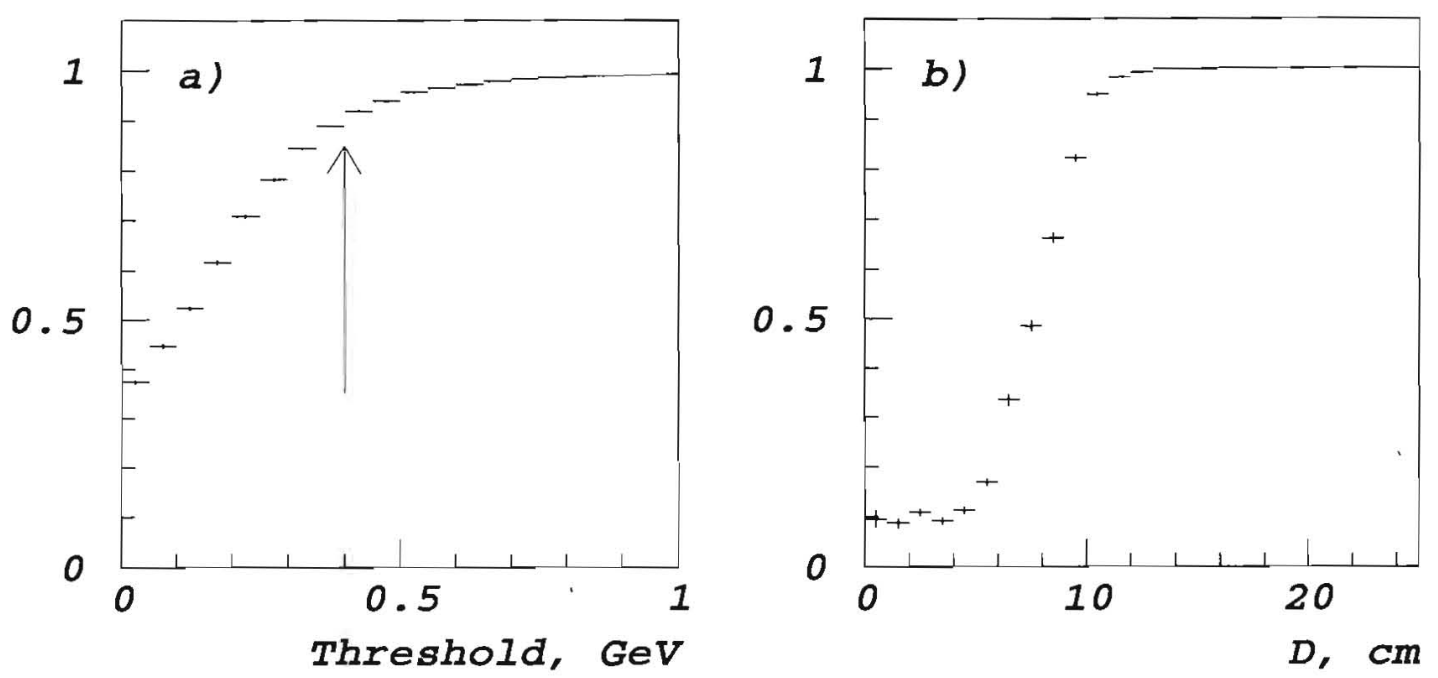

Figure 127: Measured from KTeV Data, Figure (a): Efficiency of accepting a $\pi^{+}$as a function of threshold energy of blocks outside of the central $3 \times 3$ cluster. Figure (b): The efficiency of detecting a $(1.0-2.0 \mathrm{GeV})$ photon near a $14-20 \mathrm{GeV} \pi^{+}$as a function of separation at a fixed $\pi^{+}$efficiency of $90 \%$.
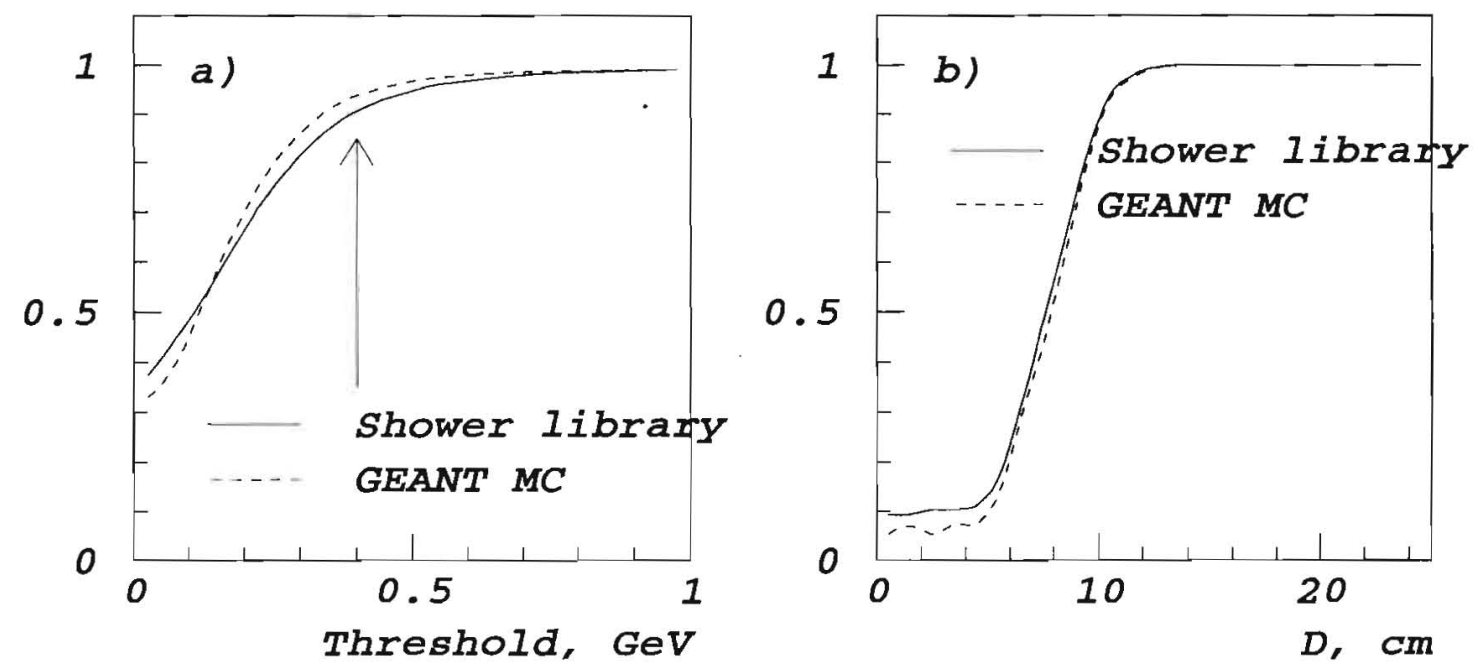

Figure 128: Overlay of the $\mathrm{KTeV}$ shower library analysis and an identical analysis of a GEANT simulation of $27 X_{0}$ CsI. Figure (a) shows $\pi^{+}$efficiency as a function of outer block energy threshold. Figure (b): The efficiency of detecting a $(1.0-2.0 \mathrm{GeV})$ photon near a $14-20 \mathrm{GeV} \pi^{+}$as a function of separation at a fixed $\pi^{+}$efficiency of $90 \%$. 

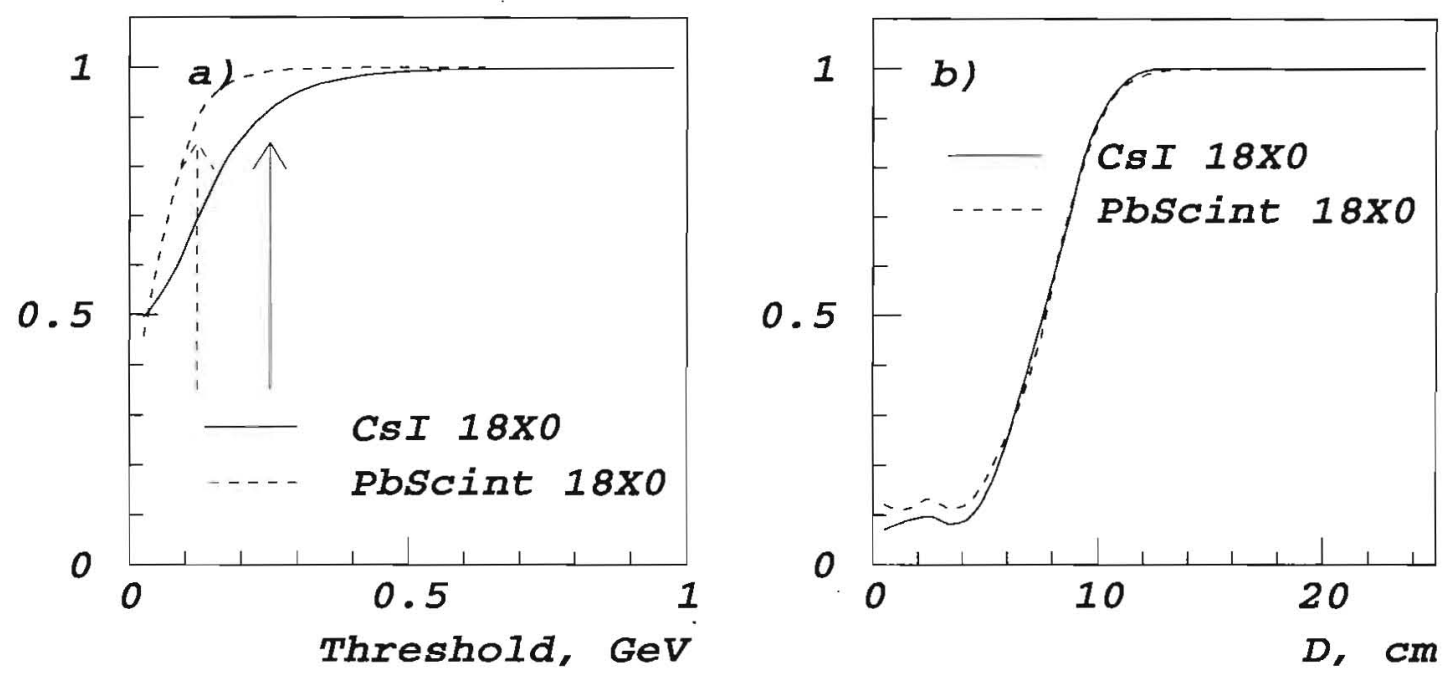

Figure 129: GEANT simulation of $18 X_{0}$ Pb-Scintillator and CsI. Figure (a) shows $\pi^{+}$efficiency as a function of outer block energy threshold. Arrows in figure (a) indicate the respective $90 \%$ efficiency points. Figure (b): The efficiency of detecting a $(1.0-2.0 \mathrm{GeV})$ photon near a $14-20 \mathrm{GeV} \pi^{+}$as a function of separation at a fixed $\pi^{+}$efficiency of $90 \%$.
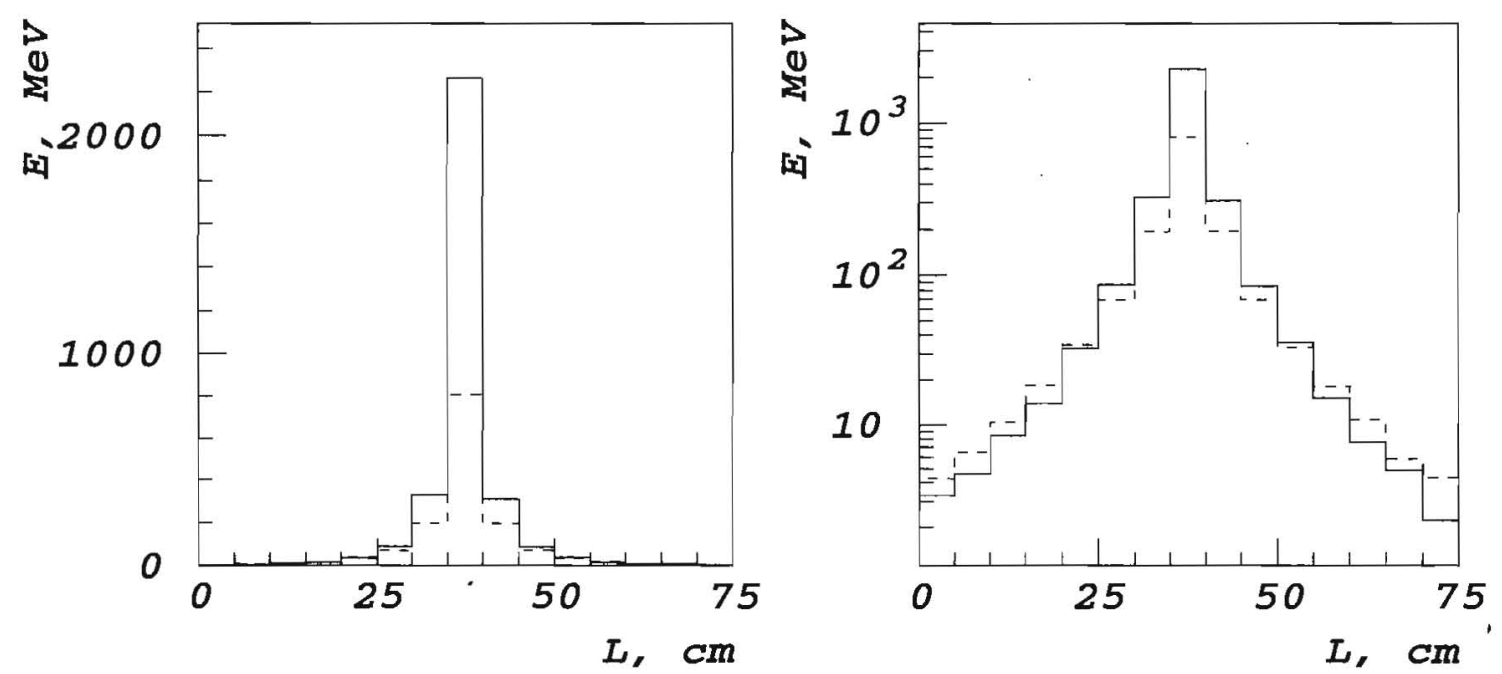

Figure 130: Liner and log transverse shower profiles of CsI (solid) and shashlik Pb-scintillator (dashed) simulations in GEANT. 
the order of $\sigma_{E} / E=5 \% / \sqrt{E} G e V$ energy resolution, $2-3 \mathrm{~mm}$ of transverse position resolution, and good linearity. This level of performance can be achieved with the default Shashlik designs we are considering, and certainly with crystals.

\subsubsection{Benefits and liabilities of longitudinal segmentation.}

In principle the $\pi^{+} / \gamma$ separation could be improved by longitudinally segmenting the FVS. We have investigated a variety of schemes, all of which come at some cost of hermeticity and calorimetric performance. Given that $18 X_{0}$ of longitudinally uniform Shashlik or crystal tower geometry can achieve the necessary $\pi^{+} / \gamma$ separation, at this time there is little motivation to explore longitudinal segmentation and the attendant complexities.

\subsubsection{Other FVS technologies being considered.}

We have considered a wide scope of possible FVS technologies, ranging from projective readout schemes to heavy liquid scintillators. One particularly novel scheme we have explored is a Shashlik scheme with liquid scintillator. Such a scheme built with large lead sheets has the potential for being very hermetic, but does come with a cost of loosely-defined transverse segmentation. We have in fact prototyped such a scheme, and although the amount of light detected is acceptable, the complexity associated with loose tower boundaries probably excludes this option.

After considering a wide range of calorimetric technologies we have found the the FVS requirements are best met with a conventional wall of Shashlik or crystal tower geometry of modest size $(2 m \times 2 m)$. There is a wealth of experience in the field with these technologies, and we expect the forward and hole veto systems can be designed, costed, built and commissioned with relatively little uncertainty.

\subsection{The Muon Veto System}

As described in section 3.4.7 the Muon Veto System (MVS) must identify muons between $14 \mathrm{GeV}$ and $22 \mathrm{GeV}$ with a muon mis-id rate of less than $1 \times 10^{-5}$ while maintaining high acceptance $(\sim 95 \%)$ for the $K^{+} \rightarrow \pi^{+} \nu \bar{\nu}$ signal.

A plausibility argument for achieving this level of mis-id integrity was presented in section 3.4.7 by considering $\mu^{-}$decay, $\mu^{-}$bremsstrahlung, and $\mu^{-}$(Deep Inelastic Scattering) (DIS) processes separately. Preliminary studies of the interplay and topology of these processes with respect to $K^{+} \rightarrow \pi^{+} \nu \bar{\nu}$ pion showers have been studied with GEANT simulations for a range stack consisting of steel plates and scintillator hodoscope planes described in section 5.10 .2 below.

In order to achieve $1 \times 10^{-5}$ muon efficiency at $95 \%$ pion acceptance it is necessary for the outer radial aperture of the MVS to be $20 \mathrm{~cm}$ greater than that of the FVS to allow for multiple scattering in the FVS and MVS. About $6 \mathrm{MHz}$ of incident muon tracks from halo and decays within the detector will penetrate to and count as hits within the $2.4 \mathrm{~m} \times 2.4 \mathrm{~m}$ MVS fiducial area. Both the FVS and MVS have a $15 \mathrm{~cm} \times 15 \mathrm{~cm}$ beam hole that passes the $50 \mathrm{MHz}$ charged beam. The transverse segmentation of the scintillator in the range stack is determined by the rate of ranging particles, and is currently under study. The counters will be readout with the both pipelined TDC and ADC systems.

\subsubsection{MVS prototype test beam results}

In a November, 2000 run of U-70 at IHEP Protvino we had 2 weeks of the beam time in the channel $4 \mathrm{~A}$ with the ISTRA spectrometer to test a Muon Veto System (MVS) prototype. The main goal of 
the run was to demonstrate that, indeed, it is possible to achieve a high rejection for muons (better than $10^{5}$ ) with a reasonable efficiency for the pions.

The negative $4 \mathrm{~A}$ beam is produced by primary $70 \mathrm{GeV}$ protons on the internal target located inside the U-70 vacuum chamber. The secondary beam included two $\sim 70 \mathrm{~cm}$ long iron beam plugs (BS1-2) to remove hadrons and four $50 \mathrm{~cm}$ long tunable vertical and horizontal collimators (K1-4). In the MVS prototype tests the beam-line was used in three main modes:

1. pion beam:

The beam was tuned for $25 \mathrm{GeV}$ negative particles $\left(\pi^{-}\right)$with $\frac{\Delta p}{p} \sim 1 \%$. The collimators were tuned to provide a narrow beam with intensity of $\sim 10^{6} /$ spill (the U-70 duty factor is $1.9 / 9$ sec.) Both beam stops were opened.

2. High intersity muon beam:

The magnets are tuned, as before, to $25 \mathrm{GeV}$ with the first collimator pair and beam stop open and the downstream collimators and beam stop closed. This tune selects muons from $25 \mathrm{GeV}$ pions which decay before the closed elements. The undecayed pion beam is absorbed with the charged daughters swept out of the beam line by the last dipole which is set to for $17 \mathrm{GeV}$. Muons of $14-20 \mathrm{GeV}$ are transmitted along the beam.

3. Low intensity muon beam:

The magnetic elements are set as before, but all collimators and beam stops are closed. This setting provides low intensity, but a very clean muon beam.

\subsubsection{The Muon Veto System prototype}

The MVS prototype is shown in Figure 131. The prototype consists of 27 planes of $600 \times 600 \times$ $41 \mathrm{~mm}^{3}$ steel plates interleaved with 26 scintillator planes $500 \times 480 \times 10 \mathrm{~mm}^{3}$. The planes are perpendicular to the beam axis, eacli scintillator plane consists of twelve $500 \times 40 \times 10 \mathrm{~mm}^{3}$ counters; two consecutive planes are arranged liorizontally and vertically with a staggering in eacll view. All the scintillators are polished and covered with mylar. The light is read-out by Russian PMT's (FEU-84) through a light guide. The signals from the PMT's are readout with ADCs. Figure 132 illustrates the location of the MVS prototype on the beam.

Elements of the ISTRA setup used during the test were: two Cherenkov's $C_{1}, C_{2}$ were filled with $0.2 \mathrm{~atm}$ of $\mathrm{CO}_{2}$ to remove low energy particles; the scirtillation counters $S_{1} \div S_{5}$ were used to define the beam, $S_{4}$ was a halo veto counter (with a hole), which was used in the anti-coincidence. $\mathrm{BPC} 1 \div \mathrm{BPC} 2-\mathrm{x}$ and $\mathrm{y}$ proportional beam chambers with a step of $1 \mathrm{~mm}$. APC1, APC2- $\mathrm{x}, \mathrm{y}$ proportional chambers with a step of $2 \mathrm{~mm}$ installed in front of the MVS. The magnet M1 deflects the beam in the vertical direction and was used to estimate the momentum of the beam particles. A large Veto counter $(\mathrm{V})$ was used to veto the particles passing through the MVS. It consists of $500 \times 500 \times 40 \mathrm{~mm}^{3}$ scintillator viewed by 4 PM's. The lead-glass counters LG0 $\div$ LG7 with the dimensions of $100 \times 100 \times 400 \mathrm{~mm}^{3}$ were used to provide a clean muon beam. In the high intensity muon runs LG0 $\div$ LG6 were moved into the beam; in the low intensity muon run- just LG7. The signals from LG's were sent to ADC's. MIP signals were required in LG's off-line; to suppress hadron and electron (from $\mu$ decays) background. The following main data sets were logged during the run:

1. High intensity muon ( 1.6 M events $)$ :

The trigger was $\mathrm{T}=C_{1} \cdot C_{2} \cdot S_{1} \cdot S_{2} \cdot S_{3} \cdot \bar{S}_{4} \cdot S_{5} \cdot \bar{V}$, the rate of the monitor $\mathrm{M}=C_{1} \cdot C_{2} \cdot S_{1} \cdot S_{2} \cdot S_{3} \cdot \bar{S}_{4} \cdot S_{5}$ was about $10^{4} /$ spill ; $\mathrm{T} / \mathrm{M} \sim 2 \%$. 

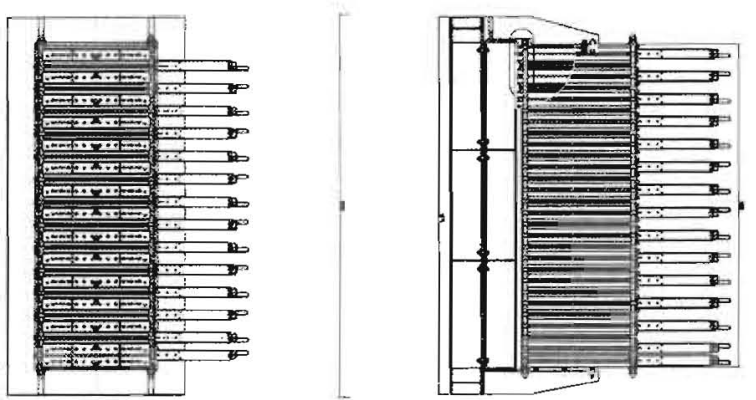

$+x+x$

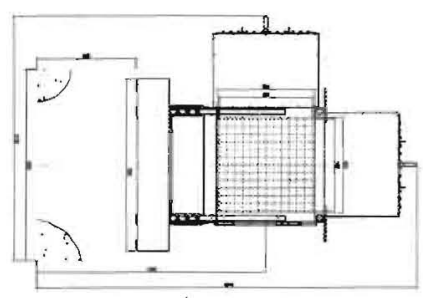

Figure 131: The MVS prototype. 

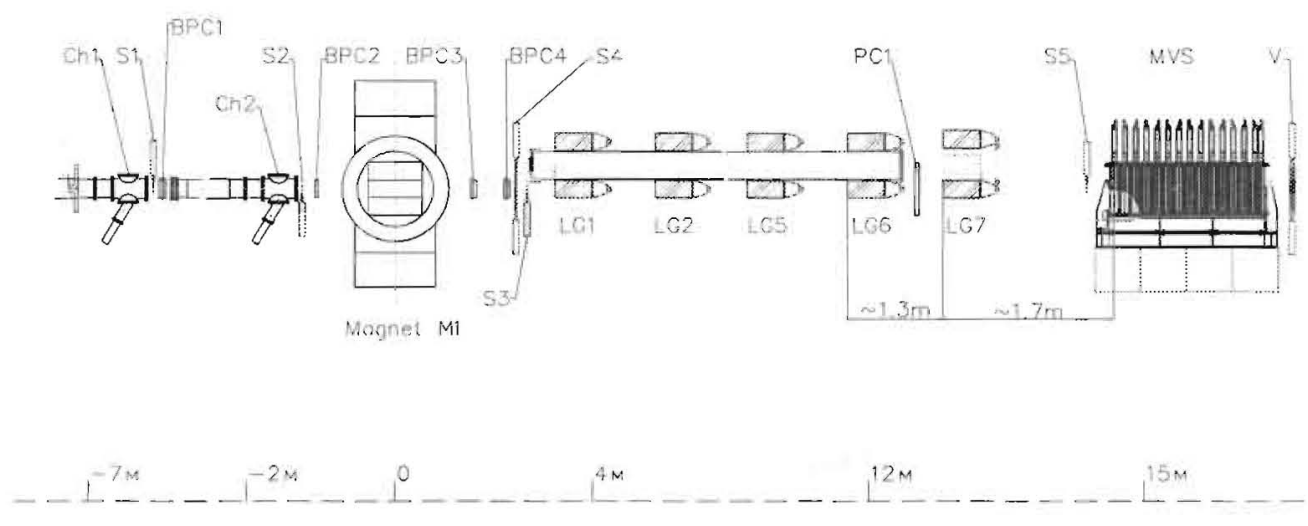

Figure 132: The MVS prototype on the ISTRA-M beam.

2. Low intensity muon $(\dot{\sim} 3.6 \mathrm{M}$ events):

$\mathrm{T}=C_{1} \cdot C_{2} \cdot S_{1} \cdot S_{2} \cdot S_{3} \cdot \bar{S}_{4} \cdot S_{5}$, the rate was $\sim 10^{3} /$ spill.

3. $25 \mathrm{GeV} \pi$ Runs $128 \div 132(3.1 \mathrm{M}$ events):

$\mathrm{T}=C_{1} \cdot C_{2} \cdot S_{1} \cdot S_{2} \cdot S_{3} \cdot \bar{S}_{4} \cdot S_{5}$, the rate was $\sim 10^{5} /$ spill

\subsubsection{Analysis}

The data processing began with the calibration of the MVS using data from a special run with a wide muon beam with the trigger of $\mathrm{T}=C_{1} \cdot C_{2} \cdot V$. The dependence of the signal on the distance from the hit to the PM was taken into account. Figure 133(left) shows the total signal in the MVS after the calibration. The resolution of $\frac{\sigma_{F}}{E}=0.15$ in good agreement with Monte Carlo is observed. In the top figure a clear signal from the muon admixture in the hadron beam is seen. Muon disappear in the bottom figure, where the absence of the signal from V-counter was required. This muon admixture was corrected for when calculating the efficiency of the selection cuts for the pions. The muon beam momentum spectrum is shown in Figure 133(right). It is perfectly consistent with the CKM working range.

Clean the inuon beam was selected by requiring in time single hits in the the beam chambers in a square of $10 \times 10 \mathrm{~cm}^{2}$ around the beam and no hits outside the square. Pulse heights in the lead glass counters (LG) consistent with a muon were also required to suppress two muon and deadtime effects.

After these beam definitions are applied, the main cuts which must suppress the muons with reasonable efficiency to pions were used. The first of this group of cuts, and the most obvious and efficient one is the cut on the amplitude of the V-counter behind the prototype which is used after 

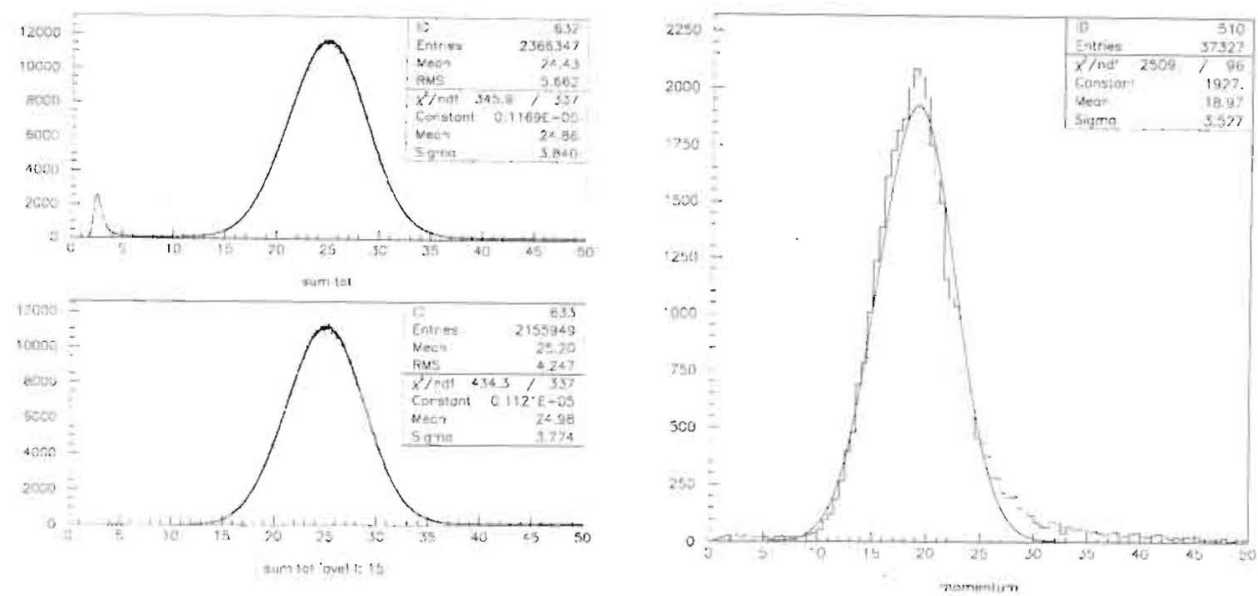

Figure 133: The total MVS signal for $25 \mathrm{GeV}$ pions(left), the muon beam momentum spectrum(right).

taking deadtime and pile-up effects into account.

To explain the logic of further cuts, Figure 134 shows $\mathrm{x}$ and y views of a typical $25 \mathrm{GeV}$ pion event in the MVS prototype and a muon event, where muon emitted bremstrahlung photon. The numbers in the pictures are uncalibrated, pedestal subtracted, ADC counts. There are 13 planes of counters in each view, 12 counters per plane. In the most of muon events clear segments of muon tracks are seen. It seems natural to introduce the following variables:
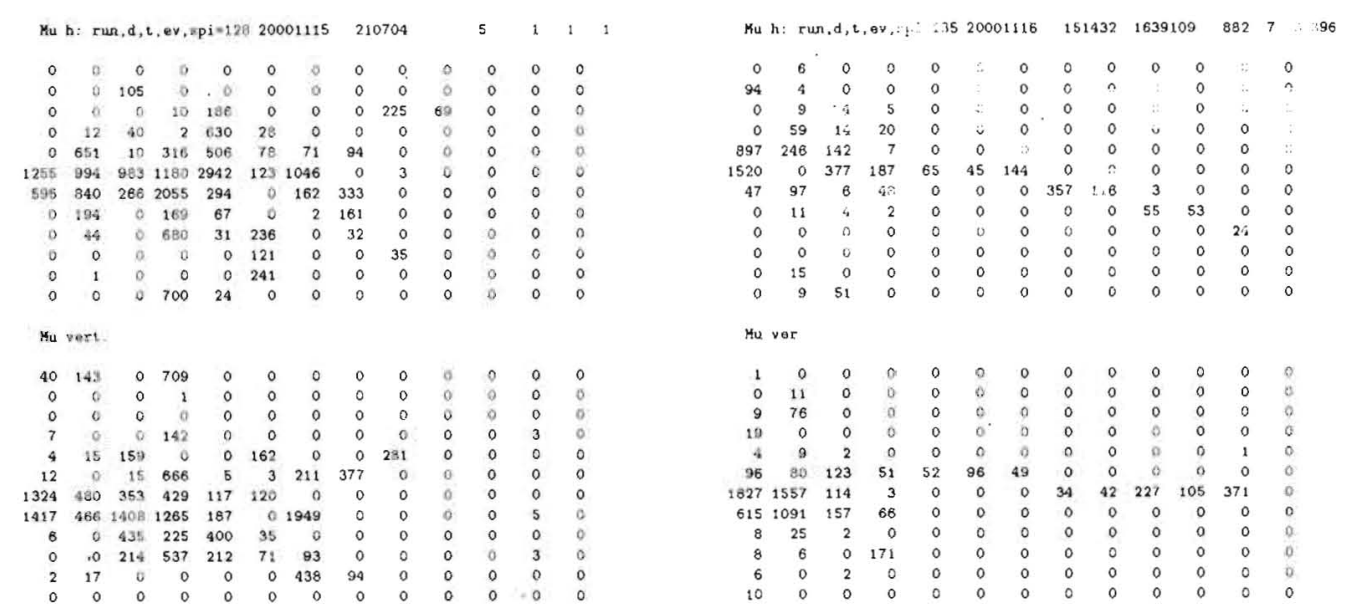

Figure 134: $\mathrm{x}$ (top) and y view of $25 \mathrm{GeV} \pi$ event (left) and muon event(right) in the MVS

- n1- number of planes (out of 26) with just one hit above some threshold (20 ADC counts).

- n3- number of planes with more than 2 hits above the threshold. 
The distributions over these variables are presented - for the real data and Monte Carlo GEANT simulation in Figures 135 and 136.
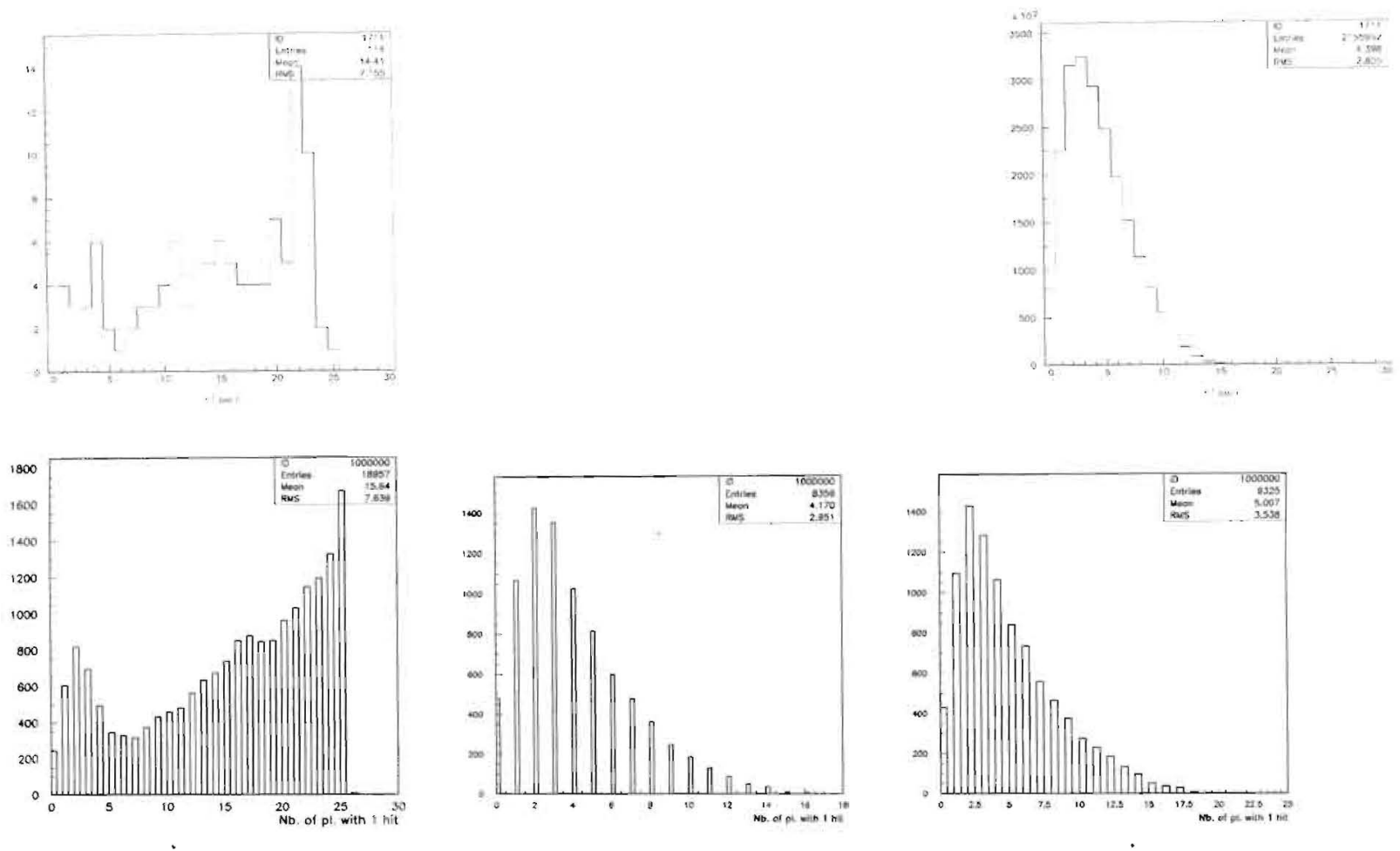

Figure 135: The n1 distributions: (above) for the low intensity muon (left) and pion data(right)// (below) GEANT Monte Carlo:muons(left), $25 \mathrm{GeV}$ pions(center), $12 \mathrm{GeV}$ pions(right)

The following cuts were selected to suppress muons: $n 1<9 ; n 3>3$. After these cuts, the remaining muon background mainly consists of events where muon decays in flight to $e \nu \bar{\nu}$. The decay may happen before the calorimeter or inside the calorimeter (see Figure 137 ). It leads to a narrow (across the beam) and relatively short along the beam) electromagnetic shower in the MVS.

To control this type of background we introduced variables $w_{t}\left(w_{l}\right)$ which give an estimate for the transversal(longitudinal) width of the shower in MVS. The following cuts were selected to suppress muons: $w_{t}>.55 ; w_{l}>1.9 \vee w_{t}>1$. The second cut is mainly against "early" muon decays before the calorimeter. As it is seen from the figures, there is a good agreement between data and $\mathrm{MC}$ for the muon data. There is a significant discrepancy for the $w_{t}$ hadron distribution. As a result, a cut of $w_{t}>.8$ is removing almost all the muon background without touching pions for the Monte Carlo case, but kills many pions in the data. That's why a cut of $w_{t}>.55$ is selected. The difference can be partially explained by somewhat different algorithms of $w_{t}$ calculations in Monte Carlo and $"$ the data. We'll certainly improve the situation in the nearest future.

\subsubsection{The results}

Table 32 shows the data reduction when applying the sequential cuts described in the previous section.

For the high intensity run (second column) the efficiency is corrected for the $\mathrm{V}$ counter which was in the trigger. In other cases the efficiency calculation is straightforward. For the $\pi$ run, the 

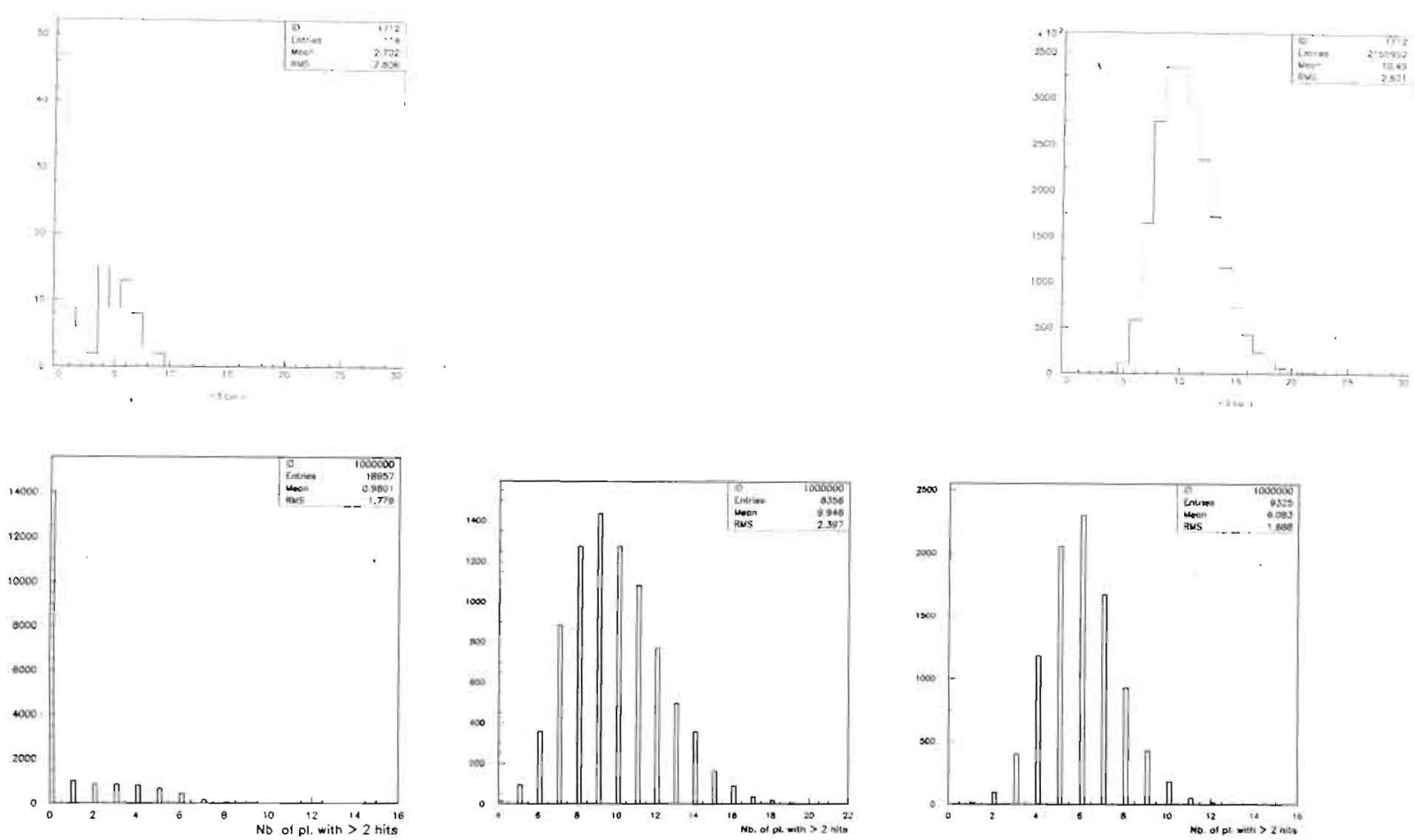

Figure 136: The $n 3$ distributions: (above) for the low intensity muon (left) and pion data(right)// (below) GEANT Monte Carlo:muons(left), $25 \mathrm{GeV}$ pions(center), $12 \mathrm{GeV}$ pions(right)
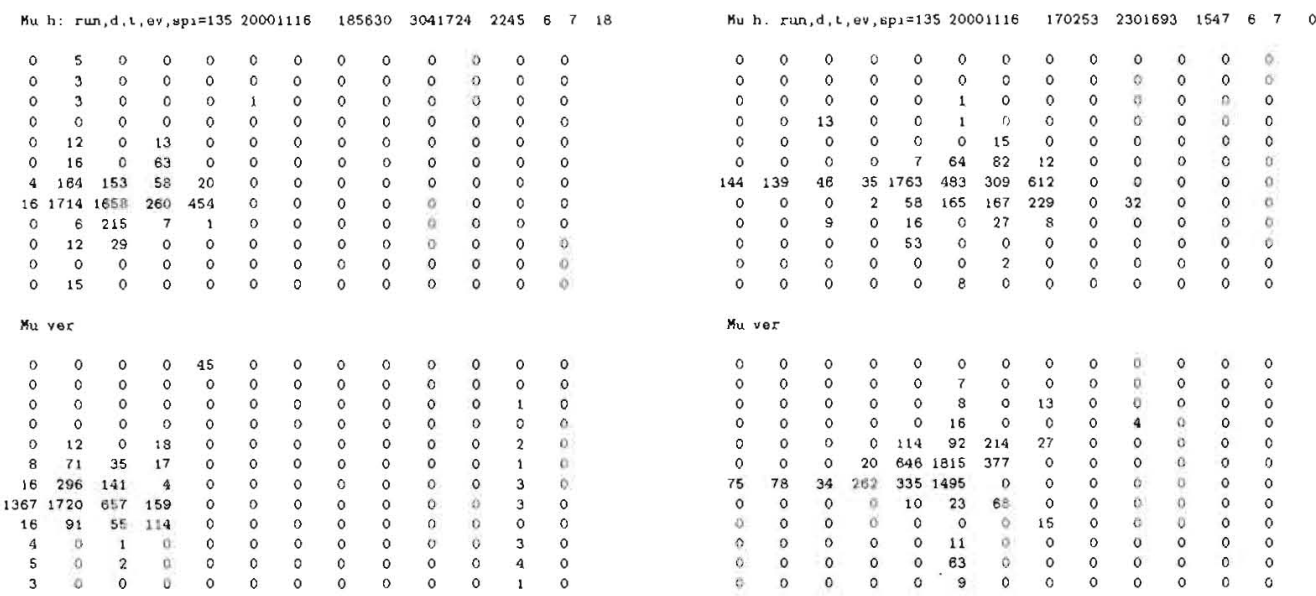

Figure 137: $\mathrm{x}$ and $\mathrm{y}$ view of the e.m. shower caused by $\mu \rightarrow e \nu \bar{\nu}$ decay before the calorimeter(left) or inside.(low intensity muon run) 


\begin{tabular}{|c|c|c|c|c|}
\hline Run & high int. $\mu$ & low int. $\mu$ & low int. $\mu$, no LG & $\pi^{-}$ \\
\hline$N_{\text {read }}$ & 1595894 & 2654965 & 1065118 & 3139913 \\
\hline Beam PC cuts & 309315 & 1480664 & 624489 & $2366359(-65448)$ \\
\hline LG cuts & 160474 & 1327330 & & \\
\hline Veto $<15$ & 1799 & 114 & 176 & 2155952 \\
\hline$n_{1}<\overline{9}$ & 1048 & 26 & 144 & 1951864 \\
\hline$n_{3}>3$ & 540 & 18 & 55 & 1951759 \\
\hline$w_{t}>.55$ & 265 & $\frac{8}{4}$ & 18 & 1839404 \\
\hline$w_{1}>1.9 \vee w_{t}>1$ & 146 & $3.6 \cdot 10^{-6}$ & $75.3 \%$ \\
\hline efficiency & $3.8 \cdot 10^{-6}$ & $3 \cdot 10^{-6}$ & $9.6 \cdot 173083$ \\
\hline
\end{tabular}

Table 32: The details of the data reduction

presence of the muon admixture in the beam was taken into account by subtracting 65448 events from 2366359 events left after the beam PC cuts. This correction was determined from the total energy spectrum of Figure 133, where the muon signal is well seen. The muon suppression for the low intensity run without Lead Glass counters in the beam is significantly worse than that for the other runs because of the obvious reason: many more muons decay to $e \nu \bar{\nu}$ in front of the calorimeter. The effective muon decay length for the high intensity run was (see Figure132 about $2.5 \mathrm{~m}$, and $1.3 \mathrm{~m}$ for the low intensity run with LG7 in the beam. Instead, it was about $15 \mathrm{~m}$ for the run without LG.

The analogous information for the Monte Carlo GEANT data is presented in Table 33

\begin{tabular}{|c|c|c|c|}
\hline Run & $\mu$ & $\pi 25 \mathrm{GeV}$ & $\pi 12 \mathrm{GeV}$ \\
\hline$N_{\text {read }}$ & $2.7 \times 10^{7}$ & $10^{4}$ & $10^{4}$ \\
\hline Veto & 18957 & 8356 & 9325 \\
\hline$n_{1}<9$ & 4219 & 7600 & 7899 \\
\hline$n_{3}>3$ & 986 & 7600 & 7510 \\
\hline$w_{t}>.8$ & 57 & 7068 & 6790 \\
\hline efficiency & $2 . \cdot 10^{-6}$ & $70 \%$ & $68 \%$ \\
\hline
\end{tabular}

Table 33: The details of the Monte Carlo GEANT data reduction

As discussed in the previous section, the $w_{t}$ distributions are somewhat different for the $\pi$ data and Monte Carlo, that is why the cuts are different for the Monte Carlo case. The effect of the Veto counter on the pions is stronger in Monte Carlo, probably because the response of the V-counter is not reproduced in details (light attenuation etc.).

\subsubsection{Further tests}

The Muon Veto System of CKM must have the same structure as in prototype but with different dimensions: 26 planes with 60 counters/plane; the dimensions of the counter $240 \times 4 \times 1 \mathrm{~cm}^{3}$. We have studied two options for the construction of scintillation counters for MVS (with slightly different geometry of counters in these preliminary tests).

- Plastic scintillator sheet with BCF92 fibers light collecting system. For purpose of the study the following materials were used: 
1. PS's scintillator sheet $\left(200 \times 40 \times 10 \mathrm{~mm}^{3}\right)$ - assembled from 4 different scintillator plates

2. wave shifting fiber - BCF92 $(1 \mathrm{~mm})$

3. phototube - FEU85

Edge surfaces of the scintillator sheet were covered with Mylar. WLS fiber was installed into 3 grooves (1 mm deep) in the scintillator sheet. The fanout end of the WLS fibers had $4 \mathrm{~cm}$ diameter loop. A prototype scintillator was tested with $p_{\mu}=5 \mathrm{GeV}$ muons triggered using a separate scintillator counters. More than 12 photo-electrons were observed with a time resolution of 2 nsec.

- Plastic scintillator with direct collection of light by a phototube. These tests were done on muons for 15 scintillators with dimensions $220 \times 2.5 \times 1 \mathrm{~cm}^{2}$. This is another type of scintillator also produced in IHEP (Protvino) by extrusion method and characterized of better transparency (light attenuation length is $\lambda \simeq 120 \mathrm{~cm}$ ). The $25-45$ photoelectrons were obtained from the remote end of scintillator. In the measurements with 2 phototubes (FEU85) at different ends of scintillator the time resolution was $\tau=1.5 \mathrm{nsec}$

Both options look quite acceptable for MVS production. All scintillator slabs were produced in IHEP 6 years ago which means that aging effect for them is not very significant.

\subsubsection{Conclusions}

The concept of the muon suppression with a finely segmented calorimeter is demonstrated by the present experimental results. We have achieved rejection of muons at the level of $\sim 3 \times 10^{-6}$ The structure of the calorimeter (sampling, scintillator strip width etc.) appear adequate. The test shows the importance of the e.m.-hadron shower separation power of the calorimeter. The low pion efficiency $\sim 70 \%$ is, in part due to the cut on the amplitude of the Veto counter, which is located after the calorimeter. This inefficiency can be easily removed by increase of the length of . the calorimeter. Then, for $25 \mathrm{GeV}$, the pion efficiency would become $83 \%$.

A scan of the muon events which survived all the cuts shows that there are still some events (3-muon tracks, for example) which can be rejected with simple additional cuts. Our estimate is that the rejection can be improved by a factor of 2 . There are some other factors, which should lead to the stronger muon suppression in the CKM: the distance between the LG-counter and the front face of the calorimeter was(minimum) $1.3 \mathrm{~m}$ in the tests. This distance is important for the muon-decay background and will be less in the CKM apparatus. We did not have a precise muon momentum measurement during the tests. In CKM, momentum-dependent cuts could be used. Finally, it was shown in a GEANT Monte-Carlo simulation that replacing the $4 \mathrm{~cm}$ thick iron absorbers in the MVS with $4 \mathrm{~cm}$ lead absorbers and using a set of fully optimized selection cuts for this configuration can increase rejection power of MVS by factor of more than a factor of $\times 4$. This enhanced rejection results from better identification of single-brem and multi-brem events.

All these additional factors together with the increased the length of calorimeter not only increase the rejection power of the Muon Veto System but open possibility for more flexible adjustment and reshaping of muon and pion separation criteria to increase the efficiency of pion registration (to be no less than $95 \%$ with muon suppression factor $>10^{5}$ ).

\subsection{The Conversion Veto Plane}

The beam transport downstream of the pion RICH and through the FVS/MVS is inside a 10 meter long atmospheric helium bag which terminates on a. $20 \mathrm{~cm} \times 20 \mathrm{~cm}$ Conversion Veto Plane (CVP) composed of scintillating fibers. 
This veto plane is crucial to veto of photons that have converted in the upstream pion RICH material, as well as tagging beam particles. This system must have excellent time resolution $( \pm 1 \mathrm{nsec})$, and have a charged particle efficiency of at least $99.0 \%$. In order to ensure excellent time resolution and high efficiency, relatively thick and bright D0 fibers ( $830 \mu \mathrm{m}, 12$ p.e./mip) will compose the CVP plane. The CVP system is readout with multi-anode PMTs instrumented with the pipeline TDC described in section 6.2.2. Immediately downstream of the CVP the beam is kicked by a Fermilab BM-109 magnet ( $1 \mathrm{GeV} p_{\perp}$-kick) to the charged beam dump 20 meters further downstream. Photons that remain in the upstream beam acceptance are intercepted by the Hole Veto System (HVS) which must provide a photon inefficiency performance listed in table 11.

\subsection{The Beam Dump}

In order to minimize back splash and the ambient neutron gas level, the charged kaon beam must terminate in a borated reentrant cave 20 meters downstream of the Hole Veto System. 


\section{The Trigger and Data Acquisition Systems}

\subsection{Experiment Requirements on DAQ and Trigger Electronics}

The electronics for CKM is used to record the signals generated by the detector. One of the key elements of CKM is the Photon Veto System, needed to reject the $K^{+} \rightarrow \pi^{+} \pi^{0}$ background, in order to make a measurement of the branching ratio $K^{+} \rightarrow \pi^{+} \nu \bar{\nu}$ on the order of $10^{-10}$. The efficiency of the Photon Veto System is crucial for the background rejection and therefore, the success of the CKM experiment. The electronics is required to faithfully record the detector signals which are needed for background rejection.

Deadtime - the inability of the electronics to record information from the detector - is a potential source of un-rejectable background. There is deadtime introduced in a channel for some period of time after it receives a signal. A detector element and its DAQ channel are temporarily dead after being hit by a particle. The channel recovers back after certain amount of time. During the recovery time, the channel functions with reduced performance. A very concerned example in CKM is that the previously hit channels in the Photon Veto. System could fail to detect the photons in the $K^{+} \rightarrow \pi^{+} \pi^{0}$ events and cause the events to have the same apparency as $K^{+} \rightarrow \pi^{+} \nu \bar{\nu}$ events. So the foremost requirement of the CKM DAQ and trigger electronics is that it must not miss any information that is needed to reject background. A coherent unrecorded deadtime of the electronics must be below the level of one part in $10^{7}$.

During the time period of about $10 \mathrm{nsec}$ around a hit in the veto system, the veto system is considered not suitable for studying next decay. The time period thus becomes a deadtime of the entire detector, which is about $15 \%$ of data taking time when the detector is operating at full rate. The DAQ and trigger system should not add additional deadtime on top of this. A possible source of deadtime can be caused by the system being unable to accept an event because all event buffers are full. The architecture of the DAQ and Trigger system must minimize the amount of deadtime. In situations where deadtime becomes unavoidable, the DAQ and trigger system has to record the source and length of deadtime.

The requirements on the electronics must come from and be compatible with the goals of the experiment. The photon veto system requires that the electronics system have a reliability that is greater than in may other experiments. There is a requirement on both single channel reliability and system reliability. Reliability issues must be brought in at all stages of the electronics architecture, design, fabrication and testing. This could be done, for example, by following the rules and procedures for building electronics for satellites. However, there is a competing requirement that can make doing this impossible - the cost of the electronics must be reasonable.

The electronics system must have adequate bandwidth to record the $50 \mathrm{MHz}$ beam rate and accept events from the level one trigger at $220 \mathrm{kHz}$.

The signals from the detector elements in CKM fall in one of three categories - signals requiring only a time stamp or TDC information, signals requiring both a time stamp and amplitude information, TDC and ADC, and signals requiring monitoring such as power supplies.

Signals from the veto systems, which require inefficiencies as small as time (TDC) and charge (ADC) information to minimize electronic inefficiencies. Since inefficiencies of the order of $10^{-2}$ can be tolerated in the tracking and RICH detectors only time (TDC) information is recorded for these systems.

As shown in Table 34 there are about 27,500 detector channels together with about 100 control and timing signals requiring TDC information alone and about 6000 detector channels requiring both TDC's and ADC's .

Instantaneous rates in the detector will also require online monitoring. While it is possible 
Table 34: CKM Detector Electronics Channel Summary: In this table, we have assumed that each TDC card or TDC+ADC card hosts 16 or 8 channels, respectively.

\begin{tabular}{|c|c|c|c|c|}
\hline Detector & $\begin{array}{r}\text { total } \\
\mathrm{ch}\end{array}$ & $\begin{array}{l}\mathrm{TDC} \\
\text { cards }\end{array}$ & $\begin{array}{r}\text { TDC \& ADC } \\
\text { cards }\end{array}$ & Notes \\
\hline$\overline{\mathrm{BTSM}}$ & 512 & $\overline{32}$ & - & TDC \\
\hline UMS & 12888 & 810 & - & TDC \\
\hline KRICH & $6 \overline{600}$ & 38 & - & $\overline{T D C}$ \\
\hline$\overline{\mathrm{K}} \overline{\mathrm{EAT}}$ & 4296 & 270 & - & TDC \\
\hline$\overline{B I V S}$ & 240 & - & 30 & $\mathrm{TDC}+\mathrm{ADC}$ \\
\hline VVS & 2176 & - & 272 & $\overline{\mathrm{TDC}}+\mathrm{ADC}$ \\
\hline $\mathrm{DMS}$ & $46 \overline{40}$ & 290 & - & $\mathrm{TDC}$ \\
\hline$\overline{\mathrm{ETP}}$ & 800 & 52 & - & $\mathrm{TDC}$ \\
\hline PRICH & 3000 & 188 & - & $\overline{\mathrm{TDC}}$ \\
\hline$\overline{\mathrm{FVS}}$ & $17 \overline{97}$ & - & 225 & $\overline{\mathrm{TDC}}+\overline{\mathrm{ADC}}$ \\
\hline CVP & 800 & 52 & & TDC \\
\hline MVS & 1920 & - & 240 & $\overrightarrow{\mathrm{TDC}}+\mathrm{ADC}$ \\
\hline $\mathrm{HVS}$ & 25 & - & 4 & $\mathrm{TDC}+\mathrm{ADC}$ \\
\hline Total & $\begin{array}{r}27536 \\
6158 \\
33694 \\
\end{array}$ & 1732 & 771 & $\begin{array}{r}\mathrm{TDC} \\
\mathrm{TDC}+\mathrm{ADC}\end{array}$ \\
\hline
\end{tabular}

that instantaneous rates can be extracted from event data it will be more straightforward to have selected signal rates recorded directly in scaler modules. The exact number of scaler channels has not yet been determined, but it is expected that on the order of 1000 signals will be rate monitored.

We expect there will be about 1000 signals from power supplies and fan units that will require monitoring. The electronics for monitoring the detector will be separate from the data acquisition system because the speed with which monitoring data needs to be collected is so much slower. It is quite likely that commercial process monitoring system, such as IFIX will fill this role.

The CKM experiment has imposed unique challenges on its DAQ and trigger system. However, we are convinced that the system can be implemented using existing electronics technology to fulfill the experiment requirements.

\subsection{Architecture of CKM DAQ and Trigger System}

The following methodologies are employed in the architecture to fulfill the experiment requirements without confronting technical challenges.

- Continuous digitization near the detector elements is utilized to preserve the data quality.

- The concept of "minimum synchronization" (see below) is adopted to eliminate the needs of time matching signal paths.

- A global level 1 trigger performs coincident and veto functions using digital timing information to avoid problems due to signal degradation in long cables.

- The DAQ logic allows to collect chronicle records around the interesting events with different levels of detail of ADC information, so that very low energy particles in the veto systems can 
be best detected.

- In addition to the level 1 and possible level 2 triggers, which make trigger decisions based on partial detector data, a re-programmable hardware trigger (L2.5) and a software trigger (L3) sitting in the DAQ stream are planned to perform data selection functions based on full detector data and are adjustable while needed.

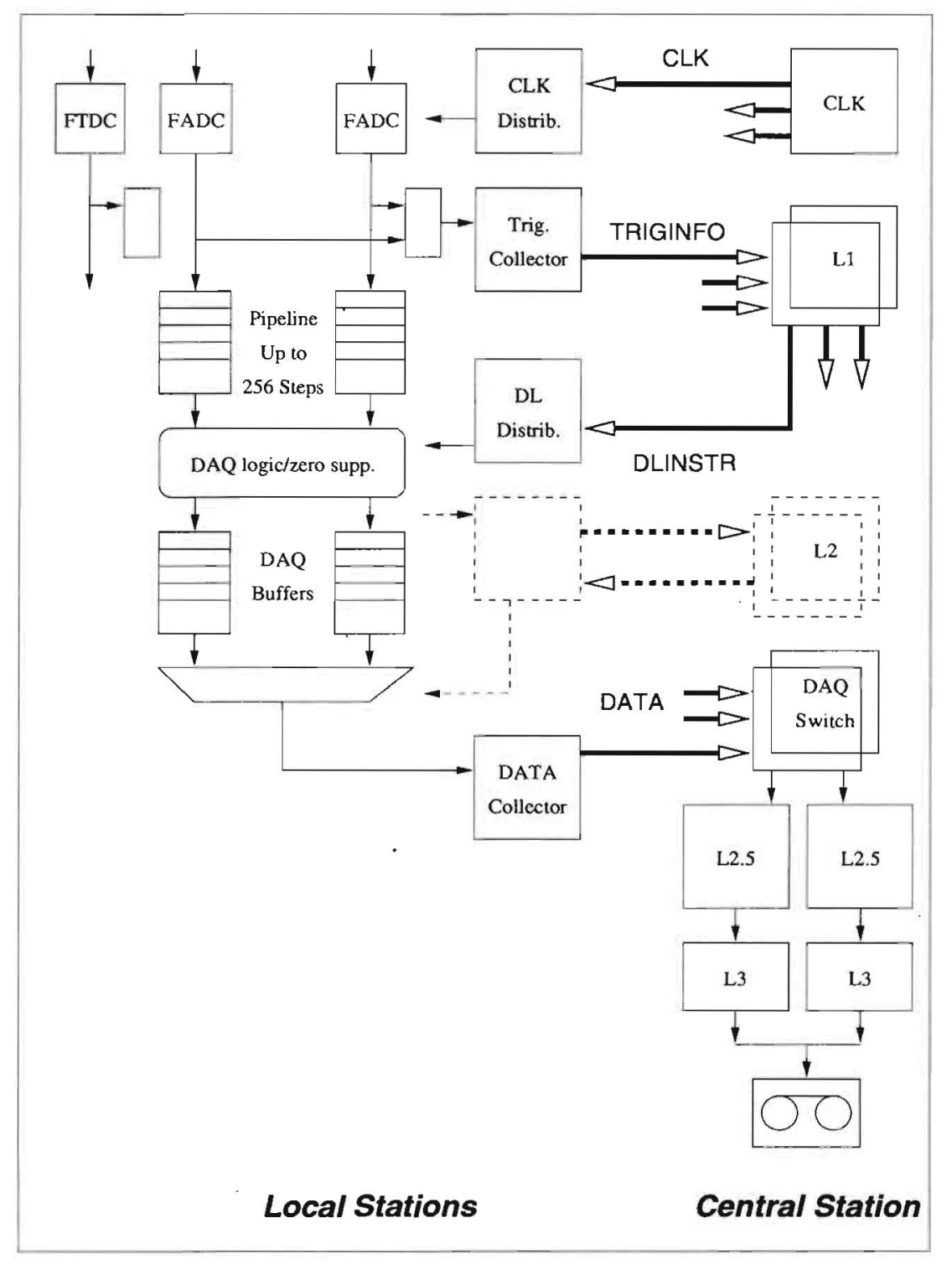

Figure 138: Block Diagram of CKM DAQ and Trigger System

The requirements for the CKM electronics - deadtime-less, high reliability, and high rate - have lead to the following overall architecture for the experiment, see Figure 138. The first step is the digitization of signals from the detector. This digitization will be continuous for both TDC and ADC information. The pulse height information will be recorded much as it would be recorded in a digital oscilloscope. A common way of recording pulse height information is to use a gated integrator feeding an ADC, in which the gate is initiated by either level 1 trigger or accelerator RF 
signal. This method results in a single digitization for a pulse. There are two reasons for not using this method in CKM. The first is that CKM uses un-bunched beams and events can come at any time. The second reason comes from needing to understand the deadtime of each channel. A large energy deposit in one section of the photon veto detector can make that part of the photon veto insensitive to small energy deposits for a short period of time. The way to best understand this is to have a record of the energy deposits in the detector for a period of time leading up to the event.

During the deadtime for single channels in the photon veto system, a $K^{+} \rightarrow \pi^{+} \pi^{0}$ may escape being vetoed. It is critical that single channel deadtime - when it occurs - not escape detection. This is accomplished by encoding the digitized signal around the period of time of the candidate $K^{+} \rightarrow \pi^{+} \nu \bar{\nu}$ decay. This leads to the definition of a CKM "event". It is not a "snap shot" of the detector but a time interval on the order of several hundred nsecaround the candidate $K^{+} \rightarrow \pi^{+} \nu \bar{\nu}$ decay.

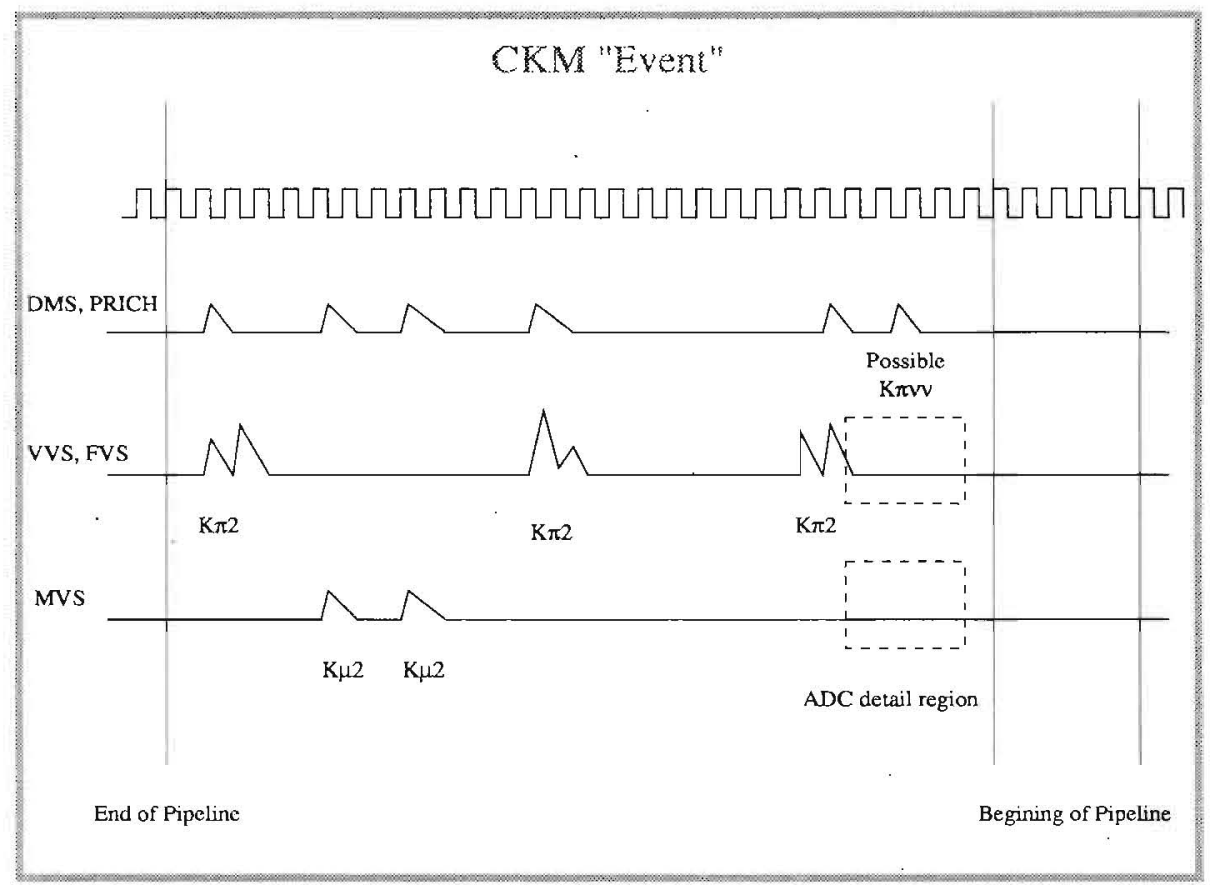

Figure 139: CKM "Event"

This concept is shown in Figure 139. The event looks similar to an oscilloscope recording of all of the data. It includes not only the candidate decay, it is a short chronicle record of the detector.

We now discuss the methods of realize this requirement. Clearly, in Figure 138, there must be a stable, known and common time reference at each local stations. The time reference is distributed from the central station via the CLK link. The planned TDC for CKM will have a LSB time bin unit, of about 1 nsec. Therefore, the relative timing reference over the entire system should have a long term correctable stability of about 0.5 nseclevel. The short term jitter of the timing signal should be less than 0.25 nsec. However, long time scale drift of the timing reference is not a constraint as long as the drift is identical throughout the system. The timing reference can be distributed via high quality media contained in a temperature controlled enclosure or liquid bath. The timing variation can also be compensated using the multi-point interval-halving technique [189] or other techniques. In addition to the compensation, the variation of the timing signal delay will also be recorded so that off line corrections based on better models and/or decay data can be done. 
The CKM electronics employs the "minimum synchronization" concept. This means that only one time reference is distributed in the system. Time stamps are kept in the local stations by counting the time reference signals. The reset and check signals for the time stamps are distributed via the same cable for time reference. All the other commands such as triggers are separately digitally issued in advance of the execution time and they are executed when the local time stamp counter matches the execution time.

In the local stations, the detector signals are continuously digitized and stored in a pipeline buffer - the level 1 FIFO. Partial of the digitized data are branched to the level 1 trigger system. The local trigger information is sent to the central station through the TRIGINFO link.

The level 1 trigger subsystem (L1) in the central station makes event selection decisions based on the trigger information from all the local stations. When a candidate decay is detected by the level 1 trigger, a down load instruction will be sent to the local stations via the DLINSTR link.

In the local stations, the download instruction will be sent to the DAQ logic requesting that the TDC and ADC data be copied into the DAQ buffer. During this operation, the ADC data of the veto systems will be collected in two different fashions. In most portion of the time interval, zero-suppression will be applied to the ADC (and TDC) data to reduce the data volume. While near the candidate $K^{+} \rightarrow \pi^{+} \nu \bar{\nu}$ event, ADC data without zero-suppression of all channels will be recorded to catch any possible very low energy particles. The data stored in the DAQ buffer are then collected and sent to the central station via the DATA link.

Although we believe it may not be needed, it is still possible to implement a level 2 (L2) trigger in this architecture (shown in dash line). Partial data can be branched to the level 2 trigger system when the data is copied to the DAQ buffer. The event data are then stored in the DAQ buffer pending a level 2 trigger decision.

The data of same event from different local stations are place together by the DAQ switch in the central station.

With the full data, further event selection algorithm can be applied in the L2.5 trigger system. The L2.5 is a collection of FPGA based trigger modules. The logic in these devices can be reprogrammed while the modules are installed in the system. In the L2.5 system here, data of all detector channels are available for the event selection. This allows the maximum flexibility of trigger adjustment in the hardware trigger stage.

Each L2.5 module sends out the selected events into a machine in a PC farm. The PC farm makes the level 3 (L3) event selection. Again, this trigger stage sees full data. The PC farm allows experimenters to run more complicate event selection algorithm which L2.5 can not run in a hurry. The data selected by the PC farm are then recorded in the massive storage media.

The DAQ system must not create significant deadtime. This means that at all levels of data buffering and recording events can be written and read simultaneously. Of course in any system there is a finite bandwidth capability and an attempt to exceed that bandwidth will cause deadtime. Therefore, both data rate and buffer lergth must be studied carefully. We have studied the data volume using GEANT simulation and we are convinced that the data rate is much lower than the planned DAQ system capability.

In case the DAQ system deadtime occurs, it is important that the amount of deadtime be accurately recorded, and the source of the deadtime be noted. Deadtime for the entire detector times when there is beam going through the detector but the electronics is unable to respond to signals from the detector - enter only in first order to reducing the total number of event observed. 


\subsubsection{Front End}

The 22,000 wires of the UMS, KEAT and DMS will be read out with chamber-mounted amplifiers and discriminators. The requirements for the system are

- the minimum threshold should be in the range of 2 to 3 femtocoulombs,

- the double pulse resolution should be less than 25 nanoseconds and

- the long ion tail component of the signal should be suppressed.

An ASIC meeting the CKM wire chamber requirements is the ASDQ that was developed for the readout of the CDF Run II central wire chamber. It contains eight channels of preamplifier, shaper, baseline restorer and discriminator in a 64-pin QFP. The discriminator has two modes, either simple time-over-threshold or a $\mathrm{dE} / \mathrm{dx}$ mode that would be disabled for use in CKM. Experience with an earlier ASD version has shown some sensitivity to system layout. Therefore it is desirable to test a fully instrumented chamber to verify that the required sensitivity can be achieved in stable operating conditions. As a back-up solution we will assume the use of the HyperCP amplifier and discriminator for the UMS and the BNL787 amplifier/discriminator for the DMS. The HyperCP electronics satisfy the noise and shaping requirements of the UMS however the double pulse resolution is about a factor of two worse. The BNL787 straw tube electronics lacks the desired sensitivity.

The 3,600 RICH PMT signals will also need local amplification and discrimination before being passed to TDC's. The typical single photoelectron signal has $10 \mu \mathrm{A}$ peak amplitude and $10 \mathrm{nsec}$ full width. The above-mentioned ASDQ might be usable here as well, although the tail cancellation filter would produce overshoot for a signal having a typical PMT pulse shape. At this moment it is unclear whether the baseline restorer could compensate for this undesirable effect. A fall back option is to build an amplifier/discriminator from commercially available transimpedance amplifiers designed for the fiber communications industry (e.g. AD815, SA5212, LM359) and comparators (e.g. AD96687, SPT9687, MAX9687).

The veto systems will employ photomultiplier tubes having reasonable gain so that only modest $(\sim 10 \times)$ electronic amplification is needed prior to conveying the signals through short cable rurs to ADCs and discriminators for TDCs. To avoid electronic inefficiency the discriminator design

must be optimized for double pulse sensitivity. Whatever electronic inefficiency remains will be reduced (at the cost of a wider veto time interval) by the continuously sampling ADC described below.

\subsubsection{TDC}

The final set of TDC requirements will be set in the Technical Design Report. The expected TDC requirements, based on our experience, are:

- Data will be digitized in 0.8 to 1.2 nanosecond time bins.

- The TDC's must run continuously storing hit times in a Level 1 buffer. It should also be able to tap this data out for the level 1 trigger.

- The TDC's must have an inefficiency in recording pulses of less than one part in $10^{7}$, regardless of the instantaneous data rate. 
- The TDC's must be able to transfer data out of the level 1 buffer while simultaneously recording new data.

- The double pulse resolution is determined by the response time of the fastest detector, and should be better than 10 nanoseconds.

While there are not any TDC devices currently available, which meet all of these criteria, we believe that such TDC devices are obtainable. The TMC TDC pipeline readout chip has been under development for more than ten years now, and is quite mature. It will be employed in the D0 muon system, and much of the ATLAS tracking system as the AMT1. The system is genuinely deadtimeless, with a base clock of $40 \mathrm{MHz}$ ( $\mathrm{LHC}$ crossing rate). Upon readout the TMC provides an ordinal number for the base clock period and a 5-bit period vernier corresponding to a least count of 0.8 nsec, with a measured $\sigma$ of 250 picoseconds. The chip has a pipeline depth of 4 microseconds, which is adequate for our application. A relevant limitation of this readout system is that the Double Pulse Resolution (DPR) is limited to the base clock period of 25 nanoseconds. That is, a hit trailing a previous one by less than 25 nanoseconds is lost without warning. The impact of this deadtime is currently under study. For veto systems that are particularly vulnerable to deadtime effects the charge pulse will be digitized with the QIE system described below as well as the TMC. The deadtimeless charge profile accessible with the QIE system should enable recovery of the TMC double-pulse deadtime loss. Another limitation with the TMC has to do with the method of internal buffering. If more hits are detected than carn be stored in internal buffers then the extra hits are discarded. For most tracking chambers this is not a severe problem, but this can be a problem with recording hits from the photon veto system.

A second candidate device is the JMC96 developed at the University of Michigan. This full custom device meets all of the basic requirements of the design. However, this is a custom chip that was built the HP 0.5 micron process and a new version of the chip would have to be made using a more modern fabrication process. This would require some modifications to the chip design and at least one prototyping cycle.

A third alternative is the TDC chip developed for the COMPASS experinient. It is designed to handle rates of about $1 \mathrm{MHz}$ per channel. Since it employs CMOS Sea-of-Gates technology it may be more readily available than full-custom chips such as the JMC96. This TDC provides 8 channels of time measurement with $100 \mathrm{psec}$ resolution and two levels of data buffering. Due to its data-driven architecture the channel density is higher than the TMC chip however it is limited in its data storage capacity. Within a chip, no more than 7 hits associated with a trigger can be stored. In addition no more than 16 hits in a channel can be stored during the time interval needed for matching hits to a trigger, which varies from 200 to about 700 nsec. Finally as with the TMC chip the double pulse resolution is about $25 \mathrm{nsec}$. Further study will be needed to determine whether the data storage limitations would be acceptable for CKM.

A fourth alternative is to implement the TDC in a commercial FPGA. This approach was first attempted by John Mann, the engineer at the University of Michigan who designed the JMC96 chip. The basic idea is to string together buffers (to first order NOT voltage-controlled, rather the standard buffer of one Xilinx CLB) just like the JMC96 chip. In this implementation two chains of logic blocks are constructed by explicit routing of the clock signals. One chain is used to record a fixed frequency clock and is used to calibrate the delay time between cells. The other chain is used to record the arrival time of the data. The delay cliains are copied to memory once per clock cycle and the bit pattern is then encoded into hit times. Such an implementation has many advantages, but it must be proven to meet all of the requirements. 


\subsubsection{ADC}

A continuously sampling ADC system together with the TDC can provide a powerful digitization of each veto channel, and would likely address any potential double-pulse inefficiency concerns of the TDC. The expected ADC requirements are:

- A dynamic range of 4-8 GeV with $0.25 \mathrm{MeV}$ least count (14-bits).

- Digitization at $100 \mathrm{MHz}$.

- The ADC must run continuously storing amplitude information in a level 1 buffer. It should also be able to tap this data out, for the level 1 trigger.

- The ADC must be able to transfer data out of the level 1 buffer while simultaneously recording new data.

This level of performance can be achieved using the "floating point" Charge Integrating Encoder (QIE) technique developed by the KTeV experiment. In the QIE the input signal is integrated on several non-overlapping ranges and continuously over successive periods of the system clock. Since all operations are pipelined there is no signal deadtime.

The current version of the QIE ASIC is being applied to the CMS hadron calorimeter (HCAL) readout. The CMS QIE chip operates at $40 \mathrm{MHz}$ providing a 14 bit dynamic range as 5 bits of mantissa and 3 range bits from the on-chip FADC for each $25 n s e c$ sample. This system has demonstrated very low noise performance in the field, so a low meaningful threshold can be imposed on the CKM veto and calorimeter systems.

In contrast to the CMS environment, signals in CKM have no synchronous relation to the system clock. Measurements on prototype chips have shown less $1 \%$ integrated charge errors for various phased signals, allowing the deposited charge to be recovered from a straight-forward sum of two contiguous samples. In CKM the required least significant count is $20 \mathrm{fC}$, about 40 times larger than for the CMS HCAL. This has allowed the designer to find modifications of the chip which, according to SPICE simulations, will allow it to run at $100 \mathrm{MHz}$ with less than $2 \%$ random phase integrated charge errors. If this performance fails to be realized a back-up solution to achieve $100 \mathrm{MHz}$ operation would be to integrate two QIE circuits in one package and clocking each circuit with opposite phases of the system clock.

Over the past few years 14-bit pipelined A/D converter chips with sampling rates greater than $50 \mathrm{MHz}$ have become commercially available, for example AD6644, CLC5958. A back-up replacement for the QIE would be a single-range integrator followed by one or two such converters. We have preliminary designs for two integrators; one composed of a two-phase integrator similar to that in the QIE and another composed of a continuous integrator with correlated double sampling. Both designs use commercially available discrete parts. They will be pursued further only if evidence develops that the neither of the QIE based solutions will meet our requirements or schedule.

\subsection{The Trigger System}

The trigger system for CKM experiment must meet the following requirements:

- It must catch $K^{+} \rightarrow \pi^{+} \nu \bar{\nu}$ events with good efficiency. The branching ration of $K^{+} \rightarrow \pi^{+} \nu \bar{\nu}$ is low (order of $10^{-10}$ ) and the acceptance of clean signal region is sniall (about $2 \%$ ). Therefore the event rate is low (about 1/week). Any additional 1\% inefficiency caused by trigger or any otler systems will cost about one week extra run time. 
- The DAQ system will collect detector hit history information before the candidate $K^{+} \rightarrow \pi^{+} \nu \bar{\nu}$ events. The trigger system must be able to handle this kind of readout sequences.

- In order to calculate branching ratio of $K^{+} \rightarrow \pi^{+} \nu \bar{\nu}$ events, one must record information about other kaon decay modes. Therefore, the trigger must be able to identified additional kaon decay modes.

- The event accept rate and rejection ratio at each level of the trigger needs to be consistent with the bandwidth of the data acquisition system and with the information available to the trigger system.

- The trigger system should have enough flexibility so that the trigger conditions can be easily improved based on early experiment data.

- The data used by the trigger to make a decision, and the result of the trigger decision including intermediate calculations will be included in the event record.

These are unique challenges but we believe that a trigger system meeting all these requirements can be implemented using existing technologies.

\subsubsection{The Level 1 Trigger}

The Level 1 trigger can only make decisions based on information it receives from the detector, and obviously better decisions can be made with better or more complete data. This is balanced by the cost needed to implement the data transmission and the time needed to process the data. The starting point for understanding the correct balance is to describe the signature required for triggering on events and signals that could be used to reject background.

A valid $K^{+} \rightarrow \pi^{+} \nu \bar{\nu}$ will have a kaon entering the detector, a pion exiting the detector, and an absence of photons or muons. The kaon entering the detector will be identified by the KRICH. The full identification of the kaon will be based on reconstructing a ring and measuring the radius. This reconstruction is probably not achievable in level 1 , so a much simpler requirement, called $\operatorname{KRICH}\left(K^{+}\right)$, that at least six phototubes from the inner kaon ring are hit will be used. The KRICH has an outer ring of phototubes that detect the ring from the $\pi^{+}$track. Signals from these phototubes would be used to veto an event; again the requirement will be met by looking for six phototubes having signals, independent of their location. This signal will be $\operatorname{KRICH}\left(\pi^{+}\right)$.

The $\pi^{+}$can similarly be identified in the PRICH. Again a full identification of the pion will involve reconstructing a ring, but the signature used in the level 1 trigger will just be that at least eight phototubes have signals, denoted as $\operatorname{PRICH}\left(\pi^{+}\right)$.

The largest decay mode producing a $\pi^{+}$is $K^{+} \rightarrow \pi^{+} \pi^{0}$ and the level 1 trigger must reject a large fraction of these events. The signal from the photon veto will be used to eliminate events where one of the photons from the decay. However the $\pi^{+}$will also go through the forward photon veto, leaving a minimum ionizing pulse. Offline this is identified by extrapolating the track of the $\pi^{+}$to the forward photon veto. This option is not available in the level 1 trigger, so instead only the vacuum photon veto system is used to reject events, VVS $(1 \mathrm{GeV})$ with the threshold set well above the noise level.

Events that have a muon, either from prompt decay $K^{+} \rightarrow \mu^{+} \nu_{\mu}$ or from the $\pi^{+}$decay in flight will be rejected. This can be done in the level 1 trigger by using a signal from the muon veto system, MVS, to veto ar event.

The level 1 trigger then can be constructed by the combination of these signals: 


$$
\mathrm{L} 1=\operatorname{KRICH}\left(K^{+}\right) * \overline{\operatorname{KRICH}\left(\pi^{+}\right)} * \operatorname{PRICH}\left(\pi^{+}\right) * \overline{\operatorname{VVS}(1 \mathrm{GeV})} * \overline{\operatorname{MuonVS}}
$$

This trigger is not synchronized with any accelerator RF signal. The TDC and ADC data of the detectors are used in the central L1 trigger station to form the combination of the above logic signals. The generation of the signals required to make the Level 1 trigger can all be local to each of the detector systems. The data rates needed for transmission of these signals can easily be accommodated by optical fibers.

The challenge is to realize this simple logic while minimizing inefficiency from the high rate sources. We assume that the detector deadtime will be 10 nsecwhich corresponds up to a $15 \%$ loss of efficiency. To determine this upper limit, we assume that the muon beam halo plus the total kaon decay rate between the upstream end of the kaon RICH and down through to the MVS can cause detector deadtime. This is clearly an overestimate, but it allows some cushion for uncertainties in the upstream beam halo and beam-detector interactions. The trigger is made based on the digitized data so it will not add extra deadtime on top of the detector deadtime.

\begin{tabular}{|l|r|}
\hline$K^{+} \rightarrow \pi^{+} \pi^{0}$ & $155 \mathrm{kHz}$ \\
\hline$K^{+} \rightarrow \pi^{0} e^{+} \nu$ & $64 \mathrm{kHz}$ \\
\hline$K^{+} \rightarrow \pi^{+} \pi^{+} \pi^{-}$ & $1 \mathrm{kHz}$ \\
\hline Total & $220 \mathrm{kHz}$ \\
\hline
\end{tabular}

Table 35: Level 1 trigger rates.

This simple level 1 trigger reduces the $\sim 12 \mathrm{MHz}$ of kaon decays occurring between the kaon $\mathrm{RICH}$ and the Muon Veto System to $220 \mathrm{kHz}$ as listed in table 35. The trigger rates listed include the effect of $15 \%$ detector deadtime. These rates are comparable to the level 1 trigger rates of the Brookhaven E871 and $\mathrm{KTeV}$ experiments. The Level 1 trigger rate can be further reduced by vetoing on the total energy in the Forward Veto System or requiring significant hadronic energy in the muon range stack. These additional criteria can reduce the level 1 rates substantially but at the risk of an acceptance loss to the signal from the showering requirements on the charged pion. These additional cuts are currently under study, and it is likely that requiring for example less than two hit clusters in the FVS or at least $5 \mathrm{GeV}$ of hadronic energy in the range stack can reduce the trigger rate to less than $100 \mathrm{kHz}$ with a small $(\sim 5 \%)$ loss of signal acceptance. In parallel with the signals from the time of the principal $K^{+} \rightarrow \pi^{+} \pi^{0}$ trigger, data of detector history will collect prescaled minimum-bias $K^{+} \rightarrow \pi^{+} \nu \bar{\nu}$ and other decay modes samples.

\subsubsection{The Level 2 and the Level 2.5 Trigger}

A hardware level 2 trigger is possible in the system architecture. This trigger system could further reduce the event rate readout over the full DAQ system if necessary. This trigger could use more detailed information from the systems in the level 1 trigger, or bring in information from the tracking system. At the moment it is thought that a level 2 trigger will not be necessary, but the hooks for it are left in the architecture.

A hardware level 2.5 trigger sitting in the DAQ data stream is planned. It has advantage of both having hardware high speed and access of full detector data, allowing maximum flexibility of 
trigger adjustment in the hardware trigger stage. It will also allow users to summarize the rejected events and keep a record for future study via the DAQ stream.

It is most likely that if the level 2 or 2.5 trigger were to be implemented that it would make use of information from the DMS. It will be difficult to use the tracking information in level 1 , but at level 2 or 2.5 reconstructing tracks in a few microseconds would be possible. The tracking information would be used to find the location of the $\pi^{+}$in the forward veto and verify that there was not an energy deposition from photons present.

\subsubsection{The Level 3 Trigger}

Once the event is readout all of the data for the event will be available for analysis. Each event will be read into one of a collection of commercial processors. The event will be analyzed and events which are candidate $K^{+} \rightarrow \pi^{+} \nu \bar{\nu}$ events, or events needed for detector monitoring will be archived to tape. The rejected events will be summarized and the brief information of them will be recorded.

\subsection{Physical Structure of CKM DAQ and Trigger System}

The exact layout of the electronics for the experiment has not been determined. We have studied several plans in order to maximize the overall system reliability.

Since the detector is about 100 meters long it is expected that the electronics will be distributed along the detector rather than placed in a central location. The electronics for each system, such as the KRICH or DMS will likely be located in one location. The distribution of electronics along the detector will require care in construction a clocking and control system to keep all of the electronics in time.

The packaging format for the electronics has not been determined. There is not a requirement for the electronics to fit in a confined space, nor is there need for high-density boards. The requirement for high reliability, and the availability of high density FPGA's leads us to expect that the most electronics especially digitizers will be built on a $6 \mathrm{U}$ standard such as VME or VXI rather than on the $9 \mathrm{U}$ standard such as VIPA. This choice will reduce the need for high pitch, high density board design and hence increase the reliability of the system. The need for high reliability also extends to cables and connectors, and the expectation is that RG58 cable and BNC connectors will be used to bring the front end signals to the electronics.

Figure 140 shows an intended plan to meet the reliability requirements.

- Circuits dealing with low level raw signals such as amplifiers, charge integrators, shaper, discriminators and double pulse detectors can be placed on chamber cards or photo multiplier bases.

- Digitizers can be placed on VME $6 \mathrm{U} \times 220 \mathrm{~mm}$ boards, so that the failure rate due to production can be minimized.

- In the local stations, some VME $9 \mathrm{U}$ crates may. be used but not best desired to host clock trigger and DAQ service modules which may need large board size.

- The clock, trigger and DAQ modules in the central station may be in VME $6 \mathrm{U}$ or $9 \mathrm{U}$ format depending on the needs.

- The level 3 PC farm can be regular personal computers.

The cost containment for electronics systems is always of great concern for new experiments. This is usually reflected in the high contingency put on electronics, often the highest contingency 


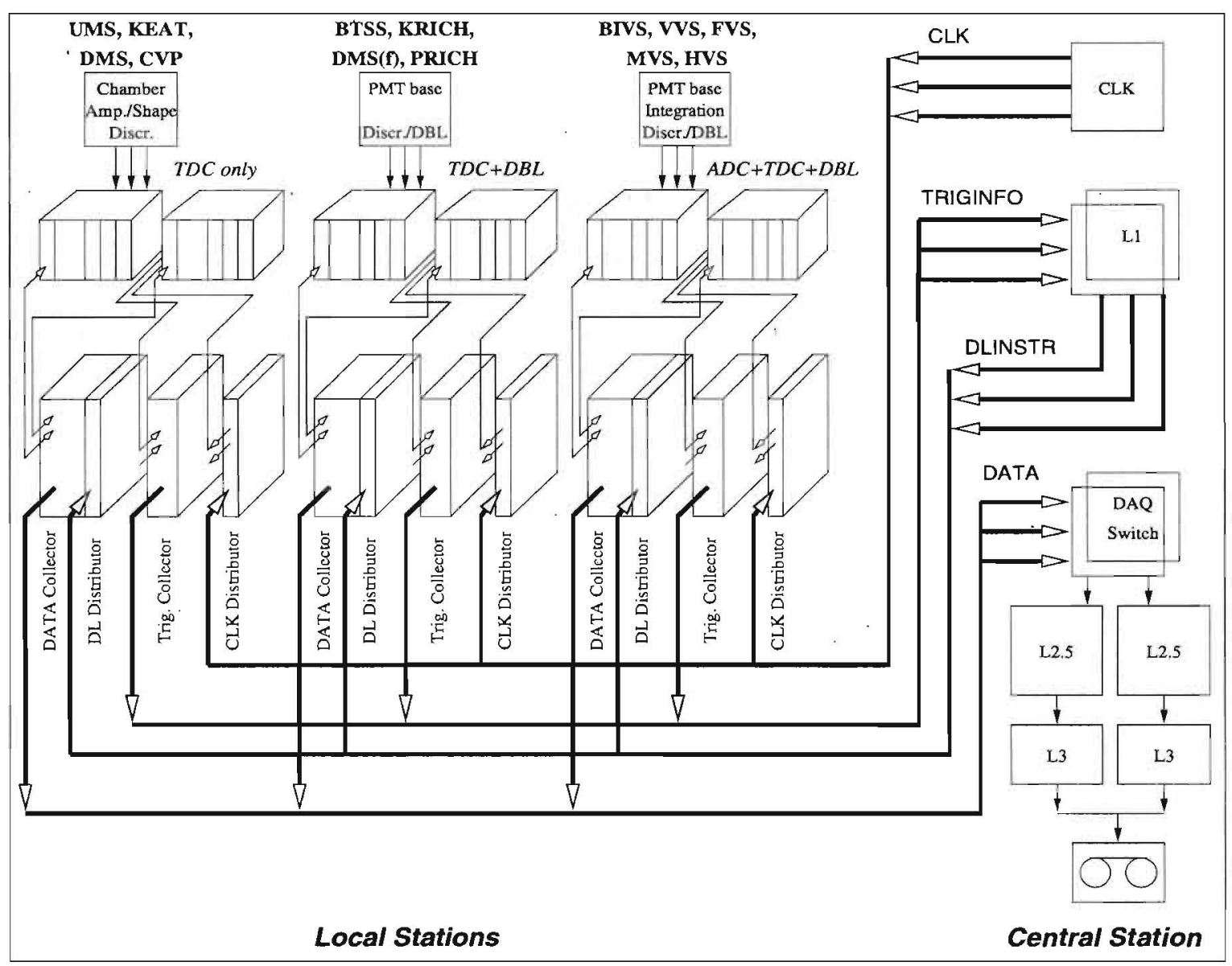

Figure 140: Physical Structure of CKM DAQ and Trigger System 
in the experiment. Many of the procedures used to ensure reliability in the production and testing of electronics are also used to tightly control the costs. These procedures include accurate record keeping, planning for verification and testability, and quality control at each step. These procedures are outlined, for example in ISO 9000:2001. Development of the electronics and DAQ system can also incur large costs. Previous experience has shown that much of the cost of development can come from producing prototypes for the wrong architecture, usually because of the pre-existence of a solution in search of a problem.

\subsection{Detector Performance Monitoring}

From the previous discussions of experimental and detector requirements it is clear that all detector systems must be monitored continuously to ensure that they are live and functioning. A particularly dramatic example of the need for continuous monitoring is in the photon veto system, where a coherent failure of just three live seconds (3 spills) per year of operation is enough to break the inefficiency budget. There are of course several methods to trap such coherent failures, and a more significant (and more likely) concern would be more subtle longer-term failures. In order to guard against such failures, every photomultiplier channel in the photon, hadron, muon veto systems will be optically flashed every second. Both RICH radiator tanks will be optically flashed every second as well in order to monitor the RICH photomultiplier arrays.

In addition to monitoring and calibration flashes, large control and calibration data sets will be acquired in parallel with the $K^{+} \rightarrow \pi^{+} \nu \bar{\nu}$ trigger. These data sets will be used to monitor the efficiency and performance of the RICH and magnetic spectrometers. These fully redundant spectrometers can continuously cross-monitor each other in the minimal bias data sets. Probe photons and muons from $K^{+} \rightarrow \pi^{+} \pi^{0}$ and $K^{+} \rightarrow \mu^{+} \nu_{\mu}$ decays will be employed to directly measure the photon and muon inefficiency of the respective veto systems. Measurement of veto inefficiencies directly from the data is crucial to demonstrate that backgrounds are under control to the required level. The direction and energy of a probe photon in $K^{+} \rightarrow \pi^{+} \pi^{0}$ decays can be calculated from the measured kinematics of the charged pion and one $\pi^{0}$ photon under a $m_{\pi^{0}}$ and $m^{+} K^{+}$mass constraint. Likewise, two-body kinematics from the magnetic and $\mathrm{RICH}$ spectrometers and a $K^{+}$mass constraint provides a clean sample of probe muons from $K^{+} \rightarrow \mu^{+} \nu_{\mu}$ decays. 


\section{$7 \quad K^{+} \rightarrow \pi^{+} \nu \bar{\nu}$ Signal Acceptance and Background Analysis.}

In the first edition of this proposal we presented results from a detailed simulation of the $K^{+} \rightarrow \pi^{+} \nu \bar{\nu}$ signal and various backgrounds. The backgrounds expressed as equivalent branching fractions are summarized below in table 36 . From table 36 one can see that the total background is dominated by $K^{+} \rightarrow \pi^{+} \pi^{0}$ decays and beam interactions with material. The total background is limited to less than $10 \%$ ( $4 \%$ from $K^{+} \rightarrow \pi^{+} \pi^{0}$ and $<6 \%$ from interactions) of a $K^{+} \rightarrow \pi^{+} \nu \bar{\nu}$ signal assuming a $1 \times 10^{-10}$ branching fraction. That full discussion of backgrounds is included as appendix in chapter 13 of this proposal. The following two important caveats (reproduced from the appendix) were called out in the first edition analysis:

- We do not simulate the tracking problem. That is, we do not generate hits in trackers along with noise hits and then reconstruct tracks. When this is done mistakes can occur that we do not account for in the current methodology. Also, non-Gaussian resolution effects need to be investigated and may need to be included.

- We have not compared FRITIOF results to tliose of another hadronic interaction generator. We have found however that the predictions of FRITIOF generally agree with published measured inclusive data for $K^{0}$ production and $\pi^{+}$production. We do not expect the range of possible errors to be large, but comparisons with other generators are an obvious check we have not yet carried out. The estimate of the $K^{+} \rightarrow \pi^{+} \pi^{0}$ background does not rely on FRITIOF in any way.

These conclusions and caveats underscore the importance of the following four simulation and analysis efforts that were launched after the first edition of the proposal:

- Develop a case based on straight-forward simulation techniques and measurements that a photon veto system can be realized with a $\pi^{0}$ (from $K^{+} \rightarrow \pi^{+} \pi^{0}$ ) inefficiency of less than $1.6 \times 10^{-7}$. We have presented this case in detail in previous discussion of the photon veto systems and are confident that the proposed system can deliver a $\pi^{0}$ inefficiency of less than $1.6 \times 10^{-7}$. In addition, we have presented a strong argument that a safety factor of at least $\times 3$ exists with the proposed system.

- Develop a case from measured data and a survey of hadronic interaction generators that beam interaction backgrounds are under control. This case is presented in following sections.

- Develop a case from measured data that the required resolution performance from the proposed velocity and magnetic spectrometers is achievable. In the previous discussions of these redundant spectrometers we have presented a strong case from the analysis of similar systems in the SELEX, Hyper $C P$, and Brookhaven BNL-871 $\left(K_{L} \rightarrow \mu e\right)$ experiments.

- Initiate development of a hit-level simulation of the detector that can be used to study reconstruction algorithms and background rejection techniques. We have recently developed a GEANT3 based full simulation of the detector that coherently meshes with the GEANT3 simulation of the beamline described in section 4 Although we have only just begun developing pattern recognition and reconstruction algorithms, we have already gained some valuable insights that feedback to the design of the detector. It is clear that this coherent "CKM_GEANT" package will provide the necessary tools to fully optimize the beamline and detector. The status of this ongoing effort is presented below. 


\begin{tabular}{|l|c|}
\hline Background Source & $\begin{array}{c}\text { Effective BR } \\
\left(\times 10^{-12}\right)\end{array}$ \\
\hline \hline$K^{+} \rightarrow \mu^{+} \nu_{\mu}$ & $<0.04$ \\
\hline$K^{+} \rightarrow \pi^{+} \pi^{0}$ & 3.7 \\
\hline$K^{+} \rightarrow \mu^{+} \nu_{\mu} \gamma$ & $<0.09$ \\
\hline$K^{+} A \rightarrow K_{L} X$ followed by $K_{L} \rightarrow \pi^{+} e^{-} \nu$ & $<0.14$ \\
\hline$K^{+} A \rightarrow \pi^{+} X$ in trackers & $<4.0$ \\
\hline$K^{+} A \rightarrow \pi^{+} X$ in residual gas $\left(10^{-6}\right.$ torr $)$ & $<2.1$ \\
\hline Accidentals $\left(2 K^{+}\right.$decays $)$ & 0.51 \\
\hline Total & $<10.6$ \\
\hline
\end{tabular}

Table 36: Background estimates in terms of effective branching ratio.

\subsection{Summary of Detector Changes Since the First Edition}

The only significant changes since the first edition of the proposed detector is a lengthening of the fiducial vacuum decay volume from $20 \mathrm{~m}$ to $23 \mathrm{~m}$, and a change in detector technology for the upstream beam trackers (UMS and KEAT). Our original design was based on double-sided silicon tracking stations in vacuum. This design was and continues to be attractive from the perspective of minimal material, but high speed readout of silicon in vacuum is a daunting task. As presented in section 5.1, fine pitch MWPCs can provide the necessary time and space resolution, have comparable material lengths, and proven solutions for high speed readout exist. One drawback of MWPC beam trackers is that the last measured space point is before the upstream vacuum window as shown in figure 76. This geometry exacerbates tracking errors from scatters in the window, and compromises the BIVS veto power of inelastic interactions in the last tracking station. We may revisit a design where the last KEAT tracking plane is in vacuum, but for the purposes of the this proposal all upstream trackers are outside the the vacuum decay volume.

\subsection{Overview of the CKM_GEANT Detector Simulation.}

The CKM_GEANT simulation tool allows us to investigate the individual performance of each sub-detector, as well as the effect of combining the results from all. A full description of each sub-detector has been presented previously. The two most relevant generation parameters to note are that electromagnetic particles (including delta rays) are fully tracked down to a threshold of 2 $\mathrm{MeV}$, and that the base hadron interaction generator is GEANT3(FLUKA). Below we summarize how the sub-detectors are implemented in CKM_GEANT.

- UMS: The simulation of this detector contains all the relevant elements of the real system, including cathode planes, gas volumes and wires. Helium volumes fill the region between chambers. Figure 54 in the UMS section shows that the momentum resolution of the UMS system alone is approximately $0.18 \%$ for $22 \mathrm{GeV} K^{+}$tracks using our analysis package on the hit output from CKM_GEANT. Tracking efficiency is essentially $100 \%$.

- RICH's: The CKM_GEANT Monte Carlo simulates the RICH detectors in detail. The windows, gas and mirrors are all good approximations of what the real elements will be. Particles are stepped through the gas volume and in each step individual Cherenkov photons are generated. These photons are reflected at the mirrors and at the light cones of the photocathode array with a realistic reflectivity. The photo-detection efficiency (as measured by SELEX) of 
the phototubes is then taken into account to determine whether or not to accept the photon as 'a hit. As shown in Figure 141 there are usually at least 5 good phototube hits in the Kaon and Pion RICH's for accepted $K^{+} \rightarrow \pi^{+} \nu \bar{\nu}$ events. The momentum resolution for the $\mathrm{Kaon} \mathrm{RICH}$ is $0.28 \%$ and its angular resolution is $202 \mu \mathrm{Rad}$. The Pion RICH has a mean momentum $(17 \mathrm{GeV} / \mathrm{c})$ resolution of approximately $0.79 \%$ and angular resolution of $146 \mu \mathrm{Rad}$.
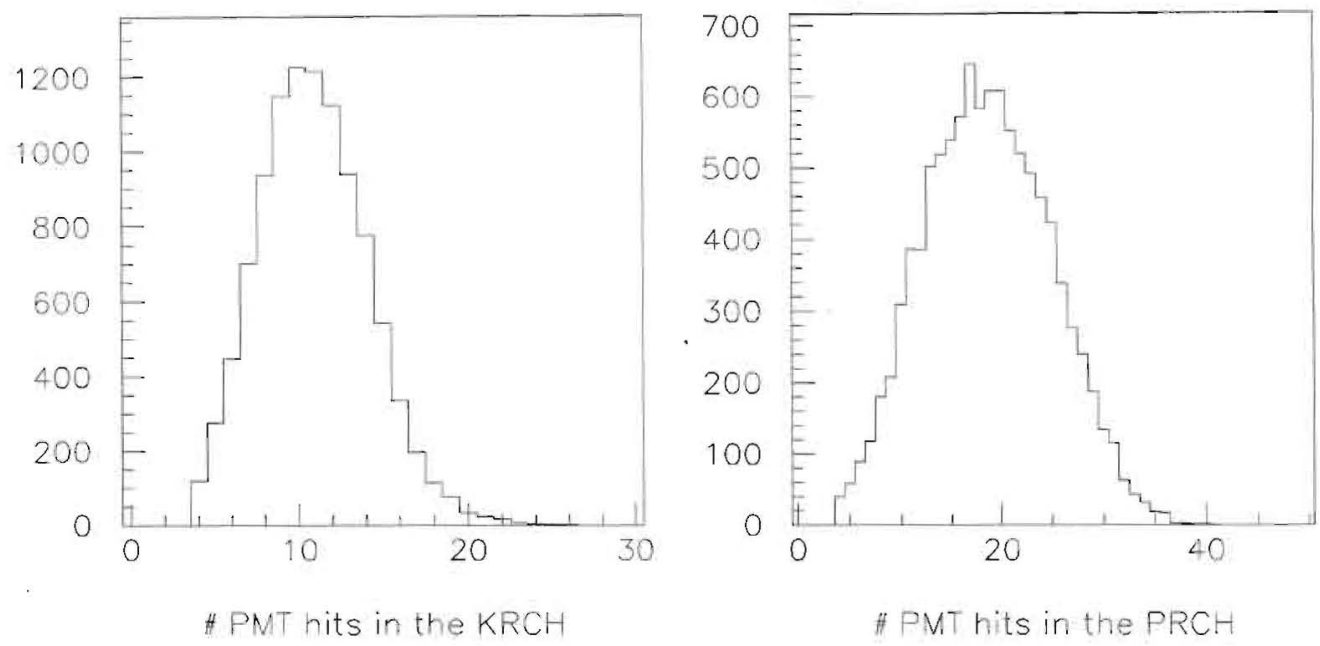

Figure 141: The number of photomultiplier tube hits in the KRICH and PRICH for reconstructed $K^{+} \rightarrow \pi^{+} \nu \bar{\nu}$ events.

- KEAT: CKM_GEANT simulates two copies of the UMS chambers just upstream of the vacuum decay volume. These two chambers recover the entrance angle of the $K^{+}$that is smeared from multiple scattering and interactions in the $\sim 8 \% X_{0}$ of the Kaon RICH. The implementation of these chambers in CKM_GEANT is identical to those in the UMS system. The KEAT chambers have a 2.6 meter separation with the intervening volume is filled with helium, and the system delivers an entrance angle resolution of $76 \mu \mathrm{Rad}$.

- BIVS: This element is used to veto any particles outside of the downstream acceptance that are produced from beam interactions in the upstream UMS/KRICH/KEAT systems. It is a $4 \mathrm{~m}$ long cylinder of lead/scintillator sandwiches with a narrow bore hole for the beam. The veto performance of BIVS is presented in a later discussion of beam interaction backgrounds. The $K^{+} \rightarrow \pi^{+} \nu \bar{\nu}$ fiducial decay volume begins at the downstream end of the BIVS.

- VVS/HVS: As presented in the photon veto section, we have simulated the VVS in great detail. We have used CKM_GEANT to show that the ultimate inefficiency floor from,electromagnetic shower fluctuations is significantly lower than our requirements. In the following $K^{+} \rightarrow \pi^{+} \pi^{0}$ background study of the spectrometers, we have assumed that the rejection of the associated $\pi^{0}$ factorizes cleanly.

- Magnets: There are two upstream magnets for the UMS system. These are identified now as being BM105 magnets. In CKM_GEANT we simulate a vertical field of $14 \mathrm{kG}$ for the $184 \mathrm{~cm}$ length of each magnet. The two fields are oppositely oriented so as to provide a measurement 
angle and then recover the original divergence of the beam. The downstream spectrometer magnet is situated between the second and third tracking stations, and will likely have to be designed specifically for CKM. For this magnet we simply simulate a 1 meter long box field of $3.33 \mathrm{kG}$ which corresponds to a $100 \mathrm{MeV} / \mathrm{c}$ transverse momentum kick.

- DMS: The straw chamber stations have been simulated in fine detail with cylindrical mylar walls, gas and wires. The separation between the chamber stations on each side of the magnet is 4 meters, while the separation between DMS2 and DMS3 is 2 meters, centered on the spectrometer magnet. This arrangement gives an optimum lever arm for measuring angles and momentum. CKM_GEANT results presented in figure 100 determine a mean track momentum resolution of $\sim 1.1 \%$ and a mean angular resolution of $37 \mu \mathrm{Rad}$.

- FVS: The Forward Veto System has been implemented in CKM_GEANT as a rectangular array of $17975 \mathrm{~cm} \times 5 \mathrm{~cm}$ blocks $210 \mathrm{~cm}$ by $215 \mathrm{~cm}$. with a $15 \mathrm{~cm} \times 15 \mathrm{~cm}$ hole offset from the center to pass the beam. An illustration of the array can be seen in figure 121. Each tower is simulated as $18 X_{0}$ of $1 \mathrm{~mm} \mathrm{~Pb}-5 \mathrm{~mm}$ Scintillator. In our $K^{+} \rightarrow \pi^{+} \pi^{0}$ background studies we do not explicitly reconstruct clusters in the FVS. Inefficiencies associated with $\pi^{+}$clustering and photon identification are discussed in section 5.9.3, and we simply scale the final acceptance with this $90 \%$ efficiency in our signal and background studies that follow.

- MVS: The discussion of test beam results in section 5.10 validates the claim that muons of momentum greater than $14 \mathrm{GeV}$ can be discriminated from pions with with a residual inefficiency of less than $1 \times 10^{-5}$ while maintaining high pion acceptance. For the purpose of background estimates in both the first edition and ongoing CKM_GEANT studies we have not explicitly simulated the MVS but rather assume a muon rejection of $1 \times 10^{-5}$ and a pion acceptance of $95 \%$.

\subsubsection{Event Reconstruction in CKM_GEANT}

The output of the GEANT simulation of CKM is a series of hits in the various detector components. These hits describe either the position of particles as they enter or leave detector boundaries (like cells in the UMS or phototubes in the RICH), or energies accumulated in the tower structures of the photon vetoes. We have a developed a set of simple analysis routines that read these hits in and fit them for particle kinematics. There are four major components of this reconstruction: 1) track reconstruction for the beam $K^{+}$in the UMS and KEAT wire chambers, 2) track reconstruction for the decay $\pi^{+}$in the DMS straw tubes, 3) ring reconstruction in the Kaon and Pion RICH's and 4) combining the results to form the event kinematics. We currently treat these reconstructions as independent. We do not reconstruct clusters of energy in any of the photon vetoes. A true reconstruction of CKM events will most likely be a global fit of all hits in the detector, including. timing information. We look forward to developing this kind of analysis program in the future. We briefly describe our current reconstruction methods below.

1. UMS/KEAT reconstruction:

The upstream track reconstruction program uses information from both the UMS and KEAT chambers. It reconstructs multi-track events and fits individual track momenta, slopes and intercepts. To start, hits are assigned to the tracks. To do that, the $x, u$ and $v$ projections of UMS and KEAT chambers are aralyzed independently and hits are fit to straight lines. If hits have small $\chi^{2}$ they are then assigned to track segments separately in the regions before and after the magnet in the UMS. Then these segments are combined into 3-dimensional 
tracks if the projection angles are consistent with the 3-dimensional track. Then tracks are fit before and after the magnet and the momentum of the track is calculated. To decrease the errors introduced by multiple scattering in the UMS and $\mathrm{KRICH}$, we then fit a separate line segment with just the two KEAT chambers. This independent line segment then determines the entrance position and angles of the candidate $K^{+}$entering the decay volume.

2. DMS reconstruction: The hit output from CKM_GEANT is the vector of the particle as it leaves each straw. In our DMS analysis package this information is extrapolated back to the $z$ position of the wire and the distance the particle was from the wire is calculated. This position is smeared with a Gaussian width of $150 \mu \mathrm{m}$. In each plane of straws, complementary pairs of hits are found which reconstruct with a good sum of distances (SOD), which is within $1 \mathrm{~mm}$ of half of the wire spacing. For each chamber, we loop over all these pairs to find good "crossings", i.e. intersections among the views that satisfy a maximum $\chi^{2}$. To have a valid crossing there must be a lit pair in each of the chamber views. Pairs cannot belong to more than one crossing. All combinations of crossings, with exactly one crossing in each chamber, are handed to the track fitter. The fitter, which minimizes the $\chi^{2}$ to a track equation, uses the underlying hits, not the crossings, to find the track. The result of the fitter is line intercepts and slopes in the $\mathrm{X}$ and $\mathrm{Y}$ view, and a curvature, which is translated into an overall momentum for the track.

3. RICH reconstruction: Reconstruction of both the Kaon RICH and Pion RICH data is essentially the same. The hit output from CKM_GEANT is a list of the phototubes that fired in the event, along with their X and Y coordinates. The RICH analysis routine demands that there be at least 4 PMT hits in a RICH for reconstruction. We simply fit a circle to all hits in each RICH that satisfy that criteria. No track information is used. The radius of the fit circle corresponds to a value of $\beta$ in each RICH. The particle is assumed to be a kaon in the $\mathrm{KRICH}$ and a pion in the PRICH. Given this assumption, the momentum of each track is calculated from $\beta$ for each RICH. The components of momentum are also calculated since the $\mathrm{X}$ and $\mathrm{Y}$ position of the center of the circle correspond to $\theta_{X}$ and $\theta_{Y}$ in lab coordinates.

4. Event kinematics After reconstruction, the missing mass variable $M_{\text {miss }}^{2}$ is calculated for the $\mathrm{RICH}$ system and the tracking system independently from the components of momentum that each system calculates. The resolution for $M_{\text {miss }}^{2}$ in simulated $K^{+} \rightarrow \pi^{+} \pi^{0}$ events is determined for each system. That resolution is used to weight the average of the two missing mass variables into a single variable:

$$
\left(M_{\text {miss }}^{2}\right)_{\text {combined }}=\frac{\frac{\left(M_{\text {miss }}^{2}\right)_{R I C H}}{\sigma_{R I C H}^{2}}+\frac{\left(M_{\text {miss }}^{2}\right)_{\text {track }}}{\sigma_{\text {track }}^{2}}}{\left(\frac{1}{\sigma_{R I C H}^{2}}+\frac{1}{\sigma_{\text {track }}^{2}}\right)}
$$

\subsubsection{CKM_GEANT $K^{+} \rightarrow \pi^{+} \nu \bar{\nu}$ Signal Selection Cuts.}

The following selection criteria employed in the CKM_GEANT simulation of $K^{+} \rightarrow \pi^{+} \nu \bar{\nu}$ and $K^{+} \rightarrow \pi^{+} \pi^{0}$ are based the analysis cuts from the first edition. These cuts have been tuned in CKM_GEANT to provide a balance between maximizing $K^{+} \rightarrow \pi^{+} \nu \bar{\nu}$ acceptance and optimizing the $M_{\nu \bar{\nu}}^{2}$ resolution.

- Aperture cuts. These simply require accepted charged particles to remain inside the outer detector boundaries and outside the beam hiole region. 
- Cleanliness cuts. These are designed to insure that the event has a clean signature in the RICH and wire chamber detectors. They consist of:

1. KRICH: between 6 and 25 phototube hits,inclusive

2. PRICH: between 6 and 40 phototube hits,inclusive

3. UMS and DMS: less than 45 hits and 1 reconstructed track in each

4. : a cut on the quality of fit in both RICH's and both UMS and DMS trackers.

- Vertex cuts. These quantities, derived from the tracker, primarily reject backgrounds from interactions in detector material. The resolution on these variables, as compared to the true CKM_GEANT quantities, are shown in Figure 142. The cuts consist of:

1. $\theta>2.5 \mathrm{mrad}$; A requirement that the reconstructed angle between the $K^{+}$and $\pi^{+}$be large enough to permit good vertex $z$ measurement.

2. $1860<Z_{v t x}<4160 \mathrm{~cm},\left|X_{v t x}\right|<10 \mathrm{~cm},\left|Y_{v t x}\right|<8 \mathrm{~cm}$; The transverse vertex location should be near the beam. The $Z_{v t x}$ limits come from demanding the vertex be at least $5 \mathrm{~m}$ from the nearest material and downstream of the BIVS. The vertex resolution as seen in Figure 142 is $32 \mathrm{~cm}$. The relatively large cut of $5 \mathrm{~m}$ away from any scattering material ensures that we are well away from potential background sources.

3. DOCA $<1 \mathrm{~cm}$; The reconstructed distance of closest approach between the $K^{+}$and $\pi^{+}$ should be small.
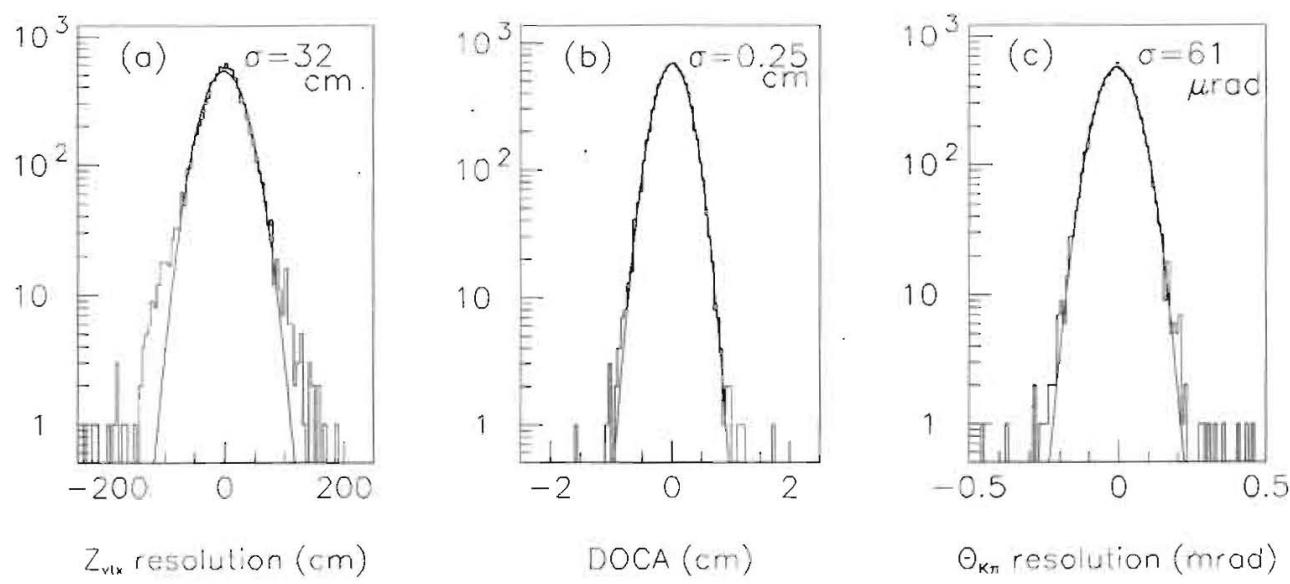

Figure 142: Reconstructed vertex variables as obtained from the tracking system in good $K^{+} \rightarrow \pi^{+} \nu \bar{\nu}$ events: a) Z vertex resolution $(\mathrm{cm})$, b) distance of closest approach (DOCA), in $\mathrm{cm}, \mathrm{c}$ ) opening angle $\theta$ resolution ( $\mathrm{mrad}$ )

- Spectrometer cuts. These cuts require the magnetic spectrometer and velocity spectrometer measurements to agree. Since the pion mass hypothesis is assumed for the velocity spectrometer, these cuts have the effect of rejecting muons based on RICH information, along with other event pathologies (e.g., $\pi^{+}$decay in flight, large scatters or energy loss in some areas, 
etc.). The minimum momentum cut on the pion assures that it is visible in the Pion RICH. The cuts are:

1. Beam particle momentum: $\left|p_{\text {magnetic }}-p_{\mathrm{RICH}}\right| / p_{\mathrm{RICH}}<0.025$

2. Secondary (decay) particle momentum: $\left|p_{\text {magnetic }}-p_{\text {RICH }}\right| / p_{\text {RICH }}<0.05$

3. Missing mass: $\left|M_{\text {magnetic }}^{2}-M_{\mathrm{RICH}}^{2}\right|<0.01 \mathrm{GeV}^{2} / c^{4}$

4. $14<p_{\mathrm{RICH}}<\left(p_{K^{+}}-1\right) \mathrm{GeV} / \mathrm{c}$

These distributions can be seen in Figure 143.

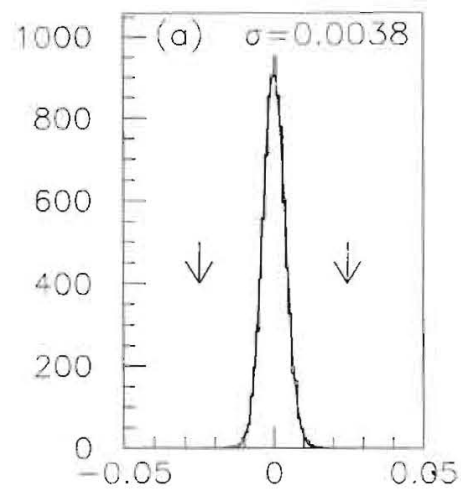

Relative diff. in $p_{k}$

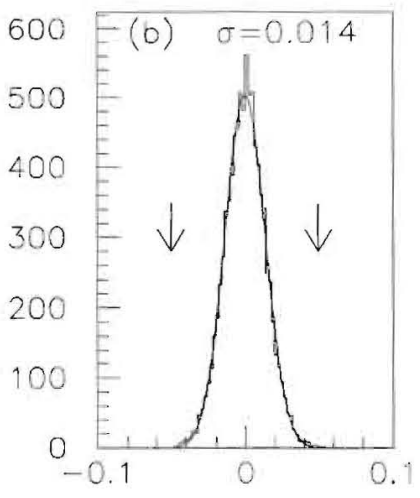

Relative diff, in $p_{\pi}$

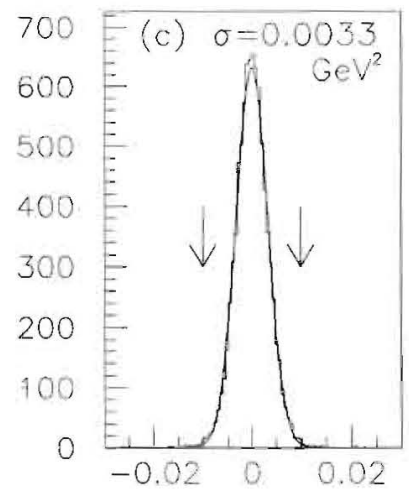

Difference in MM2 $\left(\mathrm{GeV}^{2}\right)$

Figure 143: Reconstructed spectrometer variables ill good $K^{+} \rightarrow \pi^{+} \nu \bar{\nu}$ events: a) fractional difference hetween UMS and KRCH reconstruction for $K^{+}$momentum, b) fractional difference between DMS and PRCH reconstruction for $\pi^{+}$momentum c) difference between magnetic spectrometer and RICH spectrometer for $M_{\text {miss }}^{2}\left(G e V^{2}\right)$.

- Signal region. Our signal region is defined to be: $-0.005<M_{\nu \bar{\nu} \text { combined }}^{2}<0.008 \mathrm{GeV}^{2}$. The negative non-zero limit allows for resolution smearing, and the upper limit is about $5 \sigma$ from the $m_{\pi^{0}}^{2}$ pole. The $K^{+} \rightarrow \pi^{+} \pi^{0}$ missing mass resolution line shapes of the two spectrometers are shown in Figure 144. The spectrometer consistency cuts previously listed are applied in figure 144.

\subsubsection{Developments in the $K^{+} \rightarrow \pi^{+} \nu \bar{\nu}$ Signal Acceptance.}

As discussed in section 3 , the $K^{+} \rightarrow \pi^{+} \nu \bar{\nu}$ signal is in a tightly restricted region of missing mass, $M_{\nu \bar{\nu}}^{2}$ which is redundantly measured by the magnetic and velocity spectrometers. The range of this accepted region spans from $3 \sigma$ below zero to as close as possible to $m_{\pi^{0}}^{2}$ : The missing mass of the principal kaon decay background $K^{+} \rightarrow \pi^{+} \pi^{0}$. This is vividly illustrated in the CKM_GEANT version of the "bottom-line" plot shown in figure 145. From figure 145 it is clear that the $K^{+} \rightarrow \pi^{+} \pi^{0}$ background is controlled by both minimizing the height of the $\pi^{0}$ peak through photon vetoes, and minimizing the non-Gaussian tails through faithful reconstruction of the $K^{+} \rightarrow \pi^{+} X$ kinematics. The accepted missing mass range motivated in figure 145 is shown overlaid on the 

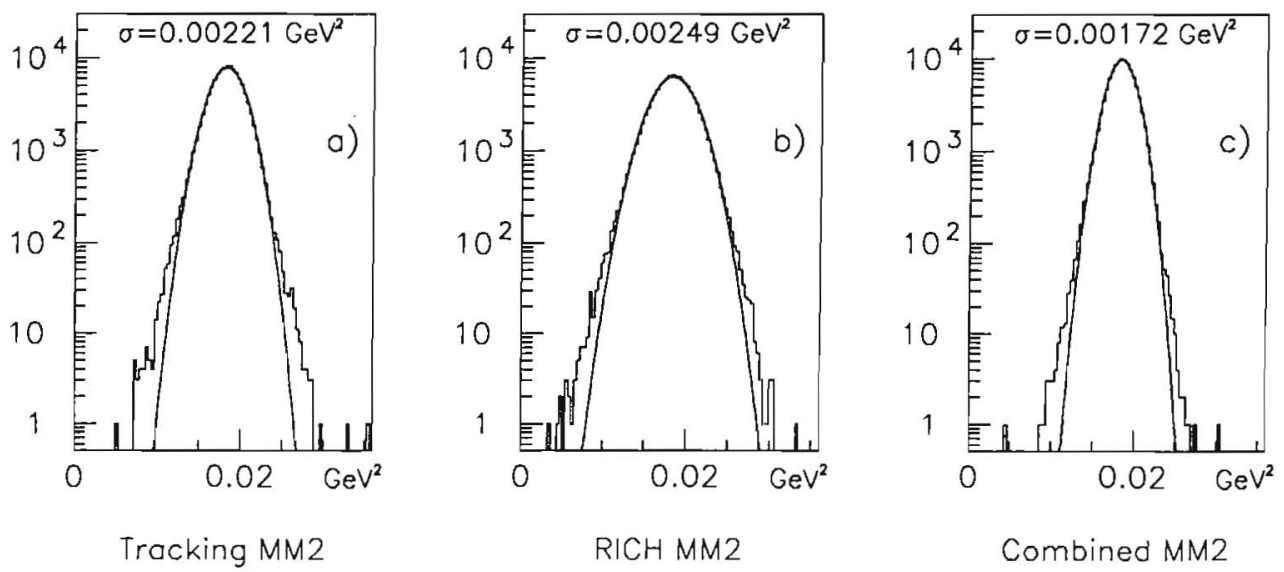

Figure 144: Reconstructed missing mass variable of $K^{+} \rightarrow \pi^{+} \nu \bar{\nu}$ events that pass the spectrometer consistency cuts listed in section 7.2.2 for: a) for the magnetic tracking spectrometer, b) for the $\mathrm{RICH}$ spectrometer, c) for the combined weighted average of both. All in $\mathrm{GeV}^{2} / c^{4}$.

$K^{+} \rightarrow \pi^{+} \nu \bar{\nu}$ signal $M_{\nu \bar{\nu}}^{2}$ distribution in figure 146 that was generated with CKM_GEANT. This narrow range of accepted $M_{\nu \bar{\nu}}^{2}$ together with the requirement of a high momentum decay pion $\left(p_{\pi^{+}}>14 \mathrm{GeV} / \mathrm{c}\right)$ are the main factors that determine the $K^{+} \rightarrow \pi^{+} \nu \bar{\nu}$ acceptance.

CKM_GEANT simulated $K^{+} \rightarrow \pi^{+} \nu \bar{\nu}$ decays within the $30 \mathrm{~m}$ decay region spanning from the upstream vacuum window to the first DMS plane. This is the same procedure we followed in the 1st edition. Within this decay volume $17 \%$ of the $22 \mathrm{GeV} \mathrm{K}^{+}$beam decays, and the previously defined cuts correspond to an acceptance of $2.3 \%$.

This acceptance is significantly larger than the acceptance of $1.8 \%$ reported in the first edition. This acceptance gain results from the following three straightforward changes to the detector geometry and analysis criteria:

1. A $23 \mathrm{~m}$ decay region was used instead of the $20 \mathrm{~m}$ nominal.

2. The upper cut on pion momentum was relaxed to be $1 \mathrm{GeV}$ or more below the kaon momentum. Previously this cut was $14<p_{\text {pion }}<20 \mathrm{GeV}$.

3. The cut on the missing mass range was expanded to include resolution smeared events. It is now $-0.005<M_{\text {miss }}^{2}<0.008 \mathrm{GeV}^{2}$. Previous this range was $0<M_{\text {miss }}^{2}<0.008 \mathrm{GeV}^{2}$.

\subsubsection{CKM_GEANT Studies of the $K^{+} \rightarrow \pi^{+} \pi^{0}$ Background Rejection.}

Applying our $\pi^{0}$ 's veto inefficiency of less than $1.6 \times 10^{-7}$, one can deduce the required kinematic rejection of $K^{+} \rightarrow \pi^{+} \pi^{0}$ decays for a fixed $K^{+} \rightarrow \pi^{+} \nu \vec{\nu}$ signal acceptance. This key factorization assumption posits that $\pi^{0}$ and kinematic rejections are independent. The Brookhaven E787 experiment has shown from their data that this factorization assumption is valid over the six orders of magnitude that correspond to their $\pi^{0}$ rejection. This is illustrated in figure 147 which demonstrates the stability of the measured $\pi^{+}$momentum line-shape of $K^{+} \rightarrow \pi^{+} \pi^{0}$ decays over the full 


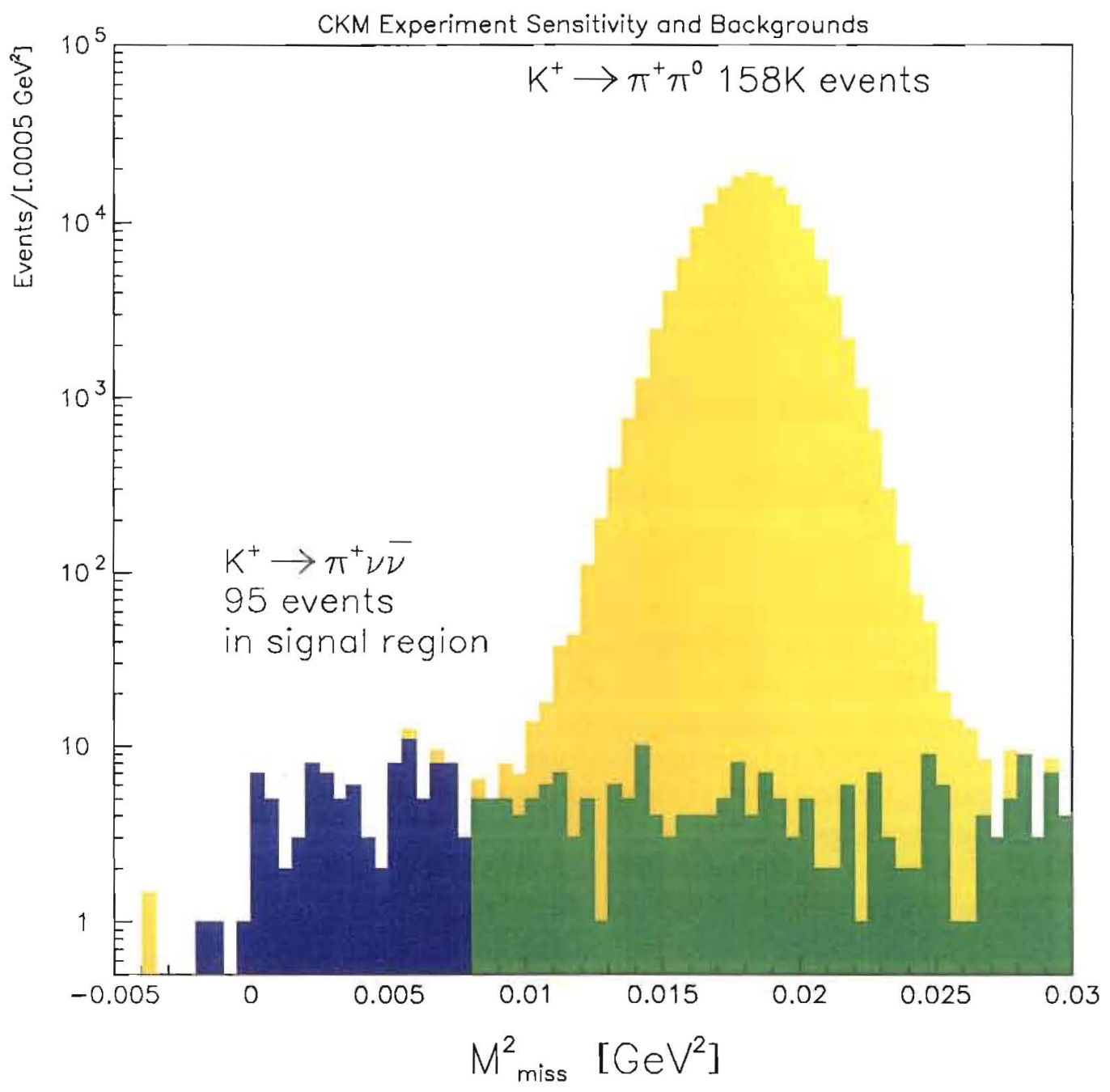

Figure 145: The combined measured missing mass squared $\left(M_{\nu \bar{\nu}}^{2}\right)$ of the two spectrometers for both the $K^{+} \rightarrow \pi^{+} \nu \bar{\nu}$ signal (light shade) and principal background $K^{+} \rightarrow \pi^{+} \pi^{0}$ are shown. The signal region is shown in dark shade. The $158 \mathrm{~K} K^{+} \rightarrow \pi^{+} \pi^{0}$ events are the residual background after all cuts expect for missing mass. 


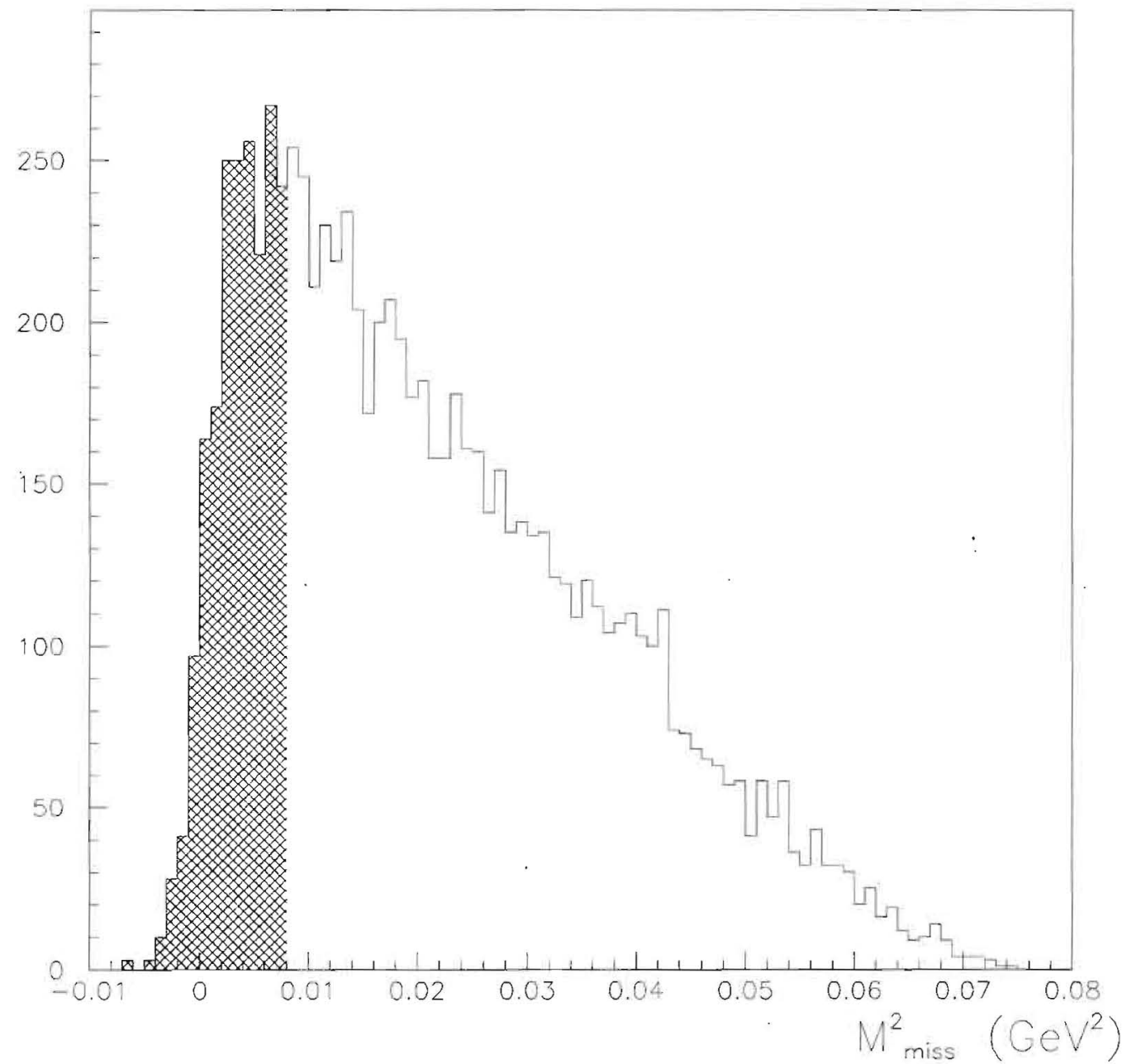

Figure 146: The combined measured missing mass squared $\left(M_{\nu \bar{\nu}}^{2}\right)$ of the two spectrometers. reconstructed in CKM_GEANT. The signal region is shaded. 
range of plioton veto cut stages. We have studied this assumption at some level with the in-flight teclinique, and find no reason to doubt it at this stage.

For a signal acceptance of $2.3 \%$, a combined rejection power against $K^{+} \rightarrow \pi^{+} \pi^{0}$ of $\times 30,000$ in missing mass is required from the spectrometers to keep this background less than $10 \%$ of the 100 -event signal sample assuming a $1 \times 10^{-10}$ branching fraction. Figure 148 shows the missing mass correlation of the two spectrometers after cuts for one million $K^{+} \rightarrow \pi^{+} \pi^{0}$ decays simulated in CKM_GEANT and reconstructed from hit-level and which pass the selection criteria listed previously. The $\mathrm{K}^{+} \rightarrow \pi^{+} \pi^{0}$ background pole at $m_{\pi^{0}}^{2}=0.0182 \mathrm{GeV} / \mathrm{c}^{2}$ clearly dominates the landscape. Most striking about figure 148 is how independent the two spectrometers are. This is particularly true far from the $m_{\pi^{0}}^{2}$ pole where it matters most. The individual and combined spectrometer resolutions are shown in figure 144.

For completeness we report that with the current reconstruction and analysis the CKM_GEANT $K^{+} \rightarrow \pi^{+} \pi^{0}$ background within the previously defined signal region corresponds to less than $10 \%$ of a $K^{+} \rightarrow \pi^{+} \nu \bar{\nu}$ signal with a $1 \times 10^{-10}$ branching fraction. It is important to emphasize that this CKM_GEANT based analysis is not yet conclusive, but rather supportive of our base background analysis reported in the first edition and summarized in table 36. As the CKM_GEANT tool matures and is fully qualified it will become the source of our base background estimates. Nevertheless, it is encouraging that at this early stage in developing the CKM_GEANT simulation environment we are consistent with the goal of keeping the principal kaon decay background controlled to less than $10 \%$ of our nominal $K^{+} \rightarrow \pi^{+} \nu \bar{\nu}$ signal.

\subsubsection{Additional Kaon Decay Backgrounds Considered.}

Since the submission of the first edition we have explicitly considered the processes $K^{+} \rightarrow \pi^{+} \gamma \gamma$ and $K^{+} \rightarrow \pi^{+} e^{+} e^{-}$as background sources to $K^{+} \rightarrow \pi^{+} \nu \bar{\nu}$. These 3 -body decays could potentially be troublesome since the $K^{+} \rightarrow \pi^{+} X$ missing mass is not restricted by two-body kinematics as in $K^{+} \rightarrow \pi^{+} \pi^{0}$, and extends through the low $M_{X^{2}}^{2} K^{+} \rightarrow \pi^{+} \nu \bar{\nu}$ signal region. For example, as previously discussed the kinematic rejection of $K^{+} \rightarrow \pi^{+} \pi^{0}$ is $\times 30,000$ whereas the kinematic rejection of $K^{+} \rightarrow \pi^{+} e^{+} e^{-}$is only $\sim \times 50$. Fortunately the branching fractions (ref [116]) of $K^{+} \rightarrow \pi^{+} \gamma \gamma$ and $K^{+} \rightarrow \pi^{+} e^{+} e^{-}$are $6 \times 10^{-7}$ and $2.9 \times 10^{-7}$ respectively, to be compared with $21 \%$ for $K^{+} \rightarrow \pi^{+} \pi^{0}$. Hence a kinematic rejection factor of only $\sim \times 50$ is adequate to control these backgrounds below a an equivalent branching fraction of $1 \times 10^{-12}$, assuming the $e^{+} e^{-}$and $\gamma \gamma \mathrm{X}$-systems can be vetoed with comparable inefficiency to $\mathrm{K}^{+} \rightarrow \pi^{+} \pi^{0}$. We lave studied the veto power of the attendant $e^{+} e^{-}$and $\gamma \gamma$ systems with CKM_GEANT and ray-tracing simulations with the appropriate measured form factors. We conclude that these processes contribute background at levels far below an equivalent branching fraction of $1 \times 10^{-12}$.

\subsection{Developments in Beam Interaction Backgrounds.}

In the first edition of the proposal the beam interaction background was shown to be dominated by $K^{+}$interactions with the last plane of material before the vacuum volume and residual gas in within the vacuum. These interactions can produce a fast forward $\pi^{+}(>14 \mathrm{GeV}, \theta<15 \mathrm{mrad}$ ) into the acceptance of the detector, and the associated particle production sometimes escape detection. The sum of these background sources were limited to less than $6 \%$ of a $1 \times 10^{-10} \mathrm{~K}^{+} \rightarrow \pi^{+} \nu \bar{\nu}$ branching fraction. The total background from these processes is proportional to the yield of fast forward pions, and the efficiency with which associated particles can be vetoed. Our analysis in the first edition relied on one specific hadronic interaction generator (FRITIOF), from which a rejection factor of $\times 10,000$ was determined for the product of forward $\pi^{+}$production and veto power of 


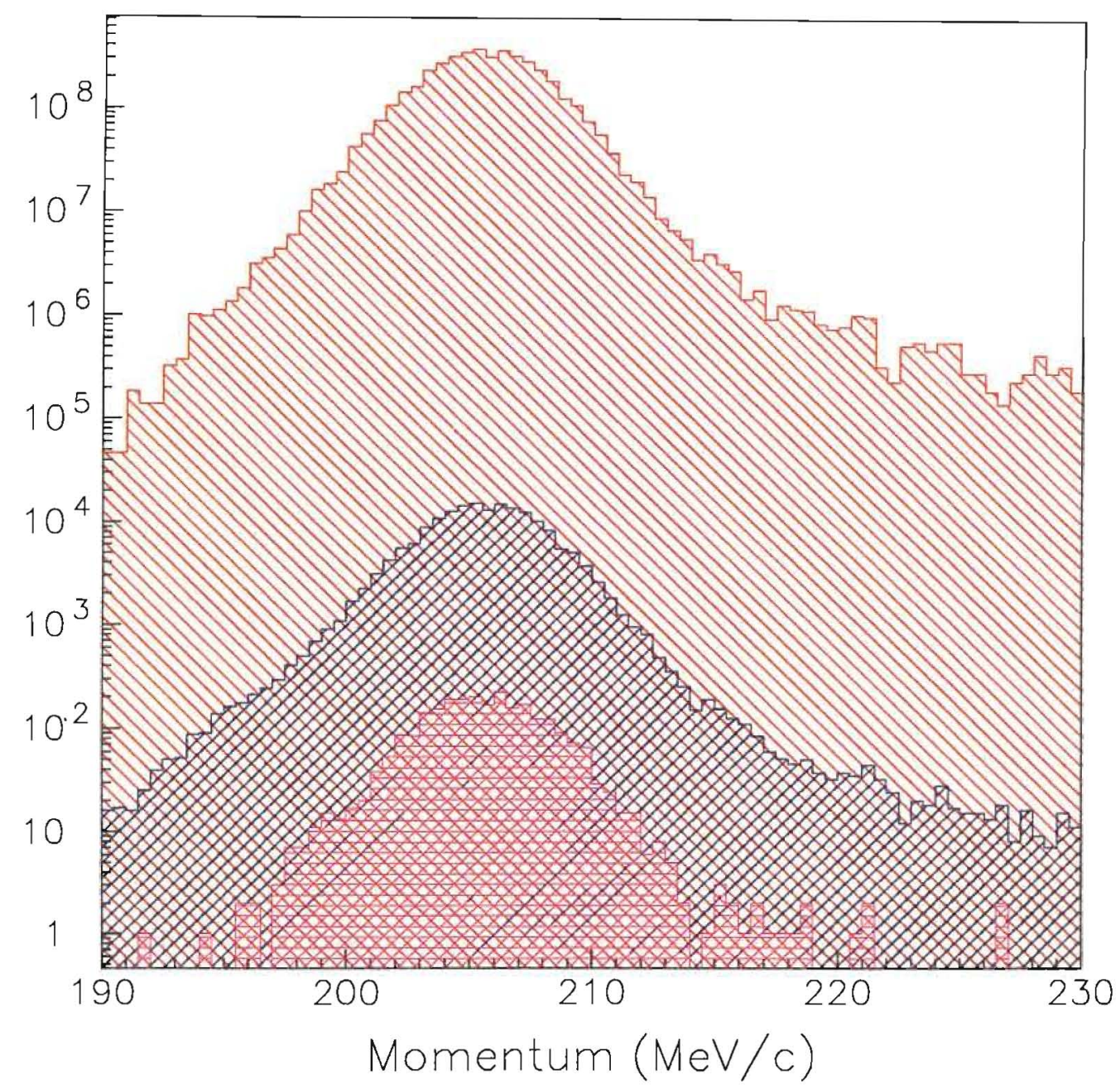

Figure 147: The measured [191] $\pi^{+}$momentum line-shape of $K^{+} \rightarrow \pi^{+} \pi^{0}$ decays from BNL experiment E787. The different curves are the evolution from no photon veto cuts, to online photon veto cuts, and finally full offline photon veto cuts. The stability of the line-shape validates the factorization hypothesis for this pair of variables. 


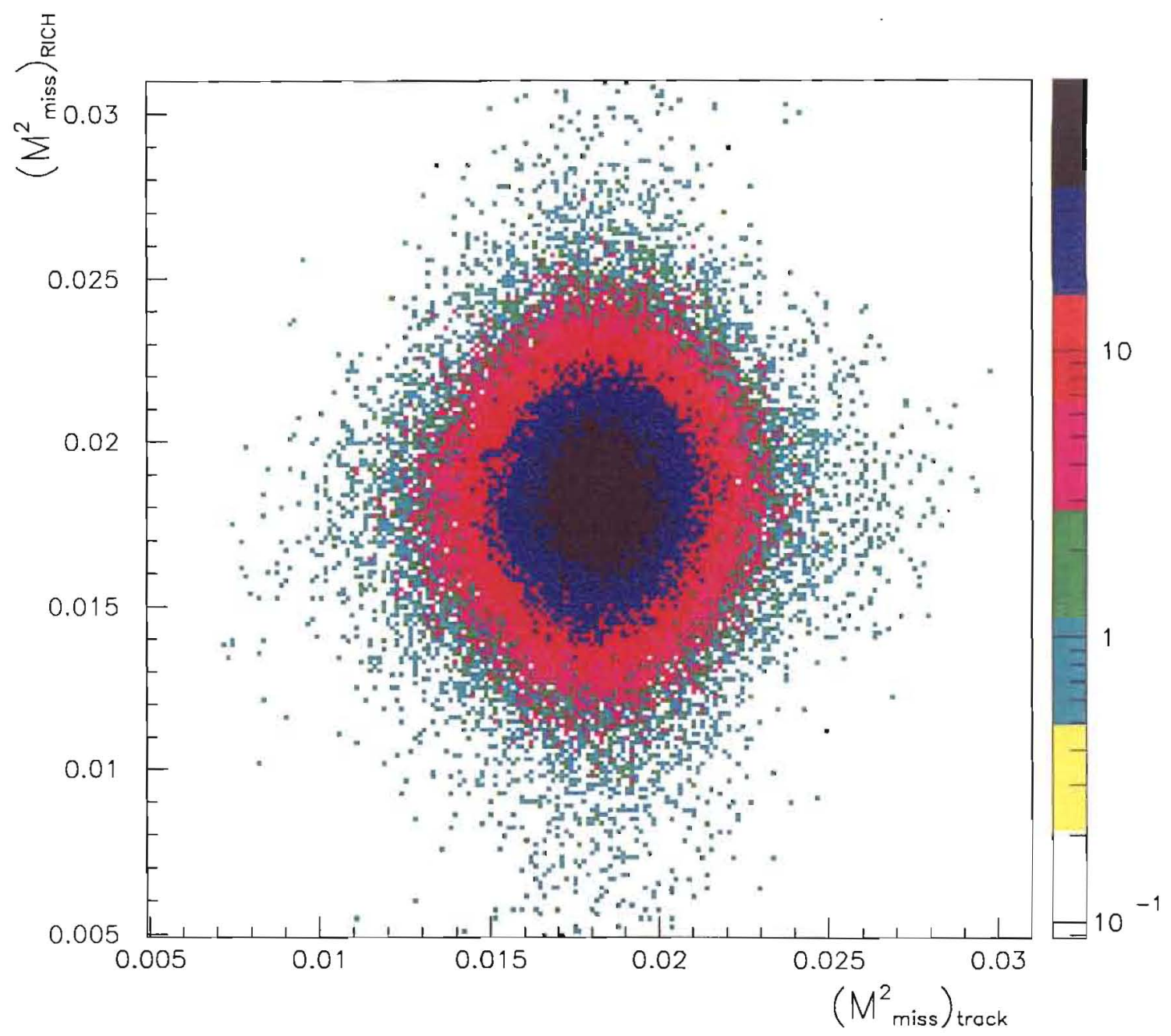

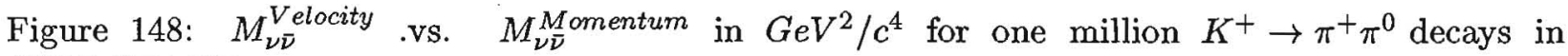
CKM_GEANT. 
associated particles. This product is the most uncertain element of the interaction background estimate presented in the appendix.

Since the first edition we have improved our estimates of this (production $\times$ veto) product with data and other hadronic interaction generators. Figure 149 shows the inclusive inelastic $\pi^{+}$production fraction as a function of momentum from an incident $22 \mathrm{GeV} K^{+}$on light nuclei. These fractions depend weakly on the atomic number of the struck nucleus. Figure 149 includes the prediction of the GEANT-FLUKA, GEANT-GEISHA, FLUKA-99 generators overlaid on measured data from the Fermilab Single Arm Spectrometer data (SAS) [123, 192]. Figure 150 shows SAS data and simulation results for $\pi^{+}$production into the forward region $(\theta<15 \mathrm{mRad})$. A significant variation between generators is evident from these figures, and it is clearly best to anchor our beam interaction background estimates on SAS data. For technical reasons we have not been able to generate a differential momentum spectrum from the FRITIOF generator, but the integral above $14 \mathrm{GeV}$ is $\times 3.9$ higher than the SAS data.

In the first edition of the proposal we estimated that the Beam Interaction Veto System (BIVS) described in section 5.5 was capable of tagging beam interactions with a residual inefficiency of less than $2.8 \%$. This estimate was based on the FRITIOF generator, the results of which were tracked from an interaction in the last KEAT plane forward into the BIVS. Given that FLUKA-99 in general matches the character of the data in this regime, we have repeated this exercise with the FLUKA-99 generator. The following results are based on this analysis.

The distribution of hit BIVS photomultipliers as a function of longitudinal position is shown in figure 151. As expected the occupancy of the upstream layers is highest, which effectively shield the downstream elements. The distribution of hit arrival time with respect to a common trigger time is shown if figure 151 as well. In order to maintain high BIVS live-time with an upstream beam interaction rate of about $50 \mathrm{MHz} \times 4 \% \lambda_{I}^{K^{+}} \sim 2 \mathrm{MHz}$, only hits within 5 nsec of a traversing beam particle are allowed to veto the event.

The veto inefficiency as a function of threshold of observed visible energy is shown in figure 152 . From figure 152 it is clear that there is little advantage to driving the veto threshold very low, which is fortunate given the high rates in this device. Running with a threshold of $(10-20) \mathrm{MeV} / \mathrm{channel}$ corresponds to a veto inefficiency of about $1.5 \%$ which permits a tolerable deadtime of $4 \%$. For comparison, the offline veto threshold on visible energy for the downstream vacuum veto system is required to be $1-2 \mathrm{MeV}$.

Although it is encouraging that different hadronic generators predict comparable BIVS efficiencies, it is useful to understand what can be learned from related veto systems. One related system is the fully active $\mathrm{KTeV}$ regenerator [169], which must efficiently veto inelastic scattered neutral kaons while maintaining high live-time for coherently regenerated forward $K_{S}$. The $\mathrm{KTeV}$ regenerator is 1.8 meters long, composed of high-speed segmented scintillator and instrumented with 180 fast photomultiplier tubes. The rate of inelastic hadron interactions in the regenerator is $\sim 1.5 \mathrm{MHz}$, and the average kaon momentum is about $50 \mathrm{GeV}$. The offline veto threshold per photomultiplier tube in the $\mathrm{KTeV}$ regenerator is $\sim 10 \mathrm{MeV}$ within a 20 nsec gate. With this veto threshold, the rejection [170] of inelastically scattered neutral kaons $\left(K_{L} A \rightarrow K^{0} X\right)$ is about $\times 100$ at the relatively low inelastic $p_{t}^{K^{0}}$ of $100 \mathrm{MeV} / c$, and continues to increase for greater $p_{i}^{K^{0}}$. The deadtime associated with this $10 \mathrm{MeV} /$ channel threshold and $\sim 1.5 \mathrm{MHz}$ rate is $\sim 5 \%$.

Clearly the veto problem of the CKM BIVS and the KTeV regenerator differ significantly in details, but the experience of the $\mathrm{KTeV}$ regenerator demonstrates that a $\times 100$ rejection level of inelastic interactions that produce relatively low $p_{t}$ very forward hadrons is plausible in a high rate environment. Equally important is the demonstration that a phototube based scintillator veto system can operate with thresholds and rates comparable to the CKM requirements.

The recent work reported here on beam interaction backgrounds has validated our upper lim- 


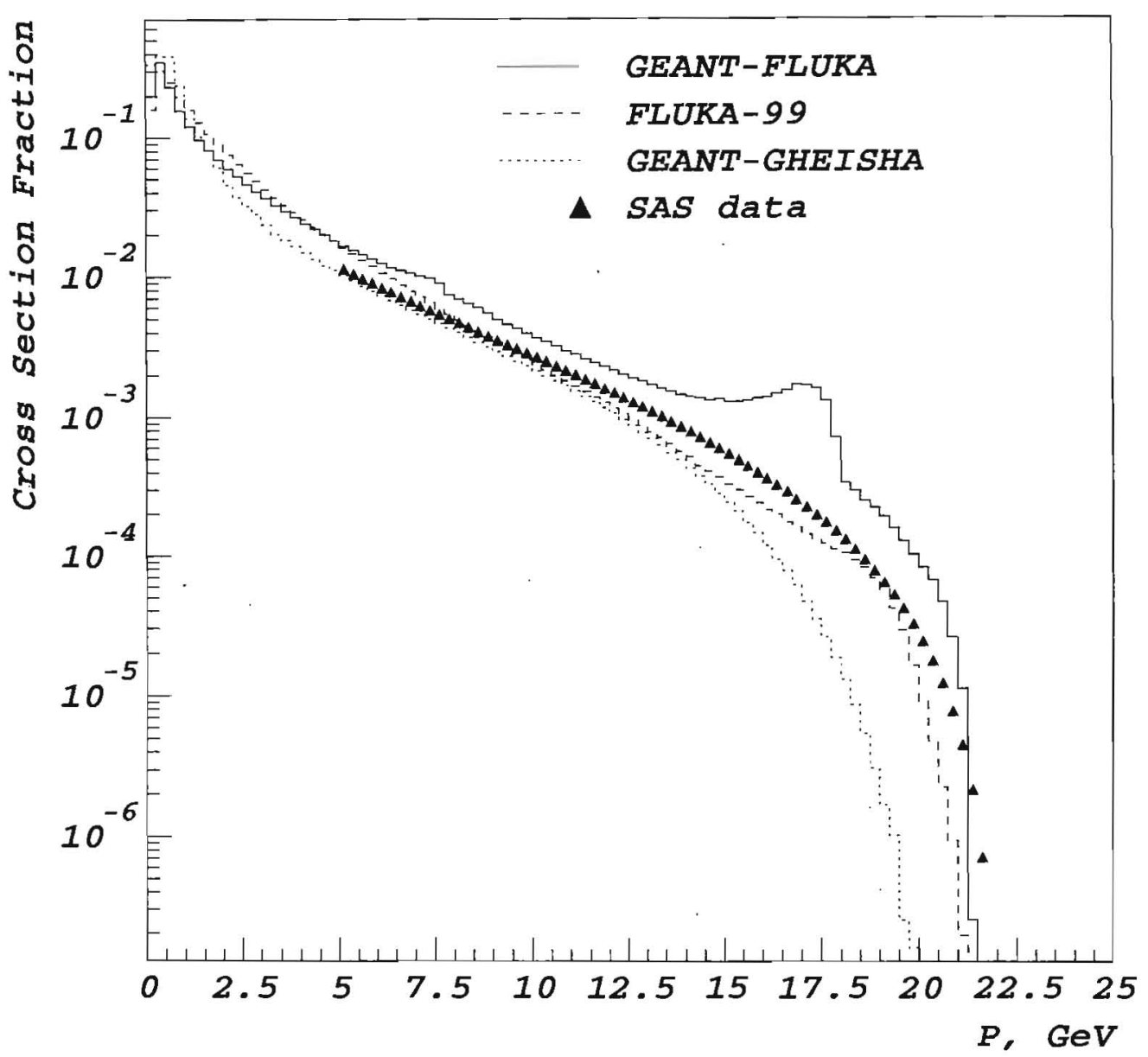

Figure 149: The inclusive inelastic $\pi^{+}$production fraction as a function of momentum from an incident $22 \mathrm{GeV} \mathrm{K}^{+}$on light nuclei. These fractions depend weakly on the atomic number of the struck nucleus. The SAS curve shown is from a straightforward fit in $\left(x_{F}, p_{t}\right)$. The curve is an extrapolation above $19 \mathrm{GeV}$. 


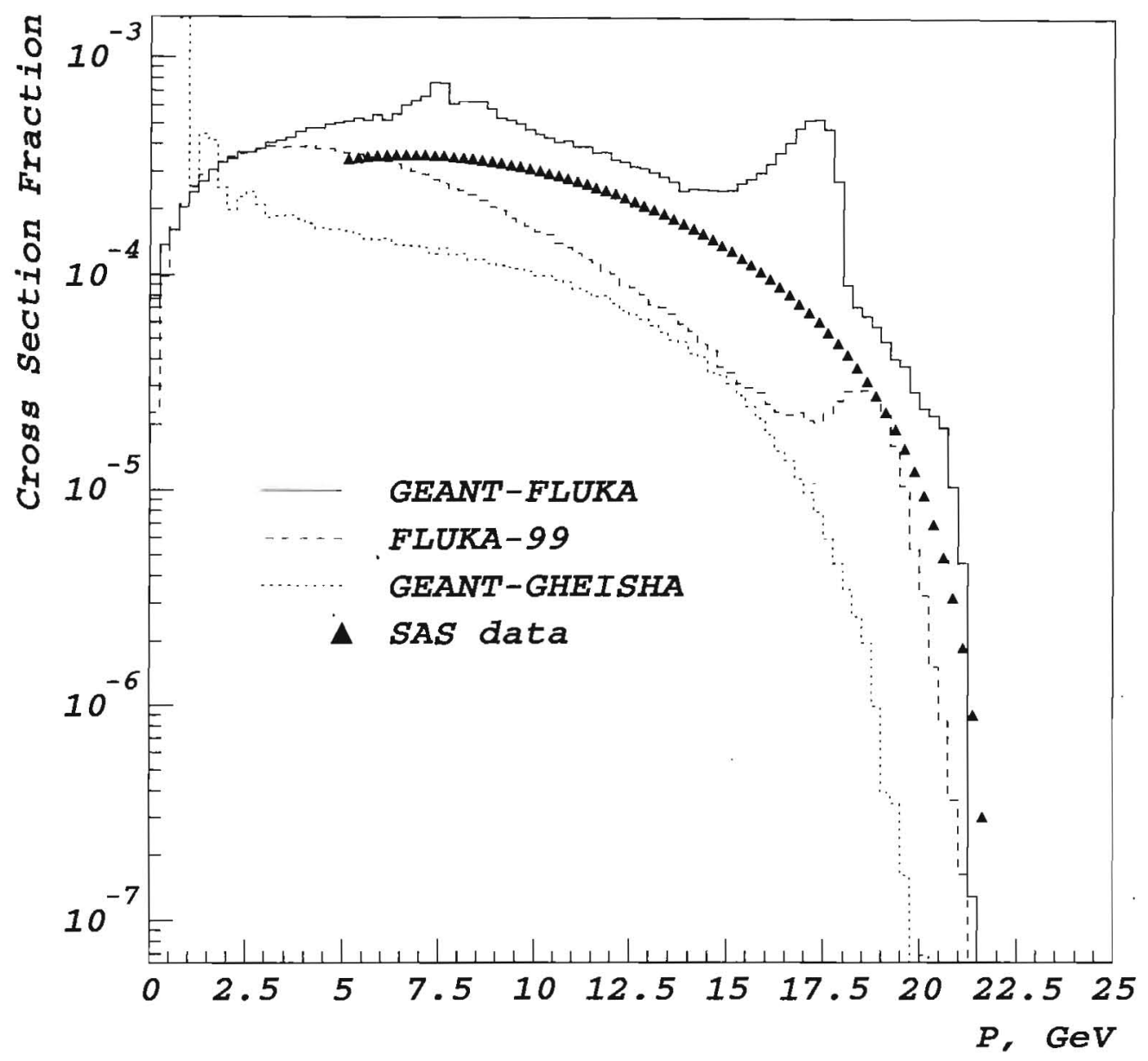

Figure 150: The SAS data and simulation results for $\pi^{+}$production into the forward region $(\theta<$ $15 \mathrm{mrad}$ ) from a $22 \mathrm{GeV} K^{+}$on light nuclei. These fractions depend weakly on the atomic number of the struck nucleus. The SAS data points are straightforward $\left(x_{F}, p_{t}\right)$ extrapolations of higher energy SAS data. The curve is an extrapolation above $19 \mathrm{GeV}$. 

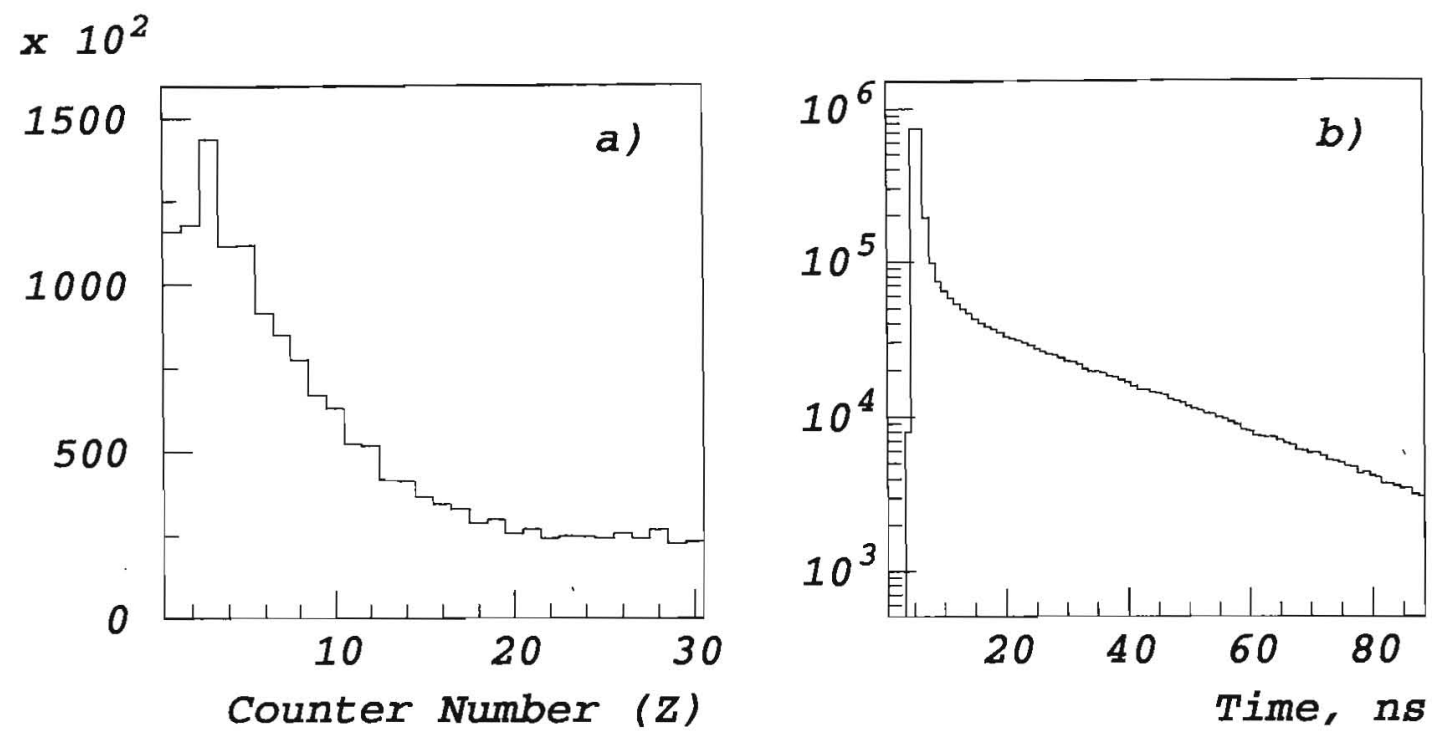

Figure 151: Figure (a) shows the distribution of hit BIVS photomultipliers as a function of longitudinal position. Figure (b) shows the distribution of hit arrival time with respect to a common trigger time.

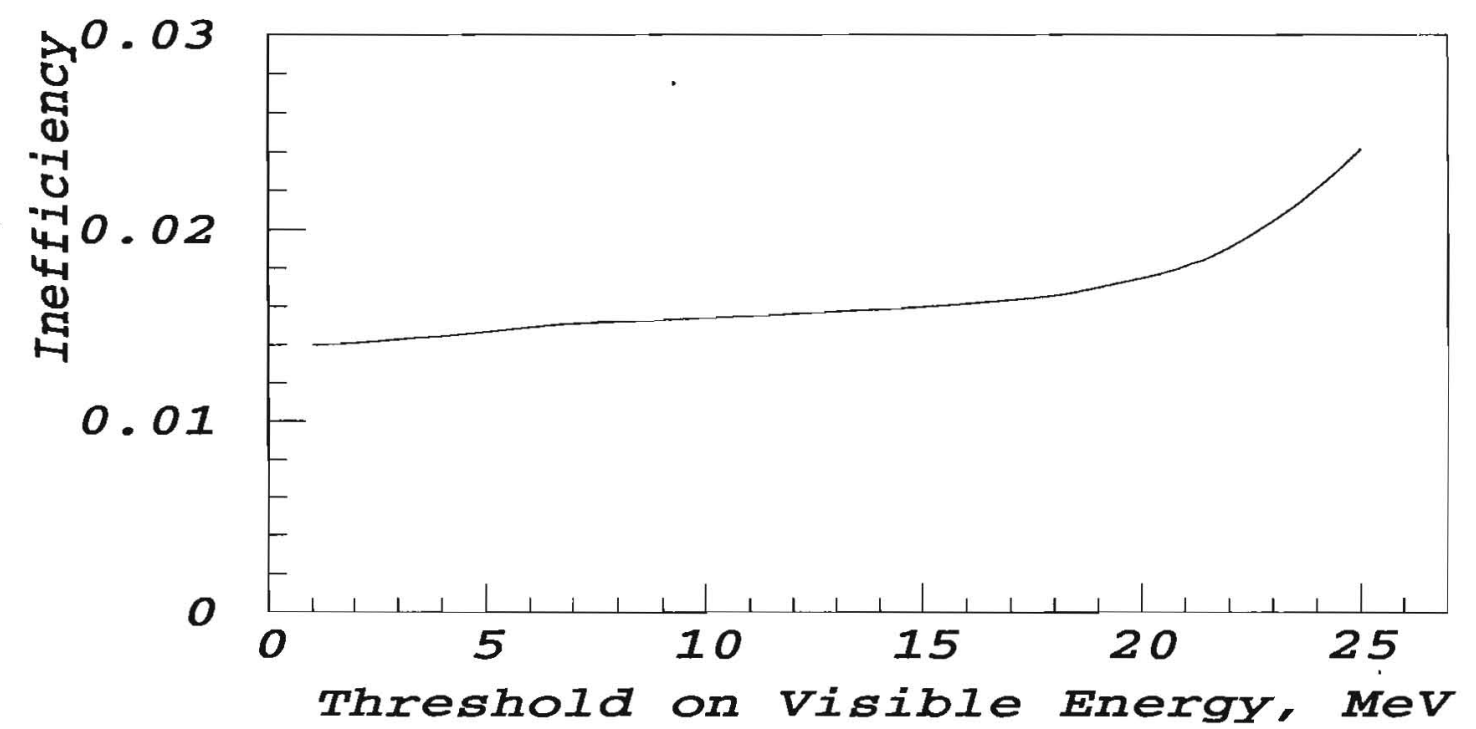

Figure 152: The dependence of BIVS residual inefficiency on the threshold of visible energy detected within a 5 nsec of $K^{+}$traversal. 
its quoted in the first edition, and significantly bolsters the case that this class of backgrounds is under control. Our current design of the KEAT and attendant vacuum window increases the relevant length of scattering from $4 \times 10^{-4} \lambda_{I}^{K^{+}}$(silicon in vacuum) to $6 \times 10^{-4} \lambda_{I}^{K^{+}}$. Our estimates of forward production yield are however considerably more robust, and are reduced by $\times 4$ with respect to our first analysis in the first edition. These factors together with the increase in $K^{+} \rightarrow \pi^{+} \nu \bar{\nu}$ acceptance reduce the level of expected beam interaction backgrounds to less than $2.5 \%$ of a $1 \times 10^{-10} K^{+} \rightarrow \pi^{+} \nu \bar{\nu}$ signal. This reduced background estimate doesn't include the reduced BIVS inefficiency as determined from FLUKA-99.

\subsection{Summary of Global Efficiency and Required Running Time.}

There are several global efficiency effects that reduce the signal acceptance presented above. These global inefficiencies generally result from reconstruction efficiencies and detector deadtimes not yet considered. Our deadtime analysis based on CKM_GEANT is presented in section 5.6.8. These deadtime sources are listed individually below in table 37 . Collectively these rate effects correspond to a global detector deadtime of $13 \%$ which is dominated by the $(6-7) \mathrm{MHz}$ halo muon rate.

In addition, our analyses have not yet included losses due to reconstruction of the $\pi^{+}$in the FVS and MVS. As discussed in section 5.9.3, our $\pi^{+} / \gamma$ separation cut has been shown to be at least $90 \%$ efficient for accepting $K^{+} \rightarrow \pi^{+} \nu \bar{\nu}$ events. As argued in section 5.10, the MVS can deliver at a. $95 \%$ pion efficiency for the required muon rejection of $1 \times 10^{-5}$.

Our base $K^{+} \rightarrow \pi^{+} \nu \bar{\nu}$ acceptance value determined from CKM_GEANT is based on simple pattern recognition and reconstruction algorithms executed on events simulated at hit level. We have yet to explore the robustness of these algorithms against rate effects that can corrupt reconstruction in the spectrometer. An example of such an effect is shown in figure 55, which illustrates broadening of the $\mathrm{K}^{+}$momentum resolution with the addition accidental tracks in the UMS spectrometer. Given the wealth of redundant information and timing resolution of each spectrometer; there is reason to be optimistic that even simple reconstruction algorithms can remain robust with rate. The algorithms employed on actual data however will no doubt exploit the \pm 1 nsec timing resolution of reconstruction primitives and the redundancy built into and between the spectrometers. We expect these advanced reconstruction techniques will come at the cost of some additional global inefficiency which we estimate at $5 \%$ in table 37 below.

These global efficiency losses collectively are $30 \%$ as shown in Table 37, reducing the final acceptance $1.6 \%$. It is worth noting that these losses affect the signal and backgrounds equivalently, so that the signal/noise ratio remains the same.

\begin{tabular}{|l|r|}
\hline Loss Mechanism & Inefficiency \\
\hline MVS deadtime (dominated by halo muons): & $4 \%$ \\
BIV deadtime (beam interactions): & $5 \%$ \\
VVS deadtime (dominated by $K^{+}$decays): & $6 \%$ \\
FVS $\pi / \gamma$ separation cuts: & $10 \%$ \\
MVS $\pi / \mu$ separation cuts: & $5 \%$ \\
Advanced Pattern Recognition Losses: & $5 \%$ \\
\hline Total global inefficiency: & $30 \%$ \\
\hline
\end{tabular}

Table 37: Losses to our signal acceptance due to deadtime and inefficiencies. 


\subsubsection{Estimated Yield of a Two-Year Run.}

To determine the estimated yield for a 2 year run, we have assumed the Main Injector can deliver $5 \times 10^{12}$ protons per 1 -second pulse with a 3 -second cycle time. For an Accelerator $\times$ Detector running efficiency we have assumed 120 hours per week (168 hours), which as a reference, is a reasonable representation of good running for the $\mathrm{KTeV}$ detector during the last fixed target runs. Further we assume that we will be running for 39 weeks/year, allowing 13 weeks/year for accelerator and detector maintenance and development. This combined clock efficiency is $54 \%$.

The nominal proton intensity corresponds to $30 \times 10^{6} \mathrm{~K}^{+} /$pulse entering the decay volume, $17 \%$ of which decay. All these factors combined correspond to a total flux of $5.8 \times 10^{13} \mathrm{~K}^{+}$decays from a two year run. Given our final acceptance of $1.6 \%$, this flux produces a yield of $95 K^{+} \rightarrow \pi^{+} \nu \bar{\nu}$ events for a $1 \times 10^{-10}$ branching ratio.

\subsection{Summary of Signal and Background Studies.}

The updated analysis of beam interaction backgrounds and the high fidelity of simple hit-based reconstruction algorithms support our assertion in the first edition of the proposal that backgrounds can be controlled in an in-flight experiment. We have augmented our Monte Carlo estimates of beam interaction backgrounds in the first edition with measured cross sections and real world experience of analogous veto systems. Further, as appropriately emphasized in the first edition, the CKM apparatus is a low-mass detector and hence beam interaction backgrounds can be directly measured by increasing material in the detector. Strategies for these measurements are discussed in section 8 .

Regarding $\mathrm{K}^{+} \rightarrow \pi^{+} \pi^{0}$ baçkground, we have shown that even the simplest hit-based reconst"'uction algorithms deliver kinematic resolution functions with a very low level of non-Gaussian t:al . Equally important we have demonstrated that the reconstructed missing mass in the two separate spectrometers are independent of each other, which is an essential criteria for redundant systems. We are continuing to refine our reconstruction algorithms and analyses to fully exploit the power of the spectrometers. As the CKM_GEANT simulation matures and we transfer our background estimates to that environment we fully expect $K^{+} \rightarrow \pi^{+} \pi^{0}$ and all other decay backgrounds to remain under control.

Complementing spectrometer development, we have shown that our proposed photon veto system performance has a significant safety factor over the nominal requirements which permits some breathing room in the tradeoff between acceptance and background rejection of $K^{+} \rightarrow \pi^{+} \pi^{0}$. This margin is a direct result performing the experiment in-flight at high energy, and is one of the key advantages of high energy. This and other safety factors are discussed in section 8 . 


\section{The Defensive Play Book}

Experimental studies of kaon decays have a history now exceeding 40 years. Indeed, without real experimental experience to this level of depth an experiment such as CKM would not be feasible. Not all of the lessons to be learned from this history are positive. Many of the ultra rare kaon decay experiments have required one or more iterations of upgrades beyond the initial plans of their proposals to achieve, or even approach, their design sensitivities. A general pattern emerges in which the real challenges presented by the backgrounds are underestimated. The acceptance left after all the cuts really required to control those backgrounds are applied falls short of the experiment's design sensitivity by a significant margin.

In this section we will address the measures we will take to avoid repeating this bit of history. Nothing here is to be taken as a claimed improvement to the experiment. Nor will anything here significantly compromise the measurement. It will illustrate that we can, and will, know our backgrounds before we spend several years taking sufficient data to achieve our sensitivity goals.

\subsection{Measuring the Backgrounds}

$K^{+} \rightarrow \pi^{+} \nu \bar{\nu}$ is a kinematically unconstrained decay mode. Everything left after the known backgrounds are extrapolated into the signal region and subtracted is the signal. It is imperative that the significant backgrounds be estimated reliably. By exploiting the redundant design of the experiment we will measure all the significant backgrounds in the factorization limit before we commit to a long data taking run to acquire the signal. By factorization we mean the selection of events clearly identified in one subsystem as background. With such samples we can safely study the behavior of the background in and near the signal region. The products of such background probabilities done with these different, samples yields an overall estimate of the background from a particular source, thus factorization.

This technique is the basis of the background estimates in BNL E787. (They call this the bifurcated analysis technique.) They have already demonstrated that backgrounds at the $1 \times 10^{-11}$ level in $K^{+} \rightarrow \pi^{+} \nu \bar{\nu}$ branching ratio can be reliably estimated in this way [6].

\subsection{1 $K^{+} \rightarrow \pi^{+} \pi^{0}$}

An application of the technique is in the $K^{+} \rightarrow \pi^{+} \pi^{0}$ which is the largest source of background. With a sample of events which contain cleanly detected photons from the $\pi^{0}$ decay we will measure the non-Gaussian resolution tails of the missing mass measurement from the pure background region near $m_{\pi^{0}}^{2}$ into the signal region near zero. Figure 147 is an example from E787 of just such an analysis, albeit in the center of mass. Conversely we will measure the $\pi^{0}$ veto inefficiency by reconstructing events with charged particle spectrometer information alone where the missing mass is consistent with only a $K^{+} \rightarrow \pi^{+} \pi^{0}$ decay. The $K^{+} \rightarrow \pi^{+} \pi^{0}$ background is the product of the $\pi^{0}$ inefficiency probability measurement and the $m_{\pi^{0}}^{2}$ mis-measurement probability measurement done with these samples.

Acquiring the data for these studies is nearly trivial. The rate of $K^{+} \rightarrow \pi^{+} \pi^{0}$ decays which pass fiducial cuts on the $K^{+}, \pi^{+}$and decay vertex is $3 \times 10^{8}$ per beam hour at full design intensity. This data volume is likely beyond the digestive capabilities of the trigger and DAQ systems. The same data set could be acquired in less than a week of data taking with unseparated beam at the same $50 \mathrm{MHz}$ total beam intensity with a kaon beam fraction of $1 \%$. Such data can be taken with a negligible number of machine protons and with a less than fully capable DAQ before the RF separation is operational, or even installed in the beam line. Such a data sample, after the strict 
cuts necessary to define it as pure $K^{+} \rightarrow \pi^{+} \pi^{0}$, would have $\sim 1 \times 10^{8}$ very clean events. These samples would yield:

- $\sim 10$ events with undetected $\pi^{0}$ 's assuming we achieved our $\pi^{0}$ specification of inefficiency of $1.6 \times 10^{-7}$

- $\sim 1000$ events with $m_{\pi^{0}}^{2}$ reconstructed in the signal region assuming we have achieved our design rejection of $3 \times 10^{-4}$.

Background to these control samples from non- $K^{+} \rightarrow \pi^{+} \pi^{0}$ decays must be kept small compared to the inefficiency under study. For the measurement of spectrometcr inefficiency using $\pi^{0}$ tagged events potential problems come from $K^{+} \rightarrow \pi^{0} \mu^{+} \nu$ with the $\mu^{+}$mis-identified. At the expected level of this inefficiency $\left[\sim 1 \times 10^{-5}\right]$ none of these backgrounds are significant. For the measurement of the $\pi^{0}$ detection inefficiency, whose design value is $2 \times 10^{-7}$, with events kinematically reconstructed with a $\pi^{0}$ missing mass the backgrounds are also well under control. The cleanliness of these samples is achieved by being profligately inefficient in utilizing these data to measure our inefficiencies: we can cut as much as necessary to get a clean sample. For example we can require a large hadronic shower in the muon veto system after a clean 1 MIP signal in the FVS to insure that the daughter charged track is really a $\pi^{+}$. A potential pitfall witl the factorization technique is that in order to be allowed to multiply the resulting probabilities they must really be independent of each other. In lowest order this is enforced in $K^{+} \rightarrow \pi^{+} \pi^{0} \rightarrow \gamma \gamma$ by the introduction of two completely uncorrelated random variables between the two decays in this chain, the CM decays angles of the $\pi^{0}$. The photon inefficiency is almost entirely controlled by the $\pi^{0}$ decay angles. We miss events with one photon nearly along the $\pi^{0}$ line of flight and the other photon at very low energies and large angles in the lab. The kinematic and measurement properties of the charged tracks are identical for symmetric and very asymmetric $\pi^{0}$ decays. Care will have to be taken to not over-estimate the mis-measurement probability due the the remnants of the electromagnetic shower of a photon in the VVS adding hits and confusing the measurement of the $\pi^{+}$in the DMS. There are no clear mechanisms by which the measurement of the charged particles can cause an apparent increase in the inefficiency of the photon veto system. There are various higher order processes, like bremsstrahlung and interactions, by which the charged tracks can decrease the veto inefficiency. These effects are both small and exactly the ones we wish to include anyway. This sample is the background we are trying to kill.

\subsubsection{Interactions}

The most difficult background sources to reliably estimate from simulations alone are those from beam particle interactions in the material of the detectors just upstream of the decay volume and from the residual gas in the decay volume. As illustrated in figures 149 and 150 neither the dynamics embodied in event generators nor the experimental data which they are tuned to reproduce are particularly robust for $22 \mathrm{GeV} K^{+}$interaction on low $\mathrm{Z}$ materials.

It is completely straightforward for us to measure these background in-situ by adding material to the beam line at the relevant places. For example, we are most sensitive to interactions in the last station of the KEAT. This detector along with the vacuum window comprise $\sim 6 \times 10^{-4} \mathrm{~K}^{+}$ interactions lengths of material. A $1 \mathrm{~cm}$ thick scintillator inserted into the beam at this point increases that interaction length by a factor of 10 . Similarly, by increasing the pressure in the vacuum decay volume about our design level we can have interactions in that gas at any level we might wish. 
The increase in multiple Coulomb scattering associated with this added material, while significant, still does not dominate the MCS budget from the RICH gas and mirrors already in the beam. The resolutions remain largely unchanged.

Recall from section 13.5 that $K^{+}$interactions in the material of the KEAT require a $\pi^{+}$scatter in the first DMS station material for the interaction to fake a decay which appears to originate in the fiducial decay volume. Again applying factorization these two processes can be measured with separate samples. The probability that a $K^{+}$interacts in KEAT material to produce a high momentum $\pi^{+}$and no detectable signal in the BIV for photon vetoes is directly measurable from events which form a vertex in the added material in the KEAT region. Since that material is scintillator, and therefore active, light from recoil protons in the scintillator can be used as a further tag of a $K^{+}$iteration Quasi-elastic processes dominate this background source. Half or more of those sliould be taggable from recoiling target fragments. The probability that $\pi^{+} s$ scatter in a DMS plane can be measured directly using $K^{+} \rightarrow \pi^{+} \pi^{0}$ events where the $\pi^{+}$scatters in the second or third station making an observable kink in the non-bend plane. Folding these two probabilities together will yield the expected rate of this background per interaction length of scintillator added.

For beam gas interactions, which already occur in the fiducial decay volume, no second scatter is required. We will measure this background rate directly as a function of decay volume gas pressure and extrapolate to our nominal operating pressure.

\subsubsection{Accidentals}

An event in CKM is a few hundred nsec time window surrounding the time of a trigger. We have, on average, a beam particle every $20 \mathrm{nsec}$ and a muon every $100 \mathrm{nsec}$. We will average several of each in each event. These data are a semi-infinite source of out of time events (accidentals) which get recorded automatically.

We will use these data in several ways. The traditional approach of adding data and random events on top of each other can be done as a function of the separation in time between the two events. We will study the slower detectors on "real" accidentals where we can resolve two particles in time in the RICHs, for example, but not in the trackers in the magnetic spectrometers.

These data also allow us to know that the detector is alive. There are, on average $\sim 10$ other beam particles and several halo muons that are accidental neighbors in time to any given trigger. They tell us about the instantaneous behavior of both the beam and the detector at the time of the trigger. We can know, for example, if one of the photon vetoes goes to lunch for a millisecond. We also have the very nearly in-time activity in the detector for each event. The allow both the identification of "dead" or "recovering" channels due to activity prior to the trigger. It also tags channels which are active in non-veto systems which are still on from earlier activity. It also tells us that at a coarse level the whole detector is on for a trigger. We sample at shorter time interval that the time constant for any detector to go off. A useful check will be to study out of time activity in the signal sample events in comparison to less refined data sets.

\subsubsection{Other Backgrounds}

The only background we understand to be significant are $K^{+} \rightarrow \pi^{+} \pi^{0}$ and interactions. The same techniques we discussed above can be adapted to deal with the other potential background sources. For example we have two muon identifiers, the pion RICH and the MVS. The factorization technique can and will be applied to background sources like $K^{+} \rightarrow \mu^{+} \nu_{\mu}$ and $K^{+} \rightarrow \mu^{+} \nu_{\mu} \gamma$ if only to confirm that they are not significant sources of background events. 


\subsection{Measuring Detector Response Functions}

The factorized data samples provide the ideal environment to directly measure the detector response functions for all the detector subsystems. Kinematically identified $K^{+} \rightarrow \pi^{+} \pi^{0}$ decay are a source of tagged $\pi^{0} \mathrm{~s}$, etc.

Measuring the VVS inefficiency is, in fact, our hardest case. We can measure the response of the FVS to tagged photons from kinematically tagged $K^{+} \rightarrow \pi^{+} \pi^{0}$ event with a clean photon observed in the VVS. Using conservation of momentum we can do a reasonable job at pointing the missing photon at the FVS; requiring, in addition, the absence of other signals in the VVS or the pion RICH to control conversions and Dalitz decays. Due to our finite momentum resolution this technique will not work for low energy photons in the VVS. We will have no ability to point photons with low energies. These are the photons we need to veto; we have full coverage for them. We won't be able to measure the differential inefficiency in energy or position. We will directly measure the relevant integral inefficiency for low energy photons. We know both the spectrum and the basic behavior of the low energy inefficiency (see figure 19) so we can model our results to extract a VVS inefficiency as a function of energy.

This will not be adequate. We have plans for a test beam effort with a real prototype VVS module in a tagged photon beam. This work has already begun in Russia with measurements using the 4.3 MeV tagged photons from a Pu-Be source.

\subsection{Other Analysis Techniques}

This measurement poses and interesting analysis challenge. We wish to measure the rate of an ultrarare, kinematically under-constrained decay mode. We have set the design goals of the experiment as if it were a search; beat the background into the ground so that any excess remaining can be confidently ascribed to the signal.

OK, let's assume we've done that. We have a sample of 104 events with a predicted background of 9. What is the optimal analysis to determine the branching ratio? The statistical uncertainty doesn't seriously degrade due to the fluctuations in the background until the signal to noise ratio approaches 1 . The cut and sample which optimize the measurement of the branching ratio might be less stringent than those required to rediscover the signal.

There are no factors of 2 to be gained here. At best there would be a small improvement in the statistical uncertainty in the branching ratio measurement. This technique might help reduce some of the systematic uncertainties by allowing one to relax cuts which are cutting hard and therefore potentially sensitive. The usefulness of this approach will depend completely on the details of the yields on signal and backgrounds in the cuts parameters to be varied.

\subsection{Safety Factors}

There are several places in the design of the experiment where we are being conservative in our claims. This is the only prudent approach given the nature of this measurement. We enumerate here this list as we understand it now.

\section{Beam Flux / Front End Bandwidth}

Our design specification for the beam line and beam detectors is $100 \mathrm{MHz}$ of secondary flux - twic sur nominal design for the experiment as a whole. This specification has two motivations; it increases the likelihood that will achieve our $50 \mathrm{MHz}$ requirement without having all systems straining to achieve their design goal. Design goals have a way of quickly becoming 
designed in upper limits. It also allows the opportunity to push the apparatus to higher rates once we have real operational experience on where it fundamental limitations really are.

It seems imprudent to design a new experiment that has fundamental limitation which limit it to using use $<20 \%$ of the potentially available beam. We understand full well that the ability to go to higher rate is necessary, but not sufficient, for us to actually get more beam.

2. High Energy Photon Inefficiency

We have made a reasonable case in section 5.6.10 that the physical limits to our high energy photon veto inefficiency are considerably below of specification of $3 \times 10^{-5}$. Our $K^{+} \rightarrow \pi^{+} \pi^{0}$ background is directly proportional to this number. We should to be able to achieve $1 \times 10^{-5}$, a factor of 3 better than our specification. If this can be demonstrated then we have a significant safety factor in the rejection of $K^{+} \rightarrow \pi^{+} \pi^{0}$ background. This will provide us with a defense against problems in the spectrometers which could limit their kinematic rejection or require us to lower their efficiency in order to achieve the necessary background rejection.

We plan to take full VVS and FVS prototypes into electron test beams in order to measure the real limits to our high energy photon inefficiency.

3. Magnetic Resolution

It is clear from figure 13 that the magnetic miss-mass resolution is dominated by the measurement of the $\pi^{+}$momentum in the DMS. In principal we can linearly improve the momentum resolution of the DMS by increasing the field of the spectrometer magnet. The present value corresponds to a $p_{T}$-kick of $100 \mathrm{MeV}$. There is no significant technical problem with a $200 \mathrm{MeV}$ $p_{T}$-kick at least as far as the magnet is concerned. The $\mathrm{KTeV}$ magnet, which is quite similar to our requirements, has a $400 \mathrm{MeV} p_{T}$-kick. The effect on the photon veto inefficiency due to photon conversion and Dalitz decays where the electrons get curled up in the field and the increase of the size of the beam hole in the FVS requires study. We will look at this again when our simulation tools are more advanced.

4. Region 2

In section 3.2 and figure 11 we discussed "Region2 "which is the kinematical region where the di-neutrino missing mass is above $m_{\pi^{0}}^{2}$ and below the $K^{+}$three body decay modes. In decay at rest experiments this is the region "below the $K^{+} \rightarrow \pi^{+} \pi^{0}$ peak" in $\pi^{+}$momentum. The combination of matrix element and available phase space makes this region potentially about as sensitive as Region 1. (Region 2 is mass range $0.028<M_{\text {miss }}^{2}<0.050$ on figure 162) The problems are a set of additional backgrounds like $K^{+} \rightarrow \pi^{+} \pi^{0} \gamma$ which inust be controlled.

This region has significant physics import even if the cuts necessary to control the backgrounds reduce the acceptance. A fundamental prediction of the theory is that the matrix elements including form-factors for $K^{+} \rightarrow \pi^{+} \nu \bar{\nu}$ and $K^{+} \rightarrow \pi^{0} e^{+} \nu$ should be identical to the levels of statistical precision we car reach. Any new physics could show itself as a deviation from this prediction. This form-factor is essentially constant across Region 1. Region 2 has a significant lever arm with which to measure a deviation from the predicted form-factor.

An analysis is underway now in E787 to attempt a search in Region 2. Assuming a successful outcome of this analysis then an extension of this study into E949 data should yield a signal. It is premature to undertake a serious study of CKM's potential in Region 2 until the physical sources of background are known from E787 and E949. We will do this when that information is knowm. It does not follow that if E787 cannot make the search then CKM 
cannot either. Several of the important backgrounds are associated with the interactions of low energy particles with the material in the E787 stopping target. These backgrounds would not necessarily limit CKM. 


\section{Siting and Conventional Construction}

\subsection{Siting Constraints}

The configuration for the CKM experiment utilizes $22 \mathrm{GeV}$ kaons from a Superconducting RF Separated beamline, designed to deliver a flux of $3 \times 10^{7}$ positively charged kaons in a 1 second spill. In the design of this proposal, the detector requirements as well as the beam requirements are developed to a stage that allowed consideration of the location of the experiment. Consideration of existing facilities in the laboratory, or the need to design new facilities, have been studied as well, keeping in mind that physical constraints of the experiment and beam are not expected to change significantly in the near future. With the uncertainty of the laboratory schedule, other commitments of facilities under consideration, and priorities of the laboratory management, this narrative makes no assumptions about the availability of present or future facilities. We have concentrated on the most compatiblc domain of existing territories in the designated Meson area.

There are four general areas of consideration, and in some cases constraints. These areas include the experimental geometry, a general description of the Meson beamline sites, preliminary ideas on construction, and initial thoughts on the environmental assessment. A more detailed analysis including more information on the muon backgrounds and shielding, are integrated into this proposal. It is important to state that one of the major fixed target areas using the Main Injector beam at Fermilab considered for future operations is the Meson area. A scaled layout of the Meson area is shown in Figure 153. The design of the beam also poses a constraint. There are three general sections located after the target and the momentum selection, namely a RF separator section, a 'clean-up bend section' of the beam to keep the secondary muon flux away from the detector, and the experimental detector region. The need for this final bend and the cost in muon contamination are discussed in Section 4.

\subsection{Geometry Constraints}

In evaluations concerned with siting the relatively recent $\mathrm{KTeV}$ facility, a number of options were considered. Primary among the constraints. regarding utilizing existing buildings was the size and shape of the detector. A long detector, large decay region, and increased beam intensity resulted in a recommendation that led to construction of a new facility, rather than the use of an existing experimental hall. This result was also highly coupled with the upgrade of an existing beamline. The proposed CKM experiment has similar requirements for the detector apparatus, and some unique requirements for a new beamline that uses Main Irjector primary protons, cryogenic and $\mathrm{RF}$ equipment, and controls.

\subsection{Superconducting RF Beamline}

A number of beamlines have operated in the Meson area over the past 28 years, including beams utilizing superconducting elements. Our present effort must couple the efficient extraction and transport of the Main Injector primary beam with the location and operation of the superconducting RF separated secondary kaon beam (SCRF). The superconducting RF separated beam has a number of features that influence the design, operational efficiency, and the possible location of this beam in existing areas. Existing locations take into account the detail beam design and constraints imposed by the requirements of the CKM experiment.

To meet the experimental requirements, the beamline should deliver a kaon rate of $30 \mathrm{MHz}$ using $5 \mathrm{E} 12$ protons per pulse from the Main Injector accelerator. The RF separated secondary beam is expected to separate out a pure Kaon flux over all other hadrons. The beam is approximately 210 

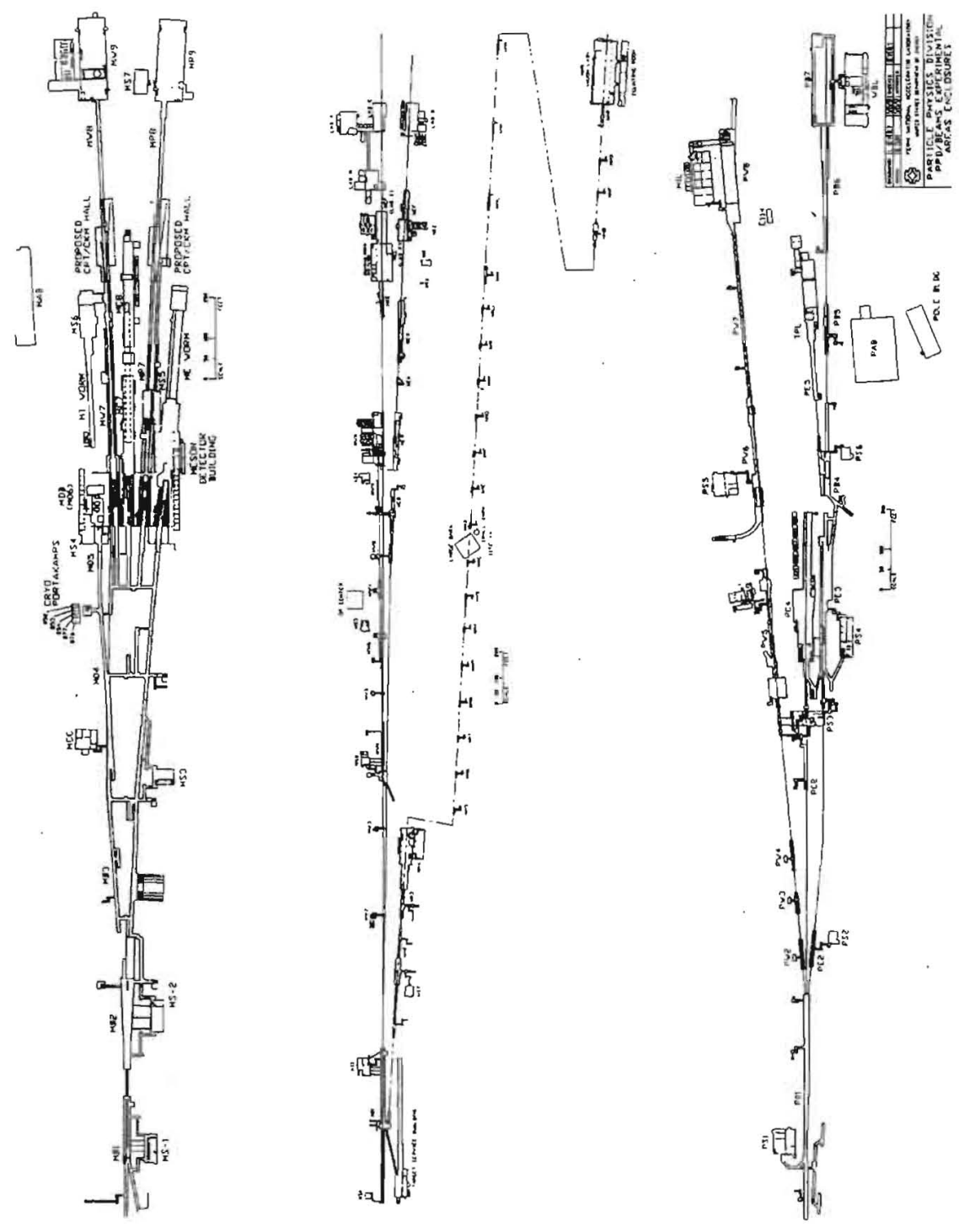

Figure 153: Fixed target experimental areas. The Meson Area is on the left. 
meters long characterized by an initial targeting and momentum selection section, followed by two superconducting RF deflector stations nearly 85 meters apart, followed by a beam plug device to absorb the unwanted particles after separation, and a final focusing section for transport to the CKM detector.

Unlike conventional fixed target beamlines, this beamline requires superconducting RF cavities, and the associated cryogenic equipnient to support and operate the cavities. An engineering sketch of the proposed cryostat units showing the configuration of two half meter RF cavities is presented in Figure 28. A station consists of 3 meters of RF cavity and contains three cryostats per station. The frequency of the cavities (designed for $3.9 \mathrm{GHz}$ ), and the separation between the RF stations is a constraint chosen to fit the particle $\mathrm{RF}$ phase requirements of the beam design. Flexibility in the properties of the beam is expected if the design goal of $\sim 5 M \mathrm{~V} / \mathrm{m}$ in cavity field strength is not met or exceeded. These particular features, along with the location of the target station and space needed for the final focus and clean-up bends, restricts the location of this beamline. The enclosure space around the RF stations must be sufficient to include the controls equipment and piping for the liquid cryogen necessary to keep the RF cavities at cryogenic temperatures.

\subsection{Meson Area Sites}

There are a number of historical beamlines in the Meson area, including in some configurations, MP (meson polarized beam), MT (meson test beam), ME (meson east beam), MW (meson west), MC (meson center), and at one time MB (meson bottom). Not all of these beamlines are compatible with the expected new fixed target configuration without extensive redesign and modification. Since the facility requirements for the RF separated beam and the proposed CKM experiment are presently highly developed, some of the possible sites in the Meson area were deemed not feasible, due to estimated large capitol cost. required to prepare these areas for Main Injector beam. Although not exhaustive, we have concentrated the limitations to a few areas of consideration, namely the connection to the Switchyard 120 design (SY120) [193], existing enclosures that would need the minimum of modification, an area for the target station, the length of an existing beamline, and in some cases a modified existing experimental hall that could accommodate the CKM experimental apparatus.

\subsubsection{MP Beamline}

The MP beamline will receive primary beam from the Main Injector through a reconfigured switchyard, included in the Switchyard 120 Project (SY120). This project is initially applicable to other beams mentioned above, but it is important to note that the preliminary SY120 design extracts beam to the Meson area, then later provides beam to the MT area, primarily. Over a year after this work, the three way split will be revised and then provide beam to three different areas. The impact of this current SY120 project plan is not expected to impose further constraints on the current proposal for CKM. The beamline enclosure extends from far upstream of the Meson Detector Building (MDB) near the M05 cross over tunnel enclosure, and runs downstream to the MP9 Experimental Hall, as shown in Figure 153. This region can accommodate either an upstream or downstream scenario for the facility, (each length in excess of 400 meters). Existing beam pipe is also in place from the switchyard to $\mathrm{MDB}$, (for all current beamlines). Portions of these spaces are sufficient for the expected 60 elements that make up the beamline. Cross sections of various tunnels may be sufficient (in the current configuration), in regions where superconducting RF cavities cryogenic equipment can be placed. This equipment is unique to some beamlines, but similar in scope to the usual elements of magnets, collimators, and monitoring devices normally found in beamlines. 
Precise locations depend on the position of the target station, elevation above the existing floor, and a final bend for muon cleanup. Figure 154 presents a sketch of the Kaon facility overlaid on existing areas of the ME beamline and MP Experimental Hall. Both the downstream region and the upstream region utilize existing space and tunnels. Although some of the additional required enclosures exist, some modification of them will be required.

\subsubsection{ME Beamline}

A region near the MS3 service building cross-over to the MDB totals approximately 200 meters. Although in some areas, the cross sections of the tunnels are smaller than the MP tunnels, there is continuous tunnel into the $\mathrm{MDB}$ an beyond. An additional 100 meters is required for the detector, as well as the needed detector environment. The area downstrearn of the MDB is known as the 'WORM'. Previous experiments have been located and operated in this area. However, an engineering assessment would be required to determine the modifications needed to construct the CKM experiment in this area. The proposed configuration of the experiment does not fit in the MDB. This condition supports a proposed location for the target building, RF separator section, and subsequent beam section and experimental hall downstream of the existing Meson detector building.

\subsubsection{Experimental Apparatus}

The geometry for the CKM experimental apparatus is shown in Figure 42. In its current design, the detector portion of this apparatus is approximately 100 meters long, with varying cross sections and a cross section limit of approximately 3 to 4 meters. The major portions of the apparatus include an upstream magnetic spectrometer including a kaon RICH counter; a vacuum decay region, which includes a downstream magnetic spectrometer system; and a downstream pion RICH counter, muon system, additional veto system, and beam dump. Preliminary engineering design of the vacuum system indicates a cylindrical geometry, about 40 meters long, with approximately a 1.9 meter diameter cross section for the vacuum tank. Completely surrounding this tank are ports and connections along the entire length (at periodic intervals) for the vacuum veto photomultiplier tubes. This geometry constrains the apparatus to be centered on the beam, but well above the floor level in order to access the surrounding detectors and match the existing elevation of the Meson area beamlines. The proposed design of the vacuum veto modules is shown in Figures 78, 79. Electronics equipment space for the four plane $\mathrm{X}-\mathrm{Y}-\mathrm{U}-\mathrm{V}$ drift chambers is also needed in this area, as well as numerous cables connecting to all of the instruments. Since many detector components are inside the vacuum, view ports will be required for drift chamber surveys, as well as sufficient transverse space and distance conducive to laser surveys and laser position monitoring. The vacuum section also includes straw tubes surrounding and bordering a magnetic field region. This section will include numerous sheets of lead and scintillator. Due to the massive and delicate nature of the instrumentation for many of these elements, a crane is required, capable of off loading and lifting the detector elements into position. This crane is also a safety constraint (and large detector units are expected). Experience gained from operating the $\mathrm{KTeV}$ experiment, and analysis of $\mathrm{KTeV}$ data, suggest that a reliable temperature controlled environment is required to maintain stability.

Figure 155 shows a 'scaled sketch' of the superposition of the CKM detector and the existing MP9 experimental hall. This is not an engineered, precise drawing, but a suggested match to the constraints already discussed in this proposal. Since the beam dump does not have significant instrumentation requirements, placing or burying this element beyond the downstream wall of the present building does not create a significant technical difficulty, but optimizes an adjustment in 


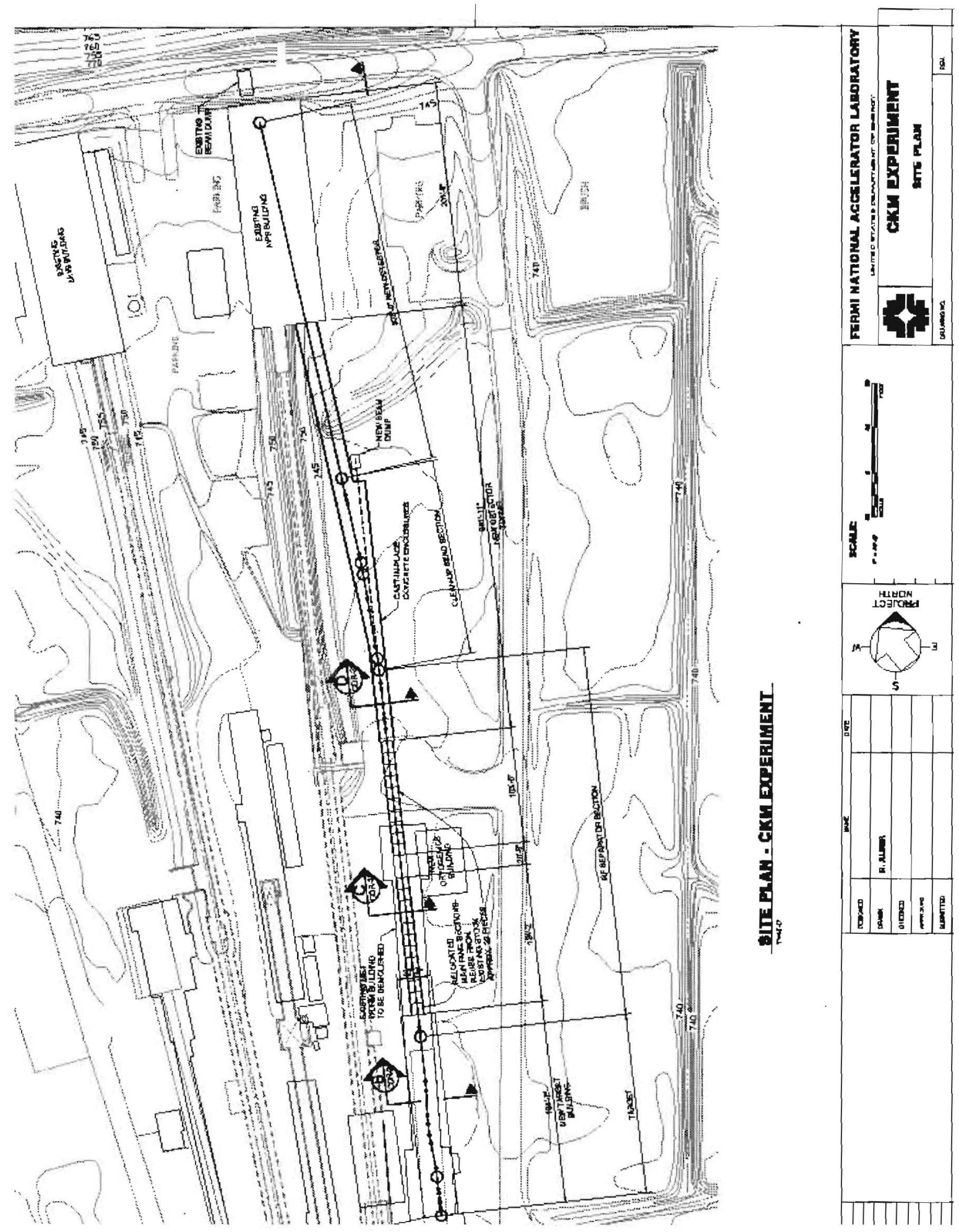

Figure 154: The MP9 experimental hall configuration. 

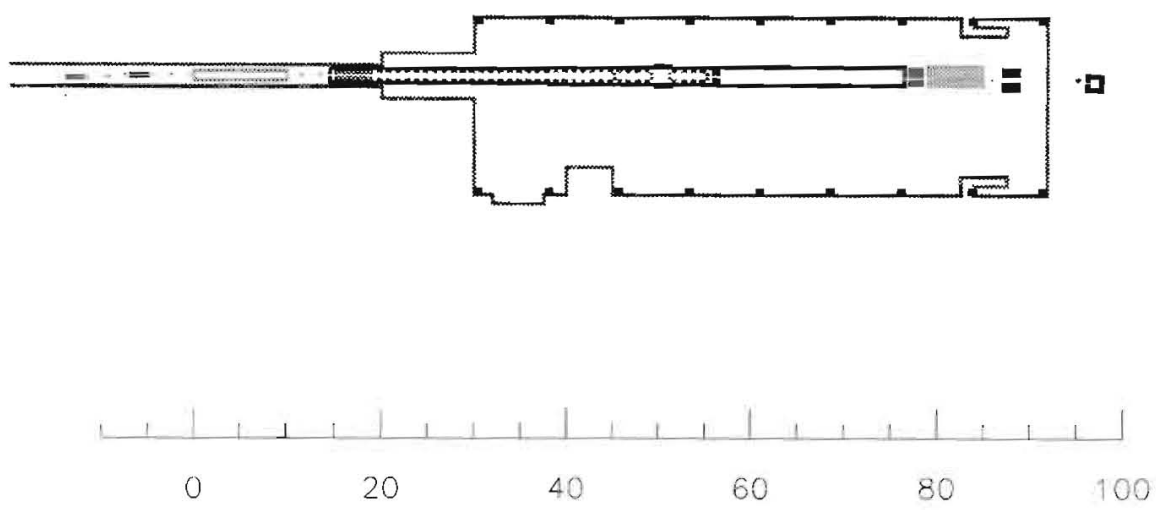

Figure 155: The CKM detector and the existing MP9 experimental hall. (meters)

position of the overall length. The upstream detector elements also fit within the existing beamline tunnel. The upstream tunnel-enclosure area cross section is approximately 3.2 meters diameter, and the far upstream beamline tunnel leading to this hall is about 2.9 meters diameter. Of course, a detailed engineering assessment of this experiment-hall configuration (or alternatives) would provide a better understanding of the possibilities, but with modifications this possible location seems to fit many needs of the CKM experiment detector. However, the competition for existing property is also a factor for consideration in the optimal use of laboratory resources. As a result of this competition, the CKM experiment also considers alternative locations for housing the experimental apparatus, requiring a new experimental hall. We have been explicitly asked by the Fermilab Directorate to consider a new experimental hall which leaves MP9 for other purposes. We discuss this option below.

\subsection{Construction}

It is expected that some construction is required for areas of the beamline; driven by the physical constraints of the beam design, and possibly radiation shielding requirements. Design characteristics, beam yields, and associated muon background fluxes studies have been extensively simulated. A combination of operations may be employed to reduce the muon background at the experiment, but also measures will be considered to contain the radiation field generated from muons all along the beamline. The bend in the beamline after the separator beam dump will limit the direction of the muons produced from that dump source.

\subsubsection{Target Enclosure}

All configurations of the beamline include the design and construction of a target station. A preliminary lay out of a target station that fits the current beamline design is shown in Figure 156. A detailed model and analysis of this target pile is discussed elsewhere. [134] The target, magnetic elements, and shielding portions fit in an area 147 feet long by 25 feet wide. This configuration is expected to maintain effective shielding for primary beam at $120 \mathrm{GeV}, 3$ sec repetition rate, $5 \mathrm{e} 12$ ppp, resulting in a residual dose of no more that $100 \mathrm{mrem}$ on contact outside of the immediate shielding wall after 30 days operation and 1 hour cool off. 


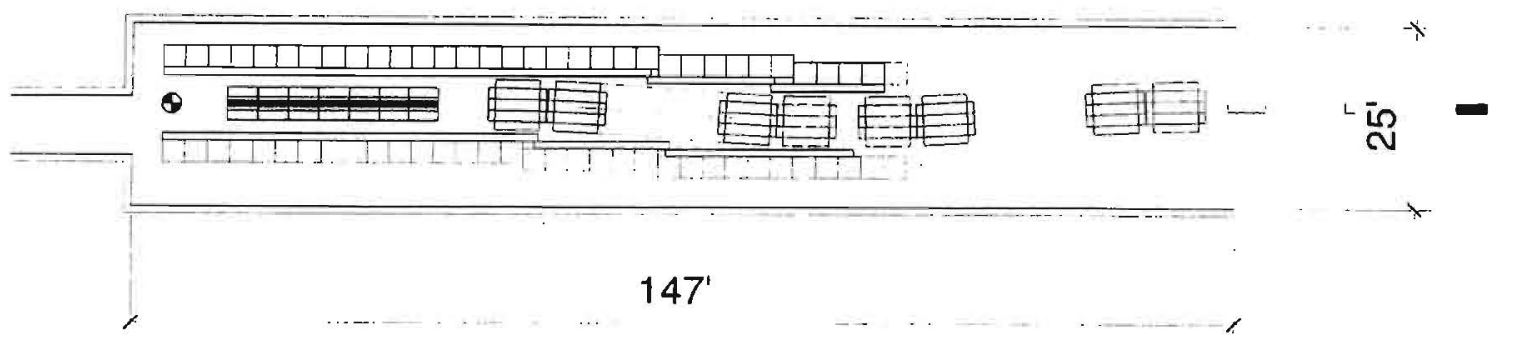

Figure 156: Target enclosure.

One of the reasons for either re-configuring or redesigning a new target station is adherence to any new regulations concerning radiation shielding; particularly ground water contamination shielding. The configuration for the target station presented here is part of a beamline that is approximately 210 meters long; stretching from the target to the last magnet element of the beamline before the detector. (Note that the detector is an additional $100 \mathrm{~m}$ long). Choosing the location of a current existing target station is problematic at best, since the length of the beamline is a constraint and adding more distance from the end of the beamline to the detector reduces the expected flux of kaons at the detector due to particle decay, and increases muon flux. For example, placing the target station in the Meson Detector Building would add nearly 40 meters to the decay length for the beamline assuming the detector was located in the nearest available existing experimental hall in the MP beamline. Therefore the proposed location for these facilities is a new target station in the ME beamline located to minimize loss of kaons from particle decay, maximize kaon beam purity and optimize the elements for radiation shielding.

\subsubsection{Experimental Hall}

It should be pointed out that the construction of a new experimental hall similar to the MP9 hall would cost approximately $\$ 2 \mathrm{M}$, according to a current FESS estimate of $\$ 150 /$ square foot. This estimate does not include EDIA, administrative contingency, or any upgraded power for experimental equipment, etc. This particular cost helps compare options such as using (and modifying if necessary) an existing experimental hall, building a new facility, or limiting the experimental requirements to fit available real estate.

A configuration is presented in Figure 154 which shows construction of the target hall in the extension downstream of the $\mathrm{MDB}$, a secondary beamline incorporating new enclosures and existing relocated main ring tunnel sections, matching into connecting space with the existing MP9 facility. This configuration also requires modification of the ME worm and some modification of the MP9 experimental hall. It should also be pointed out that this configuration does not impact other possible experiments located in the MDB. An alternative proposal is shown in Figure 157, which does not reflect utilization of the MP9 facility and illustrates the location of a new experimental hall, previously discussed. 


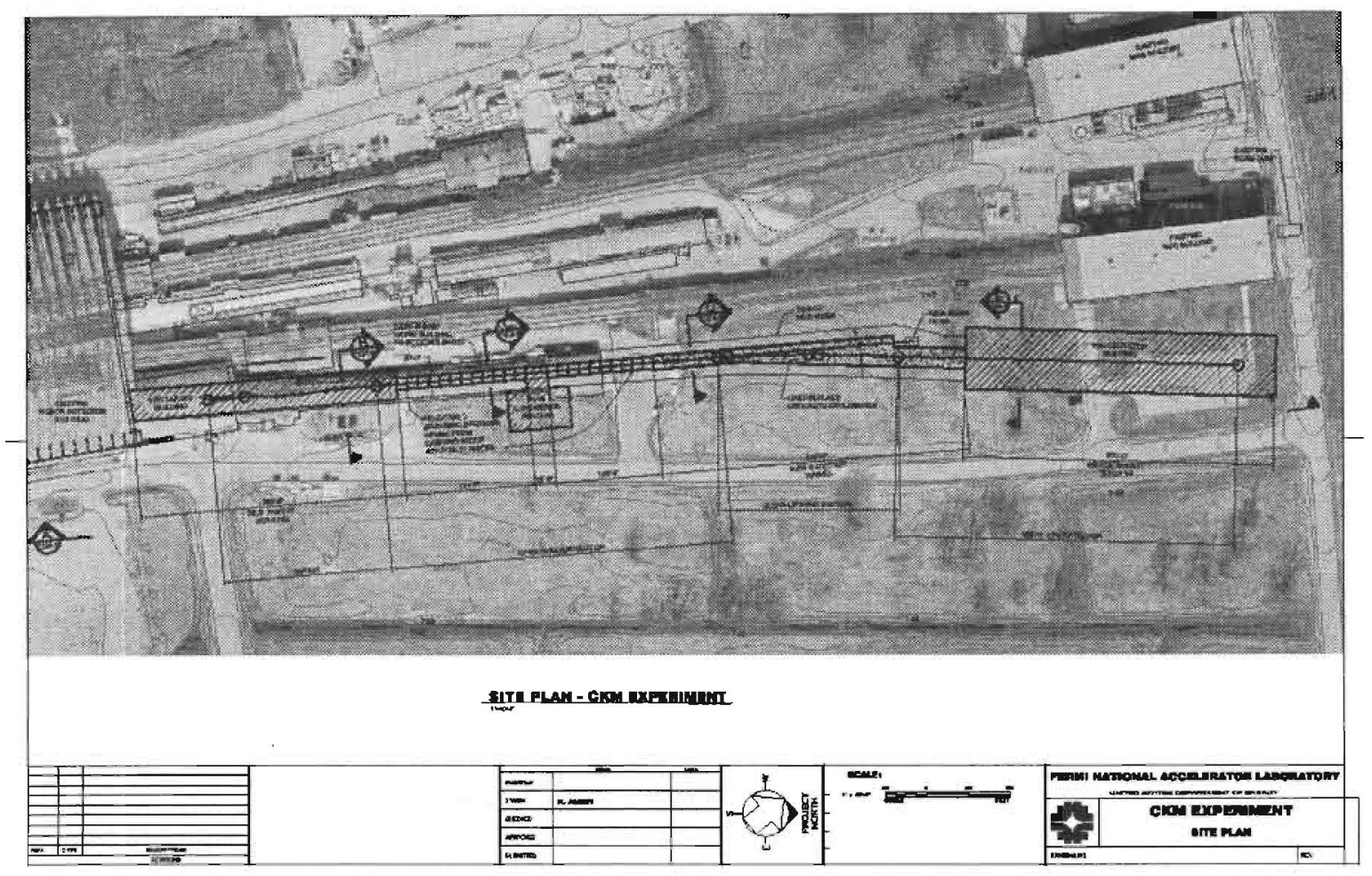

Figure 157: A new experimental hall configuration.

\subsection{Environmental Assessment}

Since the proposed action for siting the CKM experiment is in an existing research area, more general considerations for the required assessment will probably take precedent. These concerns include modifications or upgrades of existing beam enclosures, building a new target hall versus upgrades of an existing target hall, as well as assessment of any radiation shielding needs.

As discussed before, one of the preferred sites for this facility is the MP9 experimental hall, although other sites are being considered. A preliminary evaluation of these areas regarding potential environmental impact is warranted. The relevant components of such an evaluation include the need for construction, the requirements for a target station (including the protection of soil and ground water activation) and shielding to control general background rates.

The preferred construction site is in a region that contains the existing ME beam line which was associated with a previous fixed-target experiment. The primary beam transport system would be mostly constructed within existing beam line enclosures that are located in an existing berm and have radiation shielding that conforms to the criteria specified in the Fermilab Radiation Guide. Where possible, the new beamline would use components from other beamlines on site that no longer require them. Since these components and existing infrastructure for a generic beamline already exist, it is anticipated that any environmental impact will be consistent with previous use. Cryogenic components in the RF separated beamline constitute the major difference in this beamline from previous beam configurations in this location. It is expected that the environmental constraints from cryogenic operation in other parts of the laboratory will no doubt apply here as well, and those guidelines will be followed for this proposed facility.

For the proposed experiment, consideration of the configuration and location of the target 
station is constrained by the optimized conditions of the RF separated beam. Constructing a new station would be preferable from the standpoint of controlling and improving the environmental profile of this facility. One former targeting station (although partially disassembled) was located in the Meson Detector building. Thus other existing target stations, within decommissioned areas of the fixed target regions of the laboratory, can provide some components (such as target components, possibly magnets, support stands, and surrounding steel and concrete shielding). Any useful objects stored elsewhere on-site and incorporated into the new target station, where possible, will minimize the creation of new radioactive material.

Concrete and steel shielding would be installed within the enclosure to keep soil and ground water activation, primarily $3 \mathrm{H}$ and $22 \mathrm{Na}$, within the limits prescribed by the Fermilab Radiation Guide (i. e. for community drinking water supplies of $20 \mathrm{pCi}$ per $\mathrm{ml}$ for $3 \mathrm{H}$ and $0.4 \mathrm{pCi}$ per ml for $22 \mathrm{Na}$, and the discharge limits for surface waters of $2000 \mathrm{pCi}$ per $\mathrm{ml}$ for $3 \mathrm{H}$ and $10 \mathrm{pCi}$ per $\mathrm{ml}$ for $22 \mathrm{Na}$ ), and within the limits prescribed by DOE Orders.

Muon background, hadron shielding, and effects from the beam dump, will be addressed in a shielding document. At this time no extraordinary constraints exist from these elements that would go beyond the usual scope of environmental impact for this area.

\section{Cost, Schedule and Staffing Estimates}

We have been asked by the laboratory to submit the cost and schedule section of this proposal, after a review by the Particle Physics Division, in time for review by the PAC at its Aspen meeting in June 2001. This section will be completed then.

As a reminder, and to set a scale, we reproduce here the summary sections and tables from the 1st edition of the proposal.

\subsection{Costs}

In the absence of detailed engineering designs it is impossible to give precise cost estimates. Despite this, we have made an effort to estimate the capital costs for the detector elements of the CKM experiment based on the recent experience of the $\mathrm{KTeV}$ project, the SELEX experiment, and experiment 871 and Brookhaven. The costs below do not include engineering costs, SWF, or contingency. These estimates at this point serve only as a guide for the detector costs of the CKM experiment.

\subsubsection{Summary of Detector Cost Estimates}

The summary and total of all costs enumerated in the 1st edition are in Table 38.

\subsubsection{Fermilab Facility Cost Estimates}

We are told that the estimated cost for the RF separated beamline totals $\$ 4000 \mathrm{~K}$. There will be costs associated with siting the experiment. Any estimate of these costs awaits a definite location for the experiment and a siting plan. 


\begin{tabular}{|l|l|}
\hline \multicolumn{2}{|l|}{ Summary of Detector Cost Estimates } \\
\hline Detector System & Sub-cost. \\
\hline \hline BTSM System & $\$ 335 \mathrm{~K}$ \\
KMS System & $\$ 170 \mathrm{~K}$ \\
KEAT System & $\$ 80 \mathrm{~K}$ \\
BIV System & $\$ 228 \mathrm{~K}$ \\
VVS System & $\$ 2720 \mathrm{~K}$ \\
DMS System & $\$ 2100 \mathrm{~K}$ \\
Pion RICH & $\$ 1525 \mathrm{~K}$ \\
FVS System & $\$ 980 \mathrm{~K}$ \\
MVS System & $\$ 475 \mathrm{~K}$ \\
CVP System & $\$ 315 \mathrm{~K}$ \\
HVS System & $\$ 70 \mathrm{~K}$ \\
Trigger System & $\$ 300 \mathrm{~K}$ \\
DAQ System & $\$ 700 \mathrm{~K}$ \\
\hline \hline Total & $\$ 10357 \mathrm{~K}$ \\
\hline
\end{tabular}

Table 38: April 1998 cost estimate of the CKM detector systems. 


\subsection{Milestones}

\subsection{Staffing}

Our list of proponents is dominated by physicists with significant experience in doing rare and ultra-rare kaon decay experiments of this kind. The present collaboration has grown by a factor of two since the submission of our 1st edition of this proposal in April 1998. We have been joined by a group from the Brookhaven National Laboratory who are senior members of BNL787/949, and by two major University groups, Michigan and Virginia. The Fermilab group has doubled in size with the addition of two Wilson Fellows, a research associate and several others.

When the university and laboratory groups represented fill out with younger physicists and students we will have a significant fraction of the physics staff required to carry out this experiment. We could profitably add a few more groups to the collaboration. In addition to the present masthead two more groups have already applied for membership.

There seems to be no lack of interest in CKM. University groups have difficulty in joining an effort, such as ours, before it gains official recognition. First stage approval of this proposal is all we believe we require in order to strengthen our collaboration up to a level sufficient to produce a complete and credible design and cost for the experiment. The staffing of the experiment at the engineering and technical levels will require negotiation with the laboratory.

\section{Conclusion}

The measurement of the $K^{+} \rightarrow \pi^{+} \nu \bar{\nu}$ branching ratio to the $10 \%$ level of statistical precision which we are proposing is a critical next step in advancing our understanding of the source of CP violation in nature.

The decay in flight technique we advocate is a departure from all previous experiments searching for $K^{+} \rightarrow \pi^{+} \nu \bar{\nu}$. With this technique we believe that we can make a large advance in sensitivity, and achieve the first measurement with precision commensurate with the current level of theoretical understanding.

The goal we set for CKM was to observe $100 \mathrm{~K}^{+} \rightarrow \pi^{+} \nu \bar{\nu}$ events with less than $10 \%$ background in two years of data taking. This proposal achieves 95 signal events with less than 10 background events with the experiment described here. This corresponds to $1.05 \times 10^{-12}$ single event sensitivity and we assume a signal branching ratio of $1 \times 10^{-10}$.

The significant changes to this proposed experiment since the submission of the first edition of this proposal in April 1998 are few and positive. They include:

- UMS Detector Technology

We have adopted fine pitch, high rate MWPC's as the basic tracking detector for the Upstream Magnetic Spectrometer (UMS) and Kaon Entrance Angle Tracker (KEAT). These were double sided Silicon strip detectors in the first edition. The advantage is high rate performance demonstrated in HyperCP along with the group which achieved that performance. These chambers have time resolution $(\sim 5 \mathrm{nsec})$ and a maximum drift time $(\sim 25 \mathrm{nsec})$ which is better matched to our high rate environment. They do add somewhat more material in the beam in a critical region. For this reason silicon strip detectors remain an option for further study.

- Increased Signal Acceptance

Our signal acceptance has increased from $1.8 \%$ in the first edition to $2.3 \%$ now. The increase is due to three safe and simple parameter changes 
- Lengthening the fiducial decay region by $15 \%(3 \mathrm{~m})$

- Accepting resolution smeared signal events to small negative values of $M_{m i s s}^{2}$

- Accepting events with $P_{\pi}^{+}>20 \mathrm{GeV} / c$ where the $K^{+} \rightarrow \pi^{+} \pi^{0}$ 'background is kinematically forbidden.

\section{- An Enlarged Collaboration}

We now are 40 physicists from 7 institutions, twice the size of of collaboration at the time of the first edition. In addition two more groups have applied for membership in CKM.

We have supported our original background estimates with real data from previous experiments and a full beam line and detector simulation which is beginuing to approach realistic levels of complexity. We have identified several safety factors and data taking strategies which will realistically allow us to achieve our sensitivity goals the first time we take two years worth of data.

The past three years the has seen a remarkable reversal of fortune for the future of kaon physics. BNL-E949 is now commissioning with beam, and a run to reach the $\sim 1 \times 10^{-11}$ level of $K^{+} \rightarrow \pi^{+} \nu \bar{\nu}$ sensitivity will commence this summer. There are now active new programs at CERN, BNL, IHEP Protvino and KEK. There are approved and funded $K_{L} \rightarrow \pi^{0} \nu \bar{\nu}$ experiments at both BNL and KEK, and the KAMI proposal is currently under review. We must reasonably assume that the $K_{L} \rightarrow \pi^{0} \nu \bar{\nu}$ measurement will be made in a timely way by these efforts.

A decision for first stage approval of this proposal is now required. We have a strong collaboration that has worked three years now to refine the original design, and in the process develop a much deeper understanding of the technique. The experience of the CKM R\&D project has demonstrated that we cannot garner sufficient laboratory technical resources to seriously engineer and cost this experiment without a full first stage (scientific) approval.

The rest of the international kaon physics fleet has sailed. Either we're going on this race or we're not - the boat is ready!

\section{Acknowledgements}

The evolution of this proposal has and continues to be fueled by lively and fruitful discussion with our colleagues in BNL-787. In particular, discussions with L. Littenberg and M. Diwan of Brookhaven National Laboratory have been very helpful and insightful. We also wish to acknowledge many useful discussions with our KAMI colleagues who face some common technical challenges. We gratefully acknowledge the support of the $\mathrm{KTeV}$ collaboration in acquiring test beam data and for access to calibration data that has significantly bolstered the experimental case of this proposal.

We acknowledge M.G.Schepkino(ITEP) for numerous stimulating discussions of the problems of lepton flavor violation in kaon decays. The original idea to use a superconducting RF separated beam and a considerable amount of the early work on this idea was due to Gordon Thomson of Rutgers University.

We are grateful for the efforts of several groups from the Fermilab Beams, Computing and Particle Physics Divisions who have been of particular help to us. The A0 Photo-Injector group in BDIV, lead by Helen Edwards, has played the major role in advancing the R\&D on the superconducting RF for the separated beam. The Electrical Support Group in PPD, lead by Ray Yarema and Vince Pavicek's Electronic Systems Engineering Department in CDIV have both provided important and continuing support and advice to us. Cary Kendziora and his vacuum group in PPD have helped greatly with both the problems of a strawtube spectrometer in a vacuum and with the vacuum system design. John Krider, of PPD, has also been and important consultant on 
the straw tube systems. We have had excellent technical help from Robin Denham-Carter, Brian LaVoy, Bud Koecher in Lab 6 and elsewhere. The plans for siting the experiment and conventional construction have had important contributions from Russell Alber, Jeff Sims, and Tom Lackowski of FESS. Don Goloskie of PPD has brought his expertise gained in the design and construction of the $\mathrm{KTeV}$ vacuum photon vetoes and his $40+$ years designing mechanical systems for particle physicists to bear on our design problems on the VVS and FVS.

Finally we have been fortunate to have two exceptionally gifted summer students. Belinka Gonzales, from the Universidad Autonoma de Puebla in Mexico, made many contributions to the our veto scintillator studies with cosmic rays in lab6. And then there is Stephen Steffes University of Michigan class of 2002. Stephen has made so many contributions, from DAQ software through reconstruction codes, track fitting and even proposal editing that it is hard to to remember that this undergraduate does not yet have his Ph.D.

\section{Appendix I - Simulation Results Chapter from 1st Edition}

We have performed Monte Carlo simulations to evaluate the acceptance, to estimate rates, and to study backgrounds in CKM. Most of these simulations have been performed with a Monte Carlo written specifically for CKM. Some specialized issues have been addressed by feeding events from this Monte Carlo into GEANT. Over time it is likely that we will rely more heavily on GEANT, but thus far it has been more efficient to proceed in this fashion.

\subsection{Monte Carlo Overview}

The CKM Monte Carlo generates beam $K^{+}$'s with a central momentum of $22 \mathrm{GeV}$ and with distributions in position, angle, and momentum corresponding to the beam design of Doornbos. It swims these particles from the upstream end of the beam RICH detector until they decay, and it subsequently swims all the daughter particles to the face of the forward veto calorimeter. The beamline upstream of the momentum spectrometer, which is upstream of the beam RICH, is not simulated beyond the generation of a Gaussian distributed value for momentum centered at $22 \mathrm{GeV}$. In the simulation of the detector important effects such as pion decay in flight, multiple Coulomb scattering and single Coulomb scatters (Mott scattering), hadronic elastic scattering, and bremsstrahlung. are included. Some parameters used in this simulation are listed in table 39. For estimates of some backgrounds which involve inelastic hadronic interactions we have relied on the FRITIOF generator [196] to produce hadrons and photons which are then fed into this Monte Carlo.

The CKM Monte Carlo simulates the RICH detectors in some detail. Particles are stepped through the gas volume and in each step individual Cherenkov photons are generated. Of course, we do not generate photons which are not detected, so the number generated is a Poisson with the expected mean (from $N_{p e}=N_{0} L \sin \theta_{C}$ with $N_{0}=150 \mathrm{~cm}^{-1}$ ). In each step through the $\mathrm{RICH}$ multiple scattering occurs and for pions a decay to a muon is allowed with the appropriate probability. If a pion decays, then the muon is stepped forward; in the RICH it will generate photons at a different Cherenkov angle. The $\mathrm{RICH}$ simulation also includes chromatic dispersion by smearing the angle of each photon. The photons are then registered in a hexagonal array 'with the same configuration as the SELEX RICH phototube array. In the pion RICH a circular dead region is included to eliminate the hits from beamline $\pi^{+}$'s. All quantities derived from the RICHs are based on a fit to the center location of hit cells. The fit does not use extra information from any other source (such as track directions from tracking chambers). Randomly located noise hits are also included at a level of 1 per event, significantly worse than anticipated in practice. 


\begin{tabular}{|c|c|}
\hline Description & Value \\
\hline Bearn (magnetic) momentum resolution & $0.5 \%$ \\
\hline \multicolumn{2}{|l|}{ Beam RICH gas $\left(10 \mathrm{~m} \mathrm{CF}_{4}\right.$ at 0.63 atm $)$} \\
\hline Focal length & $20 \mathrm{~m}$ \\
\hline Index of refraction $(n-1)$ & $2.9 \times 10^{-4}$ \\
\hline$N_{0}\left(\right.$ as in $\left.N_{p e}=N_{0} L \sin ^{2} \theta_{C}\right)$ & $150 \mathrm{~cm}^{-1}$ \\
\hline Fraction of radiation length & $6.9 \%$ \\
\hline \multicolumn{2}{|l|}{ Beam RICH mirror (4 mm glass) } \\
\hline Fraction of radiation length & $3.3 \%$ \\
\hline \multicolumn{2}{|l|}{ Silicon vacuum trackers $(300 \mu \mathrm{m} \mathrm{Si})$} \\
\hline Position resolution ( $x$ and $y$ ) & $15 \mu \mathrm{m}$ \\
\hline Fraction of radiation length/station & $0.32 \%$ \\
\hline$K^{+}$Elastic cross section ${ }^{\dagger}$ (Si nucleus) & $80 \mathrm{mb}$ \\
\hline$K^{+}$Elastic slope parameter (Si nucleus) & $106 \mathrm{GeV}^{-2}$ \\
\hline \multicolumn{2}{|l|}{ Straw vacuum trackers (5-layer mylar straws) } \\
\hline Position resolution ( $x$ and $y$ ) per station & $120 \mu \mathrm{m}$ \\
\hline Fraction of radiation length/station & $0.22 \%$ \\
\hline$\pi^{+}$Elastic cross section ${ }^{\dagger}$ ( $\mathrm{C}$ nucleus) & $50 \mathrm{mb}$ \\
\hline$\pi^{+}$Elastic slope parameter (C nucleus) & $63 \mathrm{GeV}^{-2}$ \\
\hline \multicolumn{2}{|l|}{ Spectrometer Magnet } \\
\hline Transverse momentum kick & $100 \mathrm{MeV}$ \\
\hline \multicolumn{2}{|l|}{ Scintillating fiber tracker } \\
\hline Position resolution ( $x$ and $y$ ) per station & $120 \mu \mathrm{m}$ \\
\hline Fraction of radiation length/station & $0.7 \%$ \\
\hline \multicolumn{2}{|l|}{ Vacuum window } \\
\hline Fraction of radiation length & $0.12 \%$ \\
\hline \multicolumn{2}{|l|}{ Pion RICH gas $(20 \mathrm{~m}$ Ne at $1 \mathrm{~atm})$} \\
\hline Focal length & $20 \mathrm{~m}$ \\
\hline Index of refraction $(n-1)$ & $6.4 \times 10^{-5}$ \\
\hline$N_{0}\left(\right.$ as in $\left.N_{p e}=N_{0} L \sin ^{2} \theta_{C}\right)$ & $150 \mathrm{~cm}^{-1}$ \\
\hline Fraction of radiation length & $5.8 \%$ \\
\hline
\end{tabular}

Table 39: Parameters used in the Monte Carlo detector simulation. 
Tracking detectors are currently simulated by recording the particle's position smeared by a Gaussian resolution appropriate to that detector. Thus far no a postiori track reconstruction is performed on events and no account is made for noise hits. This is a clear area for improvement, but we do not anticipate major difficulties because of the wealth of information available to constrain track hypotheses.

The effect of the spectrometer magnet on tracks is simulated as a $100 \mathrm{MeV} p_{\perp}$-kick in the lorizontal plane. The momentum from the spectrometer is calculated from the smeared hit positions. While we have thus far only included Gaussian smearing of hits, large mismeasurements due to large scatters in matter (such as an elastic scatter of a pion in a chamber) are simulated.

Photons from kaon decays or other sources are swum by the Monte Carlo. The probability of detecting photons as a function of the photon energy is stored in a table. (Our assumptions for the photon veto systems are listed in table 11. When a photon reaches one of the three veto detectors (vacuum veto, forward veto, or beam hole veto), a weight is recorded which is the probability of not detecting that photon. If a photon misses all three detectors, a probability of unity is stored. Also, if a photon is within $10 \mathrm{~cm}$ of the charged particle at the face of the forward veto calorimeter, it is assumed to be lost (weight $=1$ ). If an event has multiple photons, the weights of each are multiplied to provide a weight for the full event. In this way the expected veto-detector response is folded with the energy and position distributions for different classes of events (such as $K^{+} \rightarrow \pi^{+} \pi^{0}$ and $\left.K^{+} \rightarrow \mu^{+} \nu_{\mu} \gamma\right)$ to obtain a veto probability appropriate to each class of events. We are careful to insure that the observed charged particle in the events satisfy the cuts we make, since any bias from the cuts on the $z$ distribution of events, energy or angle of $\pi^{0}$ 's, etc., can affect the rejection probability.

An important aspect of CKM is the redundancy provided by the magnetic momentum spectrometer and the RICH velocity spectrometer. For a correct mass hypothesis they should give similar results. Since they make measurements of the momenta of both the beam $K^{+}$and daughter $\pi^{+}$with independent hardware, it is fair to combine them. We rely on the same tracking detectors to determine the angle $\theta$ between the $K^{+}$and $\pi^{+}$independent of how we obtain the momenta, so we do not average the missing masses. But we can average the momenta from each source and then calculate a combined missing mass value based on the averaged nomenta. Figure 158 shows the result for $K^{+} \rightarrow \pi^{+} \pi^{0}$ decays for the missing mass for the three cases.

In addition to the Monte Carlo described above, a preliminary simulation of CKM has been made with GEANT in order to address some focused questions. GEANT's full simulation of physics processes such as Compton scattering, pair production, bremsstrahlung, energy loss, and decays enable the study processes that are not being addressed otherwise, such as photons interacting with pion RICH material.

Our GEANT simulation currently includes the kaon RICH, the vacuum vessel and its associated veto system, the pion RICH filled with neon (and its mirror) and the Forward Veto System. A dipole magnetic field of $6 \mathrm{kG}$ is simulated in the vacuum vessel. We expect that this simulation will evolve in detail as our understanding of the apparatus and background issues mature.

The input to the GEANT Monte Carlo is a list of kaon decays with decay vertex and daughter momenta specified. These decays have already been accepted by analysis cuts based on simulation in the CKM Monte Carlo and written to a file for input to GEANT. For example, analysis cuts on the $\pi^{+}$track in $K^{+} \rightarrow \pi^{+} \pi^{0}$ events provide a rejection of $2 \times 10^{-6}$ as described in section 13.3.1. Thus, with ten million simulated GEANT events of this type, we can study photon veto and loss mechanisms for background effects below the $10^{-12}$ level of sensitivity.

In practice we have not simulated the full shower development of photons and electrons in the vacuum and forward veto systems. Rather, primary and daughter photons and electrons are both subjected to the photon veto efficiency profile listed in table 11. This is a conservative underestimate 

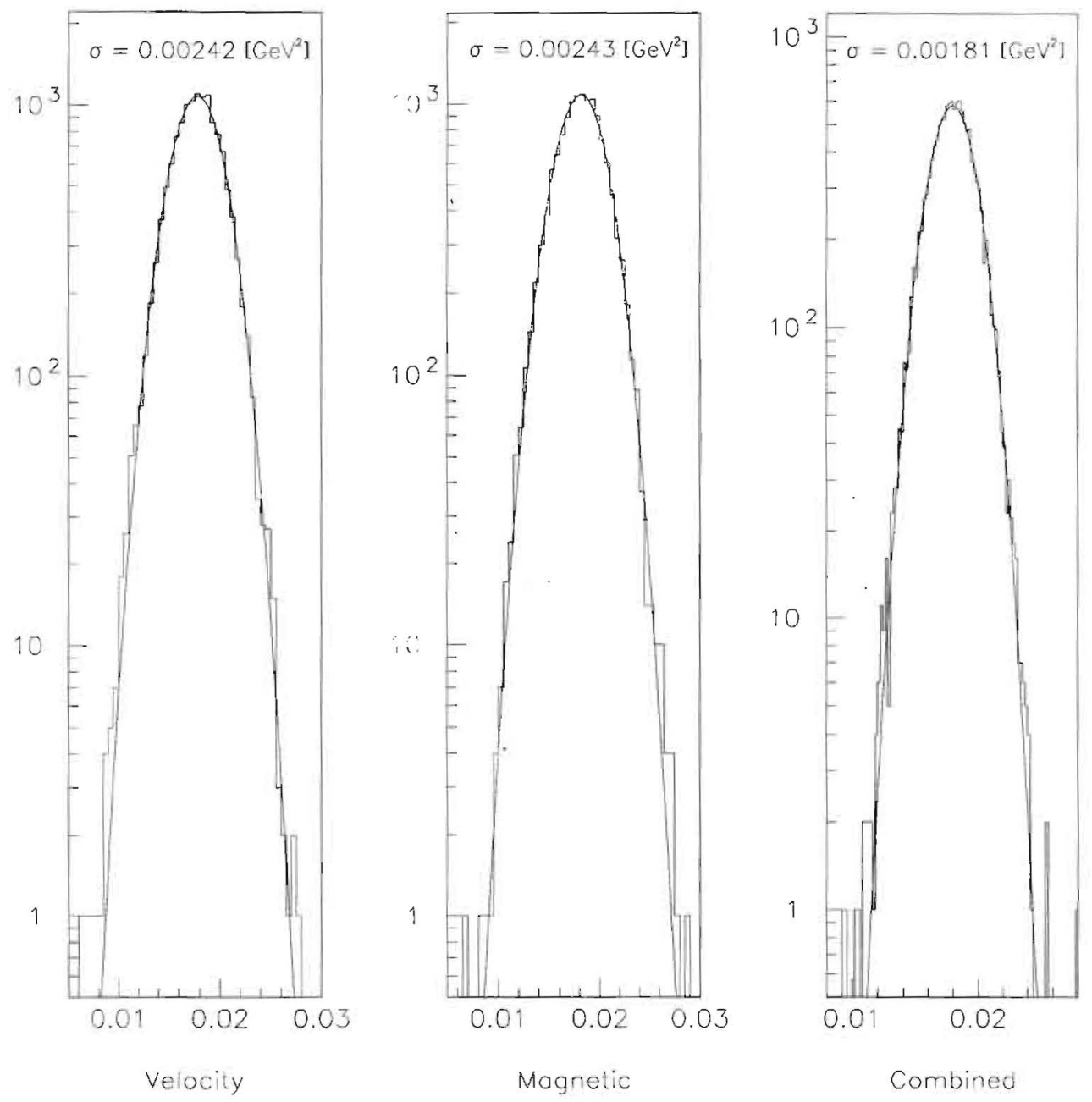

Figure 158: The resolution in missing mass squared in $K^{+} \rightarrow \pi^{+} \pi^{0}$ decays for the three methods of determining it as described in the text. About 20,000 events are shown. 
of the veto efficiency for electrons and positrons that result from the interaction of primary decay photons. Decay photons and photon shower products that intercept the Forward Veto System within a $10 \mathrm{~cm}$ radius of the charged pion track are assumed to be lost by association with the pion track cluster.

\section{2 $K^{+} \rightarrow \pi^{+} \nu \bar{\nu}$ Acceptance}

The Monte Carlo generates the $K^{+} \rightarrow \pi^{+} \nu \bar{\nu}$ decay using the $K_{e 3}$ matrix element and form factor. The acceptance for $K^{+} \rightarrow \pi^{+} \nu \bar{\nu}$ is determined by calculating the fraction of decays which pass our cuts. The detector geometry of course plays a role in the acceptance, but the detector is sized so little acceptance is lost on apertures. For example, the pion RICH has a pion Cherenkov threshold of about $12 \mathrm{GeV}$ and the number of photoelectrons detected is not adequate to be useful below about $14 \mathrm{GeV}$. Consequently our acceptance is limited by a minimum pion momentum of $14 \mathrm{GeV}$, but for pions above $14 \mathrm{GeV}$ the maximum transverse excursion is small enough that we can afford to collect almost all of them. Equally important are cuts we have devised to reject backgrounds. Two closely related examples are a cut on the reconstructed vertex location (the apparent $K^{+}$ decay position as determined by the point of closest approach of the $K^{+}$and $\pi^{+}$tracks) and on the angle between the $K^{+}$and $\pi^{+}$tracks. We require this measured decay angle to be larger than $2.5 \mathrm{mrad}$, because below that the vertex resolution (along the beam direction, $z$ ) degrades quickly. Figure 159 shows the resolution obtained on vertex $z$-position for $K^{+} \rightarrow \pi^{+} \nu \bar{\nu}$ events. We cut on the vertex location, specifically requiring it to be 5 meters away from the nearest tracking detectors, because interactions or large scatters in those detectors are a major potential sources of background. Having made these two cuts, the subsequent loss in acceptance from the inner aperture of the straw chambers is small.

We have developed a set of cuts based primarily on background rejection considerations while trying to maintain good acceptance for $K^{+} \rightarrow \pi^{+} \nu \bar{\nu}$. They fall into five categories:

- Aperture cuts. These simply require accepted charged particles to remain inside the outer detector boundaries and outside the beam hole region.

- RICH cuts. These are designed to insure good results from the RICH detectors. They consist of:

1. Beamı RICH: between 6 and 20 hits (photoelectrons).

2. Pion RICH: 8 or more hits, with at least one in each quadrant of the fit ring.

3. Both RICHs: a cut on the quality of fit.

- Vertex cuts. These primarily reject backgrounds from interactions in detector material. They consist of:

1. $\theta>2.5 \mathrm{mrad}$, a requirement that the reconstructed angle between the $K^{+}$and $\pi^{+}$be large enough to permit good vertex $z$ measurement.

2. $20 \mathrm{~m}<z_{\text {vertex }}<40 \mathrm{~m}$, a requirement that the reconstructed vertex position be $5 \mathrm{~m}$ from the nearest material. The fitted sigma vertex resolution as seen in figure 159 is $18 \mathrm{~cm}$. The relatively large cut of $5 \mathrm{~m}$ ensures that the tails visible in figure 159 are eliminated.

3. DOCA $<0.6 \mathrm{~cm}$, the reconstructed distance of closest approach between the $K^{+}$and $\pi^{+}$must be less than $0.6 \mathrm{~cm}$, as shown in figure 160 .

4. $\sqrt{x_{\text {vertex }}^{2}+y_{\text {vertex }}^{2}}<10 \mathrm{~cm}$, the vertex location should be near the beam. 


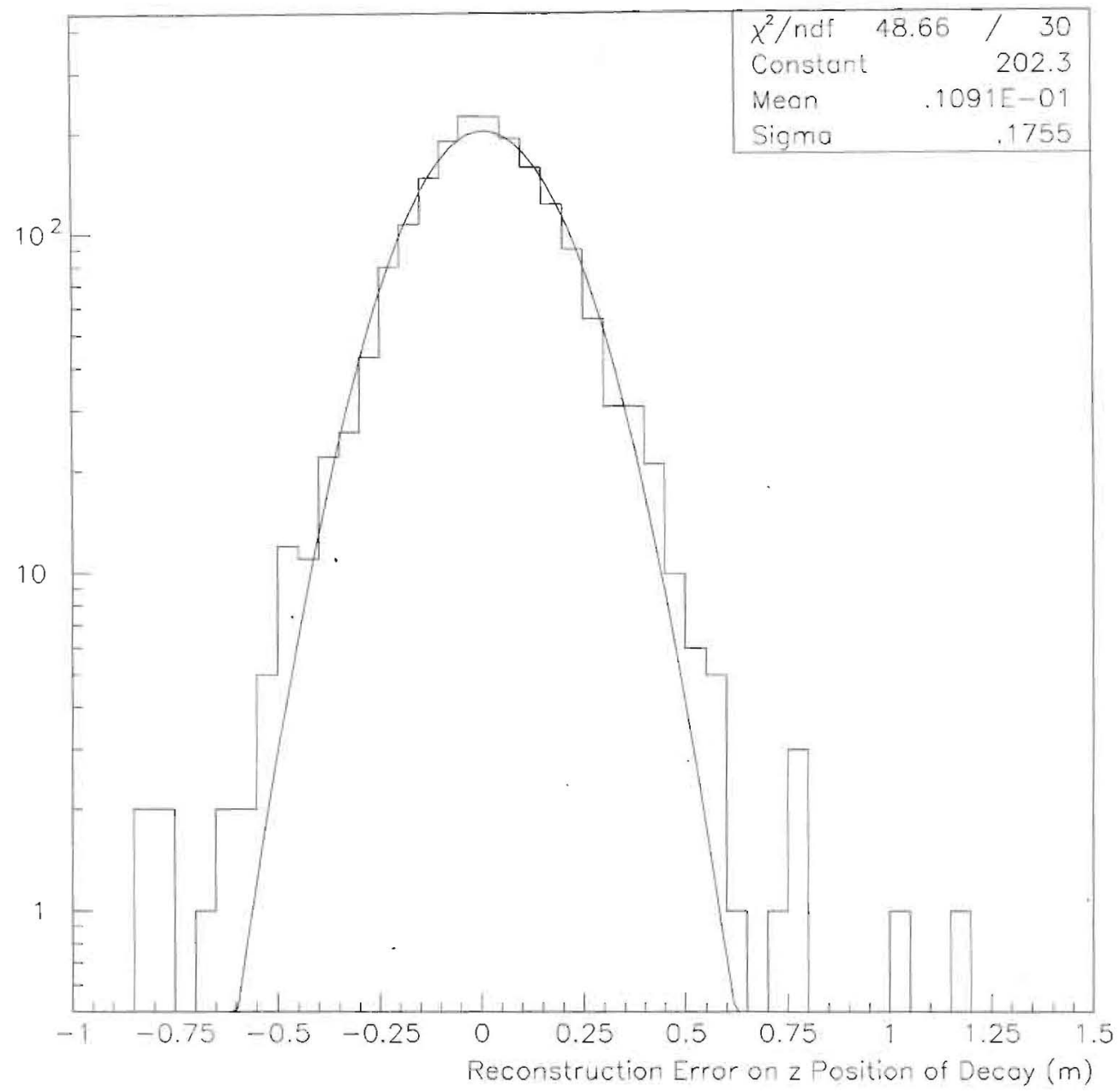

Figure 159; Distance between measured vertex location and actual decay position in $K^{+} \rightarrow \pi^{+} \nu \bar{\nu}$ events. The fitted resolution sigma is $\sim 18 \mathrm{~cm}$, with non-Gaussian tails originating from hard scatters included in the tracking simulation. 


\begin{tabular}{|l|l|}
\hline Loss Mechanism & Inefficiency \\
\hline Trigger deadtime: & $15 \%$ \\
MVS $\pi / \mu$ separation cuts: & $5 \%$ \\
UMS/DMS tracking: & $5 \%$ \\
Accidental losses at analysis: & $5 \%$ \\
(Balance after trigger deadtime losses.) & \\
Double $K^{+}$ambiguity in kRICH: & $5 \%$ \\
\hline Total miscellaneous inefficiency: & $30 \%$ \\
\hline
\end{tabular}

Table 40: Miscellaneous inefficiency table.

- Spectrometer cuts. These cuts primarily require the magnetic spectrometer and velocity spectrometer measurements to agree. Since the pion mass hypothesis is assumed for the velocity spectrometer, these cuts have the effect of rejecting muons based on RICH information, along with other event pathologies (e.g., $\pi^{+}$decay in flight, large scatters or energy loss in some areas, etc.). They consist of:

1. Beam particle momentum: $\left|p_{\text {magnetic }}-p_{\mathrm{RICH}}\right| / p_{\mathrm{RICH}}<0.025$

2. Non-bend plane track angle match through downstream spectrometer magnet: $\Delta\left|\theta_{y}\right|<$ $1.5 \mathrm{mrad}$

3. Secondary (decay) particle momentum: $\left|p_{\text {magnetic }}-p_{\mathrm{RICH}}\right| / p_{\mathrm{RICH}}<0.05$

4. Missing mass: $\left|M_{\text {magnetic }}^{2}-M_{\mathrm{RICH}}^{2}\right|<0.01 \mathrm{GeV}^{2}$ (see figure 161)

5. $14<p_{\mathrm{RICH}}<20 \mathrm{GeV}$

- Signal region. This selects the region of phase space between the $K_{\mu 2}$ and $K_{\pi 2}$ peaks and consequently rejects those decays based on two-body kinematics. The selection is based on the missing mass value from combining the momentum spectrometer and velocity spectrometer information: $0<M_{\text {combined }}^{2}<0.008 \mathrm{GeV}^{2}$

For $K^{+} \rightarrow \pi^{+} \nu \bar{\nu}$ decays within the decay volume, the set of cuts previously described accept $1.83 \%$ of events. If we are ultimately able to include events below the $K_{\pi 2}$ peak, the acceptance can be more than doubled. Figure 162 shows the missing mass distribution for $K^{+} \rightarrow \pi^{+} \nu \bar{\nu}$ events pass all cuts (except on missing mass).

Inefficiencies due to trigger deadtime, accidental losses, etc, are not simulated in this Monte Carlo. These loss mechanisms listed in table 40 have been discussed in previous sections, and correspond to an additional acceptance loss of $\sim 30 \%$. Taking this into account, the final $K^{+} \rightarrow \pi^{+} \nu \bar{\nu}$ acceptance is about $1.25 \%$. This is the number used in this proposal to calculate the CKM sensitivity.

\subsection{Kaon Decay Backgrounds}

For the signal region considered here, between the $K_{\mu 2}$ and $K_{\pi 2}$ peaks, only three kaon decays are potentially significant sources of background to a $K^{+} \rightarrow \pi^{+} \nu \bar{\nu}$ search. They are the two decays making up these peaks and the radiative tail of the lower missing mass peak. That is, we must consider $K^{+} \rightarrow \pi^{+} \pi^{0}, K^{+} \rightarrow \mu^{+} \nu_{\mu}$, and $K^{+} \rightarrow \mu^{+} \nu_{\mu} \gamma$.

As a matter of convention we will quote background estimates in terms of an "effective branching ratio." This quantity must depend inversely on the acceptance for $K^{+} \rightarrow \pi^{+} \nu \bar{\nu}$ because a smaller 


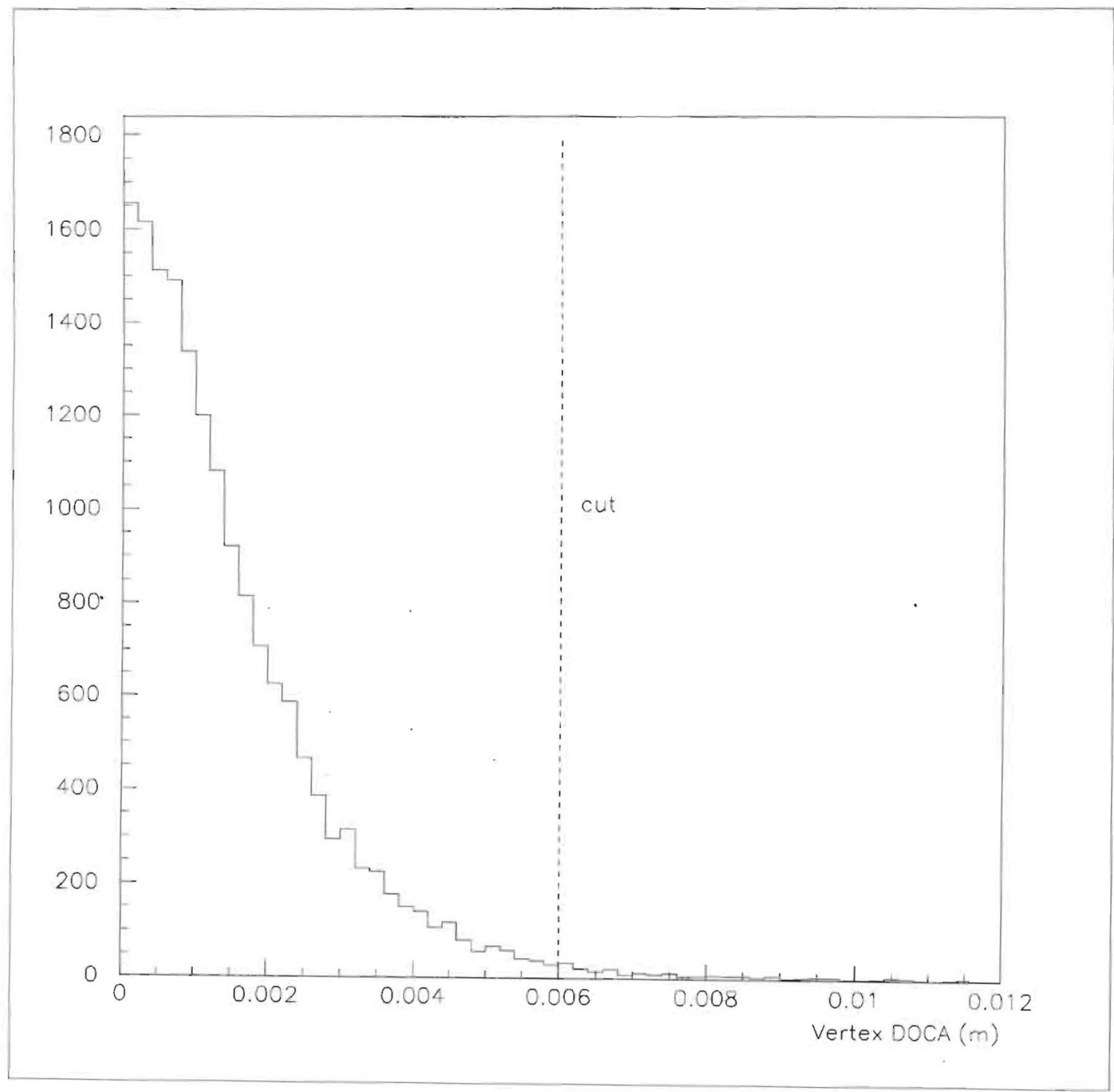

Figure 160: Measured distance of closest approach (DOCA) for the $K^{+}$and $\pi^{+}$in $K^{+} \rightarrow \pi^{+} \nu \bar{\nu}$ events. 


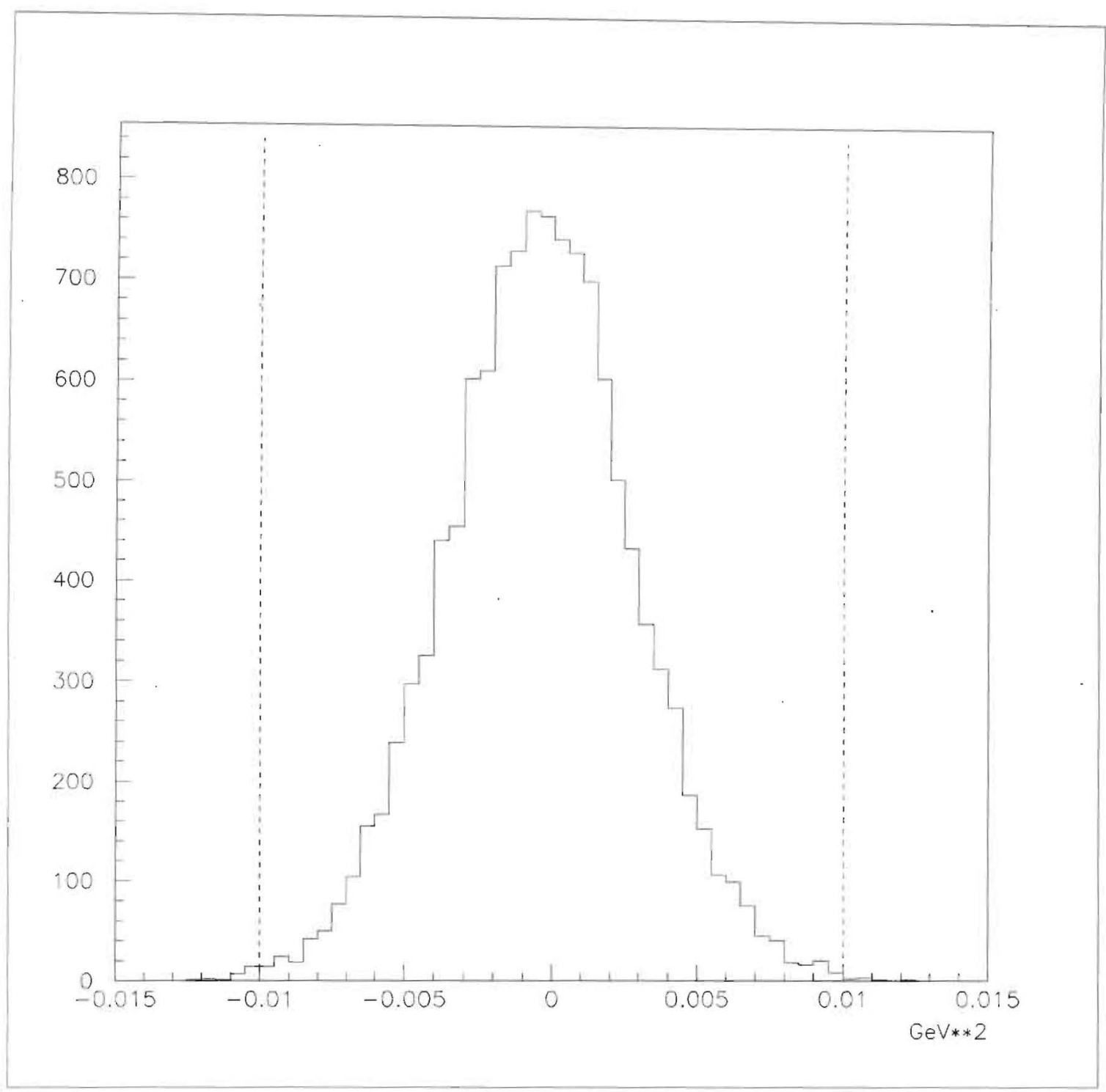

Figure 161: Difference in measured missing mass squared from the momentum and velocity measurements $\left(M_{\text {magnetic }}^{2}-M_{\mathrm{RICH}}^{2}\right)$ for $K^{+} \rightarrow \pi^{+} \nu \bar{\nu}$ events. 


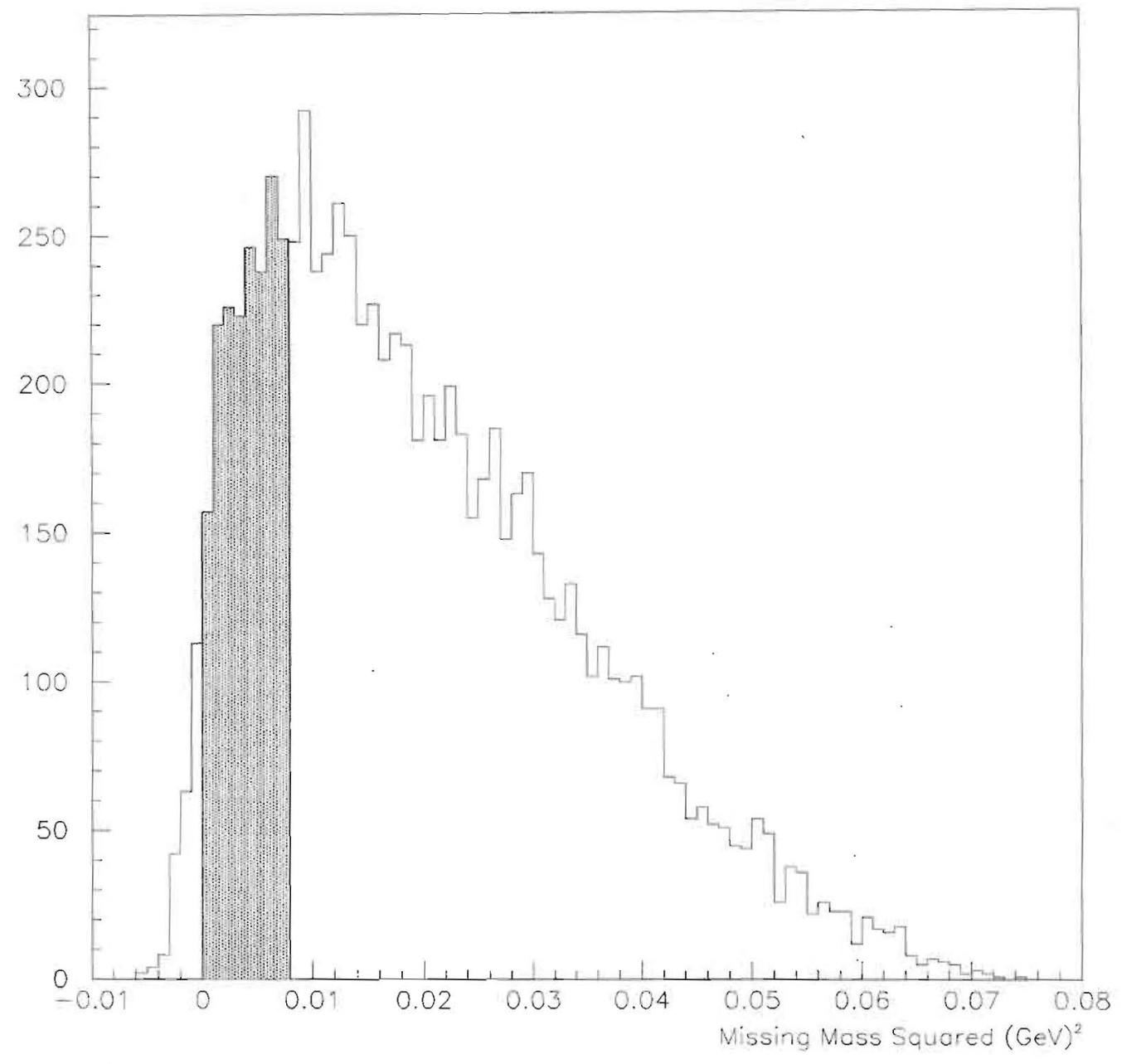

Figure 162: Measured missing mass squared $\left(M_{\text {combined }}^{2}\right)$ in $K^{+} \rightarrow \pi^{+} \nu \bar{\nu}$ events. The signal region is shaded. 
signal acceptance means it will require more $K^{+}$'s into the experiment, which in turn increases the number of background events. The effective branching ratio multiplied by the single event sensitivity for $K^{+} \rightarrow \pi^{+} \nu \bar{\nu}$ gives the number of expected events from that background. Since the deadtime and losses listed in table 40 are common to both the $K^{+} \rightarrow \pi^{+} \nu \bar{\nu}$ signal and backgrounds, we use the pre-loss $K^{+} \rightarrow \pi^{+} \nu \bar{\nu}$ acceptance for in the background estimates.

\subsection{1 $K^{+} \rightarrow \pi^{+} \pi^{0}$ Background Study}

The $K^{+} \rightarrow \pi^{+} \pi^{0}$ decay is the most serious kaon decay background for CKM. Its suppression depends critically on excellent $\pi^{0}$ rejection and on excellent kinematic reconstruction (i.e., measurement of the beam $K^{+}$and daughter $\pi^{+}$momenta and the angle between them) in order to exploit the two-body kinematics.

An attractive feature of $K^{+}$decays in-flight is the boost of most photons to relatively high energies. Even though a small fraction (about $0.1 \%$ ) of photons may have exceedingly low energy, below $20 \mathrm{MeV}$ (the minimum for $K^{+} \rightarrow \pi^{+} \pi^{0}$ decays at rest), we are assured that the other photon has energy of $2 \mathrm{GeV}$ or more. This is a simple consequence of starting with a $22 \mathrm{GeV} K^{+}$and requiring the $\pi^{+}$energy to be less than $20 \mathrm{GeV}$. As a result the sum of the energies of the two photons must be $2 \mathrm{GeV}$ or more.

We have estimated the $\pi^{0}$ rejection in $K^{+} \rightarrow \pi^{+} \pi^{0}$ via Monte Carlo by using a sample of $K^{+} \rightarrow \pi^{+} \pi^{0}$ decays that pass all cuts, except the $0<M_{\text {combined }}^{2}<0.008 \mathrm{GeV}^{2}$ cut which needlessly lowers statistics. For these events both photons are swum from the point of the $K^{+}$decay until they intercept veto detectors. Then a weight, equal to the probability of not rejecting the photon, is determined for the particular veto system it hit based on the energy of the photon. Photon veto probabilities are listed in table 11 for each veto system. The two weights are multiplied together to get the probability of not vetoing that event. Over a large event sample we determine the appropriate $\pi^{0}$ rejection for this class of events.

Some photons, only rarely, may miss all veto detectors; this can occur if the decay occurs far downstream of the required $20 \mathrm{~m}<z_{\text {vertex }}<40 \mathrm{~m}$ fiducial region. In such events a wide-angle photon can miss both the vacuum veto and the forward veto by exiting the pion RICH at a large angle. This type of event only occurs when the vertex position is badly mismeasured, not as a result of simple resolution smearing. In particular, large hadronic elastic scattering of the $\pi^{+}$in the first straw cliamber can create this type of mismeasurement. This effect is included in the Monte Carlo. Any such photons are given a weight of unity.

Another source of photons being missed is coalescence with the $\pi^{+}$in the forward veto detector. It may or may not ultimately be possible to make a cut on electromagnetic energy deposited close to a $\pi^{+}$, but for now we have made the conservative assumption that any photon within $10 \mathrm{~cm}$ of the $\pi^{+}$is lost.

Two topologies of $K^{+} \rightarrow \pi^{+} \pi^{0}$ events limit $\pi^{0}$ rejection in CKM: (1) those with one photon below $20 \mathrm{MeV}$ that is unlikely to be vetoed and (2) those with one photon within $10 \mathrm{~cm}$ of the $\pi^{+}$. In both cases the $\pi^{0}$ rejection depends on vetoing the other photon. Our Monte Carlo result for $\pi^{0}$ rejection in $K^{+} \rightarrow \pi^{+} \pi^{0}$ events is $1.6 \times 10^{-7}$.

We have considered some additional scenarios not simulated in the MC and have used GEANT to estimate them. A photon may also convert to an $e^{+} e^{-}$pair as it traverses tracking planes, the vacuum window, the RICH gas and mirror, and any other material the detector presents to it. In most cases the rejection will not be degraded because the $e^{+} e^{-}$pair will reach the veto detector and deposit energy very much as the photon would have. Also, if a relatively energetic photon converts in the pion RICH gas, it is likely to be detected by the RICH itself. However, in a small number of cases, typically where the photon had little energy, the $e^{+} e^{-}$pair may be lost. We have 
investigated these losses using GEANT (see section 13.4). Our conclusion is that the effect of these losses enters below the $10^{-7}$ level in $\pi^{0}$ rejection.

Also, in $K^{+} \rightarrow \pi^{+} \pi^{0}$ the $\pi^{0}$ may Dalitz decay, giving a photon and $e^{+} e^{-}$pair instead of two photons. Most of the comments above concerning external conversion apply here as well. However, almost all external conversions will occur downstream of the spectrometer magnet, while all the relevant Dalitz decays will occur upstream of the magnet. Consequently the effect of the magnetic field on the $e^{+} e^{-}$pair will be more important for these events. An example of such a Dalitz event is shown in figure 165. This issue has also been addressed using GEANT with the result that Dalitz decay also enters below the $10^{-7}$ level in $\pi^{0}$ rejection.

We have simulated $5 \times 10^{6} K^{+} \rightarrow \pi^{+} \pi^{0}$ decays. The analysis cuts we make were listed in section 13.2. Figure 163 shows the missing mass distribution for events passing all cuts (except the requirement on missing mass itself). Ten events appear in the signal region $0<M_{\text {miss }}^{2}<0.008 \mathrm{GeV}^{2}$. From this we can estimate our overall $\mathrm{K}^{+} \rightarrow \pi^{+} \pi^{0}$ rejection in terms of an effective branching ratio:

$$
\begin{gathered}
B_{\text {eff }}=\left(\frac{1}{\epsilon_{\pi \nu \nu}}\right)\left(B\left(K^{+} \rightarrow \pi^{+} \pi^{0}\right)\right)\left(\text { fraction } K^{+} \rightarrow \pi^{+} \pi^{0} \text { passing cuts }\right)\left(\pi^{0} \text { rejection }\right) \\
B_{\text {eff }}=\left(\frac{1}{0.018}\right)(0.21)\left(\frac{10}{5 \times 10^{6}}\right)\left(1.6 \times 10^{-7}\right)=3.7 \times 10^{-12} .
\end{gathered}
$$

Mismeasurement of the angle $\theta$ between the $K^{+}$and $\pi^{+}$, due primarily to hadronic elastic scattering in the trackers, is a limiting contribution to the kinematic rejection of events. Inelastic hadronic interactions may also corrupt events. These have not been included in the $K^{+} \rightarrow \pi^{+} \pi^{0}$ simulation thus far. We believe the contribution of inelastic interactions to backgrounds should be significantly less than elastic scatters.

Several features of inelastic events will provide additional rejection. If the $K^{+}$interacts inelastically in the last tracker, evidence via extra hadrons will show up in the Beam Interaction Veto System. The energy of the $K^{+}$will be reduced in the interaction before it decays, so that the DMS and beam RICH will have overestimated the momentum of the $K^{+}$when it decays. This has the effect of increasing the calculated missing mass and moving it farther from our signal region. If the daughter $\pi^{+}$interacts inelastically in the first vacuum straw chamber, it will lose energy before its momentum is measured, so the effect will be a mismeasurement on the low side. This again favors background rejection since it increases the missing mass and moves it farther from the signal region. And of course, such an inelastic interaction will produce additional particles in the detector that will provide significant rejection. Needless to say, the contribution to $K^{+} \rightarrow \pi^{+} \pi^{0}$ backgrounds from inelastic interactions needs to be addressed in a more quantitative fashion and that is our plan. However, it is unlikely to compete with the processes we have simulated.

\subsection{2 $K^{+} \rightarrow \mu^{+} \nu_{\mu}$}

The most common $K^{+}$decay is $K^{+} \rightarrow \mu^{+} \nu_{\mu}$ and it is a potentially serious background to $K^{+} \rightarrow \pi^{+} \nu \bar{\nu}$. As a two-body decay, it can be rejected kinematically. Also, CKM will have powerful rejection against muons because of both the pion RICH and the muon veto system. Potential background arises primarily when either the beam $K^{+}$or the $\mu^{+}$scatters at large angle and introduces a mismeasurement of the angle between the $K^{+}$and $\mu^{+}$. In fact, the probability of a large hadronic scatter of the $K^{+}$is significantly larger than a large $\mu^{+}$Mott scatter. The locations where such scatters are dangerous are in the last tracking plane the $K^{+}$traverses and in the first tracking: plane the $\mu^{+}$traverses (scatters in other locations do not induce a mismeasurement of the angle between the $K^{+}$and $\mu^{+}$). Consequently these trackers must be very low-mass devices. Cuts on vertex quality are highly effective in rejecting such events. 


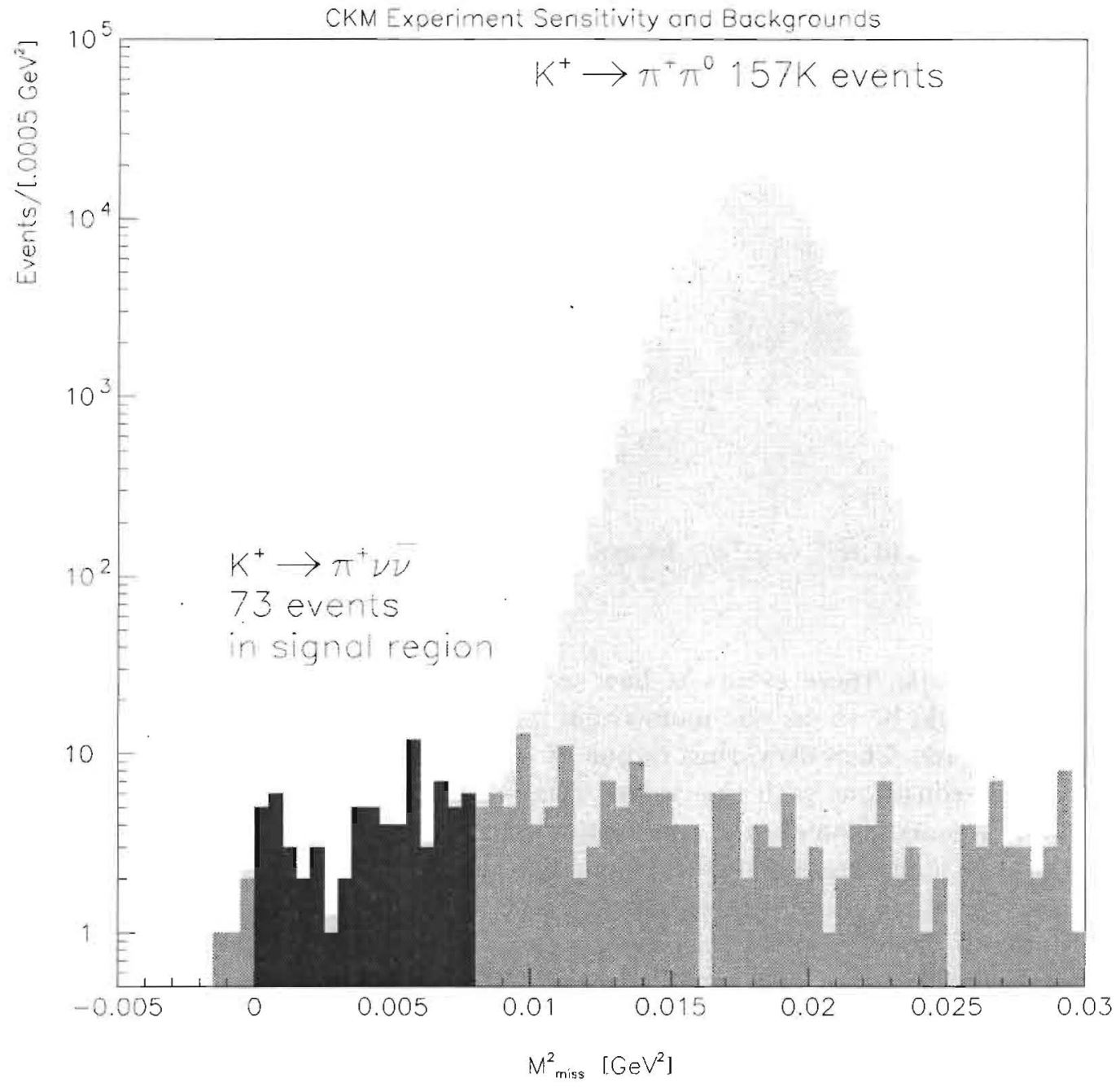

Figure 163: Missing mass squared $\left(M_{\text {combined }}^{2}\right)$ for $K^{+} \rightarrow \pi^{+} \pi^{0}$ events passing all cuts superimposed on the signal (light shading). The sensitivity shown in this plot corresponds to the experimental sensitivity of $73 \mathrm{~K}^{+} \rightarrow \pi^{+} \nu \bar{\nu}$ events in our mass window (single event sensitivity of $1.4 \times 10^{-12}$ ) which are indicated with the dark shading. 


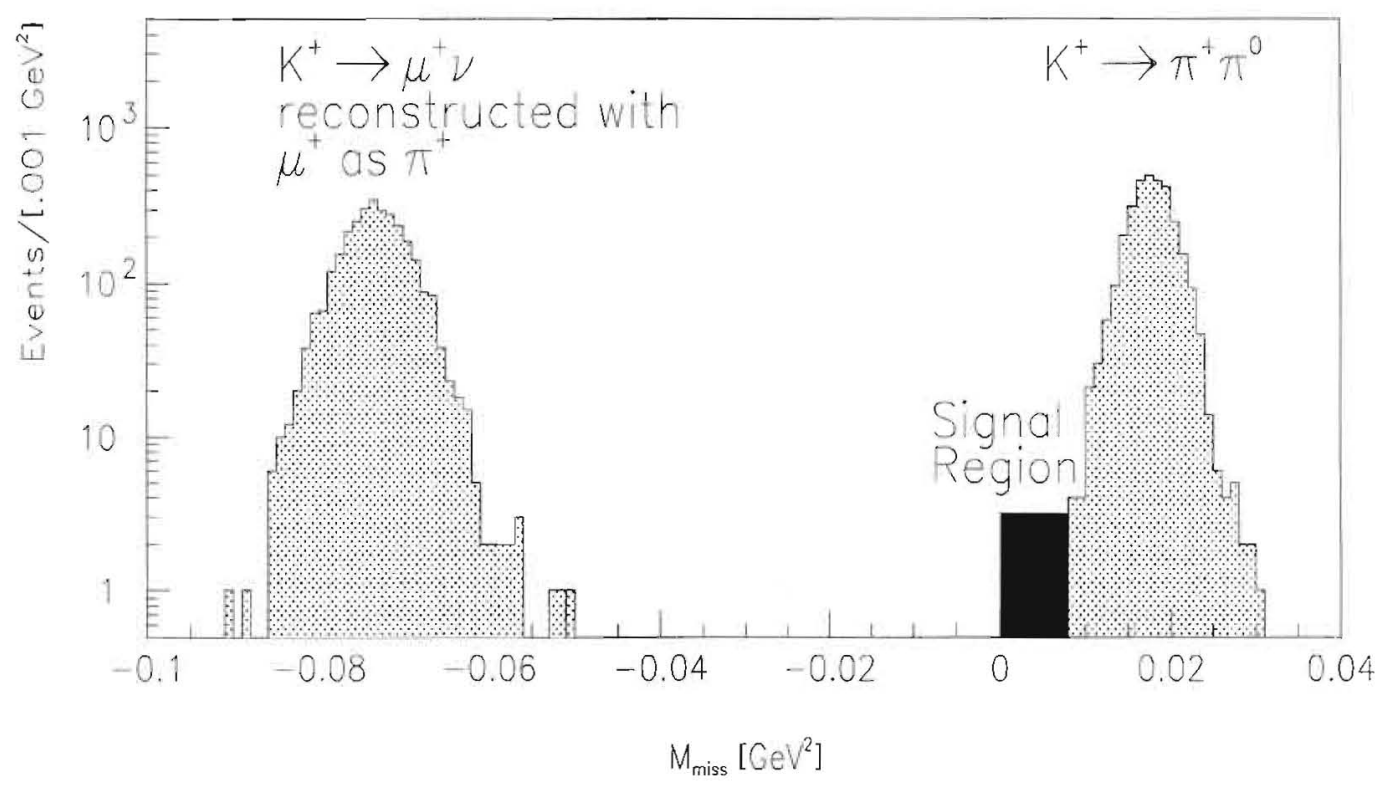

Figure 164: Missing mass of $K^{ \pm} \rightarrow \mu^{ \pm} \nu_{\mu}$ decays where the muon is reconstructed under the pion mass hypothesis.

One issue in analyzing these events is how to include the $\mathrm{RICH}$ rejection of muons. The traditional method would be to use the momentum measurement from the magnetic spectrometer to estimate the expected Cherenkov ring radius of a pion, and then to reject events when the measured ring is not consistent with this value. Here we choose to use the RICH information to calculate the event kinematics under the assumption that the observed particle is a pion. Then we can compare these (velocity spectrometer) kinematic quantitics to the same quantities based on the magnetic spectrometer and reject events where they differ significantly.

This may seem at first an unnatural way to perform the analysis. Perhaps it would be if $K^{+} \rightarrow \mu^{+} \nu_{\mu}$ were the only source of events (other than $K^{+} \rightarrow \pi^{+} \nu \bar{\nu}$ ). We make this choice because we wish to use measurements from the velocity spectrometer to improve our kinematic resolution by combining that information with measurements from the magnetic spectrometer. For decays such as $K^{+} \rightarrow \pi^{+} \pi^{0}$, where the assumed mass hypothesis is correct, the improvement in kinematic resolution is significant. When the mass hypothesis is wrong, the kinematic quantities will differ wildly and we can reject the events on that basis. Figure 164 shows the momentum and missing mass determined from both sources for a sample of $K^{+} \rightarrow \mu^{+} \nu_{\mu}$ decays and their differences.

An important part of our rejection of $K^{+} \rightarrow \mu^{+} \nu_{\mu}$ comes from the muon rejection of the Muon Veto System. We will use the conservative value of $1 \times 10^{-5}$ for muons in the range of 14 to $20 \mathrm{GeV}$.

To reach a simulation sensitivity of $10^{-12}$ we would need to simulate $5 \times 10^{8}$ events based on this level of muon rejection. Instead we have simulated a smaller number of $K^{+} \rightarrow \mu^{+} \nu_{\mu}$ decays in which large scatters are forced, due to hadronic elastic scattering for the $K^{+}$and single Coulomb (Mott) scattering for the $\mu^{+}$. We have simulated these two scenarios separately, running $2 \times 10^{6}$ events of the first kind and $1 \times 10^{6}$ events of the second, much less likely, kind. We apply the cuts 


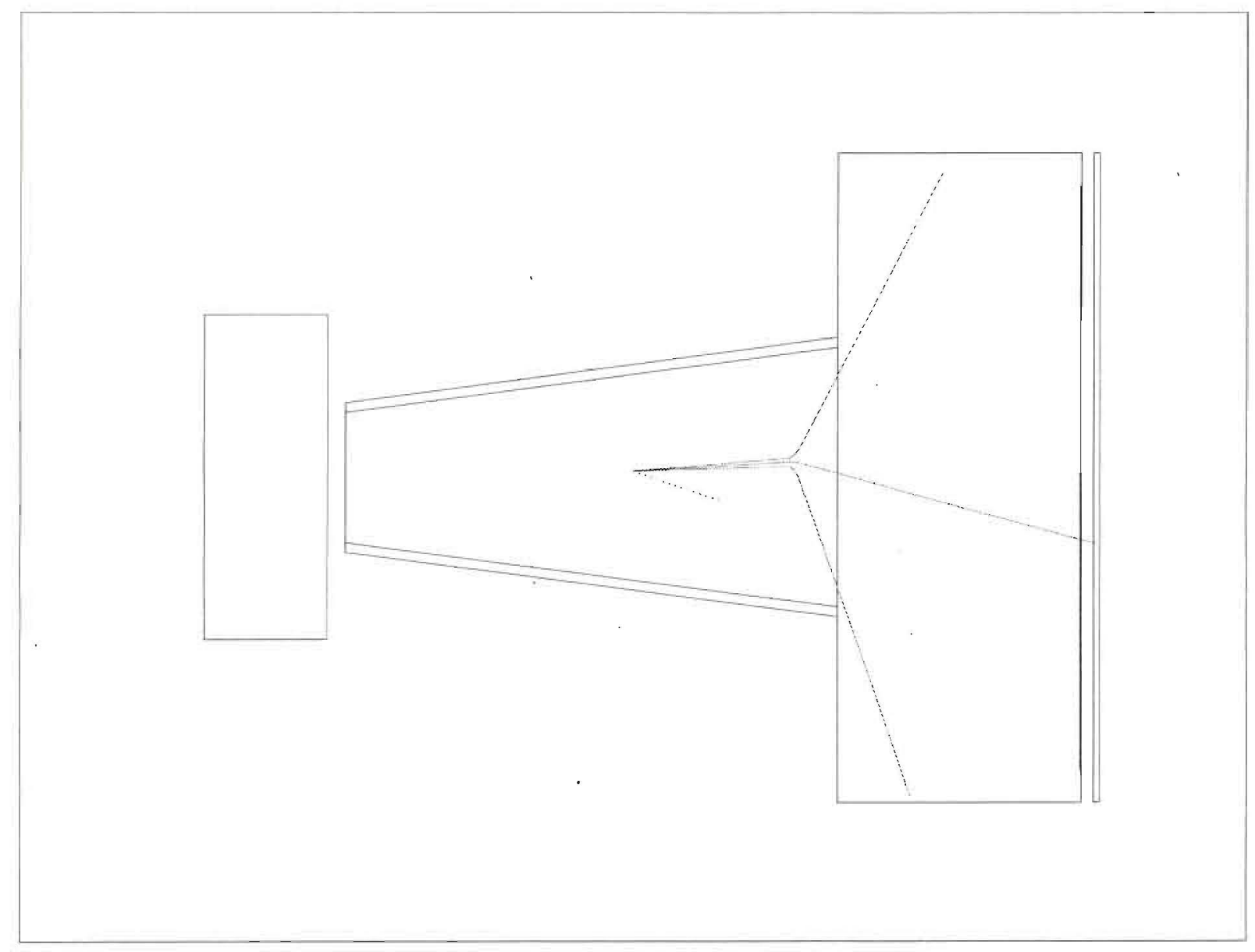

Figure 165: A GEANT simulation of a common Dalitz decay topology where the electron and positron tracks are kicked out of the photon veto system coverage by the $100 \mathrm{MeV} / c p_{\perp}$-kick magnet. Fortunately the rejection of the remaining photon together with the low Dalitz decay branching fraction set the scale of this problem topology to be low at the outset. 


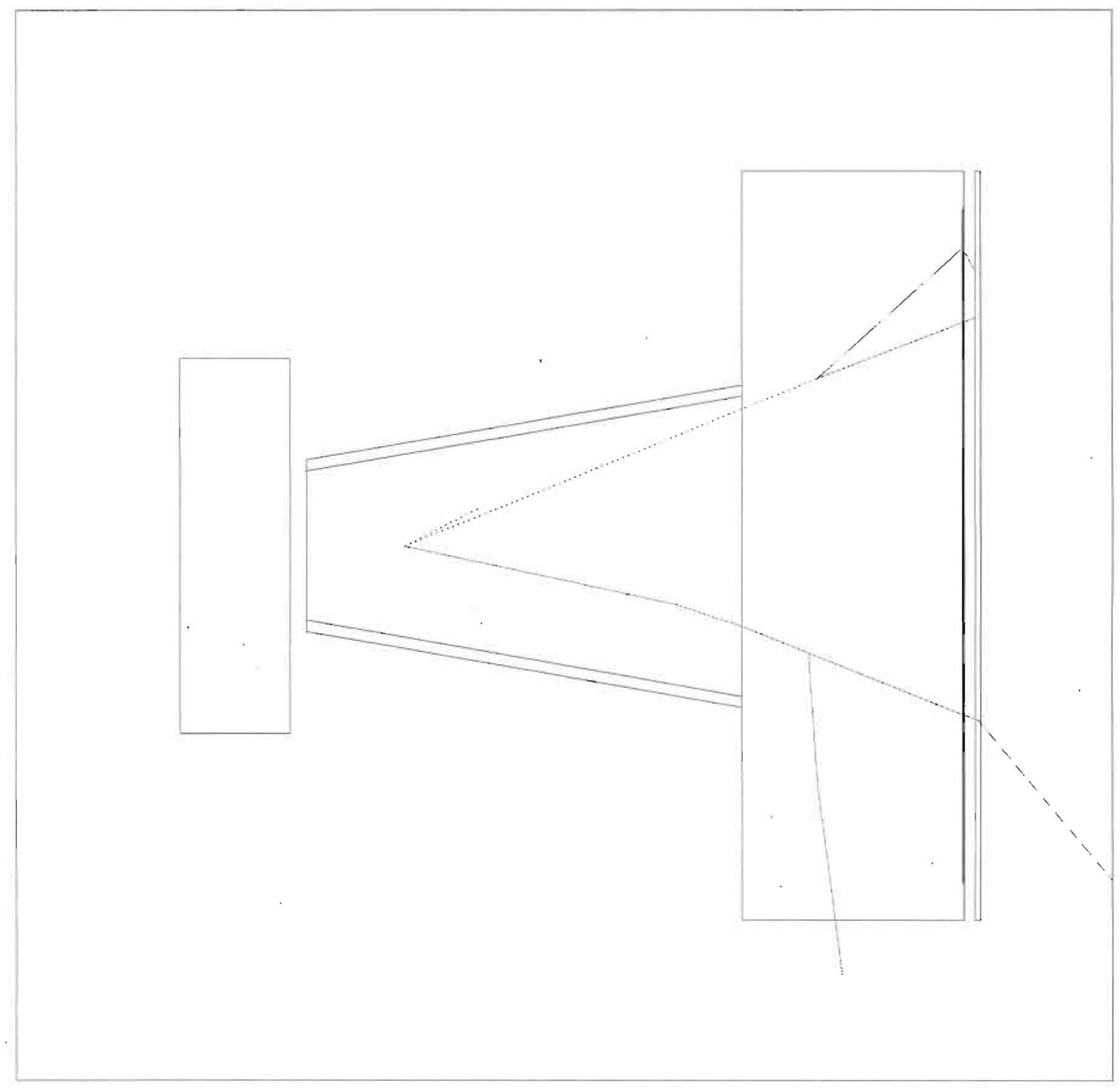

Figure 166: A typical GEANT event where one of the decay $K^{+} \rightarrow \pi^{+} \pi^{0}$ photons converts in the pion RICH gas. Photons are dot-trajectories and charged particles are dashed-trajectories. The photon entering the pion $\mathrm{RICH}^{*}$ volume is seen to convert in the neon gas, and the charged pion (lower track) produces a hard delta-ray in the neon gas as well. 
listed in section 13.2 to determine the fraction of events of either type which satisfy all analysis cuts and fall inside the signal region. No background events appear at this level. The probability of an elastic $K^{+}$scatter in the last silicon plane is $1.2 \times 10^{-4}$ and the probability for a Mott scatter in the first straw chamber is $3.6 \times 10^{-5}$. (The Mott cross section goes as $1 / q^{4}$ and this probability corresponds to a small angle cut-off of $2 \mathrm{mrad}$ in the center of mass.) Thus, the effective branching ratio limit on this background is:

$$
\begin{gathered}
B_{\mathrm{eff}}=\left(\frac{1}{\epsilon_{\pi \nu \nu}}\right)\left(B\left(K^{+} \rightarrow \mu^{+} \nu_{\mu}\right)\right)\left(\text { fraction } K^{+} \rightarrow \mu^{+} \nu_{\mu}\right. \text { events passing cuts)(muon rejection) } \\
B_{\mathrm{eff}}=\left(\frac{1}{0.018}\right)(0.64)\left[\left(1.2 \times 10^{-4}\right)\left(\frac{<1}{2 \times 10^{6}}\right)+\left(3.6 \times 10^{-5}\right)\left(\frac{<1}{1 \times 10^{6}}\right)\right]\left(1 \times 10^{-5}\right)<3.4 \times 10^{-14} .
\end{gathered}
$$

\subsection{3 $K^{+} \rightarrow \mu^{+} \nu_{\mu} \gamma$ Background Study}

The $K^{+} \rightarrow \mu^{+} \nu_{\mu} \gamma$ decay with branching fraction $5 \times 10^{-3}$ is potentially dangerous because it is not a two-body decay. That is, the presence of the photon has the effect of removing the constraint of two-body kinematics. It can populate the signal region we have defined. Some addition rejection is available because of the photon, but that rejection is limited by a couple of factors: (1) the photon tends to have low energy and (2) the photon tends to be collinear with the $\mu^{+}$so that our non-coalescence criterion ( $10 \mathrm{~cm}$ separation at the forward photon veto) may not be satisfied; of course the spectrometer magnet helps by deflecting the muon. Our calculated rejection for the $K^{+} \rightarrow \mu^{+} \nu_{\mu} \gamma$ photon is $6 \times 10^{-2}$.

We have simulated $2 \times 10^{6} \dot{K}^{+} \rightarrow \mu^{+} \nu_{\mu} \gamma$ events in CKM. At this level no events pass all analysis cuts. Thus, the effective

$$
\begin{gathered}
B_{\mathrm{eff}}=\left(\frac{1}{\epsilon_{\pi \nu \nu}}\right)\left(B\left(K^{+} \rightarrow \mu^{+} \nu_{\mu} \gamma\right)\right)\left(\text { fract } K^{+} \rightarrow \mu^{+} \nu_{\mu} \gamma \text { pass cuts }\right)(\gamma \text { rejection })(\mu \text { rejection }) \\
B_{\mathrm{eff}}=\left(\frac{1}{0.018}\right)\left(5 \times 10^{-3}\right)\left(\frac{<1}{2 \times 10^{6}}\right)\left(6 \times 10^{-2}\right)\left(1 \times 10^{-5}\right)<8.3 \times 10^{-14} .
\end{gathered}
$$

\subsection{Photon loss mechanisms in detector material}

Decay photons from $K^{+} \rightarrow \pi^{+} \pi^{0}$ and $K^{+} \rightarrow \mu^{+} \nu_{\mu} \gamma$ that are born in the vacuum decay volume must traverse the material of the downstream spectrometer and the pion RICH before intercepting the Forward Veto System. Since the walls of the pion RICH are inactive, it is possible for decay photons to get lost in the pion RICH through conversion, scattering, and energy loss mechanisms. In principle the pion RICH itself can contribute to the identification of conversion electrons and positrons if they are produced above Cherenkov threshold ( $41 \mathrm{MeV}$ ) and within the angular acceptance of the RICH. To be conservative we have ignored this potential rejection power in evaluating this loss mechanism.

Figure 166 shows one such $K^{+} \rightarrow \pi^{+} \pi^{0}$ decay in our GEANT simulation, with one of the photons from the $\pi^{0}$ decay interacting in the gas inside the pion RICH detector. Despite the clear presence of events of this nature, ten million GEANT events were simulated and only two events were not vetoed. These two events were lost to the nominal inefficiencies listed in table 11, and not to material interactions in the pion RICH. This inefficiency level of $\sim 2 \times 10^{-7}$ is consistent with the $K^{+} \rightarrow \pi^{+} \pi^{0}$ background estimate described early based on a weighted Monte Carlo technique. Further investigation into the loss mechanisms for photons will be performed using this simulation. 


\subsection{Beam-Detector Interaction Backgrounds}

Hadronic interactions of a beam $K^{+}$or $\pi^{+}$, or of a daughter $\pi^{+}$from a $K^{+}$decay, can create backgrounds in a $K^{+} \rightarrow \pi^{+} \nu \bar{\nu}$ search. Hadronic interactions were identified as a problem in the 1989 Main Injector Workshop report [117] on $K^{+} \rightarrow \pi^{+} \nu \bar{\nu}$ in-flight (which referred to "window scattering events") and were deemed to be the limiting background for an in-flight $K^{+} \rightarrow \pi^{+} \nu \bar{\nu}$ search. While further improvement is planned in our simulation of these effects, we have gone far beyond any previous effort to understand how they affect an in-flight $K^{+} \rightarrow \pi^{+} \nu \bar{\nu}$ search.

It should be emphasized that hadronic scattering in $K^{+} \rightarrow \pi^{+} \pi^{0}, K^{+} \rightarrow \mu^{+} \nu_{\mu}$, and $K^{+} \rightarrow \mu^{+} \nu_{\mu} \gamma$ decays was accounted for in the background estimates already discussed. Here we are concerned with other background scenarios where a hadronic interaction causes an event to fake $K^{+} \rightarrow \pi^{+} \nu \bar{\nu}$. To study these problems, we have used the same Monte Carlo described earlier to swim particles and to simulate the CKM detector. However, as a starting point (generator) for inelastic hadronic interactions we have used the FRITIOF program [196]. This is a standard program for hadronnucleus interactions which is part of the Lund Monte Carlo family.

We have taken steps in the CKM design to minimize hadronic interactions in critical locations by putting tracking detectors inside vacuum and minimizing the mass of those detectors. As will be discussed below, the most dangerous location for inelastic hadronic interactions is in the last beam vacuum tracker. To achieve extra rejection for these events, the CKM design includes a special veto device (Beam Interaction Veto System) immediately downstream of the last beam tracker. Most hadronic interactions will create extra particles, and the Beam Interaction Veto System is designed to detect the low-energy, wide-angle charged and neutral hadrons coming from hadronic interactions in the last beam vacuum tracker.

\subsubsection{Charge-Exchange}

The most complicated potential background occurs when a beam $K^{+}$hadronically interacts in material upstream of the decay volume to produce a high momentum $K_{L}$ which subsequently decays via $K_{L} \rightarrow \pi^{+} e^{-} \nu$ inside the decay volume. The resulting $\pi^{+}$comes from a three-body kaon decay with two effectively massless partners, so kinematically it is similar to $K^{+} \rightarrow \pi^{+} \nu \bar{\nu}$. The extra electron may provide a veto signature, but in rare cases the electron may be missed. It is conventional to refer to this as the charge-exchange background.

Because the $K_{L}$ is long-lived, the offending $K^{+}$interaction can occur far upstream of the detector. However, in that case it is necessary for the interaction also to create another charged particle to provide the requisite hits in the beam vacuum trackers. Interactions in the beam RICH may accomplish this, but if the $K^{+}$interacts at an early point in the beam $\mathrm{RICH}$ it is unlikely to satisfy the minimum requirement of $6 \mathrm{RICH}$ hits. Also, these far-upstream interactions will be the easiest to reject based on tracking and vertex quality cuts. Far more dangerous is an interaction in the last beam tracker. Our cuts have been designed with effects such as this in mind. In particular the vertex $D O C A<0.6 \mathrm{~cm}$ cut and $20 \mathrm{~m}<z_{\text {vertex }}<40 \mathrm{~m}$ (no vertex with $5 \mathrm{~m}$ of nearby material) provide powerful rejection.

To pose a potential threat to CKM the $K_{L}$ must have momentum above about $16 \mathrm{GeV}$, a fact we have determined with Monte Carlo studies. Fortunately this process is suppressed in CKM because any $K^{0}$ produced in an interaction with momentum close to the beam $K^{+}$momentum is likely to carry the $\bar{s}$ quark from the $K^{+}$. This is equivalent to saying the dilution factor $D$ is near unity in $K^{+} \rightarrow K^{0}$ for high momentum $K^{0}$ 's. It is $(1-D)$ that figures in the background process in CKM. In that case it will mostly decay to $\pi^{-} e^{+} \nu$ rather than $\pi^{+} e^{-} \nu$ provided the decay occurs within the first several $K_{S}$ lifetimes. For a typical case in CKM, a $19 \mathrm{GeV} K^{0}$ has $c \tau \gamma$ of about 
$1 \mathrm{~m}$, so $\pi^{+} e^{-} \nu$ decays are suppressed for decays within $10 \mathrm{~m}$ or so of the point of production.

The rejection of this background has been calculated in three pieces, all starting witl a set of FRITIOF generated $K^{+} \rightarrow K^{0}$ interactions. One piece is the probability for an event of this type to satisfy our analysis cuts on the $K^{+}$and $\pi^{+}$. Another piece is the probability for a photon, charged pion, or proton, to reach the Beam Interaction Veto System. The third piece is the probability that the electron in the event can be detected and provide the basis of a veto.

Initially we generated events in which the interaction took place anywhere in the material upstream of the decay volume. Figure 167 shows the $z$ distribution of such events, with a flat region from $-14 \mathrm{~m}$ to $-4 \mathrm{~m}$ showing interactions in the beam $\mathrm{RICH}$ gas, a spike at $-4 \mathrm{~m}$ corresponding to the beam RICH mirror, and two smaller spikes at $-2 \mathrm{~m}$ and $0 \mathrm{~m}$ indicating the locations of the vacuum trackers. We verified that any background contribution from this source was dominated by interactions in the most downstream material. Specifically, applying only our vertex cuts to the events shown in figure 167 removes all events where the interaction occurred at upstream of the last beam vacuum tracker.

In fact the $2 \mathrm{~m}$ gap between the beam RICH and the vacuum trackers was introduced specifically to move beam $\mathrm{RICH}$ interactions upstream to a location where they were readily suppressed. A simulation of $2 \times 10^{6}$ events was run. After all cuts 277 events appear in the signal region. The Beam Interaction Veto System detects particles emerging from the last beam tracker at relatively large angles. Its purpose is to flag events where hadronic interactions occur in that tracker. We use the extra hadrons generated by FRITIOF to estimate the efficacy of this device in the same events. Using FRITIOF, we count photons with energy above $20 \mathrm{MeV}$ into the BIVS, and hadrons reaching the BIVS within a 5 nsec time window (the limiting factor for detecting soft charged hadrons is not their energy, which is adequate, but the delay in their arrival because $\beta<1$ ). Since detection of these types of particles should be fairly efficient, we can estimate the inefficiency of this device from the fraction of events where no associated particle intercepts the BIVS to be $3.5 \%$.

The extra electron in these events can also be detected in most cases, providing additional rejection. A potential danger is that the very low-energy electrons will not be detected. They emerge from the decay at large laboratory angles and strike the Vacuum Veto System. For electron rejection we have followed the assumptions outlined in the discussion in section 3.4.6. That is, below $2 \mathrm{MeV}$ an electron is lost, between 2 and $20 \mathrm{MeV}$ it is detected with $90 \%$ efficiency, between 20 and $40 \mathrm{MeV}$ it is detected with $99 \%$ efficiency, and above that the efficiency is the same as for photons. To estimate a rejection factor we have used the energy spectrum of the electrons weighted by these factors. The resulting rejection is $2 \times 10^{-3}$.

To estimate the effective branching ratio of this background we also need to account for the following factors: (1) the events are induced by undecayed beam $K^{+}$'s $\left(17 \%\right.$ of $22 \mathrm{GeV} K^{+}$'s decay in decay volume.) (2) the interaction probability in the relevant tracker is $4.0 \times 10^{-4}$, (3) the fraction of inelastic $K^{+}$interactions that produce a $K_{L}$ with sufficient momentum to induce this scenario ( $16 \mathrm{GeV}$ from studying the events) is 0.012 , (4) the $K_{L}$ decay rate is a factor of 4.2 lower than the $K^{+},(5)$ the mean momentum of the relevant $K_{L}$ 's is about $19 \mathrm{GeV}$ rather than $22 \mathrm{GeV}$, and (6) the $K_{L} \rightarrow \pi^{+} e^{-} \nu$ branching ratio is $0.39 / 2$. The various factors may be combined to provide an estimate of the effective branching ratio of this background scenario:

$$
\begin{aligned}
B_{\mathrm{eff}}= & \left(\frac{1}{0.018}\right)\left(\frac{1}{0.17}\right)\left(4 \times 10^{-4}\right)(0.012)\left(\frac{0.17}{4.2}\right) \times \\
& \left(\frac{22}{19}\right)\left(\frac{0.39}{2}\right)\left(\frac{277}{2 \times 10^{6}}\right)\left(2 \times 10^{-3}\right)(0.035)<1.4 \times 10^{-13} .
\end{aligned}
$$

We regard this as an upper bound because we have not included the suppression due to the dilution 


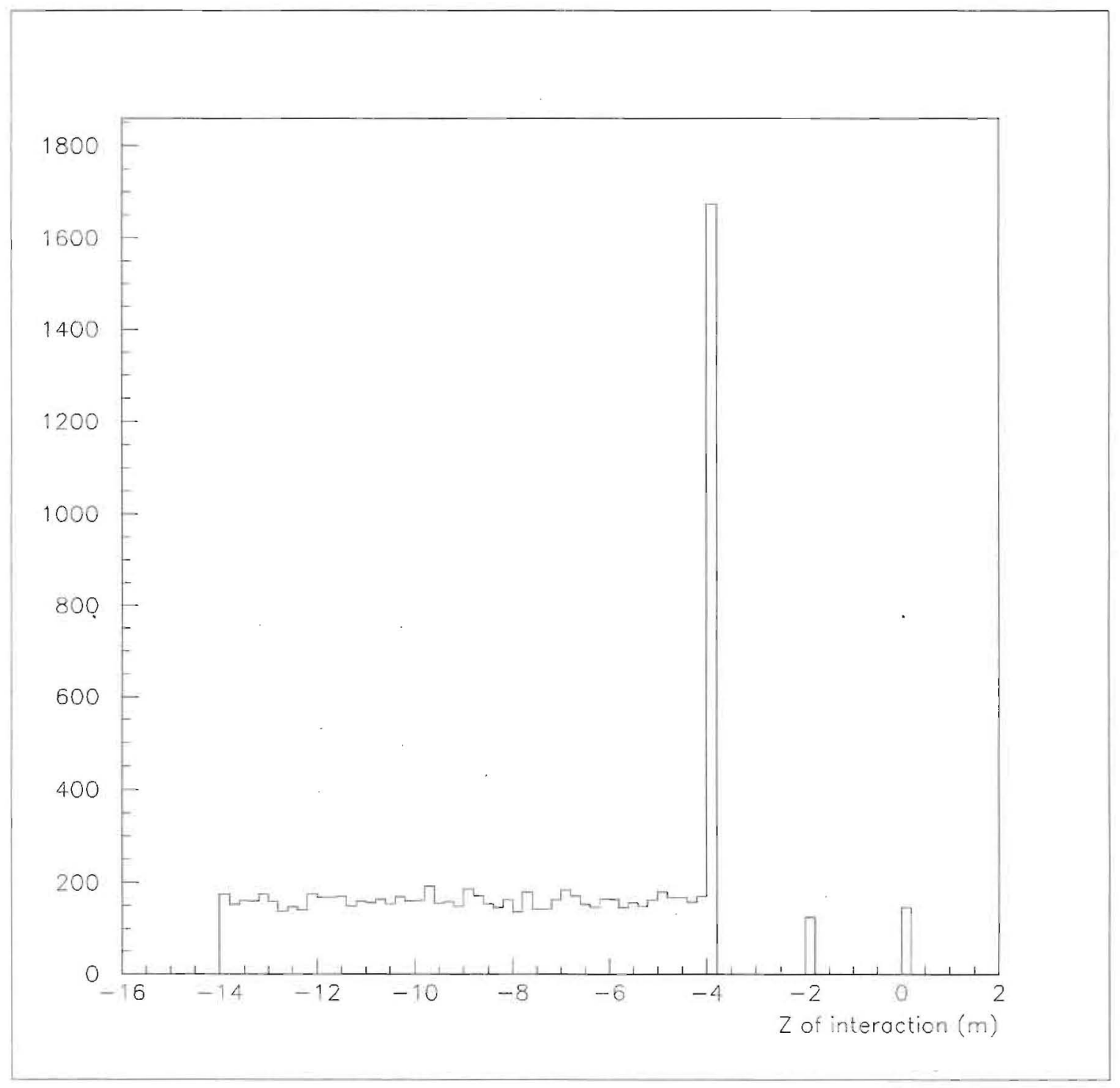

Figure 167: The location of 10,000 hadronic interactions in beamline material. In the $z$ scale of this figure (not the same as figure 42) the beam RICH spans the range $-14 \mathrm{~m}<z<-4 \mathrm{~m}$, with the mirror located at $z=-4 \mathrm{~m}$. The two beam vacuum trackers are located at $z=-2 \mathrm{~m}$ and $z=0$. 
factor (the $(1-D)$ effect). This will be significant since events which pass our vertex quality cuts are strongly biased toward the upstream part of the decay volume.

\subsubsection{Other Interactions in Detectors}

Other "window scattering" scenarios, i.e., hadronic interactions in the material immediate upstream and downstream of the decay volume, must be considered. The dominant scenario would be an inelastic beam $K^{+}$interaction in either the last beam vacuum tracker or the first straw chamber that produces a fast forward $\pi^{+}$which is a candidate for the $\pi^{+}$from a signal event. For now we assume there is a beam hole in the first downstream straw chamber so $K^{+}$'s cannot interact in it. For $K^{+}$interactions in the last beam vacuum tracker (KEAT) plane, substantial rejection comes from cuts on vertex quality and location $\left(D O C A<0.6 \mathrm{~cm}\right.$ cut and $20 \mathrm{~m}<z_{\text {vertex }}<40 \mathrm{~m}$, i.e., no vertex with $5 \mathrm{~m}$ of nearby material). Indeed, the only situation that leads to such an egregious mismeasurement of the vertex location occurs when the $\pi^{+}$hadronically scatters in the first straw chamber so that the direction of the $\pi^{+}$is corrupted.

This class of events of is dominated by charge-exchange processes in the KEAT material where a fast forward pion is created as well as a moderate energy $K^{0}$. In these events additional rejection comes from the BIVS because extra particles such as the exchange $K^{0}$ are usually produced along with the $\pi^{+}$.

We have generated $2 \times 10^{5}$ events with a $\pi^{+}$with momentum greater than $14 \mathrm{GeV}$, the minimum value allowed for the $K^{+} \rightarrow \pi^{+} \nu \bar{\nu}$ analysis, and a production angle less than $15 \mathrm{mrad}$. (The angular acceptance of the CKM detector, as simulated here, is about $13 \mathrm{mrad}$, defined by the size of the first straw chamber at a distance of $30 \mathrm{~m}$ from the production point. A typical hadronic $p_{\perp}$ of $300 \mathrm{MeV}$ at a momentum of $14 \mathrm{GeV}$ corresponds to a 21 mrad production angle, which over the $30 \mathrm{~m}$ to the first straw tracker is $64 \mathrm{~cm}$, far beyond the edge of the chamber.) In the Monte Carlo we force the $\pi^{+}$to elastically scatter in the first straw tracker, an effect that should occur with probability of $2.8 \times 10^{-4}$. The estimation procedure for this background is similar to that for the charge exchange case. The additional pieces of information needed are: (1) the probability of an inelastic $K^{+}$interaction producing a $\pi^{+}$with $p>14 \mathrm{GeV}$ with $\theta<15 \mathrm{mrad}$, which is $7.5 \times 10^{-3}$ according to FRITIOF, (2) in this Monte Carlo sample events pass all cuts on the $K^{+}$and $\pi^{+}$and fall inside the signal region, and (3) the BIVS/VVS inefficiency for this class of interactions is at most $2.75 \%$. Consequently, the effective branching ratio of this background scenario is:

$$
B_{\text {eff }}=\left(\frac{1}{0.018}\right)\left(\frac{1}{0.17}\right)\left(4.0 \times 10^{-4}\right)\left(7.5 \times 10^{-3}\right)\left(2.8 \times 10^{-4}\right)\left(\frac{106}{2 \times 10^{5}}\right)(0.0275)<4.0 \times 10^{-12} .
$$

The interaction of a beam $\pi^{+}$in the last vacuum tracker could also create a $\pi^{+}$in the acceptance of the CKM detector. This is removed by the requirement placed on beam RICH hits. A pion far off-momentum (at about $6 \mathrm{GeV}$ ) could fake a $K^{+}$, but it could not produce a $14 \mathrm{GeV}$ pion in an interaction.

\subsubsection{Beam Gas Interactions}

An interaction of a $K^{+}$with residual gas in the vacuum decay volume can produce a $\pi^{+}$that falls into the acceptance of the CKM detector. Vertex quality cuts will not assist in rejecting these events. The best protection, of course, is a good vacuum. We assume $10^{-6}$ torr, which has been achieved in $\mathrm{KTeV}$. Any such interaction will also produce other observable particles, and most beam gas interactions can be rejected on that basis. 
The $K^{+}$interaction length in air at 1 atm is about $470 \mathrm{~m}$, so at $10^{-6}$ torr it is smaller by a factor of $10^{-6} / 760=1.3 \times 10^{-9}$. Hence, the interaction probability in $30 \mathrm{~m}$ is

$$
\left(1.3 \times 10^{-9}\right)\left(\frac{30}{470}\right)=8.4 \times 10^{-11} .
$$

We have simulated 12,000 beam gas interactions, using FRITIOF to generate $K^{+}$interactions that produce a $\pi^{+}$with $p>14 \mathrm{GeV}$. Of all $K^{+}$interactions in FRITIOF, $2.5 \%$ produce such a $\pi^{+}$. The interactions are generated flat in $z$ between the last vacuum tracker and the first straw tracker. The events are subjected to the standard cuts, with 70 passing. Also, $48 \%$ of the interactions produce ${ }^{0}$ 's for which the veto probability is effectively unity. The effective branching ratio of this backgrom is then:

$$
\begin{gathered}
B_{\mathrm{eff}}=\left(\frac{1}{\epsilon_{\pi \nu \nu}}\right)\left(\frac{1}{K^{+} \text {decay }}\right)(\text { prob interact })\left(\text { fract } p_{\pi}>14 \mathrm{GeV}\right)(\text { frac pass cuts })\left(\pi^{0} \text { rejection }\right) \\
B_{\mathrm{eff}}=\left(\frac{1}{0.018}\right)\left(\frac{1}{0.17}\right)\left(8.4 \times 10^{-11}\right)(0.025)\left(\frac{70}{12000}\right)(0.52)<2.1 \times 10^{-12}
\end{gathered}
$$

We consider this to be a conservative upper limit since charged hadrons from beam gas interactions will also be used to veto.

\subsection{Accidental Background Sources}

By accidentals background we mean any process by which two beam particles conspire to fake $K^{+} \rightarrow \pi^{+} \nu \bar{\nu}$. The two beam particles must be close in time, yet one must go undetected. Both $K^{+}$and $\pi^{+}$will be detected by the beam RICH as well as the beam trackers, so the a priori probability is small for conditions to exist for an accidental background event to occur. Even if a $K^{+}$or $\pi^{+}$enters the decay volume undetected, there is no problem unless it decays. If a $\pi^{+}$decays, it almost always produces a muorl, for which CKM has enormous rejection. The detected $K^{+}$must also decay, since we will veto events with beam particles in-time with $K^{+} \rightarrow \pi^{+} \nu \bar{\nu}$ candidates. We can project the $K^{+}$trajectory from the beam vacuum trackers to the scintillating fiber tracker which spans the beam and make a requirement that no beam particle consistent with a non-decayed $\mathrm{K}^{+}$appear. Even if the scintillating fiber tracker suffers some level of inefficiency, the Charged Veto Plane will also have a shot at it, making a complete miss unlikely.

Thus we are led to conclude two $K^{+}$decays are the most plausible candidate accidental background. To be outside the scope of prior estimates, the event must be confused by associating the $\pi^{+}$ from one decay (the undetected $K^{+}$) with the the detected $K^{+}$, whose decay products must escape completely unnoticed. One can imagine a $K^{+} \rightarrow \mu^{+} \nu_{\mu}$ decay where the muon is very low-energy in the laboratory. Then the undetected $K^{+}$should decay via $K^{+} \rightarrow \pi^{+} \pi^{0}$ or $K^{+} \rightarrow \pi^{+} \pi^{+} \pi^{-}$in order to provide a $\pi^{+}$candidate. However, one can see from figure 11 that $K^{+} \rightarrow \pi^{+} \pi^{+} \pi^{-}$cannot produce a $\pi^{+}$with $p>14 \mathrm{GeV}$, so we will focus on $K^{+} \rightarrow \pi^{+} \pi^{0}$.

So, we need: (1) the probability of detecting only one of two $K^{+}$'s that enter CKM close in time, (2) the probability that one will decay with no observed products, and (3) the probability that a $K^{+} \rightarrow \pi^{+} \pi^{0}$ decay will pass all cuts when its kinematics are corrupted by using the wrong beam $K^{+}$.

It is reasonable to take the probability of a second $K^{+}$in-time with the first to be about $1 \mathrm{nsec} / 30 \mathrm{nsec}$, roughly the timing resolution divided by the time between $K^{+}$'s on average. Also, we expect a tracking inefficiency of about $0.1 \%$ for beam particles due to algorithm inefficiencies (silicon is intrinsically very efficient). Also, we will reject events in which the beam RICH has 
too many hits, above 20 , which rejects about half of these events. Thus, we take factor (1) to be $1.7 \times 10^{-5}$.

For factor (2) we have simulated $K^{+} \rightarrow \mu^{+} \nu_{\mu}$ events to determine the fraction of decays where a mion (for which the minimum momentum is $1 \mathrm{GeV}$ in $22 \mathrm{GeV} K^{+}$decays) below RICH threshold misses both the Vacuum Veto System and the Forward Veto System. This is a conservative estimate since we do not utilize available tracker information. With this fraction, $9.5 \%$, we estimate factor (2) to be $(0.21)(0.64)(0.095)=0.013$. The first factor is the decay probability upstream of the scintillating fiber tracker and the second is the branching ratio.

To evaluate factor (3) we have used the Monte Carlo and simply calculated all quantities from one $K^{+} \rightarrow \pi^{+} \pi^{0}$ decay mixed with another beam $K^{+}$. We have generated $5 \times 10^{6}$ such events and found that 1288 events pass all cuts on the (wrong) $K^{+}$and $\pi^{+}$and fall in the signal region. Of course the $\pi^{0}$ in these events provides addition rejection. We have determined the $\pi^{0}$ rejection for these events using the same method described earlier. The rejection is not as good as in a correctly measured event, since there is some probability that the decay occurs farther downstream than $z=25 \mathrm{~m}$, making it possible for photons to escape at wide angles out the side of the pion RICH. The $\pi^{0}$ rejection of these events is found to be $3.3 \times 10^{-4}$.

These factors can be combined to obtain an estimate of the effective branching ratio of the accidentals background:

$$
\begin{gathered}
B_{\mathrm{eff}}=\left(\frac{1}{\epsilon_{\pi \nu \nu}}\right)\left(B\left(K^{+} \rightarrow \pi^{+} \pi^{0}\right)\right)(\text { prob miss } 2 \text { nd } K) \times \\
\left(\text { prob miss } K^{+} \rightarrow \mu^{+} \nu_{\mu}\right)(\text { frac pass cuts })\left(\pi^{0} \text { rejection }\right) \\
B_{\mathrm{eff}}=\left(\frac{1}{0.018}\right)(0.21)\left(1.7 \times 10^{-5}\right)(0.012)\left(\frac{1288}{2 \times 10^{6}}\right)\left(3.3 \times 10^{-4}\right)=5.1 \times 10^{-13} .
\end{gathered}
$$

Clearly this is in many respects an imperfect estimate. The point of it is that with reasonable assumptions a plausible accidental scenario does not pose a problem. That does not prove some scenario which has been overlooked cannot pose a problem, but it indicates that accidentals do not seem to be an area of special danger.

\subsection{Background Summary}

We collect the background estimates made in earlier sections in table 41 . Our conclusion is that $K^{+} \rightarrow \pi^{+} \pi^{0}$ decays and tracker-window beam interactions are are the most challenging. We must clearly work hard to maintain our specs for kinematic resolution, photon veto rejection, and the ability to veto particles associated in beam interactions with detector material.

At this point we will identify the two areas where our background simulations seem to us the weakest:

1. We do not simulate the tracking problem. That is, we do not generate hits in trackers along with noise hits and then reconstruct tracks. When this is done mistakes can occur that we do not account for in the current methodology. Also, non-Gaussian resolution effects need to be investigated and may need to be included.

2. We have not compared FRITIOF results to those of another hadronic interaction generator. We have found however that the predictions of FRITIOF generally agree with published measured inclusive data for $K^{0}$ production and $\pi^{+}$production. We do not expect the range of possible errors to be large, but comparisons with other generators are an obvious check we have not yet carried out. The estimate of the $K^{+} \rightarrow \pi^{+} \pi^{0}$ background does not rely on FRITIOF in any way. 


\begin{tabular}{|l|c|}
\hline Background Source & $\begin{array}{c}\text { Effective BR } \\
\left(\times 10^{-12}\right)\end{array}$ \\
\hline $\bar{K}^{+} \rightarrow \mu^{+} \nu_{\mu}$ & $<0.04$ \\
\hline$K^{+} \rightarrow \pi^{+} \pi^{0}$ & 3.7 \\
\hline$K^{+} \rightarrow \mu^{+} \nu_{\mu} \gamma$ & $<0.09$ \\
\hline$K^{+} A \rightarrow K_{L} X$ followed by $K_{L} \rightarrow \pi^{+} e^{-} \nu$ & $<0.14$ \\
\hline$K^{+} A \rightarrow \pi^{+} X$ in trackers & $<4.0$ \\
\hline$K^{+} A \rightarrow \pi^{+} X$ in residual gas $\left(10^{-6}\right.$ torr $)$ & $<2.1$ \\
\hline Accidentals $\left(2 K^{+}\right.$decays $)$ & 0.51 \\
\hline \hline Total & $<10.6$ \\
\hline
\end{tabular}

Table 41: Background estimates in terms of effective branching ratio.

We expect the quality of our studies will be improved over time and that we will be able to assuage the concerns of a skeptical reviewer before any metal is cut.

Finally, it should be emphasized that an important aspect of CKM is the ability to enhance interaction-induced backgrounds in a controlled way by increasing the material in the path of particles. This provides an extremely powerful tool for measuring backgrounds.

\section{References}

[1] G. Buchalla and A.J. Buras, Phys. Rev. D54, 6782 (1996).

[2] D. Rein and L.M. Sehgal, Phys. Rev. D39, 3325 (1989).

[3] J.S. Hagelin and L.S. Littenberg, Prog. Part. Nucl. Phys. 23, 1 (1989).

[4] M. Lu and M. Wise, Phys. Lett. B324, 461 (1994).

[5] S. Fajfer, Nuovo Cim. 110 A, 397 (1997).

[6] S. Adler, et.al., Phys. Rev. Lett. 79, 2204 (1997). S. Adler, et.al., Phys. Rev. Lett. 84, 3768 (2000).

[7] T. Inami and C. S. Lim, Prog. Theor. Phys. 65, 297 (1981).

[8] J. Ellis, J. S. Hagelin, Phys. Lett. B192, 201 (1983);

Nucl. Phys. B217, 189 (1983).

[9] C. O. Dib et al., Mod. Phys. Lett. A6, 3573 (1991).

[10] W. Marciano, Z. Parsa, Phys. Rev. D53, R1 (1996).

[11] G. Buchalla et al., Rev. Mod. Phys. 68, 1125 (1996).

[12] A. J. Buras hep-ph/9711217, TUM-HEP-299/97. CKM Matrix: Present and Future Andrzej J. Buras (Technische Universitaet Muenclien, Physik Department) Invited talk given at the Symposium on Heavy Flavours, Santa Barbara, July 7-July 11, 1997, to appear in the proceedings. 
[13] A. J. Buras, R. Fleischer, hep-ph/9704376, TUM-HEP-275/97, TTP 97-15. Quark mixing, $\mathrm{CP}$ violation and rare decays after the top quark discovery. To appear in Heavy Flavours II, World Scientific (1997), Eds. A.J.Buras and M. Lindner.

[14] A. J. Buras, hep-ph/9806471, TUM-HEP-316/98. Weak Hamiltonian, CP Violation and Rare Decays Les Houches Lectures, to appear in "Probing the Standard Model of Particle Interactions", F.David and R. Gupta, eds, Elsevier Science B.V.

[15] G. Buchalla, A. J. Buras, hep-ph/9901288, Nucl. Phys. B548, 39 (1999).

[16] A. J. Buras, hep-ply/9905437. Lectures at 14 Lare Louise Winter Institute, February 1999.

[17] A. Falk, A. Lewandowski, A. Petrov, hep-ph/0012099, Dec 2000.

[18] G. Buchalla, hep-ph/9912369, CERN-TH/99-325;

CP Violation and Rare Kaon Decays, Plenary talk at EPS-HEP99, Tampere, Finland. G. Buchalla, hep-ph/0002207, CERN-TH/2000-057. Theoretical Update on Rare K Decays, Invited talk presented at Daphne 99, Frascati, Nov. 1999.

[19] G. Isidori, hep-ph/9902235;

Precision tests and searches for New Physics with K decays, talk presented at the "International Workshop on CP Violation in K", 18-19 December 1998, KEK-Tanashi, Tokyo.

G. Isidori, hep-ph/9908399;

Standard Model vs New Physics in Rare Kaon Decays, talk presented at KAON $99-21-26$ Jun 1999- Chicago Univ.

[20] A. R. Barker, S. H. Kettell, Anı.Rev.Nucl.Part.Sci.50: 249-297, 2000, hep-ph/0009024; BNL 67590.

[21] S. Bosch et al., Nucl. Phys., B565, 3 (2000).

[22] G. C. Branco et al., CP violation, Clarendon Press, Oxford, 1999.

[23] D. E. Groom et al., Europ. Phys. Journ. C15, 1 (2000).

[24] I. I. Bigi, A. I. Sanda, CP violation, Cambridge University Press, 2000.

[25] N. Cabibbo, Phys. Rev. Lett. 53, 531 (1963).

[26] M. Kobayashi, and T. Maskava, Prog. Theor. Phys. 49, 652(1973).

[27] L. Wolfenstein, Phys. Rev. Lett. 51, 1945 (1983).

[28] M. Bargiotti et al., Riv. Nuov. Cim. 23, 1 (2000), hep-ph/0001293.

[29] P. Faccioli, hep-pl//0011269. CKM matrix: the "over-consistent" picture of the unitarity triangle; Contribution to Beauty 2000, September 13-18 2000, Kibbutz Maagan, Israel;' Proceedings (Nucl. Instr. Meth. A).

[30] A. Stocchi, hep-ph/0012215. Determination of Unitarity Triangle parameters: where do we stand; Invited talk given at the 7 th International Conference on B-Physics at Hadron Machines-Beauty2000, Kibbutz Maagan, Israel, September 13-18, 2000-Proceedings (Nucl. Instr. Meth. A). 
[31] M. Ciuchini et al. hep-ph/0012308, LAL 0077. 2000 CKM-Triangle Analysis A Critical Review with Updated Experimental Inputs and Theoretical Parameters, M. Ciuchini et al. Submitted to JHEP.

[32] S. Mele hep-ph0103040, Indirect Determination of the Vertex and Angles of the Unitarity Triangle. Presented at the 5th International Symposium on Radiative Corrections (RADCOR-2000) Carmel CA, USA, 11-15 September,2000.

[33] L. Littenberg, Phys. Rev. D39, 3322 (1989).

[34] Y. Grossman, Y. Nir, Phys. Lett. 398, 163 (1997); hep-ph/9701313.

[35] A. J. Buras et al., Nucl. Phys. B423, 349 (1994).

[36] J. F. Donoghue, F. Gubbiani, Phys. Rev. D51, 2187 (1995).

[37] E. Cheu et al. , Fermilab KAMI Proposal, 1997. "An Expression of Interest to Detect and Measure the Direct CP Violating Decay $K_{L} \rightarrow \pi^{0} \nu \bar{\nu}$ and Other Rare Decays at Fermilab Using the Main Injector," Fermilab-Pub-97-321-E.

[38] I. H. Chiang et al., KOPIO

a Search for $k \rightarrow \pi \nu \bar{\nu}$, NSF proposal, November (1999).

[39] Y. Nir, hep-ph/9911321, Lectures at the XXVII SLAC Summer School Institute on Particle Physics, 1999;

Y. Nir, hep-ph/9810520, Lectures at the 1998 Europ. School of High Energy Phys., Univ. of St. Andrews, Scotland.

[40] A. L. Kagan, M. Neubert, hep-ph/0007360;

J. P. Silva, L. Wolfenstein, hep-ph/0008004;

G. Eyal et al., hep-ph/0008009;

A. J. Buras, R. Buras, hep-pl//0008273.

[41] S. Bergman, G. Perez, JHEP 08(2000)034,hep. Standard Model correlations between $K->$ $\pi \nu \bar{\nu}$ decays and observables in B Physics-hep-ph/0007170.

[42] T. G. Rizzo, hep-ph/9809526. Rare K Decays and New Physics Beyond the Standard Model, "Proceedings of the Workshop on CP Violation", University of Adelaide, Adelaide, Australia, 3-8 July, 1998.

[43] J. L. Hewet et al., hep-ph/9603391, SLAC-PUB-7088, (1996). Indirect Probes of New Physics. In Electroweak Symmetry Breaking and Beyond the Standard Model.

[44] T. Hattori et al., hep-ph/9804412. $K^{+} \rightarrow \pi^{+} \nu \bar{\nu}$ with four generations and its effects on unitarity triangle,(Univ. Tokushima).

[45] G. Collangelo, G. Isidori, JHEP, 09, 009 (1998).

[46] A. J. Buras, L. Silvestrini, Nucl. Phys. B546, 299 (1999).

[47] A. J. Buras et al., Nucl. Phys. B566, 3 (2000), hep-ph/9908371. 
[48] L. Silvestrini, hep-ph/0009284. epsilon'/epsilon and Rare K Decays in the Standard Model and Supersymmetry, talk given at the 4th International Conference On Hyperons, Charm And Beauty Hadrons, 27-30 June 2000, Valencia, Spain. Nucl.Phys.Proc.Suppl. 93 (2001) $247-252$.

[49] A. J. Buras et al.,Phys.Lett. B500 (2001) 161-167 Universal Unitarity Triangle and Physics Beyond the Standard Model A.J. Buras, P. Gambino, M. Gorbahn, S. Jager, L. Silvestrini, hep-ph/0007085;

A. J. Buras et al., Nucl.Phys. B592 (2001) 55-91, epsilon'/epsilon and Rare K and B Decays in the MSSM, hep-ph/0007313.

[50] G. Isidori, hep-ph/0101121, SUPERSYMMETRIC EFFECTS IN RARE SEMILEPTONIC DECAYS OF B AND K MESONS. CERN-TH-2001-003, Sep 2000, Presented at 5th International Symposium on Radiative Corrections (RADCOR 2000): Applications of Quantum Field Theory to Phenomenology, Carmel, California, 11-15 Sep 2000.

[51] J. Ellis, hep-ph/0011396. Highlights of CP 2000, talk at International Conference On CP Violation Physics, Ferrara, Sept. 2000.

[52] G. Buchalla, G. Isidori,Phys.Lett. B440 (1998) 170-178. The CP Conserving Contribution to $K_{L} \rightarrow \pi^{0} \nu \bar{\nu}$, hep-ph/9806501.

[53] S. Baek et al., hep-ph/9907572 (?);

Fully supersymmetric CP violations in the kaon system, Contribution to the Proceedings of ICHEP2000, Osaka, 2000, hep-ph/0009105.

[54] Z. Xiao et al.,Eur.J.Phys. C7 (1999) 487-499, The rare K-decays in the Multiscale Walking Technicolor Model hep-ph/9903347.

[55] Y. Kiyo, T. Morozumi, hep-ph/9805307 RARE DECAY OF K MESON. HUPD-9809, Mar 1998, Talk given at Workshop on Fermion Mass and CP Violation, Hiroshima, Japan, 5-6 Mar 1998.

[56] G. Burdman, Phys.Rev. D59 (1999) 035001 Triple Gauge Boson Couplings in Rare B and K Decays hep-ph/9806360;

Gustavo Burdman hep-ph/9811457. Theoretical Issues in Rare K, D and B Decays, Talk presented at the Workshop on Heavy Quarks at Fixed Target, Fermilab, Batavia IL, October 10-12, 1998.

[57] G. Perez,JHEP 0002 (2000) 043. The $K_{L} \rightarrow \pi^{0} \nu \bar{\nu}$ Decay in Models of Extended Scalar Sector, hep-ph/0001037.

[58] G.-Ch. Cho, Eur. Phys. J. C5, 525 (1998).

[59] A. Alvari-Harati et al., Phys. Rev. (2000) (in press).

[60] A. Alvari-Harati et al., Phys.Rev.Lett.86:397-401,2001 SEARCH FOR THE DECAY $K_{L} \rightarrow$ $\pi^{0} e^{+} e^{-}$,By KTeV Collaboration ,FERMILAB-PUB-00-225-E, Sep 2000. hep-ex/0009030.

[61] N. Arkani-Hamed et al., Phys. Rev. Lett. B429, 263 (1998);hep-ph/9807344.

[62] F. Wilczek, Phys. Rev. Lett. 49, 1549 (1982). 
[63] S. Adler et al., Phys. Rev. Lett. 79, 4756 (1997).

[64] A. Masiero, O. Vives, Phys.Rev.Lett. 86 (2001) 26-29 Kaon vs. Bottom: Where to look for a general MSSM, hep-ph/0007320.

[65] P. Langacker, S.U. Sankar and K. Schilcher, Phys. Rev. D38, 2841 (1998)

[66] A. Acker and S. Pakvasa, Mod. Phys. Lett. A7, 1219 (1992).

[67] R. L. Kang, PhD. Thesis, U. of California at Irvine, Section 2.1.1.

[68] A. Belyaev et al., hep-ph/0008276. Charged-Lepton-Flavour Violation in Kaon Decays in Supersymmetric Theories, Prepared for the Kaon Physics Working Group as part of the ECFA/CERN study of Neutrino Factory 8 Muon Storage Rings At CERN.

[69] L. Littenberg, R. Shrock, Phys. Lett. B491, 285 (2000).

[70] R.Barbieri et al., Nucl. Phys. B449, 437 (1995).

[71] Z. Gagyi-Palffy et al., Nucl. Phys. B513, 517 (1998);

Phys.Lett. B343, 275 (1995).

[72] R.N. Cahn, H. Harari, Nucl. Phys. B176, 135 (1980).

[73] S. Davidson et al., Z.Phys. C61, 613 (1994).

[74] L.M. Barkov et al., Research Proposal to PSI (1999); http://www.icepp.s.u-tokyo.ac.jp; M. Bachman et al., MECO Proposal to BNL (1997); http://meco.ps.uci.edu.

[75] R. Appel et al., Phys. Rev. Lett. 85, 2450 (2000).

[76] R. Appel et al., Phys. Rev. Lett. 85, 2877 (2000).

[77] D. Ambrose et al., Phys. Rev. Lett. 81, 5734 (1998).

[78] KTeV preliminary result, shown at the conference of the APS Division of Particles and Fields, Columbus Ohio, Aug 9-12, 2000.

[79] A.R. Zhitnitskii, Sov.J.Nucl.Phys (in Russian) 32, 224 (1980); V.P.Efrosinin et al., hepph/0008199.

[80] G. Bekanger, C. Geng, Phys.Rev. D44, 2789 (1991);

R. Peccei, hep-ph/9909236, Overview of Kaon Physics to be published in the Proceedings of Kaon99, Chicago, Illinois, June 1999; Y. Grossman, Nucl. Phys. B426, 355 (1994).

[81] S. Blatt et al., Phys. Rev. D 27, 1056 (1983).

[82] M. Abe et al., Phys. Rev. Lett. 83, 4253 (1999).

[83] L.Littenberg, International Workshop on Kaon, Muon, and Neutrino Physics and the Future at KEK, Tsukuba, Japan, 31 Oct - 1 Nov 1997. BNL-65188, hep-ex/9802014.

[84] J. Gevais et al., Phys. Rev. D, 1020 (1966).

[85] H.Y. Cheng, Phys. Rev. D 26, 143 (1982). 
[86] L.G. Landsberg, V.F. Obraztsov, Proc. of the Workshop on K Physics (KAON-99), Chicago, USA, June 1999;

V.F. Obraztsov, L.G. Landsberg, hep-ex/0011033; Proc. Int. Conf. CP-Violation Physics, Ferrara, Italy, September 2000;

L.G. Landsberg, Preprint IHEP 2000-27, Protvino, 2000.

[87] V.N. Bolotov et al., Yad. Fiz. 44, 108 (1998).

[88] G. D’Ambrosio, G. Isidori Int. Journ. of Mod. Phys. A13, (1998);

Phys. Lett. B480, 164 (2000).

[89] L. Maiani, N. Paver The Second DAPHNE Physics Handbook, INFN Frascati, Italy, pg. 51 (1995).

[90] E. Shabalin, Preprint ITEP 8-98, 1998.

[91] V.V. Ammosov et al., Preprint IHEP 98-2, Protvino 1998 (in Russian);

A. Belkov et al., Proceedings of the ICHEP96 v.II, p.1204, World Scientific, 1997.

[92] W.T. Ford et al., Phys. Rev. Lett. 25, 1370 (1970).

[93] S. White et al., Nucl. Phys. Proc. Suppl. B71, 451 (1999);

Proc. of the Workshop on K Physics (KAON-99), Chicago, USA, June 1999,

N. Leros, Proc. Int. Conf. on CP-violation Physics, Ferrara, Italy, September 2000.

[94] R. Batley et al., CERN/SPSC/P253 add.3, 2000.

[95] The Second DAFNE Physics Handbook, v.1, IFNF, Frascati, Italy (1995).

[96] E. Shabalin, Pis'mo Zh. Eksp. Theor. Fiz., 22, 117 (1975).

[97] C. Dib et al., Phys.Lett. B493, 82 (2000).

[98] M. Schepkin, Private communication.

[99] K. Zuber, Phys.Lett. B479, 33 (2000).

[100] C. Picciotto, Phys. Rev. D56, 1612 (1997).

[101] R. Appel et al., Phys. Rev. Lett. 83, 4482 (1999).

[102] H. Ma et al., Phys. Rev. Lett. 84, 2580 (2000).

[103] G. D'Ambrosio et al., JHEP 9808,004 (1998).

[104] H. Burkhardt et al., hep-ph/0011345 (2000).

[105] D.E. Neville, Phys. Rev. 124, 2037 (1961); more recently, see K.A. Milton and W.W. Wada, Phys. Lett. B98, 367 (1981); J.N. Huang and C.Y. Lee, Phys. Rev. D 27,2227 (1983); D. Bryman, P. Depommier and C. Leroy, Phys. Rep. 88, 151 (1982). J. Bijnens, G. Ecker, and J. Gasser, Nucl. Phys B396, 81 (1993); J. Bijnens et al., in The Second DAPHNE Physics Handbook, INFN Frascati, Italy, pg. 315 (1995).

[106] S. Adler et al, Phys. Rev. Lett., 85, 2256 (2000). 
[107] J. Heintze et al, Nucl. Phys., B149, 365 (1979).

[108] Y. Akiba et al., Phys. Rev. D32, 2911 (1985);

V.S. Demidov et al., Sov.J. Nucl. Phys. 52, 1006 (1990).

[109] C. Milstene, P. Cooper, M. Moinester, hep-ex/9803033 presented at the Main Injector Workshop Fermilab, May 1997.

[110] J. Engelfried et al., The E781 (SELEX) RICH Detector. FERMILAB-CONF-97/210, Nucl.Instrum.Meth.,A409, 439 (1998).

[111] "HERA-B Status: A RICH Target", D. Broemmelsiek, Accelerator Physics and Technology Seminar, Fermilab, November 28,2000.

[112] J. Engelfried et al.,Nucl.Instrum.Meth.,A431, 53 (1999).

[113] The first large phototube based RICH system that we are aware of was employed in the SPHINX experiment. D. Vavilov et al. Yad. Fiz 57, 241 (1994) (in Russian), translation, Phys.At.Nucl, 57, 227 (1994).

[114] Specres, an analytical calculation of the magnetic spectrometer resolution, Peter Cooper, CKM internal memo.

[115] B. Rossi and K. Greisen, Rev. Mod. Physics, textbf13, 240 (1941).

[116] Review of Particle Physics, M.Baldo Ceolin et al., The European Physics Journal C 15 1$4,(2000)$.

[117] "Report of the Working Group on $K^{+}$Decay in-Flight", Collins, et al., from the Proceedings of the Workshop on Physics at the Main Injector, May 16th, 1989.

[118] L. Littenberg, BNL E787, private communication.

[119] J. Ritchie, CKM Note 26, Beam Energy Considerations for CKM, September 1998.

[120] The "MIST" workshop was held in May of 1997 and focused on the fixed target physics opportunities with the Fermilab Main Injector. Proceeding of the Workshop on Fixed Target Physics at the Main Injector, G.J. Bock and J.G. Morfin, ed., Fermilab-Conf-97/279 (1997).

[121] Discussions with Phil Martin and Chandra Bhat at the "Workshop on Fixed Target Physics at the Main Injector", May 1-4,1997.

[122] BNL E777, C. F. Campagnari, Ph.D. thesis, Yale University (1988).

[123] A. Brenner et al, Phys.Rev. D26,1497,(1982).

[124] A.J.Malensek,Fermilab FN-341, Oct.1981.

[125] P. Bernard et al, Particle Separation with Two- and Three-Cavity Separators at CERN,CERN 68-29,1-61 (1968).

[126] W.K.H. Panofsky, Stanford Report HEPL-82 (1956).

[127] B.W. Montague, CERN Internal Report PS/Int.AR/PSep/60-1 (1960). 
[128] W. Schnell, CERN 61-5 (1961).

[129] AGS 1965 rf sep.beam to 80B̈ubble Chamber $12.8 \mathrm{GeV} / \mathrm{c}$.

[130] P. Bramham et al, Physics Letters 15, 290 (1965).

[131] P. Bernard et al, Proc.Int.Conf. on High-Energy Accelerators, Cambridge (1967), 190.

[132] A. Citron et al, Nucl. Instr. and Meth. 155(1978)93-96 and A. Citron et al, Nucl. Instr. and Meth. 164(1979) 31-55.

[133] J. Doornbos, "Possibilities for a 15-25 GeV/c RF-separated charged kaon beam", NIM A $455(2000) 253-270$.

[134] T. Kobilarcik, CKM Note 37, Shielding Concerns for the RF Separated Beam, July 2000.

[135] T. Kobilarcik, CKM Note 38, Shielding Assessment for CKM, September 2000.

[136] TRANSPORT,K. L. Brown, F. Rothacker,D. C. Carey, Ch. Iselin, A Computer Program for Designing Charged Particle Beam Transport Systems, SLAC Report No 91(1977).

[137] C.T. Murphy, Fermilab, private communication.

[138] D. Edwards, editor. FERMILAB-TM-2060 (Oct. 1998).

[139] M. McAshan and R. Wanzenberg, "RF Design of a Transverse Mode Cavity for Kaon Separation", (Nov. 2000).

[140] http://www-bd.fnal.gov/Documents/3.9GHz_Scrf/.

[141] B. Aune et al, DESY 00-031 (Feb. 2000).

[142] H. Padamsee, J. Knobloch and T. Hays, "RF Superconductivity for Accelerators", Wiley (1998).

[143] T. Kobilarcik, CKM Note 26, CKM Beamline Design, March 2000.

[144] TURTLE, D. C. Carey, K. L. Brown, Ch. Iselin, September, 1999, Fermilab-Pub-99/232.

[145] GEANT version 3.21, CERN.

[146] P. Lebrun, Fermilab, private communication.

[147] R. Coleman,T. Kobilarcik,C. Milstene, CKM Note 29, GEANT simulation of RF separated beam; June 2000.

[148] J. Fisher, A. Hrisoho, V. Radeka, and P. Rehak, Nucl. Instr. and Meth. A238 (1985) 249.

[149] Bicron, 12345 Kinsman Rd., Newbury OH 44065.

[150] Hamamatsu Corp., 360 Foothill Rd., Bridgewater, N.J 08807-0910.

[151] B. Baumbaugh et. al., Nucl. Instr. and Meth. A 345 (1994) 271-278.

[152] http://www-d0.fnal.gov/ sgrue/vlpc.

[153] Kuraray International Corp., 30th Floor, PanAm Bldg., 200 Park Avenue, NY 10166. 
[154] A. Bross et. al., Nucl. Instr. and Meth. A 394 (1997) 87-96.

[155] M.P. Maia et al., Nucl.Instrum.Meth.,A326, 496 (1993).

[156] The RICH Detector of the SELEX Experiment, Nuclear Instruments and Methods A433(1999)149-152 Presented Paper from the 3. International Workshop on Ring Imaging Cherenkov Detectors, RICH98, Ein Gedi, Dead Sea, Israel, November 15-20, 1998.Preprint FERMILAB-Conf-98/399-E

[157] A. Bideau-Mehu, PhD Thesis University Brest (France) 1982, unpublished.

[158] J.Koch Über due Dispersion des Lichtes in gasförmigen Körpern innerhalb des ultravioletten Spektrums. Ark. Mat. Astr. Fys. 9(1913) Nr. 6, 1-11.

[159] R. Abjean, A. Bideau-Mehu and Y. Guern, Nucl.Instrum.Meth.A354:417-418,1995, REFRACTIVE INDEX OF HEXAFLUOROETHANE (C-2F-6) IN THE 300-NM TO 150-NM WAVELENGTH RANGE.

[160] T. Inagaki et al., "Measurement of the $K \rightarrow$ pinuñu decay", KEK proposal, (June 1996).

[161] Figure courtesy of Taku Yamanaka; draft in preparation.

[162] Dave Pushka (Fermilab), personal communication. Based on an analysis of published outgassing data.

[163] Dave Pushka (Fermilab), personal communication. Based on an analysis of the KTeV vacuum system design and performance.

[164] P.C. Bergbusch, Ph.D. thesis, University of British Columbia, 2000.

[165] A Study of Photon Detection Inefficiency using real $K^{+} \rightarrow \pi^{+} \pi^{0}$ Data, Takashi Nakano and Yoshi Kuno, E787 Internal Techriical Note.

[166] For an excellent series of reviews of $(\rho, \omega, \phi)$ photoproduction see the proceedings of the International Symposium on Electron-Photon Interactions at High Energy, (1967).

[167] A. M. Boyarski et al., Phys. Rev. Lett. 20, 300 (1968).

[168] For a discussion of the $\mathrm{KTeV}$ BA system see for example the PhD thesis of B. Quinn, University of Chicago (2000).

[169] For a discussion of the KTeV BA system see for example the $\mathrm{PhD}$ thesis of $\mathrm{P}$. Shawhan, University of Chicago (1999).

[170] KTeV internal note and personal communication, R. Kessler, Univeristy of Chicago.

[171.] Greg Graham on the KTeV TRD wires, Private Communication.

[172] Gas Filtration for Straws, Martin R. Marcin, University of Texas, Austin. KL-407. UTKL-016. Sept. 211992.

[173] A Straw Tracking System to Study Rare $K_{L}$ Decays, S. Graessle, et. al., NIM, A367 (1995) 138-142.

[174] David Alan Ambrose, Ph.D. Thesis, University of Texas, Austin (1998). Unpublished. 
[175] Study of Straw Proportional Tubes for a TRT/Tracker at LHC, T. Akesson, et. al., CERNPPE/94-224, December 1994.

[176] Systematic Study of Straw Proportional Tubes for the ATLAS Inner Detector, RD6 Collaboration, ATLAS Internal Note, INDET-No-19, 1993.

[177] Kapton Straw Chambers for a Transition Radiation Detector, V. Bondarenko, et. al., Eagle Internal Note, INDET-No-010, 1992.

[178] Karol Lang, Private Communication.

[179] John Krider on studies of the KTeV TRD window, Private Communication.

[180] DMS reconstruction, Hogan Nguyen, CKM internal memo, CKM Note 40, January24,2001.

[181] Roy Kang Lee, Ph.D. Thesis, University of California at Irvine, (1998). Unpublished.

[182] Roy Kang Lee, Private Communication.

[183] Draft preprint in preparation, courtesy of S. White, BNL.

[184] A scintillator - lead photon calorimeter using optical fiber readout. H. Fessler et al., Nucl.Instrum.Meth. A240, 284 (1985).

Lead scintillator electromagnetic calorimeter with wavelength shifting fiber readout. G.S. Atoyan et al., Nucl.Instrum.Meth. A320, 144 (1992).

[185] Fermilab Technical Memo in preparation.

[186] A vme 32-channel pipeline tdc module with tmc lsis. IEEE Trans.Nucl.Sci. bf431799(1996). Conceptual design of straw tube readout with tmc. By SDC Collaboration (Y. Arai et al.). SDC-91-00063, Aug 1991. 39pp.

[187] Atlas detector at LHC/CERN design report.

[188] The performance of a high speed pipelined photomultiplier readout system in the fermilab KTEV experiment. By Juliana Whitmore (Fermilab). FERMILAB-CONF-97-272, Aug 1997. 6pp. To be published in the proceedings of 7th Pisa Meeting on Advanced Detector: Frontier Detectors for Frontier Physics, La Biodola, Isola d'Elba, Italy, 25-31 May 1997.

[189] W. Grover, "A New Method for Clock Distribution", IEEE Transactions on Circuits and Systems-I 41, 149, (1994)

[190] Review of Particle Physics, M.Baldo Ceolin et al., The European Physics Journal C 15 1$\overline{4,(2000)}$, T.G.Tripp,pp $\overline{505}$.

CKM Note 39, P.S. Cooper, January $15,2001, K_{e 3}$ Matrix Element.

[191] Provided by S. Kettell (Brookhaven), personal communication.

[192] D.S. Barton et al.,Phys.Rev.D27:2580,1983

[193] "Switchyard in the Main Injector Era Conceptual Design Report" C. Brown, T. Kobilarcik, P. Lucas, A. Malensek, C.T. Murphy, M.J.Yang, FERMILAB-TM-2014, August 1997

[194] Herman White's presentation- January 1998 Fermilab PAC meeting. 
[195] A. Schi: et al, Phys.Rev. D21,3010,(1980).

[196] Computer Physics Communications, North-Holland Volume 71 (1992) P. 173 